\title{
SHYSTER: A Pragmatic Legal Expert System
}

JAMES DAVID POPPLE

A thesis submitted for the degree of Doctor of Philosophy of

The Australian National University

April 1993 
(C) James Popple 1993

Doonesbury (page 131) copyright G. B. Trudeau Reprinted with permission of Universal Press Syndicate All rights reserved

Earlier versions of some parts of this thesis have appeared in:

Proceedings of the Thirteenth Australian Computer Science Conference

(C) Australian Computer Science Association 1990

Advances in Computing and Information: Proceedings of the International Conference on Computing and Information

(C) International Conference on Computing and Information 1990

The Australian Computer Journal

(c) Australian Computer Society Inc. 1991

National Library of Australia Cataloguing-in-Publication entry

Popple, James David, 1964-

SHYSTER: a pragmatic legal expert system.

Bibliography.

Includes indexes.

ISBN 0731518276.

1. SHYSTER (Computer file). 2. Law - MethodologyData processing. 3. Expert systems (Computer science). I. Australian National University. Faculty of Engineering and Information Technology. Dept of Computer Science. II. Title. 
Except where otherwise indicated, this thesis is my own original work.

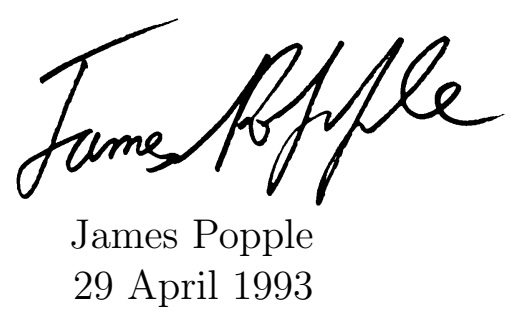



shy.ster \'shīstə(r) \n-s [prob. after Scheuster fl1840 Am. attorney frequently rebuked in a New York court for pettifoggery] : one who is professionally unscrupulous esp. in the practice of law or politics ...

Webster's Third New International Dictionary $(1961)^{1}$

shyster ('farstə(r)) ... [Of obscure origin.

It might be f. SHY $a$. (sense 7, disreputable) + -ster; but this sense of the adj. is app. not current in the U.S.]

... 'A lawyer who practises in an unprofessional or tricky manner; especially, one who haunts the prisons and lower courts to prey on petty criminals; hence, any one who conducts his business in a tricky manner' (Funk's Stand. Dict. 1895). Also attrib. or adj. Orig. and chiefly U.S. slang...

The Oxford English Dictionary $(1989)^{2}$

shyster. An unscrupulous lawyer (note that the definition presumes the existence of scrupulous ones) ...

The term does not come from - as suggested in various dictionaries - the surname Scheuster, supposedly a lawyer noted for shyster-like practices; from the name of the Shakespearean character, Shylock; ... or from any of the various meanings of shy (e.g., to be shy of money). Rather ... shyster evolved from the underworld use of shiser, a worthless fellow, which derived in turn from the German scheisse, excrement, via scheisser, an incompetent person (specifically, one who cannot control his bodily functions) ...

A Dictionary of Invective $(1991)^{3}$ 



\section{Abstract}

Most legal expert systems attempt to implement complex models of legal reasoning. But the utility of a legal expert system lies not in the extent to which it simulates a lawyer's approach to a legal problem, but in the quality of its predictions and of its arguments. A complex model of legal reasoning is not necessary: a successful legal expert system can be based upon a simplified model of legal reasoning.

Some researchers have based their systems upon a jurisprudential approach to the law, yet lawyers are patently able to operate without any jurisprudential insight. A useful legal expert system should be capable of producing advice similar to that which one might get from a lawyer, so it should operate at the same pragmatic level of abstraction as does a lawyer - not at the more philosophical level of jurisprudence.

A legal expert system called SHYSTER has been developed to demonstrate that a useful legal expert system can be based upon a pragmatic approach to the law. SHYSTER has a simple representation structure which simplifies the problem of knowledge acquisition. Yet this structure is complex enough for SHYSTER to produce useful advice.

SHYSTER is a case-based legal expert system (although it has been designed so that it can be linked with a rule-based system to form a hybrid legal expert system). Its advice is based upon an examination of, and an argument about, the similarities and differences between cases. SHYSTER attempts to model the way in which lawyers argue with cases, but it does not attempt to model the way in which lawyers decide which cases to use in those arguments. Instead, it employs statistical techniques to quantify the similarity between cases. It decides which cases to use in argument, and what prediction it will make, on the basis of that similarity measure. 
SHYSTER is of a general design: it can provide advice in areas of case law that have been specified by a legal expert using a specification language. Hence, it can operate in different legal domains. Four different, and disparate, areas of law have been specified for SHYSTER, and its operation has been tested in each of those domains.

Testing of SHYSTER in these four domains indicates that it is exceptionally good at predicting results, and fairly good at choosing cases with which to construct its arguments. SHYSTER demonstrates the viability of a pragmatic approach to legal expert system design. 


\section{Acknowledgements}

Thanks ...

TO the members of my supervisory panel-Robin Stanton, Roger Clarke, Peter Drahos and Malcolm Newey: for their counsel and encouragement.

To Robin Creyke and Phillipa Weeks: for giving so freely of their time and legal expertise.

TO Peter Bailey, David Cullen, Kate Lazenby-Cohen and Kevin Popple: for their comments on various parts of various drafts.

To Graham Jefferson, Chris Johnson, Brendan McKay, Gavin Michael, Bob Moles, Neil Tennant and Trevor Vickers: for their suggestions and advice.

To Sally Begg, Jeannie Haxell, Bev Johnstone and Jacquie Wilson: for administrative assistance.

TO David Hawking and the programming staff: for technical assistance.

TO Richard Walker and Markus Zellner: for TEXnical assistance.

To Zdzisław Łoboz, Rachel Oñate, Dharmendra Sharma, Wang Xiaoji and Yang Jian: for sharing, with me, different offices at different times.

TO Diane Hutchens: for all her help during my time with PARSA.

TO my parents-Marli and Kevin: for their unfailing love and support.

AND TO Paula Fearn: for whom, but for whom ...

This research was supported by an Australian National University PhD Scholarship, funded by the Centre for Information Science Research. Additional financial support was provided by Sigma Data Corporation. 



\section{Contents}

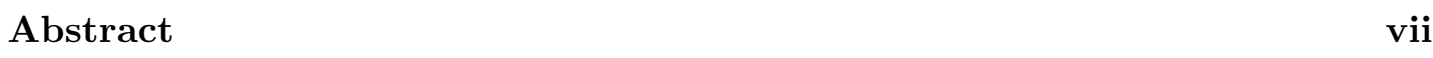

$\begin{array}{ll}\text { Acknowledgements } & \text { ix }\end{array}$

Figures $\quad$ xxi

Chapter 1: Introduction 3

1.1 The aims of this thesis $\ldots \ldots \ldots \ldots \ldots \ldots \ldots \ldots \ldots \ldots \ldots \ldots \ldots$

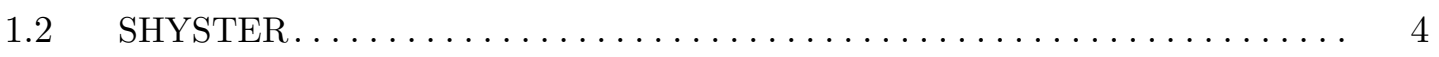

1.3 The structure of this thesis $\ldots \ldots \ldots \ldots \ldots \ldots \ldots \ldots \ldots \ldots \ldots \ldots \ldots \ldots \ldots \ldots$

$\begin{array}{ll}\text { Chapter 2: Legal analysis systems } & 7\end{array}$

$2.1 \quad$ Introduction $\ldots \ldots \ldots \ldots \ldots \ldots \ldots \ldots \ldots \ldots \ldots \ldots \ldots \ldots$

2.1.1 Sources of law ........................... 9

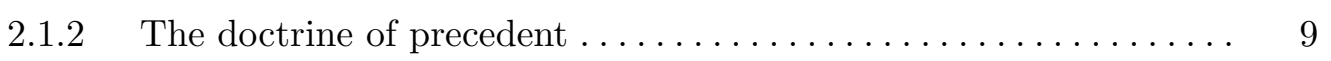

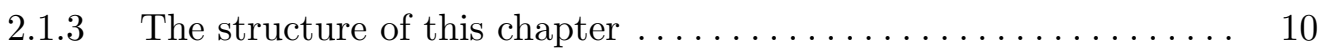

2.1 .4 Terminology ......................... 11

2.2 Jurisprudence $\ldots \ldots \ldots \ldots \ldots \ldots \ldots \ldots \ldots \ldots \ldots \ldots \ldots \ldots$

2.2.1 The importance of jurisprudence . . . . . . . . . . . . . . 11

2.2.2 Scientific and mechanical jurisprudence . . . . . . . . . . . . . 13

2.2 .3 Judgment machines $\ldots \ldots \ldots \ldots \ldots \ldots \ldots \ldots \ldots \ldots \ldots \ldots \ldots$

2.2 .4 Petrifaction of the law ...................... 16

$2.2 .5 \quad$ Clear rules and clear cases $\ldots \ldots \ldots \ldots \ldots \ldots \ldots \ldots \ldots \ldots$

2.2.6 Legal realism and rule scepticism $\ldots \ldots \ldots \ldots \ldots \ldots \ldots \ldots$

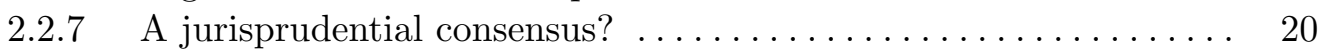

$2.3 \quad$ Jurimetrics and the behaviourists . . . . . . . . . . . . . . . . . 23

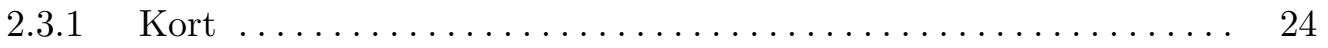

$2.3 .2 \quad$ Lawlor............................ 24 


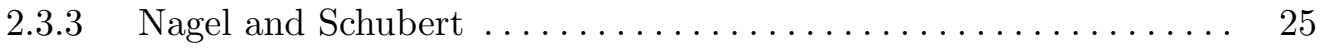

2.3.4 Haar, Sawyer and Cummings ..................... 25

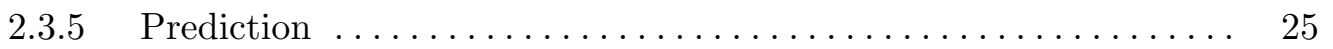

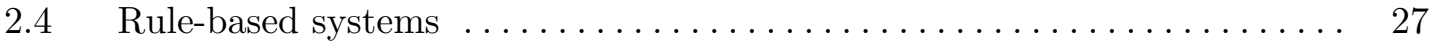

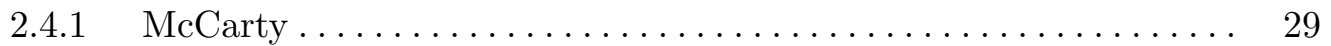

2.4.2 Bench-Capon, Kowalski and Sergot................... 32

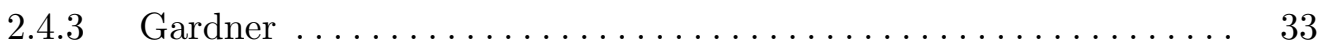

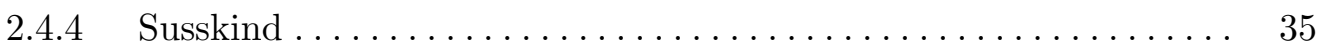

2.4.5 Knowledge acquisition and representation $\ldots \ldots \ldots \ldots \ldots \ldots \ldots$

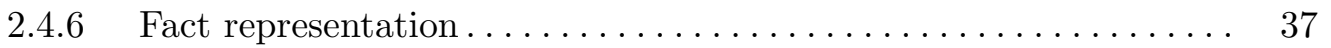

2.4.7 The inadequacy of rules for case law $\ldots \ldots \ldots \ldots \ldots \ldots \ldots \ldots$

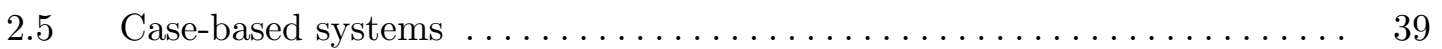

2.5.1 Nearest neighbour analysis . . . . . . . . . . . . . . 41

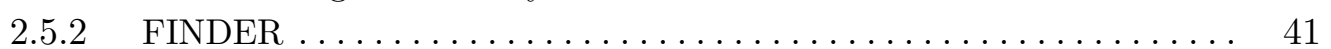

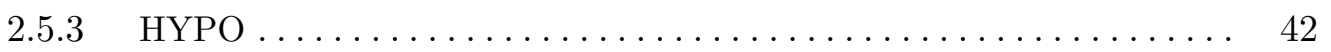

2.5.4 The inadequacy of semantic networks ............... 44

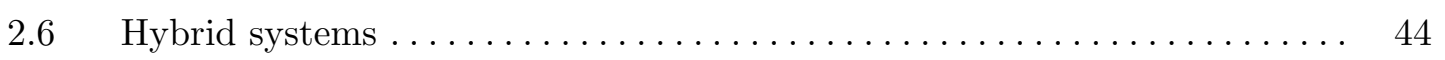

2.7 Conceptual models: deep and shallow $\ldots \ldots \ldots \ldots \ldots \ldots \ldots \ldots \ldots .46$

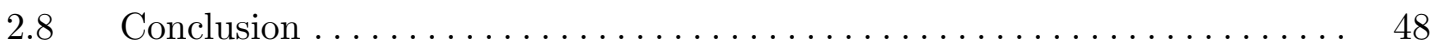

Chapter 3: A pragmatic approach to case law 51

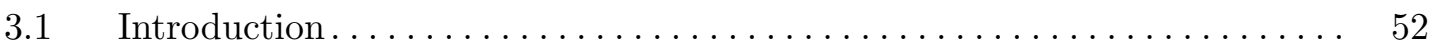

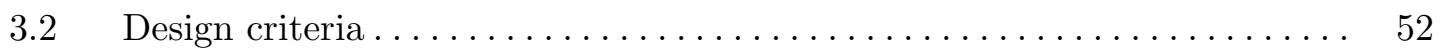

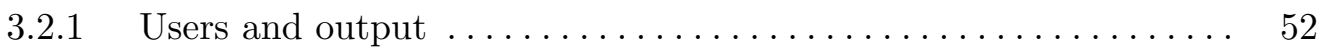

$3.2 .2 \quad$ A pragmatic model of legal reasoning . . . . . . . . . . . . 53

$3.2 .3 \quad$ Knowledge representation . . . . . . . . . . . . . . . . . 53

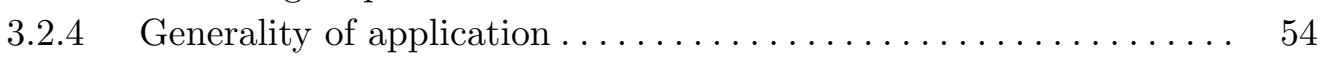

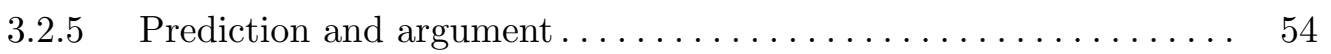

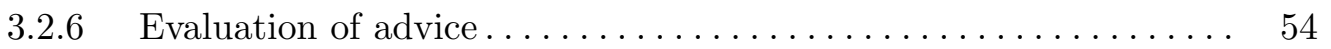

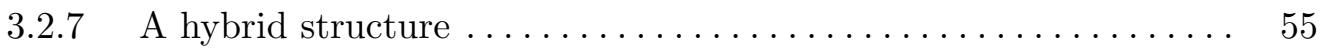

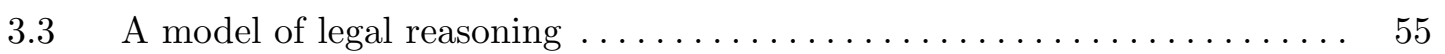

3.3.1 Private and public law ....................... 55

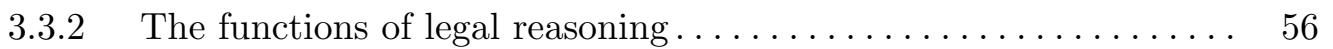

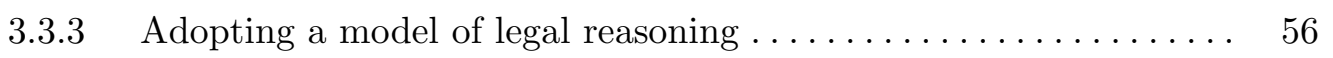

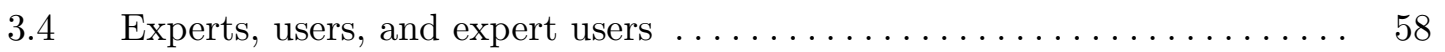

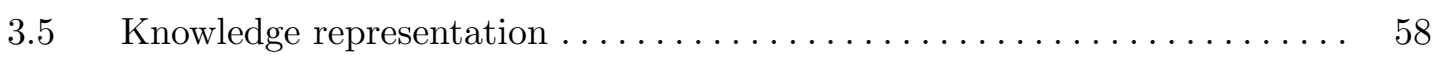

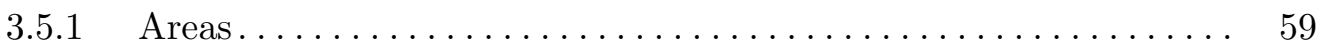

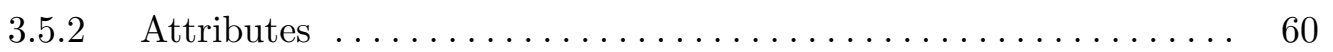

3.5.3 Leading cases and attribute values $\ldots \ldots \ldots \ldots \ldots \ldots \ldots \ldots$

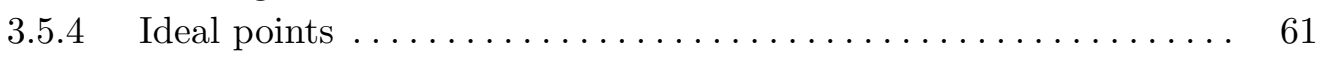

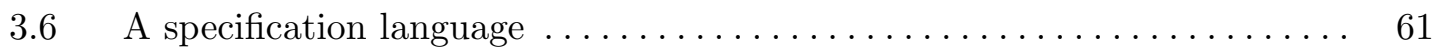




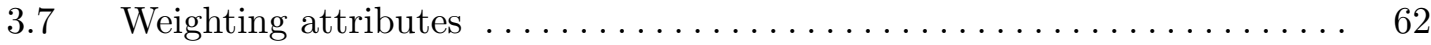

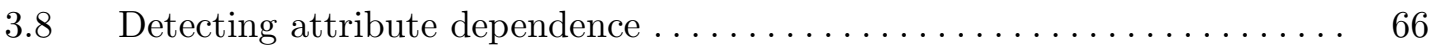

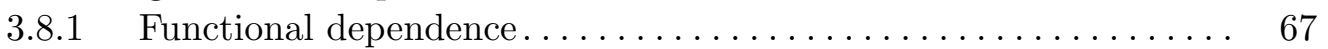

$3.8 .2 \quad$ Stochastic dependence . . . . . . . . . . . . . . . . . . . 67

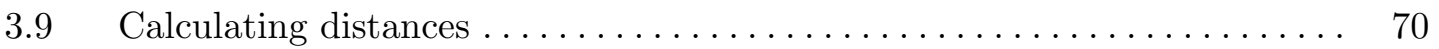

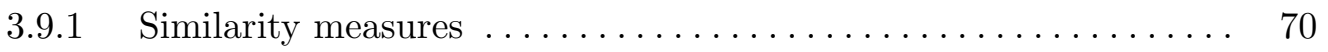

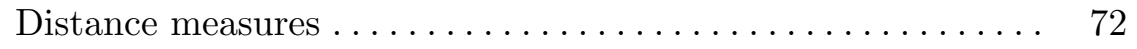

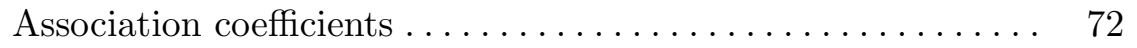

Correlation coefficients ................... 74

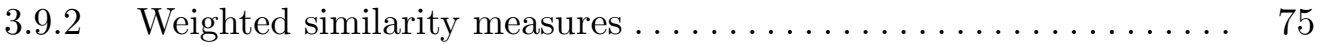

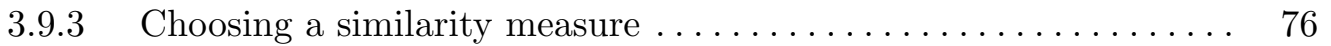

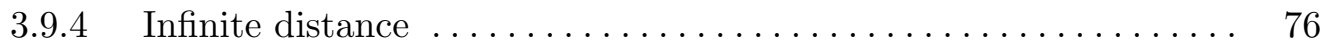

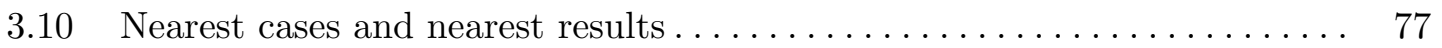

3.10 .1 Nearest cases . . . . . . . . . . . . . . . . . . . . . . 77

3.10 .2 Nearest results. . . . . . . . . . . . . . . . . . . . . 77

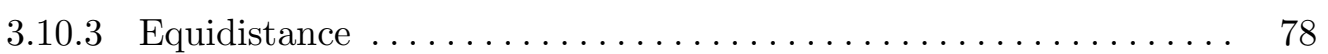

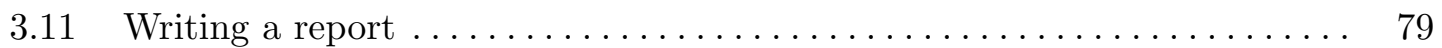

3.11.1 Arguing with the instant case $\ldots \ldots \ldots \ldots \ldots \ldots \ldots \ldots \ldots . \ldots \ldots$

Choosing cases ......................... 80

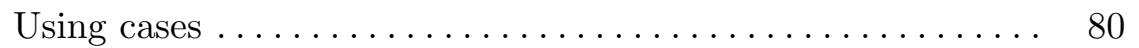

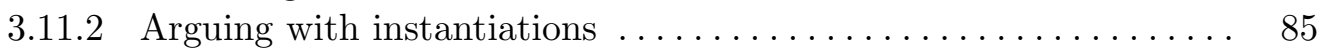

3.11.3 Arguing with hypotheticals ..................... 86

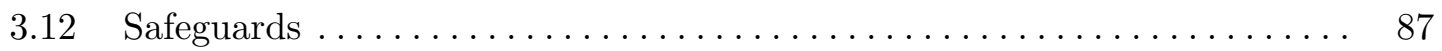

3.12 .1 Extra similarity measures $\ldots \ldots \ldots \ldots \ldots \ldots \ldots \ldots \ldots \ldots \ldots . \ldots \ldots$

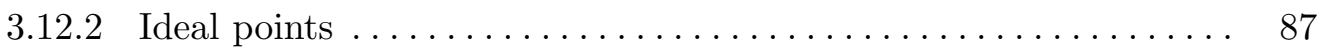

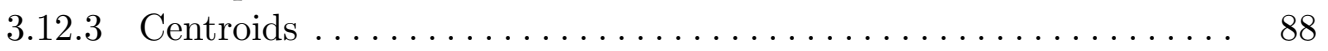

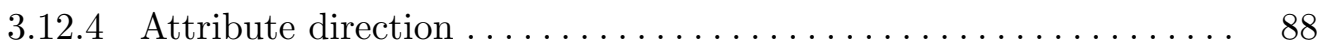

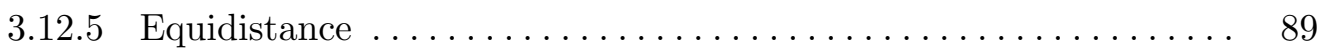

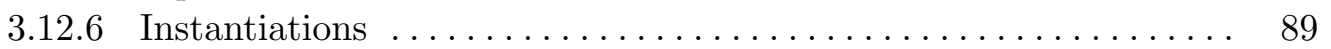

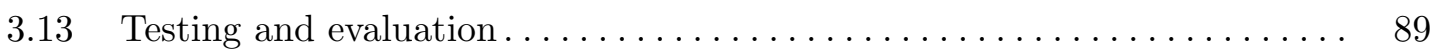

3.13 .1 Choosing a test domain . . . . . . . . . . . . . . . . . 90

3.13 .2 Evaluating SHYSTER's opinion $\ldots \ldots \ldots \ldots \ldots \ldots \ldots \ldots \ldots \ldots$

3.13 .3 The paucity of test cases . . . . . . . . . . . . . . . 92

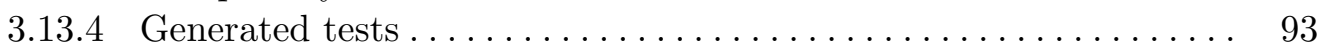

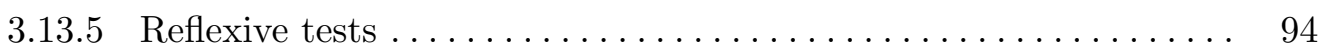

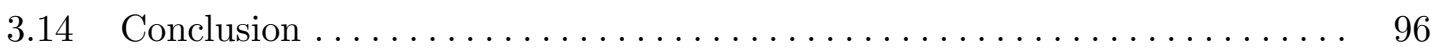

3.14.1 Comparisons with other approaches $\ldots \ldots \ldots \ldots \ldots \ldots \ldots \ldots . \ldots 9$

$3.14 .2 \quad$ A Sisyphean journey? . . . . . . . . . . . . . . . . . . . 99

$\begin{array}{ll}\text { Chapter 4: Implementing SHYSTER } & 103\end{array}$

$4.1 \quad$ Introduction . . . . . . . . . . . . . . . . . . . . . . . . . . . . . . 104

4.1.1 Internal representation. . . . . . . . . . . . . . . . . . . . 104

4.1 .2 Output files . . . . . . . . . . . . . . . . . . . . . 105

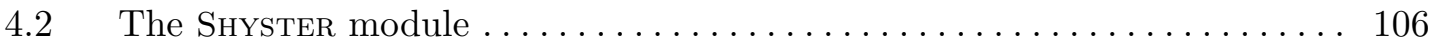

4.3 The Statutes module . . . . . . . . . . . . . . . . . . . . . . . . 106 


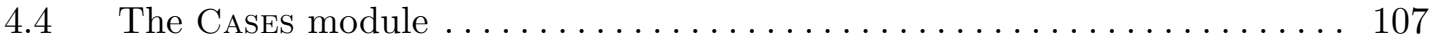

4.5 The Tokenizer module . . . . . . . . . . . . . . . . . . . . . . . . . 108

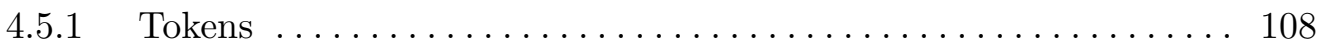

4.5.2 Comments and whitespace ...................... 108

4.6 The PARser module . . . . . . . . . . . . . . . . . . . . . . . . . . . . . . . . . . . . . . . . 109

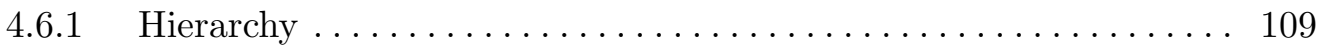

$4.6 .2 \quad$ Areas.................................. 111

4.6.3 Attributes ................................ 112

Local attributes .......................... 112

External attributes ....................... 112

4.6.4 Leading cases . . . . . . . . . . . . . . . . . . . . . . . 113

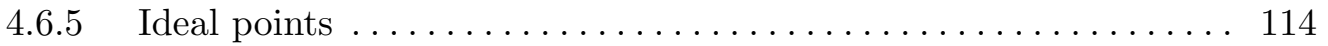

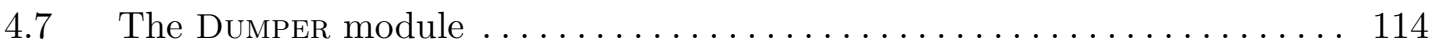

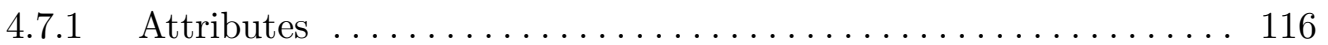

Local attributes .......................... 116

External attributes $\ldots \ldots \ldots \ldots \ldots \ldots \ldots \ldots \ldots \ldots \ldots$

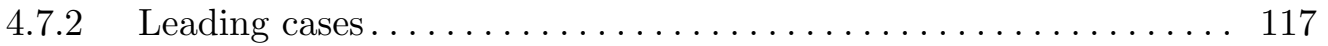

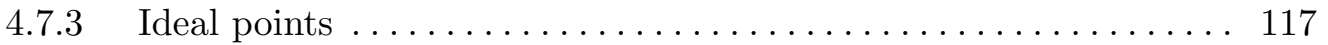

4.8 The Checker module . . . . . . . . . . . . . . . . . . . . . . . . . . . . . 117

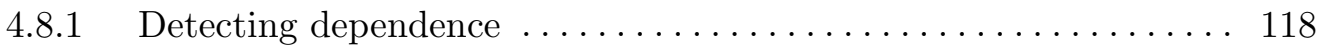

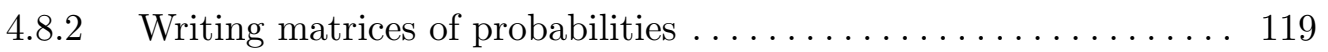

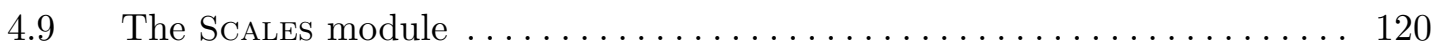

4.9.1 Calculating weights . . . . . . . . . . . . . . . . . . . . 120

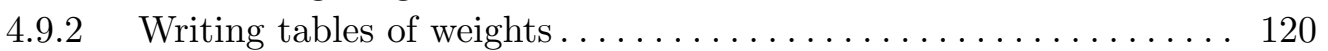

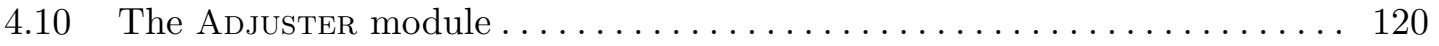

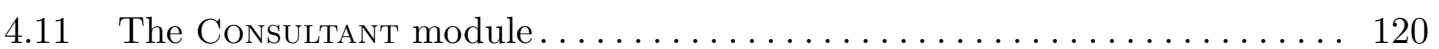

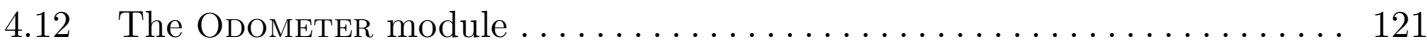

4.12 .1 Calculating distances . . . . . . . . . . . . . . . . . . . . . 122

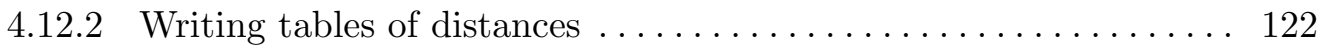

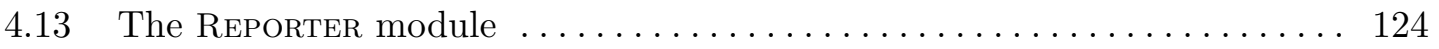

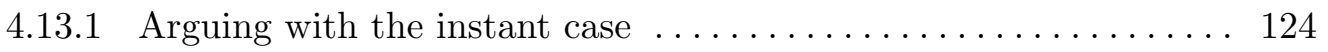

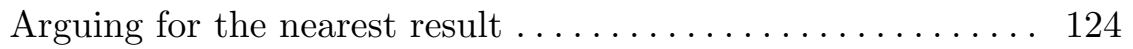

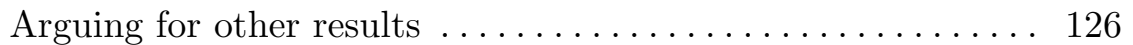

Variations . . . . . . . . . . . . . . . . . . . . . . . 127

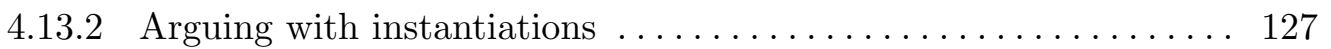

4.13.3 Arguing with hypotheticals . . . . . . . . . . . . . . . . . . . . . 127

4.13 .4 Safeguards . . . . . . . . . . . . . . . . . . . . . . . . 128

4.14 Conclusion ....................................... 130

$\begin{array}{ll}\text { Chapter 5: Case studies } & 131\end{array}$

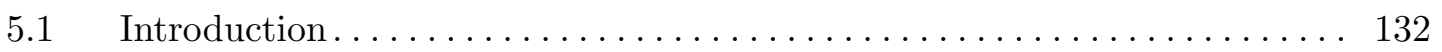

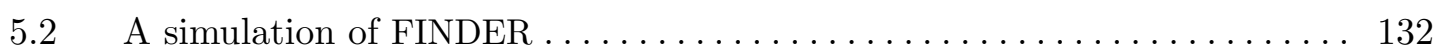

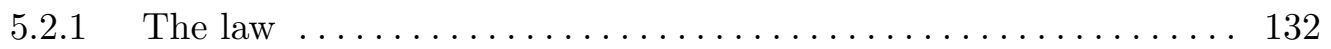


5.2 .2 The FIndER specification $\ldots \ldots \ldots \ldots \ldots \ldots \ldots \ldots \ldots \ldots \ldots \ldots$

Results............................. 135

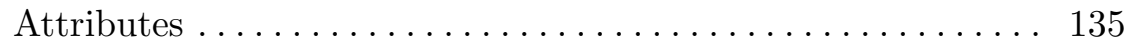

Leading cases . . . . . . . . . . . . . . . . . . 136

Attribute dependence ... . . . . . . . . . . . . . . . . 136

Weights . . . . . . . . . . . . . . . . . . . . 137

$5.2 .3 \quad$ Test case . . . . . . . . . . . . . . . . . . . . 138

Parker v. British Airways ................... 138

5.2 .4 Conclusion ........................... 140

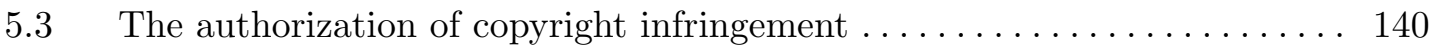

5.3 .1 The law ............................ 140

5.3 .2 The Authorization specification .................... 144

Results............................. 144

Attributes ............................... 144

Leading cases . . . . . . . . . . . . . . . . . . 146

Attribute dependence ... . . . . . . . . . . . . . . 147

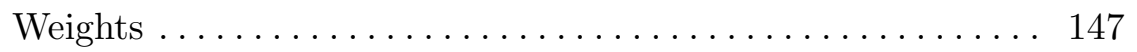

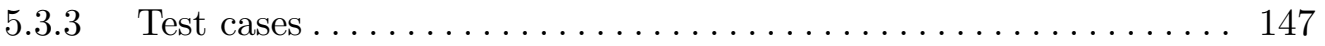

CBS Inc. v. Ames Records and Tapes Ltd. . . . . . . . . . . . 148

WEA International Inc. v. Hanimex Corporation Ltd . . . . . 149

CBS Songs Ltd v. Amstrad Consumer Electronics plc . . . . . 151

Australasian Performing Right Association Ltd v. Jain . . . . . 152

Hypothetical case $1 \ldots \ldots$. . . . . . . . . . . . . . . . . . 152

Hypothetical case $2 \ldots \ldots \ldots \ldots \ldots \ldots \ldots$

5.3 .4 Generated tests . . . . . . . . . . . . . . . . . . . . . . . 154

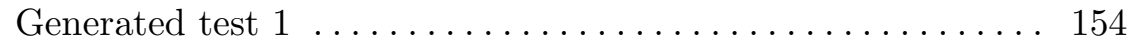

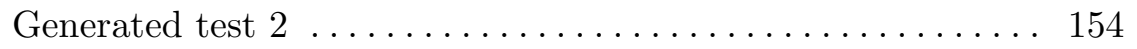

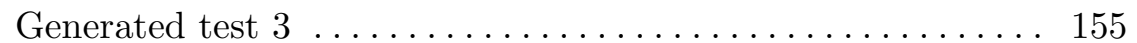

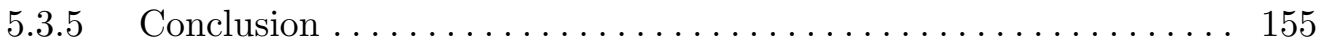

$5.4 \quad$ Employees and independent contractors . . . . . . . . . . . . . . . 155

5.4 .1 The law ............................ 156

5.4 .2 The Employe specification $\ldots \ldots \ldots \ldots \ldots \ldots \ldots \ldots \ldots \ldots \ldots$

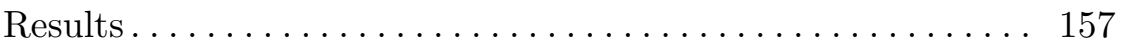

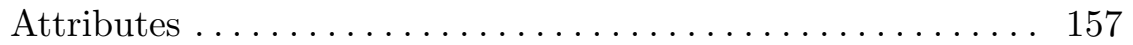

Leading cases . . . . . . . . . . . . . . . . . . . . 159

Attribute dependence . . . . . . . . . . . . . . . . . . . . . . 159

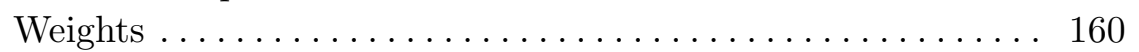

$5.4 .3 \quad$ Test cases . . . . . . . . . . . . . . . . . . . . . . . . 160

Narich Pty Ltd v. Commissioner of Pay-roll Tax . . . . . . . . 160

Stevens v. Brodribb Sawmilling Company Pty Ltd.......... 163

Re Porter; Re Transport Workers Union of Australia ....... 163

Building Workers' Industrial Union of Australia v. Odco

Pty Ltd . . . . . . . . . . . . . . . . . . . . . . . . . . . . . 164

Hypothetical case $3 \ldots \ldots \ldots \ldots \ldots \ldots$. . . . . . . . . . . . . . . . . . . . . . . . . . . . . 165

Hypothetical case $4 \ldots \ldots \ldots \ldots$. . . . . . . . . . . 165 
5.4 .4 Generated tests . . . . . . . . . . . . . . . . . . 166

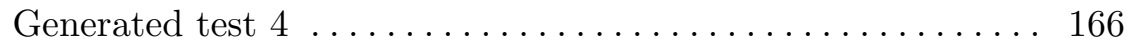

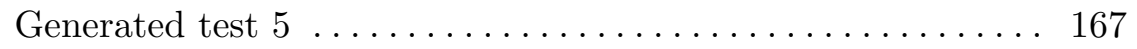

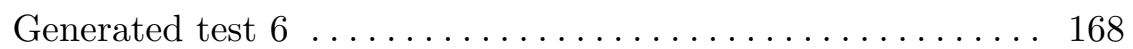

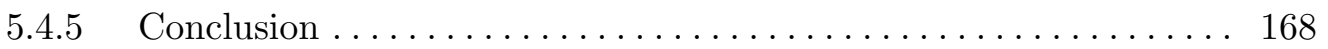

$5.5 \quad$ The implication of natural justice $\ldots \ldots \ldots \ldots \ldots \ldots \ldots \ldots \ldots \ldots \ldots \ldots$

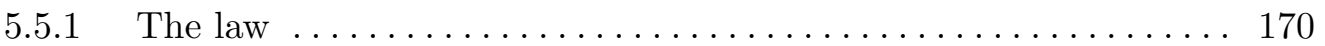

5.5 .2 The Natural specification $\ldots \ldots \ldots \ldots \ldots \ldots \ldots \ldots \ldots \ldots \ldots \ldots$

Natural area .......................... 173

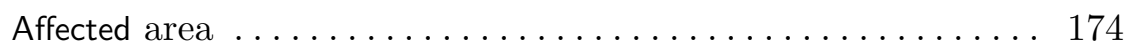

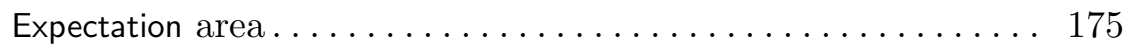

Leading cases ......................... 176

Attribute dependence ...................... 176

Weights .................................. 178

$5.5 .3 \quad$ Test cases . . . . . . . . . . . . . . . . . . . . . . . . . . . . . . 179

Twist v. Council of Municipality of Randwick .......... 179

Salemi v. MacKellar ......................... 182

Heatley v. Tasmanian Racing and Gaming Commission ..... 183

Cole v. Cunningham .......................... 183

Ackroyd v. Whitehouse (Director of National Parks 86

Wildlife Service) ............................. 184

Hodgens v. Gunn ......................... 185

Western Australia v. Bropho ..................... 186

Ainsworth v. Criminal Justice Commission ............ 187

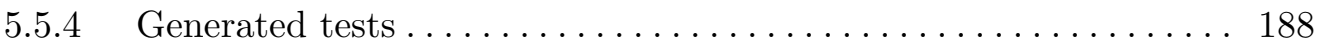

Generated test $7 \ldots \ldots \ldots \ldots \ldots \ldots \ldots \ldots \ldots \ldots \ldots$

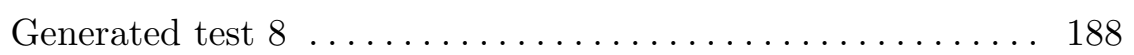

5.5 .5 Conclusion ............................. 189

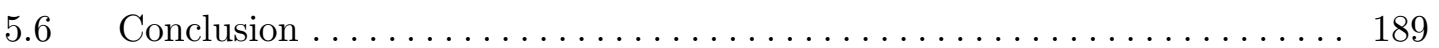

$5.6 .1 \quad$ Test cases . . . . . . . . . . . . . . . . . . . . . . . . . . . . . . . 190

5.6 .2 Generated tests . . . . . . . . . . . . . . . . . . . . . . . . 192

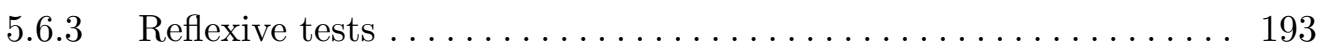

$\begin{array}{ll}\text { Chapter 6: Conclusion } & 195\end{array}$

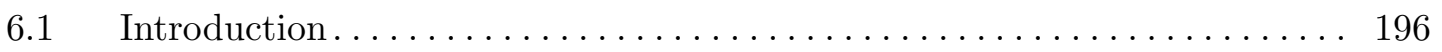

6.2 Evaluating SHYSTER . . . . . . . . . . . . . . . . . . . . 196

$6.2 .1 \quad$ A useful, working system...................... 196

$6.2 .2 \quad$ A general approach $\ldots \ldots \ldots \ldots \ldots \ldots \ldots \ldots \ldots \ldots \ldots \ldots \ldots \ldots \ldots$

6.2 .3 Good advice............................. 198

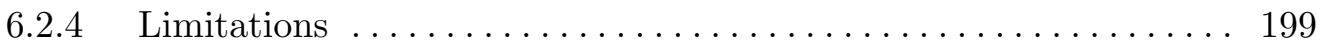

6.2.5 Possible enhancements . . . . . . . . . . . . . . . . . . . . . . . 200

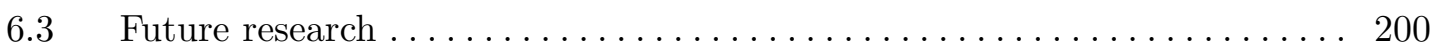

6.4 The contribution of this thesis . . . . . . . . . . . . . . . . . . . 202 


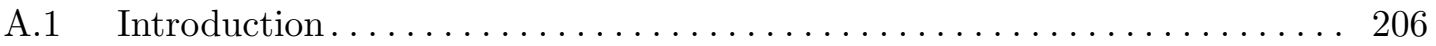

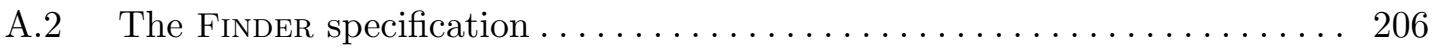

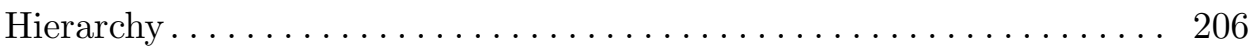

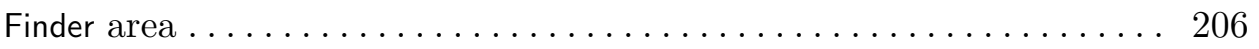

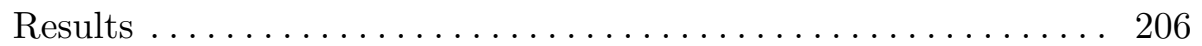

Attributes ............................... 207

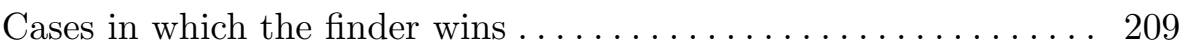

Cases in which the finder loses . . . . . . . . . . . . . . 211

A.3 The Authorization specification ...................... 216

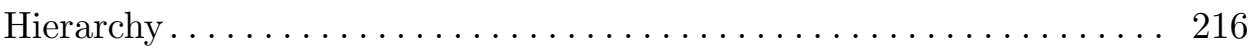

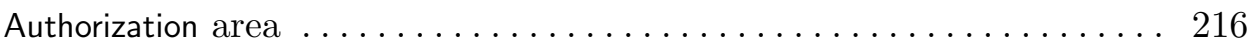

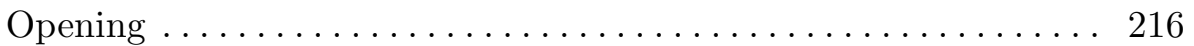

Results .................................. 217

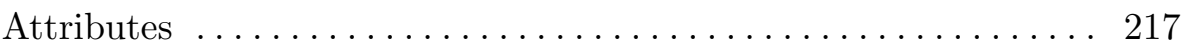

Cases in which the accused authorized the infringement ....... 219

Cases in which the accused did not authorize the infringement . . 222

Cases in which the accused is liable (directly or vicariously) for the infringement ....................... 225

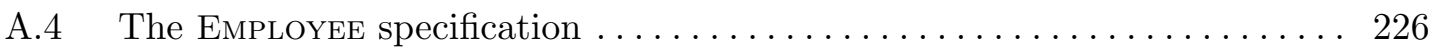

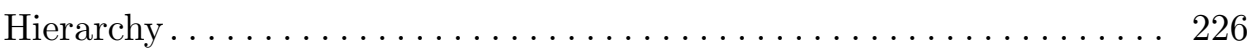

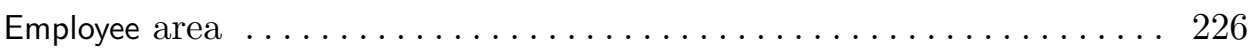

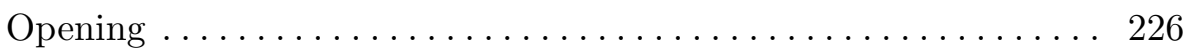

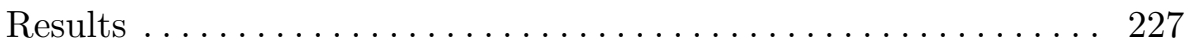

Attributes .............................. 227

Cases in which the worker is an employee ............. 231

Cases in which the worker is an independent contractor ........ 240

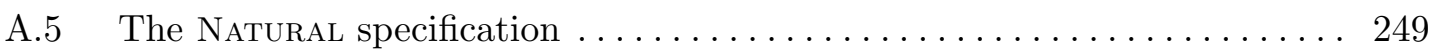

Hierarchy .................................. 249

Natural area ................................ 249

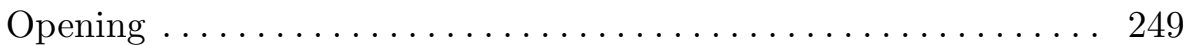

Results ................................... 250

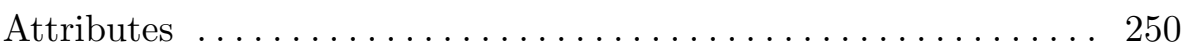

Cases in which a duty to observe natural justice is implied . . . . 252

Cases in which a duty to observe natural justice is not implied. . 259

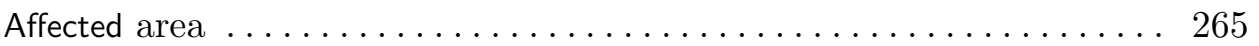

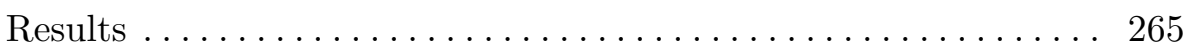

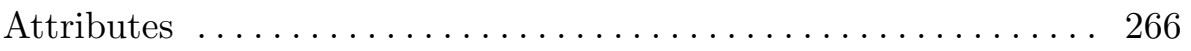

Cases in which the decision affected the property, right, interest, status, or legitimate expectation of the applicant ..... 267

Cases in which the decision did not affect the property, right, interest, status, or legitimate expectation of the applicant 273 


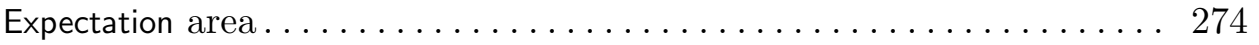

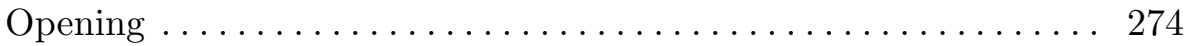

Results .......................... 274

Attributes .............................. 275

Cases in which the applicant had a legitimate expectation which was affected by the decision . . . . . . . . . 276

Cases in which the applicant did not have a legitimate expectation which was affected by the decision . . . . . . . 280

Appendix B: Example reports $\quad 283$

B.1 Introduction. . . . . . . . . . . . . . . . . . . . . . . . . . . . . . . . . . 284

B.2 Report file for Parker v. British Airways ..................... 284

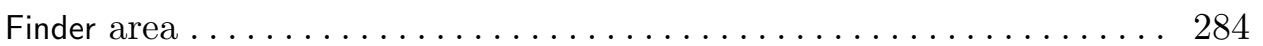

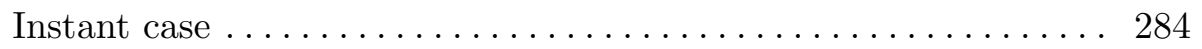

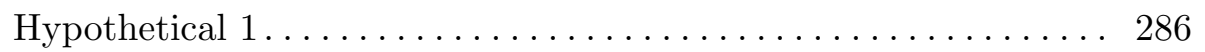

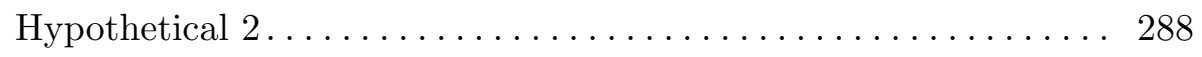

B.3 Report file for APRA v. Jain . . . . . . . . . . . . . . . . . . . . . 289

Authorization area ............................. 289

Instant case . . . . . . . . . . . . . . . . . . . . . . . 289

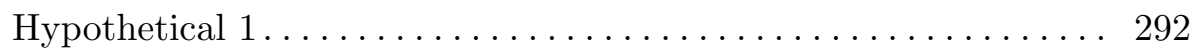

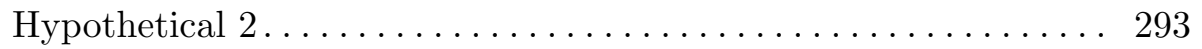

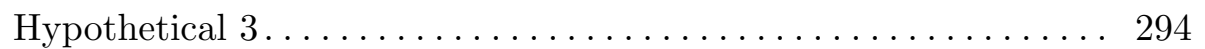

B.4 Report file for Re Porter; Re $T W U \ldots \ldots \ldots \ldots \ldots \ldots \ldots \ldots \ldots \ldots \ldots . \ldots . \ldots . \ldots 296$

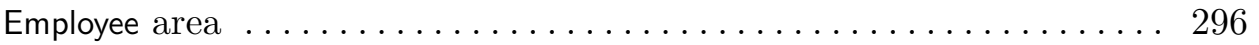

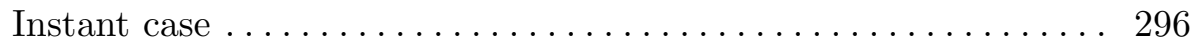

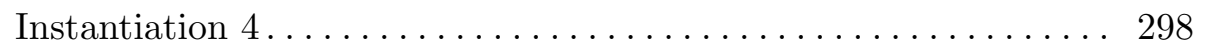

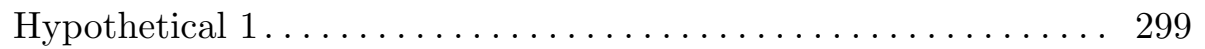

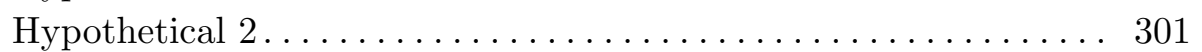

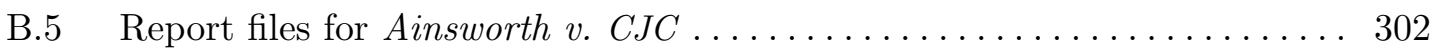

Expectation area ................................ 302

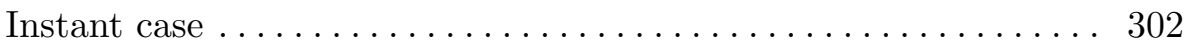

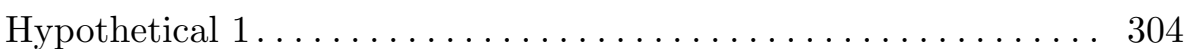

Affected area .................................. 304

Instant case ... . . . . . . . . . . . . . . . . . . . . . . . . . 304

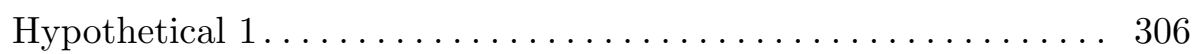

Natural area . . . . . . . . . . . . . . . . . . . . . 307

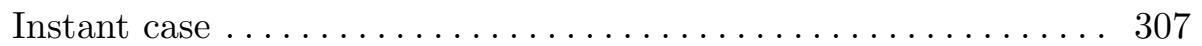

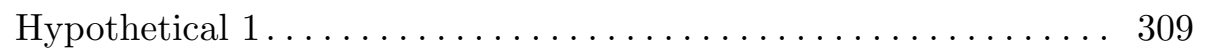

$\begin{array}{ll}\text { Appendix C: A complete example } & 311\end{array}$

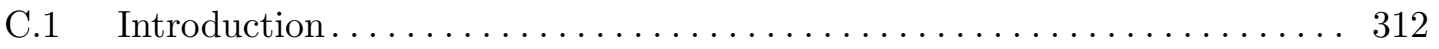

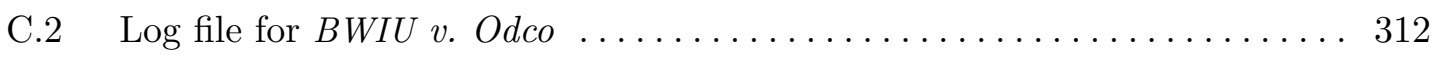

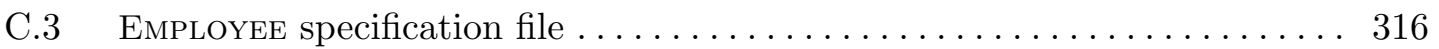


C.4 Dump file for Employee specification ( $\mathrm{LAT}_{\mathrm{E}} \mathrm{X}$ input) $\ldots \ldots \ldots \ldots \ldots \ldots 328$

C.5 Dump file for Employee specification ( $\mathrm{LAT}_{\mathrm{E}} \mathrm{X}$ output) [see $\left.\S \mathrm{A} .4\right] \ldots \ldots \ldots 331$

C.6 Probabilities file for Employee specification ( $\mathrm{LAT}_{\mathrm{E}} \mathrm{X}$ input) $\ldots \ldots \ldots \ldots \ldots . \ldots 332$

C.7 Probabilities file for EmployeE specification ( $\mathrm{LAT}_{\mathrm{E}} \mathrm{X}$ output) $\ldots \ldots \ldots \ldots \ldots 333$

Employee area ................................ 333

C.8 Weights file for EmploYeE specification ( $\mathrm{LAT}_{\mathrm{E}} \mathrm{X}$ input) $\ldots \ldots \ldots \ldots \ldots \ldots$

C.9 Weights file for EmployeE specification ( $\mathrm{LAT}_{\mathrm{E}} \mathrm{X}$ output) $\ldots \ldots \ldots \ldots \ldots \ldots$

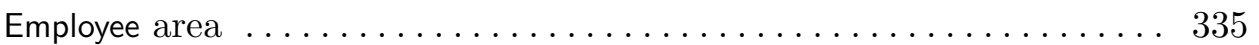

C.10 Distances file for $B W I U$ v. Odco ( $\mathrm{LAT}_{\mathrm{E}} \mathrm{X}$ input) .................. 335

C.11 Distances file for BWIU v. Odco ( $\mathrm{LAT}_{\mathrm{E}} \mathrm{X}$ output) $\ldots \ldots \ldots \ldots \ldots \ldots \ldots 336$

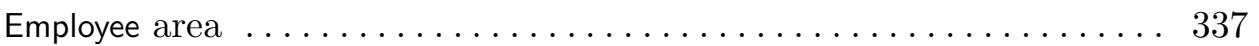

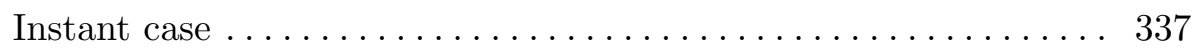

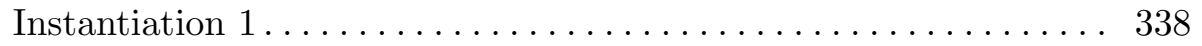

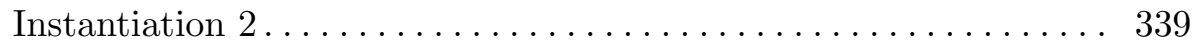

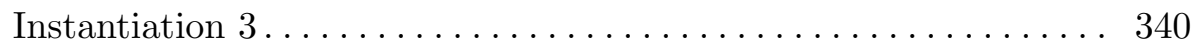

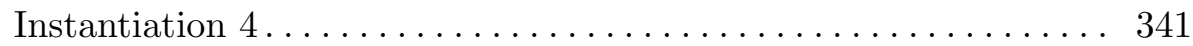

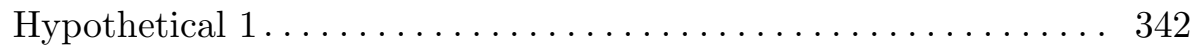

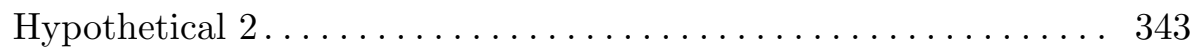

C.12 Report file for $B W I U$ v. Odco ( $\mathrm{ET}_{\mathrm{E}} \mathrm{X}$ input) $\ldots \ldots \ldots \ldots \ldots \ldots \ldots \ldots \ldots \ldots$

C.13 Report file for $B W I U$ v. Odco ( $\mathrm{ET}_{\mathrm{E}} \mathrm{X}$ output) $\ldots \ldots \ldots \ldots \ldots \ldots \ldots \ldots$

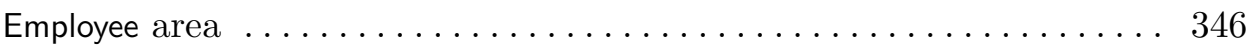

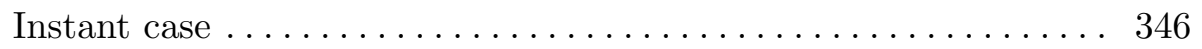

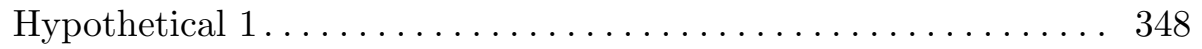

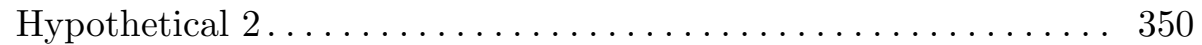

Appendix D: Reflexive tests 353

D.1 Introduction. . . . . . . . . . . . . . . . . . . . . . . . . . . . 354

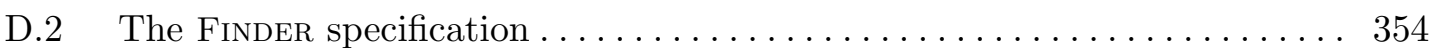

Hannah v. Peel .................................. 354

Bridges v. Hawkesworth ................................ 354

Armory v. Delamirie .............................. 355

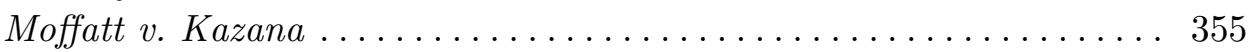

City of London Corporation v. Appleyard (1)................. 355

City of London Corporation v. Appleyard (2)................. 356

South Staffordshire Water Co. v. Sharman................... 356

Elwes v. Brigg Gas Co........................... 356

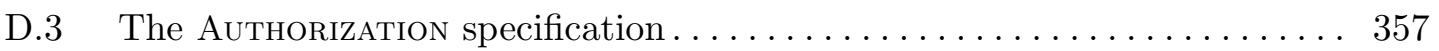

University of New South Wales v. Moorhouse ................ 357

Australasian Performing Right Association Ltd v. Canterbury-

Bankstown League Club Ltd ........................ 357

Winstone v. Wurlitzer Automatic Phonograph Co. of Australia

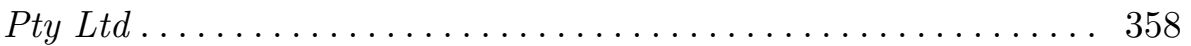

Mellor v. Australian Broadcasting Commission .............. 358 
Falcon v. Famous Players Film Co....................... 358

RCA Corporation v. John Fairfax and Sons Ltd ............... 359

Performing Right Society Ltd v. Ciryl Theatrical Syndicate Ltd ..... . 359

A $\mathscr{E} M$ Records Inc. v. Audio Magnetics Inc. (UK) Ltd ............. 359

Australasian Performing Right Association Ltd v. Miles ........... 360

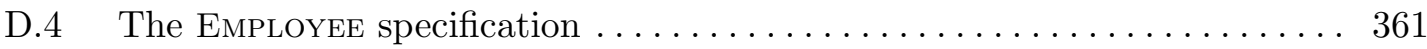

Zuijs v. Wirth Brothers Pty Ltd ..................... 361

Cam and Sons Pty Ltd $v$. Sargent ..................... 361

Federal Commissioner of Taxation v. J. Walter Thompson

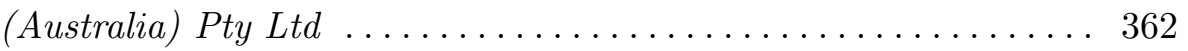

Australian Timber Workers Union v. Monaro Sawmills Pty Ltd ..... 362

Ferguson v. John Dawson \& Partners (Contractors) Ltd .......... 362

Stevenson Jordon and Harrison Ltd v. Macdonald and Evans (2) .... 363

Performing Right Society Ltd v. Mitchell \& Booker (Palais de

Danse) Ltd .............................. 363

Humberstone $v$. Northern Timber Mills................... 363

Queensland Stations Pty Ltd v. Federal Commissioner of Taxation... 364

Price v. Grant Industries Pty Ltd ........................ 364

Australian Mutual Provident Society v. Chaplin ................ 364

Massey v. Crown Life Insurance Co. ........................ 364

Stevenson Jordon and Harrison Ltd v. Macdonald and Evans (1) . . . 365

Ready Mixed Concrete (South East) Ltd v. Minister of Pensions

and National Insurance .......................... 366

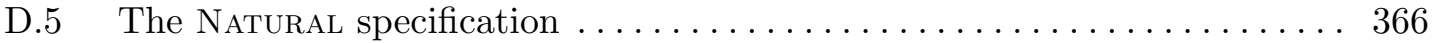

FAI Insurances Ltd $v$. Winneke ...................... 366

Haoucher v. Minister of State for Immigration and Ethnic Affairs . . 366

Annetts v. McCann ............................... 367

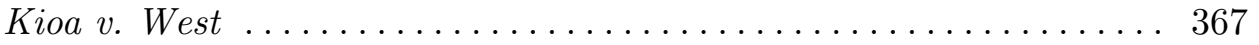

Commissioner of Police v. Tanos ........................ 367

Marine Hull \& Liability Insurance Co. Ltd v. Hurford . . . . . . . . . . 367

Macrae v. Attorney-General for New South Wales .............. 368

Attorney-General of Hong Kong v. Ng Yuen Shiu............... 368

Durayappah v. Fernando .......................... 368

South Australia v. O'Shea .............................. 369

Bread Manufacturers of New South Wales v. Evans .............. 369

Minister for Arts Heritage and Environment v. Peko-Wallsend Ltd . 369

Nashua Australia Pty Ltd v. Channon .................... 370

Council of Civil Service Unions v. Minister for the Civil Service..... 370

McInnes v. Onslow Fane ......................... 370

$\begin{array}{ll}\text { Notes } & 375\end{array}$

$\begin{array}{lr}\text { Cases } & 403\end{array}$

$\begin{array}{ll}\text { Statutes } & 409\end{array}$

$\begin{array}{ll}\text { Bibliography } & 411\end{array}$ 


\section{Figures}

Figures marked with a star are extracted from SHYSTER output.

$3.1 \quad$ Forms of functional dependence $\ldots \ldots \ldots \ldots \ldots \ldots \ldots \ldots \ldots \ldots \ldots \ldots \ldots$

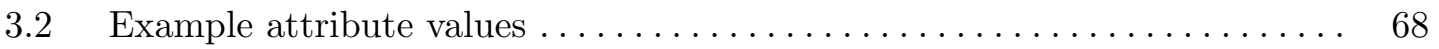

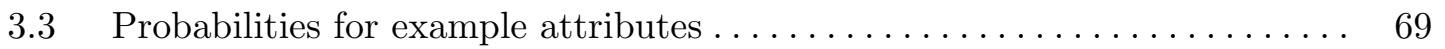

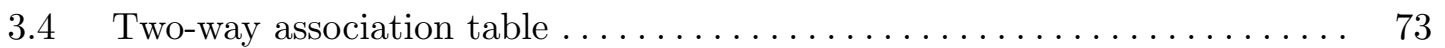

$3.5 \quad$ Example distances . . . . . . . . . . . . . . . . . . . . . . 78

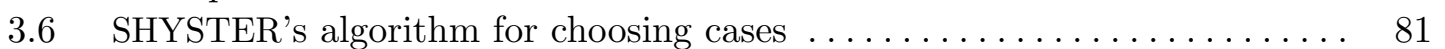

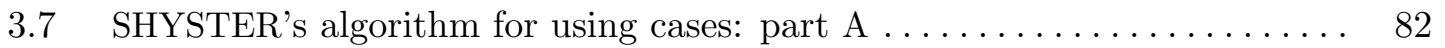

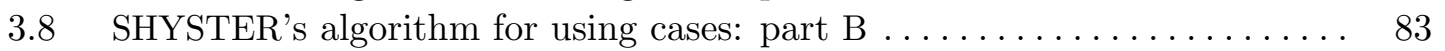

3.9 SHYSTER's algorithm for using cases: part C $\ldots \ldots \ldots \ldots \ldots \ldots \ldots . \quad 84$

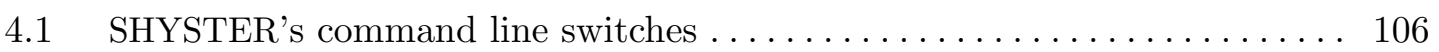

4.2 Keywords in SHYSTER's case law specification language............. 108

4.3 EBNF description of SHYSTER's case law specification language . . . . . . 110

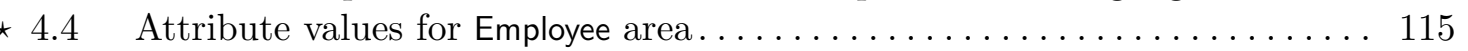

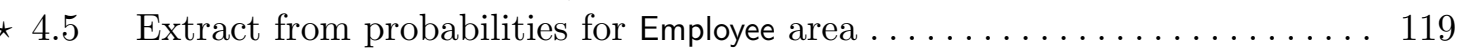

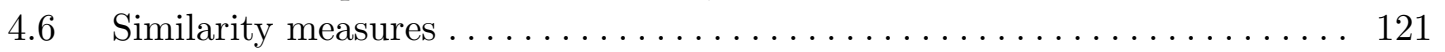

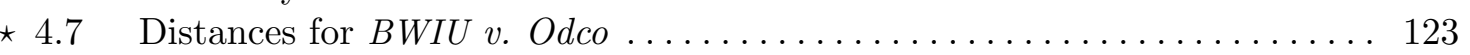

$\star 4.8$ Extract from $\log$ file for $B W I U$ v. Odco ......................... 129

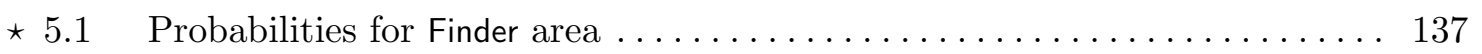

$\star 5.2 \quad$ Weights for Finder area . . . . . . . . . . . . . . . . . . . . . . 138

$\star 5.3$ Distances for Parker v. British Airways ....................... 141

$\star 5.4$ Probabilities for Authorization area ...................... 146

$\star 5.5$ Weights for Authorization area ........................... 147

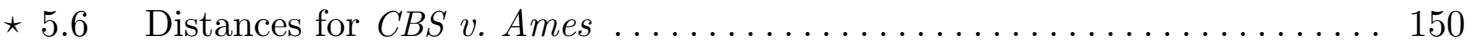

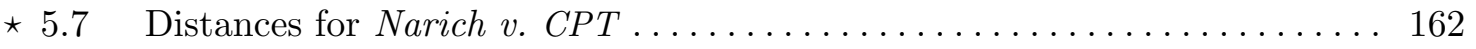

5.8 Relationship between areas in NaturaL specification . . . . . . . . . . . 173

$\star 5.9$ Probabilities for Natural specification $\ldots \ldots \ldots \ldots \ldots \ldots \ldots \ldots \ldots \ldots \ldots \ldots$ 
$\star 5.10$ Weights for Natural specification . . . . . . . . . . . . . . . . . . 178

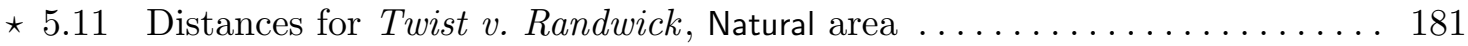

5.12 Summary of test cases . . . . . . . . . . . . . . . . . . . . . . . 191

5.13 Summary of generated testing ............................. 193

D.1 Summary of reflexive testing of FindER specification $\ldots \ldots \ldots \ldots \ldots \ldots 356$

D.2 Summary of reflexive testing of Authorization specification . . . . . . . . 360

D.3 Summary of reflexive testing of EMPLOYEe specification ............. 365

D.4 Summary of reflexive testing of Natural specification . . . . . . . . . . . 371 
Chapters 



\section{Introduction}

Dicke the Butcher: "The first thing we do, let's kill all the Lawyers."

William Shakespeare (1591)

Henry VI, Part $2^{4}$

Attending a Cabinet when there was a tendency to Mutiny in the Fleet, Sir Thomas Troubridge, ... who was a most excellent Officer, was asked his opinion what was best to be done. He said let me hang a hundred Lawyers, and we shall hear no more of the business. I asked what he could mean - what were these People that he called Lawyers. He replied, Fellows that can read and write. They are the Fellows, that I call Lawyers, and make the whole of the Mischief.

Lord Eldon (1827) Lord Eldon's Anecdote Book ${ }^{5}$

To his colleagues, [he] was a lonely and solitary nut. To an objective outsider he might have seemed a bold crusader, waging war against the forces of darkness. The truth was somewhere in between. He was in the early stages of a $\mathrm{PhD}$ thesis.

Alan Plater (1985)

The Beiderbecke Affair 6 


\subsection{The aims of this thesis}

The history of the development of legal expert systems has, for the most part, been characterized by the development and implementation of complex models of legal reasoning.

This thesis aims to show that a legal expert system need not be based upon a complex model of legal reasoning in order to produce useful advice. It advocates a pragmatic approach to legal expert system design based on the way in which lawyers deal with the law on a day-to-day basis.

It argues that a system based upon a simple model of legal reasoning can still produce good advice, where that advice is evaluated by reference to the accuracy of its predictions and to the quality of its arguments. Furthermore, such a system, with its simpler knowledge representation structure, makes commensurately simpler the process of knowledge acquisition.

These arguments are made theoretically, and then by example. A legal expert system which is based on a pragmatic approach to the law has been developed by the author. The development and testing of that system, called SHYSTER, is described in this thesis.

\subsection{SHYSTER}

SHYSTER was developed to demonstrate that a useful, working legal expert system could be based upon a pragmatic approach to the law. SHYSTER is of a general design, so that it can operate in different legal domains. It was designed to provide advice in areas of case law that have been specified by a legal expert using a specially developed specification language.

SHYSTER is a case-based legal expert system. Its knowledge of the law is acquired, and represented, as information about cases. It produces its advice by examining, and arguing about, the similarities and differences between cases. By contrast, a rule-based expert system represents the law using rules. A hybrid system uses both rule-based and case-based techniques. SHYSTER has been designed so that it can be linked with a rule-based system to form a hybrid legal expert system.

Although SHYSTER attempts to model the way in which lawyers argue with cases, it does not attempt to model the way in which lawyers decide which cases to use in those arguments. It uses statistical techniques to quantify the similarity between cases, and chooses cases on the basis of that similarity measure.

SHYSTER's representation structure was designed so as to be as simple as possible while complex enough to allow SHYSTER to produce good advice. This simple structure greatly simplifies the process of knowledge acquisition. 


\subsection{The structure of this thesis}

The body of this thesis is divided into six chapters:

- Chapter 2 discusses previous work of relevance to the development of legal analysis systems, especially legal expert systems. The value of jurisprudence to legal expert system development is also discussed, and the adoption of a pragmatic approach (as opposed to a jurisprudentially pure approach) is recommended. This approach holds that legal expert systems should operate at the same level of abstraction at which lawyers operate on a day-to-day basis.

- In chapter 3, a pragmatic approach is proposed for developing legal expert systems. It incorporates a simple model of legal reasoning, and uses a simple knowledge representation structure. Comparisons are made between this approach, and approaches adopted by other legal expert system developers. This approach is adopted for the development of SHYSTER. Specific design criteria for SHYSTER are detailed, and methods of testing and evaluating the system are discussed.

- The implementation of SHYSTER is explained in chapter 4. The twelve modules that comprise SHYSTER are described, and demonstrated using examples.

- Four different specifications have been written for SHYSTER, and these are used as the basis of case studies in chapter 5. Each specification represents a different area of case law. Several different methods are employed to test SHYSTER and these specifications.

- In chapter 6, conclusions are drawn about SHYSTER and its approach to case law. Some enhancements to SHYSTER are suggested, and avenues of future research are identified. Finally, the nature of the contribution made by this thesis is discussed.

There are four appendices to this thesis. Appendix A contains each of the four specifications used to test SHYSTER in chapter 5. Six example reports, demonstrating the use of each of these four specifications, are given in appendix B. Each report is SHYSTER's opinion on one of the test cases used in chapter 5. A complete example of SHYSTER's input and output files for another of those test cases is given in appendix C. Appendix D gives details of one of the methods of testing, as applied to each of the four specifications.

The thesis concludes with the endnotes to the chapters and appendices, a table of cases, a table of statutes, and a list of bibliographical references. 



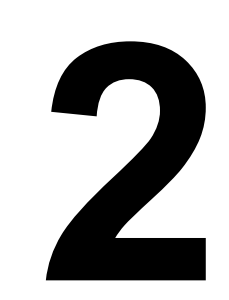

\section{Legal analysis systems}

In the yahoo world of computer public relations, it is often the loudest mouths which lead the unsuspecting computer user into that lonely canyon of empty pockets and broken promises. It now seems ... that parts of the academic world are fast approaching that decibel level so far achieved only by computer salesmen. Thus ... it seems that space can only be booked on the band-wagon if one is prepared to make more outrageous claims than the next man. And such claims are being made for the application of AI to the law.

Philip Leith $(1986)^{7}$

The computer scientists, encouraged by the modern positivists, fail to recognize ... that law, positive morality and ethics are inseparably connected parts of a vast organic whole. Judgments are involved at every stage of the legal process and machines cannot make judgments. In stating that legal rules can be applied without further judgment; that they apply in an all or nothing fashion; that legal decision making follows the form of the syllogism or that it is a patternmatching routine, the modern positivists, joined now by the computer scientists take us along a dangerous road.

Robert N. Moles (1987)

Definition and Rule in Legal Theory:

A Reassessment of H. L. A. Hart and the Positivist Tradition ${ }^{8}$

Aussi, lorsqu'un homme se rend plus absolu, songe-t-il d'abord à simplifier les lois. On commence, dans cet État, à être plus frappé des inconvénients particuliers, que de la liberté des sujets dont on ne se soucie point du tout.
Thus when a man takes on absolute power, he first thinks of simplifying the law. In such a state one begins to be more affected by technicalities than by the freedom of the people, about which one no longer cares at all.

Montesquieu (1748) De l'Esprit des lois 9 


\section{$2.1 \quad$ Introduction}

The range of computer applications in the law is wide. It extends from general applications, of use to lawyers, to applications designed specifically for the law. This thesis is concerned only with a subset of those systems that make use of artificial intelligence (AI) techniques to solve legal problems.

Legal AI systems can usefully be divided into two categories: legal retrieval systems and legal analysis systems. ${ }^{10}$ Legal retrieval systems allow lawyers to search through databases containing details of statutes and decided cases. AI techniques may be employed to simplify this task: e.g. by searching for keywords which have not been input by the user but are deduced to be equivalent to, or sufficiently related to, the input keywords. ${ }^{11}$ Legal analysis systems take information about a set of facts and determine the ramifications of those facts in a given area of law.

Mehl claims that there is no fundamental difference between these two categories - that the difference is one of degree only. ${ }^{12}$ Shannon and Golshani suggest that the difference between systems based on a "conceptual model of legal analysis" and text retrieval systems is that the latter do not "understand" any area of the law. ${ }^{13}$ Similarly, Susskind says that "knowledge-based" systems, as opposed to "database" systems are capable of "applying their knowledge of the law to the problem data presented to them." 14

A better distinction - one which avoids the vexed question of whether any AI system can be said to really know or understand anything - can be made by reference to the output of the system. The output from a legal analysis system is such that, if it had been produced by a human, that human would be said to have legal expertise. By contrast, the output from a legal retrieval system could be produced by a human possessed of no legal expertise; such output is used in, and is not the product of, legal analysis. ${ }^{15}$

This thesis is concerned only with legal analysis systems.

Legal analysis systems can be divided into two categories: judgment machines and legal expert systems.

A judgment machine is a machine designed to replace a human judge. Such machines were first proposed over forty years ago, though no such proposals have been made in the last decade. Writings on judgment machines are discussed in $\S 2.2 .3$ because they are of historical interest, and because the idea of a judgment machine raises some issues which are also relevant to the second category of legal analysis systems: legal expert systems.

A legal expert system, as the term is used in this thesis, is a system capable of performing at a level expected of a lawyer. AI systems which merely assist a lawyer in coming to legal conclusions or preparing legal argument are not here considered to be legal expert systems; a legal expert system must exhibit 
some legal expertise itself. This definition does not exclude systems that can only be used by legal experts; several systems - including SHYSTER - have been developed for exclusive use by lawyers. ${ }^{16}$

\subsubsection{Sources of law}

The law in so-called "common law countries" - e.g. Australia, Britain, Canada, USA - is derived from legislation and from case law.

Legislation, also referred to as statute law, consists of statutes and delegated legislation. In Australia, statutes are made by Federal Parliament, or a State Parliament, and enacted when given royal assent by the Governor-General, or a State Governor. Delegated legislation (rules, regulations, ordinances, by-laws, etc.) is made by a person or body to whom legislative power has been delegated by statute.

Case law, or the common law, is judge-made law: judicial resolutions of specific disputes. The sources of case law are the published reports of the cases as heard before various courts.

Legislation takes precedence over case law; ${ }^{17}$ parliaments can override judgemade law by legislative enactment. However, judges have the task of determining the meaning of legislation.

A legal analysis system that seeks to deal with the law in a common law country must account for both statute law and case law.

\subsubsection{The doctrine of precedent}

The development of case law is based upon the principle of stare decisis which holds that courts should apply the doctrine of precedent. Morris et al. summarize the general rules of the doctrine of precedent as follows:

- each court is bound by decisions of courts higher in its hierarchy;

- a decision of a court in a different hierarchy may be of considerable weight, but will not be binding;

- only the ratio decidendi of a case is binding;

- any relevant decisions, although not binding, may be considered and followed; and

- precedents are not necessarily abrogated by lapse of time. ${ }^{18}$

(The ratio decidendi of a case is, literally, the reason for deciding; rationes are "pronouncements of legal principle necessary for the judge's decision on the established facts of the case". ${ }^{19}$ They are different from pronouncements of legal principle which may be illustrative or clarifying, but are not strictly relevant to the case in issue. These are called obiter dicta, and are not binding on other courts.) 
Courts have a tendency to follow similar cases, even when not strictly bound to do so. According to Cross:

It is a basic principle of the administration of justice that like cases should be decided alike. This is enough to account for the fact that, in almost every jurisdiction, a judge tends to decide a case in the same way as that in which a similar case has been decided by another judge. ${ }^{20}$

There is considerable argument about the extent to which judges actually apply the doctrine of precedent. ${ }^{21}$ Some legal theorists contend that the doctrine is simply part of the public discourse that judges use to justify their decisions. Stone claims that "the degree of certainty and stability in the law secured by the doctrine of stare decisis is far less than it appears to be," and that one of the important social functions of the doctrine is "to maintain at a maximum the feeling and appearance of certainty and stability." ${ }^{22}$ Yet, judges of the highest court in Australia have been known to follow previous cases which they believed to be wrongly decided. ${ }^{23}$

What is clear is that judges use previously decided cases to justify their decisions - if not to reach them - and that lawyers use previously decided cases in legal argument. Any legal analysis system which deals with case law assumes the application of the doctrine of precedent, at least to this extent.

\subsubsection{The structure of this chapter}

This chapter reviews previous work of relevance to the design of legal expert systems. Jurisprudence and its value to the development of such systems is discussed (§2.2) and the field of "jurimetrics" and some behaviouristic research is examined ( $\S 2.3)$. The bulk of this chapter concerns the development of legal expert systems. This is discussed under three headings: rule-based, case-based, and hybrid systems.

Rule-based systems are examined first (§2.4), and special attention is paid to the work of four projects $(\S 2.4 .1-\S 2.4 .4)$. The problems of knowledge acquisition and representation, and fact representation are also discussed (§2.4.5 and §2.4.6). Rule-based systems have been used to represent statutes and cases. However, as explained in $\S 2.4 .7$, rule-based systems are fundamentally inadequate for representing case law.

Case-based systems are examined $(\S 2.5)$ and the work of three projects is focused upon $(\S 2.5 .1-\S 2.5 .3)$. Systems that use rules in order to represent case law are considered to be rule-based systems; only systems which adopt case-based reasoning methods are considered to be case-based. Applying this distinction is usually straightforward, though not always. ${ }^{24}$ Attempts have been made to use semantic networks to represent case law but, as explained in $\S 2.5 .4$, they are not suited to the task.

Hybrid systems (§2.6) employ both rule-based and case-based methods. 
The search for conceptual models of legal reasoning is discussed (§2.7). Finally, conclusions are drawn from the literature as to the best approach to the design of legal expert systems $(\S 2.8)$.

(The development of legal expert systems raises legal questions itself. For example, who - if anyone - is liable for "bad" advice provided by such a system? These questions are beyond the scope of this thesis, but are examined elsewhere. ${ }^{25}$ )

\subsubsection{Terminology}

For consistency, the following three terms are used wherever possible in this thesis for a variety of terms that are used throughout the literature. An attribute is a legally important fact; some use the words "descriptor," "dimension," "factor," "feature" or "variable." The instant case is the situation about which the expert system is interrogated: this is sometimes called the "actual case," the "current fact situation," the "hypothetical case" or the "new case." Leading cases make up the case base of a legal expert system; some call these the "reference set" or the "training set."

\subsection{Jurisprudence}

For centuries, lawyers and philosophers have written on, and argued about, the nature of human laws. This field is called jurisprudence. It would seem sensible for the developer of a legal expert system to have regard to jurisprudential theory before designing her/his system. However, as Susskind complained in 1987, most of the published research on various legal expert systems makes no use of jurisprudential resources. ${ }^{26}$

\subsubsection{The importance of jurisprudence}

Some expert system researchers have been doubtful as to the value of jurisprudence; Niblett claims that "a successful expert system is likely to contribute more to jurisprudence than the other way round". ${ }^{27}$ Susskind disagrees:

It is beyond argument ... that all expert systems must conform to some jurisprudential theory because all expert systems in law necessarily make assumptions about the nature of law and legal reasoning. To be more specific, all expert systems must embody theories of legal knowledge, legal science, the structure of rules, the individuation of laws, legal systems and sub-systems, legal reasoning, and of logic and the law (as well perhaps as elements of a semantic theory, a sociology, and a psychology of law), theories that must all themselves rest on more basic philosophical foundations. If this is so, it would seem prudent that the general theory of law implicit in expert systems should be explicitly articulated using (where appropriate) 
the relevant works of seasoned theoreticians of law. Perhaps the reason that there is, as yet, no overwhelmingly successful system is that the vast corpus of apposite jurisprudential material has not yet been tapped in the construction process. ${ }^{28}$

Although it is true that all legal expert systems necessarily make assumptions about the nature of law and legal reasoning, it does not follow that they must conform to some jurisprudential theory. A lawyer must have a model of the law (maybe unarticulated) which includes assumptions about the nature of law and legal reasoning, but that model need not rest on basic philosophical foundations. It may be a pragmatic model, developed through experience within the legal system. Many lawyers perform their work with little or no jurisprudential knowledge, ${ }^{29}$ and there is no evidence to suggest that they are worse, or better, at their jobs than lawyers well-versed in jurisprudence.

Susskind concedes that it is possible to build a legal expert system "without jurisprudential insight", but suggests that such a system would be of very poor quality:

Because successful legal knowledge engineering presupposes so profound a familiarity with the nature of law and legal reasoning, it is scarcely imaginable that such a mastery could be gained other than through immersion in jurisprudence. ${ }^{30}$

Harris, himself a jurisprudent, takes a different view:

People acquire those technical skills of legal reasoning and legal argumentation which make up the concept of 'good lawyer' by immersing themselves in substantive legal subjects. Jurisprudence has to do, not with the lawyer's role as a technician, but with any need he may feel to give a good account of his life's work - either to fellow citizens, or to himself, or to any gods there be. ${ }^{31}$

If Harris is right - and the existence of good (though jurisprudentially illiterate) lawyers suggests that he is - then the importance of jurisprudence to legal expert system design is questionable. A legal expert system need only operate at the same level of abstraction as does a lawyer, rather than at the philosophical level of a jurisprudent. The fact that many lawyers have mastered the process of legal reasoning, without having been immersed in jurisprudence, suggests that it may indeed be possible to develop legal expert systems of good quality without jurisprudential insight.

This does not mean that legal expert systems designers should completely ignore jurisprudential literature. However, as a legal expert system need not conform to any jurisprudential theory, a pragmatic approach to expert system design may be preferable to a jurisprudentially pure one. Susskind, as Niblett complains, "gives the role of jurisprudence in the design of legal expert systems a greater significance than it deserves." 32 
It is well beyond the scope of this thesis to cover "the vast corpus of apposite jurisprudential material", ${ }^{33}$ however several areas of jurisprudence of relevance to expert system design are discussed below.

\subsubsection{Scientific and mechanical jurisprudence}

The dictionary defines jurisprudence as:

The science which treats of human laws (written and unwritten) in general; the philosophy of law. ${ }^{34}$

Pound refers to the "scientific character" of the law:

Sir Frederick Pollock gives us the clew when he defines the reasons that compel law to take on this scientific character as three: the demand for full justice, that is for solutions that go to the root of controversies; the demand for equal justice, that is a like adjustment of like relations under like conditions; and the demand for exact justice, that is for a justice whose operations, within reasonable limits, may be predicted in advance of action. In other words, the marks of a scientific law are, conformity to reason, uniformity, and certainty. ${ }^{35}$

This approach to the law has been much criticized. Frank contends that uncertainty is inherent in the legal process, and that seeking certainty in general legal principles is simply "an expression of infantile emotional attitudes which have persisted into adulthood." 36

In his 1908 paper, Mechanical Jurisprudence, Pound distinguishes scientific jurisprudence from mechanical jurisprudence:

Roman law in its decadence furnishes a striking example [of mechanical jurisprudence]. The Valentinian "law of citations" made a selection of jurisconsults of the past and allowed their writings only to be cited. It declared them, with the exception of Papinian, equal in authority. It confined the judge, when questions of law were in issue, to the purely mechanical task of counting and of determining the numerical preponderance of authority. Principles were no longer resorted to in order to make rules fit cases. The rules were at hand in a fixed and final form, and cases were to be fitted to the rules. ${ }^{37}$

By contrast, Pound says, scientific law is "a reasoned body of principles for the administration of justice". 38

Loevinger also argues a role for science in law, but sees little science in jurisprudence. In 1949 he proposed an alternative to jurisprudence which he termed jurimetrics:

The next step forward in the long path of man's progress must be from jurisprudence (which is mere speculation about law) to jurimetrics - which 
is the scientific investigation of legal problems ... The inescapable fact is that jurisprudence bears the same relation to a modern science of jurimetrics as astrology does to astronomy, alchemy to chemistry, or phrenology to psychology. It is based upon speculation, supposition and superstition; it is concerned with meaningless questions; and, after more than two thousand years, jurisprudence has not yet offered a useful answer to any question or a workable technique for attacking any problem. ${ }^{39}$

This proposal spawned a new field of study, some work from which is discussed in $\S 2.3$ below.

Loevinger calls those who fear the dangers of mechanized jurisprudence "quixotic and uncomprehending" 40 _ yet he was not a mechanical jurisprudent himself (as explained in §2.3). Others, however, saw the development of computers as an opportunity to develop a judgment machine: a machine that could replace a judge.

\subsubsection{Judgment machines}

In 1955, Lasswell predicted that:

When machines are more perfect $[s i c]$ a bench of judicial robots ... can be constructed. The machine would apply a system of "weights" to allegations of fact made by parties to a controversy, and also to the justifications advanced in support of the claims put forward by participants. Litigation can proceed by counsel for the plaintiff and the defendant pressing buttons that translate their cases into the physical signs built into the machine. Many results would be "no decision." However, the machine could be designed to settle a controversy of this kind with a "random" operation (by lot). ${ }^{41}$

He was not altogether serious:

It is a challenging task for legal historians to assist in constructing a robot whose weights would give substantially the same result as those produced by the [US Supreme] Court at various periods. The task would not be too difficult for some justices on some issues. But a robot facsimile of the less repetitive members of the Court would provide a genuine challenge to the engineers. ${ }^{42}$

However, some have taken the idea of a judgment machine very seriously indeed. In 1949, Frank wrote that a "logic machine" might "disclose all possible available alternative legal rules," although judges would still have to exercise the "the sovereign prerogative of choice" between the rules on the basis of the judges' "conscious or unconscious notions of policy." 43 Similarly Mehl wrote, in 1959, that a machine could perform some of the functions of a judge, but that a role for humans would remain because "the solution to a legal problem may depend upon extra-rational factors, involving the whole of human experience". ${ }^{44}$ 
As recently as 1977, it has been seriously suggested that a judgment machine could replace a human judge. D'Amato proposed that a machine could take the relevant facts of a case as input and produce a number in the range -1 to 1 (where a positive number indicates a victory for the plaintiff). Given the multiplicity of factors, he claims, a result of zero would be extremely unlikely, ${ }^{45}$ although it is not clear why this would be so. These facts would be determined by a jury, but the law would be decided by the machine. Somewhat grudgingly, he allows for some vestige of human control: an appeal court could review all of the machine's determinations in a certain numerical range (e.g. -0.05 to 0.05 ) within which the cases would be so close that a re-examination might be required. The review court's subsequent decision would then be incorporated into the system. ${ }^{46}$

The idea of human judges being replaced by machines has been trenchantly criticized. According to Weizenbaum:

The very asking of the question, "What does a judge ... know that we cannot tell a computer?" is a monstrous obscenity. That it has to be put into print at all, even for the purpose of exposing its morbidity, is a sign of the madness of our times.

Computers can make judicial decisions ... They can flip coins in much more sophisticated ways than can the most patient human being. The point is that they ought not be given such tasks. They may even be able to arrive at "correct" decisions in some cases - but always and necessarily on bases no human being should be willing to accept. ${ }^{47}$

But D'Amato sees advantages in replacing human judges by machines:

Would we lose a judge's "judgment," and how important would such a loss be to our legal system? Surely computers do not make "judgments" the way humans do, and so we would lose the "human" aspect of legal judgments. But what specifically do we lose when we lose the humanness of judgments? Is human judgment just a euphemism for arbitrariness, discretion, or bias? ${ }^{48}$

By contrast, Stone stresses the importance of legal change springing from "deviance, tentativeness, and even indecision in judgment."

It is these phenomena above all which promote a judge's sensitivity to new ideas or to newly perceived social situations and stir Hamlet-like introspection. On these there rest some of the main foundations of the social good we call "justice." 49

Proponents of the idea of automated judges claim that such systems would reduce the cost of the legal system, find inconsistencies in the law, and provide a level of certainty in the law which does not exist at present. ${ }^{50}$ D'Amato claims that a judgment machine would allow people to live under the rule of law and not under the "rule of persons." 51 
D'Amato's argument is based upon "two bold initial premises." He assumes that, from a jurisprudential point of view, the law has been made completely determinable. He also assumes that, if a computer can be programmed to make judicial decisions, human "discretion" will have been completely removed. ${ }^{52}$

His views on judgment machines are extreme, and have not been widely accepted. For the most part, expert system designers have focused on building systems which provide advice to lawyers or to laypeople, rather than usurping the role of judges. However (as is discussed in $\S 2.4$ below) many expert systems designers have made D'Amato's first assumption - that the law is completely determinable - often tacitly.

\subsubsection{Petrifaction of the law}

The idea of a judgment machine removing uncertainty and ambiguity in the law raises the possibility of the petrifaction of the law. This possibility is also relevant to legal expert system design.

D'Amato claims that, once programmed, the law would become settled. The computer would stop "progress," although the legislature could always step in if anomalous results were being produced. ${ }^{53}$ Similarly, Kayton believes that the use of propositional logic could identify legal ambiguity and provide guidelines for its resolution. He asks:

Would not each concrete demonstration of ambiguity and its resolution tend to rigidify the law and eventually destroy the very flexibility which has made the common law viable? ... Despite the dangers of generalization, it is submitted that ... [this question] should be answered in the negative. Information brought to bear in a rational pursuit is always better than ignorance or confusion. The elimination of ambiguities, rather than ossify the law, would produce an optimum condition for the purposeful, intelligent, and efficient development of the law. ${ }^{54}$

Schubert, another early researcher in this field, is not convinced of the possibility, or the importance, of certainty in law. He writes:

... the ideal of certainty in law is tolerable only in the context of an empirical world in which forces inducing change are so manifold that the attainment of the goal is never possible. ${ }^{55}$

Tyree argues that a deterministic computer judge could "overrule himself" if cases are added as they are decided. ${ }^{56}$ But as Pound pointed out more than ninety years ago, the problem is more than mere ossification of the law:

The effect of all system is apt to be petrifaction of the subject systematized. Perfection of scientific system and exposition tends to cut off individual initiative in the future, to stifle independent consideration of new problems and of new phases of old problems, and to impose the ideas of one generation upon another. ${ }^{57}$ 
This problem is inherent in expert system design - in the law and in other domains. There are dangers, for example, in the use of a legal expert system by judges. Stone considers the use of predictive techniques in the law and warns that:

... reliance at the judgment seat on new predictive techniques would seriously threaten the judge's central concern with justice. For if the results thus predicted for him do affirmatively guide him in present decisions, each judge will tend to vote somewhat more consistently with his past record. The margin of deviation would accordingly disappear in deference to predicted patterns, aided by the human tendency to follow the less agonizing because already trodden path ... ${ }^{58}$

Judges, Stone says, have a duty to strive wholeheartedly for justice "at the moment of judgment." 59 Although an expert system may be of some use to judges of lower courts, there is a critical border between a machine serving the legal order and the dangers of subversion of that order.

For whenever an appellate judge faces the duty to do justice in this sense, he must address himself to it with his own present experience and insights. It would be a corruption of justice for him to shortcut this duty by resting on predictions of his future decisions based on his own past behavior - still more so if the basis is the average past behavior of a group of judges. ${ }^{60}$

So, as Stone points out, the "pioneers and experts of the new techniques" must face "the limits of the contributions they can make." ${ }^{61}$

\subsubsection{Clear rules and clear cases}

H. L. A. Hart was the most famous of the positivist legal theorists. His major work, The Concept of Law, was published in 1961. Hart realizes that because the law is expressed in natural language, it is subject to considerable semantic indeterminacy. This he terms the open texture of law. However, he claims that it is possible to use rules deductively to solve "clear cases": that is "those in which there is general agreement that they fall within the scope of a rule." ${ }^{2}$ He also contends that:

... the result of the English system of precedent has been to produce, by its use, a body of rules of which a vast number, of both major and minor importance, are as determinate as any statutory rule. They can now only be altered by statute, as the courts themselves often declare in cases where the 'merits' seem to run counter to the requirements of the established precedents. ${ }^{63}$ 
As Susskind writes, one ramification of Hart's analysis is that:

... all expert systems in law whose inference procedures are solely deductive will function exclusively in the clear case domain, and will be of no aid in the solving of "problems of the penumbra." 64

But what constitutes a "clear case" is itself far from clear. As Hart concedes:

... it is a matter of some difficulty to give an exhaustive account of what makes a 'clear case' clear or makes a general rule obvious and uniquely applicable to a particular case. ${ }^{65}$

Moles disputes the very existence of clear rules and clear cases. He performs a detailed analysis of the application of an example of an ostensibly clear rule in a British statute: a provision which prescribes the circumstances in which an injunction should be issued in domestic violence cases. ${ }^{66}$ Moles summarizes the effect of three cases as follows:

(1) Before $B v \cdot B^{67}$ we are concerned with a statutory provision which on its face appears to be clear and comprehensible (at least to non-lawyers) and it would appear that an injunction should issue in cases such as those we have looked at.

(2) After Cantliff, ${ }^{68}$ the provision has been considered twice by the [English] Court of Appeal within two weeks. All of the six judges who considered the matter are in agreement that the injunction should not issue and that the rule is clear.

(3) The matter is further considered by a specially constituted Court of Appeal of five, ${ }^{69}$ who decide that the rule is clear, but different from that in (2), and that the injunction should issue. ${ }^{70}$

Moles also analyses the judicial application of the rule of precedent-which, with this statutory rule, "must be regarded as amongst the clearest available to us" ${ }^{1}$ to demonstrate that "our experience of the legal system bears little relationship to Hart's account of it". ${ }^{2}$

Leith developed a legal expert system which operated on "clear rules" in the law. ${ }^{73}$ He later recanted, saying that:

... the very idea of a clear rule is inherently confusing and is not observable in the real operation of the judicial process ... judicial creativity is not an aberation $[s i c]$ of the legal process, but (as Moles ... suggests), the very heart of the law. ${ }^{74}$

As is explained in $\S 2.4$ below, many expert system designers have adopted a view of the law that allows for clear rules and clear cases - without considering the possibility that the law may not be like that at all. 


\subsubsection{Legal realism and rule scepticism}

In the 1920s and 1930s, a movement called legal realism developed in America. ${ }^{75}$ Realists rejected the importance that mechanical jurisprudence placed on rules. However, the rule scepticism of the American Realists is meek by comparison with the strong indeterminacy thesis. Drahos and Parker explain that:

According to Karl Llewellyn a characteristic feature of Realism was the rejection of simple (by which he meant general) rules and the substitution of more detailed classificatory schemes which better captured the specific nature of judicial rule-making ... On Llewellyn's account, rule scepticism emerges as a set of doubts about the veracity of legal actors' claims to be following the legal rules they say they are. This is not rule scepticism in the strong sense of denying the existence of rules, however. Legal actors may simply be following some other rules. The rule scepticism of American Realism could perhaps be more accurately described as rule cynicism. ${ }^{76}$

Kripke, by contrast, is a true rule sceptic. His argument is an example of the Wittgensteinian Paradox. ${ }^{77}$ Consider the two functions "plus" $(+)$ and "quus" $(\oplus)$. The + function is the mathematical function, addition. The $\oplus$ function is defined as follows:

$$
x \oplus y= \begin{cases}x+y, & \text { if } x, y<57 \\ 5, & \text { otherwise. }\end{cases}
$$

Suppose that Kripke has used + in the past, but always with values of $x$ and $y$ smaller than 57. He performs the computation $68+57$ and gets a result of 125 . Yet it could be said that when he thought he was using the "plus" function in the past, he was in fact using "quus." As Kripke explains:

... in this new instance, I should apply the very same function or rule that I applied so many times in the past. But who is to say what function this was? In the past I gave myself only a finite number of examples instantiating this function. So perhaps in the past I used 'plus' and ' + to denote ... 'quus' ... Who is to say that [' $\left.\oplus^{\prime}\right]$ is not the function I previously meant by ' + '? 78

There is no justification for Kripke answering 125 rather than 5. There is no way of determining (from his past behaviour - even his past thoughts) whether by "plus" Kripke meant + or $\oplus$. Rules are derived from a finite number of examples; for any given rule, there is always an alternative rule which also explains those examples.

Mathematicians could counter that the meaning of + is well defined. But Kripke argues that:

... scepticism about arithmetic should not be taken to be in question: we may assume, if we wish, that $68+57$ is $125 \ldots$ I cannot doubt coherently that 'plus', as I now use it, denotes plus! Perhaps I cannot ... doubt this 
about my present usage. But I can doubt that my past usage of 'plus' denoted plus ...

... There is no objective fact - that we all mean addition by ' + ', or even that a given individual does - that explains our agreement in particular cases. Rather our license to say of each other that we mean addition by ' + ' is part of a 'language game' that sustains itself only because of the brute fact that we generally agree. ${ }^{79}$

Drahos and Parker write that:

The consequences of this argument, if valid, are shattering. Rules turn out to be no more than leaps in the dark and the whole notion of rule following seems illusory. ${ }^{80}$

The implications of Kripke's argument have been realized by legal theorists. ${ }^{81}$ As Drahos and Parker point out:

Saying that there are no rules to follow, only social practices, means that propositions about the law are potentially open to wild fluctuations. The argument also puts paid to any possibility of a correspondence theory of truth in law. ${ }^{82}$

They propose a solution to this problem:

The Kripkean argument does not prevent people from saying that they are following rule X. Rather it stops them from being able to justify the existence of that rule by reference to some objective meaning. This still leaves the practice, as opposed to the justification, of successful rule following to be accounted for. ${ }^{83}$

Their solution is to view the law as a set of rules and conventions: "a type of rule used to fix or interpret the meaning of other rules." 84 They claim there is a distinction between rule knowledge and rule understanding, the latter being "a matter of absorbing conventions relating to rule use." 85 A lawyer requires knowledge of the rules, and understanding as to how to apply those rules properly. Drahos and Parker argue that the problems facing designers of legal expert systems "flow from the difficulties of representing rule understanding rather than rule knowledge." Rules can be used to represent rule knowledge; the problem is "how to represent with tolerable accuracy ... conventions which confer rule understanding." 86

But they concede that, as with other rules, conventions have to "run the gauntlet of scepticism". ${ }^{87}$

\subsubsection{A jurisprudential consensus?}

The only major examination of the role of jurisprudence in the development of legal expert systems is Susskind's 1987 book Expert Systems in Law: A Jurisprudential Inquiry. ${ }^{88}$ As mentioned in $\S 2.2 .1$ above, he argues that all expert systems must conform to some jurisprudential theory. 
He also sets out to find consensus in jurisprudential theory. As there is far too much literature in the field for one person to cover it all, he limits his choice of material: "the vast majority" of sources that he uses are British analytical jurisprudential writings since the mid-fifties and early sixties, "the impetus for which", he concedes, "was derived very largely from the publications of H. L. A. Hart." 89

Susskind concludes that "there are no theoretical obstacles, from the point of view of jurisprudence, to the development of rule-based expert systems in law of limited scope." 90 He also claims that the divergence of views within jurisprudence has been overstated because legal theorists tend to focus on the differences. He claims that there is a jurisprudential consensus, "albeit of mundane and limited application". 91

Susskind's work provided the theoretical justification for not only his own development work, ${ }^{92}$ but for much of the subsequent work of other developers of legal expert systems. It could also be said to have provided, retrospectively, legal theoretical justification for most of the work on rule-based expert systems that preceded his book.

However, Susskind's approach to jurisprudence is fundamentally flawed. As Moles points out:

[Susskind] said that he would carry out a survey of the jurisprudential literature. He acknowledged, of course, that it would not be possible to survey the whole of the jurisprudential literature. In fact, he determined that law was a system of rules by "surveying" only those whose avowed position was based on the fact that the law was a system of rules ...

I would venture to suggest that this is in fact a misuse of the survey technique ... Susskind was perfectly familiar with the work of Hart and his followers, and was well able, therefore, to find any number of books and articles which supported the "law as rules" view. He then developed his position ... on the basis of what this purported consensus within jurisprudence had to say.

... Of course, Susskind was telling certain sections of the AI and Law community what they wanted to hear, and hence their enthusiasm for it. ${ }^{93}$

Moles also notes Susskind's admission that "the most rigorous of these writings constituted the source materials with greatest potential given the overall purpose of the project." 94 Because the first objective of Susskind's work was to design, develop and implement an expert system in law, ${ }^{95}$ Moles cites this admission as evidence that Susskind prejudged his survey.

In the light of this criticism, it is ironic to note Susskind's own caution that:

... a little jurisprudential knowledge can be a dangerous thing! It is tempting for the jurisprudential neophyte to become an ardent devotee of a particular school of thought within legal theory and to go on from there to implement all and only the teachings of that school. This course of action 
should be avoided at all costs. Familiarity with a wide range of works should be achieved prior to commitment to any particular jurisprudential posture. ${ }^{96}$

Clark, another critic of Susskind, says that:

The shortcomings of the book coincide with the limits of positivist legal theory, and even then some doubt must remain as to whether expert systems in law mark a revival of the kind of "mechanical jurisprudence" which Hart opposed so vigorously from within the confines of positivist legal thought. ${ }^{97}$

But the strongest criticism of Susskind comes from Leith:

... [Susskind] believes that he can come to some sort of compromise with the various theoretical positions taken by the renowned thinkers of the field and produce "a general theory" (an indication, I might suggest, of [his] poor theoretical conceptions) ...

... Susskind sees jurisprudence as providing a variety of theoretical models which can be modelled mathematically and translated into computer programs.

... of necessity formal specification requires a formalisation of law; there can be no "informal models" which are mysteriously formalised into a computer model ...

... Therefore, in order to use formal specifications, Susskind must provide a formal specification of law which can then be incorporated into the rule-format of a computer program ... And, of course, formal specifications of law are renowned for their theoretical and practical inadequacy. Legal formalism can, surely, hardly be the compromise he wishes might arise from the conflicting positions of Kelsen, Hart, Dworkin et al. ${ }^{98}$

... if he really does believe that his informal theoretical models can be transformed into formal theoretical models without loss of their informal attributes, then I must suggest that he has really little understanding of the discipline of computer science. ${ }^{99}$

Leith's comments are consistent with the point made in $\S 2.2 .1$ above: lawyers and legal expert systems operate at a lower level of abstraction than the philosophical level of jurisprudence.

Susskind's claim to have found a consensus in jurisprudence - even one of mundane and limited application - is absurd. As shown in $\S 2.2 .5$ above, the views of just two jurisprudents (Hart and Moles) are completely irreconcilable. Similarly, Hart's views are totally at odds with those of Kripke (as explained in $§ 2.2 .6$ ). Susskind states his theory, and develops an expert system which conforms to that theory. His mistake is to claim that his theory is definitive, in that it represents a jurisprudential consensus. 


\subsection{Jurimetrics and the behaviourists}

As mentioned in $§ 2.2 .2$, the term "jurimetrics" was coined by Loevinger. He defines "jurimetrics" as "the study of law and legal problems by scientific methods and concepts, the employment of science in law to the extent that it is applicable or adaptable." ${ }^{1}$ Loevinger is neither a mechanical jurisprudent nor a positivist:

To begin with we must be clear that science offers us neither ultimate nor certain answers to legal problems. The dream that science might someday tell us which of several competing interests is the more important is a vain one. Science essays no such answers in any field. Science does not assign social or ethical values. Science may, indeed, provide data from which social or ethical judgments may be made; but the judgments will remain with man. ...

There is no prospect of any process that will preclude consideration of social desirability or wisdom. The opportunity will always be available to argue that precedent should not be followed, and that considerations of policy, or expediency, require a different rule or a special result ...

... Science does not and will not offer us any law machines that give automatic answers to specific questions put to them, whether as to particular cases or as to ultimate legal issues such as the relative importance of interests that may be in conflict. By the same token, science will provide us with no formulae or calculus that will give us certainty either of prediction, analysis or answers to ultimate questions such as which interest is to be preferred or which desire has greater social value. ${ }^{2}$

Although these comments are eminently reasonable, some of Loevinger's turns of phrase are exasperating. In 1949 he claimed that putting the law on a "rational basis" was the "indispensable condition" of the survival of the human race. ${ }^{3}$ In the light of such a ridiculous statement, and after his trenchant criticism of the value of jurisprudence (quoted in $\S 2.2 .2$ above), it is not surprising that, as Gardner says:

The attitudes Loevinger and his colleagues expressed were never adopted by the legal profession generally ... As a movement within the legal profession, jurimetrics has not been much heard from since the early 1970s. ${ }^{4}$

Nevertheless, some of the work of his colleagues in predicting judicial decisions is worthy of comment here.

A number of researchers in the early 1960s focused on the statistical analysis of the behaviour of judges. On the basis of this analysis, they claimed that they could predict the future behaviour of individual judges, and of courts. ${ }^{5}$ Such predictions were justified on the basis of the application of the doctrine of precedent. As Lawlor writes:

Even if they are man-made, the principles of stare decisis are akin to the all embracing assumption of uniformity of natural science. Without such a principle to guide us, prediction of legal decisions is impossible. ${ }^{6}$ 


\subsubsection{Kort}

Kort uses mathematical expressions to represent judicial decisions. ${ }^{7}$ He identifies attributes which are of importance and represents decided cases in the following form:

$$
\sum_{i=1}^{n} A_{i j} w_{i}=V_{j}
$$

where $n$ is the number of attributes, $A_{i j}$ is the value of the $i$ th attribute for the $j$ th case (attribute values being 0 or 1 ), $w_{i}$ is the weight of the $i$ th attribute, and $V_{j}$ is the number of votes of judges favourable to the party seeking redress in the $j$ th case. This approach is justified on the basis that the decisions of split courts do not necessarily constitute two opposite extremes, but represent certain degrees of support for one party. ${ }^{8}$

By solving these simultaneous equations, weights are obtained for the attributes. The sum of the weights of those attributes present in the instant case is, according to Kort, the number of likely judicial votes in favour of the party seeking redress.

\subsubsection{Lawlor}

Lawlor uses logical expressions to represent previously decided cases. ${ }^{9}$ He analyzes right-to-counsel cases heard before the US Supreme Court over thirty years, and finds that in all of these cases each judge behaved consistently with his own "personal stare decisis." He identifies legally significant attributes and builds a logical expression which represents the behaviour of each judge. A program uses these logical expressions to predict the likely outcome, given a composition of the Court specified by the user.

Lawlor's system successfully predicted the US Supreme Court's overruling of Betts v. Brady ${ }^{10}$ in Gideon v. Wainright. ${ }^{11}$ But it predicted a 5:4 majority; the Supreme Court's decision was unanimous.

Applying "traditional" stare decisis (i.e. considering the court as a whole, rather than the decisions of individual judges) Lawlor's system did not predict the Supreme Court's change. Given the same cases, Kort's approach did not predict the change either.

This failure draws criticism from those who disagree with such a "logarithmic approach to justice." 12 Wiener complains that "advocates of the computer" rest their arguments on an assumption that courts will adhere to the doctrine of stare decisis, which does not always hold. ${ }^{13}$

In defence of the behaviourists, Kayton refers to their failure to predict the decision in Gideon v. Wainright and writes:

Should we have expected otherwise? Of course not! A reversal is by definition a logical inconsistency. That which by stare decisis had been called black is now called white. ${ }^{14}$ 
The developers of these prediction systems accept that they rely on the doctrine of precedent. Hence, a reversal of previous authority will always be beyond the predictive capacity of these systems - as it is beyond the predictive capacity of most lawyers.

\subsubsection{Nagel and Schubert}

Nagel and Schubert ${ }^{15}$ examine the personal attitudes of US Supreme Court judges towards various political and economic relations, and claim that these "off-thebench" attitudes (as Nagel calls them) affect the judges' decision-making.

Correlations were ... made between responses to specific items and various decisional propensities. For example, there was a high and statistically significant correlation between disagreeing with [one questionnaire item] ("Our treatment of criminals is too harsh; we should try to cure, not to punish them") and being above the average of one's court with regard to the proportion of times one voted for the prosecution in criminal cases. Off-the-bench judicial attitudes thus do seem to correlate in a meaningful way with on-the-bench judicial decisions. ${ }^{16}$

\subsubsection{Haar, Sawyer and Cummings}

In the mid-1970s Haar, Sawyer and Cummings made use of regression analysis to build a predictive model for zoning amendment cases in Connecticut. ${ }^{17}$ Regression analysis is a statistical technique for analyzing the relationship of a set of independent variables to a dependent variable. For Haar et al. the dependent variable is the outcome of the cases, the independent variables are attributes.

They identify 167 attributes which "appeared to be important" to the courts, 40 of which were deemed significant using a $\chi^{2}$ ("chi-square") test for association. This is "too many to use in a regression analysis", ${ }^{18}$ so Haar et al. employ two different methods to reduce this number further: grouping attributes on the basis of "experience, knowledge, and intuition", ${ }^{19}$ and factor analysis.

\subsubsection{Prediction}

The behaviourists' predictive research has been criticized because they do not attempt to model legal reasoning. ${ }^{20}$ But, in not so doing, the behaviourists just reflect the influence upon them of the American Realists. They adopt Holmes's analysis that for any individual "the law is simply a prediction of the way in which the public force possessed by the government will act upon him." ${ }^{21}$ Loevinger claims that some method of legal prediction is "indispensable" ${ }^{22}$ Lawlor says that the ultimate goal of all scientific methods is reliable prediction of future events and "[r]eliable prediction is also one of the ultimate goals of law." ${ }^{23}$ Susskind (certainly no behaviourist) also stresses the importance of the prediction of judicial decisions. ${ }^{24}$ 
Tapper writes that:

Although the statistical techniques employed by some of these workers have been criticized, there can be no real doubt that this work provides a successful approach to the analysis of the decisions which have been reached in the past, and at least as satisfactory a method of predicting future decisions as can be arrived at by native wit and unaided intuition. ${ }^{25}$

However, he criticizes the conclusions that have been drawn using these techniques:

Either the behaviourist is to content himself with observing the objective phenomena, in which case he can conclude nothing as to motivation, or he is to ascribe motivation to the phenomena, in which case he ceases to be objective. It is precisely at the point at which decisions of the [US] Supreme Court, for example, are characterized as pro-civil liberties or anti-labour that doubts arise as to the real objectivity of the studies.

The same general line of argument may be advanced against the factoriented approach ... Here the point at which the study loses its objectivity is in the characterization of the facts present in the case. ${ }^{26}$

(Although this may be a valid criticism of a behaviouristic study, it is not an argument against the use of predictive techniques where the characterization of facts has been performed - subjectively, admittedly - by a legal expert, as is done for SHYSTER.)

Gardner is also critical of the jurimetric programs:

... programs, done from a political science viewpoint rather than a legal one, in which the data concern legally irrelevant matters such as the ideology and social background of individual judges. ${ }^{27}$

By "legally irrelevant matters" Gardner means those matters to which judges do not - and/or should not - explicitly have regard when coming to their decisions. But if a system is designed to predict decisions then any attribute which assists in prediction should be included - regardless of whether regard ought to be had to that attribute. These "legally irrelevant matters" are not necessarily irrelevant to the prediction of judicial decisions.

Stone dismisses concern about "jurimetric" prediction: "the behavioralists [sic] may have no conscious designs on the integrity of the decisional process. The judgment of justice may be of no concern to them." ${ }^{28}$

He defines the "judgment of justice" by reference to a situation where a judge makes a decision which:

... does not merely declare the existing law but decides what justice requires that the law should be. (... [W] include tacitly here the reversal of earlier decisions, that is, creative decisions which unsettle and resettle law: this is a fortiori creative.) It is this kind of creative judgment which we have here termed "a judgment of justice." ${ }^{29}$ 
The behaviourists are, Stone says:

... only observers looking at what has already been done in judgment. And when in the course of prediction they turn their attention to future judgments, it is to ask not what the judge should do to further justice but only what kind of decision he will give if he acts consistently with values attributed to him on the basis of his past decisions. ${ }^{30}$

In other words, their work attempted to be predictive not normative.

When a lawyer gives advice, she/he is expected to make some prediction as to the likely result. This prediction need not be emphatic: it may be no more than a tentative statement about the strength of a person's legal position. Hence, legal expert systems should have some degree of predictive capacity. Like the behaviourists' research, a legal expert system's predictive power is a projection based on past cases. There is - and can be - no allowance for changing social mores. This is not a serious limitation upon such a system's utility: predicting a judge-made change in the law is beyond all but the very best lawyers.

However, as discussed in $\S 2.5$ (especially $\S 2.5 .2$ ) below, the justification that a legal expert system provides for its prediction is also important.

\subsection{Rule-based systems}

There are many examples of rule-based legal expert systems, the most important of which are discussed in detail below: the work of McCarty ( $\$ 2.4 .1)$, BenchCapon, Kowalski and Sergot (§2.4.2), Gardner (§2.4.3) and Susskind (§2.4.4).

The first proposal for a rule-based legal expert system was made by Buchanan and Headrick in 1970. They complained that interdisciplinary work between lawyers and computer scientists had "floundered on the misconceptions that each has of the other's discipline", ${ }^{31}$ and suggested that the computer modelling of legal reasoning would be a fruitful area for research. ${ }^{32}$

Their proposal asserts that "[i]n the absence of any reason to speculate on how they carry on their work, [lawyers] now apply complex sets of rules without being aware of the rules themselves." 33 They make no reference to jurisprudential writing, but they do make it clear that their approach is based upon two assumptions about human problem-solving in general: "(1) problems can be broken down into a set of subproblems, and (2) the solution to any subproblem requires a series of decisions that are governed by decision rules." 34

The literature is replete with examples of projects in which the assumption that lawyers work with rules is unstated or unsupported. Maggs and deBessonet, for example, tacitly make this assumption. ${ }^{35}$ The law, they claim, can be expressed as rules using propositional calculus. A program which implements these rules could answer questions from a user, and allow the checking of statute law for redundancy and contradiction. (It is not clear what role case law plays in their system, or indeed in their model of the law.) 
Popp and Schlink's JUDITH system ${ }^{36}$ uses rules to represent parts of the German Civil Code. The principles behind JUDITH are "strikingly similar" to those of the MYCIN system (an expert system dealing with bacterial infections ${ }^{37}$ ) - so similar that, its developers claim, it would be possible to create a legal knowledge base for MYCIN and a medical knowledge base for JUDITH. ${ }^{38}$ Michaelsen and Michie's TAXADVISOR system ${ }^{39}$ is implemented using MYCIN. ${ }^{40}$ TAXADVISOR was designed to assist lawyers to advise clients on taxation and estate planning. ${ }^{41}$

Meldman's system uses two different kinds of rules: general rules which define the elements of the claim, and specific rules extracted from cases. ${ }^{42}$ Things and relations are used to represent the "everyday world of human affairs", ${ }^{43}$ and are classified hierarchically into categories. A fact comprises two things and a relation between them; facts are assembled into situations. These situations are compared with the situation of the instant case, and the system determines the extent to which the instant case falls within or near the law of intentional torts (e.g. assault and battery).

Waterman and Peterson's LDS system ${ }^{44}$ is a rule-based system for the field of product liability. It does not determine whether liability exists, but is designed to assist legal experts in settling product liability cases. LDS is a typical example of a system which developed without any legal theoretical justification. After admitting that there is no "deep model of the legal process", ${ }^{45}$ Waterman and his colleagues proceed on the unstated assumption that any such model must involve rules:

One might expect that the large body of legal rules and regulations that have been accumulated and formalized in the legal domain would make expert system development easier. Unfortunately, this is not the case. Instead, this characteristic of the domain, having rules that already exist, has led to trouble ... First, the formal rules that define and regulate legal activity are often ambiguous, contradictory and incomplete. And second, there exists a body of informal rules or procedures about how to access, interpret and use the 'formal' rules. Without these informal rules the formal rules can not be used in any efficient or cost-effective way. ${ }^{46}$

This body of rules, they write, "needs to be mapped into code" 47 before a legal expert system can be built.

Bing's SARA system ${ }^{48}$ is designed to analyze discretionary decisions. It uses rules to represent legal norms: some strict and some discretionary. These discretionary norms are weighted using correlation techniques. SARA allows a lawyer "to back up his qualitative legal reasoning by quantitative indications." 49

Stamper's LEGOL language ${ }^{50}$ also uses rules to represent legal norms. Pattison and Ciesielski use a rule-based system to review contracts. ${ }^{51}$ SoftLaw's STATUTE, a commercially successful system, uses rules to represent statutes, regulations and departmental guidelines in taxation, social security and veterans' affairs law. ${ }^{52}$ 


\subsubsection{McCarty}

McCarty has been described as "the father of AI and law". ${ }^{53}$ His TAXMAN project $^{54}$ was concerned with the development of a computational theory of legal reasoning, using corporate tax law as an experimental problem domain.

He claims that a computer-based legal consultation system must be able to represent the "facts," at some comfortable level of abstraction, and the "law," which would consist of a system of "concepts" and "rules." These concepts and rules are relatively abstract (i.e. they subsume large classes of lower-level factual descriptions), and they have normative implications (i.e. they specify which actions are permitted and which are obligatory).

Legal analysis, in its simplest form, would then be a process of applying the "law" to the "facts". Put this way, the paradigm seems to be an ideal candidate for an artificial intelligence approach: the "facts" would be represented in a lower-level semantic network, perhaps; the "law" would be represented in a higher-level semantic description; and the process of legal analysis would be represented by a pattern-matching routine. ${ }^{55}$

However, McCarty concedes, the representation of facts in such a system is more difficult than in other expert systems, because "the facts of a legal case typically involve all the complexities of daily life: human actions, beliefs, intentions, motivations, etc." 56 Even if the facts can be represented, he writes, the rules will often be problematic:

Some rules, usually those embodied in statutes, have a precise logical structure, and this makes them amenable to the existing artificial intelligence techniques. But it is a commonplace among lawyers that the most important legal rules do not have this form at all: instead they are said to have an "open texture"; their boundaries are not fixed, but are "constructed" and "modified" as they are applied to particular factual situations. A sophisticated legal consultation system would not be able to ignore these complexities, but would have to address them directly. ${ }^{57}$

McCarty also makes the startling claim that the "simplest" problems for firstyear law students are the hardest for an AI system because the student draws upon ordinary human experience:

Paradoxically, the cases that are most tractable for an artificial intelligence system are those cases, usually involving commercial and corporate matters, which a lawyer finds most complex. There is a simple reason why this is so. A mature legal system in an industrialized democracy is composed of many levels of legal abstractions ... Because of their technical complexity, the legal rules at the top levels of this conceptual hierarchy are difficult for most lawyers to comprehend, but this would be no obstacle for an artificial intelligence system. ${ }^{58}$ 
McCarty chose the area tax law for his system because "commercial abstractions, in fact, are artificial and formal systems themselves, drained of much of the content of the ordinary world" and, by legal standards, well structured. ${ }^{59}$ The field of corporate tax law, he says, is "very near the apex of the hierarchy of commercial abstractions" 60 _ "whatever that means", comments Moles. ${ }^{61}$

In TAXMAN I, McCarty's first prototype system, the basic "facts" of a corporate case are captured in a relatively straightforward representation (e.g. a corporation issues securities). Below this level is an expanded representation of the meaning of various entities (e.g. a security interest) in terms of their component rights and obligations. Above this level-presumably above both levels, although this is not made clear -is the "law" (statutory rules which classify transactions as taxable or non-taxable etc.). ${ }^{62}$

McCarty found that, although the rules are complex, the underlying representations are manageable. He concludes from his early work that "the construction of an expert consultation system in this area of the law is a feasible proposition." 63

McCarty sees the development of legal expert systems as an opportunity to contribute to jurisprudence. Although the jurisprudential literature includes "many illuminating examples and many valuable insights about the structure and dynamics of legal concepts", he complains that "taken as a whole" it is "notoriously imprecise". 64

The TAXMAN system adds a strong dose of precision and rigor to these discussions of linguistic and conceptual problems. Its critical task is to clarify the concepts of corporate reorganization law in such a way that they can be represented in computer programs. ${ }^{65}$

Moles is extremely critical of rule-based expert systems designers, and McCarty in particular. McCarty takes a positivist approach to the law, and Moles complains that:

The computer scientists have taken the de-humanizing aspects of modern positivism to their extreme. ...

The computer scientists, encouraged by the modern positivists, fail to recognize the point which Austin ${ }^{66}$ correctly emphasized throughout his work - that law, positive morality and ethics are inseparably connected parts of a vast organic whole. ${ }^{67}$

Most damning is Moles's statement that:

The sad thing is that [McCarty] has not shown the slightest awareness of the nature of the legal enterprise. Far from having emphasized any difficulties, he shows that he simply does not understand what they are. ${ }^{68}$ 
McCarty does identify two major limitations to the approach taken in TAXMAN I. Firstly, he concedes that the factual descriptions, although manageable in the corporate domain, would be too complex in (for example) the average contract or tort problem. Secondly, the higher-level conceptual representations are not adequate for all domains because judicially created concepts are incurably open-textured, have a dynamic structure with the capacity to evolve and adapt to new situations, and the evolution of these concepts is governed by a sense of purpose. ${ }^{69}$ Having recognized these problems, McCarty set out to solve them with TAXMAN II.

TAXMAN II ${ }^{70}$ uses "prototypes and deformations" to represent a legal concept by specifying the prerequisite conditions for that concept, a set of cases (real and hypothetical) in which that concept does or does not apply, and a set of transformations for getting from one case to another. If a given case (representing a legal concept) can be transformed into the instant case, while still satisfying the prerequisite conditions for the concept, then it can be used in argument as an example of that concept.

Ashley sees McCarty's work as a significant advance, but points to several shortcomings of TAXMAN II:

The work is largely an exercise in knowledge representation. McCarty does not set forth a control or process model that clarifies how a program would actually generate a legal argument ... The reported research involves a hand simulation of the arguments in one US Supreme Court case ${ }^{71}$ and work done on hand simulations of several subsequent cases.

... [TAXMAN II] has no mechanisms for comparing cases in terms of how on point they are, for distinguishing cases, or selecting the best precedents ...

McCarty's model assumes a much neater domain than exists in law. He assumes that in reality, legal cases are consistently allocated as positive and negative exemplars of concepts. They are not. He assumes that there is a near match between concepts and the features of a case that are relevant to the concept. There is not. ${ }^{72}$

McCarty's definition of legal primitives has also been criticised. Moles writes:

McCarty appears not to appreciate that 'corporations', 'securities', 'property', 'dividends' and so on are not subsumed 'beneath the law', but are each the products of complex legal analysis. The question of whether certain transactions are taxable or not is intimately tied into that legal analysis. ${ }^{73}$

Ashley makes a similar point:

The problem with such primitives, if they are taken seriously as a means for defining concepts, is that they assume what is to be shown. Far from being a primitive, that someone has a right or a duty in a given fact situation is an arguable legal conclusion that must be justified by citing authorities. ${ }^{74}$ 


\subsubsection{Bench-Capon, Kowalski and Sergot}

Bench-Capon, Kowalski, Sergot and their colleagues use PROLOG to model statutes. $^{75}$ Their approach to legislation is the most extreme of all expert system developers, due to their attitude towards knowledge acquisition. They write:

The formalisation of legislation by means of rules has almost all the characteristics of an expert system. It differs, however, in one important respect. In a classical expert system, before knowledge can be formalized, it has to be elicited from the subconscious of an expert. Eliciting this knowledge is generally regarded as the main bottle-neck in the construction of expert systems. It is entirely absent, however, in the case of legislation which is already formulated and written down. Thus the use of expert system techniques for representing legislation has virtually all the advantages of expert systems without the attendant disadvantages of eliciting the knowledge. ${ }^{76}$

This statement is nothing less than astounding. Even if it is accepted that statute law can be represented using PROLOG clauses, it is a bold claim indeed to assert that constructing those clauses requires no expertise. As Moles says, "[c]learly these researchers do not distinguish between the writing (which is the legislation) and the meaning of that writing." 77

Bench-Capon et al. have worked with several statutes. Most famous is their work with the British Nationality Act 1981 (UK). ${ }^{78}$ Consistent with their approach to knowledge acquisition, their PROLOG representation of the British Nationality Act was implemented in two months by a student, "without any expert legal assistance." 79

They also represented the Supplementary Benefits Act 1976 (UK) and its regulations using a similar method, and made a similar claim about the importance of legal expertise:

For our project, the accuracy of the representation was not a critical consideration at this [early] stage. Our formalisation could therefore be undertaken with no expert legal assistance ... In general, accuracy of the formalisation is, of course, critical, particularly if one were constructing a representation to be used in practice. ${ }^{80}$

Predictably — and correctly — this approach has been strongly criticized. Moles points out that:

This is to assume ... that the problem with regard to "accuracy" is merely a matter of changing the detail of content. It fails to appreciate that an expert may have a great many useful things to say about how one goes about the process of interpretation. The expert advice will therefore have implications for the method being employed and the way in which the knowledge is structured. ${ }^{81}$ 
Kowalski and Sergot make this assumption explicit:

Access to an expert adviser might well have changed the exact form of the rules in our program, but it would not have changed the method we used to formulate and compute with the rules. ${ }^{82}$

But how can they possibly be sure?

On the need for legal expertise in the development of legal expert systems, Susskind quotes Hayes-Roth, Waterman and Lenat:

It is very easy to be deluded into thinking one knows a great deal about the domain. Remember: the expert became one only after years of training and experience. ${ }^{83}$

The work of Bench-Capon et al. has been most vehemently criticised by Leith. He quotes them:

The formalisation of the British Nationality Act is an axiomatic theory similar, for example, to an axiomatisation of Euclidean Geometry. In principle, any logical consequence of the axiomatisation can be generated and tested mechanically $\ldots{ }^{84}$

then comments:

Does this sound like a wild claim? I suspect so. No lawyer, with whom I have so far discussed Kowalski's "legal" work, has been impressed in the least: the common reply is, "Surely he doesn't believe that?" And this is the rub. In AI research, funders are prepared to provide research funding for the most inane of ideas without appealing to those with some expertise in the area of "intelligence" being researched. ${ }^{85}$

\subsubsection{Gardner}

The aim of Gardner's research ${ }^{86}$ is not to develop a program that "solves" legal problems:

Instead, the objective is to enable the program to recognize the issues a problem raises and to distinguish between those it has enough information to resolve and those on which competent human judgments might differ. Toward this end a heuristic distinction between hard and easy questions is proposed. ${ }^{87}$

Her chosen domain is an aspect of the law of contract that is dominated by case law: offer and acceptance. She claims that, although an area based on statute might seem easier for an AI program to handle, the reverse is true. Case law must be taken into account in statutory areas too, and statutory interpretation raises its own problems. Hence, she concludes, "[b]eginning from statutes therefore seems likelier to add a layer of complication than to remove one." 88 Susskind 
agrees: "Gardner ... is right in recognising that it is unrealistic to focus on statute at the expense of precedent. There is a lesson here for all workers in the field." 89

Gardner's system has four different levels, the last of which is not implemented. The network level is an augmented transition network which represents legal states and events. The rule level is a set of rules which operate on objects at the first level. These rules are "definitions of the major concepts" 90 in the law of offer and acceptance. The third level is a set of examples which explain those predicates in the rule level which are undefined, but whose resolution is "clear"; this includes (non-legal) common sense knowledge. Gardner, citing the legal positivists, assumes that open texture does not render all cases hard:

The first three levels of the program are devoted to identifying the hard questions. From an opposite viewpoint, the first three levels try to identify the easy questions and resolve them. ${ }^{91}$

The fourth (unimplemented) level would deal with the hard questions.

Susskind comments that:

Ultimately, the human expertise that Gardner tries to encapsulate in heuristic form is the ability to assess, in advance (in a sense), whether a case does indeed raise easy or hard questions. This will indicate if a conclusion may be inferred without further ado or whether human judgement is required. She makes a brave attempt, but ... I was left wondering about the generalisability of her ideas and their applicability in other, far more extensive, branches of law. ${ }^{92}$

Ashley, too, is critical of Gardner's approach:

... an attorney distinguishes hard from easy questions in terms of comparing the strengths of the best argument he or she can make with the best arguments an opponent can make. Gardner's program provides no measure for evaluating the strengths of competing arguments ...

Even if Gardner's program evaluated case-based arguments, it could not do so very realistically given the way cases are represented. Distinguishing, for example, is not possible because there is nothing to distinguish. ${ }^{93}$

Unlike McCarty and Bench-Capon et al., Gardner does confront the arguments of the (American) Realists:

If legal realism is right, it appears to make the AI paradigm of rule-based expert systems inappropriate, at least with any simple mapping from legal rules to knowledge-base rules. There are several directions one might go instead. One direction would emphasize the idea that it is individual decisions, not general rules, that have authoritative status as law. With this emphasis, one might look for a method of reasoning from the decisions in 
past cases to a conclusion in a present case $\ldots{ }^{94}$ But this cannot be the whole story. If it were, why would present-day law professors, thoroughly aware of the insights of realism, continue to expect their students to know rules? ${ }^{95}$

This is a strange argument; it says more about the way in which the law is taught than it does about whether a rule-based approach to the law is appropriate. (And it must be remembered that the American Realists were not true rule sceptics, as discussed in $\S 2.2 .6$ above.)

Gardner identifies two other directions to take "if legal realism is right": emphasizing the behaviouristic side of legal realism (e.g. the jurimetrics research discussed in §2.3), and a third direction which Gardner herself adopts - "to retain an important place for legal rules but to reinterpret their significance." 96 She claims that:

Rulelike sentences can be understood as useful cognitive constructs, needed to find order in (or impose order upon) an unwieldy mass of individual decisions. Once articulated, they can provide guidance as to how future decisions can be kept in some rough conformance with this order; or, if the articulated rule seems to be a bad rule, it can suggest a way of saying how the course of decisions ought to be changed. ${ }^{97}$

This does not really represent a reinterpretation of the significance of rules. Only the most ardent rule-follower would suggest that rules were anything else.

Gardner develops a rule-based model for offer and acceptance cases, but makes the significant concession that:

... a rule-based model of case law must be understood, like any academic legal writing, as a secondary source. The official sources are the decisions. There has never been agreement on what it would mean for rulelike generalizations from decisions to be both accurate and appropriate. Thus, the basis for even uncontroversial rules remains undefined in legal theory. ${ }^{98}$

This is an interesting, and quite pragmatic concession, coming as it does from someone whose work has been characterized as "fairly clearly purist in nature". 99

\subsubsection{Susskind}

Susskind's work on the importance of jurisprudence to the development of legal expert systems is discussed in detail in $\S 2.2 .1$ and $\S 2.2 .7$ above. He chose the Scottish law of divorce as his first experimental legal domain. ${ }^{1}$ He has also been involved in the development of the Latent Damage System: a legal expert system concerned with the time periods within which claimants may start negligence proceedings where they have suffered latent damage or loss. ${ }^{2}$ Capper and Susskind claimed in 1988 that this was the first legal expert system built in the UK by lawyers for lawyers. ${ }^{3}$ 
Susskind believes that statutes and some cases - the clear cases - can and should be represented using rules. With clear cases, he claims, it is possible to "draw legal conclusions on the basis of literal interpretations of the formal legal sources." 4 However:

... it should not be taken for granted that the entire common law system can be reduced to a collection of rules ... Simpson has forcefully contended that such a reductionist model misrepresents the common law and is inconsistent with its development, content, and scope ... ${ }^{5}$

Other methods, he says, would be required in order to represent cases which are not clear: methods which reason with uncertainty and draw "probabilistically phrased conclusions." 6 Yet, he contends:

... although the common law may not be sufficiently represented in terms of rules, it cannot be doubted that it is invariably possible, desirable, and necessary to interpret individual cases in the form of individuated rules. ${ }^{7}$

The author doubts that it is possible, disputes that it is desirable, and demonstrates - with SHYSTER - that it is not necessary to represent cases using rules.

\subsubsection{Knowledge acquisition and representation}

Feigenbaum wrote in 1981 that "[t]here are many important problems of knowledge representation, utilisation, and acquisition that must be solved, but the acquisition problem is the most critical 'bottleneck' problem." 8 As discussed in $§ 2.4 .2$ above, Bench-Capon et al. claim that there is no such bottleneck for knowledge acquisition from statute law because legislation is "already formulated and written down." 9 However, this demonstrates a serious misunderstanding of the law. Knowledge acquisition is as much a problem in the legal domain (statute and case law) as it is in any other. The author proposes a solution to this problem, at least in regard to case law, in chapter 3.

In order to avoid the knowledge acquisition bottleneck, some researchers have examined the possibility of the machine-processing of statutes. If the only input is the words of the statute itself then this problem is one of understanding natural language - a research field in which results have not fulfilled original hopes.

A more feasible approach is to convert the words of the statute into a machinereadable form. Allen has developed one such form and claims that if statutes were drafted using this form, not only would the automatic logical analysis of their contents be possible, but humans would be able to read and work with them more easily. ${ }^{10}$ Unless and until legislation is expressed in machine-readable form-an extremely unlikely event, the desirability of which is far from clear-builders of legal expert systems which deal with statute law must use human expertise to extract knowledge from statutes. 
The process of acquiring knowledge from statutes is usually seen as a process of writing rules. However, Shannon and Golshani warn that doing this in an ad hoc fashion is unsatisfactory because the rules cannot be checked for correctness, and such an approach may lead to dissimilar rule formulations which do not work well together. ${ }^{11}$ Instead they recommend following precise and consistent methods (like those of Allen) to formulate rules. This, they say, would reduce the scope for dissimilar rule formulations. ${ }^{12}$

Shannon and Golshani's belief that there is a "correct" interpretation of a statute is shared by many researchers in this field. In believing this, they go even further than the legal positivists on whose theories much of their research is based. ${ }^{13}$

\subsubsection{Fact representation}

The representation of legal facts in a rule-based system-indeed any expert system - is difficult. As with the representation of statutes, researchers have developed normalized forms for fact representation. An example is deBessonet and Cross's atomically normalized form (ANF). ${ }^{14}$ Shannon and Golshani use a modified example, taken from some of deBessonet and Cross's work modelling the Louisiana law of causality, to demonstrate how a statement of fact can be represented using ANF.

The statement:

A lessor believes that the lessee caused a defect in the leased premises which requires that the lessee fix the defect

is represented in ANF as:

(Lessor Believes

((Lessee Caused (Property Has Defect))

Causes

(Lessee Must-Fix Defect))).

This decomposes into clauses as follows:

"Lessor Believes $A$," where

$A$ is: "B1 Causes B2," and

$B 1$ is: "Lessee Caused $C$," and

B2 is: "Lessee Must-Fix Defect," and

$C$ is: "Property Has Defect." 15

In this ANF representation, and the decomposed clauses, the lessor believes that the lessee caused a defect in the leased premises and that (as a result) the lessee is required to fix the defect. 
There is at least one other interpretation of the original statement:

"Lessor Believes B1" and " $A$ "

where $A$ and $B 1$ have the same meanings as above. This interpretation may not be "better" than Shannon and Golshani's, but it is certainly plausible. In attempting to demonstrate the uses of one method of fact representation they (unwittingly) provide an excellent example of the enormous difficulties that representing facts can present. ${ }^{16}$

It makes no more sense to say that any given representation of the facts is "correct" than it does to make the same claim of a representation of the law.

\subsubsection{The inadequacy of rules for case law}

Although several developers have used rule-based systems to model statute law and case law, rules are fundamentally inadequate for representing cases.

Tyree, Greenleaf and Mowbray claim that it is inappropriate to model case law using a rule-based system: not because it is theoretically impossible, but that "it is not the natural way in which lawyers reason with cases." 17 As discussed in $\S 2.2 .6$ above, it may be theoretically impossible to write such rules: the sceptical view of rules applies to representing statutes and cases. But there is a fundamental difference between these two sources of law which means that, even if rules are appropriate for representing statutes, they are not appropriate for representing cases.

Lawyers apply statutes in a rule-like fashion. This is understandable given the rule-like form in which they are written. However, lawyers reason with cases by arguing about their similarities and differences. As Tyree et al. explain, it used to be thought that each decided case stood for a rule of law. "It is now clear that any interpretation of the legal significance of a case must be in the larger context of the legal material in which it is embedded". ${ }^{18}$ In support of this assertion, they cite Stone who argues that:

... however much we try to conceal the truth by using singular terms like "case", "precedent", "decision" or "holding", the truth is that the ratio decidendi of a case has always to be sought in a body of judicial discourse, that is, of communications by judges which enter the legal materials as a more or less complex collocation of words in a written report. ${ }^{19}$

Perhaps, Tyree et al. suggest, this is why reasoning with case law using the usual production rule formulation has had little success. ${ }^{20}$ (The way in which lawyers argue with statutes and cases is examined further in §3.3.)

The fact that case law is embodied in cases makes knowledge acquisition for a rule-based system extremely problematic. Extracting rules from case law is difficult for a legal expert, because that is not the way in which legal experts 
view case law. Kowalski identifies this "profiling" of cases as the bottleneck in the construction of case-based reasoning legal expert systems. ${ }^{21}$

It is not possible to automate this knowledge acquisition process using inductive methods because the number of decided cases in any given area of law is usually so small that inductive inference algorithms cannot be used. ${ }^{22}$ Even if an area of law has a sufficiently large number of cases, those cases are, in a sense, unrepresentative of that area: so-called "problems of the penumbra" 23 are more likely to require determination by the courts than are straightforward cases. Once such a problem has been resolved, other cases in which the same problem arises are less likely to be taken to court - if they are taken to court they are less likely to be reported. (Reasons for the paucity of reported cases are discussed in detail in §3.13.3.) This further complicates the induction of sensible rules. And, of course, it is not at all clear how any such rules could be used in legal reasoning with cases even if they could be induced.

Developers of case-based systems like FINDER (§2.5.2) and HYPO (§2.5.3), and, to some extent, the hybrid CABARET system $(\S 2.6)$ have recognized the inadequacy of rules for representing case law. However this view has not been universally accepted. Notable amongst its critics is Berman. His argument against case-based reasoning is examined, and refuted, in $\S 3.14 .2$ - after the pragmatic approach to case law adopted in chapter 3 has been fully explained.

\subsection{Case-based systems}

Bench-Capon and his colleagues chose the British Nationality Act as an object of research because, as a fairly recently enacted statute, they claim it was "free of the complicating influence of case law." ${ }^{24}$

In Australian courts, even new Acts may be interpreted in the light of previously decided cases. This applies to decisions interpreting an expression in a similar statute, in the same or in a different jurisdiction, and (to a lesser extent) to the re-enactment of a statutory provision after a judicial decision as to its meaning. ${ }^{25}$

If Bench-Capon et al. had sought expert advice they would have learnt that, similarly, in the United Kingdom the prior legal history of the language and concepts used in a statute are relevant to its interpretation. ${ }^{26}$

A statute - even a newly enacted statute - must always be interpreted in the light of case law.

In 1988, according to Pearce and Geddes, approximately $50 \%$ of recent reported Australian cases required the court to rule upon the meaning of some legislative instrument. In a further $25 \%$ of cases courts were required to apply legislation, "its meaning this time not being in dispute." 27 Clearly, any useful legal expert system must be able to take account of the legal effect of previously decided cases. 
Ashley claims that the following operations are general to all case-based reasoning:

... (1) ordering relevant cases and potentially relevant cases in terms of how analogous they are to the problem situation, (2) selecting the most analogous cases, (3) identifying configurations of counterexamples, (4) hypothetically modifying the problem situation to explore contingencies, and (5) comparing case-based analyses of different problem situations to explain differences. ${ }^{28}$

In order to have some basis upon which to determine whether two cases are similar, information about certain attributes of those cases must be gathered. As Lambert and Grunewald explain:

The first task of [case-based] reasoning is to pick, from the infinite number of respects in which cases can be similar and dissimilar, a manageable set of [attributes] that could support a conclusion that one case is so similar to another that it will likely have the same outcome. Without such constraint, one would be faced with the commonsense impracticability, if not the jurisprudential impossibility, of defining the entire set of [attributes] that any case in the domain can have, together with the full ranges of possible values that those [attributes] could take.

Taking this arbitrarily restricted, but practically necessary, case structure, one could in principle generate the complete set of cases belonging to the domain and produce thereby a case base containing one case that would be exactly the same as any possible test case in the domain. But the size of such casebases would be intractable for case structures possessing more than an extremely small number of [attributes]. Therefore the next task of the reasoner is to install in the case base a set of cases considered typical of those one is likely to encounter in the domain. These can be either real or hypothetical cases that an expert concludes collectively capture the essence of the domain. ${ }^{29}$

As with statute law, there is no "correct" answer to a question of case law. So, as Ashley writes, a case-based legal expert system:

... does not "decide" a case; it makes arguments on behalf of the respective parties but does not necessarily determine a winner. The program would be useful as an attorney's assistant, spotting issues, strengths, weaknesses, and precedents that an attorney representing a client in the [instant case] would want to take into account, or as part of a legal tutoring system. ${ }^{30}$

Nevertheless, as discussed in $§ 2.3 .5$ above, an ability to predict the likely outcome of a case is a component of legal advice, both human and computerized.

Three examples of case-based systems are discussed below: the nearest neighbour analysis of Mackaay and Robillard (§2.5.1), and the FINDER (§2.5.2) and HYPO (§2.5.3) systems. These examples are compared with SHYSTER in $§ 3.14 .1$. 


\subsubsection{Nearest neighbour analysis}

Mackaay and Robillard were the first to examine the use of nearest neighbour analysis in predicting judicial decisions. ${ }^{31}$ Nearest neighbour analysis is a statistical technique first developed in the early 1950s. ${ }^{32}$ As Cover and Hart describe it:

The nearest neighbor decision rule assigns to an unclassified sample point the classification of the nearest of a set of previously classified points ...

If it is assumed that the classified samples ... are independently identically distributed ... it is reasonable to assume that observations which are close together (in some appropriate metric) will have the same classification, or at least will have almost the same posterior probability distributions on their respective classifications. ${ }^{33}$

Mackaay and Robillard chose as their domain Canadian capital gain cases decided in the ten years before 1968. These same cases were the subject of earlier research by Lawlor. ${ }^{34}$ They screened the set of "initial or standard" cases and removed those in which the decision did not coincide with the majority decision amongst its nearest neighbours. This, they claim, "has the advantage of purifying the standard or reference cases by eliminating those that appear to be erroneous in relation to the other ones." 35

Mackaay and Robillard used, as their similarity metric, the number of different attributes. They weighted each attribute equally, on the basis that such a method is more reliable than differential weighting via multiple regression. ${ }^{36}$ Their results compare favourably with those of Lawlor, using the same cases. ${ }^{37}$

\subsubsection{FINDER}

Tyree, Greenleaf and Mowbray use nearest neighbour analysis in their FINDER system. ${ }^{38}$ FINDER is a case-based system which gives advice in the law of troverthe law concerning the rights of the finders of lost chattels. This area of law is unusual in that it is based entirely on cases.

FINDER has a database of leading trover cases, and a set of attributes which were of legal significance in those cases: e.g. "Was the chattel attached to the land or premises where it was found?" For each of the leading cases, FINDER has a vector of attribute values; each attribute value (YES or NO) answers the corresponding attribute's question for that case. Hence, each vector of attribute values represents the facts of that case. The user provides FINDER with the facts of the instant case by giving a YES or NO answer to each of the attribute questions.

FINDER assigns a weight to each attribute, equal to the inverse of the variance of the values of that attribute across all the cases. ${ }^{39}$ FINDER uses these weights to find the weighted Euclidean distance between the instant case and each of the leading cases. It uses the nearest case, and the nearest case with the opposite result, to build an argument about the likely result in the instant case: i.e. whether 
or not the finder should be allowed to keep the found chattel. Several statistical techniques are employed to reduce the possibility of giving bad advice.

FINDER was designed to provide a comprehensive report for the user as to its opinion. As Tyree explains:

Usually explanation and justification are provided in the [non-legal expert] system as a means of establishing the user's confidence in the advice which is given by the system ...

By contrast, the legal expert system provides the justification, that is, the reasoning process, as one of its major products. The user, particularly if a professional, may care little for the prediction of the system, but the reasons provided for the prediction could be useful even if the predictions were always wrong. If the expert system can provide good arguments, then these are useful as a product in themselves. ${ }^{40}$

Tyree understates the role of prediction in a legal expert system. Certainly, a legal expert system's argument is important; so, too, is its predictive capacity (as discussed in $§ 2.3 .5$ above).

SHYSTER adopts and expands upon FINDER's approach to case law. SHYSTER's approach is detailed in chapter 3. Further details of FINDER are explained in $\S 3.14 .1$ by comparison with SHYSTER. FINDER has been simulated using SHYSTER as described in $§ 5.2 .^{41}$

\subsubsection{HYPO}

Ashley and Rissland's HYPO system is a case-based legal expert system which makes use of hypotheticals in building its arguments. ${ }^{42}$ Their aim was to build a working model of "making reasonable arguments in law," a "messy domain ... that lacks a strong theoretical model that would support deductive reasoning techniques." 43

HYPO has four knowledge sources. Its case representation language - the first knowledge source - has two tiers: "legal case frames" are used to store basic information about cases (including the instant case) and hypotheticals, and the factual objects and relations that are important in those cases; "factual predicates" are used to summarize the facts of a case represented by the legal case frames. "They are generalized factual statements that confirm whether certain legally significant relationships are true in the case". ${ }^{44}$

HYPO's case base - its second knowledge source - contains thirty legal cases (including hypotheticals) concerning trade secrets law.

Its third knowledge source are its dimensions. These are constructs for representing factors: "stereotypical facts of legal cases important for the strength of a plaintiff's position on a particular kind of claim." ${ }^{45}$ A claim can be thought of as a result that one of the parties desires. ${ }^{46}$ 
Each dimension has a list of prerequisite factual predicates: information about those facts which make a case more or less extreme along the dimension, and how a change in those facts makes the case better or worse for the plaintiff along the dimension. A dimension is applicable if all of its prerequisites are satisfied; a dimension is a near miss if all of its prerequisites are satisfied except those associated with facts which locate the instant case somewhere along the dimension. These dimensions:

... are not definitional elements of a claim; they do not purport to specify necessary and sufficient conditions for determining the existence of a claim. Instead, they represent collections of facts that tend to strengthen or weaken an assertion that the claim applies to a fact situation. ${ }^{47}$

HYPO's fourth knowledge source is a set of criteria for evaluating the strength of an argument: e.g. if one case is more "on point" than another case, the former case is better. ${ }^{48}$

Given the facts of an instant case, HYPO selects the relevant cases (the cases which share at least one dimension with the instant case) and generates an argument based on those cases. HYPO's arguments are "three-ply"; it makes a point for one side ("drawing the analogy between the problem and the precedent" ${ }^{49}$ ), responds with a point for the other side (attempting to distinguish the cited case, and citing other cases as counterexamples), then makes a final rebuttal (attempting to distinguish the counterexamples).

The best cases to cite in the first instance are those in which the result favours the chosen side, and which share (with the instant case) at least one applicable dimension favouring that side. HYPO also uses "most-on-point near-miss cases" in its arguments. These are cases that would be analogous to the fact situation if all of the dimensions that were near misses applied.

Of course, as Ashley points out, there may be more than one analogous case. Because the evaluation criteria are not well defined, the choice of most analogous case may depend on which evaluation technique is used. "Thus it may be useful for decision-making or explanation to define 'most analogous' less restrictively to yield a larger set of alternatives." 50 The most analogous cases may lead to conflicting results. However:

... comparing and contrasting the conflicting most analogous cases in a symbolic way can help educate the decision maker. She or he sees the alternative ways of answering a question and is better prepared to make a wise decision. She or he also sees how small changes in the problem could lead to different results. The law's adversarial system institutionalized this approach to decision making. ${ }^{51}$

HYPO also generates hypothetical variants of the instant case; variants that would strengthen/weaken the case for each side. ${ }^{52}$

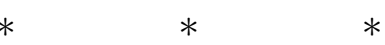


HYPO is a sophisticated case-based system. But, its complicated structure for knowledge representation means that knowledge acquisition is difficult. Although HYPO is based on a general theory of law, it was developed and tested using only one domain: American trade secrets law. The applicability of HYPO's approach in other domains has not been demonstrated.

\subsubsection{The inadequacy of semantic networks}

Semantic networks represent another potential approach to representing case law. Hafner's LRS system, for example, uses a semantic network to represent statutes and cases of relevance to negotiable instruments law. ${ }^{53}$ Branting cites two reasons for using a semantic network, rather than a "feature-vector" representation of cases, in his GREBE system: ${ }^{54}$

First, any particular set of case features can represent only a small portion of the nearly limitless variety of event sequences that can give rise to legal claims. A second and more fundamental reason is that determining the legally relevant aspects of a new case is frequently the most difficult step in legal reasoning. Systems limited to case descriptions consisting of sets of legally relevant features ignore this step or force it onto the user. The capacity to represent and create multiple, competing arguments about the legal consequences of a set of facts depends on a representation that is free of bias towards any particular analysis. Any such unbiased representation must be of a finer granularity than legally relevant features. ${ }^{55}$

The author contends that no representation can be unbiased. Furthermore, representations with exceptionally fine granularity face the same criticism as do deep conceptual models of legal reasoning (discussed in $\S 2.7$ below): viz. they operate at too high a level of abstraction for use in an expert system.

Using semantic networks raises other problems. As Branting concedes:

Unfortunately, representing complex cases in a semantic network formalism is extremely laborious and difficult. Knowledge representation is a sufficiently immature field that each new case may raise representational issues that are more difficult than the legal issues posed by the case. ${ }^{56}$

Semantic networks are of dubious use for representing case law. At the very least, their use is not consistent with a pragmatic approach to case law which, it is argued, is appropriate for expert system design.

\subsection{Hybrid systems}

The law in Australia and other common-law countries is based on both statutes and cases. For a legal expert system to be of use in most legal domains, it must be able to take account of statute law and case law. 
The first legal expert systems used rule-based methods to represent both statutes and cases. It was not until the late 1980s that a few researchers examined the possibility of combining rule-based and case-based methods to produce a hybrid legal expert system. Rissland and Skalak wrote in 1989 that "[t]his sort of hybrid architecture ... has not been much researched to date", ${ }^{57}$ and only a few projects have arisen since.

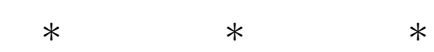

The most important of these hybrid systems is CABARET. ${ }^{58}$ CABARET deals with a small area of US taxation law: home office deductions. It treats its rule-based and case-based systems as co-reasoners, each capable of operating on its own. Some thirty heuristics control how the two systems work together. For example, some of its heuristics concern how to "broaden" a near miss rule (i.e. one in which all but one conjunct can be established):

- Use CBR [case-based reasoning] to find cases where the rule did not fire, but the consequent of the rule still held. (That is, show that the missing conjunct is not necessary to fire the rule.)

- Use CBR to find cases where the rule did fire, and point out the similarities between those cases and the present case. (Show that effectively you have the missing conjunct.)

- Use CBR to find similar cases where the rule did not fire, but the ultimate disposition of the case was consistent with the user's point of view. (Show that the rule firing is not necessary for the ultimate result the user wants. $)^{59}$

As can be seen from these heuristics, CABARET treats cases as examples of rules firing or not firing. CABARET's hybrid structure is a mixed one: it mixes rules and cases.

Branting's GREBE system ${ }^{60}$ deals with Texas worker's compensation law. GREBE is a hybrid system. Its rule base contains statutory rules and common-law rules. It uses a semantic network to represent case facts, ${ }^{61}$ and utilizes precedent constituents: "Each precedent constituent acts as a warrant connecting some subset of the facts of a precedent to one of eight distinct legal predicates." ${ }^{2}$ These precedent constituents are (effectively) rules which allow GREBE to use portions of precedents. Branting claims that this improves the system's case matching capacity; it can match portions of cases where the entire cases would not match.

Of course, all case-based systems should be capable of matching cases which are not completely identical. GREBE's precedent constituents simply constitute a different approach to the notion of similarity between cases than that adopted by (for example) FINDER and SHYSTER. 
PROLEXS (in its earlier versions ${ }^{63}$ ) was a hybrid system. If the sum of the weights of a list of weighted factors in the instant case exceeded a certain threshold, the instant case was said to match the "case-prototype" and "other types of (mostly rule-based) reasoning took over". ${ }^{64}$ The weights and the threshold were determined by the knowledge engineer.

However, the developers have since adopted a different approach. PROLEXS now uses a neural network to model the Dutch law of "suitable employment." 65 Its developers claim that neural networks have advantages over rule-based systems because a neural network generalizes from the examples it is provided with, though they concede that a neural network cannot explain its decision by referring to explicit rules since its knowledge is not symbolic. ${ }^{66}$

PROLEXS's developers comment that "the generalizing capacity of the network is already evident: in a sense the network has discovered a new rule." ${ }^{67}$ Clearly PROLEXS's approach to the law is, now, rule-based.

All of these, and other, ${ }^{68}$ hybrid systems use rules and cases to represent case law. A cleaner division between the rule base and the case base - one which does not mix rules and cases - is proposed in chapter 3 (see especially §3.2.7).

\subsection{Conceptual models: deep and shallow}

As Susskind says, "purely rule-based systems can cope only with problems for which they have explicitly represented and applicable rules". ${ }^{6}$ In response to this problem - "to fill the gaps in rule-based legal knowledge bases" 70 _ several researchers have sought to develop conceptual models of the legal domain. ${ }^{71}$ Foremost amongst these is McCarty ${ }^{72}$ who identifies "the construction of a conceptual model of the relevant legal domain" as "the most critical task in the development of an intelligent legal information system". ${ }^{73}$

McCarty advocates the development of what he calls "deep conceptual models," as opposed to "shallow" ones. As Greenleaf, Mowbray and Tyree comment, "[t]he meaning to be given to 'deep' and 'shallow' is not always clear." ${ }^{4}$ It seems that by "deep," McCarty means a conceptual model that is detailed enough to express the important facts about a particular legal world, yet abstract enough to suppress the irrelevant detail. ${ }^{75}$ According to Shannon and Golshani, truly deep conceptual models closely approach human reasoning, because they model the meaning behind words, not just the words themselves. ${ }^{76}$

Greenleaf et al. claim that there are at least three levels at which a legal expert system might be expected to model legal knowledge:

(i) the system could include only the heuristics of legal experts as to the outcomes which are likely in particular situations, but without provision of any justification based on primary legal sources; 
(ii) the representation could include justification based on the primary legal sources, but without any explicit model of those sources; certain heuristics concerning the relationship between these sources, e.g., principles of interpretation, may be implied in the representation or the inference system;

(iii) the system could include an explicit causal model which serves to define the relationships among the concepts employed in the primary sources. Justification would then, presumably, be based on the model. ${ }^{77}$

A "deep" model, then, would be one of type (iii).

Susskind claims that the results obtained by researchers who are trying to develop conceptual models "are not universalizable in so far as they do not seem to be offering any coherent guidance regarding the development of conceptual models in other legal domains." 78

But the problem is more fundamental than this. Deep conceptual models of legal reasoning, like jurisprudence, operate at too high a level of abstraction to be of use in legal expert system development.

Furthermore, developing a conceptual model of legal reasoning amounts to the writing of meta-rules ${ }^{79}$ meta-rules have all the limitations inherent in rule-based systems, with the single exception that they can cope with problems for which a rule-based system does not have explicit rules. A conceptual model does not meet the arguments put by rule sceptics (see $\S 2.2 .6$ above).

The author agrees with Greenleaf et al.:

We believe that the absence of anything even resembling a "deep" model of more than the smallest subset of the legal domain means that expert systems of type (iii) are far in the future and will require very substantial resources to build. Whether the expenditure of these resources is necessary or even justified, at least for the purpose of building expert systems, will depend upon the performance of level (ii) type systems. This is an empirical problem which may only be resolved by building systems and evaluating their performance. ${ }^{80}$

SHYSTER is a system of type (ii).

As to the value of developing conceptual models of the law, it is worth noting the words of Stone:

When excessive pretensions are avoided, it may be a worthwhile intellectual activity to construct general concepts of law, or of particular notions found within legal orders, and to draw from these logical implications concerning the conceivably possible arrangements and contents of legal orders ...

Such jurisprudential activity ... does not, despite many misconceptions, help to discover or create any actual law. Its raison d'être is basically to extend knowledge, and to order complex legal materials for mnemonic purposes of legal study and legal reform. Of course, like all efforts to extend knowledge, analytical jurisprudence also serves to sharpen the mind. Also, 
since it exposes premises from which existing legal rules may claim to have been inferred, logical analysis may provide a basis for substantive criticism of law which includes the rationalising and the testing of rationalisations offered for such rules. These are all legitimate outcomes of logical analysis; but they must always be carefully distinguished from erroneous uses of these outcomes. Among these erroneous uses is their tactical use in legal tasks, for instance to persuade courts to a particular view of the law. ${ }^{81}$

But it is just these legal tasks with which a legal expert system should be designed to deal.

McCarty describes his "language for legal discourse" as taking "a first concrete step" towards the realization of a deep conceptual model. ${ }^{82}$ Moles is disparaging: "McCarty is only taking his first steps after some 15 years (although apparently he is going to continue to use the same tools as previously)." 83

\subsection{Conclusion}

Susskind argues that "jurisprudence can and ought to supply the models of law and legal reasoning that are required for computerized implementation in the process of building all expert systems in law." 84 In fact, jurisprudence is of limited value to developers of legal expert systems. For a legal expert system to be capable of producing advice similar to that which one might get from a lawyer, it needs to operate at the same pragmatic level of abstraction as does a lawyernot at the philosophical level of jurisprudence. Many lawyers operate without jurisprudential insight; why not, then, develop a legal expert system based upon a similar pragmatic approach?

Jurisprudence is of greater value to developers of judgment machines; machines concerned more with the nature of law and justice than with the nature of lawyers' arguments. But the social desirability of judgment machines is questionable, and whether such machines are possible is debatable. It is doubtful that anyone would seriously advocate their development today.

The expectations of legal analysis systems have changed since the proposals of the 1940s and 1950s. Interest now focuses not upon judgment machines but upon legal expert systems: systems which are designed not to pass judgment but to provide legal advice. Developers have, as Stone urged them to in 1964, faced the limits of the contributions they can make. ${ }^{85}$

Providing legal advice, in an adversarial legal system like that in Australia, requires the construction and analysis of arguments and counter-arguments. It also requires prediction of the likely outcome, or at least some comment on the relative strengths of the arguments. The jurimetrics researchers focused on prediction; case-based systems like FINDER and HYPO focus on argument construction, although they can identify one side's argument as being stronger than another's. 
Although a legal expert system should have a degree of predictive power, it should not be normative. Its predictions must be based on projections from the past. It need make no allowance for new issues of policy, changing social mores, or other factors to which a judge may, openly or otherwise, have regard when coming to a decision. Without regard to these factors, a legal expert system can still provide useful legal advice. Taking all of these factors into account requires a level of predictive skill which is beyond all expert systems - and beyond all but the most prescient of lawyers.

Susskind blames the lack of successful working expert systems on the developers' failure to use jurisprudence. ${ }^{86}$ Moles attributes it to the developers' "uncritical acceptance of law as a system of rules." 87 Certainly, most legal expert system designers have embraced the work of Hart and the legal positivists, without considering the work of the legal realists or the rule sceptics.

Although a rule-based approach may be appropriate for representing statute law, it is not appropriate for representing case law, and a case-based approach is clearly inappropriate for representing statute law. A legal expert system should account for both statute law and case law. A hybrid approach, utilizing rule-based and case-based techniques, addresses these representational problems.

Despite what Bench-Capon et al. say, ${ }^{88}$ legal expertise is essential in the development of a legal expert system. Knowledge acquisition is as much a problem for the expert system designer in the legal domain as it is in any other; legal expert systems are subject to the knowledge acquisition bottleneck.

Deep conceptual models of legal reasoning are inappropriate for legal expert systems. They contribute to the difficulty of knowledge acquisition. They also operate at the same level of abstraction as jurisprudence, so they have little relevance to the pragmatic level of abstraction at which lawyers operate. A conceptual model of legal reasoning attempts to precisely model a process which is not fully understood. Hence, developers of these deep models confuse precision with accuracy.

A legal expert system should operate at the same pragmatic level of abstraction as does a lawyer. The approach taken in the development of SHYSTER, detailed in the next chapter, is based upon this conclusion. 



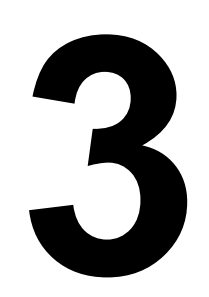

\section{A pragmatic approach to case law}

Hector Frome: "Justice is a machine that, when someone has once given it the starting push, rolls on of itself."

John Galsworthy (1910)

Justice $^{89}$

Then there is the doctrine of precedent, one of my favourite doctrines. I have managed to apply it at least once a year since I've been on the Bench. The doctrine is that whenever you are faced with a decision, you always follow what the last person who was faced with the same decision did. It is a doctrine eminently suitable for a nation overwhelmingly populated by sheep. As the distinguished chemist, Cornford, said: "The doctrine is based on the theory that nothing should ever be done for the first time."

Lionel Murphy (1979)

The Responsibility of Judges ${ }^{90}$ 


\subsection{Introduction}

The adoption of a pragmatic approach to legal expert system design is advocated in the previous chapter. This chapter describes a pragmatic approach to case law - an approach which was adopted for the development of SHYSTER.

SHYSTER's design criteria are set out in $\S 3.2$. SHYSTER is based upon a model of legal reasoning which is described in $§ 3.3$. The need for users of a system like SHYSTER to have some legal expertise is explained ( $\$ 3.4)$.

SHYSTER's knowledge representation structure is described in $\S 3.5$. This structure was designed to facilitate specification of different areas of case law using a specification language which is described in $§ 3.6$. Areas of case law are specified in terms of the cases and attributes of importance in those areas. SHYSTER weights its attributes (as described in $\S 3.7$ ) and checks for dependence between them (§3.8).

In order to choose cases upon which to construct its opinions, SHYSTER calculates distances between cases $(\S 3.9)$ and uses these distances to determine which are the nearest leading cases to the instant case (§3.10). SHYSTER uses information about these cases to construct a report (§3.11). Several safeguards are employed so as to warn the user when SHYSTER's opinion may be suspect (§3.12). Methods of testing and evaluating SHYSTER's performance are discussed in $\S 3.13$.

Conclusions are drawn in $§ 3.14$. SHYSTER's approach to case law is compared with those of other systems (§3.14.1), and an argument that an approach like SHYSTER's is inappropriate for case law is examined and refuted (§3.14.2).

\subsection{Design criteria}

The following design criteria were used in the development of SHYSTER. They are based upon the conclusions drawn in chapter 2 (see $\S 2.8$ ), and are presented here in the future tense.

\subsubsection{Users and output}

SHYSTER will be designed to be used by lawyers. Hence, SHYSTER will attempt to imitate the manner in which lawyers write advice for their clients, and for each other; its output will be constructed so as to resemble legal advice produced by and for a lawyer.

The user will be assumed to have legal expertise, though no specific expertise in the area of law about which SHYSTER is interrogated. This approach will not significantly restrict the utility of the system, and has been adopted by other expert system developers. Susskind, for example, suggests that:

... the users of expert systems in law should be lawyers, or at least those with considerable familiarity with the workings of the legal and court systems ... for a system to be used responsibly, the user must be aware of 
the possible role in legal reasoning of 'principles' ... and of 'purpose' ... Moreover, he must be sensitive to the drawbacks and implications of 'compartmentalizing' the law ... and capable too of recognizing those occasions when some legal aid cannot help him with any problem at hand ... ${ }^{91}$

A system designed to be used exclusively by lawyers is still a legal expert system as defined in $\S 2.1$.

(The role of legal expertise in the development and use of a legal expert system is further discussed in $\S 3.4$ below.)

\subsubsection{A pragmatic model of legal reasoning}

As SHYSTER's advice will be in a form that might be produced by a lawyer, SHYSTER will be designed to operate at the same level of abstraction as does a lawyer.

Lawyers operate on a day-to-day basis at a pragmatic level of abstraction which is different to the philosophical level of a jurisprudent. A legal expert system should be built upon a model of legal reasoning, but this model need not conform to any jurisprudential theory about the nature of law. The model of legal reasoning adopted for SHYSTER will reflect the way in which lawyers reason with statutes and cases in areas of private law. ${ }^{92}$

(The model of legal reasoning adopted for SHYSTER is explained in $\S 3.3$ below.)

\subsubsection{Knowledge representation}

The representational structure used for SHYSTER will be as simple as possible while complex enough to allow SHYSTER to produce good advice.

Simple knowledge representation is consistent with the choice of a pragmatic model of legal reasoning over a more complex, or deeper, model. Furthermore, complex knowledge representation requires commensurately complex knowledge acquisition. A simpler representation - one which makes no attempt to model accurately the way lawyers represent legal problems - will obviate, to some extent, the knowledge acquisition problem.

The knowledge representation structure will be similar to that used for the FINDER system (§2.5.2): cases will be represented as points in space, the dimensionality of which is the number of relevant attributes. Like FINDER, SHYSTER will allow attributes to have YES or NO values. In addition, SHYSTER will allow an attribute's value to be unknown. The smaller the distance between two points in this space, the more similar they will be considered to be.

Using such a structure, SHYSTER will choose cases to use in argument on the basis of similarity. This structure will be less sophisticated than, for example, HYPO (§2.5.3). However, this simplicity can be justified on the grounds that it will greatly simplify the knowledge acquisition process - avoiding, to some degree, 
the knowledge acquisition bottleneck. Despite this simpler structure, SHYSTER will still be capable of arguing with hypotheticals like HYPO, though not necessarily with the same sophistication.

(SHYSTER's knowledge representation structure is detailed in $\S 3.5$ below. Differences between SHYSTER and FINDER are explained in §3.14.1.)

\subsubsection{Generality of application}

SHYSTER will be of general design, so that it can operate in more than one legal domain. The only restriction upon applicable domains will be that they conform to the model of legal reasoning adopted for the system.

Generalizability has been claimed by several legal expert system developers, ${ }^{93}$ but to date none has been demonstrably generalizable (or even demonstrably widely applicable). SHYSTER will be designed so that once information about different areas of case law has been specified for it, it can be interrogated as to the ramifications of any or all of those areas of law for a given situation.

(SHYSTER's case law specification mechanism - a specification language - is introduced in $\S 3.6$, and its use is illustrated by example in $\S 4.5$ and $\S 4.6$.)

\subsubsection{Prediction and argument}

SHYSTER will be designed to make a prediction about the likely result in a case. This prediction will be based upon previously decided cases, assuming (as must any legal case-based expert system) the application of the doctrine of precedent. SHYSTER will also produce legal argument supporting, and opposing, the predicted outcome. The calculations which SHYSTER uses to reach its conclusions and to construct its legal arguments will not be part of those arguments, although they will be accessible.

A legal expert system's predictive ability and its ability to construct legal argument are both important: prediction is a valuable component of legal advice, but the nature of the adversarial system requires that a lawyer be able to argue a case, and be prepared to respond to counter-arguments.

Of course, SHYSTER will not be normative. A prediction will merely be a statement about the likely outcome - a statement about the relative strengths of the arguments that are constructed.

(SHYSTER's method of constructing arguments is explained in §3.11.)

\subsubsection{Evaluation of advice}

SHYSTER's advice will be evaluated by reference to the accuracy of its predictions and the quality of its arguments. These are two of the three criteria by which a lawyer's advice is evaluated. (The third criterion is the cost of the advice which, for the purposes of this thesis project, is ignored.) 
As a principal method of evaluating its advice, SHYSTER will be given details on real cases and its output compared with the results and legal arguments in those cases. (This and other methods of testing and evaluation are discussed in detail in $\S 3.13$.

\subsubsection{A hybrid structure}

SHYSTER will be designed so that it can be linked with a rule-based system to form a hybrid system.

A case-based system is inappropriate for representing statute law (see §2.4.7). Yet some researchers have recommended the use of both rules and cases to represent case law. As discussed in $\S 2.4 .4$, Susskind advocates the use of rules to represent statutes and clear cases, and hints that other methods of representation could be employed for the hard cases. In effect he proposes dividing a rule-based system from a case-based system at the boundary between clear and hard cases. The major drawback with this approach is that, as explained in $\S 2.2 .5$, there is considerable doubt as to whether there is such a thing as a clear case - and even if there is, no-one has devised a method of identifying one.

CABARET (see §2.6) uses rules and cases to represent case law, but its structure is a mixed one. Rules are used to guide case-based arguments. Although it is not clear exactly where the two systems meet, the division falls somewhere in case law. The problem with a mixed approach is that it complicates the model of legal reasoning and, consequently, the system's knowledge representation.

Deciding exactly how to divide the law into rule-based and case-based sources is an arbitrary process. For SHYSTER, a clean and intuitive approach will be adopted. Rule-based techniques will be used only for the representation of statute law; case-based techniques will be employed for case law. This division can be clearly defined because the sources of law (statutes and cases) are clearly defined. It also simplifies the knowledge acquisition process.

\subsection{A model of legal reasoning}

A legal expert system must be based upon a (possibly implicit) model of legal reasoning. For the development of SHYSTER, a pragmatic model of legal reasoning was adopted. That model is explained and set out here.

\subsubsection{Private and public law}

Traditionally, a distinction has been drawn between private and public law. ${ }^{94}$ Private law concerns relationships between citizens; public law concerns relationships between citizens and the state. Private law is characterized by commercial law; public law is characterized by constitutional law or international law. 
This distinction has been rejected by some jurisprudents. ${ }^{95}$ However, some areas of law are more overtly concerned with matters of policy than are others. In these areas - public law areas - precedent is given less weight than it is in private law areas. In areas of private law, where predictability is crucial, the doctrine of precedent is given greater weight (at least ostensibly ${ }^{96}$ ).

This distinction is not sharp: precedent still applies in public law, and matters of policy underlie much of private law. The difference is a matter of emphasis. However, this difference in emphasis affects the way in which lawyers reason in different areas of law. SHYSTER's model of legal reasoning assumes the application of the doctrine of precedent, and has no regard to matters of policy. This model reflects the way in which lawyers reason with statutes and cases in areas of private law.

\subsubsection{The functions of legal reasoning}

Susskind identifies three functions of legal reasoning: justification, prediction and persuasion. Judges, he says, need to provide "at least ostensible reasons" 97 to justify their decisions. Lawyers try to predict judicial or official behaviour, and try to persuade the courts. ${ }^{98}$ These three functions, Susskind points out, are "not fundamentally incompatible with one another". ${ }^{99}$ Indeed a legal expert system designer does not have to choose one of these three functions:

For there is an underlying, more restricted, and yet fundamental, model of legal reasoning, common to all three accounts of function just noted, that should be at the core of all current systems. ${ }^{1}$

Susskind, it must be remembered, claims to have found a consensus in jurisprudential theory. ${ }^{2}$ This explains his reference to $a$ model. There is no jurisprudential consensus, and there is no single model of legal reasoning. However, he is correct in that if a model of legal reasoning is to be used for the development of an expert system, it should be "common to all three accounts of function" noted above.

\subsubsection{Adopting a model of legal reasoning}

For the purposes of the development of SHYSTER, the following model of the process of reasoning with statutes and case law was adopted. ${ }^{3}$

A lawyer examines the facts of the case in question - the instant case - and determines which area of law, and which statutes (if any) apply. These statutes are applied to the facts of the instant case. The meaning of a concept in a statute may be open-textured, and may determine the result of the application of that statute to the instant case.

A lawyer argues about the meaning of an open-textured concept by reference to the facts of the instant case and those of previously decided cases. The results of some cases are desirable in that they ascribe a meaning to an open-textured 
concept which (when the statute is applied) leads to a desired result in the instant case. No two cases can be completely identical, given the plethora of facts associated with any given case. Some of these differences may be insignificant, and much of a lawyer's reasoning by analogy concerns the legal significance of these differences.

A lawyer argues with cases in the following fashion:

- If the result of a previously decided case is desirable, she/he argues that there are no legally significant differences between the previous case and the instant case, so the previous case should be followed.

- If the result of a previously decided case is undesirable, she/he argues that there is some legally significant difference between the previous case and the instant case upon which the previous case should be distinguished.

However, as Hart points out, "the class of such differences can never be exhaustively determined." 4

This model allows that some concepts in a statute may be open-textured, but assumes that these concepts are amenable to full definition-at some level of abstraction - by reference to case law. That is, open-textured statutory concepts can be defined by arguing with cases, a process which (in turn) may involve arguing with more open-textured concepts. These open-textured case-based concepts are also assumed to be amenable to definition by further reference to case law. The model does not allow that a case-based open-textured concept may be defined by reference to statutes. This assumption simplifies the model and is not a significant restriction upon its application.

The appropriate level of abstraction below which a concept is considered to be fully defined depends upon the legal expertise of the user (as explained in $\S 3.4$ below).

That part of this model that deals with cases is consistent with what Ashley describes as the standard model of analogical legal reasoning. ${ }^{5}$ This model has three steps: identifying a proper precedent; analyzing the facts and comparing and contrasting the precedent with the instant case; and determining whether the factual similarities or the differences are more important under the circumstances (i.e. deciding whether to follow or distinguish the precedent).

As Ashley points out, this model provides no guidance as to which similarities and differences are more important, or for deciding between competing analogies. He argues that "HYPO's model of analogical legal reasoning meets both of these criticisms", ${ }^{6}$ and he is right in the sense that HYPO has a well-defined algorithm for choosing important similarities and differences and for choosing between the most analogous precedents. So too with SHYSTER, as explained in $\S 3.7$ and $\S 3.9$ below. 


\subsection{Experts, users, and expert users}

As already discussed, ${ }^{7}$ legal expertise is essential to the development of a legal expert system. The author, who developed SHYSTER, is a computer scientist and a lawyer. ${ }^{8}$ He tested SHYSTER with the assistance of three lawyers expert in different areas of law. ${ }^{9}$

In the model of legal reasoning described in $\S 3.3 .3$ above, the level of abstraction at which open-textured concepts are considered to be fully defined depends on the level of legal expertise possessed by the user of the system.

Take, for example, Shannon and Golshani's discussion of the development of a rule-based expert system. They identify one of the major problems of expert system design as "the overwhelming problem of open texture, illustrated by the swelling volume of case law." ${ }^{10}$ In fact, they don't see the problem as being literally overwhelming, for they propose a solution. If a predicate is open-textured, they say, the designer has two options: to rely on the user's assessment of whether the predicate is true, or to produce new rules which define that open-textured concept.

As explained in $\S 2.4 .7$, the second option is inappropriate. The first option represents a very high level of abstraction at which open-textured statutory concepts are deemed to be fully defined. It is, of course, not a very practical level of abstraction to choose: the only people qualified to use such a system would have no need of it.

The approach adopted in SHYSTER is to assume that the user has legal expertise, though no specific expertise in the area of law that is represented. ${ }^{11}$

Legal expertise is required at the first step of the model of legal reasoning described above: i.e. determining which statute applies. Unless an expert system can cover the whole field of the law — an unlikely prospect, discussed in $\S 3.5 .1-$ then legal expertise is required at the top level to choose which expert system applies.

SHYSTER's ability to argue with instantiations of the facts of the instant case means that a user who is unable to answer any of SHYSTER's questions can force SHYSTER to consider all the possibilities (see $\S 3.11 .2$ below).

For the remainder of this thesis, the term the legal expert is used to refer to the person who specifies areas of law for SHYSTER. The user of the system-also a legal expert, though with different expertise - is called the user.

\subsection{Knowledge representation}

SHYSTER adopts and expands upon the approach to case law adopted by FINDER (§2.5.2). However, where FINDER gives advice only in the law of trover, SHYSTER gives advice in an area of case law specified by a legal expert. SHYSTER's method 
of representing knowledge about case law was designed so as to be complex enough to allow the production of good advice, yet simple enough to facilitate knowledge acquisition and avoid the knowledge acquisition bottleneck.

A program written in SHYSTER's case law specification language is called a specification. A specification may contain any number of areas. Each area is specified in terms of the attributes in that area, leading cases, hypothetical cases (ideal points), ${ }^{12}$ and relationships between these entities.

The legal expert may also specify a hierarchy of courts which applies in all areas of the specification. This hierarchy allows SHYSTER to take account of the relative ranking of leading cases when constructing its opinion.

\subsubsection{Areas}

For SHYSTER, an area of law represents an open-textured concept. Each area has at least two results; a result is a possible resolution of the open-textured concept that the area represents. ${ }^{13}$

If the attributes within an area are also open-textured, they may be defined by reference to other areas in the same specification. For example, an area may be specified in order to define a statutory open-textured concept. That area may have several open-textured attributes which are linked to other areas, which in turn may include open-textured attributes. This linkage is achieved by binding the result from one area to a value for the open-textured attribute.

There is no theoretical limit to the number of levels of areas that may be used, but every open-textured attribute must be defined, at some level, in terms of areas which have no open-textured attributes; circular definitions are not allowed. The same area may be linked to more than one attribute.

This structure can be thought of as a directed acyclical graph. Each area is an internal node, with a child node for each of its attributes. The root node is the top level-usually statutory - open-textured concept. The user need only provide values for the leaf nodes; SHYSTER determines a value for each internal node until a value is obtained for the root node. ${ }^{14}$

In 1959, Mehl suggested that "a machine covering the whole field of law would be simpler and less cumbersome than a series of machines handling separate legal sectors." ${ }^{15}$ Susskind disagrees:

... the practical problems faced in engineering a system to function even in a limited legal domain of application are so numerous that it is likely that the only way 'a vast field of law' [Mehl's words ${ }^{16}$ ] could be catered for is through the networking of smaller systems. ${ }^{17}$

The approach to case law adopted for SHYSTER lies somewhere between these two. Theoretically a single specification could represent a vast field of law using a large number of areas. 


\subsubsection{Attributes}

For SHYSTER, there are two types of attribute. An open-textured attribute is called an external attribute because it is defined by reference to another area of case law: an external area. Its value is determined by the result in that external area. The value of a local attribute must be input by the user.

Each external attribute has an association between each of the possible results in its external area and an attribute value. When the result of the external area is determined, the appropriate value is given to the attribute. Each local attribute has an associated question. The user gives the attribute its value by answering that question.

An area's attributes should represent all of (what the legal expert deems to be) the relevant similarities and differences between cases in that area. These may be questions of fact or-because an attribute may represent an open-textured concept - questions of law. A question of fact is represented by a local attribute; a question of law can be represented by a local or an external attribute, depending on the extent of its open-texture and the assumed legal expertise of the user. A point of law which is below the appropriate level of abstraction is considered to be fully defined (i.e. answerable by the user) and represented by a local attribute despite its open texture.

In allowing attributes to be questions of fact and of law, this approach differs from those discussed in chapter 2 where an attribute is considered to be a "legally important fact." However, this approach is a natural consequence of the model of legal reasoning adopted in $\S 3.3 .3$ above. ${ }^{18}$

An attribute may be a political attribute: i.e. one which Gardner would characterize as "legally irrelevant" (see $§ 2.3 .5$ ). The fact that an attribute ought not to be relevant is not a reason to exclude it; SHYSTER is predictive, not normative. An attribute need not have been judicially enunciated for the legal expert to include it in the specification. ${ }^{19}$ So, for example, if the legal expert advises that the skin colour of the person seeking relief in a certain area of law has proved to be relevant in the leading cases, an appropriate attribute should be included despite the fact that it ought not to be relevant.

Attribute direction allows any value for any attribute (local or external) to be "directed" towards a result (or results). Attribute direction indicates that the occurrence of that value for that attribute suggests that result (or results). Such an occurrence is not conclusive, merely suggestive. SHYSTER uses attribute direction as a safeguard, as explained in $\S 3.12 .4$ below.

\subsubsection{Leading cases and attribute values}

Each of SHYSTER's areas includes details of the important cases decided in that area of the law. These are the leading cases. Not all cases in the area should be specified, only the important ones: the best cases for SHYSTER to use in legal argument. 
By letting the legal expert decide which are the important cases, SHYSTER avoids the need to "screen" or "purify" the leading cases, as Mackaay and Robillard do. The legal expert's choice of cases is assumed to be a good one, even if some cases "appear to be erroneous in relation to the other ones." 20

For each leading case, the legal expert specifies various items of information including a fact vector. The fact vector is a vector of attribute values: one value for each attribute specified in the area. An attribute may have one of three values: YES, NO, or UNKNOWN. The fact vector represents the relevant facts of the case - although some of these "facts" may actually be questions of law. ${ }^{21}$

Every attribute has a value in the fact vector (wherever possible, a known value: YES Or NO) even if that attribute was not the subject of legal argument and/or was not judicially considered in the case. This is because lawyers in their arguments, and judges in their judgments, may not address all of the matters of legal significance in a case. ${ }^{22}$ Furthermore, if a case is decided before the case in which an attribute is first judicially enunciated, that attribute is assumed to have always been important, even though not mentioned until the later case.

This assumption is consistent with the declaratory theory of law. ${ }^{23}$ That theory has been strongly criticised, ${ }^{24}$ but this assumption is justified on the basis that the previously decided cases used in a specification are assumed to be consistent with each other and (taken together) to represent the law as it is, not as it used to be. ${ }^{25}$ This is not to deny that judges make law, and that case law evolves over time. It is simply assumed that the body of cases which the lawyer will use in argument in a given area of law form a consistent whole.

A result must be associated with each case. This corresponds to the decision reached by the court.

\subsubsection{Ideal points}

The legal expert may also specify ideal points. An ideal point represents the best case for a given result. ${ }^{26} \mathrm{~A}$ fact vector is specified, representing the ideal combination of attribute values for that result. For an ideal point, an attribute value of UNKNOWN indicates that the value of that attribute does not matter.

Only one ideal point may be specified for each result. ${ }^{27}$ It is useful to have ideal points in the case base because the leading cases may represent extreme combinations of attribute values. SHYSTER uses ideal points to provide a safeguard against giving erroneous advice (see $§ 3.12 .2$ below).

\subsection{A specification language}

A language was designed in which areas of case law can be specified for use by SHYSTER. Programs written in this language - specifications - reflect the structure of the SHYSTER's knowledge representation (described in $\S 3.5$ above). 
Each specification can contain any number of areas. Each area contains information on results, attributes, cases and ideal points. Results are represented as strings of characters: statements in English as to the effect of that outcome. Attributes are represented by a collection of strings explaining the effect of each possible attribute value. Local attributes have another string: a question to ask the user when determining the value of this attribute for the instant case.

Leading cases are represented by citation information (names, dates, etc.) and a vector of attribute values: one value for each of the attributes specified in this area. A vector is used to represent each ideal point.

Identifiers are used to link these concepts. So, for example, each result has an identifier and each leading case is linked to a result by use of one of those identifiers. Areas also have identifiers, so they can be accessed by name by a rule-based system and/or linked to external attributes in other areas. The result from an area is indicated to the rule-based system, or the external attribute in the case-based system, that invoked it using a result identifier. Identifiers are attached to the attribute values for an external attribute so that the result from one area can be bound to a value for that external attribute. Identifiers are also used to indicate attribute direction (linking a value for a particular attribute to a particular result).

Each specification can include a hierarchy of courts with a list of strings: each describing a court and each with an identifier. Any case in any area in the specification can be linked to a court in that hierarchy using its identifier.

In this fashion, identifiers are used to link entities within, and between, areas in the same specification.

The use of this language to specify areas of case law for SHYSTER is illustrated by example in $\S 4.5$ and $\S 4.6$. A formal definition of the syntax of SHYSTER's specification language is given in figure 4.3.

\subsection{Weighting attributes}

In order to construct its opinion, SHYSTER quantifies the "distance" between the instant and the leading cases. These calculations, explained in $\S 3.9$ below, use weighted attributes. The question of how - indeed, whether - to weight attributes to account for their relative importance is controversial.

One approach is to ask the legal expert to quantify the weight to be given to each attribute. For example, the developers of LESTER ${ }^{28}$ assign weights that, they say, "reflect the associations and relative significance an expert would attach to particular case features, with some adjustments we have arrived at through trial and error." ${ }^{29}$ But lawyers are not used to thinking in this fashion. Ashley and Rissland write:

In the legal domain, attorneys do know what [attributes] are important in a particular legal claim. Although they may be willing to say in the abstract 
that a certain [attribute] is more important than other [attributes], they almost never will venture numerical weights to distinguish the [attributes'] importance. ${ }^{30}$

Lawyers are unwilling, or unable, to give numerical weights to attributes. If attributes are to be weighted then their weights should be determined without assistance from the legal expert.

Aldenderfer and Blashfield warn:

While the concept of weighting is simple, its practice is difficult, and very few guidelines exist. Williams ${ }^{31}$ describes five types of weighting, the most common being the a priori manipulation of [attributes]. Sneath and Sokal ${ }^{32}$... argue strongly against a priori weighting, and suggest that the appropriate way to measure similarity is to give all [attributes] equal weight. ${ }^{33}$

However, Everitt points out that:

... the consequences of choosing one or other of the plethora of similarity indices are in many cases equivalent to the adoption of different schemes of [attribute] weighting, and so the concept of "equal weighting" is not as simple as it seems at first sight. It should also be remembered that [attributes] not included in the analysis are effectively given a zero weighting compared with those included. ${ }^{34}$

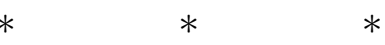

SHYSTER adopts the approach to attribute weighting used by FINDER. Each attribute value of YES is assigned a value of 1 ; each NO is assigned a value of 0 . (These values are completely arbitrary - although they must be different - as they form the basis of calculations to determine relative weights of attributes.) Each attribute is assigned a weight equal to the inverse of the variance of the numerical values of that attribute across all the leading cases.

UNKNOWN values are ignored for this purpose. It would be inappropriate to give UNKNOWN a numerical value of (say) 0.5 on the basis that it is neither YES nor NO. UNKNOWN is not a halfway point between YES and NO; it simply indicates that a value is not known.

Using the inverse of the variance is diametrically opposite to the standard approach adopted in statistical classification problems which deems high-variance variables to be the most important. ${ }^{35}$ As Tyree explains:

It is not that low-variance facts are of themselves important, but that low-variance relevant facts are more important than high-variance relevant facts. They are the facts which have been included by the expert in spite of the fact that they do not appear to assist greatly in the separation of the cases into two classes. ${ }^{36}$ 
The aim is to quantify the importance that the law attaches to an attribute, not that attribute's efficiency in discriminating between the leading cases. ${ }^{37}$

The variance $\sigma_{i}{ }^{2}$ of the numerical values of an attribute $A_{i}$ across $n$ cases is defined as follows: ${ }^{38}$

$$
\sigma_{i}^{2}=\frac{1}{n} \sum_{j=1}^{n}\left(A_{i j}-\bar{A}_{i}\right)^{2}
$$

where $A_{i j}$ is the value of the $i$ th attribute for the $j$ th case, and $\bar{A}_{i}$ is the mean of all attribute values for the $i$ th attribute. Because $A_{i j}$ is either 0 or $1, \sigma_{i}{ }^{2}$ ranges from 0 to 0.25 . Consequently an attribute's weight ranges from 4 to infinity.

An attribute with all known values the same has zero variance and is assigned infinite weight. ${ }^{39}$ This seems paradoxical, but is actually appropriate. Consider an area of law in which a value of YES for an attribute $A_{X}$ is enormously suggestive of a result $R .^{40}$ In an instant case in which $A_{X}=$ YES it may be clear that, on the strength of $A_{X}$ alone, the likely result is $R$. Such a case may never reach a court; a result of $R$ may be so likely that no-one seeks judicial determination of the instant case. So, it is possible that $A_{X}=$ No in all of the leading cases in this area. If SHYSTER is asked for its opinion on an instant case where $A_{X}=$ YES, it is appropriate that SHYSTER treats that attribute value as being of considerable importance.

HYPO does not assign weights to attributes because, according to Ashley, the concept of an attribute's weight "though intuitively attractive, is, on closer view, highly problematic." 41 He gives five reasons for not giving attributes numerical weights. Three of these can be easily countered in SHYSTER's case.

- Domain experts may not reason in terms of weighting schemes, especially numerical ones. Attorneys generally concede that [attributes] are useful in analyzing legal problems, but they rarely are willing to apply weights or probabilities to those [attributes].

For this reason, as discussed above, SHYSTER does not require the legal expert to weight attributes.

- Weighting [attributes] is not justified by any authoritative means. Even if attorneys did assign weights to [attributes,] they would disagree on what those weights should be. In addition, attorneys would not actually be able to cite [attribute] weights in their arguments to a court because weighting is not an accepted kind of argument.

Bing is unconvinced by Ashley's reference to "authoritative means", which he sees as criticizing a weighting scheme "for not being an objective method, or a method which is related to the legal argument." ${ }^{42}$ SHYSTER's method is objective, though, Bing claims, "its relation to legal argument is less obvious." 43 
And, as explained in $\S 3.13 .2$ below, SHYSTER's advice is evaluated by reference to the accuracy of its predictions and the quality of its arguments. It does not use attribute weights in its arguments, only in choosing the cases to use in those arguments, so the extent to which its weighting method relates to legal argument is irrelevant.

Furthermore, the fact that lawyers would disagree on weights is no reason not to weight attributes. It means that no weighting method can be definitive; it does not mean that no weighting method can be effective.

- Reduction to numerical weights obliterates information needed for symbolic comparison of cases. The business of attorneys is arguing about the competing [attributes] in the light of the precedents. If the [attributes] are collapsed into a number, there is nothing left to argue about.

Although this may be true given HYPO's approach to argument (discussed below, and in $§ 2.5 .3)$ it is not true of SHYSTER. SHYSTER does not collapse attribute information into a number, but uses numbers to decide which cases to use in argument. The choice of cases determines the manner in which attributes are used in argument.

Two of Ashley's reasons for not weighting attributes (both closely related) do apply to SHYSTER:

- [An attribute's] weight is highly contextual and depends on individual problem situations. Although an attorney may consider one factor generally to be more important than another, she or he is always mindful of peculiar cases where the opposite is true ...

- Premature commitment to a weighting scheme may cut off fruitful lines of inquiry. A rigid scheme may cause an attorney to overlook a factor that, although not generally important, is crucial in a particular situation. ${ }^{44}$

To address these concerns, Ashley and Rissland advocate a "symbolic least commitment approach" to attribute weighting, in which weighting is postponed for as long as feasible. ${ }^{45}$ This approach is adopted in HYPO. As Ashley explains:

HYPO clusters the applicable [attributes] according to how they appear in the most-on-point cases, interprets the effect of the clustered [attributes] in the light of the most-on-point cases, and criticizes and tests the interpretations in the light of the salient differences among the most-on-point cases by distinguishing precedents, citing counterexamples, and posing hypotheticals that change magnitudes and combinations of [attributes] in the problem ...

Although by the end of [this process] HYPO has not actually assigned weights to the competing [attributes], it has dealt symbolically with the problem of weighting. It has generated precedent-citing arguments in favor of alternative interpretations of the weights of the [attributes] within the context of the problem. ${ }^{46}$ 
SHYSTER's simple knowledge representation means that HYPO's approach to weighting is inappropriate for SHYSTER. Nevertheless, there can be no doubt that, in the legal domain, some attributes are of greater importance than others. For this reason, and despite the problems identified by Ashley, SHYSTER weights its attributes. However, it is important not to lose sight of what Lambert and Grunewald call "the necessary arbitrariness of any weighting function." 47

SHYSTER's approach to weighting is discussed in $\S 6.3$ in the light of the case studies performed in chapter 5 .

\subsection{Detecting attribute dependence}

A functional dependence exists between two attributes when there is a function which maps the values of one of the attributes directly to the values of the other. A stochastic dependence exists when the occurrence of one event affects the probability of the occurrence of another event. Functional dependence or stochastic dependence between attributes may indicate shortcomings in the legal expert's specification.

Consider this (extreme) example. The legal expert chooses two attributes which, although worded differently, are identical: i.e. they ask precisely the same question, in different ways. The values of these two attributes across the leading cases are identical, and each attribute is assigned the same weight. By effectively choosing the same attribute twice, the legal expert has given that attribute twice its appropriate weight.

In this example, there is both a functional dependence and a stochastic dependence between the two attributes. ${ }^{48}$ Alternatively, if two attributes differ only very slightly in their values across the cases then there is a stochastic dependence (though no functional dependence) between them: i.e. the occurrence of a given value for one attribute affects the probability of the occurrence of a given value for the other. The legal expert may have chosen two very similar attributes. Dependency also exists where two attributes are completely, or very nearly completely, different.

For every pair of attributes there is a pair of attribute values corresponding to each of the leading cases. SHYSTER detects attribute dependence by examining the known pairs (pairs where both the attribute values are known) for each pair of attributes in the area. If either, or both, of a pair of attribute values is UNKNOwN then both values are ignored. (An UNKNOWn value is not a value that could form part of a dependency; it is an absence of known values.)

Attribute dependence does not necessarily mean that the specification must be re-written. Even if two attributes have exactly the same values across the leading cases, the legal expert may decide that they do not ask the same question: i.e. that a case can be imagined where the values of those two attributes are different, despite the fact that they do not differ in any of the leading cases. ${ }^{49}$ SHYSTER 


$\begin{array}{lll}\text { Equivalence function: } & \mathrm{YES} \mapsto \mathrm{YES}, \quad \mathrm{NO} \mapsto \mathrm{NO} \\ \text { Inverse function: } & \mathrm{YES} \mapsto \mathrm{NO}, \quad \mathrm{NO} \mapsto \mathrm{YES} \\ \text { YES function: } & \mathrm{YES} \mapsto \mathrm{YES}, \quad \mathrm{NO} \mapsto \mathrm{YES} \\ \text { NO function: } & \mathrm{YES} \mapsto \mathrm{NO}, \quad \mathrm{NO} \mapsto \mathrm{NO}\end{array}$

Figure 3.1: The four forms of functional dependence. Only the equivalence and inverse functions are detected by SHYSTER; the YES and No functions do not indicate a relationship between two attributes.

warns the legal expert of attribute dependence so that she/he can reconsider her/his choice of attributes.

It is not possible to prove that two attributes are stochastically independent, hence it is not possible to detect stochastic dependence with complete certainty. However, SHYSTER can detect evidence of stochastic dependence, and warn the legal expert that such evidence exists.

\subsubsection{Functional dependence}

Detecting functional dependence between attributes is straightforward. There are only four functions which map an attribute $A_{X}$ to an attribute $A_{Y}$. These functions - the four forms of functional dependence - are described in figure 3.1. The equivalence function produces the same value in $A_{Y}$ as in $A_{X}$; the inverse function produces the opposite value; the YES function sets $A_{Y}$ to YES regardless of the value of $A_{X}$; and the NO function sets $A_{Y}$ to NO regardless of the value of $A_{X}$.

SHYSTER checks all pairs of attributes, and warns of any equivalence function, or inverse function. YES functions and NO functions are ignored. Each is a constant function: i.e. each produces the same result regardless of its argument. Hence, a YES or No function does not indicate a relationship between two attributes. $^{50}$

\subsubsection{Stochastic dependence}

Two events $E_{1}$ and $E_{2}$ are said to be stochastically independent if the probability of their both occurring is equal to the product of the probabilities of each of them occurring:

$$
P\left(E_{1} \wedge E_{2}\right)=P\left(E_{1}\right) P\left(E_{2}\right) .
$$

There are four types of known pair: YES/YES, YES/NO, NO/YES, and NO/NO. Let $N$ be the number of known pairs in the attributes $A_{X}$ and $A_{Y}$. Let $x$ be the number of yess in $A_{X}$, and let $y$ be the number of yess in $A_{Y}$. Let $n$ be the 
$A_{X}:$ NO NO NO NO NO YeS YeS YeS YeS YeS NO NO YeS

$A_{Y}:$ No yes NO NO NO NO YeS YeS YeS YeS YeS NO YeS

Figure 3.2: Example attribute values for two attributes $A_{X}$ and $A_{Y}$.

number of YES/YEs pairs for the attributes $A_{X}$ and $A_{Y}$. Because known attribute values are binary, the number of any type of known pair is linked to the numbers of all other types, and can be expressed in terms of $N, x, y$ and $n$ :

$$
\begin{aligned}
& \text { number of YES/YES pairs }=n \quad \text { (by definition), } \\
& \text { number of YES/NO pairs }=x-n, \\
& \text { number of NO/YES pairs }=y-n, \\
& \text { number of NO/NO pairs }=N-x-y+n .
\end{aligned}
$$

So, in order to detect evidence of stochastic dependence it is only necessary to examine the expectation and actual occurrence of one of the four possible pairs. SHYSTER examines only the YES/YES pairs.

Let $P(n)$ be the probability of there being exactly $n$ YES/YES pairs given $N$, $x$ and $y$, and assuming random data:

$$
\begin{aligned}
P(n) & =\frac{\text { number of configurations with exactly } n \text { YES/YES pairs }}{\text { total number of configurations }} \\
& =\frac{\left(\begin{array}{l}
N \\
n
\end{array}\right)\left(\begin{array}{l}
N-n \\
x-n
\end{array}\right)\left(\begin{array}{l}
N-x \\
y-n
\end{array}\right)}{\left(\begin{array}{l}
N \\
x
\end{array}\right)\left(\begin{array}{l}
N \\
y
\end{array}\right)} \\
& =\frac{\left(\begin{array}{l}
y \\
n
\end{array}\right)\left(\begin{array}{l}
N-y \\
x-n
\end{array}\right)}{\left(\begin{array}{l}
N \\
x
\end{array}\right)}
\end{aligned}
$$

Because the variables $N, x, y$, and $n$ specify a combination of attribute values, $P(n)$ is the probability of that combination occurring.

The manner in which SHYSTER uses this formula for $P(n)$ to check for evidence of stochastic dependence between attributes is best illustrated by example.

Consider two attributes $A_{X}$ and $A_{Y}$, with known attribute values as shown in figure 3.2. ${ }^{51}$ There are thirteen known pairs: $N=13$. There are six yess in $A_{X}: x=6$. There are seven Yess in $A_{Y}: y=7$. The formula for $P(n)$ can be used to calculate the probability that $n=i$ for $i=0 \ldots N$. Figure 3.3 lists these probabilities in the column labelled " $P(n=i)$ ".

There are five Yes/yes pairs in $A_{X}$ and $A_{Y}: n=5$. Figure 3.3 shows that the probability of there being exactly five YES/YES pairs (given the distribution of YESs and NOs in $A_{X}$ and $A_{Y}$, and assuming random data) is 0.0734 . 


\begin{tabular}{|c|c|c|c|}
\hline$i$ & $P(n=i)$ & $P(n \leq i)$ & $P(n \geq i)$ \\
\hline \hline 0 & 0.0006 & 0.0006 & 1.0000 \\
1 & 0.0245 & 0.0251 & 0.9994 \\
2 & 0.1836 & 0.2087 & 0.9749 \\
3 & 0.4079 & 0.6166 & 0.7913 \\
4 & 0.3059 & 0.9225 & 0.3834 \\
5 & 0.0734 & 0.9959 & 0.0775 \\
6 & 0.0041 & 1.0000 & 0.0041 \\
7 & 0.0000 & 1.0000 & 0.0000 \\
$\vdots$ & $\vdots$ & $\vdots$ & $\vdots$ \\
13 & 0.0000 & 1.0000 & 0.0000 \\
\hline
\end{tabular}

Figure 3.3: The probabilities for the example attributes $A_{X}$ and $A_{Y}$ defined in figure 3.2. $N=13, x=6, y=7$.

But, the probability of there being exactly five YES/YES pairs is not of much use on its own. What is important is whether the number of YES/YES pairs is unusually high, or unusually low.

The " $P(n \leq i)$ " column gives the probability of there being $i$ YES/YES pairs or fewer: i.e. the cumulative total of the " $P(n=i)$ " column. The " $P(n \geq i)$ " column gives the probability of there being $i$ YES/YEs pairs or more: i.e. the cumulative total of the " $P(n=i)$ " column, summing backwards from the bottom.

The probability of there being five YES/YES pairs or fewer is 0.9959. The probability of there being five YES/YEs pairs or more is 0.0775 . It is necessary to choose a threshold of likelihood, below which a given number of YES/YES pairs will be considered unusually high or unusually low.

The method that SHYSTER uses to detect stochastic dependence is analogous to using the $\chi^{2}$ ("chi-square") test for independence, ${ }^{52}$ except that that test assumes a normal distribution and, hence, a large sample while the test used by SHYSTER calculates probabilities exactly and does not require a large sample. As such SHYSTER's method is an example of what is called Fisher's exact test. ${ }^{53}$

When using the $\chi^{2}$ test, it is common practice to use a threshold of 0.05. Using this threshold, the occurrence of five YEs/yes pairs in $A_{X}$ and $A_{Y}$ is neither unusually high nor unusually low. This means that the combination of attribute values in those attributes is not unusual: there is no evidence of stochastic dependence between $A_{X}$ and $A_{Y}$.

Had there been six YES/YES pairs, that would have been unusually high because $P(n \geq 6)=0.0041$. One or no YEs/Yes pairs would have been unusually low, because $P(n \leq 1)=0.0251$. 


\subsection{Calculating distances}

SHYSTER's choice of the cases with which to construct its arguments is based upon a notion of similarity between cases. SHYSTER quantifies this similarity using distance measures: the smaller the distance between two cases, the more similar they are.

SHYSTER calculates two different types of distance: the known distance is defined as the sum of the weights of every attribute for which those two cases have different known values; the unknown distance is defined as the sum of the weights of every attribute for which either of the two cases has an unknown value.

A known distance of zero indicates that the two cases are identical - at least as far as the known attributes are concerned. A large unknown distance indicates that the values of some important (i.e. heavily weighted) attributes were unknown, casting some doubt on the reliability of the known distance calculation for those two cases. The unknown distance can be thought of as a measurement of possible error: it is the maximum distance that could be added to the known distance if all of the unknown attribute values were known.

Known and unknown distances are calculated between the instant case and each of the leading cases. These distance measurements are treated as characteristics of each leading case. So, for example, a statement that case $j$ has a smaller known distance than case $k$ means that the known distance between case $j$ and the instant case is less than the known distance between case $k$ and the instant case.

Statisticians make use of many different similarity measures. However, two aspects of SHYSTER's approach to case law mean that each of the commonly used similarity measures reduces to one of three measures - six, allowing for attribute weighting. This is because SHYSTER's known values are binary, and because its choice of cases is based on the relative distance/similarity between cases: i.e. it has regard to the fact that a case $j$ is further from a case $k$ than a case $\ell$, but not how much further.

The decision to use known and unknown distance as SHYSTER's similarity measure is explained in $\S 3.9 .3$ below, after a brief survey of the different techniques of measuring similarity.

\subsubsection{Similarity measures}

Cluster analysis is the separation of data into groups on the basis of similarity. Entities are grouped so that two entities in the same group are more similar than two entities in different groups. Similarity measures are used to quantify the similarity between every entity and every other entity. SHYSTER does not 
perform cluster analysis, but it does quantify the similarity between the instant case and each of the leading cases. This process takes $O(n)$ time, where $n$ is the number of cases, whereas cluster analysis takes $O\left(n^{2}\right)$ time.

Many different types of similarity measure have been developed. The most widely used measures are called metrics. Aldenderfer and Blashfield explain:

The quantitative estimation of similarity has been dominated by the concept of metrics; this approach to similarity represents cases as points in a coordinate space such that the observed similarities and dissimilarities of the points correspond to metric distances between them ... The dimensionality of the space is determined by the number of [attributes] used to describe the cases. ${ }^{54}$

For a similarity measure to be a metric it must satisfy the following four criteria: $:^{55}$

- Given two cases $j$ and $k, d_{j k}=d_{k j} \geq 0$, where $d_{j k}$ is the distance between case $j$ and case $k$.

- Given three cases $j, k$ and $\ell, d_{j k} \leq d_{j \ell}+d_{k \ell}$ (this is called the triangle inequality or the metric inequality).

- Given two cases $j$ and $k$, if $d_{j k} \neq 0$, then $j$ is not identical to $k$.

- Given two identical cases $j$ and $j^{\prime}, d_{j j^{\prime}}=0$.

Many researchers have argued against the use of similarity measures which do not meet these criteria. Aldenderfer and Blashfield point out that:

[Measures] that are not metrics may not be jointly monotonic; that is, the values of different [measures] used with the same data will not necessarily vary conjointly, raising the disturbing issue that these [measures] could suggest quite different relationships among the entities. ${ }^{56}$

However, it is not essential that a similarity measure be a metric. For example, the Pearson product-moment correlation coefficient ("a popular similarity measure" ${ }^{57}$ discussed below) is not a metric.

There are four different kinds of similarity measures: distance measures, association coefficients, correlation coefficients, and probabilistic similarity coefficients.

Strictly speaking, probabilistic similarity coefficients do not actually calculate the similarity between two cases. They take into account the distribution of the frequencies of the attribute values over all the cases, and are calculated during the formation of clusters. ${ }^{58}$ Probabilistic similarity coefficients are inappropriate for use with SHYSTER because it does not perform cluster analysis.

The three kinds of similarity measure appropriate for SHYSTER are distance measures, association coefficients, and correlation coefficients. 


\section{Distance measures}

Two popular distance measures are Euclidean distance and Manhattan distance. The Euclidean distance between two cases $j$ and $k$ is defined as

$$
\sqrt{\sum_{i=1}^{n}\left(A_{i j}-A_{i k}\right)^{2}}
$$

where $A_{i j}$ is the value of the $i$ th attribute for the $j$ th case, ${ }^{59}$ and $n$ is the number of attributes. ${ }^{60}$ The Manhattan distance is defined as

$$
\sum_{i=1}^{n}\left|A_{i j}-A_{i k}\right|
$$

Both Euclidean distance and Manhattan distance are specific examples of the class of metric distance functions known as Minkowski metrics. The Minkowski metric is defined as

$$
\left(\sum_{i=1}^{n}\left|A_{i j}-A_{i k}\right|^{K}\right)^{1 / K}
$$

where $K$ is some constant: for Euclidean distance, $K=2$; for Manhattan distance, $K=1$. In the binary case (e.g. SHYSTER),

$$
\left|A_{i j}-A_{i k}\right|^{K}= \begin{cases}0, & \text { if } A_{i j}=A_{i k} \\ 1, & \text { if } A_{i j} \neq A_{i k}\end{cases}
$$

Hence, for binary attribute values, the Minkowski metric reduces to

$$
\Delta_{j k}^{1 / K}
$$

where $\Delta_{j k}$ is the number of differences in the corresponding attribute values of case $j$ and case $k$.

For increasing values of $\Delta_{j k}$, this metric always increases regardless of the value of $K$. As SHYSTER is concerned only with the relative distance between cases, it does not matter which value of $K$ is used. Choosing $K=1$ gives a distance measure $d_{j k}$ :

$$
d_{j k}=\Delta_{j k} .
$$

This distance measure is a metric as it satisfies all four metric criteria. It ranges from 0 to $n$ : the smaller the value of $d_{j k}$, the nearer case $j$ is to case $k$.

\section{Association coefficients}

Association coefficients are used to describe similarity between cases with binary attributes. These coefficients are usually expressed in terms of $a, b, c$ and $d$; the two-way association table in figure 3.4 defines these variables. 


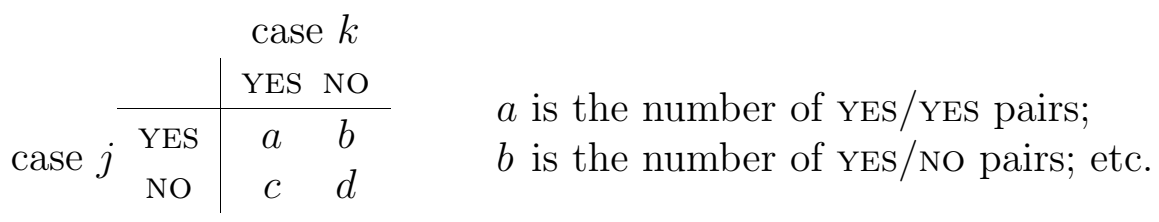

Figure 3.4: A two-way association table defining $a, b, c$ and $d$.

Many different association coefficients have been proposed-Aldenderfer and Blashfield state that there are more than thirty. ${ }^{61}$ According to Everitt, the reason for this proliferation of coefficients is:

... uncertainty over how to incorporate negative matches $[d]$ into the coefficients, and also whether or not matched pairs of [attributes] are equally weighted, or carry twice the weight of unmatched pairs, or unmatched pairs carry twice the weight of matched pairs. ${ }^{62}$

Everitt gives several examples of association coefficients. Jaccard's coefficient,

$$
\frac{a}{a+b+c}
$$

is one measure of the association between two cases $j$ and $k$. It ignores $d$ - the number of NO/NO pairs. This coefficient was developed for use in applications where it would be inappropriate to treat two cases as being similar for lacking the same features as well as sharing the same features. ${ }^{63}$ Similarly,

$$
\frac{2 a}{2 a+b+c} \quad \text { and } \quad \frac{a}{a+2(b+c)}
$$

ignore negative matches - and give double weight to matched pairs and unmatched pairs, respectively. And

$$
\frac{a}{a+b+c+d}
$$

gives no positive significance to negative matches.

Coefficients such as these are inappropriate for use by SHYSTER because the occurrence of NO/NO pairs is as important as the occurrence of YES/YES pairs. An attribute value of no does not indicate the lack of a feature; it means that the answer to the attribute's question is "no." Simply rephrasing attribute questions could turn all YES/YES pairs into NO/NO pairs. 
The other two example coefficients that Everitt gives are

$$
\frac{a+d}{a+b+c+d} \quad \text { and } \quad \frac{2(a+d)}{2(a+d)+b+c} .
$$

The first, called the simple matching coefficient, is the number of matching pairs as a fraction of the total number of pairs. The second gives matching pairs twice the weight of non-matching pairs. Coefficients such as these are appropriate for use by SHYSTER because they do not distinguish between YES/YES pairs and NO/NO pairs.

As $\Delta_{j k}=b+c$ and $n=a+b+c+d$, coefficients of this kind can be generalized to

$$
\frac{K\left(n-\Delta_{j k}\right)}{K\left(n-\Delta_{j k}\right)+\Delta_{j k}}
$$

where $K$ is some constant. For increasing values of $\Delta_{j k}$, this coefficient always decreases regardless of the value of $K$. Because SHYSTER is concerned only with relative measures of similarity, it does not matter which value of $K$ is used. The simplest is $K=1$, giving

$$
\frac{n-\Delta_{j k}}{n}
$$

which ranges from 0 to 1 . Subtracting it from 1 yields an inversely proportional association coefficient $S_{j k}$ which satisfies all four metric criteria:

$$
S_{j k}=\frac{\Delta_{j k}}{n}
$$

This association coefficient also ranges from 0 to 1: the smaller the value of $S_{j k}$, the nearer case $j$ is to case $k$. (Note that, in SHYSTER, $n$ may vary within the same area of law because $n$ is the number of pairs of attributes with known values. Hence, $S_{j k}$ is not proportional to $d_{j k}$ as derived above.)

\section{Correlation coefficients}

The most popular correlation coefficient is Pearson's product-moment correlation coefficient. ${ }^{64}$ This coefficient is defined as

$$
r_{j k}=\frac{\sum_{i=1}^{n}\left(A_{i j}-\bar{A}_{j}\right)\left(A_{i k}-\bar{A}_{k}\right)}{\sqrt{\sum_{i=1}^{n}\left(A_{i j}-\bar{A}_{j}\right)^{2} \sum_{i=1}^{n}\left(A_{i k}-\bar{A}_{k}\right)^{2}}}
$$

where $n$ is the number of attributes, $A_{i j}$ is the value of the $i$ th attribute for the $j$ th case, and $\bar{A}_{j}$ is the mean of all attribute values for the $j$ th case. 
In general, this correlation coefficient has several limitations. ${ }^{65}$ It is not a true metric. ${ }^{66}$ Furthermore, as Aldenderfer and Blashfield explain:

... the use of [this] method to calculate the correlation of cases does not make statistical sense, because one must obtain the mean value across different [attribute] types rather than across cases, as in the standard use of the method. The meaning of the "mean" across these [attributes] is far from clear. ${ }^{67}$

Although this is true in general, in SHYSTER all attributes are of the same type. Hence it does make sense to calculate the mean across the attributes.

Despite its drawbacks this coefficient has been widely used. It ranges from -1 to $1: 68$ the larger the value of $r_{j k}$, the nearer case $j$ is to case $k$.

\subsubsection{Weighted similarity measures}

Each of the three similarity measures discussed in $\S 3.9 .1$ above can be weighted to take account of the importance of each attribute.

The weighted distance measure $d_{j k}^{\prime}$ is

$$
d_{j k}^{\prime}=\sum_{i=1}^{n}\left|A_{i j}-A_{i k}\right| \times w_{i}
$$

where $w_{i}$ is the weight of the $i$ th attribute. It ranges from 0 to $\sum_{i=1}^{n} w_{i}$ : the smaller the value of $d_{j k}^{\prime}$, the nearer case $j$ is to case $k$.

The weighted association coefficient $S_{j k}^{\prime}$ is

$$
S_{j k}^{\prime}=\frac{\sum_{i=1}^{n}\left|A_{i j}-A_{i k}\right| \times w_{i}}{\sum_{i=1}^{n} w_{i}}
$$

It ranges from 0 to 1 : the smaller the value of $S_{j k}^{\prime}$, the nearer case $j$ is to case $k$.

The weighted correlation coefficient $r_{j k}^{\prime}$ is

$$
r_{j k}^{\prime}=\frac{\sum_{i=1}^{n}\left(A_{i j} \times w_{i}-\bar{A}_{j}^{\prime}\right)\left(A_{i k} \times w_{i}-\bar{A}_{k}^{\prime}\right)}{\sqrt{\sum_{i=1}^{n}\left(A_{i j} \times w_{i}-\bar{A}_{j}^{\prime}\right)^{2} \sum_{i=1}^{n}\left(A_{i k} \times w_{i}-\bar{A}_{k}^{\prime}\right)^{2}}}
$$

where $\bar{A}_{j}^{\prime}$ is the weighted mean of all attribute values for the $j$ th case. It ranges from -1 to 1 : the larger the value of $r_{j k}^{\prime}$, the nearer case $j$ is to case $k$. 


\subsubsection{Choosing a similarity measure}

For SHYSTER - and allowing for the weighting of attributes - each of the commonly used similarity measures reduces to one of six measures: $d_{j k}, d_{j k}^{\prime}, S_{j k}, S_{j k}^{\prime}$, $r_{j k}$ and $r_{j k}^{\prime}$.

Mackaay and Robillard (§2.5.1) use $d_{j k}$ as their similarity measure; FINDER $(\S 2.5 .2)$ uses $d_{j k}^{\prime}$.

According to Ashley, HYPO (§2.5.3) uses four comparison metrics: ${ }^{69}$ The basic measure is on pointness: the degree of overlap of dimensions shared by the instant case and a given case, relative to that of other cases. HYPO also has regard to the outcome of a leading case (because "depending on the procedural context of the case, some outcomes are more determinative than others" ${ }^{70}$ ), the magnitude of shared dimensions, and a case's potential relevance as a near miss. A near miss case is examined to see whether a small hypothetical change would make that case more on point.

Strictly speaking, HYPO uses only one similarity measure: on-pointness. The outcome of a case and the magnitude of shared dimensions are weighting considerations; ${ }^{71}$ making hypothetical changes to a case is an argument technique. None of these three is a similarity measure (in the sense that that term is used in this thesis).

When choosing an appropriate similarity measure for a particular set of data, it is important to have regard to the sort of data, and to what is to be measured. Because SHYSTER treats cases as points in $n$-dimensional space, where $n$ is the number of attributes, a metric should be an appropriate similarity measure. As discussed in $\S 3.7$ above, attribute weighting is also appropriate.

SHYSTER's known and unknown distance measures are variations on the weighted distance measure $d_{j k}^{\prime}$ which is a metric. However, it is important to note Sneath and Sokal's warning that "when all is said and done, the validation of a similarity measure ... in a given field has so far been primarily empirical". ${ }^{72}$

The testing of SHYSTER is described in chapter 5. In order to compare empirically the various similarity measures, SHYSTER also calculates values of $d_{j k}$, $S_{j k}, S_{j k}^{\prime}, r_{j k}$ and $r_{j k}^{\prime}$. These extra measures are used as safeguards (see $§ 3.12 .1$ below).

\subsubsection{Infinite distance}

As explained in $\S 3.7$ above, each attribute's weight ranges from 4 to infinity. In determining a distance - known or unknown-SHYSTER may have to deal with one or more attributes with infinite weight.

To handle such possibilities, SHYSTER's distances have an infinite and a finite component. The infinite component is the number of infinitely weighted attributes that differ between the two cases. A distance of " $2 \infty+x$ " is considered to be 
greater than " $\infty+y$ ", regardless of the values of $x$ and $y$ (the finite components). The infinite component of this first distance should not be thought of as being the sum of two infinities. It represents the effect, upon the distance between two cases, of an infinite weight in each of two dimensions in $n$-dimensional space.

\subsection{Nearest cases and nearest results}

Having determined the distance between the instant case and each of the leading cases, SHYSTER can decide which of the leading cases is nearest to the instant case. Having chosen a nearest case, SHYSTER can decide upon the most likely result: the result in the nearest case.

In fact, SHYSTER deals with several nearest cases and can deal with several cases which are equidistant from the instant case.

\subsubsection{Nearest cases}

The nearest known neighbour is the case with the smallest known distance, and with no unknown distance (i.e. with an unknown distance of zero). The nearest unknown neighbour is the case which has the smallest sum of the known and unknown distances, and non-zero unknown distance. The nearest of these, the case with the smallest sum of known and unknown distances, is called the nearest neighbour.

Consider the example distances in figure 3.5, taken from one of the tests performed in chapter $5 .^{73}$ There are fourteen cases: $C_{1} \ldots C_{14}$. Each case has a known and an unknown distance. Zero distances are indicated by "-". The nearest known neighbour (and the nearest neighbour) is $C_{5}$; the nearest unknown neighbour is $C_{3}$.

\subsubsection{Nearest results}

The result of the nearest neighbour is termed the nearest result. Applying the doctrine of precedent, SHYSTER assumes that the decision in the instant case will be the same as that in the nearest neighbour. SHYSTER also finds the nearest known and nearest unknown neighbours for every other result-i.e. from amongst the cases in which another result was reached. These are termed the nearest known other and the nearest unknown other. The nearest of these cases is called the nearest other; there is a nearest other for each other result.

The example cases whose distances are given in figure 3.5 are grouped by result: the first seven cases have one result, the second seven have another. ${ }^{74}$ The nearest result is the result of the nearest neighbour: $C_{5}$. The nearest known other (and the nearest other) is $C_{12}$; the nearest unknown other is $C_{13}$. 


\begin{tabular}{|c|c|c|}
\hline Case & $\begin{array}{c}\text { Known } \\
\text { distance }\end{array}$ & $\begin{array}{c}\text { Unknown } \\
\text { distance }\end{array}$ \\
\hline \hline$C_{1}$ & 43.57 & - \\
$C_{2}$ & 36.45 & - \\
$C_{3}$ & 17.76 & 5.63 \\
$C_{4}$ & 42.85 & 4.02 \\
$C_{5}$ & 17.09 & - \\
$C_{6}$ & 14.92 & 62.65 \\
$C_{7}$ & 59.32 & - \\
\hline$C_{8}$ & 54.48 & - \\
$C_{9}$ & 71.79 & - \\
$C_{10}$ & 55.74 & - \\
$C_{11}$ & 55.21 & - \\
$C_{12}$ & 46.13 & - \\
$C_{13}$ & 28.54 & 54.97 \\
$C_{14}$ & 49.58 & - \\
\hline
\end{tabular}

Figure 3.5: Distances for fourteen example cases. The cases are grouped by result: the first seven have one result, the second seven have another. The nearest known neighbour (and the nearest neighbour) is $C_{5}$; the nearest unknown neighbour is $C_{3}$. The nearest known other (and the nearest other) is $C_{12}$; the nearest unknown other is $C_{13}$.

\subsubsection{Equidistance}

In each comparison made during these classifications of cases and results it is possible that the distances being compared are equal. If two distances are the same then their cases (or results, as applicable) are said to be equidistant, because they are equidistant from the instant case.

SHYSTER allows any number of equidistant cases in all of the categorizations explained above. Equidistant cases are used in argument (one after another, in order of importance) wherever a single case would be used. SHYSTER also allows equidistant results - results whose nearest neighbours are equidistant-but only if those results are "other" results. There can only be one nearest result so that the open-textured concept can be defined for use by other areas in the case base or by a rule-based system.

Mackaay and Robillard consider the problem of equidistance and propose two solutions. ${ }^{75}$ The nearest result can be that which has the greatest number of equidistant cases. If there is no majority, then no prediction can be made. Alternatively, the set of nearest neighbours can be repeatedly extended to include 
the next nearest neighbour(s) until a majority is achieved. This approach is not adopted for use with SHYSTER. If there is a great distance between the nearest neighbours and the next nearest neighbours, it is inappropriate to use those next nearest neighbours to resolve the problem.

SHYSTER chooses between equidistant results by reference to the relative importance of the equidistant cases and (if further resolution is required) the year in which those cases were decided (more recently decided are considered to be more important). If the equidistance remains unresolved, SHYSTER refuses to venture an opinion, writes an error message and stops. It was intended to add further comparisons, resolving equidistance by reference to the nearest ideal points, the nearest centroids (centroids are described in $\S 3.12 .3$ below), and attribute direction. However, testing of SHYSTER indicates that the two stage approach adopted is adequate. The utility of going further is doubtful; to make further distinctions would be to split a very fine hair.

SHYSTER uses equidistance as a safeguard against giving bad advice (see $\S 3.12 .5$ below).

\subsection{Writing a report}

SHYSTER constructs a report - a legal opinion - about the instant case. This opinion includes a statement as to the likely result in the instant case, and justification of that statement. This justification is crucially important. As Tyree, Greenleaf and Mowbray point out:

... the justification in a legal system is the main product, for the justification is no more and no less than the legal arguments which support the suggested outcome. It is in the nature of legal reasoning that these arguments must also address the support for the opposite outcome. It is these arguments which, if the matter goes to court, must be presented for adjudication. ${ }^{76}$

SHYSTER's approach to report generation is based on that of FINDER, but SHYSTER takes account of unknown distance and equidistance, and argues about the effect upon its argument of hypothetical changes to the attribute values of the instant case. This last feature allows SHYSTER to adopt, to some extent, aspects of HYPO's approach to arguing with hypotheticals.

HYPO:

Summarizes the cases that can be cited in favor of a position, characterizes how strongly they support the position, focuses the attorney's attention on the most significant cases and hypotheticals, ... and facilitates comparing arguments between cases and hypotheticals ... ${ }^{77}$

So too does SHYSTER, although the sophistication of its reporting is limited by its main advantage: the simplicity of its knowledge representation. 


\subsubsection{Arguing with the instant case}

SHYSTER opens its report with some introductory comments about the area of law, then boldly declares its opinion ${ }^{78}$ that the result in the instant case will be the nearest result. It then uses the nearest neighbours and nearest results to justify that statement, before closing with some concluding remarks about the area. The opening and closing remarks are general comments provided by the legal expert.

How SHYSTER chooses the cases to use in argument, and how those cases are used, is described below.

\section{Choosing cases}

Figure 3.6 gives a pseudo-code description of SHYSTER's algorithm for choosing leading cases to use in argument, and the order in which to use those cases. SHYSTER will always use the nearest known neighbour in argument. It will also use the nearest unknown neighbour in two circumstances: if it is the nearest neighbour, or if it would be the nearest neighbour but for its unknown distance. As defined in $\S 3.9$ above, the unknown distance can be thought of as a measurement of error: it is the maximum distance that could be added to the known distance if all of the unknown attribute values were known. Whichever of the nearest known neighbour and the nearest unknown neighbour is the nearer is used first.

A slightly different approach is used with the nearest others. For every other result SHYSTER uses the nearest known other in argument. The nearest unknown other is used in two circumstances: if it is the nearest other, or if it would be nearer the instant case than the nearest neighbour - not just the nearest otherwere it not for unknown distance. The nearest unknown other is used in this second circumstance because, if all of the unknown attribute values were known, the nearest unknown other could be the nearest neighbour and SHYSTER's opinion as to the likely result would be different. The nearest known other is used in argument before the nearest unknown other, except in either of these two circumstances.

This description is simplified in one respect: it assumes that there is only one nearest known case and one nearest unknown case for each result. If there are two or more equidistant cases, SHYSTER uses each case - one after the other, in order of importance.

\section{Using cases}

How each leading case is used in argument varies depending on several factors. Figures 3.7, 3.8 and 3.9 give pseudo-code descriptions of SHYSTER's algorithm for using cases. Each description is a refinement of some steps in the algorithm 
FOR the nearest result DO

IF the nearest known neighbour is the nearest neighbour THEN use the nearest known neighbour; IF (were it not for unknown distance) the nearest unknown neighbour would be nearer the instant case than the nearest neighbour THEN END use the nearest unknown neighbour;

ELSE

$\left.\begin{array}{l}\text { use the nearest unknown neighbour; } \\ \text { use the nearest known neighbour; }\end{array}\right\}$ B END

END

FOR every other result DO

IF the nearest unknown other is the nearest other OR (were it not for unknown distance) the nearest unknown other would be nearer the instant case than the nearest neighbour THEN use the nearest unknown other; END

use the nearest known other; END

Figure 3.6: SHYSTER's algorithm for choosing the cases upon which to base its opinion.

The nearest known neighbour is the case with the smallest known distance, and with no unknown distance; the nearest unknown neighbour is the case which has the smallest sum of known and unknown distances, and non-zero unknown distance. The nearest neighbour is the case with the smallest sum of known and unknown distances. The nearest result is the result of the nearest neighbour. Similarly, for every other result there is a nearest known other and a nearest unknown other; the nearest of these is the nearest other.

The steps marked $\mathrm{A}$ are refined in figure 3.7; $\mathrm{B}$ in figure 3.8; $\mathrm{C}$ in figure 3.9. For simplicity, this description and the descriptions in these other figures assume no equidistance.

for choosing cases described in figure 3.6. Words within quotation marks are paraphrasings of words that SHYSTER uses in its opinion.

Each case is summarized, then the similarities and differences between the case and the instant case are explained. If the case is an unknown case, the attributes for which values are unknown are also detailed. 
IF (were it not for unknown distance) the nearest unknown neighbour would be nearer the instant case than the nearest neighbour THEN predict that the result will be the nearest result, citing the nearest known neighbour and the nearest unknown neighbour;

ELSE

predict that the result will be the nearest result, citing just the nearest known neighbour;

END

FOR the nearest known case DO summarize the case;

IF there is no distance between the case and the instant case THEN "the two cases are identical";

ELSE

list the similarities between the two cases;

list the differences between the two cases;

"nevertheless, the case should still be followed"; END

END

IF (were it not for unknown distance) the nearest unknown neighbour would be nearer the instant case than the nearest neighbour THEN cite the nearest unknown neighbour as another case in which the nearest result was reached;

FOR the nearest unknown neighbour DO

summarize the case;

IF there is some known distance between the case and the instant case THEN

list the similarities between the two cases;

list the differences between the two cases;

ELSE

"the two cases may be identical, and ...";

END

"I would have suggested, that this case be followed instead

of the nearest neighbours except that ...";

list the unknown attributes; END

END

Figure 3.7: Part of SHYSTER's algorithm for using cases in argument: a refinement of the steps marked $\mathrm{A}$ in the algorithm described in figure 3.6. 
predict that the result will be the nearest result, citing the nearest unknown neighbour and the nearest known neighbour;

FOR the nearest unknown neighbour DO summarize the case;

list the similarities between the case and the instant case; list the differences between the two cases;

list the unknown attributes;

"nevertheless, the case should still be followed"; END

FOR every nearest known neighbour DO summarize the case;

list the similarities between the case and the instant case; list the differences between the two cases; "nevertheless, the case should still be followed"; END

Figure 3.8: Part of SHYSTER's algorithm for using cases in argument: a refinement of the steps marked $B$ in the algorithm described in figure 3.6.

For the nearest result, it is argued that (because of the similarities, and despite the differences) the result in the instant case should be the same. For every other result, it is argued that (because of the differences, and despite the similarities) the result in the instant case should be different.

If SHYSTER's opinion is a desirable result for the user, she/he can use SHYSTER's discussion of the nearest case, and the differences between the nearest case and the instant case, as the basis for a legal argument. Alternatively, if SHYSTER's opinion is not a desirable result for the user, she/he can base a legal argument upon SHYSTER's discussion about the nearest others.

This description also assumes that there is only one nearest known case and one nearest unknown case for each result. Two or more equidistant cases are used one after the other.

The report makes no reference to "nearest cases," "nearest others," etc. No weights or distance measures are included or discussed. The calculations which SHYSTER uses to reach its conclusions are not part of its report, although they are written to various intermediate files. The report refers only to the similarities and differences between the instant case and the leading cases. 
IF the nearest unknown other is the nearest other OR

(were it not for unknown distance) the nearest unknown other would be nearer the instant case than the nearest neighbour THEN

"if the nearest unknown other and the nearest known other are followed then the result will be this (other) result";

IF (were it not for unknown distance) the nearest unknown other would be nearer the instant case than the nearest neighbour THEN

FOR the nearest unknown other DO summarize the case;

IF there is some known distance between the case and the instant case THEN

list the similarities between the two cases;

list the differences between the two cases; ELSE

"the two cases may be identical, and ..."; END

"I would have suggested, that this case be followed instead of the nearest neighbours except that ...";

list the unknown attributes;

"nothing in the case warrants changing the prediction"; END

ELSE

FOR the nearest unknown other DO

summarize the case;

list the similarities between the case and the instant case;

list the differences between the two cases;

list the unknown attributes;

"nothing in the case warrants changing the prediction"; END

END

ELSE

"if the nearest known other is followed then

the result will be this (other) result";

END

FOR the nearest known other DO

summarize the case;

list the similarities between the case and the instant case;

list the differences between the two cases;

"nothing in the case warrants changing the prediction"; END

Figure 3.9: Part of SHYSTER's algorithm for using cases in argument: a refinement of the steps marked $\mathrm{C}$ in the algorithm described in figure 3.6. 
Both FINDER and HYPO report on the similarities and differences in this fashion. However, as Ashley concedes:

We expect more from law students' explanations ...

(and, presumably, from lawyers' explanations)

... of why a precedent should or should not be followed than a discussion of the superficial, factual similarities and differences associated with factors. We expect their explanations to invoke some principled analysis of why the similarities and differences matter and to structure their explanations to reflect the relevant statutes and court-made rules. Thus, at first glance, HYPO's approach to identifying important similarities and differences may not seem philosophically satisfying.$^{79}$

The same criticism applies to SHYSTER, but is countered on the pragmatic grounds that the aim is to produce a working expert system, and to obviate the problem of knowledge acquisition. Similarly, Ashley makes a pragmatic argument in defence of HYPO:

Undoubtedly fundamental legal principles play a role in legal analogical reasoning ... But one cannot hope to model that kind of adversarial reasoning until one understands the simpler, more factual analogical comparisons among precedents ... ${ }^{80}$

\subsubsection{Arguing with instantiations}

Apart from using unknown distance, SHYSTER has a second method of taking UNKNOWN attribute values into account.

SHYSTER instantiates the unknown attribute values in the instant case to create instantiations of the instant case in which all the attribute values are known. SHYSTER treats each instantiation as if it were a new instant case, and determines the nearest cases and nearest results.

Because there are only two known values there are $2^{n}$ different instantiations, where $n$ is the number of unknOwns in the instant case. To avoid writing unnecessarily long reports, SHYSTER only reports on an instantiation if its nearest result is different to that of the instant case.

Instantiation is a useful feature. If the user is unable to answer an attribute question, she/he simply answers "unknown." If having known values for the UNKNOWN attribute values could make a difference to the result, the relevant instantiations are reported on in full. ${ }^{81}$ This feature can also be used where the user knows the answer to an attribute question, but wants to test the effect of providing a different answer. ${ }^{82}$

Instantiation is used as a safeguard against giving erroneous advice (see $\S 3.12 .6$ below). It is also used in chapter 5 to perform generated tests (which are described in $§ 3.13 .4)$. 


\subsubsection{Arguing with hypotheticals}

HYPO generates hypotheticals: hypothetical variations of the instant case that are stronger or weaker for a particular side. There are five heuristics for modifying the instant case: make a near-miss dimension apply; strengthen or weaken a case along an applicable dimension; move a case along a related dimension; make a case extreme along a dimension; and make a case into a near-win given a target. $^{83}$ Ashley identifies several uses for hypotheticals in legal argument: to factor a complex situation into component parts (e.g. by exaggerating strengths, weaknesses, or by hypothetically eliminating features); to create a test case that puts an issue or pits competing attributes against each other; to present, support, and attack positions in an argument (e.g. by testing consequences of a tentative conclusion); etc. ${ }^{84}$

Ashley describes hypothetical reasoning as the key to "exploring the dialectics between cases and principles or between cases and rules." 85 Whether or not it is appropriate to think of cases as rule exemplars (as Ashley seems to), SHYSTER's simplified representation of case law does not allow it to reason with hypotheticals to the same extent as does HYPO. However, SHYSTER does examine hypothetical variations in order to alert the user to the effect of such variations. Showing how a case can be strengthened and weakened can be particularly useful where there is some uncertainty surrounding one or more "known" attribute values.

For SHYSTER, the number of possible hypothetical variations upon instant case is $2^{n}$, where $n$ is the number of known attribute values. ${ }^{86}$ Generating all the possible hypothetical variations is of little use, as well as being computationally intensive. Instead, SHYSTER examines all possible variations which can be achieved by making no more than a certain number of changes to the known attribute values in the instant case; that number is specified by the user. ${ }^{87}$

As with instantiations, SHYSTER treats each hypothetical as if it were a new instant case, and determines the nearest cases and nearest results. A hypothetical is considered eligible to be reported on if its nearest result is different to that of the instant case, or if it has the same nearest result but its nearest neighbour is nearer the instant case than that of the instant case: i.e. it is a better case for the nearest result. Of these eligible hypotheticals, only the nearest are chosen to be reported on - up to a certain (user-specified) number for each result.

The hypothetical reports give the user information about how the argument about the instant case can be strengthened and weakened by changing only a specified number of attributes. (If the user wishes to examine the effect of varying a specific attribute or attributes, she/he can use SHYSTER's instantiation feature.) 


\subsection{Safeguards}

SHYSTER employs several safeguards so that it can warn the user in situations where its advice may be suspect. In certain circumstances, the results of these safeguards are logged, for the user, in a log file. Only in some of these circumstances is a warning issued.

SHYSTER's safeguards are based on the extra similarity measures, ideal points specified by the legal expert, centroids (described in $§ 3.12 .3$ below), attribute direction, equidistance and instantiations.

\subsubsection{Extra similarity measures}

As well as calculating known and unknown distance, SHYSTER also determines the similarity between the instant case and each of the leading cases using the extra similarity measures discussed in $\S 3.9 .3$ above: viz. $d_{j k}, S_{j k}, S_{j k}^{\prime}, r_{j k}$ and $r_{j k}^{\prime}$. If any of these measures suggests different nearest neighbours or nearest others then those differences are logged. In this way, cases which might be useful to the user's argument, but are not mentioned in SHYSTER's report, are brought to the user's attention.

A warning is issued if either of the extra weighted measures (the weighted association coefficient or the weighted correlation coefficient) suggests that a case with a different result to that of the nearest neighbour ought to be the nearest neighbour. This may be cause for concern about SHYSTER's opinion as to the likely result.

A different result suggested by one of the unweighted measures is not considered important enough to warn the user about, on the basis that attribute weighting is essential in the legal domain (see $\S 3.7$ above).

\subsubsection{Ideal points}

As discussed in $§ 3.5 .4$ above, the legal expert may specify ideal points. SHYSTER treats each ideal point as if it were one of the leading cases, and determines the distance between it and the instant case.

It is reasonable to expect that the result in the nearest ideal point will be the same as that of the nearest neighbour; after all, the nearest ideal point represents the ideal combination of attributes for its result. If the nearest ideal point has a different result to that of the nearest neighbour, this fact is logged. 
More serious would be a situation where an ideal point with a different result is at least as near to the instant case as is the nearest neighbour. If this occurs, a warning is issued.

\subsubsection{Centroids}

For each result, SHYSTER calculates the mean of each attribute for the cases with that result and creates an average attribute value vector for that result. ${ }^{88}$ These vectors of attribute value are called centroids. SHYSTER treats each centroid as if it were one of the leading cases, and determines the distance between it and the instant case.

As with ideal points, it is reasonable to expect that the result in the nearest centroid will be the same as that of the nearest neighbour. The nearest centroid represents the average combination of attributes for its result. If the nearest centroid has a different result to that of the nearest neighbour then this fact is logged because, as Tyree et al. explain, the instant case "is, in some sense, near the common boundary of the [result] groups and so must be considered as a 'difficult' case." 89 However, a warning is not issued unless a centroid with a different result is at least as near to the instant case as is the nearest neighbour.

\subsubsection{Attribute direction}

As mentioned in $§ 3.5 .2$ above, SHYSTER allows the legal expert to specify attribute directions. These indicate that the occurrence of a certain value for a certain attribute suggests a certain result or results.

For each result, SHYSTER sums the weights of each attribute for which the value of that attribute in the instant case is directed towards that result. ${ }^{90}$ This sum is termed a direction. The larger - the stronger - a direction, the greater the extent to which the attributes in the instant case "direct" SHYSTER towards that result.

There are three types of attribute direction for each result. The specified direction is calculated using the legal expert's attribute direction in the case law specification. The ideal point direction is calculated using ideal points; if an ideal point is the only ideal point in the area with a given value for an attribute then that value for that attribute is considered to be directed towards the ideal point's result. By an analogous method, the centroid direction is calculated using each result's centroid.

For each type of direction, the result with the strongest direction is said to be suggested by that direction. If any of these three directions suggest a result different to the nearest result, that fact is logged. Only if the specified direction 
suggests a different result is a warning issued. This distinction between directions is made because the specified direction, based as it is on information provided by the legal expert, is less likely than the other directions to suggest a different result anomalously.

\subsubsection{Equidistance}

As explained in $\S 3.10 .3$ above, SHYSTER chooses between equidistant results by reference to the relative importance of the equidistant cases and the year in which those cases were decided. If equidistance has to be resolved in such a fashion, SHYSTER issues a warning; equidistant results cast doubt on SHYSTER's choice of nearest result.

\subsubsection{Instantiations}

As explained in $\S 3.11 .2$ above, SHYSTER creates instantiations of the instant case in which all attribute values are known, and treats each as if it were a new instant case.

SHYSTER reports on any of these instantiations of the instant case which have a different result to that of the uninstantiated instant case. In such an event, doubt is cast on SHYSTER's conclusion; there is a combination of known attribute values which is consistent with the instant case but which leads SHYSTER to a different result, so a warning is issued.

\subsection{Testing and evaluation}

SHYSTER's general structure allows the testing of SHYSTER's approach to case law in different areas of law. Testing, using four different case law specifications, is described in chapter 5 . The choice of test domains - deciding which areas of law to specify for testing purposes - is discussed in $\S 3.13 .1$ below.

The principal testing method involves specifying an area of law for SHYSTER, then giving it information about a case which was actually decided in that area but which is not one of the leading cases in the specification. Unfortunately there is a paucity of such test cases, as explained in $\S 3.13 .3$. Even if the specification is written so as to represent the law as it was a few years ago, the number of cases decided since then (all potential test cases) is small.

So other testing methods are also employed. Generated testing (§3.13.4) uses SHYSTER's ability to generate instantiations to create many different imaginary cases. Reflexive testing ( $\S 3.13 .5)$ involves removing a leading case and testing it using the case base from which it was removed. (Cases used with the principal testing method are referred to as "test cases", to distinguish them from those used in generated tests or reflexive tests.) 
Because all three methods of testing make use of a specification, the quality of SHYSTER's advice is a function of both SHYSTER's approach to case law and of the quality of the specification; even assuming that SHYSTER's approach is a good one, its advice is only as good as its specification. Methods of evaluating the quality of SHYSTER's advice are examined in $\S 3.13 .2$.

For simplicity, the tests described in chapter 5 are often referred to as tests of their specification: of course, those tests are actually testing SHYSTERi.e. SHYSTER's approach to case law - using that specification. (This is true for test cases and generated tests. For reasons discussed in $\S 3.13 .5$ below, reflexive testing does not test SHYSTER's approach although it does provide some information about the specification.)

\subsubsection{Choosing a test domain}

Susskind suggests that (insofar as any area of the law is self-contained) an area of law chosen as a test domain should be relatively autonomous: its sources should be limited in number and reasonably well defined. It should be small enough to allow extensive coverage and its problems should not require the use of "a great deal of 'common-sense' knowledge":91 not because reasoning in the law doesn't require common sense - on the contrary - but because artificial intelligence techniques cannot cope satisfactorily with common sense. Given Susskind's consensual approach to jurisprudence (discussed in $§ 2.2 .7$ ), it is not surprising that he also suggests that there should be agreement amongst experts as to the scope and content of the test domain. ${ }^{92}$

Furthermore, he says, the domain must be one in which problem solving requires expertise. He is critical of Leith's ELI system, ${ }^{93}$ which deals with British welfare rights - a domain which was:

... chosen because of its simplicity which allowed Leith (not himself trained in law) to "become 'expert' in it." The system was not constructed, therefore, with the assistance of a legal expert, there could not have been any inclusion of experts' heuristics, and this factor might incline us to doubt whether the designation "expert system" is appropriate. Moreover, if a non-lawyer could, in a fairly short period, develop expertise in an area of law, then we might justifiably query whether that chosen area is indeed a suitable domain of application. For the chosen legal domain ought to be one whose problems do indeed require expertise (normally acquired over many years), and not relatively brief research, for their resolution. ${ }^{94}$

As Clark comments, Susskind's criteria of suitability "would seem to narrow the scope of expert systems in law quite considerably." 95

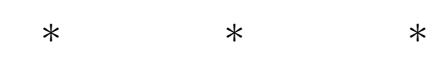


Each of the four specifications used to test SHYSTER is quite different from the others. The FINDER specification ( $\$ 5.2)$ is a simulation of the FINDER system, and deals with a completely case-based area of law. The Authorization specification ( $(5.3)$ deals with the definition of a specific open-textured statutory concept, and is an example of an area of law in which there are more than two possible results. The EMPLOYEe specification ( $\$ 5.4)$ defines an open-textured concept which is common to several different statutes and areas of case law.

Gardner justifies her choice of an area of the law on the grounds that it is "relatively well developed and stable." 96 Although that is true of the first three specifications discussed in this chapter, it is not true of the fourth. The Natural specification ( $\S 5.5)$ deals with a recently developed (and still developing) area of Australian administrative law.

These four specifications satisfy most of Susskind's criteria. They are all specified in terms of a limited number of well-defined cases. Each domain is fairly small, yet sufficiently complex that solving problems requires legal expertise. However, no claim is made that any of these specifications embodies a legal consensus as to what the law is. Apart from the Finder specification, each was developed by the author - a lawyer - with the assistance of an expert in the relevant field. As such, each specification represents one interpretation of its field.

\subsubsection{Evaluating SHYSTER's opinion}

There are a number of levels at which SHYSTER's opinion can be evaluated. It states its prediction as to the likely outcome, on the assumption that the result will be the same as it was in the case which it deems most similar to the instant case. SHYSTER's prediction of the likely result is considered "good" if it is the same as the result in the actual case, and "bad" otherwise. ${ }^{97}$

In their written judgments, judges will often explicitly follow, or refer favourably, to certain cases before coming to their conclusion. Sometimes they will explicitly refuse to follow a case on the basis that it is distinguishable on its facts. Some reported judgments also include precis of the arguments put to the judges by counsel for the parties involved.

SHYSTER's opinion is only as good as the cases it chooses upon which to base its arguments. If a case which SHYSTER chooses is referred to by a judge, or cited in argument before the court, then it is considered a "good" case for SHYSTER to have chosen. This is true for cases upon which SHYSTER bases its arguments and its counterarguments. ${ }^{98}$ SHYSTER is proposing these cases as the best arguments for each of the possible results.

For example, a judge may explicitly follow a case in coming to her/his conclusion. If SHYSTER chooses that same case as the basis of its counterargument then it will come to a bad conclusion, by following another case. However, it has identified a good case upon which to base an argument for one party; its conclusion is bad, but at least one of its chosen cases is good. 
Generally, SHYSTER's choice of a case is considered "bad" if that case was neither cited in forensic argument nor in judgment. In some circumstances, however, it is argued that SHYSTER's choice of a case is good even though it was not used by counsel or by the court. All such divergences from the general rule are discussed in detail in chapter 5 .

Sometimes the case which is used as a test was decided before one of the leading cases that SHYSTER chooses. If this happens, SHYSTER's choice is only characterized as "good" or "bad" on the advice of the legal expert.

As discussed in $\S 3.12$ below, SHYSTER employs several safeguards to protect against giving bad advice. If SHYSTER issues a warning and SHYSTER's prediction as to the likely result is bad, that warning is characterized as "good"; if the predicted result is good, the absence of a warning is also considered to be "good." If SHYSTER issues a warning even though SHYSTER's prediction is good, the warning is "bad"; similarly if the prediction is bad and SHYSTER issues no warning, the absence of a warning is deemed "bad."

When lawyers argue with a case, it may be that only one aspect of that case is considered. This is true of the test cases, and of the leading cases that make up the specifications in appendix A. For example, Salemi v. MacKellar ${ }^{99}$ is used as a test of the NATURAL specification in $\$ 5.5 .3$ because the High Court had to determine whether Salemi had a right to be heard before the Minister ordered his deportation. In that case, the Court also considered the issue of whether news releases were "instruments under the hand of the Minister." But that issue is ignored in the discussion of Salemi v. MacKellar in chapter 5. It is not relevant to the specification that the case is used to test, and is not taken into account when evaluating SHYSTER's opinion.

\subsubsection{The paucity of test cases}

One of the major obstacles to developing ${ }^{1}$ and testing a case-based legal expert system like SHYSTER is the paucity of reported cases. This can be attributed to the filtering effect of various stages of the legal system.

Consider a client who seeks legal advice as to her/his legal options in some matter. Good legal advice will filter out a hopeless case. ${ }^{2}$ If, for example, a lawyer recognizes the matter as being identical to a previously decided case which suggests that the client will lose, her/his advice will probably be not to proceed. So, for any given leading case in a SHYSTER specification, there may be many substantially identical cases which proceeded no further than a lawyer's office.

Even if the client's legal case is a strong one, there are many reasons why it might never reach court. Most people find the cost of legal redress prohibitively expensive. Further, the time delay involved may well dissuade a person from 
taking legal action, or defending an action. For any number of non-legal reasons, prospective parties to a case may choose not to proceed, and/or to "settle out of court."

Finally, not all cases that are decided in court are reported. Only those cases which the court reporters deem significant are included in the law reports. The legal domain is quite different from other areas of case-based expert system development where the developer may have access to a large number of cases which are relatively unfiltered.

In the face of this lack of test cases, Gardner used examination problems to test her system on the basis that, although they may not be as complex as real cases, they are reasonably difficult and the sorts of questions that lawyers are expected to be able to handle. ${ }^{3}$ This is certainly true - and SHYSTER is tested with some hypothetical cases in this way - but the author contends that actual cases make better tests. Although the legal system greatly restricts the number of cases that are reported, it ensures that those cases that are reported make good tests. If a case had been straightforward, it is highly unlikely that it would have proceeded through the various filters that the legal system provides to emerge as a reported judgment.

The four specifications described in chapter 5 are tested with a total of seventeen previously decided cases which are not amongst the specified leading cases. Although a small number, it compares well with the testing of other case-based systems. Tyree, Greenleaf and Mowbray tested FINDER with one case. ${ }^{4}$ Ashley evaluated HYPO's performance using only four cases - all of which were taken from the case base. ${ }^{5}$ In addition to the real cases used to test SHYSTER, there are four hypothetical test cases. These were proposed by the legal experts as interesting tests that have not yet been before the courts.

\subsubsection{Generated tests}

Generated tests are performed using SHYSTER's capacity to argue with instantiations (see $\S 3.11 .2$ above). SHYSTER can be used to generate, and give advice on, all possible cases in an area. However, there is no point in generating the entire search space in this fashion. In an area of reasonable size, the search space would be so large as to make evaluation of SHYSTER's advice in each case impractical. ${ }^{6}$

Instead, the legal expert is asked to specify a number of attribute values whose presence in a case means that a certain result, or results, follows (i.e. that result, or results, would be reached if that case were to be heard by a court). A fact vector is constructed containing these known attribute values, with the remaining attribute values UNKNOWN, and SHYSTER is made to generate all instantiations of that fact vector. 
Although it is not possible to evaluate SHYSTER's choice of cases in all of these instantiations - these generated cases - it is to be hoped that SHYSTER will choose a good result (i.e. one of the results specified by the legal expert) in most, if not all, of them. The number of good choices of result as a fraction of the number of generated cases is SHYSTER's success rate for a generated test. Some of these generated cases may represent paradoxes: combinations of attribute values which are impossible. Paradoxical generated cases are ignored when determining SHYSTER's success rate. ${ }^{7}$

SHYSTER's ideal point warnings ${ }^{8}$ are of particular importance to the generated tests. Many of the generated cases may be extremely unusual: i.e. combinations of attribute values that are very unlikely to occur, though not impossible. Assuming that its leading cases are well chosen, SHYSTER is more likely to choose a bad nearest case when given an extremely unlikely and unusual instant case than if it were given a realistic combination of attribute values as its instant case. Ideal point warnings are designed to detect these extremes, and are taken into account when evaluating the results of generated tests.

\subsubsection{Reflexive tests}

A reflexive test is performed in the same fashion as a test performed using the principal testing method, except that one of the leading cases in the current specification is used as the test case.

Of course, if SHYSTER is presented with a fact vector which is identical to one of the leading cases in its specification it will simply follow that leading case: the two cases are identical. Such a test would demonstrate nothing. So, before a reflexive test is performed, the leading case that is to be used as a test case is removed from the specification. SHYSTER is effectively asked "if this case were decided now, in the light of all the leading cases except itself, how would it have been decided?" (The term "reflexive" is coined to describe such a test because the case is being applied to the specification whence it came.)

Mackaay and Robillard criticize Lawlor for adopting a reflexive approach to testing. They point out that:

... when all cases are used to determine optimal [attribute] weights ... there can be no surprise to find that on the basis of those weights each case is correctly classified; the results would look unduly promising. ${ }^{9}$

This criticism does not apply to reflexive testing of SHYSTER because SHYSTER recalculates attribute weights (without the removed leading case) for each of the reflexive tests. 
The specification that is used for a reflexive test is diminished: it excludes the test case. It must be assumed that the case that has been removed belongs in the specification, otherwise the legal expert would not have included it. Hence the diminished specification no longer represents the area of law that it was written to represent.

This means that the result of a reflexive test does not assist in evaluating SHYSTER's approach to case law: SHYSTER's opinion and the cases it chooses cannot sensibly be compared with the judgment in the actual case. However, the results of reflexive tests do provide information about the specification itself.

It is very unlikely that a specification of reasonable size would have a leading case for every possible combination of attribute values. Yet it is highly desirable that a specification be capable of handling new cases which are not identical to any of the leading cases.

Consider a specification in which conducting a reflexive test for every leading case in that specification yields a good result. All of the leading cases contribute to the extent that each case's result can be determined from the other cases. Such a set of results would indicate that the specification is successful at handling new combinations of attributes. Conversely, consider a specification in which every reflexive test yields a bad result. That specification is clearly very poor at handling new fact situations.

In reality, a given specification is unlikely to reach good conclusions in all of its reflexive tests, or in none of them. The number of good conclusions will probably lie somewhere in between. If a large proportion of a specification's reflexive tests yield a good result, that is indicative of that specification's suitability to handle new cases.

It is important to note the following points about reflexive testing.

Even if SHYSTER's opinion in a reflexive test is very good-i.e. it comes to the same conclusion as did the court in that case, and for the same reasons - that is no reason to remove that case from the specification permanently. It does not follow that, because the diminished specification is all SHYSTER needs to reach a good conclusion, the excluded case adds nothing to the specification. A new case could occur in which the best opinion would be to follow the excluded case, and not the cases that SHYSTER chooses in the reflexive test. This will almost certainly be true for a new case with the same attributes as the excluded case, but the attribute values need not be identical. The diminished specification may reach a good conclusion, but not necessarily for good reasons. ${ }^{10}$

Removing a case from a specification can introduce attribute dependencies which were not there before. It is also quite likely that one of the cases which SHYSTER chooses upon which to base its opinion was decided after the instant case. If that happens, it is impossible to evaluate SHYSTER's choice of cases. 
The weight given to the decisions of a given court may vary between jurisdictions. For example, decisions of the English House of Lords are binding on lower courts in England, but only strongly persuasive in Australian courts. Three of the four specifications discussed in chapter 5 use Australian and English cases, ${ }^{11}$ and each specification aims to represent the Australian law. If an English case is used for a reflexive test, SHYSTER may choose a case which was not referred to in the test case but which might have been a good case to choose had the test case been heard in an Australian court.

Reflexive tests are not tests of SHYSTER's approach to case law, though these tests do provide information about a particular specification. A reflexive test is performed for every leading case in each of the four specifications discussed in chapter 5 . The results of these tests are set out and discussed in appendix D.

\subsection{Conclusion}

SHYSTER treats cases as points in space, the dimensionality of which is the number of attributes. The instant case is placed at its appropriate point in this space, and the nearest leading cases are determined. These nearest cases are used to produce an argument (based on similarities and differences between the cases) about the likely outcome in the instant case. That argument relies on the doctrine of precedent; it assumes that the instant case will be decided the same way as was the nearest case.

SHYSTER can also instantiate unknown attributes, thus testing all possible configurations of the instant case. A limited number (specified by the user) of hypothetical variations of the instant case can also be tested, to see whether the case can be strengthened toward some result. SHYSTER also applies several safeguards and the user is warned if SHYSTER has some doubt about the veracity of its advice.

The report that SHYSTER generates makes a prediction and justifies that prediction by reference only to cases and their similarities and differences: the numbers which SHYSTER uses in coming to its opinion do not appear in that opinion.

SHYSTER models legal knowledge at the second of the three levels that Greenleaf, Mowbray and Tyree identify (as quoted in $\S 2.7$ ): its representation includes justification based on the primary legal sources, but without any explicit model of those sources; principles of interpretation are implied in the representation. ${ }^{12}$ 
By taking a pragmatic approach to representing case law, SHYSTER follows one of the approaches that Gardner recommends taking "if legal realism is right": emphasizing the behaviouristic side of legal realism. ${ }^{13}$ Gardner herself takes a different approach, ${ }^{14}$ and says of the behaviourists' programs:

These are not AI programs ... The programs are concerned with predicting judicial decisions, or more generally with analyzing judicial behavior, from a data base in which legal rules have no role. Traditional modes of reasoning are replaced by mathematical methods $\ldots{ }^{15}$

Gardner does not explain why she thinks that these "prediction programs" are not AI programs. If a program can successfully predict judicial decisions - even without adopting a "traditional mode of reasoning" - it is at least arguable that it is artificially intelligent.

Areas are used in SHYSTER to represent open-textured concepts: statutory or case-based. This structure facilities the linking of a rule-based system with SHYSTER's case-based system to form a hybrid system, capable of dealing with statutes and case law. ${ }^{16}$

A hybrid approach has a number of benefits. It has the advantage of approximating the approach which a lawyer would take when given a legal problem. The rules, derived from a statute, are applied until the meaning of some opentextured concept is required. Faced with this problem, a lawyer would turn to the common law in order to further clarify the meaning of the statute. So, too, does SHYSTER: the lawyer's two-stage approach is clearly modelled.

A consequence of SHYSTER's approach is that it returns a result from each area of case law, for use in another area, or by a statutory rule base. It is certainly true, as Ashley points out, that "the goal of a theory of analogical legal argument should not be to explain what the right answer is." 17 But in returning a result from an area, SHYSTER does not pretend to be giving the "right answer"; it is merely attempting to predict the "likely answer," applying the principle of stare decisis. SHYSTER's opinion is predictive, not normative, and (as explained in $§ 2.3 .5)$ a good legal expert system should have predictive power.

Gardner cites various writers who identify the following as difficulties associated with this approach to case law: separating findings of fact from legal conclusions; determining what the judges in the leading cases believed the facts to be; categorizing facts appropriately; and deciding what aspects of the facts should be included. ${ }^{18}$ But these difficulties are not peculiar to legal expert systems which 
take a statistical approach to case law: all of these difficulties are inherent in the problem of dealing with case law. SHYSTER expects the legal expert to have regard to these difficulties when specifying an area of case law. This is not an unreasonable expectation: knowing how to overcome these difficulties is one of the characteristics of legal expertise.

\subsubsection{Comparisons with other approaches}

SHYSTER adopts and expands on the nearest neighbour approach to case law taken by FINDER. Mackaay and Robillard apply nearest neighbour techniques to cases, too, although they did not actually develop an expert system. SHYSTER also adopts aspects of HYPO's approach to reasoning with hypotheticals.

One difference between SHYSTER and both FINDER and HYPO is SHYSTER's generality; while FINDER deals only with the law of trover and HYPO deals with trade secrets law, SHYSTER allows a legal expert to define an arbitrary number of areas of case law in each specification.

SHYSTER's knowledge representation is more complex than FINDER's, but simpler than HYPO's. ${ }^{19}$ Unlike SHYSTER, FINDER does not allow the specification of unknown attribute values: unknown values are entered as nos. Tyree suggests that an alternative approach, "possibly better," would be to select a value at random $;{ }^{20}$ SHYSTER's use of UNKNOWNs is better still.

SHYSTER allows any number of possible results in an area. Both HYPO and FINDER treat all cases (including the instant case) as having one of two possible results. Ashley claims that:

... subject to some qualification, there are only two possibilities: either the plaintiff won the case or did not. The qualification is that depending on the procedural context of the case, some outcomes are more determinative than others. ${ }^{21}$

This qualification relates not to the number of possibilities but to the importance of the leading cases (which is captured by SHYSTER using its hierarchy of courts). Although there may be only two possible results in the areas of law covered by FINDER and HYPO, this is not true in other areas. ${ }^{22}$

Because of its approach to weighting attributes, SHYSTER assumes attribute independence, and checks for attribute dependence in all areas. SHYSTER uses the same method to weight its attributes as does FINDER; hence, FINDER also assumes attribute dependence, although it does not address the problem. Fortunately, for FINDER, there is neither functional dependence, nor evidence of stochastic dependence, between its attributes. ${ }^{23}$ SHYSTER, like FINDER but unlike HYPO, assumes that each attribute has the same weight in all cases (see $\S 3.5 .3$ above). 
SHYSTER's distance metric is similar to FINDER's, except that it takes account of unknown distance and infinitely weighted attributes. ${ }^{24}$ FINDER does not use extra similarity measures, specified directions, equidistance or instantiations as safeguards, as does SHYSTER. FINDER does use centroids as a safeguard. The use of ideal points as a safeguard is suggested by Tyree et al. but is not implemented in FINDER. ${ }^{25}$

Apart from a few cosmetic additions, the structure of SHYSTER's reports is similar to those of FINDER. However, SHYSTER's algorithm for choosing which cases to use in argument is more complicated because of the need to account for unknown distance. Unlike FINDER, SHYSTER handles equidistant cases and argues with instantiations and hypotheticals.

Two of SHYSTER's features - specified direction and hypotheticals - are designed to incorporate some of HYPO's functionality, without the need for HYPO's more complex knowledge representation.

HYPO represents the attributes that favour each side, and treats a problem as a collection of possibly competing attributes. All attributes are required to favour one side or the other. ${ }^{26}$ Similarly, SHYSTER's specified direction allows the legal expert to specify a result favoured by a certain value for a certain attribute. However, SHYSTER does not require that all attributes (or attribute values) be directed, for the simple reason that not all attribute values can be directed towards a result or results. ${ }^{27}$

SHYSTER's reasoning with hypotheticals is not as sophisticated as HYPO's, due to SHYSTER's simpler representation of case law. However, SHYSTER is able to examine limited hypothetical variations, and inform the user if the effect of these variations is to strengthen or weaken its argument about the instant case.

\subsubsection{A Sisyphean journey?}

Now that SHYSTER's approach to case law has been explained, it is possible to mount a detailed refutation (foreshadowed in \$2.4.7) of Berman's argument that case-based reasoning techniques are inappropriate for modelling case law. Berman is a proponent of deep rule-based models. He believes that "lawyers make their decisions on the basis of their judgment as to whether a particular rule will be applied to the facts of a specific case." ${ }^{28}$ It is argued elsewhere that developers of conceptual models of legal reasoning confuse precision with accuracy. ${ }^{29}$ The refutation that follows deals only with Berman's arguments against case-based reasoning, with particular reference to SHYSTER.

Berman claims that the choice for the developer in the legal domain is a "Sisyphean journey" with case-based reasoning or "down hill with rules". ${ }^{30}$ He refers to work in case-based legal reasoning employing "frame-based structures, transition 
nets, semantic networks, discrimination trees, connectionist models, etc. ${ }^{31}$ and claims that:

To legal scholars well versed in the subtleties of legal reasoning these particular representations of legal cases, though seminal works of considerable scientific importance, constitute a mere simulacrum of legal thought.

First, the models do not contain choice of law rules to account for those legal cases that implicate the law of more than one jurisdiction ...

This could be achieved using a hybrid system. "Choice of law" rules could be implemented in the rule base (or a meta-rule base) to ensure that the appropriate areas of case law were applied. Alternatively, assuming that the user is an expert, the user could make this choice her/himself (as discussed in $\S 3.4$ above, such an assumption is reasonable).

Second, the models do not account for the fact that some precedents are weakened by divided courts ...

SHYSTER allows the legal expert to specify a hierarchy of courts. Several courts in this hierarchy could be different compositions of the same court (e.g. one, three, five or seven judges of the High Court of Australia). The legal expert could even include a minority judgment as a separate case in the specification if it was deemed sufficiently important. SHYSTER only uses its hierarchy for resolving equidistance, but there is no reason why a case-based system could not make more use of such a hierarchy in its operation.

Third, the models do not take into consideration that judicial opinions carry varying precedential values...

SHYSTER takes this into consideration in two ways: by use of a hierarchy, and by allowing a legal expert to choose the important cases and, by implication, to reject the less important cases.

Fourth, the models do not account for the fact that the precedential weight may turn on when the case was decided ...

As with the relative importance of a case, SHYSTER takes the year in which a case was decided into account when resolving equidistance - there is no reason why a case-based reasoning system could not give the time since a case was decided more importance than does SHYSTER. Further, the legal expert should be expected not to include cases which are so old as to be of little precedential weight.

Fifth, the models do not account for sub silentio overruling - the disregard of precedents which have been so often distinguished or ignored that they lack precedential value.

SHYSTER assumes that all of the cases chosen by the legal expert are "good law." Disregarded precedents should be disregarded. 
Sixth, except for the works of McCarty ... the models lack mechanisms for resolving tensions between conflicting lines of authority.

But conflicting lines of authority are unresolvable. The appropriate response to a conflicting line of authority is to present all the arguments to assist the user in constructing her/his argument.

Seventh, the model does not account for judicial decisions motivated by political considerations unarticulated in opinions ...

Political considerations, if the legal expert deems them important, can be included as attributes in one of SHYSTER's case law specifications. ${ }^{32}$

Eighth, these models have not provided for the computational representation of legal fictions where the concepts of contracts, easements and notice become spurious easements, quasi contracts, and constructive notice.

Why these examples could not be represented in a case-based system is not clear.

Ninth, these models do not consider that the precedential value of a case may turn on the prestige of the judge who wrote the opinion.

As discussed above, under Berman's second point, this presents no difficulty for SHYSTER.

Tenth, and most importantly, the model does not represent accurately the procedural posture of a case so that the resulting arguments fail to distinguish cases in which courts have ruled on matters of law from cases where appellate courts have merely affirmed findings of fact ... ${ }^{33}$

If a case involves merely an affirmation of a finding of fact, and no ruling on a matter of law, then the legal expert is unlikely to choose it as a leading case.

Berman confuses the role of a case-based system with the role of a legal expert in the knowledge acquisition process. With the possible exceptions of BenchCapon et al. (\$2.4.2), no-one would deny that legal expertise is required in the development of a legal expert system. Hence, it is reasonable to use the fact of legal expert specification in SHYSTER to refute several of Berman's points.

Berman concedes that rules "fall far short of fully representing legal knowledge", but:

For developers, as contrasted to researchers, the issue is not whether the resulting base is "complete" or even "accurate" or "self-modifying"—but whether the resulting rule base is sufficiently complete and accurate to be "useful". 34

The author agrees that usefulness should be the principal criterion in legal expert system design, but argues that, in a case law domain at least, a case-based system can better satisfy that criterion. 



\section{Implementing SHYSTER}

I know you Lawyers can, with ease,

Twist words and meanings as you please;

That language, by your skill made pliant,

Will bend to favour ev'ry client;

That 'tis the fee directs the sense

To make out either side's pretense.

When you peruse the clearest case,

You see it with a double face;

For scepticism's your profession;

You hold there's doubt in all expression.

Hence is the bar with fees supply'd,

Hence eloquence takes either side...

John Gay (1732)

The Dog and the Fox ${ }^{35}$

He is no lawyer who cannot take two sides.

Charles Lamb $(1833)^{36}$

Thelma Todd: "I didn't know you were a lawyer. You're awfully shy for a lawyer." Groucho Marx: "You bet I'm shy. I'm a shyster lawyer." 


\subsection{Introduction}

SHYSTER's approach to case law is described in the previous chapter. In this chapter, the implementation of that approach is described, and illustrated using examples. SHYSTER is implemented using a dozen modules, written in ISO C. ${ }^{38}$ (The code for each module is listed in full elsewhere. ${ }^{39}$ ) The structure of this chapter mirrors that of SHYSTER, with the description of the implementation divided into descriptions of each module.

The Shyster module $(\S 4.2)$ is the top-level module for the whole system. The Statutes module $(\S 4.3)$ is the top-level module for a rule-based system, presently unimplemented. The CASES module $(\S 4.4)$ is the top-level module for the case-based system. The Tokenizer and PARser modules ( $\S 4.5$ and $\S 4.6)$ tokenize and parse a program written in SHYSTER's case law specification language. The Dumper module ( $(4.7)$ displays the information that has been parsed. The CHEcker module $(\S 4.8)$ checks for evidence of dependence between the attributes. The Scales module $(\S 4.9)$ determines the weight of each attribute. The Adjuster module ( $(4.10)$ allows the legal expert to adjust the weights of the attributes. The Consultant module $(\$ 4.11)$ interrogates the user as to the attribute values in the instant case. The Odometer module $(\S 4.12)$ determines the distances between the leading cases and the instant case, and the REPORTER module (§4.13) writes SHYSTER's legal opinion.

Except where otherwise indicated, the examples used in this chapter are taken from the EMPLOYEe specification, which is explained in $\S 5.4$ and appears in $\S$ A.4. The Employee specification is used as the basis of the complete example in appendix $\mathrm{C}$.

\subsubsection{Internal representation}

SHYSTER uses records to represent entities (courts, areas, results, attributes, cases, etc.) and makes multiply linked lists of these entities to reflect the relationships between them.

For example, each area record has a pointer to the head of a linked list of result records. Each result record has a pointer to the head of a linked list of case records. Each case record has a pointer to the head of a linked list of attribute value records. Each attribute value record is an element in two lists, linked by case and by attribute, forming part of a matrix of attribute values.

Using linked lists allows the manipulation of these entities and relationships without requiring the imposition of any limits upon their numbers. The only limits that affect the user of SHYSTER or the writer of specifications are on the maximum length of a filename, and the maximum length of an identifier. ${ }^{40}$

The information contained in each record, and the links between those records, are built up by each of SHYSTER's modules until, by the time that the REPORTER module is invoked, the structure is complete. 


\subsubsection{Output files}

Each time SHYSTER's case-based system is invoked it writes the following files:

- a log file (introduced in §3.12) which summarizes SHYSTER's operation and includes any warnings that have been issued;

- a dump file which is a dump of SHYSTER's internal representation of the case law specification;

- a probabilities file which gives probability figures for each attribute pair in each area;

- a weights file which gives details of the weights that SHYSTER gives to each attribute in each area;

- a distances file for each invoked area which includes distances and other similarity measures for the instant case, instantiations and hypotheticals; and

- a report file for each invoked area which is SHYSTER's legal opinion-its argument about the likely result in that area for the instant case.

Only the first and the last of these are intended for the user. SHYSTER's report files are completely self-contained; the user need have no knowledge of SHYSTER and its operation in order to understand its reports. The log file should be comprehensible to anyone with only passing acquaintance with SHYSTER's operation. A user who finds the information contained in a log file arcane may safely ignore the file: of its contents, only the warnings are crucial, and these are also written to the standard error stream. ${ }^{41}$

All other files are intermediate files that SHYSTER produces on its way to its report. They provide details about SHYSTER's internal workings for the information of the knowledge engineer and the legal expert.

All of these output files are plain text files. With the exception of the log file, they are all in $\mathrm{LAT}_{\mathrm{E}} \mathrm{X}$ format: i.e. they are suitable for processing by the $\mathrm{HAT}_{\mathrm{E}} \mathrm{X}$ document processor. ${ }^{42}$ This contributes to SHYSTER's portability, as $\mathrm{LAT}_{\mathrm{E}} \mathrm{X}$ is widely available on many platforms. Using $\mathrm{LAT}_{\mathrm{E}} \mathrm{X}$ simplifies the footnoting of text, allows some data to be displayed in a clear and economical tabular format, and ensures the aesthetic quality of the output. All of the examples of SHYSTER output in this thesis - the dump files in appendix A, the example reports in appendix B, the "LAT $\mathrm{T}_{\mathrm{E}} \mathrm{X}$ output" files in the complete example in appendix $\mathrm{C}$, and extracts in fourteen of the figures in this chapter and the next ${ }^{43}$-appear exactly as produced by SHYSTER after processing by $\mathrm{LAT}_{\mathrm{E}} \mathrm{X} .{ }^{44}$ 


-a
-c specification
-d distances
-D dump
-e
$-\mathrm{h} r c$
$-\mathrm{i}$
$-\mathrm{I}$ log
$-\mathrm{p}$ probabilities
-q
- $\mathrm{r}$ report
$-\mathrm{w}$ weights

Enable weight adjustment (see $§ 4.10)$

Read the case law specification from "specification.cls"

Write each distances file to "distances-area.tex"

Write the dump file to "dump.tex"

Enable echo mode (see $\S 4.11$ )

Hypothesize, reporting on $r$ hypotheticals per result with a limit of $c$ changes

Write $\mathrm{IAT}_{\mathrm{E}} \mathrm{X}$ code that can be included in another $\mathrm{LAT}_{\mathrm{E}} \mathrm{X}$ document (i.e. not stand-alone code)

Write the $\log$ file to "log. $\log "$

Write the probabilities file to "probabilities.tex"

Enable quiet mode (don't summarize cases, etc.)

Write each report file to "report-area.tex"

Write the weights file to "weights.tex"

Figure 4.1: The UNIX command line switches and arguments recognized by SHYSTER. Apart from the $-c$ switch, all switches are optional.

\subsection{The SHYSTER module}

The SHYster module is the top-level module for SHYSTER. It extracts the options and arguments from the UNIX command line, initializes the rule-based system and the case-based system, then invokes the rule-based system. Figure 4.1 lists the command line switches and arguments that SHYSTER recognizes.

Only the -c switch must be used; all other switches are optional. So, for example, if the $-\mathrm{d}$ switch is not used, no dump file is written. However, if the -1 switch is not used then the information that would have been written to the log file is written to the standard output stream. ${ }^{45}$

\subsection{The Statutes module}

The Statutes module is the top-level module for a rule-based system. This module provides a skeletal structure within which a rule-based system could be developed, and linked with the case-based system to form a hybrid system.

The module has two functions.

The Initialize_Statutes function presently returns a pointer to a dummy structure. If implemented, it would initialize the rule-based system, by reading a statute law specification, and return a pointer to SHYSTER's internal representation of that specification. 
The Statute_Law function would invoke the rule-based system proper. Each statutory open-textured concept in the rule base would be associated with an identifier corresponding to an area in the case base. The rule-based system would invoke the case-based system using that identifier. The case-based system returns an identifier corresponding to the result from the appropriate area. That result would be bound in the rule-base to a value for the statutory open-textured concept.

At present, the Statute_Law function prompts the user for an identifier, then invokes the case-based system seeking advice in the area corresponding to that identifier. The result returned by the case-based system is written to the log file.

\subsection{The CASES module}

The CASES module is the top-level module for the case-based system. Its two major functions correspond to the two functions in the Statutes module.

The Initialize_Cases function calls the Tokenizer and PARser to read the case law specification and build an internal representation of that specification. The Dumper is invoked to dump that internal representation to the dump file. The Checker is used to check for attribute dependence. Finally, the Scales module is called to assign weights to all the attributes. The function returns a pointer to SHYSTER's internal representation of the specification with all attributes weighted.

The Case_Law function takes a pointer to that internal representation, and an identifier corresponding to one of the areas in that specification. If weight adjustment is enabled, it calls the Adjuster. It then invokes the Consultant to interrogate the user as to the attribute values in the instant case. (It is the Consultant which recursively invokes the Case_Law function, if required, to resolve open textured - external-attributes.)

Having ascertained the attribute values, the Case_Law function calls the Odometer to calculate the distances between the instant case and the leading cases, and to determine the nearest cases and results. Then the REPORTER is invoked to write a report about the instant case.

The CASEs module instantiates any unknown variables in the instant case and invokes the Odometer and the REPORTER to recalculate the distances and argue using the instantiation. If the user has requested it, the CASEs module also makes hypothetical variations to the instant case and invokes the ODOMETER and the REPORTER yet again to recalculate distances and argue with the hypothetical.

Output from these invocations of the ODOMETER and the REPORTER is written to the distances file and report file for the current area. 


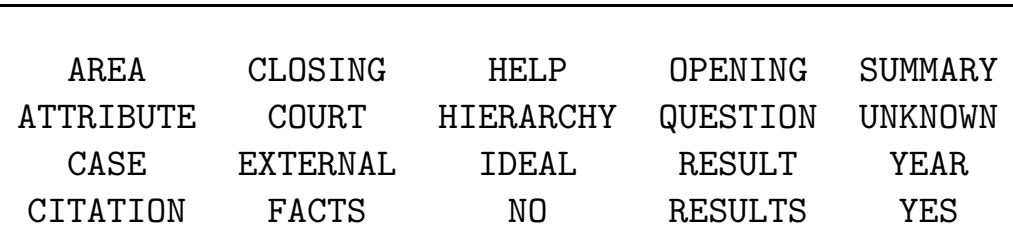

Figure 4.2: The keywords in SHYSTER's case law specification language.

\subsection{The ToKenIZER module}

The Tokenizer module reads the case law specification file, and breaks it into tokens. The Tokenizer is invoked repeatedly by the PARser; each time it is invoked, it returns the next token in the specification.

\subsubsection{Tokens}

There are seven different valid types of token.

An identifier is any sequence of alphabetic characters, numeric characters, and the - character (starting with an alphabetic character). If an identifier is more than 16 characters long it is truncated to its first 16 characters and a warning is issued. An identifier's case is significant.

A keyword is an identifier that has a special meaning to SHYSTER. There are twenty keywords, and they are listed in figure 4.2. Keywords are reserved: i.e. they cannot be used as identifiers.

A string is a sequence of characters, enclosed between a pair of " characters. ${ }^{46}$ There must be at least one character between the two quotation marks, but SHYSTER imposes no upper limit on the length of a string. SHYSTER converts a pair of consecutive " characters within a string into a single " character. ${ }^{47}$

A year is a positive integer of up to four digits.

An attribute vector is a sequence of $\mathrm{Y}, \mathrm{N}$ and $\mathrm{U}$ characters within parentheses. ${ }^{48}$

The remaining two tokens are the $=$ character and a token which indicates that the end of the specification file has been reached.

\subsubsection{Comments and whitespace}

When the TOKEnIzen reads a \% character in the specification (except in a string), it skips over the rest of that line: any characters between the $\%$ and the next carriage return are ignored. This allows the legal expert to put comments in the specification file.

Whitespace $^{49}$ is required between adjacent identifiers/keywords, and between adjacent strings. The Tokenizer treats each occurrence of whitespace in the specification as a single space. Hence, extra whitespace can be freely added 
between tokens making the specification easier to read without changing the way that it is tokenized. This also applies inside strings; if a string is too long to fit on a single line it can be split over several lines.

\subsection{The PARSER module}

The PARSER module parses ${ }^{50}$ the case law specification using the tokens provided by the Tokenizer. A formal definition of the syntax of the specification language is given, in Extended Backus-Naur Form (EBNF) ${ }^{51}$ in figure 4.3. The specification of case law using this language is best illustrated by example.

\subsubsection{Hierarchy}

A specification starts with an (optional) hierarchy. This binds a court identifier to a string which describes that court. For example:

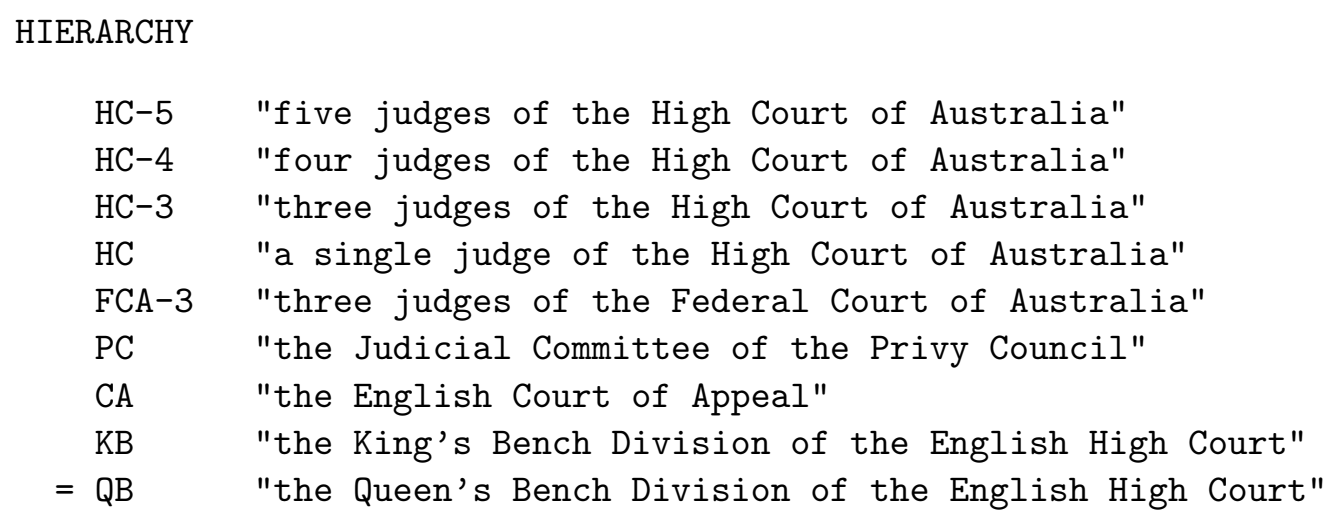

The courts are listed in descending order of seniority; the earlier in the list that a court appears, the better the authority of its cases. An = character separates courts of equivalent rank. It is not necessary to specify every court in the jurisdiction, because courts are ranked relatively: the above example tells SHYSTER that FCA-3 is more important than PC - but not how much more important. Each court identifier in the hierarchy must be unique.

Note that there are three different sorts of identifier: court identifiers, area identifiers and result identifiers. SHYSTER knows which sort of identifier to expect in various places in the specification. Hence, the same identifier can be used (for example) to refer to a result and to a court in the same area. Court identifiers apply in all areas in the specification. The scope of a result identifier is the area in which it is specified: i.e. the same identifier can be used for two different results in two different areas. ${ }^{52}$ 


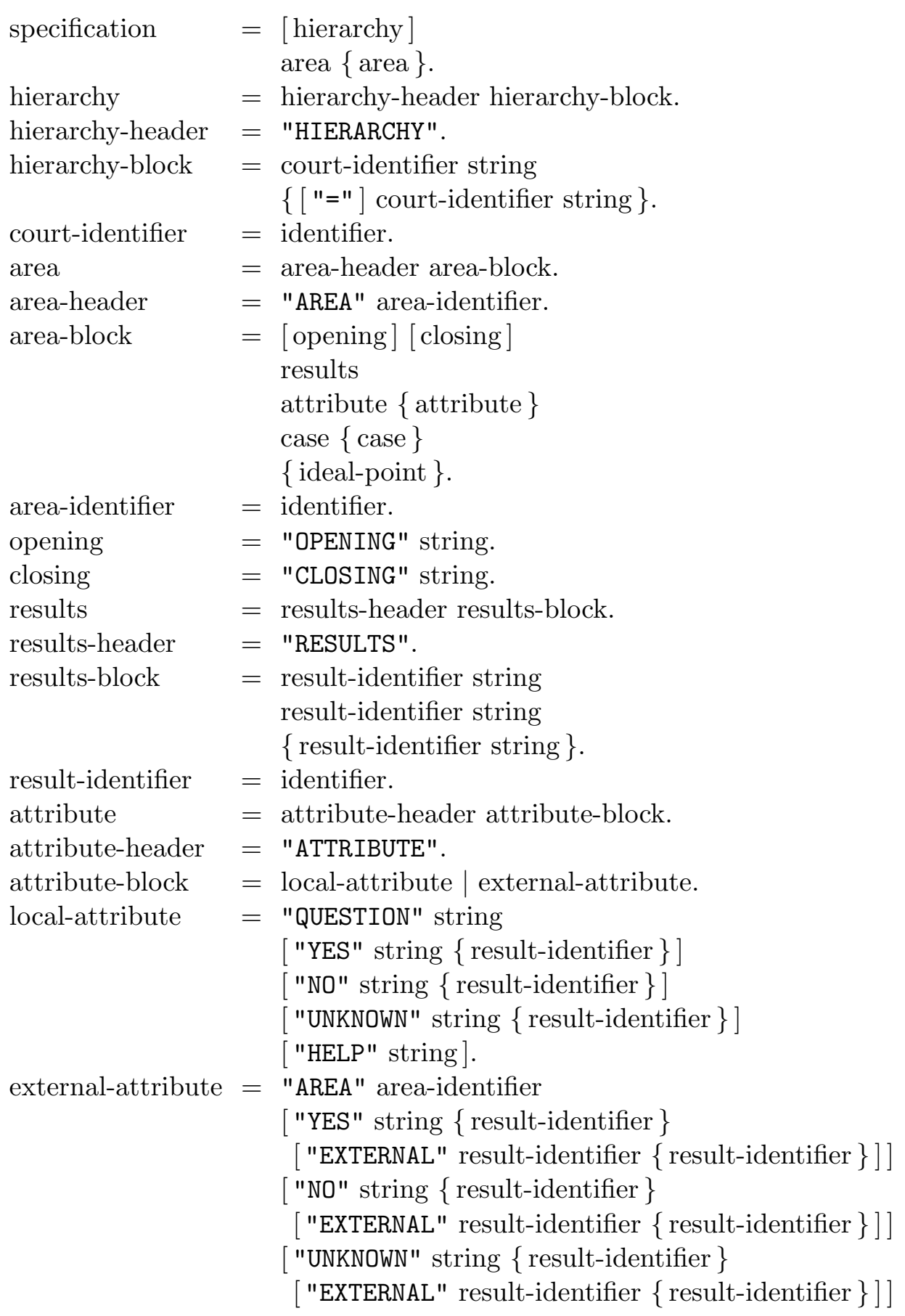

Figure 4.3: A formal definition, in Extended Backus-Naur Form (EBNF), of the syntax of SHYSTER's case law specification language. CONTINUED NEXT PAGE 


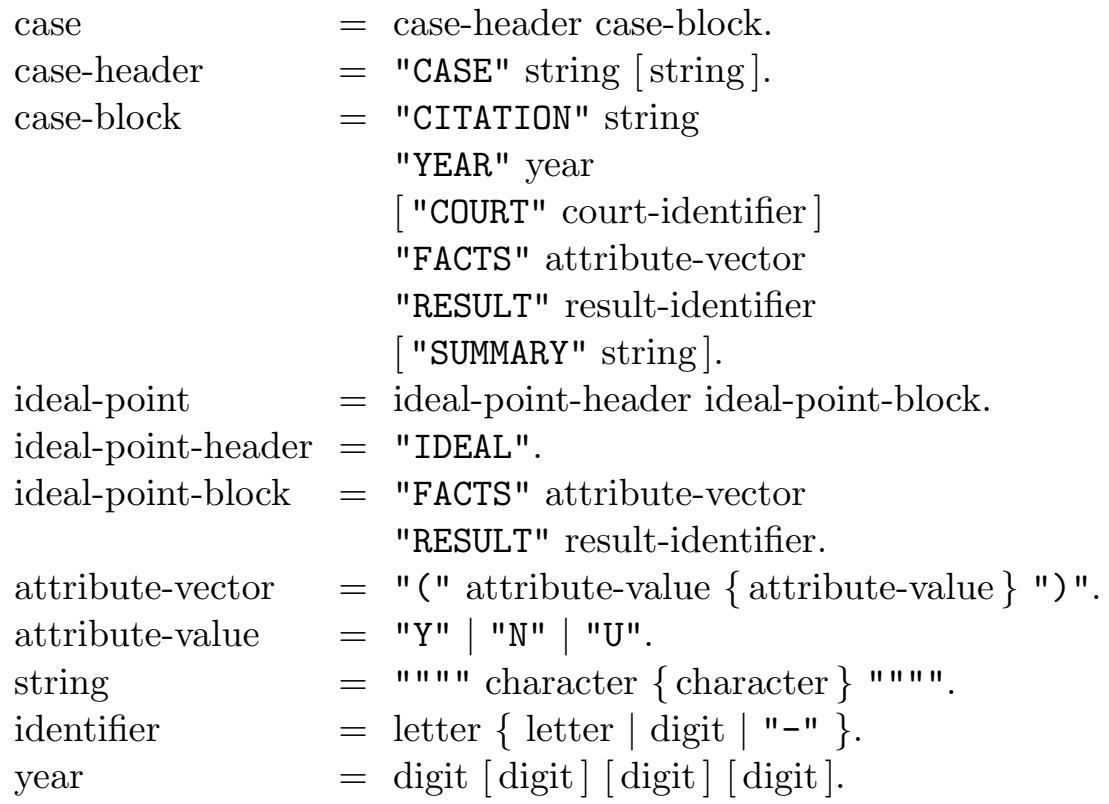

Figure 4.3 (continued).

\subsubsection{Areas}

A specification can contain any number of areas. An area commences with an area header:

\section{AREA Employee}

which binds an identifier to the area that is about to be specified: the identifier with which the case-based system is invoked in order to use this area to define an open-textured concept.

An opening string may be specified. This should be a brief introduction appropriate to any opinion given in this area. It is written at the beginning of SHYSTER's report for this area. Similarly a closing string may be specified, containing concluding remarks.

At least two results must be specified; this involves binding identifiers to strings. For example:

RESULTS

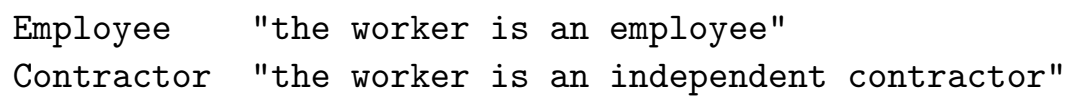

Each string is a statement which holds when the relevant result occurs. It must be cast so that is makes sense when prefixed with the following: "If case is followed then ..." 


\subsubsection{Attributes}

Any number of attributes may be specified. Each attribute must be either local or external.

\section{Local attributes}

For a local attribute, the first keyword after ATTRIBUTE is QUESTION. For example: ${ }^{53}$

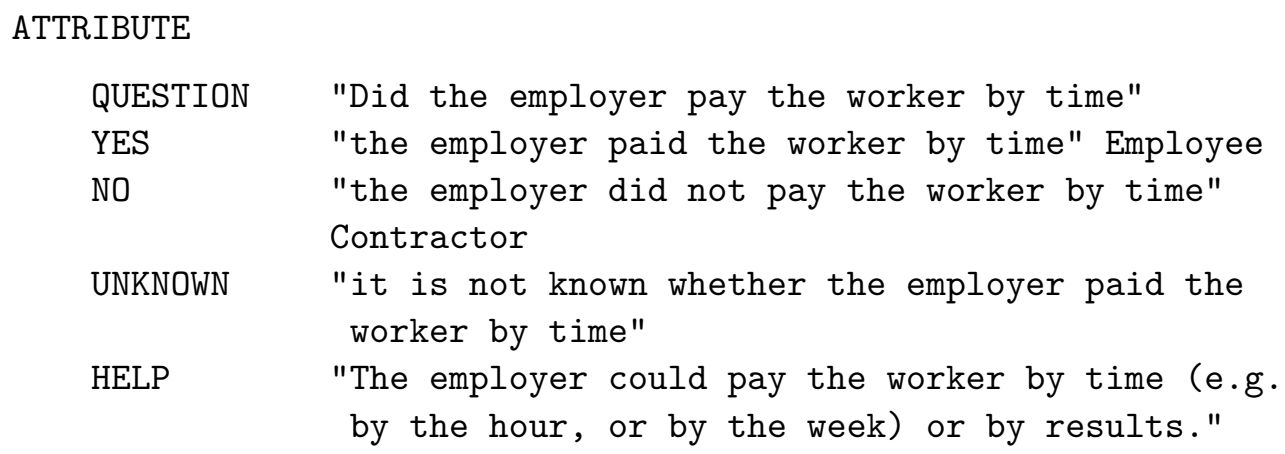

The QUESTION string is the question that the user will be asked if the value of the attribute needs to be determined. The YES, NO and UNKNOWN strings are optional, but the user will only be allowed to answer the question with a value for which there is a string: e.g. if no UNKNOWN string is defined, the user will not be allowed to answer UNKNOWN. The HELP string is displayed at the user's request, and should provide further information to assist her/him in answering the question.

The result identifiers after the strings specify attribute direction: A value of YES for this attribute is directed towards the Employee result; a value of NO is directed towards Contractor.

\section{External attributes}

For an external attribute, the first keyword after ATTRIBUTE is AREA. This example is taken from another specification: ${ }^{54}$

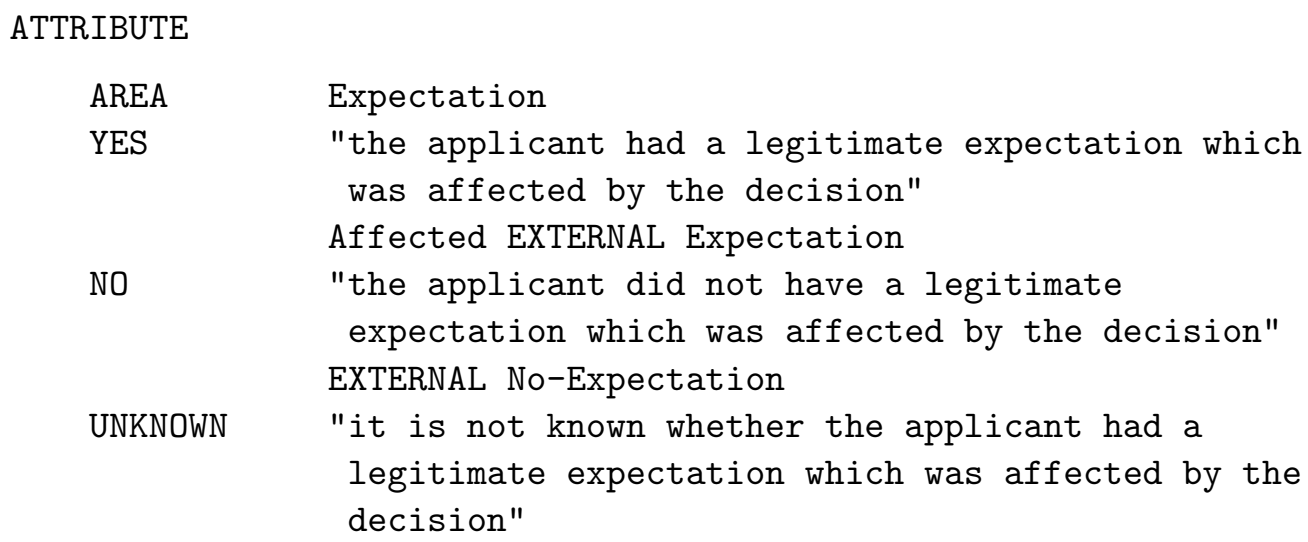


The value of this attribute is to be resolved by reference to the Expectation area. ${ }^{55}$ The identifiers after the EXTERNAL keywords are external result identifiers: result identifiers in the external area. These results are associated with values for this attribute. If SHYSTER returns a result of Expectation for the Expectation area, the value of this attribute is set to YEs; if it returns a result of No-Expectation, the value is set to No. ${ }^{56}$ If it returns any other identifier, SHYSTER exits with an error. $^{57}$

A value of YEs for this attribute is directed towards the Affected result in this area.

\subsubsection{Leading cases}

Any number of leading cases may be specified as follows: ${ }^{.8}$

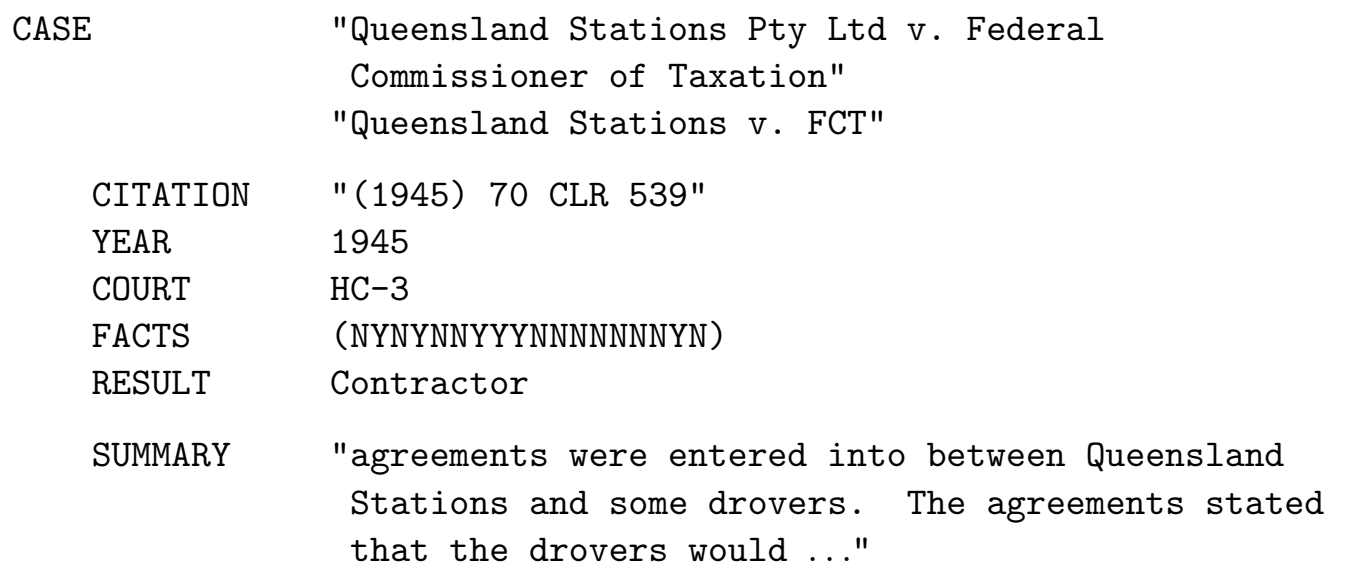

The first CASE string is the full name of the case; the second is a shorter version of the name, and is optional. The CITATION string is the case citation, and the YEAR is the year of the decision - not necessarily the same as the year in the citation. ${ }^{59}$ The (optional) COURT identifier links the case to the court in which it was decided. The FACTS attribute vector has one attribute value for each attribute defined in the area. The order of these values corresponds to the order in which the attributes have been defined. The $\mathrm{Y}, \mathrm{N}$ and $\mathrm{U}$ characters represent the values YES, NO and UNKNOWN, respectively.

The SUMMARY string is the legal expert's summary of the case. The string may contain many sentences, but must be cast so as to make sense when prefixed with the following: "In case, a decision of court, ..." This string may (but need not) include $\mathrm{IAT}_{\mathrm{E}} \mathrm{X}$ commands which will be processed when the case is listed in the dump file, and if the case is used in a report file: e.g. \footnote.

A warning is issued if a result has been specified with no cases, or (worse still) with neither cases nor an ideal point. ${ }^{60}$ And if two cases in an area have the same attribute values, or the same attribute values except for unknown values, a warning is issued. If these two cases have different results, this is also mentioned 
in the warning. If two or more leading cases have identical attribute values but different results then one of those cases was wrongly decided or the area needs another attribute to distinguish between those two cases. ${ }^{61}$

\subsubsection{Ideal points}

Ideal points are specified as follows:

IDEAL

FACTS (YNYNYYYNNYYNYYYYU)

RESULT Employee

This represents the ideal combination of attribute values for the specified result.

\subsection{The DuMPER module}

The Dumper module writes the dump file: a formatted version of the case law specification that has just been read. This file is easier to read (once processed by $\mathrm{LAT}_{\mathrm{E}} \mathrm{X}$ ) than its corresponding specification, simplifying the development and amendment of a specification. It also reflects SHYSTER's internal representation of the specification. Each of the four specifications given in appendix $\mathrm{A}$ is a complete dump file.

The Dumper begins the dump file by displaying the hierarchy of cases in a tabular format. The courts are numbered; courts of the same rank share the same number.

For each area in the specification, the DUMPER starts by displaying the cases and their attribute values in a matrix format. An example is given in figure 4.4. The attribute values are represented by a $\bullet$ symbol for YES, a $\times$ symbol for NO, and a blank space for unKNOwn. ${ }^{62}$

The attributes are named $A_{1} \ldots A_{18}$ in the order of their appearance in the specification. The cases have been grouped by their result, and named $C_{1} \ldots C_{14}$ in the order of their importance within their group. (The more important of two cases is considered to be the one decided by the more important court in the hierarchy or, if their courts are equally important, the case that is more recent.) The ideal points are also represented: $I_{\text {Employee }}$ and $I_{\text {Contractor }}$. The rank of the court in which each case was decided is indicated in the column labelled " $c$ "; these numbers correspond to those in the hierarchy display. This matrix allows the user easily to compare the attribute values in all the specified cases and ideal points.

The Dumper writes out the opening and closing strings (if they were specified). It then writes the result identifiers and their strings. 


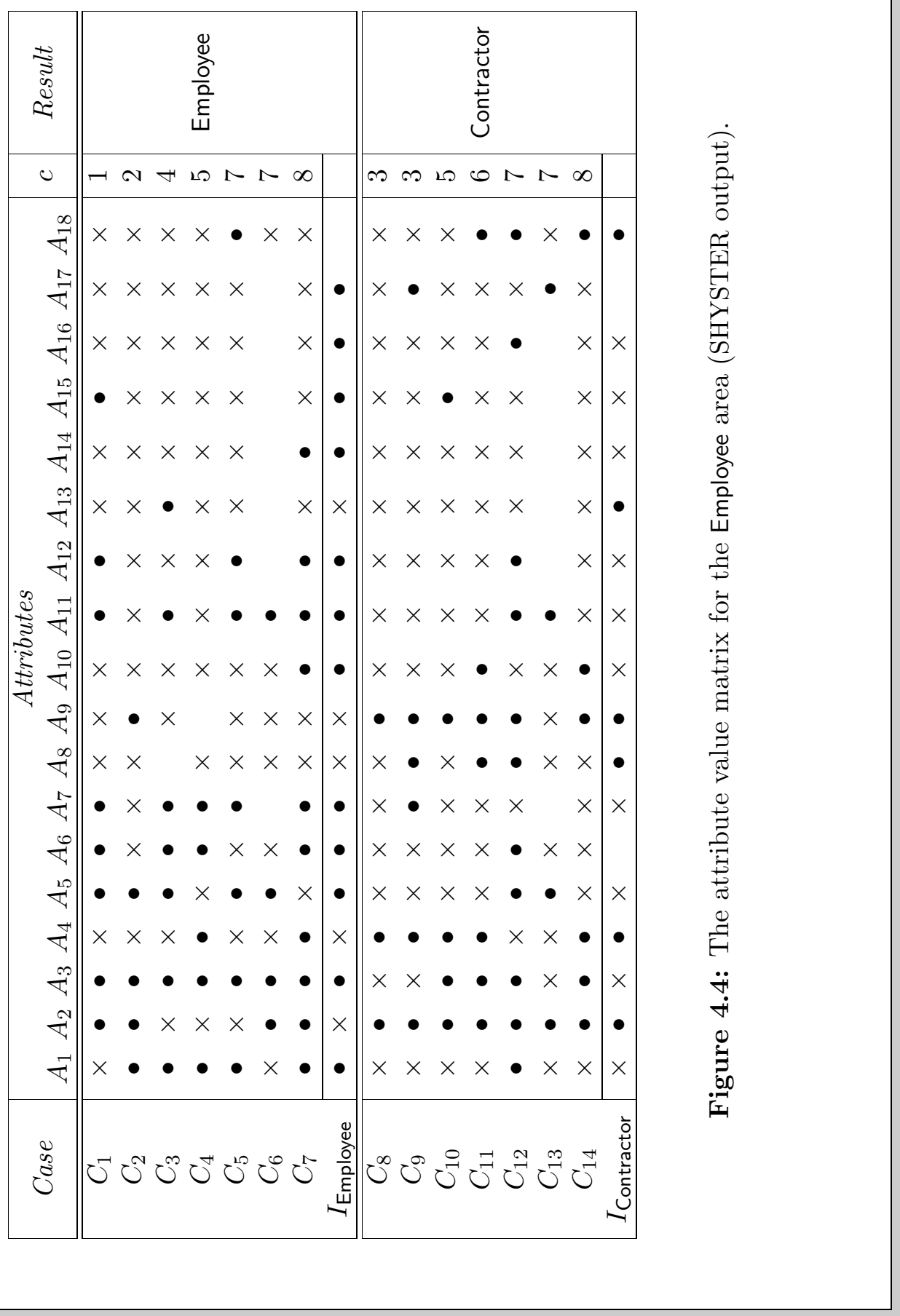




\subsubsection{Attributes}

The Dumper writes details on all attributes: local and external.

\section{Local attributes}

Local attributes are displayed in the following format: ${ }^{63}$

$A_{12}$ : Did the employer pay the worker by time?

YES: the employer paid the worker by time.

$$
\Rightarrow \text { Employee }
$$

NO: the employer did not pay the worker by time.

$$
\Rightarrow \text { Contractor }
$$

UNKNOWN: it is not known whether the employer paid the worker by time.

The employer could pay the worker by time (e.g. by the hour, or by the week) or by results.

Specified direction is indicated using a $\Rightarrow$ symbol: e.g. a value of yES is directed towards the Employee result. ${ }^{64}$ The last paragraph is the help string.

\section{External attributes}

External attributes are displayed in the following format: ${ }^{65}$

$A_{4}: \Leftrightarrow$ Expectation area

YES: the applicant had a legitimate expectation which was affected by the decision.

$\Leftarrow$ Expectation

$\Rightarrow$ Affected

NO: the applicant did not have a legitimate expectation which was affected by the decision.

$\Leftarrow$ No-Expectation

UNKNOWN: it is not known whether the applicant had a legitimate expectation which was affected by the decision.

The link between this attribute and the Expectation area is indicated using a $\Leftrightarrow$ symbol. The association of results from the external area with values of this attribute is indicated using $\mathrm{a} \Leftarrow$ symbol: e.g. if the Expectation area returns a result of Expectation the value of this attribute is set to YES. As with local attributes, specified direction is indicated using a $\Rightarrow$ symbol. 


\subsubsection{Leading cases}

Leading cases appear grouped under the appropriate result heading: e.g. "Cases in which the worker is an independent contractor." They are displayed in the following format:

$C_{9}$ : Queensland Stations Pty Ltd v. Federal Commissioner of Taxation (1945) 70 CLR 539 ("Queensland Stations v. FCT")

$A_{1}$ : the employer did not direct the manner in which the work was to be done.

$A_{2}$ : the worker was allowed to use her/his own discretion in doing an aspect of the work that was not specified beforehand.

$A_{18}$ : the employer and the worker did not express any intention that the relationship would be one of principal and independent contractor.

In Queensland Stations Pty Ltd v. Federal Commissioner of Taxation, ${ }^{66}$ a 1945 decision of three judges of the High Court of Australia, agreements were entered into between Queensland Stations and some drovers. The agreements stated that the drovers would ...

The short case name appears in parentheses after the full citation. The attribute values are described in full. The summary is formatted as it would be if the case were used in a report, complete with footnotes - in the above excerpt, an endnote.

\subsubsection{Ideal points}

Ideal points are displayed using a similar format to that used for leading cases $(\S 4.7 .2)$. Details of the ideal point's attribute values are given under an appropriate result heading. For example:

$I_{\text {Employee }}$ (the ideal case in which the worker is an employee):

\subsection{The ChECKER module}

The CHECKER module examines every pair of attributes in each area for functional dependence and evidence of stochastic dependence. 


\subsubsection{Detecting dependence}

Detecting functional dependence is straightforward. Detecting evidence of stochastic dependence (as explained in $\S 3.8 .2$ ) involves calculating the probability $P(n)$ of there being exactly $n$ YES/YES pairs, assuming random data:

$$
P(n)=\frac{\left(\begin{array}{l}
y \\
n
\end{array}\right)\left(\begin{array}{l}
N-y \\
x-n
\end{array}\right)}{\left(\begin{array}{l}
N \\
x
\end{array}\right)}
$$

where $N$ is the number of known pairs in two attributes $A_{X}$ and $A_{Y}, x$ is the number of yess in $A_{X}$, and $y$ is the number of yess in $A_{Y}$.

For every pair of attributes, the CHECKER counts the actual number of YES/YES pairs and calculates the probability of there being that number of YES/YES pairs or fewer, and the probability of there being that number of YES/YES pairs or more.

The Checker does not need to build a complete probability table, like that in figure 3.3, in order to calculate these two probabilities for each attribute pair. The probability of $i$ YeS/YEs pairs or fewer is $P(n \leq i)=\sum_{n=0}^{i} P(n)$ and the probability of $i$ YES/YES pairs or greater is $P(n \geq i)=1-\sum_{n=0}^{i-1} P(n)$. So, $P(n)$ needs only to be calculated for $n=0 \ldots i$.

The CHecker does not use the formula for $P(n)$ directly when calculating probabilities. Because $n \geq \max (0, x+y-N)$, the first non-zero probability is either $P(0)$ or $P(x+y-N)$. By substitution into the formula for $P(n)$,

$$
P(0)=\frac{(N-x) !(N-y) !}{N !(N-x-y) !}, \quad x+y \leq N
$$

and

$$
P(x+y-N)=\frac{x ! y !}{N !(x+y-N) !}, \quad x+y \geq N
$$

The CHecker starts with the first non-zero probability and successively multiplies it by $P(i+1) / P(i)$ : by substitution,

$$
\frac{P(i+1)}{P(i)}=\frac{(i-x)(i-y)}{(i+1)(N-x-y+i+1)} .
$$

This is a more efficient method of calculating each probability than applying the formula for $P(n)$ directly.

$P(i+1) / P(i)$ becomes zero when $i=x$ or $i=y$, so all values of $P(i)$ for $i>\min (x, y)$ are zero. This limit on $i$ is never exceeded because $n \leq \min (x, y)$ and, as explained above, the CHECKer only calculates $P(n)$ for $n=0 \ldots i$. 


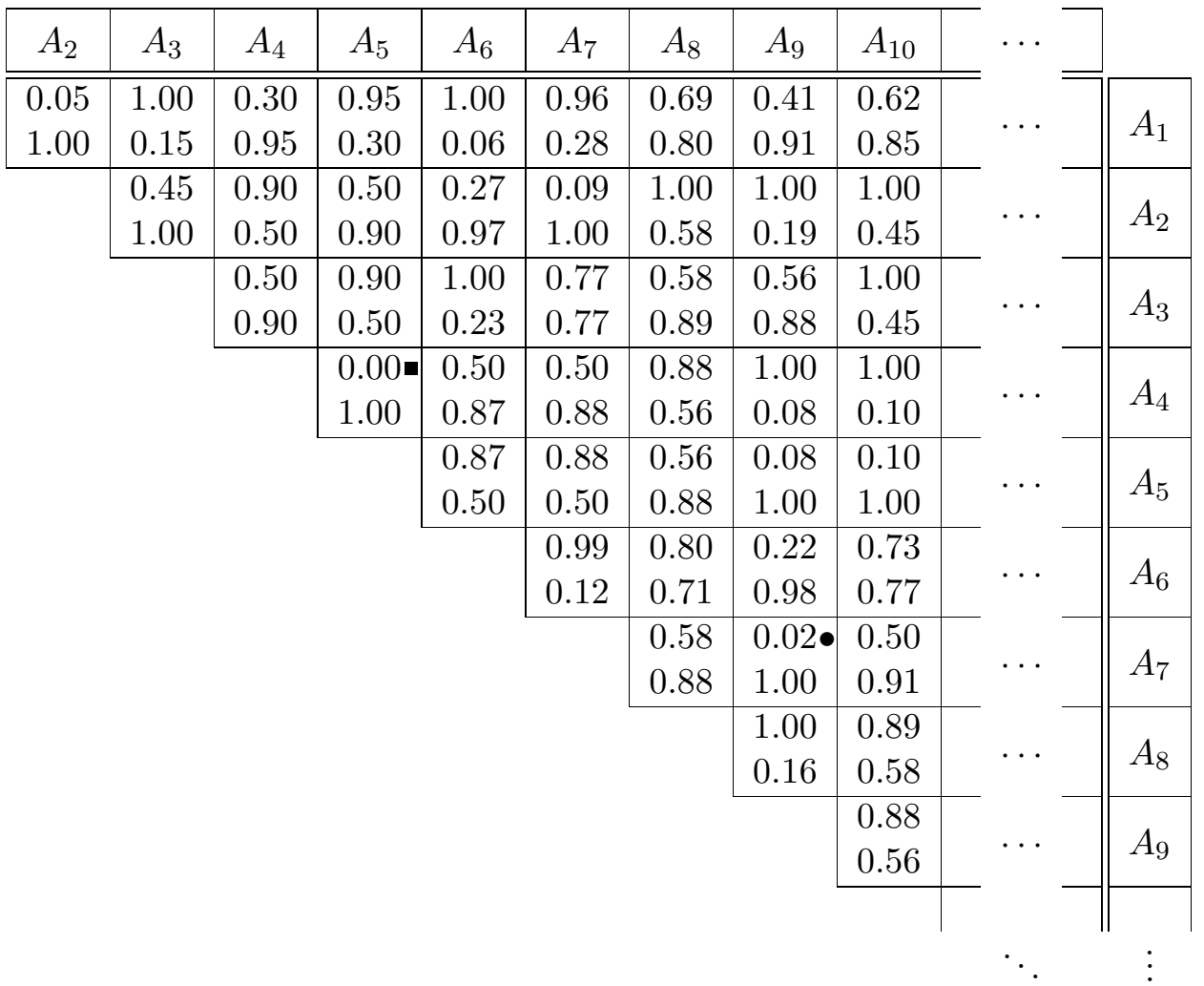

Figure 4.5: An extract from the probabilities matrix for the Employee area (SHYSTER output). The Employee area has 18 attributes; only 45 of the 153 cells in the complete matrix are shown here.

\subsubsection{Writing matrices of probabilities}

Probability figures are written, in matrix form, to SHYSTER's probabilities file. Each cell in this matrix has two probabilities: ${ }^{67}$

1.00 probability of the actual number of YES/YES pairs or fewer; 0.08 probability of the actual number of YES/YES pairs or more.

If a probability figure is not above the threshold of 0.05 (chosen in $§ 3.8 .2$ ) it is marked with a $\bullet$ symbol and a message is written to the log file to warn the legal expert that there is evidence of stochastic dependence. If there is an equivalence function or an inverse function mapping one attribute to the other, the first probability is marked with a symbol and a warning message is written.

An extract from the probabilities matrix for this example is given in figure 4.5. (The complete probabilities file is in $\S$ C.7.) There is functional dependence between attributes $A_{4}$ and $A_{5}$. There is evidence of stochastic dependence between attributes $A_{7}$ and $A_{9}$. 


\subsection{The Scales module}

The ScAles module assigns weights to attributes and writes, to the weights file, a table of weights for each area in the specification.

\subsubsection{Calculating weights}

For each attribute, the SCALEs module calculates a weight and some result weights (one for each result).

The weight (as defined in $\S 3.7$ ) is the inverse of the variance ${ }^{68}$ and it ranges from 4 to infinity. ${ }^{69} \mathrm{~A}$ warning is issued if an attribute has infinite weight.

A result weight is the inverse of the variance of the attribute values for that result. Result weights are used to calculate the strength of attribute directions which represent the extent to which the attribute values of the instant case suggest a given result (see $\S 3.12 .4$ ).

\subsubsection{Writing tables of weights}

The Scales module writes to the weights file a table of weights for each area in the specification. The weights file for the EmpLOYEe specification is given in $\S$ C.9. Each table has columns for the mean $\mu$, variance $\sigma^{2}$ and weight $w$ for each result and for the attribute as a whole. The mean column for each result is that result's centroid (see $\S 3.12 .3$ ).

\subsection{The Adjuster module}

The Adusster module allows any of the attribute weights (including result weights) in the current area to be set to any desired value. This feature is intended for use by the legal expert - not the user, who need not be aware of the manner in which SHYSTER comes to its conclusions. Using the ADJUSTER, the legal expert can test the effect of changing weights during the development of a specification.

The ADJuster is not invoked unless weight adjustment is enabled using the -a switch on the command line. If a weight is changed, an adjusted weights file is written for the current area.

\subsection{The Consultant module}

The Consultant module determines the attribute values for the instant case in the current area. If an attribute is external, the Consultant invokes the CASES module again and assigns the attribute a value on the basis of the result identifier that is returned. If an attribute is local, the user is interrogated as to the attribute values. 
distance

measure:

$$
d_{j k}=\Delta_{j k}
$$

association

coefficient:

$$
S_{j k}=\frac{\Delta_{j k}}{n}
$$

correlation

$$
r_{j k}=\frac{\sum_{i=1}^{n}\left(A_{i j}-\bar{A}_{j}\right)\left(A_{i k}-\bar{A}_{k}\right)}{\sqrt{\sum_{i=1}^{n}\left(A_{i j}-\bar{A}_{j}\right)^{2} \sum_{i=1}^{n}\left(A_{i k}-\bar{A}_{k}\right)^{2}}}
$$

weighted

distance

measure:

$$
d_{j k}^{\prime}=\sum_{i=1}^{n}\left|A_{i j}-A_{i k}\right| \times w_{i}
$$

weighted association

coefficient:

$$
S_{j k}^{\prime}=\frac{\sum_{i=1}^{n}\left|A_{i j}-A_{i k}\right| \times w_{i}}{\sum_{i=1}^{n} w_{i}}
$$

weighted correlation coefficient:

$$
r_{j k}^{\prime}=\frac{\sum_{i=1}^{n}\left(A_{i j} \times w_{i}-\bar{A}_{j}^{\prime}\right)\left(A_{i k} \times w_{i}-\bar{A}_{k}^{\prime}\right)}{\sqrt{\sum_{i=1}^{n}\left(A_{i j} \times w_{i}-\bar{A}_{j}^{\prime}\right)^{2} \sum_{i=1}^{n}\left(A_{i k} \times w_{i}-\bar{A}_{k}^{\prime}\right)^{2}}}
$$

Figure 4.6: Measures of similarity between a case $j$ and a case $k$ (discussed in $\S 3.9 .1$ and $\S 3.9 .2) . \quad \Delta_{j k}$ is the number of differences in the corresponding attribute values of the two cases; $n$ is the number of attributes; $A_{i j}$ is the value of the $i$ th attribute for the $j$ th case; $\bar{A}_{j}$ is the mean of all attribute values for the $j$ th case; $w_{i}$ is the weight of the $i$ th attribute; and $\bar{A}_{j}^{\prime}$ is the weighted mean of all attribute values for the $j$ th case.

At present, the Consultant uses a simple scrolling prompted dialogue. If the user has enabled echo mode (using the -e switch on the command line) then the Consultant echoes the user's input by writing the appropriate attribute string to the terminal. This ensures that the user understands the meaning of the attribute value that she/he has entered. SHYSTER's modular design is such that any desired interface could be employed by changing just this module.

\subsection{The Odometer module}

The Odometer module performs all of SHYSTER's distance calculations, and writes tables of distances to a distances file. 


\subsubsection{Calculating distances}

As explained in $\S 3.9$, for SHYSTER each of the commonly used similarity measures reduces to one of six measures, and a variant of the weighted distance metric $d_{j k}^{\prime}$ was chosen. All six similarity measures are summarized in figure 4.6 (see previous page).

The Odometer calculates the distances (known and unknown) between the instant case and the leading cases, the ideal points, and the centroids. It also calculates values for the extra similarity measures, and the strength of the attribute directions. Using the known and unknown distances it determines which are the nearest cases and the nearest result.

If necessary, it resolves equidistant results by reference to the rank of the courts involved in the nearest cases, and the recentness of those cases. A warning is issued if equidistance has to be resolved in this fashion.

SHYSTER uses a precision threshold of two decimal places in all of its comparisons. This allows for the possibility of rounding errors having been introduced in SHYSTER's arithmetic, and recognizes the danger of relying too much on precise quantification of abstract notions. In addition there is a second precision threshold for use in distance comparisons. ${ }^{70}$ This is the threshold within which two cases will be considered equidistant.

The Odometer is invoked for the instant case, and for each instantiation and each hypothetical. ${ }^{71}$ It treats each instantiation or hypothetical as if it were the instant case for the purposes of performing its calculations.

\subsubsection{Writing tables of distances}

The Odometer writes, to the distances file, a table of distances for the instant case, each instantiation, and each of the chosen hypotheticals. ${ }^{72}$

A distances file for the Employee area is given in full in $\S \mathrm{C} .11$; the instant case is Building Workers' Industrial Union of Australia v. Odco Pty Ltd (discussed in detail in \$5.4.3). An extract from that file - the table of distances for the uninstantiated and unhypothesized instant case - is given in figure 4.7. Tables of distances extracted from other distances files can be found in figures 5.3, 5.6, 5.7 and 5.11 .

The table in figure 4.7 includes an attribute value matrix, similar to the one in figure 4.4, with the addition of the instant case $C_{\text {Instant }}$ and a centroid for each result: $\mu_{\text {Employee }}$ and $\mu_{\text {Contractor. }}$ As before, the rank of the court that decided each case is in the column labelled " $c$ ".

The known and unknown distances are in columns labelled " $d_{\mathrm{K}}$ " and " $d_{\mathrm{U}}$ ". Unweighted distance measures are in the column labelled " $\Delta$ " —not " $d$ ", to avoid confusion with the known and unknown distances. Values for the other similarity measures $\left(S, S^{\prime}, r\right.$ and $\left.r^{\prime}\right)$ are labelled appropriately. ${ }^{73}$ 


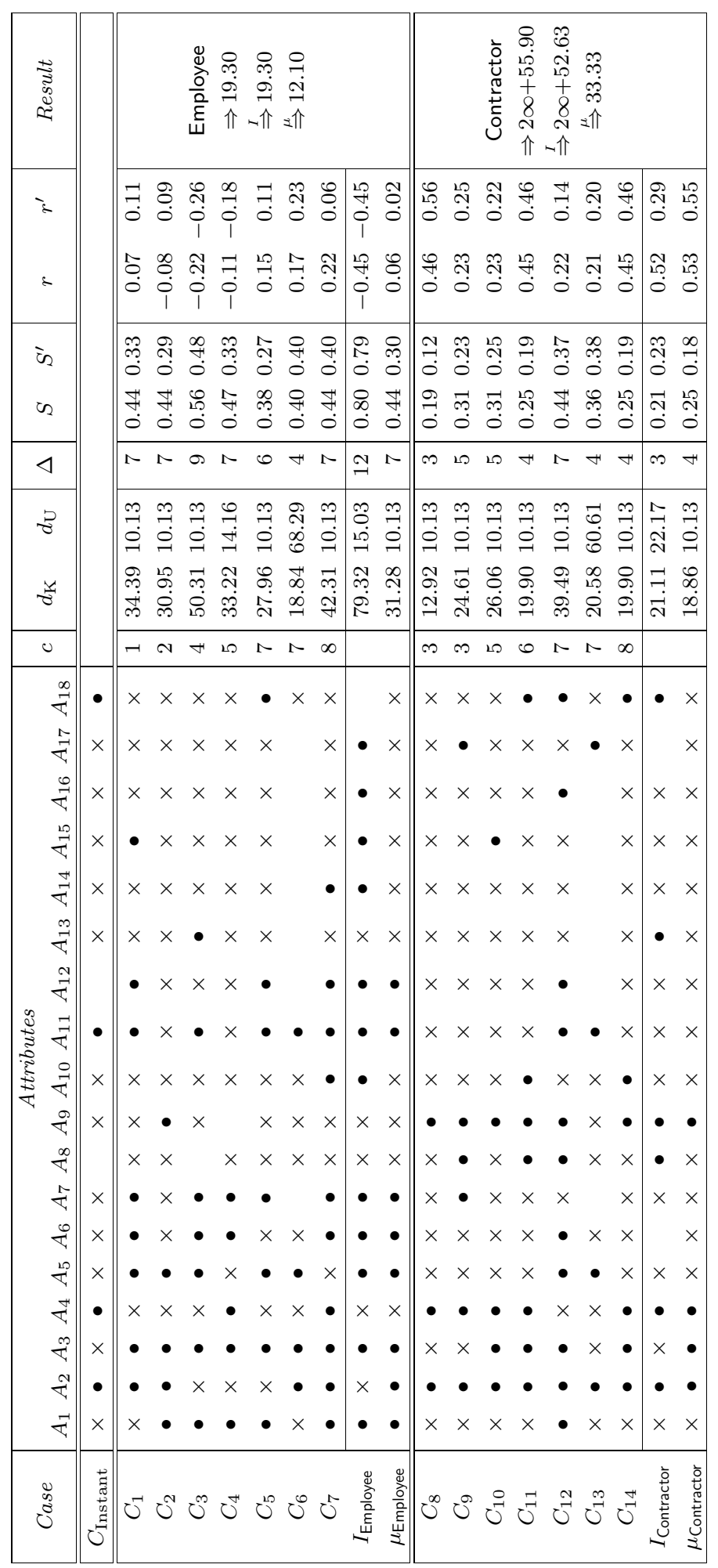

空

a

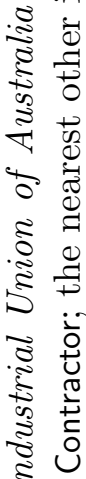

है.

के

है

3

. ¿

సิำ

రై

y $0^{\infty}$

:

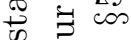

.马

पे

욤

茟苟

焉.

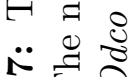

-

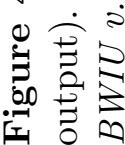


The strength of each non-zero attribute direction is displayed in the "Result" column. $\mathrm{A} \Rightarrow$ symbol indicates specified direction; $\stackrel{I}{\Rightarrow}$ symbol indicates ideal point direction; a $\stackrel{\mu}{\Rightarrow}$ symbol indicates centroid direction. (If all three directions are of the same strength, $\mathrm{a} \stackrel{\star}{\Rightarrow}$ symbol and a single number appear in their place.) Each number represents the strength of the direction towards that result in the instant case.

\subsection{The REPORTER module}

The principal output from SHYSTER is contained in its report file, written by the REPORTER module. This module also implements SHYSTER's safeguards and writes information about them, together with a general summary of its report, to the log file.

In each area, the REPORTER is invoked once for the instant case, and once for each instantiation and for each chosen hypothetical. ${ }^{74}$ Only if an instantiation has a different result to that of the instant case does the REPORTER write a report on that instantiation.

A report file for the Employee area is given in full in $\S$ C.13. Again, the instant case is BWIU v. Odco. Except where otherwise indicated, the examples in this section are taken from that report file. Other example report files are given in appendix B.

\subsubsection{Arguing with the instant case}

The REPORTER argues that the result in the instant case will be the nearest result, then builds a counterargument for each of the nearest other results. This same process is also used to argue with instantiations and with hypotheticals (see $\S 4.13 .2$ and $\S 4.13 .3$ below).

\section{Arguing for the nearest result}

For the instant case, the REPORTER starts by writing the opening string to the report file. It then states the facts of the instant case and declares its opinion as to the likely result - the nearest result:

In my opinion-following Humberstone v. Northern Timber Mills - the worker is an independent contractor.

Humberstone v. NTM ( $C_{8}$ in figure 4.7) is the nearest (unknown) neighbour. There are no nearest known cases; all of the leading cases have some unknown distance because of the two UNKNOWN attribute values in the instant case. 
The REPORTER then summarizes the nearest neighbour: ${ }^{75}$

In Humberstone v. Northern Timber Mills, ${ }^{76}$ a 1949 decision of three judges of the High Court of Australia, Humberstone carried goods for NTM. He had originally held himself out as a carrier, ...

If the case is identical to the instant case - if it has the same attribute valuesthen the REPORTER announces that the two cases are "on all fours." 77 If there is no known distance between the cases but there is some unknown distance, it declares that the two cases "may be on all fours" and explains its reservation on the basis of the unknown attributes. In this example there are some known differences. First, the REPORTER lists all of the similarities:

There are several significant similarities between the instant case and Humberstone $v$. NTM: the employer did not direct the manner in which the work was to be done; the worker was allowed to use her/his own discretion in doing an aspect of the work that was not specified beforehand; ...

Three or more similarities are characterized as "significant"; two similarities are "very significant"; a solitary similarity is called "extremely significant," as it must be for such a dissimilar case to be the nearest neighbour.

Then the REPORTER lists all of the differences:

However, the instant case is not on all fours with Humberstone v. NTM. In that case the worker was not in business on her/his own account; the worker was allowed to employ others to assist with her/his work; the worker was not required to work at specified times; the employer did not pay the worker by time; and the employer and the worker did not express any intention that the relationship would be one of principal and independent contractor.

And if there are any unkNOwns in the leading case - there are none in this example - then they are explained too.

Despite the differences, SHYSTER stands by its original statement:

Nevertheless, I believe that Humberstone v. NTM should be followed.

If there are equidistant nearest neighbours they are all handled in this fashion, with a linking paragraph written between each pair of cases. This paragraph explains which case is the more important and why, as in this example taken from the report on the second hypothetical for BWIU v. Odco:

In 1967, Ready Mixed Concrete (South East) Ltd v. Minister of Pensions and National Insurance ${ }^{78}$ was decided by the Queen's Bench Division of the English High Court. (A case decided by the Queen's Bench Division of the English High Court is not as good authority as a case decided by the Judicial Committee of the Privy Council-like AMP v. Chaplin; furthermore Ready Mixed v. Minister is 11 years older than AMP v. Chaplin.) 


\section{Arguing for other results}

For every other result, the REPORTER discusses the nearest other. It explains the effect of following the nearest other instead of the nearest neighbour, then summarizes the nearest other:

If Ferguson v. John Dawson $\mathcal{B}$ Partners (Contractors) Ltd is followed then the worker is an employee.

In Ferguson v. John Dawson $\&$ Partners (Contractors) Ltd, ${ }^{79}$ a 1976 decision of the English Court of Appeal, Ferguson fell off a roof while removing some scaffolding boards. He claimed damages against John Dawson (the building contractors) for breach of statutory duty relying on ...

Once again all of the similarities are listed, but this time the differences are decisive:

However, there are several significant differences between the instant case and Ferguson v. Dawson. In that case the employer directed the manner in which the work was to be done; the worker was not allowed to use her/his own discretion in doing an aspect of the work that was not specified beforehand; the worker was an integral part of the employer's business; ...

(Like the similarities in the nearest neighbours, the differences in the nearest others are characterized as "significant," "very significant" or "extremely significant," depending on their number.)

The REPORTER also compares the importance of this case with that of the nearest neighbour, ${ }^{80}$ before reiterating its opinion:

Note also that Ferguson v. Dawson is only a decision of the English Court of Appeal and not as good authority as a case decided by three judges of the High Court of Australia-like Humberstone v. NTM.

Consequently, there is nothing in Ferguson v. Dawson to warrant any change in my conclusion.

The REPORTER is not swayed from its conclusion on the basis of the importance of the nearest other. For example, in the report on the first hypothetical for $B W I U$ v. Odco it writes:

Despite the fact that Cam v. Sargent is a decision of four judges of the High Court of Australia (and better authority than a case decided by three judges of the High Court of Australia-like Humberstone v. NTM), there is nothing in Cam v. Sargent to warrant any change in my conclusion.

Equidistant nearest others are handled in a similar fashion to that used for equidistant nearest neighbours. The report on the instant case concludes with the closing string. 


\section{Variations}

Although the REPORTER relies heavily on the strings supplied by the legal expert in the specification, each report is more than a mere regurgitation of these strings. The structure of each report varies depending on the circumstances. For example, where a case would have been the nearest case if not for its unknown distance, the REPORTER makes this clear-without any mention of unknown distance. This example is taken from another report file: ${ }^{81}$

I would have suggested that Stevenson v. Macdonald (1) be followed (instead of Massey v. Crown Life) except that it is not known whether the employer supervised or inspected the work; it is not known whether the employer paid the worker by time; it is not known whether the money that the employer paid to the worker was stated to be a "fee"; ...

Different situations are handled in different ways at several stages in the process of preparing the report. For this reason, there are many different possible reports, even ignoring the difference in case names, summaries, and other strings. This means that SHYSTER's reports read quite well. Although their style is a little stilted, it is just possible that they could be mistaken for the work of a lawyeralthough that is not one of the aims of this thesis project.

\subsubsection{Arguing with instantiations}

For the purposes of writing reports on instantiations, the REPORTER treats each instantiation as if it were the instant case and follows the steps outlined in $\S 4.13 .1$ above. ${ }^{82}$ The only differences are in the introductory comments, and in the fact that the instant case is referred to as the "instantiated case."

For example, a report on an instantiation from another test case commences as follows: ${ }^{83}$

It may be that the following is true of the instant case: the employer would not make a profit/loss if the work performed by the worker cost less/more than expected; and the employer neither supervised nor inspected the work.

If that is so then in my opinion - following Ready Mixed Concrete (South East) Ltd v. Minister of Pensions and National Insurance - the worker is an independent contractor.

\subsubsection{Arguing with hypotheticals}

As with instantiations, to write a report on a hypothetical the REPORTER treats that hypothetical as if it were the instant case and follows the steps outlined in $§ 4.13 .1$ above. ${ }^{84}$ Again, the introductory comments are different, and the instant case is referred to as the "hypothetical case." 
The report on the first hypothetical in the report file for BWIU v. Odco starts with these words:

Consider the instant case changed so that the following is true: the worker was allowed to employ others to assist with her/his work; and the employer and the worker did not express any intention that the relationship would be one of principal and independent contractor.

If that were so then I would be more strongly of the opinion thatfollowing Humberstone v. Northern Timber Mills - the worker is an independent contractor.

If the hypothetical variations lead SHYSTER to a different conclusion, as in the second hypothetical in the example report file, the words are different again:

Consider the instant case changed so that the following is true: the worker was not allowed to use her/his own discretion in doing an aspect of the work that was not specified beforehand; and the worker was an integral part of the employer's business.

If that were so then my opinion would be that-following Ferguson $v$. John Dawson $\&$ Partners (Contractors) Ltd-the worker is an employee.

\subsubsection{Safeguards}

As well as constructing its reports, the REPORTER implements the safeguards explained in $\S 3.12$. It determines which would be the nearest neighbours applying, in turn, each of the extra similarity measures: $d_{j k}, S_{j k}, S_{j k}^{\prime}, r_{j k}$ and $r_{j k}^{\prime}$. If these measures suggest different nearest neighbours from those chosen by SHYSTER using its known and unknown distance, that fact is noted in the log file.

Figure 4.8 is extracted from the log file for this example (the complete log file is in $\S$ C.2). The fact vector for the instant case is given, then the short case names of the nearest neighbour and the nearest other. All of the extra similarity measures suggest the same nearest neighbour; there are no safeguard warnings. The nearest result is declared to be Contractor (this is the identifier that is returned by this invocation of the case-based system).

The fact vector of the first instantiation is given, and the differences between it and the instant case are marked with carets. In this instantiation, the nearest neighbour and nearest other are the same as in the uninstantiated instant case. However, some of the extra similarity measures now disagree with the choice of nearest neighbour. These disagreements are set out under the "Safeguards" heading. ${ }^{85}$ Extra cases which the extra measures suggest as the nearest neighbour are marked with $\mathrm{a}^{+}$; if an extra case has a different result to that of the instantiation, it is marked with a $*$ and its result appears in parentheses after the case name. If an extra measure does not suggest one of the nearest neighbours it is marked with a -. 


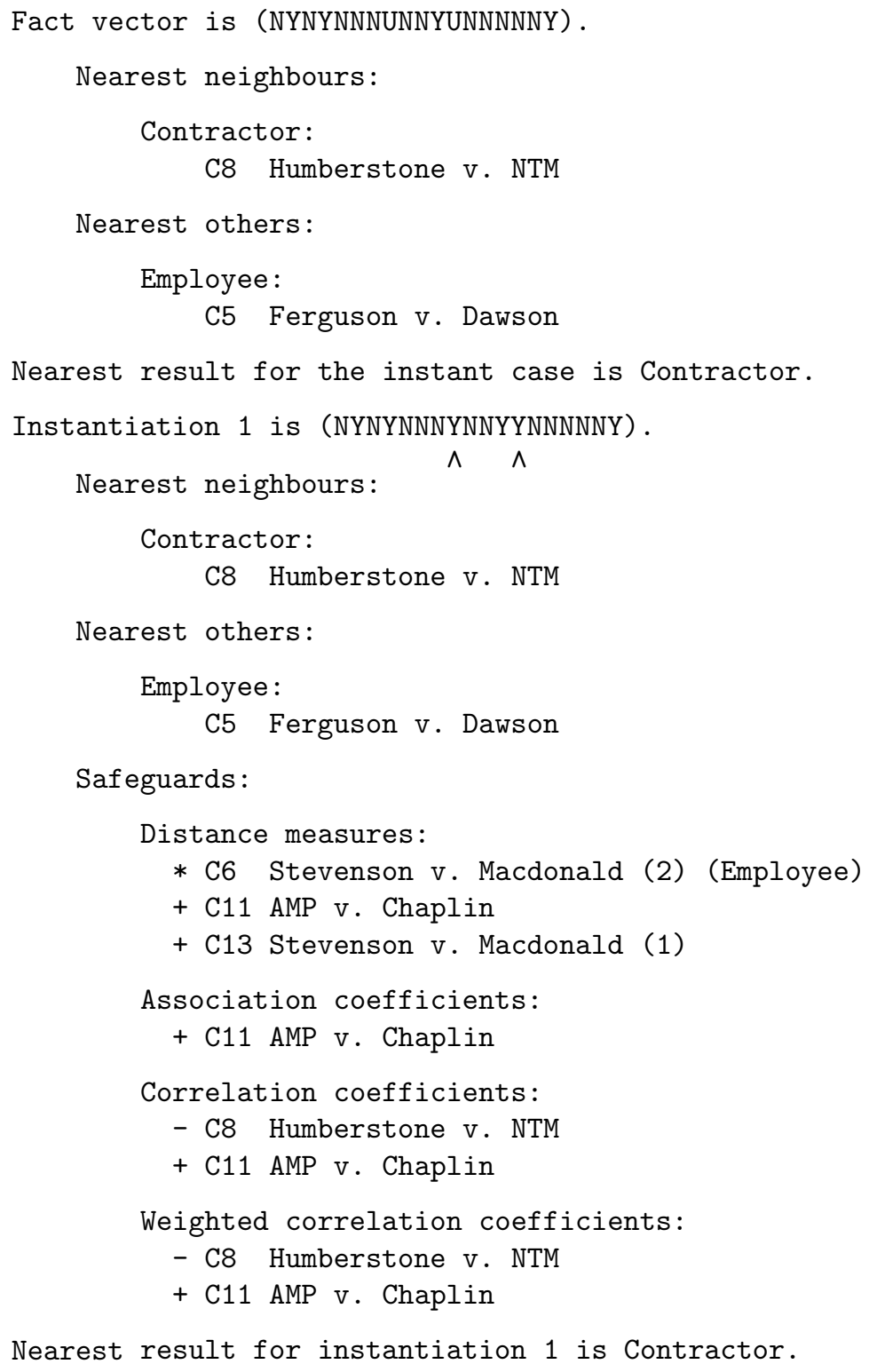

Figure 4.8: An extract from the log file for Building Workers' Industrial Union of Australia v. Odco Pty Ltd (SHYSTER output).

All of these differences are logged, but only some of them will cause a warning to be issued. If one or both of the weighted safeguard metrics suggest that a case with a different result should be the nearest neighbour then a warning is issued. In this example, only the (unweighted) distance measures suggest a different result, so no warning is issued. 
The Reporter also writes to the log file if the ideal points, centroids or any of the three attribute directions (specified, ideal point and centroid directions) suggest a different result. However, a warning is not issued unless an ideal point or a centroid with a different result is at least as near to the instant case (or instantiation or hypothetical) as is the nearest neighbour, or if the specified directions suggest a different result. Warnings are written to the log file and to the standard error stream.

In summary, SHYSTER will issue a warning in each of the following circumstances:

- the weighted association coefficients suggest that a case, with a different result than that of the nearest neighbour, ought to be the nearest neighbour;

- the weighted correlation coefficients suggest that a case, with a different result than that of the nearest neighbour, ought to be the nearest neighbour;

- an ideal point suggesting a different result is at least as near to the instant case as is the nearest neighbour;

- a centroid suggesting a different result is at least as near to the instant case as is the nearest neighbour; or

- the specified directions suggest a different result or results.

As explained in $§ 3.12 .5$, SHYSTER will also issue a warning if:

- there are two or more equidistant results.

And, as explained in $\S 3.12 .6$, a warning is issued if:

- an instantiation of the instant case has a different result to that of the uninstantiated instant case.

\subsection{Conclusion}

SHYSTER is a working implementation of the pragmatic approach to case law adopted, and justified, in the previous chapter. It has been designed to allow a legal expert to specify areas of case law using a simple specification language. SHYSTER's case-based system has been constructed so as to facilitate its linking with a rule-based system to form a hybrid legal expert system.

Within the limitations imposed by its internal representation of case law, SHYSTER is capable of producing sophisticated reports on the likely outcome in user-specified instant cases. In the process of constructing its report, SHYSTER produces several intermediate files containing information of use to the knowledge engineer and the legal expert.

The next chapter discusses the specification of areas of law and the use of those specifications, and various testing methods, to evaluate SHYSTER's output - to evaluate SHYSTER's approach to case law. 


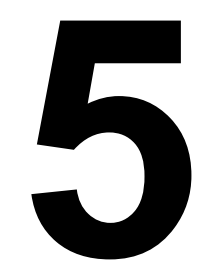

\section{Case studies}

... the notion that a computer can predict the course of judicial decision rests on assumptions that are demonstrably untenable, does violence to the very nature of law, and is moreover certain to blunt the professional techniques of any lawyer who relies on machines rather than on his own powers of reasoning and advocacy.

Frederick Bernays Wiener (1962) Decision Prediction by Computers: Nonsense Cubed-and Worse ${ }^{86}$
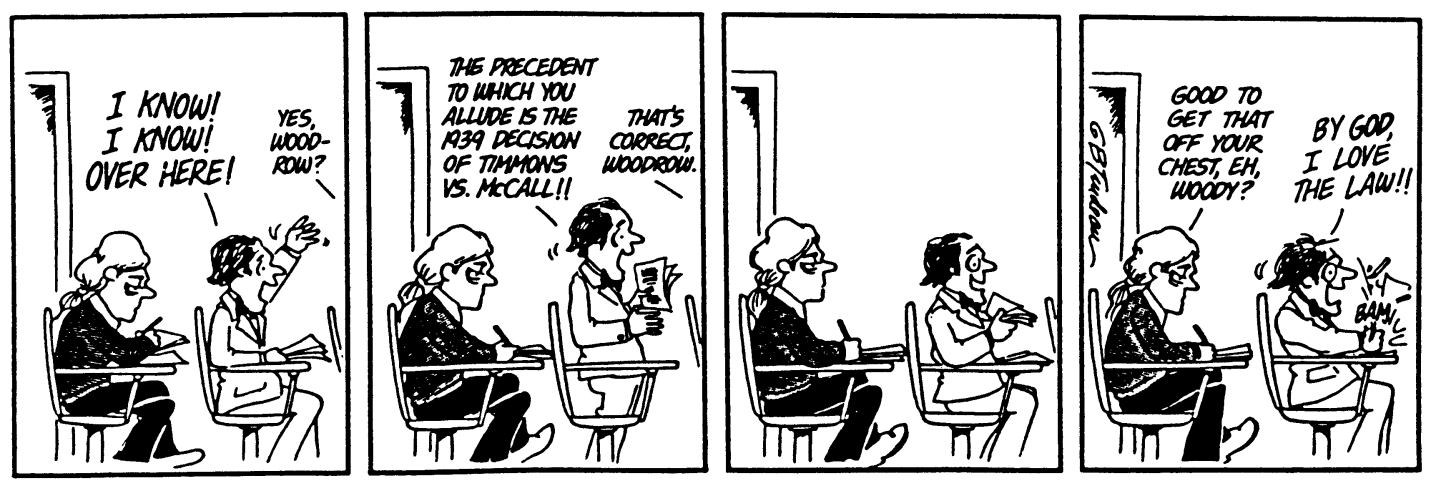

G. B. Trudeau (1975)

The Doonesbury Chronicles ${ }^{87}$ 


\section{$5.1 \quad$ Introduction}

SHYSTER is unusual amongst legal expert systems in that it has not been developed specifically for a given area of the law. Instead, its case-based system has been designed to allow the specification of different areas of law. In order to test SHYSTER, and its approach to case law, four specifications were written. Each specification aims to represent an area of Australian law.

The complete specifications are given in appendix A, and discussed in detail in $\$ 5.2-\S 5.5$ below. For each specification, the law is briefly stated, and the specification and its testing are described. The methods used to test each specification, and SHYSTER, are explained in $\S 3.13$.

The facts of cases - cases which are not part of the specification - were given to SHYSTER, and SHYSTER's opinions were compared with the judgments in those cases. If a specification includes ideal points, those ideal points were also used as test cases. Each of these tests is discussed, individually, below. The results of these tests are summarized in figure 5.12 near the end of this chapter, and conclusions are drawn from them in $\S 5.6 .1$.

Generated tests (explained in $\$ 3.13 .4$ ) were performed for each specification for which legal expertise was available: i.e. all but the FINDER specification. Generated testing is also discussed below, and summarized in figure 5.13 at the end of this chapter. Conclusions are drawn from the results of these tests in $\S 5.6 .2$.

The results of reflexive testing (explained in §3.13.5) of every leading case in each of the four specifications are set out and discussed in appendix D, and summarized in the four figures in that appendix. Conclusions are drawn from these results in $§ 5.6 .3$.

Conclusions are drawn from all of this testing in the next chapter.

\subsection{A simulation of FINDER}

FINDER $(\S 2.5 .2)$ is a case-based legal expert system. Because SHYSTER's approach to case law expands upon that of FINDER, a specification can be written which causes SHYSTER to simulate FINDER.

The simulation does not make use of all of SHYSTER's features (as explained in $§ 5.2 .2$ below). However, SHYSTER's safeguard mechanisms, and the opportunity to perform reflexive tests, mean that FINDER's approach to case law can be tested more extensively using SHYSTER than was possible using FINDER itself.

\subsubsection{The law}

The law of trover - the law concerning the rights of the finders of lost chattelsis unusual in that it is based entirely on cases. The 1772 case of Armory $v$. Delamirie $^{88}$ established that the finder of any article which has been lost has a general right to that article as against all the world except the true owner. ${ }^{89}$ Only 
a few reported trover cases have been decided in the 270 years since. Although they are few, these cases have qualified considerably the general rule stated in Armory $v$. Delamirie, and the law of trover has become complicated.

In 1948, Lord Goddard CJ of the King's Bench Division of the English High Court referred to several trover cases (Bridges v. Hawkesworth, ${ }^{90}$ Elwes v. Brigg Gas Co. ${ }^{91}$ South Staffordshire Water Co. v. Sharman ${ }^{92}$ and Hannah v. Peel ${ }^{93}$ ) and said:

These cases, or rather the first three of them, have long been the delight of professors and text writers, whose task it often is to attempt to reconcile the irreconcilable. It is, however, right to say that in recent years both the Corpus Professor of Jurisprudence at Oxford and the Professor Emeritus of English Law at Cambridge have expressed the opinion that Bridges v. Hawkesworth was wrongly decided. If it was, the difficulty largely disappears. But that much-battered case has lately been re-invigorated by Birkett J's decision in Hannah v. Peel, and I am glad to think that ... it is still for wiser heads than mine to end a controversy which will no doubt continue to form an appropriate subject for moots till the House of Lords lays it to rest for all time. ${ }^{94}$

Although wiser heads than Lord Goddard's have not yet laid it to rest for all time, Donaldson LJ made what has been called "an unusually clear statement of the principles to be applied" 95 in the 1982 case of Parker v. British Airways. ${ }^{96}$ He listed the following rights and obligations of the finder:

1. The finder of a chattel acquires no rights over it unless (a) it has been abandoned or lost and (b) he takes it into his care and control.

2. The finder of a chattel acquires very limited rights over it if he takes it into his care and control with dishonest intent or in the course of trespassing.

3. Subject to the foregoing and to point 4 below, a finder of a chattel, whilst not acquiring any absolute property or ownership in the chattel, acquires a right to keep it against all but the true owner or those in a position to claim through the true owner or one who can assert a prior right to keep the chattel which was subsisting at the time when the finder took the chattel into his care and control.

4. Unless otherwise agreed, any servant or agent who finds a chattel in the course of his employment or agency and not wholly incidentally or collaterally thereto and who takes it into his care and control does so on behalf of his employer or principal who acquires a finder's rights to the exclusion of those of the actual finder.

5. A person having a finder's rights has an obligation to take such measures as in all the circumstances are reasonable to acquaint the true owner of the finding and the present whereabouts of the chattel and to care for it meanwhile. ${ }^{97}$ 
His lordship then listed the following rights and obligations of the occupier of the premises where the chattel was found:

1. An occupier of land has rights superior to those of a finder over chattels in or attached to that land and an occupier of a building has similar rights in respect of chattels attached to that building, whether in either case the occupier is aware of the presence of the chattel.

2. An occupier of a building has rights superior to those of a finder over chattels on or in, but not attached to, that building if, but only if, before the chattel is found, he has manifested an intention to exercise control over the building and the things which may be on or in it.

3. An occupier who manifests an intention to exercise control over a building and the things which may be on or in it so as to acquire rights superior to those of a finder is under an obligation to take such measures as in all the circumstances are reasonable to ensure that lost chattels are found and, on their being found, whether by him or by a third party, to acquaint the true owner of the finding and to care for the chattels meanwhile. The manifestation of intention may be express or implied from the circumstances including, in particular, the circumstance that the occupier manifestly accepts or is obliged by law to accept liability for chattels lost on his 'premises' e.g. an innkeeper's or carrier's liability.

4. An 'occupier' of a chattel, e.g. a ship, motor car, caravan or aircraft, is to be treated as if he were the occupier of a building for the purposes of the foregoing rules. ${ }^{98}$

It is clear that the question of who owns a found chattel remains, in Lord Goddard's words, "a really difficult area of law". 99

\subsubsection{The FINDER specification}

The dump file for the FINDER specification is given in full in $\S$ A.2. The specification contains a single area: the Finder area. This area does not make use of all of SHYSTER's features - it has no opening string, no closing string, no help strings, no UNKNOWN attribute values, no ideal points and no attribute direction-because FINDER does not have these features.

Only two features of this specification are not part of FINDER. First there is the inclusion of a hierarchy of courts. This is not strictly necessary; SHYSTER does not require that a hierarchy be specified. The effect of this addition is merely cosmetic: it allows SHYSTER to mention the court in which a case was heard when it discusses that case. There are only three courts in this hierarchy representing two of the three divisions of the English High Court: the Chancery division, and the King's/Queen's Bench division. All three courts are of equal importance; the inclusion of the hierarchy does not affect which cases SHYSTER chooses to justify its opinions. 
The second feature of this specification that is not part of FINDER is the inclusion of UNKNOwN strings in each attribute. This allows the user to answer "unknown" to any of the attribute questions, and force SHYSTER to examine instantiations of the instant case.

\section{Results}

The Finder area has two results: Win and Lose. These correspond to FINDER's two possible outcomes: "the finder wins" and "the finder loses." The area-like FINDER - actually represents only part of the law of trover: the resolution of conflict between the finder of a chattel and another person who is not the true owner of the chattel.

\section{Attributes}

There are ten attributes, whose questions are taken (slightly reworded) from the questions asked by FINDER:

$A_{1}$ : Was the finder the occupier of the premises where the chattel was found?

$A_{2}$ : Was the chattel attached to the land or premises where it was found?

$A_{3}$ : Was the other claimant (the non-finder) the owner of the premises where the chattel was found?

$A_{4}$ : Was the other claimant the true owner of the chattel or did she/he claim through the rights of the true owner?

$A_{5}$ : Did the finder hand over the chattel to the other claimant after the finding?

$A_{6}$ : Did one of the parties rely on the terms of an agreement made with the other which purported to give her/him the right to the chattel?

$A_{7}$ : Was the finder a servant of the other claimant?

$A_{8}$ : Was the chattel hidden or in a position so as to be difficult to find?

$A_{9}$ : Was an attempt made to find the true owner of the chattel or, alternatively, was the chattel clearly abandoned?

$A_{10}$ : Did either of the parties know of the existence of the chattel prior to the finding?

Answering these questions should be fairly straightforward. Only the use of the word "servant" in $A_{7}$ could cause difficulty. Lawyers use the word to refer to an employee as opposed to an independent contractor - a distinction which is the basis of the EMPLOYEE specification described in $\S 5.4 .2$ below. Tyree suggests that 
"servant" can be read as "employee". ${ }^{1}$ However, in one (and perhaps both) of the FINDER cases in which the value of $A_{7}$ is YES, the finder was an independent contractor of the other claimant. ${ }^{2}$ Clearly "servant" has a broad meaning in FINDER; it would probably be better to ask "Was the finder an employee of, or independent contractor to, the other claimant?" However, in the interests of an accurate simulation, the question for $A_{7}$ has not been rephrased.

\section{Leading cases}

The same cases used by FINDER are used as leading cases in the specification. As well as the 270 year old case of Armory v. Delamirie, ${ }^{3}$ there are three nineteenth century cases-Bridges v. Hawkesworth, ${ }^{4}$ Elwes v. Brigg Gas Co. ${ }^{5}$ and South Staffordshire Water Co. v. Sharman ${ }^{6}$-and three twentieth century cases - Hannah v. Peel, ${ }^{7}$ City of London Corporation v. Appleyard ${ }^{8}$ and Moffatt $v$. Kazana. ${ }^{9}$ All of these cases are English. Nevertheless, they represent the law as it applies in Australia.

London v. Appleyard is used twice. In that case some workers (including Appleyard) were employed by a firm which was engaged by another firm (Yorkwin Investments Ltd) to perform some construction work on premises leased by Yorkwin from the owner (the City of London). ${ }^{10}$ The workers found some banknotes during construction. These banknotes were claimed by Appleyard (and his colleagues), by Yorkwin and by the City of London. Before McNair J of the Queen's Bench Division of the English High Court the case was considered as two separate conflicts: Appleyard (as finder) against Yorkwin, and Yorkwin (as finder) against the City of London. Hence the case appears in the specification as City of London Corporation v. Appleyard (1) and as City of London Corporation v. Appleyard (2). ${ }^{11}$ SHYSTER treats each as a separate case; the facts are different, although the result happens to be the same in both cases.

Unlike SHYSTER, FINDER does not allow the specification of UNKNOwN attribute values: unknown values are coded as Nos in FINDER. ${ }^{12}$ Two of the No values in FINDER are actually unknown, ${ }^{13}$ and two other attribute values in FINDER are incorrect. ${ }^{14}$ Nevertheless, the fact vectors in the specification are those used in FINDER; in the interests of accurate simulation, no UNKNOWNs are included and the errors are not rectified. As it happens, including UNKNOwn values and rectifying errors changes neither SHYSTER's opinion nor the cases SHYSTER chooses to justify its opinion in $\S 5.2 .3$ below.

The summary strings for each case are taken (slightly reworded) from FINDER.

\section{Attribute dependence}

SHYSTER detects no functional dependence, and no evidence of stochastic dependence, between the attributes in this specification. The probabilities matrix for the Finder area is given in figure 5.1. 


\begin{tabular}{|c|c|c|c|c|c|c|c|c|c|}
\hline$A_{2}$ & $A_{3}$ & $A_{4}$ & $A_{5}$ & $A_{6}$ & $A_{7}$ & $A_{8}$ & $A_{9}$ & $A_{10}$ & \\
\hline 0.93 & 0.82 & 1.00 & 0.71 & 1.00 & 0.36 & 1.00 & 1.00 & 0.89 & \multirow[b]{2}{*}{$A_{1}$} \\
\hline 0.50 & 0.71 & 0.38 & 0.82 & 0.11 & 1.00 & 0.36 & 0.63 & 0.64 & \\
\hline & 0.93 & 0.50 & 0.50 & 1.00 & 1.00 & 1.00 & 1.00 & 0.21 & \multirow{2}{*}{$A_{2}$} \\
\hline & 0.50 & 1.00 & 0.93 & 0.21 & 0.21 & 0.21 & 0.50 & 1.00 & \\
\hline & & 0.38 & 0.82 & 1.00 & 0.64 & 0.89 & 1.00 & 0.11 & \multirow{2}{*}{$A_{3}$} \\
\hline & & 1.00 & 0.71 & 0.36 & 0.89 & 0.64 & 0.38 & 1.00 & \\
\hline & & & 0.63 & 0.75 & 0.75 & 1.00 & 1.00 & 1.00 & \multirow{2}{*}{$A_{4}$} \\
\hline & & & 1.00 & 1.00 & 1.00 & 0.75 & 0.88 & 0.25 & \\
\hline & & & & 0.89 & 0.36 & 0.11 & 0.38 & 0.89 & \multirow{2}{*}{$A_{5}$} \\
\hline & & & & 0.64 & 1.00 & 1.00 & 1.00 & 0.64 & \\
\hline & & & & & 0.54 & 1.00 & 1.00 & 0.54 & \multirow{2}{*}{$A_{6}$} \\
\hline & & & & & 1.00 & 0.54 & 0.75 & 1.00 & \\
\hline & & & & & & 1.00 & 1.00 & 0.54 & \multirow{2}{*}{$A_{7}$} \\
\hline & & & & & & 0.54 & 0.75 & 1.00 & \\
\hline & & & & & & & 1.00 & 0.46 & \multirow{2}{*}{$A_{8}$} \\
\hline & & & & & & & 0.25 & 0.96 & \\
\hline & & & & & & & & 0.25 & $A_{0}$ \\
\hline & & & & & & & & 1.00 & 199 \\
\hline
\end{tabular}

Figure 5.1: The probabilities matrix for the Finder area (SHYSTER output).

\section{Weights}

The table of weights for the Finder area, as extracted from the weights file, is given in figure 5.2. The rightmost column indicates that two attributes are of equal greatest importance: ${ }^{15}$

$A_{4}$ : Was the other claimant the true owner of the chattel or did she/he claim through the rights of the true owner?

$A_{9}$ : Was an attempt made to find the true owner of the chattel or, alternatively, was the chattel clearly abandoned?

That SHYSTER deems $A_{4}$ to be important is pleasing. The finder has a general right to the found article as against all the world except the true owner. If the other claimant is the true owner then the finder should lose (as happened in Moffatt v. Kazana). Although $A_{9}$ is important, it probably does not deserve to be considered as important as $A_{4}$. 


\begin{tabular}{|c|ccc|ccc|ccc|}
\hline \multirow{2}{*}{ Attr. } & \multicolumn{3}{|c|}{ Win } & \multicolumn{3}{|c|}{ Lose } & & \multirow{2}{*}{$\sigma^{2}$} & $w$ \\
& $\mu$ & $\sigma^{2}$ & $w$ & $\mu$ & $\sigma^{2}$ & $w$ & & & \\
\hline \hline$A_{1}$ & 0.00 & 0.00 & $\infty$ & 0.60 & 0.24 & 4.17 & 0.38 & 0.23 & 4.27 \\
$A_{2}$ & 0.00 & 0.00 & $\infty$ & 0.80 & 0.16 & 6.25 & 0.50 & 0.25 & 4.00 \\
$A_{3}$ & 0.67 & 0.22 & 4.50 & 0.60 & 0.24 & 4.17 & 0.63 & 0.23 & 4.27 \\
$A_{4}$ & 0.00 & 0.00 & $\infty$ & 0.20 & 0.16 & 6.25 & 0.13 & 0.11 & 9.14 \\
$A_{5}$ & 0.67 & 0.22 & 4.50 & 0.20 & 0.16 & 6.25 & 0.38 & 0.23 & 4.27 \\
$A_{6}$ & 0.00 & 0.00 & $\infty$ & 0.40 & 0.24 & 4.17 & 0.25 & 0.19 & 5.33 \\
$A_{7}$ & 0.00 & 0.00 & $\infty$ & 0.40 & 0.24 & 4.17 & 0.25 & 0.19 & 5.33 \\
$A_{8}$ & 0.33 & 0.22 & 4.50 & 1.00 & 0.00 & $\infty$ & 0.75 & 0.19 & 5.33 \\
$A_{9}$ & 0.67 & 0.22 & 4.50 & 1.00 & 0.00 & $\infty$ & 0.88 & 0.11 & 9.14 \\
$A_{10}$ & 0.33 & 0.22 & 4.50 & 0.20 & 0.16 & 6.25 & 0.25 & 0.19 & 5.33 \\
\hline
\end{tabular}

Figure 5.2: The table of weights for the Finder area (SHYSTER output).

\subsubsection{Test case}

A major drawback of the Finder area as a case study for SHYSTER is the dearth of finder cases. Only one such case has been decided since the most recent of FINDER's leading cases.

\section{Parker v. British Airways}

In Parker v. British Airways, ${ }^{16}$ Parker (an airline passenger) found a gold bracelet in the British Airways international executive lounge at Heathrow Airport, London. He handed the bracelet to a British Airways employee, and left his name and address, asking that the bracelet be returned to him if the true owner could not be found. ${ }^{17}$

The owner never claimed the bracelet, and British Airways sold it and kept the proceeds: $£ 850$. When Parker discovered this, he sued British Airways in the Brentford County Court and was awarded $£ 850$ damages plus $£ 50$ interest. British Airways appealed to the English Court of Appeal claiming that, as occupiers of the premises, they had rights superior to Parker's.

Counsel for Parker relied heavily on Bridges v. Hawkesworth. ${ }^{18}$ Counsel for British Airways, on the other hand, submitted that Bridges could be distinguished in favour of South Staffordshire Water Co. v. Sharman.

The three judges who heard Parker v. British Airways in the Court of Appeal unanimously found for Parker. British Airways was held not to have sufficiently manifested an intention to exercise control over lost property before it was found in the executive lounge. 
The English Court of Appeal is the highest court to have heard a case in this area. Although not strictly bound by the previously decided cases, the court took those cases into account. Donaldson LJ, who delivered the major judgment, exhaustively discussed the authorities before stating the rights and obligations of the finder and of the occupier in such cases (as quoted in $\S 5.2 .1$ above). He gave considerable emphasis to Bridges v. Hawkesworth. ${ }^{19}$ Eveleigh LJ and Sir David Cairns agreed, Sir David declaring that Bridges v. Hawkesworth "is the closest case on its facts to the present case." 20

The complete report file for Parker $v$. British Airways is given as an example in $\S$ B.2. SHYSTER's fact vector is (NNNNYNNNYN); to quote the report file:

... the finder was not the occupier of the premises where the chattel was found; the chattel was not attached; the other claimant was not the owner of the premises where the chattel was found; the other claimant was not the true owner of the chattel and was not claiming through the rights of the true owner; the finder handed over the chattel to the other claimant after the finding; neither party relied on the terms of an agreement regarding the right to the chattel; the finder was not a servant of the other claimant; the chattel was not hidden and was not in a position so as to be difficult to find; an attempt was made to find the true owner of the chattel or, alternatively, the chattel was clearly abandoned; and neither party knew of the existence of the chattel prior to the finding.

Of these attribute values, only the value of $A_{9}$ (an attempt was made to find the true owner of the chattel) is contentious. This contention could be important; $A_{9}$ is one of the most important attributes, according to SHYSTER.

The British Airways employee to whom Parker gave the bracelet handed it to the company's lost property department. The true owner never claimed it. It is not clear from the report of the case whether Parker or British Airways made any attempt to find the true owner beyond leaving the bracelet in the lost property department for some time (e.g. they may have advertised the fact that the bracelet had been found, in the executive lounge). It is at least arguable that the correct value of $A_{9}$ is UNKNOwn, although all three judges who decided the case believed that all reasonable steps to find the true owner had been taken. ${ }^{21}$ In any event, changing the value of $A_{9}$ from YES to UNKNOWn makes no difference despite that attribute's weight; SHYSTER comes to the same conclusion on the basis of the same cases, and both instantiations have the same nearest result as does the uninstantiated instant case. 
SHYSTER concludes that Parker should win. SHYSTER's table of distances, as extracted from the distances file, is given in figure 5.3. The nearest neighbour is Bridges $v$. Hawkesworth $\left(C_{2}\right)$; the nearest other is City of London Corporation $v$. Appleyard (1) $\left(C_{5}\right)$. SHYSTER issues no warnings.

SHYSTER comes to a good conclusion, and its choice of nearest neighbour is a good one. Its choice of nearest other is not unjustifiable: the court in London $v$. Appleyard (1) explicitly followed South Staffordshire v. Sharman - the case upon which British Airways relied. ${ }^{22}$ As can be seen in figure 5.3, South Staffordshire $v$. Sharman $\left(C_{7}\right)$ is SHYSTER's (equal) second-nearest other.

SHYSTER reports that Parker $v$. British Airways is not on all fours with Bridges $v$. Hawkesworth because, in that case, the other claimant was the owner of the premises where the chattel was found. Nevertheless, SHYSTER states its opinion that Bridges v. Hawkesworth should be followed. SHYSTER's report on this case is substantially identical to that of FINDER given the same facts, ${ }^{23}$ although this is hardly surprising.

\subsubsection{Conclusion}

SHYSTER is able to simulate FINDER. However due to an almost total lack of test cases, the FINDER specification is not of much use in the testing of SHYSTER.

The reflexive tests performed in $\S$ D.2, and summarized in figure D.1, indicate that the FINDER specification is capable of handling new fact situations.

\subsection{The authorization of copyright infringement}

The Authorization specification deals with the meaning of "authorization" in the Australian Copyright Act. This specification is designed to be used with a rule-based system representing the Copyright Act.

\subsubsection{The law}

The Copyright Act 1968 (Cth) is the statutory component of the law of copyright as it applies in Australia. Sections 13(2), 36(1) and 101(1) forbid the authorization of copyright infringement:

13. (2) For the purposes of this Act, the exclusive right to do an act in relation to a work, an adaptation of a work or any other subject-matter includes the exclusive right to authorize a person to do that act in relation to that work, adaptation or other subject-matter. 


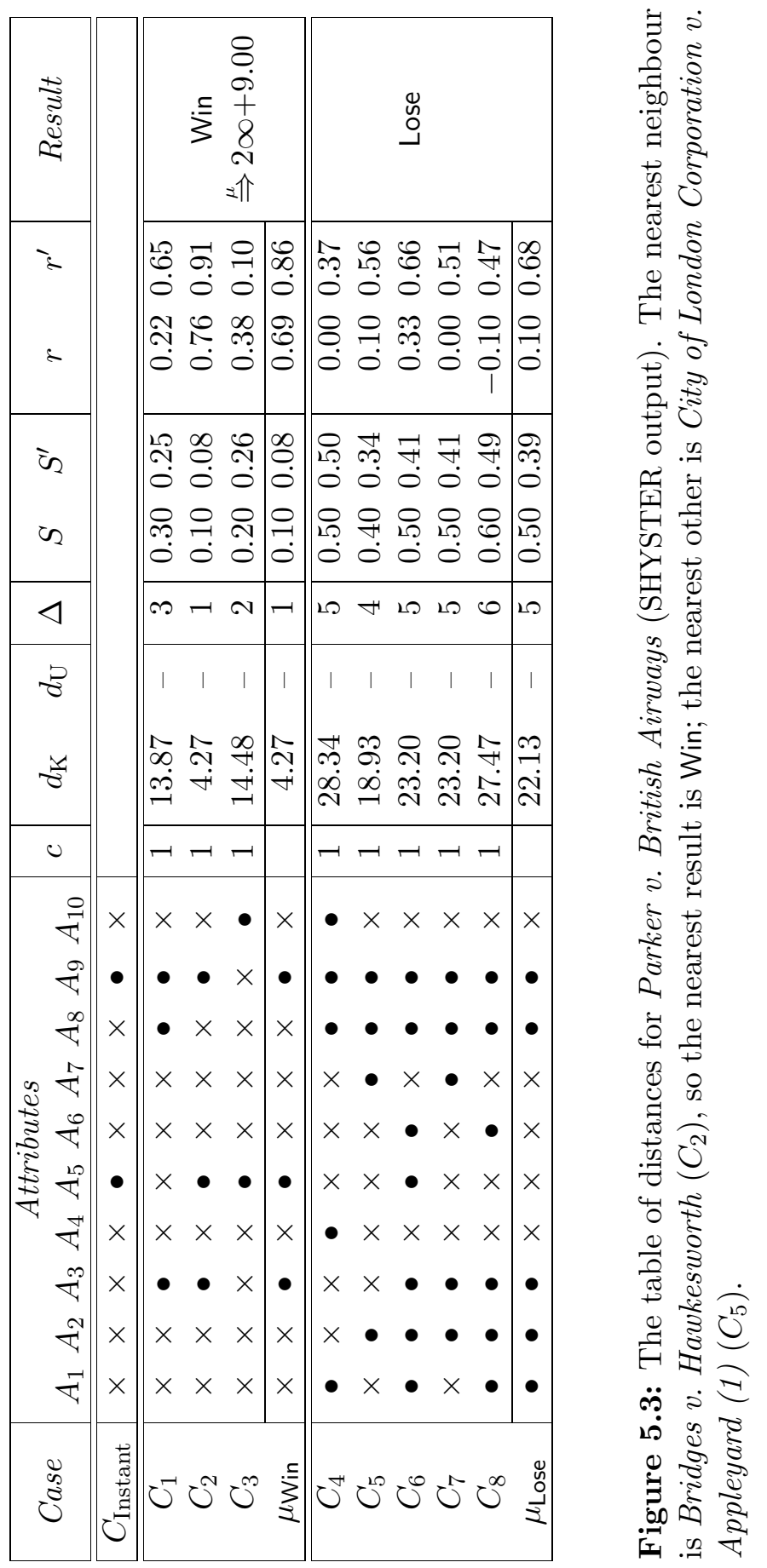


36. (1) Subject to this Act, the copyright in a literary, dramatic, musical or artistic work is infringed by a person who, not being the owner of the copyright, and without the licence of the owner of the copyright, does in Australia, or authorizes the doing in Australia of, any act comprised in the copyright.

101. (1) Subject to this Act, a copyright subsisting by virtue of this Part [copyright in subject-matter other than works] is infringed by a person who, not being the owner of the copyright, and without the licence of the owner of the copyright, does in Australia, or authorizes the doing in Australia of, any act comprised in the copyright.

Nowhere in the Act is there any other reference to authorization; the meaning of the word is left to the courts to interpret.

From 1912, until the 1968 Act came into force (in 1969), the Copyright Act 1911 (UK) was in force in Australia by virtue of the Copyright Act 1912 (Cth). Section 1(2) of the UK Act also forbids authorization of copyright infringement:

1. (2) For the purposes of this Act, "copyright" means the sole right to produce or reproduce the work or any substantial part thereof in any material form whatsoever, to perform, or in the case of a lecture to deliver, the work or any substantial part thereof in public; if the work is unpublished, to publish the work or any substantial part thereof; and shall include the sole right,- -

(a) to produce, reproduce, perform, or publish any translation of the work;

(b) in the case of a dramatic work, to convert it into a novel or other non-dramatic work;

(c) in the case of a novel or other non-dramatic work, or of an artistic work, to convert it into a dramatic work, by way of performance in public or otherwise;

(d) in the case of a literary, dramatic, or musical work, to make any record, perforated roll, cinematograph film, or other contrivance by means of which the work may be mechanically performed or delivered,

and to authorize any such acts as aforesaid.

As with the 1968 Act, "authorize" is not defined in the UK Act.

Since early this century there have been several cases in which courts have had to decide whether copyright infringement had been authorized under one of these Acts. Because of the similarities between the UK Act and the 1968 Act, Australian courts have taken into account cases concerning the former when interpreting the latter. $^{24}$ 
In one of the earliest of these authorization cases, Performing Right Society Ltd v. Ciryl Theatrical Syndicate Ltd, Scrutton LJ expressed his view that the words "to authorize any such acts as aforesaid" at the end of s. 1(2) of the UK Act "are superfluous and add nothing to the definition." ${ }^{25}$ He considered them to be a reference to acting through an agent. However, only two years later Bankes LJ in Falcon v. Famous Players Film Co. decided that "authorize" should be given its ordinary dictionary meaning of "sanction, approve, countenance". ${ }^{26}$ Bankes LJ's interpretation was adopted by Isaacs J of the High Court of Australia in 1928, ${ }^{27}$ and has been used ever since. As Ricketson points out, this means that:

... the notion of "authorisation" extends beyond the authority given to an agent. Thus, if an infringing act is committed by an agent acting within his real or ostensible authority, his principal will be directly liable on the ordinary rules governing the relationship of principal and agent. The same, of course, applies where the infringing act is committed by an employee acting within the course of his employment. ${ }^{28}$

Gibbs J discussed the meaning of authorization in some detail in University of New South Wales v. Moorhouse. ${ }^{29}$ He pointed out that "authorize" can also mean "permit," though this is tempered by the principle that a person cannot be said to authorize an infringement of copyright unless she/he has the power to prevent it. ${ }^{30}$ Furthermore:

Express or formal permission or sanction, or active conduct indicating approval, is not essential to constitute an authorization ... However, the word "authorize" connotes a mental element and it could not be inferred that a person had, by mere inactivity, authorized something to be done if he neither knew nor had reason to suspect that the act might be done. ${ }^{31}$

Having cited several authorities, Gibbs J summarized the law as follows:

It seems to me to follow from these statements of principle that a person who has under his control the means by which an infringement of copyright may be committed ... and who makes it available to other persons, knowing, or having reason to suspect, that it is likely to be used for the purpose of committing an infringement, and omitting to take reasonable steps to limit its use to legitimate purposes, would authorize any infringement that resulted from its use. ${ }^{32}$

However, he warned that:

... the question of whether one person authorizes another to commit an infringement depends upon all the facts of the case so that a decision on a particular set of circumstances may be of no assistance in other cases. ${ }^{33}$ 


\subsubsection{The Authorization specification}

The author-having consulted a legal textbook, ${ }^{34}$ the published judgments in the leading cases to which that textbook refers, and a legal expert ${ }^{35}$-wrote a specification of the meaning of "authorization" in the Copyright Act. This specification was written so as to represent the law as it was in 1983 - the textbook states the law as it was then ${ }^{36}$ — so that important cases which have been decided since that time can be used as test cases.

The dump file for the Authorization specification is given in full in $\S$ A.3. It contains a single area: the Authorization area. This area makes use of several of the features not used in the FINDER specification. There is a hierarchy which ranks seven courts at six distinct levels, there is an opening string (although there is no closing string), attributes have help strings, some of the attribute values of the leading cases are UnKNOwn, and ideal points and attribute direction are included.

\section{Results}

The Authorization area has three results: Auth, Not-Auth and Liable. These correspond to "the accused authorized the infringement," "the accused did not authorize the infringement" and "the accused is liable (directly or vicariously) for the infringement." (An employer is vicariously liable for an employee's infringing act if the employee is acting within the course of her/his employment.) These are three quite distinct results; the Authorization area demonstrates the need for a case-based system to be able to handle more than two possible outcomes, as discussed in $§ 3.14 .1$.

\section{Attributes}

There are seven attributes:

$A_{1}$ : Was the infringer an employee of the accused?

$A_{2}$ : Was the infringer an independent contractor to the accused?

$A_{3}$ : Did the accused sell or hire the infringer the means of infringing?

$A_{4}$ : Did the accused have the power to prevent the infringement?

$A_{5}$ : Did the accused take reasonable steps to avoid the infringement?

$A_{6}$ : Did the accused know, or have reason to anticipate or suspect, that the infringing act was to be, or was likely to be, done?

$A_{7}$ : Was the specific infringement causally related to an incitement to infringe on the part of the accused? 
Although there will be times when the answers to $A_{1}$ and $A_{2}$ are obvious, these questions are not always easy to answer. The EMPLOYEe specification described in $\S 5.4 .2$ below is concerned solely with deciding whether a worker is an employee or an independent contractor. If the Employee area from that specification were included in the Authorization specification then either or both of $A_{1}$ and $A_{2}$ could be made external attributes; i.e. rather than asking the user a single question, the attribute value would be determined by reference to the Employee area, which has 18 attributes. ${ }^{37}$ The Natural specification described in $§ 5.5 .2$ illustrates this process: it has three areas, two of which have external attributes.

However, a simpler approach was adopted for the Authorization specification. Both $A_{1}$ and $A_{2}$ have help strings which give assistance to the user in answering the attribute question. For example, the help string for $A_{1}$ is:

If the accused had control over the infringer's manner of doing her/his work then the infringer was an employee of the accused. If the infringer undertook to do something for the accused and had discretion as to the manner in which it was to be done then the infringer was an independent contractor to the accused, not an employee.

(This is a greatly simplified statement of the law, as can be seen by comparison with the EMPLOYEe specification.)

Of the other attribute questions, only $A_{5}$ should present any difficulty. This attribute is very important. If the accused did take reasonable steps to avoid the infringement then she/he will not be said to have authorized the infringement, ${ }^{38}$ although she/he could still be directly or vicariously liable. In all of the cases that make up the Authorization area the value of $A_{5}$ is No: i.e. in none of the cases did the accused take reasonable steps. This is not surprising; if reasonable steps had been taken, the cases would probably never have been taken to court. It also means that SHYSTER assigns $A_{5}$ infinite weight.

The attribute has a fairly unhelpful help string: "Whether particular steps were reasonable depends on the facts of the case." But the nebulous concept of "reasonableness" is one with which lawyers very often have to deal. A lawyer would probably not have much difficulty in answering this question, and she/he always has the option of answering UNKNOwn and forcing SHYSTER to examine instantiations.

If $A_{5}$ is changed to UNKNOWN in all of the test cases in $§ 5.3 .3$, SHYSTER comes to the same conclusion on the basis of the same cases. This does not mean that $A_{5}$ is irrelevant; its inclusion (and its infinite weighting) means that if the accused did not take reasonable steps to avoid the infringement, the specified directions and ideal point directions will strongly suggest Not-Auth. ${ }^{39}$

In theory, it should be possible to specify another area of case law which defines this very open-textured concept of reasonableness, however that might prove practically impossible. ${ }^{40}$ 


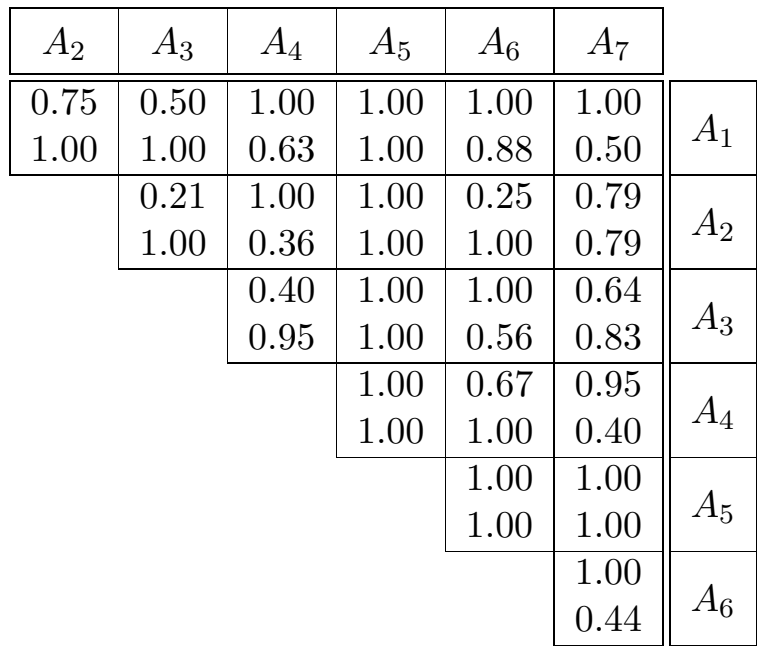

Figure 5.4: The probabilities matrix for the Authorization area (SHYSTER output).

\section{Leading cases}

There are nine leading cases in the Authorization area. Three of them arose under the Copyright Act 1911 (UK): Performing Right Society Ltd v. Ciryl Theatrical Syndicate Ltd ${ }^{41}$ Falcon v. Famous Players Film Co. ${ }^{42}$ and A\&M Records Inc. $v$. Audio Magnetics Inc. (UK) Ltd. ${ }^{43}$ Four arose under the UK Act, in force in Australia by virtue of the Copyright Act 1912 (Cth): Mellor v. Australian Broadcasting Commission, ${ }^{44}$ Winstone v. Wurlitzer Automatic Phonograph Co. of Australia Pty Ltd, ${ }^{45}$ Australasian Performing Right Association Ltd v. Miles ${ }^{46}$ and Australasian Performing Right Association Ltd v. Canterbury-Bankstown League Club Ltd. ${ }^{47}$ And two arose under the Copyright Act 1968 (Cth): University of New South Wales v. Moorhouse ${ }^{48}$ and RCA Corporation v. John Fairfax and Sons Ltd. ${ }^{49}$

SHYSTER warns that APRA $v$. Canterbury-Bankstown and Mellor v. ABC have identical facts (except for unknown values), and that APRA v. CanterburyBankstown and APRA v. Miles have identical facts (except for unknown values) and different results.

The first draft of the specification included an early King's Bench Division case: Performing Right Society Ltd v. Bradford Corporation. ${ }^{50}$ That case has an identical fact vector to that of Mellor $v$. $A B C$. On reflection, the former case was omitted in favour of the latter, later, and more important, Privy Council decision. (See $§ 5.5 .2$ below for a discussion of identical cases.) 


\begin{tabular}{|c|ccc|ccc|ccc|ccc|}
\hline \multirow{2}{*}{ Attr. } & \multicolumn{4}{|c|}{ Auth } & \multicolumn{4}{|c|}{ Not-Auth } & \multicolumn{3}{|c|}{ Liable } & \multicolumn{3}{|c|}{$\sigma^{2}$} & \multirow{2}{*}{$w$} \\
& $\mu$ & $\sigma^{2}$ & $w$ & $\mu$ & $\sigma^{2}$ & $w$ & $\mu$ & $\sigma^{2}$ & $w$ & & & \\
\hline \hline$A_{1}$ & 0.00 & 0.00 & $\infty$ & 0.00 & 0.00 & $\infty$ & 1.00 & 0.00 & $\infty$ & 0.13 & 0.11 & 9.14 \\
$A_{2}$ & 0.25 & 0.19 & 5.33 & 0.33 & 0.22 & 4.50 & 0.00 & 0.00 & $\infty$ & 0.25 & 0.19 & 5.33 \\
$A_{3}$ & 0.60 & 0.24 & 4.17 & 0.33 & 0.22 & 4.50 & 0.00 & 0.00 & $\infty$ & 0.44 & 0.25 & 4.05 \\
$A_{4}$ & 0.80 & 0.16 & 6.25 & 0.33 & 0.22 & 4.50 & 1.00 & 0.00 & $\infty$ & 0.67 & 0.22 & 4.50 \\
$A_{5}$ & 0.00 & 0.00 & $\infty$ & 0.00 & 0.00 & $\infty$ & 0.00 & 0.00 & $\infty$ & 0.00 & 0.00 & $\infty$ \\
$A_{6}$ & 1.00 & 0.00 & $\infty$ & 0.67 & 0.22 & 4.50 & 1.00 & 0.00 & $\infty$ & 0.89 & 0.10 & 10.13 \\
$A_{7}$ & 0.80 & 0.16 & 6.25 & 0.00 & 0.00 & $\infty$ & 1.00 & 0.00 & $\infty$ & 0.56 & 0.25 & 4.05 \\
\hline
\end{tabular}

Figure 5.5: The table of weights for the Authorization area (SHYSTER output).

\section{Attribute dependence}

SHYSTER detects no functional dependence, and no evidence of stochastic dependence, between the attributes in this specification. The probabilities matrix for the Authorization area is given in figure 5.4.

\section{Weights}

The table of weights for the Authorization area, as extracted from the weights file, is given in figure 5.5. The rightmost column of the table indicates that, as discussed above, $A_{5}$ is given infinite weight. ${ }^{51}$ The next most important attributes are:

$A_{6}$ : Did the accused know, or have reason to anticipate or suspect, that the infringing act was to be, or was likely to be, done?

$A_{1}$ : Was the infringer an employee of the accused?

These attributes are indeed important, although $A_{4}$ ("Did the accused have the power to prevent the infringement?") should probably be more heavily weighted.

\subsubsection{Test cases}

The Authorization specification was written so as to represent the law as it was in 1983. Three authorization cases have been decided since then, and they are used to test SHYSTER. Another case was decided before 1983 but was not considered important enough to include in the specification. That case, $C B S$ Inc. v. Ames Records and Tapes $L t d,{ }^{52}$ is used as a test case too. There are also two hypothetical test cases, chosen by the legal expert. The results of all these tests are summarized in figure 5.12. 
The Authorization area has three results, and SHYSTER will always look for nearest cases for each result. So, SHYSTER argues about the Liable result even in those test cases where the courts did not explicitly consider the possibility that the accused was directly liable. It is not inappropriate to argue about the Liable result in such cases; the judges must have (at least tacitly) rejected that conclusion themselves. It would only be cause for concern if SHYSTER's opinion were that the accused was directly liable in such a case - but it does not make that mistake in any of these tests.

\section{CBS Inc. v. Ames Records and Tapes Ltd}

In CBS Inc. v. Ames Records and Tapes $L t d,{ }^{53}$ Ames operated a record lending library. As well as hiring records, Ames sold blank cassettes. CBS sold records to Ames, and held the copyright in those sound recordings. CBS claimed that Ames knew that it was likely that their customers would copy the records onto blank cassettes at home, and that hence Ames was "authorizing" infringement within the meaning of 1(2) of the Copyright Act 1956 (UK).

Before Whitford J of the Chancery Division of the English High Court, CBS relied on Falcon v. Famous Players Film Co., Vigneux v. Canadian Performing Right Society Lt ${ }^{54}$ (not one of the leading cases in the Authorization specification), Winstone v. Wurlitzer Automatic Phonograph Co. of Australia Pty Ltd and University of New South Wales v. Moorhouse. They urged that A\&BM Records Inc. v. Audio Magnetics Inc. (UK) Ltd be distinguished on the basis that "here the intention and desire was to take advantage of home taping." 55

Ames argued that, applying the dictionary meaning of authorization as did Bankes LJ in Falcon v. Famous Players, they had not authorized any infringement. They also claimed that UNSW $v$. Moorhouse was wrongly decided. ${ }^{56}$ Ames relied on $A \mathscr{E} M \quad v$. Audio Magnetics, pointing to the absence of evidence of a particular case of infringement.

Whitford J agreed with Ames, although he made no mention of A\&M v. Audio Magnetics in his judgment.

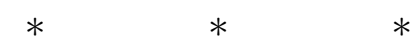

SHYSTER's fact vector is (NNYNNYN); to quote its report file:

... the infringer was not an employee of the accused; the infringer was not an independent contractor to the accused; the accused sold or hired the infringer the means of infringing; the accused did not have the power to prevent the infringement; the accused did not take reasonable steps to avoid the infringement; the accused knew, or had reason to anticipate or suspect, that the infringing act was to be, or was likely to be, done; and the specific infringement was not causally related to an incitement to infringe on the part of the accused.

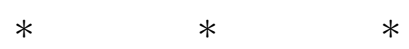


SHYSTER agrees with Whitford J that Ames did not authorize the infringement. SHYSTER's table of distances, as extracted from the distances file, is given in figure 5.6. The nearest neighbour is $A \mathscr{E} M v$. Audio Magnetics $\left(C_{8}\right)$ : one of the cases upon which Ames relied; the nearest others are Falcon v. Famous Players $\left(C_{5}\right.$ : Auth) - upon which CBS relied-and Australasian Performing Right Association Ltd v. Miles ( $C_{9}$ : Liable). SHYSTER warns that the specified directions suggest the Auth result, as can be seen in figure 5.6: the strength of the specified direction for Auth $(3 \infty+4.16)$ is greater than that for the other two results.

\section{WEA International Inc. v. Hanimex Corporation Ltd}

In WEA International Inc. v. Hanimex Corporation Ltd, ${ }^{57}$ Hanimex advertised one of their cassette products on radio. These humorous advertisements implied that, while the cassette recordings of some artists' songs would melt in a hot car, Hanimex's cassettes would not: "If you don't want your favourite recordings ruined, use Fuji GTI Car Tapes."

WEA owned the copyright in sound recordings made by the singer Madonnaone of the artists mentioned by name in the advertisements. WEA claimed that Hanimex had authorized members of the public to infringe their copyright, contrary to the Copyright Act 1968 (Cth).

The case was heard in the Federal Court of Australia by Gummow J. His judgment includes a long history of authorization provisions in copyright legislation since the turn of the century. Of the recent cases, he referred to University of New South Wales v. Moorhouse, CBS Inc. v. Ames Records and Tapes Ltd and RCA Corporation v. John Fairfax and Sons Ltd. ${ }^{58}$ He pointed out that RCA v. Fairfax was authority for the proposition that authorization of copyright infringement requires an actual act of infringement, and observed that:

... it has not even been shown that there has been any unauthorized reproduction by any particular person of any of the sound recordings in which [WEA] hold copyright. ${ }^{59}$

In addition, Gummow J held that the advertisements were not "an invitation or incitement to or approval of" copyright infringement. ${ }^{60}$

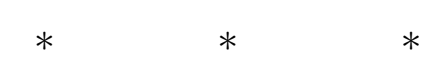

SHYSTER's fact vector is (NNYNNYN); as it is in CBS v. Ames. Hence, SHYSTER's distances and report files are identical to those for that case. SHYSTER agrees that Hanimex did not authorize the infringement, although it warns that the specified directions suggest Auth.

The nearest neighbour is A\&M Records Inc. v. Audio Magnetics Inc. (UK) Ltd. That this case was not mentioned by Gummow $\mathrm{J}$ in his judgment is most surprising. The facts of $A \mathscr{E} M \mathrm{v}$. Audio Magnetics are very similar to those of 


\begin{tabular}{|c|c|c|c|c|c|c|c|}
\hline 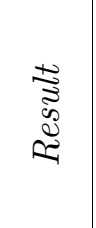 & & 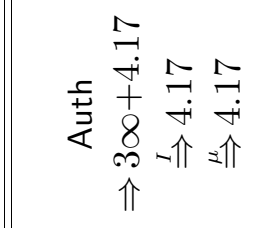 & & 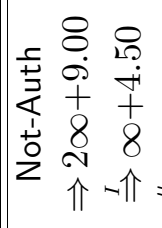 & $\begin{array}{l}\stackrel{?}{+} \\
+ \\
+ \\
8 \\
\Uparrow\end{array}$ & & \\
\hline$=$ & & 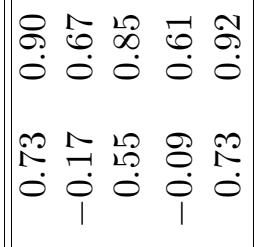 & $\left|\begin{array}{cc}1 & \Lambda \\
1 & \infty \\
0 & 0 \\
0 & 0 \\
& \overrightarrow{0} \\
0 & 0 \\
0 & 0\end{array}\right|$ & 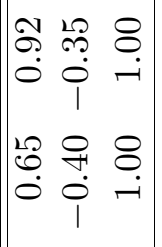 & 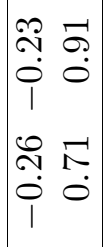 & $\mid \begin{array}{l}0 \\
0 \\
0 \\
0 \\
0 \\
0 \\
0 \\
1\end{array}$ & \begin{tabular}{ll}
0 & 0 \\
0 & $?$ \\
0 & 0 \\
0 & 0 \\
7 & 0 \\
\hdashline & 0 \\
0 & 0 \\
& 1
\end{tabular} \\
\hline os & & 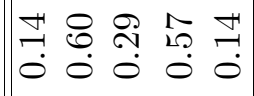 & ঙr & 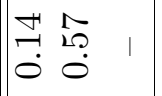 & 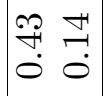 & $\mid \begin{array}{l}1 \\
20 \\
0 \\
0\end{array}$ & 우 \\
\hline$\triangleleft$ & & $\neg \infty \propto ⿻ 尸 一$ & $\infty$ & $\| \begin{array}{lll}-r & 1\end{array}$ & $\infty-1$ & $F$ & $N \forall$ \\
\hline 苑 & & 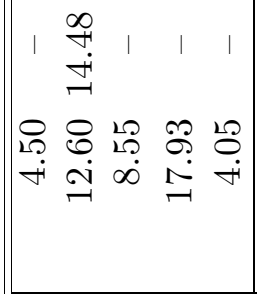 & $\mid$\begin{tabular}{ll}
1 & 1 \\
& \\
$\infty$ & 20 \\
$\infty$ & 2 \\
0 & 0 \\
\hdashline & $\infty$ \\
-1 & 0
\end{tabular} & 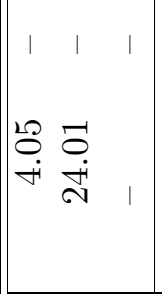 & 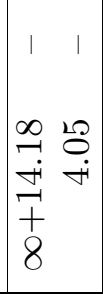 & 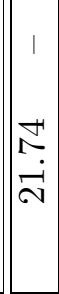 & 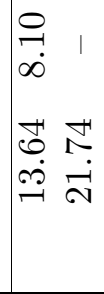 \\
\hline u & & $\neg \propto ⿻ 上 丨$ & & $\infty\llcorner 0$ & & $\infty$ & \\
\hline 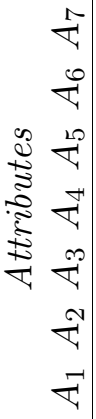 & $\begin{array}{l}x \\
\bullet \\
x \\
x \\
\bullet \\
x\end{array}$ & 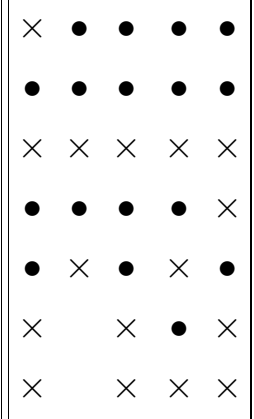 & \begin{tabular}{ll|}
$\bullet$ & $\bullet$ \\
$\bullet$ & $\bullet$ \\
$\times$ & $\times$ \\
$\bullet$ & $\bullet$ \\
$\bullet$ & $\bullet$ \\
$\bullet$ & $\times$ \\
$x$ & $\times$
\end{tabular} & $\mid \begin{array}{lll}x & x & \times \\
\bullet & x & \bullet \\
x & x & \times \\
x & \bullet & x \\
x & x & \bullet \\
x & \bullet & \times \\
x & x & x\end{array}$ & $\begin{array}{ll}x & x \\
x & \bullet \\
\bullet & x \\
x & x \\
x & x \\
\times & \times \\
x & x\end{array}$ & $\begin{array}{l}\bullet \\
\times \\
\times \\
\bullet\end{array}$ & $\begin{array}{ll} & \bullet \\
\bullet & \bullet \\
\times & \times \\
\bullet & \bullet \\
& \times \\
\times & \times \\
\bullet & \bullet\end{array}$ \\
\hline 离 & 䲩 & $\mho^{-1} \mho^{N} \mho^{\infty} U^{\star}$ & 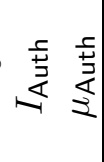 & $\mho^{\infty} \mho^{\wedge} \mho^{\infty}$ & 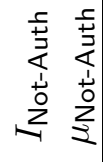 & & $\frac{\frac{0}{0}}{\stackrel{\frac{0}{\sigma}}{-\frac{\pi}{-1}}}$ \\
\hline
\end{tabular}

官苛总

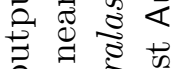

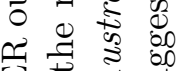

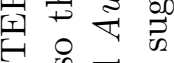
당 \& 0 市䒕 近已

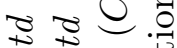
겅ㅇㅇㅇ \&ै: औ

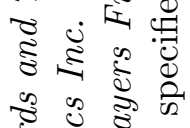

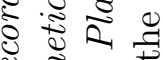
영 औ क से है है:

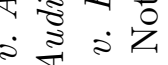

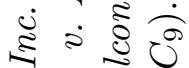
का نि ठी है

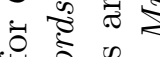
\& 记 专焉

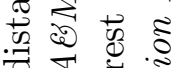
प. 효 焉芒 ज焉急 0. ० बू. है है $\exists \pm$ के कo \& है

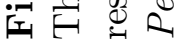


WEA v. Hanimex: they both concern advertisements claiming high durability of blank cassettes and referring to specific recording artists. A $\dot{\xi} \mathrm{v}$. Audio Magnet$i c s$ is a decision of the Chancery Division of the English High Court, and not as good authority in Australia as a decision of the NSW Supreme Court, like RCA $v$. Fairfax. Nevertheless, it is amazing, given the length of his discussion of the development of the law in Australia, England and the US, that Gummow J did not refer to $A \& M v$. Audio Magnetics.

Both A\&\&M v. Audio Magnetics and RCA v. Fairfax were decided on the same point: that there must be an actual infringing act before someone can be said to have authorized an infringement. The judge in $R C A v$. Fairfax explicitly followed $A \mathscr{B} M$ v. Audio Magnetics, amongst other cases, in coming to his decision. ${ }^{61}$ Hence, the author claims that SHYSTER's choice of nearest neighbour is a good one, despite the fact that it was not referred to in WEA $v$. Hanimex.

Gummow J made no reference to SHYSTER's nearest others: Falcon v. Famous Players Film Co. (Auth) and Australasian Performing Right Association Ltd $v$. Miles (Liable).

CBS Songs Ltd v. Amstrad Consumer Electronics plc

In CBS Songs Ltd v. Amstrad Consumer Electronics plc, ${ }^{62}$ Amstrad manufactured twin-deck tape-recording machines. They were designed so as to facilitate tapeto-tape copying, and were advertised in a manner that was likely to encourage home taping and copying. However, the advertisements warned that some copying could not be done without permission, and made it clear that Amstrad had no authority to grant such permission.

CBS and other record companies sued Amstrad claiming that they had authorized users of their twin-deck recorders to infringe copyright. Before the House of Lords, CBS cited many cases in argument, including Falcon v. Famous Players Film Co. Amstrad relied on A\&MM Records Inc. v. Audio Magnetics Inc. (UK) $L t d$, amongst others.

As in CBS Inc. v. Ames Records and Tapes Ltd, A\&̋M v. Audio Magneticsalthough used in argument - was not referred to in the judgments. Instead, Lord Templeman (with whom the other four law lords agreed) found for Amstrad on the basis of Bankes LJ's definition of authorization in Falcon v. Famous Players, and following CBS Inc. v. Ames Records and Tapes Ltd.

SHYSTER's fact vector is (NNYNNYN); as it is in CBS v. Ames and WEA International Inc. v. Hanimex Corporation Ltd. 
SHYSTER agrees that Amstrad did not authorize the infringement. Its choice of cases is good: the nearest neighbour is $A \mathscr{E} M v$. Audio Magnetics; the nearest others are Falcon v. Famous Players (Auth) and Australasian Performing Right Association Ltd v. Miles (Liable). However, SHYSTER warns that the specified directions suggest Auth.

Australasian Performing Right Association Ltd v. Jain

In Australasian Performing Right Association Ltd v. Jain, ${ }^{63}$ Jain was the director and principal executive officer of a company (Valamo Pty Ltd) which was the proprietor of a tavern. Live bands, and a video music system, performed works in which the APRA held copyright. It was conceded that Valemo had infringed copyright, but Jain claimed that he was not personally liable. The day-to-day operations of the tavern were controlled by another person (the licensee). Jain claimed that the licensee was responsible for all the music at the tavern, and that he (Jain) only took an interest if takings were down, in which case he would advise the licensee to engage a different band.

The Full Court of the Federal Court of Australia held that Jain had authorized the infringement. Sheppard, Foster and Hill JJ held that Jain had allowed:

... a situation to develop and to continue in which he must have known that it was likely that the [APRA's] music would be played without any licence from it. It was within his power to control what was occurring but he did nothing at all. ${ }^{64}$

In coming to their conclusion, their honours mentioned many cases. To the extent that they followed any particular case, they followed University of New South Wales v. Moorhouse. ${ }^{65}$

$* \quad * \quad *$

The complete report file for APRA $v$. Jain is given as an example in $\S$ B.3. SHYSTER's fact vector is (NYNYNYN). SHYSTER agrees with the Federal Court that Jain authorized the infringement. However, none of the cases chosen by SHYSTER was mentioned by the Court. The nearest neighbour is Mellor v. Australian Broadcasting Commission; the nearest others are RCA Corporation v. John Fairfax and Sons Ltd (Not-Auth) and Australasian Performing Right Association Ltd $v$. Miles (Liable). There are no warnings.

\section{Hypothetical case 1}

The legal expert suggested a hypothetical case for testing the Authorization specification: a fact situation about which he had been asked for advice, but on which no court has been forced to rule. 
A residential college on a university campus provides personal computer facilities for the use of its residents. It also makes available to the residents several items of software. Neither the college nor the residents hold the copyright in this software, although the software could easily be copied. The college is concerned as to whether, if a resident were to copy an item of software, the college could be said to have authorized that infringement.

The legal expert advises that the college could be said to have authorized the infringement.

SHYSTER's fact vector is (NNNYNYN). SHYSTER's conclusion is that the college would have authorized the infringement. The nearest neighbour is University of New South Wales v. Moorhouse; the nearest others are RCA Corporation v. John Fairfax and Sons Ltd (Not-Auth) and Australasian Performing Right Association Ltd v. Miles (Liable). However, it warns that the specified directions suggest Liable.

The legal expert advises that SHYSTER's choice of cases, nearest neighbour and nearest others, is good.

\section{Hypothetical case 2}

The legal expert suggested a second hypothetical authorization case. A language school lends audio tapes to its students and allows them to take those tapes home. One of the language school's employees gives a student one of these tapes and tells him that "lots of people copy them." Could the school be said to have authorized an infringement if the student makes a copy of the tape?

The legal expert advises that the language school would probably not be held to have authorized the infringement.

SHYSTER's fact vector is (NNNNNYY). Its conclusion is that the language school would not have authorized the student's infringement. The nearest neighbour is RCA Corporation v. John Fairfax and Sons Ltd; the nearest others are Falcon $v$. Famous Players Film Co. (Auth) and Australasian Performing Right Association Ltd $v$. Miles (Liable).

However, SHYSTER warns that two of these cases are equidistant from the instant case; it chooses RCA v. Fairfax because it is a decision of the New South Wales Supreme Court whereas Falcon v. Famous Players is merely a decision of the English Court of Appeal. The weighted correlation coefficients suggest that Falcon v. Famous Players should be the nearest neighbour, and the specified directions suggest Liable.

Again, the legal expert advises that SHYSTER's choice of cases is good. 


\subsubsection{Generated tests}

The legal expert provided three examples for generating tests using the AutHoRIZATION specification: generated tests 1,2 and 3. The results of these tests are summarized in figure 5.13.

\section{Generated test 1}

The legal expert advises that, if the following is true, either the accused authorized the infringement, or the accused is directly or vicariously liable for the infringement:

... the infringer was an employee of the accused; the infringer was not an independent contractor to the accused; and the accused did not take reasonable steps to avoid the infringement.

Given the fact vector (YNUUNUU), SHYSTER generates 16 instantiations: one eighth of the total search space. In all but two of these instantiations, SHYSTER's chosen results are good (i.e. Auth or Liable).

In the two instantiations in which the result is Not-Auth, the nearest ideal point is that of a good result, but those ideal points are not nearer the instant case than the nearest neighbour so no warnings are issued. In both of those instantiations, the accused did not have the power to prevent the infringement, and the specific infringement was not causally related to an incitement to infringe on the part of the accused. The legal expert agrees that these attribute values justify SHYSTER's choice of result in both instantiations.

\section{Generated test 2}

The legal expert also advises that, if the following is true, the accused did not authorize the infringement:

... the infringer was not an employee of the accused; the accused did not have the power to prevent the infringement; and the accused took reasonable steps to avoid the infringement.

The fact vector is (NUUNYUU). SHYSTER generates 16 instantiations, in all but four of which SHYSTER's chosen result is good (i.e. Not-Auth).

In each of those four instantiations, SHYSTER warns that an ideal point suggesting the good result is at least as near to the instant case as is the nearest neighbour. The only attribute for which these four instantiations have common values (apart from the three attributes with known values in the above fact vector) is the last: in each, the specific infringement was causally related to an incitement to infringe on the part of the accused. Although this attribute value does suggest Auth or Liable, ${ }^{66}$ the three known attribute values in the above fact vector are more persuasive: SHYSTER's choice of results in these four instantiations is bad. 


\section{Generated test 3}

The legal expert advises that, if the accused took reasonable steps to avoid the infringement, she/he cannot have authorized the infringement (although she/he may be directly or vicariously liable for the infringement). SHYSTER's fact vector is (UUUUYUU). It generates 64 instantiations: half the search space. In 13 of these 64 instantiations, SHYSTER's chosen result is bad (i.e. Auth).

In all 13, SHYSTER warns that an ideal point suggesting a good result is at least as near to the instant case as was the nearest neighbour. The fact that, in each of these 13 instantiations, the infringer was not an employee of the accused, does not justify SHYSTER's choice of result in these instantiations.

\subsubsection{Conclusion}

In the test cases described in $\S 5.3 .3$, SHYSTER chooses a good result every time, and $75 \%$ of its chosen cases are good. However, the small number of attributes specified for the Authorization area means that SHYSTER has difficulty distinguishing between cases: e.g. the first three test cases share the same fact vector. This may indicate a deficiency in the specification, or it may be indicative of a feature of the area.

As shown in figure 5.13, SHYSTER's choice of result is good in $80.21 \%$ of the instantiations in all three generated tests. If a bad choice of result is considered good where an ideal point warning is issued, SHYSTER's success rate rises to $87.50 \%, 100.00 \%$ and $100.00 \%$ for each test: $97.92 \%$ overall. (In none of the instantiations in these three tests does SHYSTER issue a warning about a nearer ideal point when the choice of results is good; i.e. none of SHYSTER's ideal point warnings is bad.)

The Authorization area is a good example of an area which could be used to define an open-textured statutory concept in a rule base: the meaning of "authorization" is undefined in sections 13(2), 36(1) and 101(1) of the Copyright Act. It is also a good example of an area of law in which there are more than two possible results.

SHYSTER (when using the AuthorizATion specification) produces good advice in the test cases, and chooses good results in almost all of the generated tests. The reflexive tests performed in $\S$ D.3, and summarized in figure D.2, indicate that the Authorization specification is capable of handling new fact situations.

\subsection{Employees and independent contractors}

The EMPLOYEE specification deals with the legal categorization of a worker as an employee or as an independent contractor. 


\subsubsection{The law}

If a person works for another, one of two relationships exists: either they are employer and employee - and theirs is a contract of service - or they are principal and independent contractor - and theirs is a contract for services.

(The parties to a contract of service used to be called "master" and "servant," but this terminology is no longer used. ${ }^{67}$ When it is not yet clear which sort of relationship exists, it is convenient to use generic terms: "employer" and "worker" are used to mean "employer" and "employee" - in the case of a contract of service - or "principal" and "independent contractor" - in the case of a contract for services.)

This distinction is important; the law of employment is concerned almost totally with contracts of service. ${ }^{68}$ As mentioned in $§ 5.3 .2$ above, an employer is vicariously liable for the actions of an employee if the employee is acting within the course of her/his employment. A principal would not normally be vicariously liable for the actions of an independent contractor.

The distinction also affects the terms that will be implied into the employment contract in the absence of an express agreement between the parties, the applicability of industrial awards, the applicability of statutes which may affect workers' compensation, occupational health and safety, long-service leave, annual holidays, unfair dismissal, payroll tax, fringe benefits tax, etc. ${ }^{69}$

As Creighton, Ford and Mitchell point out:

It is most important therefore to be able to determine whether any given worker is engaged under a contract of employment. Unfortunately neither courts nor parliaments have been consistent in the attitude they have adopted to this process of categorization. In particular they have contrived to make it virtually impossible to draw a clear and consistent distinction between 'employees' and 'independent contractors.'

... Parliaments tend to adopt one of two approaches to the process of categorization. The first, and simplest, is to ignore the issue entirely, and to leave the matter to be determined by reference to common law criteria ... The other approach is to include some form of definition, usually in the interpretation section. In some instances this definition is purely circular in form ... whereas in others it consists of either a broadening or a narrowing of the common law concept (sometimes going as far as to include 'independent contractors'). Whatever approach is adopted, the final answer is to be found in the common law. ${ }^{70}$

The most commonly applied criterion for distinguishing between a contract of service and a contract for services is the "control test." If an employer has a right of control over a worker's manner of doing her/his work then the control test suggests that the contract is of service and that the worker is an employee. Conversely, if a worker has discretion as to the manner in which the work is to be done then the test suggests that the contract is for services. ${ }^{71}$ 
However, courts have explicitly had regard to many different factors in deciding whether a worker is an employee or an independent contractor. In Price $v$. Grant Industries Pty Ltd, for example, the Full Court of the Federal Court examined in detail fifteen points which Price claimed were indicative of a contract of service; Grant Industries relied on eleven different points which it claimed were indicative of a contract for services. ${ }^{72}$ This is an area of the law in which many factors have to be balanced before a conclusion can be reached.

\subsubsection{The EMPLOYEE specification}

The author-having consulted a legal textbook, ${ }^{73}$ Halsbury's, ${ }^{74}$ the published judgments in the leading cases to which they refer, and a legal expert ${ }^{75}$ — wrote a specification of the distinction between an employee and an independent contractor. This specification was written so as to represent the law as it was in 1982 so that important cases which have been decided since then can be used as test cases. $^{76}$

The Employee specification is used as the basis of the complete example of SHYSTER's output files in appendix C. The specification file, written in SHYSTER's case law specification language and input by SHYSTER, is in $\S$ C.3. The dump file for the EMPLOYEe specification is given in full in $\S$ A.4.

The specification contains a single area: the Employee area. There is a hierarchy which ranks nine courts appropriately, and there is an opening string (although there is no closing string).

\section{Results}

The Employee area has two results: Employee and Contractor. These correspond to "the worker is an employee" and "the worker is an independent contractor."

\section{Attributes}

In keeping with the large number of important factors in this area, there are eighteen attributes:

$A_{1}$ : Did the employer direct not only what work was to be done, but also the manner in which it was to be done?

$A_{2}$ : Was the worker allowed to use her/his own discretion in doing an aspect of the work that was not specified beforehand?

$A_{3}$ : Was the worker an integral part of the employer's business?

$A_{4}$ : Did the worker own the tools or provide the transport with which she/he performed the work?

$A_{5}$ : Would the employer make a profit/loss if the work performed by the worker cost less/more than expected? 
$A_{6}$ : Was the work performed on the employer's premises?

$A_{7}$ : Did the employer supervise or inspect the work?

$A_{8}$ : Was the worker in business on her/his own account?

$A_{9}$ : Was the worker allowed to employ others to assist with her/his work?

$A_{10}$ : Was the worker obliged to work only for the employer?

$A_{11}$ : Was the worker required to work at specified times?

$A_{12}$ : Did the employer pay the worker by time?

$A_{13}$ : Was the money that the employer paid to the worker stated to be a "fee"?

$A_{14}$ : Was the money that the employer paid to the worker stated to be "wages" or "salary"?

$A_{15}$ : Did the employer deduct PAYE ${ }^{77}$ tax instalments from the worker's pay?

$A_{16}$ : Did the employer pay the worker sick pay or holiday pay?

$A_{17}$ : Did the employer and the worker express an intention that the relationship would be one of employer and employee?

$A_{18}$ : Did the employer and the worker express an intention that the relationship would be one of principal and independent contractor?

Answering these questions should be fairly straightforward. Only $A_{3}$ could cause difficulty. The help string for that attribute suggests that:

If the worker was "part and parcel" of the employer's business then she/he was an integral part of the business, not merely accessory to it.

And the user always has the option of answering UNKNOwn if unsure.

The first draft of the specification included three extra attributes:

- Did the employer have to pay the costs of performing the work?

- Did the employer stand to make a profit if the work performed by the worker cost less than expected?

- Did the employer bear the risk of loss if the work performed by the worker cost more than expected?

These attributes were chosen because courts have had regard to these questions in employee/independent contractor cases. However, SHYSTER detected functional dependence between each of these attributes and the other two: viz. every case in 
the specification had the same attribute values for each of these three attributes. It was decided that these three attributes were, in fact, three different ways of asking the same question, so they were removed and replaced with a single attribute:

$A_{5}$ : Would the employer make a profit/loss if the work performed by the worker cost less/more than expected?

\section{Leading cases}

There are thirteen leading cases in the Employee area. Three of them concerned the applicability of an industrial award: Cam and Sons Pty Ltd v. Sargent, ${ }^{78}$ Australian Timber Workers Union v. Monaro Sawmills Pty Ltd ${ }^{79}$ and Price v. Grant Industries Pty Ltd. ${ }^{80}$ Three others concerned the payment of tax or payments to a government contribution scheme: Federal Commissioner of Taxation v. J. Walter Thompson (Australia) Pty Ltd, ${ }^{81}$ Queensland Stations Pty Ltd v. Federal Commissioner of Taxation ${ }^{82}$ and Ready Mixed Concrete (South East) Ltd v. Minister of Pensions and National Insurance. ${ }^{83}$ Two concerned workers' compensation: Zuijs v. Wirth Brothers Pty Ltd ${ }^{84}$ and Humberstone v. Northern Timber Mills. ${ }^{85}$ Two arose out of copyright claims: Stevenson Jordon and Harrison Ltd $v$. Macdonald and Evans ${ }^{86}$ and Performing Right Society Ltd v. Mitchell \& Booker (Palais de Danse) $L_{t d .}{ }^{87}$ One was a claim for damages for breach of statutory duty: Ferguson v. John Dawson 8 Partners (Contractors) Ltd. ${ }^{88}$ Another concerned a worker's entitlement to long service leave: Australian Mutual Provident Society v. Chaplin. ${ }^{89}$ And one was a claim for compensation for unfair dismissal: Massey v. Crown Life Insurance Co. ${ }^{90}$

Stevenson v. Macdonald is used twice. In that case Macdonald and Evans claimed copyright in a book because its author had written it while he was working for them. The book was divided into sections, which were written under different circumstances. The English Court of Appeal looked at each section separately, to determine whether that section had been written by its author as an employee of Macdonald and Evans. The case appears as Stevenson $v$. Macdonald (1) — which deals with the first section — and as Stevenson v. Macdonald (2) - which deals with the second. SHYSTER treats each as a separate case; the facts and the result are different in both.

\section{Attribute dependence}

The probabilities file for the EMPLOYEe specification is given in full in §C.7. SHYSTER detects functional dependence between $A_{4}$ and $A_{5}$. Although, in the leading cases, the inverse function maps the attribute values of one to those of the other, the two attributes represent different questions: there is no reason to believe that there is any relationship between the worker not owning the tools 
or providing the transport with which she/he performs the work $\left(A_{4}\right)$, and the employer standing to make a profit/loss if the work performed by the worker costs less/more than expected $\left(A_{5}\right)$. Hence, both attributes remain in the specification.

SHYSTER also detects evidence of stochastic dependence between $A_{3}$ and $A_{17}$, $A_{4}$ and $A_{11}, A_{5}$ and $A_{11}, A_{7}$ and $A_{9}, A_{9}$ and $A_{11}$, and $A_{11}$ and $A_{12}$. Only in two of these pairs - the first and the last - is there any danger that the attributes are actually asking significantly similar (or dissimilar) questions. After careful consideration it was decided that all of these attributes, even those in the first and last pairs, are different questions and important in distinguishing between employees and independent contractors. So these attributes, too, remain in the specification.

\section{Weights}

The weights file for the Employee specification is given in full in $\S$ C.9. The rightmost column of the table of weights indicates that three attributes are of equal greatest importance in the Employee area: ${ }^{91}$

$A_{13}$ : Was the money that the employer paid to the worker stated to be a "fee"?

$A_{14}$ : Was the money that the employer paid to the worker stated to be "wages" or "salary"?

$A_{16}$ : Did the employer pay the worker sick pay or holiday pay?

The fact that the courts have had regard to so many different factors when deciding employee cases, makes it difficult to judge SHYSTER's choice of most important attributes. However, $A_{1}$ and $A_{2}$ should probably be more heavily weighted than they are.

\subsubsection{Test cases}

The EMPLOYEe specification was written so as to represent the law as it was in 1982. Four actual cases are used to test the EMPLOYEE specification-cases which were decided after that time. There are also two hypothetical cases, chosen by the legal expert. The results of all these tests are summarized in figure 5.12.

Narich Pty Ltd v. Commissioner of Pay-roll Tax

In Narich Pty Ltd v. Commissioner of Pay-roll Tax ${ }^{92}$ Narich held the Australian franchise of Weight Watchers. Lecturers taught classes, following a program detailed in the Weight Watchers handbook, and deducted their fees from the 
money that they collected from class members. Clause 3 of the agreement between the lecturers and Narich stated that the lecturers were not employees of Narich, but independent contractors.

The Commissioner claimed that the lecturers were actually employees of Narich, and that Narich was liable, under the Pay-roll Tax Act 1971 (NSW), to pay payroll tax on the money paid to the lecturers.

The Judicial Committee of the Privy Council cited Australian Mutual Provident Society $v$. Chaplin as authority for the principle that a statement in an agreement that a worker is an "independent contractor" or an "employee" is not decisive. Their lordships held that, despite clause 3, the effect of the agreement between the lecturers and Narich was that the lecturers were employees of Narich.

SHYSTER's fact vector is (YNYNYNNNNNYYYNNNNY); to quote its report file:

... the employer directed the manner in which the work was to be done; the worker was not allowed to use her/his own discretion in doing an aspect of the work that was not specified beforehand; the worker was an integral part of the employer's business; the worker neither owned the tools nor provided the transport with which she/he performed the work; the employer would make a profit/loss if the work performed by the worker cost less/more than expected; the work was not performed on the employer's premises; the employer neither supervised nor inspected the work; the worker was not in business on her/his own account; the worker was not allowed to employ others to assist with her/his work; the worker was not obliged to work only for the employer; the worker was required to work at specified times; the employer paid the worker by time; the money that the employer paid to the worker was stated to be a "fee"; the money that the employer paid to the worker was not stated to be "wages" or "salary"; the employer did not deduct PAYE tax instalments from the worker's pay; the employer paid the worker neither sick pay nor holiday pay; the employer and the worker did not express any intention that the relationship would be one of employer and employee; and the employer and the worker expressed an intention that the relationship would be one of principal and independent contractor.

SHYSTER agrees with the Privy Council that the lecturers are employees, although it warns that the specified directions suggest Contractor. Its table of distances, as extracted from the distances file, is given in figure 5.7. None of SHYSTER's chosen cases was cited in the judgment: the nearest neighbour is Ferguson v. John Dawson 8 Partners (Contractors) Ltd $\left(C_{5}\right)$; the nearest other is Massey v. Crown Life Insurance Co. $\left(C_{12}\right)$. 


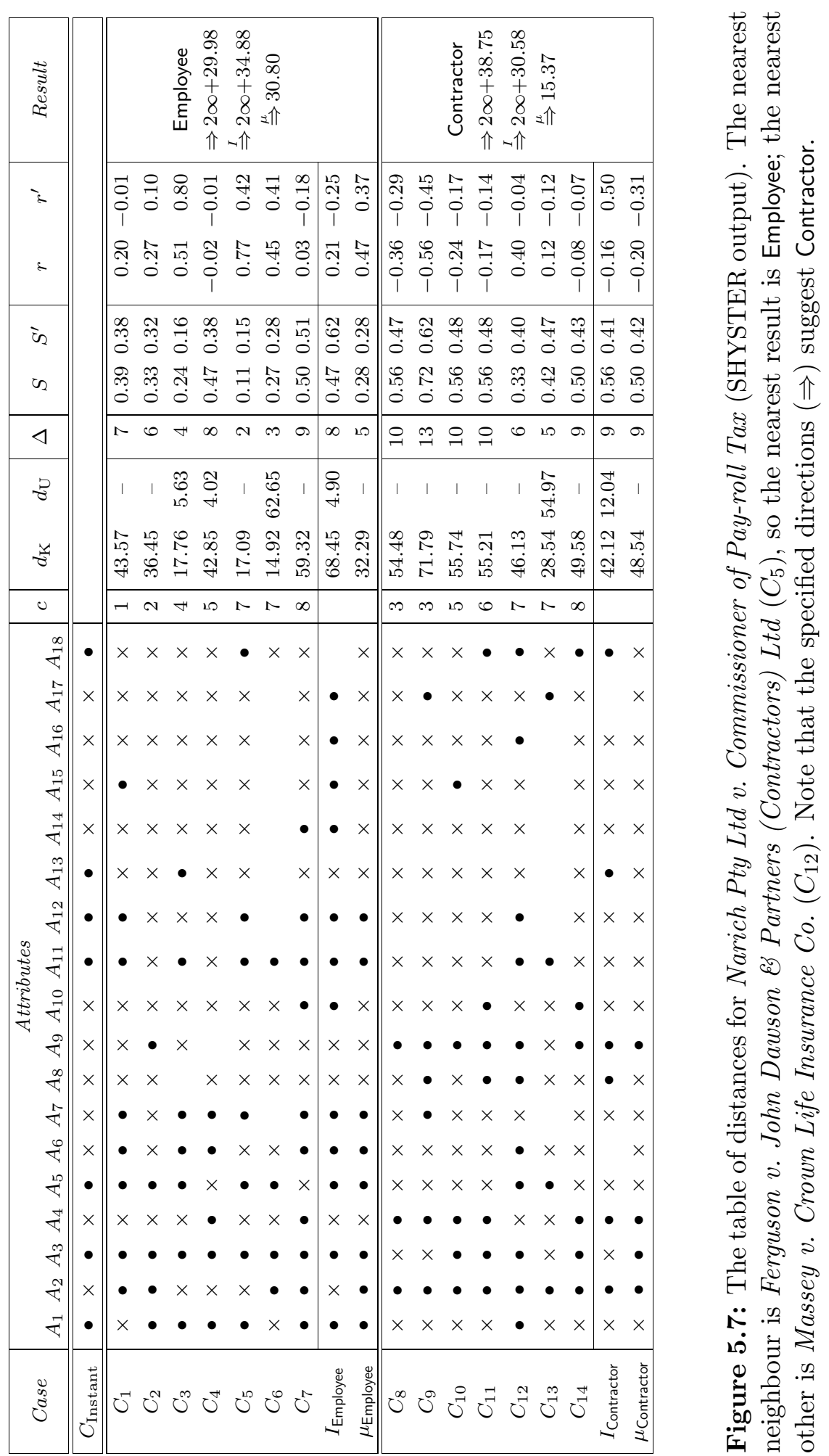


In Stevens v. Brodribb Sawmilling Company Pty Ltd, ${ }^{93}$ Brodribb employed sniggers to fell trees, and truck drivers to carry the trees to the sawmill. They used their own vehicles, set their own hours of work, and were paid according to the volume of timber that they delivered to the sawmill. Stevens, a truck driver, was injured due to the negligence of a snigger. He claimed that the snigger was an employee of Brodribb and, hence, Brodribb was vicariously liable for the snigger's negligence. He also claimed that he was an employee of Brodribb and, hence, Brodribb was personally liable to him for breaching the duty of care owed by an employee to another employee.

Five judges of the High Court of Australia applied Humberstone v. Northern Timber Mills and Australian Mutual Provident Society v. Chaplin, and decided that neither the truck driver nor the snigger were Brodribb's employees. ${ }^{94}$

SHYSTER's fact vector is (NYYYNUNYYNNNNNNNNN). SHYSTER agrees with the High Court that Stevens was an independent contractor. The nearest neighbour is Australian Mutual Provident Society v. Chaplin: one of the cases that the High Court applied. But none of their honours referred to SHYSTER's nearest other: Cam and Sons Pty Ltd v. Sargent. SHYSTER issues no warnings.

\section{Re Porter; Re Transport Workers Union of Australia}

In Re Porter; Re Transport Workers Union of Australia, ${ }^{95}$ five truck drivers, who owned their own trucks, had nominated for various offices in the Victorian branch of the TWU. The returning officer rejected their nominations on the grounds that only members who were employees were eligible for election, and they were independent contractors. The drivers challenged the returning officer's decision.

In the Federal Court, the TWU relied heavily on four Australian cases (only one of which is part of the EMPLOYEE specification) in which owner-drivers of trucks had been held not to be employees of the company for whom they worked: Humberstone v. Northern Timber Mills, Wright v. Attorney-General for Tasmania, ${ }^{96}$ Barro Group Pty Ltd v. Fraser ${ }^{97}$ and Stevens v. Brodribb Sawmilling Company Pty Ltd. The union also relied on the English case of Ready Mixed Concrete (South East) Ltd v. Minister of Pensions and National Insurance. Gray J said that, despite the consistency of these cases, "a balancing exercise is always involved in the determination whether an employment relationship exists." 98 On balance, he held, the five drivers were employees. 
The complete report file for Re Porter; Re $T W U$ is given as an example in $\S$ B.4. SHYSTER's fact vector is (YNYYUNUNNYNYNNYNNY) ${ }^{99}$ SHYSTER agrees with Gray J that the drivers were employees. The nearest neighbour is Ferguson v. John Dawson \& Partners (Contractors) Ltd, which was not mentioned in the case. The nearest other is Ready Mixed v. Minister: one of the cases cited by the TWU.

SHYSTER warns that one of the instantiations has a different result to that of the instant case. If the employer would not make a profit/loss when the work performed by the drivers cost less/more than expected $\left(A_{5}\right)$, and the employer neither supervised nor inspected the drivers' work $\left(A_{7}\right)$-attributes with UNKNOWN values in the instant case - then SHYSTER's opinion would be that the drivers were independent contractors (following Ready Mixed v. Minister). The legal expert confirms that this is a sensible distinction to make.

\section{Building Workers' Industrial Union of Australia v. Odco Pty Ltd}

In Building Workers' Industrial Union of Australia v. Odco Pty Ltd, ${ }^{1}$ Odco provided labour to builders in Victoria. ${ }^{2}$ In the agreement between Odco and the workers it was stated that the workers were contractors, not employees. They undertook to work for an agreed hourly rate, regardless of any industrial awards which might apply. Odco encountered "hostility from within the trade union movement, especially amongst officials of building unions" 3 and "incidents" occurred which led Odco to bring an action against the union for breach of s. 45D of the Trade Practices Act 1974 (Cth). The question of whether the workers were employees or independent contractors of Odco was relevant to establishing a defence for the union under the Act.

The case was heard by the Federal Court. Wilcox, Burchett and Ryan JJ applied Stevens v. Brodribb Sawmilling Company Pty Ltd and held that the workers were not employees of Odco. ${ }^{4}$ The fact that parties to an agreement label workers as employees or as independent contractors is not decisive - their honours cited Ferguson v. John Dawson $\&$ Partners (Contractors) Ltd ${ }^{5}$-however they held that, in this case, the "contractor" label was correct.

$B W I U v$. Odco is used as the test case in the complete example in appendix C. SHYSTER's fact vector is (NYNYNNNUNNYUNNNNNY). ${ }^{6}$ The distances file for $B W I U v$. Odco is given in full in $\S \mathrm{C} .11$. The report file is in $\S \mathrm{C} .13$.

SHYSTER's conclusion is that the workers were independent contractors. Its choice of cases is good. The nearest neighbour is Humberstone $v$. Northern Timber Mills $\left(C_{8}\right)$, which was applied by the High Court in Stevens v. Brodribb which was 
applied by the Federal Court in BWIU v. Odco (Stevens v. Brodribb is not one of the leading cases in the Employee specification). The nearest other is Ferguson $v$. John Dawson $\&$ Partners (Contractors) Ltd $\left(C_{5}\right)$, which their honours cited in their judgment. No warnings are issued.

\section{Hypothetical case 3}

Both hypothetical case 3 and case 4 are taken from a question set in an examination for a university employment law course. ${ }^{7}$ A secretary, Amber, is employed in the Department of Legal Studies in an Australian university. The written contract between the university and Amber provides that salary, hours, allowances, and leave shall be in accordance with the terms of a specified award. Both the university and Amber contribute to a superannuation scheme for Amber's benefit. Amber's appointment is to continue until she is 65 years of age, after an initial probationary period. There is a duty statement for Amber's position, and she performs duties as required by the Head of the Department.

It is clear, and the legal expert confirms, that Amber is an employee of the university.

SHYSTER's fact vector is (YNYNYYYNNUYNNYYYYN); SHYSTER agrees that Amber is an employee. The nearest neighbour is Performing Right Society Ltd v. Mitchell E Booker (Palais de Danse) Ltd; the nearest other is Massey v. Crown Life Insurance Co. Neither is a good choice.

SHYSTER warns that the weighted correlation coefficients suggest that Stevenson Jordon and Harrison Ltd v. Macdonald and Evans (1), in which the worker was an independent contractor, should be the nearest neighbour.

\section{Hypothetical case 4}

From the same examination question as hypothetical case 3 comes another hypothetical case.

Bonny works as a secretary in the same department as Amber. Bonny is registered with an employment agency. The Department and the employment agency entered into a contract in which the agency agreed to provide a competent secretary for a three month period, and retained the right to provide a substitute at any time. The university indemnified the employment agency for any liability arising out of Bonny's work.

When Bonny registered with the employment agency she signed a contract which stated that any work which she procured through the agency she would perform as an independent contractor. Bonny is paid a specified daily rate, is not eligible for any leave, and is responsible for injury insurance and taxation liabilities. Bonny has had her work period extended several times, and has worked 
in the Department of Legal Studies for a year. Bonny and Amber have similar duties and patterns of work. They both follow guidelines issued by the Head of the Department.

The legal expert advises that, unlike Amber and on the basis of BWIU v. Odco, Bonny is an independent contractor.

SHYSTER's fact vector is (YNYNYYYNNNYYNNNNNY); SHYSTER disagrees with the expert, and concludes that Bonny is an employee of the university. The nearest neighbour is Ferguson v. John Dawson 8 Partners (Contractors) Ltd. As in this hypothetical case, both the employer and the worker in Ferguson v. Dawson agreed that the worker was an independent contractor. ${ }^{8}$ In fact the only difference that SHYSTER sees between Ferguson $v$. Dawson and hypothetical case 4 is that in this case the work was performed on the employer's premises. Nevertheless, SHYSTER's choice of nearest neighbour is a bad one. The nearest other is Massey v. Crown Life Insurance Co., a good choice. In Massey v. Crown Life, Massey was an employee for two years, then performed the same duties for another two years as an independent contractor (for tax purposes). The relationship between Massey as employee and Massey as independent contractor, is similar to that between Amber and Bonny. SHYSTER issues no warnings.

The legal expert advises that one of the aims of this examination question was to demonstrate the absurdity of the law in this area: Amber and Bonny perform very similar jobs, yet the law sees them as having quite different relationships with the university. Hypothetical case 4 is an example of a tripartite employment relationship: a relationship where one party pays an employment agency which pays the worker. BWIU v. Odco was the first case in which a court had to determine the employment status of workers in such relationships. It was decided in 1991 and so is not part of the EMPLOYEE specification. SHYSTER's failure in this hypothetical case illustrates the unsuitability of the EMPLOYEE specification for providing advice on such tripartite relationships.

\subsubsection{Generated tests}

The legal expert provided three examples for generating tests using the EMPLOYEE specification: generated tests 4,5 and 6 . The results of these tests are summarized in figure 5.13 .

\section{Generated test 4}

The legal expert advises that, if the following is true, the worker is an employee:

... the employer directed the manner in which the work was to be done; the worker was an integral part of the employer's business; the employer would make a profit/loss if the work performed by the worker cost less/more than 
expected; the employer supervised or inspected the work; the worker was not allowed to employ others to assist with her/his work; the money that the employer paid to the worker was stated to be "wages" or "salary"; the employer deducted PAYE tax instalments from the worker's pay; and the employer paid the worker sick pay or holiday pay.

SHYSTER's fact vector is (YUYUYUYUNUUUUYYYUU). It generates 1024 instantiations: a mere 1/256th of the search space. However, it is not possible that the money that the employer paid to the worker was stated to be both a "fee" and "wages." This paradox occurs whenever the values of both $A_{13}$ and $A_{14}$ are YES. Because $A_{14}$ is always YES, setting $A_{13}$ to NO avoids these impossible cases and halves the size of the search space. In 149 of the 512 instantiations in this reduced search space, SHYSTER's choice of result is bad (i.e. Contractor): a success rate of $70.90 \%$.

In all 149 the nearest ideal point is that of the expected result; and in all but 4 of those 149, SHYSTER warns that that ideal point is at least as near to the instant case as is the nearest neighbour. There is no (originally unkNOwn) attribute for which these 149 instantiations have common values. However, in 131 (or 87.92\%) of them the worker was in business on her/his own account. The legal expert advises that although this (in combination with the originally specified attribute values) does not present a paradox, it is an almost inconceivable set of attributes.

\section{Generated test 5}

The legal expert also advises that, if the following is true, the worker is an independent contractor:

... the employer would not make a profit/loss if the work performed by the worker cost less/more than expected; the worker was in business on her/his own account; the worker was allowed to employ others to assist with her/his work; the employer did not deduct PAYE tax instalments from the worker's pay; and the employer paid the worker neither sick pay nor holiday pay.

Given the fact vector (UUUUNUUYYUUUUUNNUU), SHYSTER generates 8192 instantiations: 1/32nd of the search space. Once again, a paradox occurs whenever the values of both $A_{13}$ and $A_{14}$ are YEs. Because both attributes are UnKNown, removing these impossible cases reduces the size of the search space by a quarter. In 1946 of the 6144 instantiations in this reduced search space, SHYSTER's choice of result is bad (i.e. Employee): a success rate of $68.33 \%$. 
Despite the fact that the nearest ideal point is that of the expected result in 1353 of those 1946, only 492 of them cause SHYSTER to issue a warning. Furthermore, in 111 instantiations SHYSTER issues an ideal point warning, even though the choice of result is good.

\section{Generated test 6}

The legal expert further advises that, if the following is true, the worker is an independent contractor:

... the worker was allowed to use her/his own discretion in doing an aspect of the work that was not specified beforehand; the worker owned the tools or provided the transport with which she/he performed the work; the employer would not make a profit/loss if the work performed by the worker cost less/more than expected; the worker was in business on her/his own account; the money that the employer paid to the worker was stated to be a "fee"; the employer did not deduct PAYE tax instalments from the worker's pay; and the employer paid the worker neither sick pay nor holiday pay.

SHYSTER's fact vector is (UYUYNUUYUUUUYUNNUU). It generates 2048 instantiations. As with generated test 4, removing the paradoxical cases halves the size of the search space. In 149 of the 1024 instantiations in this reduced search space, SHYSTER's choice of result is bad (i.e. Employee): a success rate of $85.45 \%$.

In all 149 the nearest ideal point is that of the expected result; and in all but 37 of these 149, SHYSTER warns that that ideal point is at least as near to the instant case as is the nearest neighbour. The fact that, in 120 (or 80.54\%) of these 149, the work was performed on the employer's premises, is not enough to justify SHYSTER's choice of result in these instantiations.

\subsubsection{Conclusion}

In all but one of the tests described in $\S 5.4 .3$ above (i.e. 83.34\%) SHYSTER reached a good conclusion, but in only $41.67 \%$ of those tests did it choose good cases to use in argument.

As shown in figure 5.13, SHYSTER's choice of result is good in only $70.78 \%$ of the instantiations in all three generated tests. (This takes into account the reduction of the size of the search space due to the removal of instantiations where the values of both $A_{13}$ and $A_{14}$ are yEs. Such instantiations do not represent plausible cases. ${ }^{9}$ ) 
This performance is dominated by generated test $5:{ }^{10}$ the test which produced, from SHYSTER, the worst performance of all the generated tests described in this chapter. Alone amongst all the generated tests, this test causes SHYSTER to issue ideal point warnings for instantiations in which its choice of result is good. If a bad choice of result is considered good where an ideal point warning is issued and a good choice of result is considered bad where an ideal point warning is issued, SHYSTER's success rate for the three generated tests of the EMPLOYEE specification rises to $99.22 \%, 74.53 \%$ and $96.39 \%$, respectively: $79.09 \%$ overall.

There are two tripartite employment cases amongst the test cases: BWIU v. Odco and hypothetical case 4 . In both of these tests, it is important to be clear as to who is the "employer" for the purposes of answering SHYSTER's questions: in $B W I U$ v. Odco it is Odco (the employment agency); in hypothetical case 4 it is the university. Although SHYSTER's choices of result and cases for BWIU v. Odco are good, the ability of the EMPLOYEE specification to handle tripartite employment relationships is stretched by hypothetical case 4 .

The Employee area also demonstrates one of SHYSTER's minor shortcomings. In its report, SHYSTER's summary of the facts in a case can make difficult reading when, as here, there is a large number of attributes. The wording of the strings for the last two attributes means, for example, that the statement of facts in SHYSTER's report on Stevens v. Brodribb Sawmilling Company Pty Ltd is a 253-word sentence that concludes:

... the employer and the worker did not express any intention that the relationship would be one of employer and employee; and the employer and the worker did not express any intention that the relationship would be one of principal and independent contractor.

It is to be hoped that no human legal expert would express the facts so clumsily. However, this problem is inherent in SHYSTER's method of writing reports; possible solutions are discussed in $\S 6.2 .5$.

The Employee area could be linked to several statutory open-textured concepts, as represented in a rule-based system. It could also be linked to areas (like the Authorization area) in which the distinction between employees and independent contractors is important. 
SHYSTER (when using the EMPLOYEE specification) is capable of providing good advice in cases involving bipartite employment relationships (as it was designed to do). It is not so well suited to cases involving tripartite relationships. The reflexive tests performed in $\S$ D.4, and summarized in figure D.3, indicate that the EMPLOYEe specification is capable of handling new fact situations.

The specification chooses good results in most of the generated tests. It performs fairly well in an area of law in which, according to Clark and Wedderburn, the juridical concepts "approach anarchy." 11

\subsection{The implication of natural justice}

The last specification discussed in this chapter concerns the implication of natural justice principles in administrative decision-making.

\subsubsection{The law}

The law in Australia has developed the notion of affording natural justice to a person who is affected by an administrative decision. Natural justice principles might require, for example, that the person affected be given the right to a hearing - to make representations - before the decision is made. Or the decisionmaker might be obliged to give reasons for the decision, after it has been made. These requirements are sometimes called requirements of "procedural fairness."

The development of this area of law has been convoluted:

The evolution of the doctrine of natural justice has been a journey in fluctuation, typified by the regular pitch of basic principles from one leaning to another. ${ }^{12}$

In Kioa v. West, Mason J said:

The law has now developed to a point where it may be accepted that there is a common law duty to act fairly, in the sense of according procedural fairness, in the making of administrative decisions which affect rights, interests and legitimate expectations, subject only to the clear manifestation of a contrary statutory intention. ${ }^{13}$

But McMillan asserts that:

The main residual difficulty concerning the implication of natural justice has been the tendency to apply uncritically [this] superficial test ... the affect $[$ sic $]$ of a decision on a person's interest is only one of many factors that is relevant to deciding whether a natural justice obligation attaches to the decision. ${ }^{14}$ 
The notion of a "legitimate expectation" — or a "reasonable expectation"15 _ expands the area considerably: it has been judicially stated that legitimate expectations "are capable of including expectations which go beyond enforceable legal rights, provided they have some reasonable basis". ${ }^{16}$

McMillan discusses different ways of determining whether natural justice principles apply to a given administrative procedure:

An option at one end of the spectrum has always been to treat the obligation of procedural fairness as attaching universally to all decisions, and to concentrate instead on the practical content of that obligation in any particular instance. This option ... was described by Deane J in Haoucher as "conceptually more satisfying". ${ }^{17}$

However, that approach is too broad. As Wilcox J points out in Minister for Arts Heritage and Environment v. Peko-Wallsend Ltd:

... the law has not yet reached the stage of applying the obligation of natural justice to every decision which disadvantages individuals. ${ }^{18}$

McMillan proposes an alternative:

... a preferable approach would be to determine whether natural justice applied by examining in each case a number of different factors. While the weight attached to some factors is clearly greater, it is the overall balance that is important. This approach was propounded by the Privy Council in Durayappah v. Fernando, ${ }^{19}$ was initially applied in Australia in Salemi v. MacKellar (No. 2) ${ }^{20}$ and FAI Insurances Ltd v. Winneke, ${ }^{21}$ but has not been developed further. ${ }^{22}$

His factors are:

- the nature of the property, right, interest, status, or reasonable expectation;

- the effect or impact of the decision;

- the nature of the power being exercised;

- the statutory and factual criteria according to which the decision was made;

- the nature of the officer making the decision;

- the statutory procedural framework under which the decision was made; and

- the circumstances in which the decision was made. ${ }^{23}$ 
McMillan discusses these factors in detail. It is worth discussing here the law relating to one of these factors as it significantly affects one of the test cases in $§ 5.5 .3$ below. ${ }^{24}$

The statutory procedural framework under which the decision was made may grant a person a right to appeal against a decision. The existence of a statutory right to appeal used to suggest that natural justice would not be implied. For example, in Twist v. Council of Municipality of Randwick, Mason J stated:

Having regard to the subject matter of the section, ... and more particularly the comprehensive nature of the appeal to a District Court judge, I am of opinion that s. 317B(5) [granting the right of appeal] should be read as providing the exclusive remedy available to an owner who wished to challenge the validity or correctness of an order made under s. $317 \mathrm{~B}(1) \cdot{ }^{25}$

And, in the first Marine Hull $\&$ Liability Insurance Co. Ltd v. Hurford, Wilcox J held that the Treasurer should have applied the principles of natural justice before making the contested direction - however, because the relevant Act provided for a review of any such directions by the Administrative Appeals Tribunal:

... the legislature must be taken to have evinced an intention that, in the event of the Treasurer failing to so act, the directions are not to be regarded as being invalid in law. They are merely susceptible of challenge before the Tribunal. ${ }^{26}$

Recently, there has been a tendency to interpret the existence of a statutory right to appeal as indicating that natural justice should be implied. In the second Marine Hull \& Liability Insurance Co. Ltd v. Hurford, for example, Davies J stated that:

The existence of a right to have a matter reconsidered and of a right to have a matter reviewed by the Administrative Appeals Tribunal may well affect the nature of the procedures which ought to be adopted in complying with the rules of natural justice but, ordinarily, it does not exclude them. ${ }^{27}$

And in Bropho v. Western Australia, Rowland $\mathrm{J}$ held that the existence of a right of appeal to the Supreme Court indicated that the decision-maker should be influenced by the same considerations as would a judge of that Court; it indicated that questions of natural justice should not be excluded. ${ }^{28}$

Rowland J's approach is diametrically opposed to that of the High Court in Twist v. Randwick, decided fourteen years earlier. His decision was overturned on appeal by the Full Court of the Supreme Court of Western Australia in Western Australia v. Bropho ${ }^{29}$ (another of the test cases in $\S 5.5 .3$ below). So, although the law may be evolving towards Rowland J's interpretation, it has not yet reached that stage. 


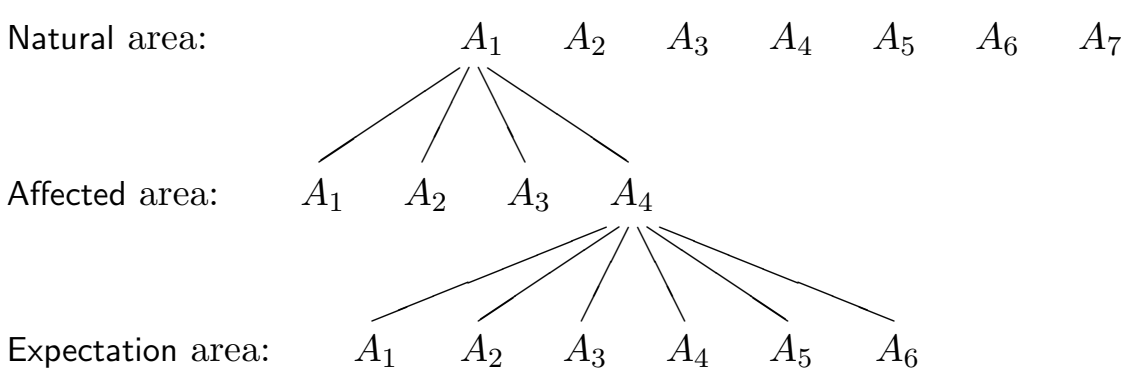

Figure 5.8: The relationship between the attributes in the three areas which make up the NATURAL specification.

\subsubsection{The NATURAL specification}

The author - having consulted a legal expert, ${ }^{30}$ and the published judgments in the leading cases - wrote a specification of the implication of the duty to observe natural justice. The specification is based on the factors identified by McMillan. ${ }^{31}$

The dump file for the NATURAL specification is given in full in $\S \mathrm{A} .5$. It contains three areas: the Natural area, the Affected area and the Expectation area. The relationship between the attributes in these areas is indicated in figure 5.8. There is a hierarchy which ranks nine courts, and the Affected and Expectation area both have opening strings.

\section{Natural area}

The Natural area has two results: Implied and Not-Implied. These correspond to "a duty to observe natural justice is implied" and "a duty to observe natural justice is not implied."

There are seven attributes in the Natural area, corresponding to the seven factors identified by McMillan. The term applicant is used to refer to the person who seeks the implication of natural justice:

$A_{1}$ : Did the decision affect the property, right, interest, status, or legitimate expectation of the applicant?

$A_{2}$ : Is the decision apt to have a discrete impact on the interests of the applicant?

$A_{3}$ : Is the power of a nature that would suggest that procedural fairness would be applied?

$A_{4}$ : Did the statutory or factual criteria focus on matters which were discrete to the interests of the applicant?

$A_{5}$ : Was the decision-maker a high-level policy-maker? 
$A_{6}$ : Is there a statutory right to appeal against the decision? ${ }^{32}$

$A_{7}$ : Were there circumstances which make an obligation to observe natural justice inappropriate?

Only $A_{6}$ is straightforward. But (with the exception of $A_{1}$ ) answering the other questions - with the assistance of the help strings which are included in the specification - should present no major difficulties. For example, the help string for $A_{4}$ is:

The decisional criteria are of two kinds: the spectrum of considerations to which the decision maker was authorized to have regard (the statutory criteria), and the specific considerations to which regard was had in fact (the factual criteria). Either set of criteria can focus on matters which are discrete to the interests of the applicant, or on matters of policy or public interest.

And the help string for $A_{5}$ explains that:

Ministers, members of Cabinet, Governors and the Governor-General are high-level policy-makers.

However, $A_{1}$ is such a difficult question to answer that it is defined as an external attribute, linked to the Affected area. The user is never asked the $A_{1}$ question, but is asked questions from the Affected area instead.

\section{Affected area}

The Affected area has two results: Affected and Unaffected. These correspond to "the decision affected the property, right, interest, status, or legitimate expectation of the applicant" and "the decision did not affect [those interests]." A result of Affected produces a value of YEs for $A_{1}$ in the Natural area; a result of Unaffected produces a value of no.

There are four attributes in the Affected area:

$A_{1}$ : Did the decision affect a financial, property or occupational interest of the applicant?

$A_{2}$ : Did the decision affect the applicant's personal liberty?

$A_{3}$ : Did the decision affect the applicant's reputation?

$A_{4}$ : Did the applicant have a legitimate expectation which was affected by the decision?

Only $A_{4}$ is not a straightforward question. It, too, is defined as an external attribute, and linked to the Expectation area. 


\section{Expectation area}

The Expectation area also has two results: Expectation and No-Expectation. These correspond to "the applicant had a legitimate expectation which was affected by the decision" and "the applicant did not have a legitimate expectation which was affected by the decision." A result of Expectation produces a value of YEs for $A_{4}$ in the Affected area; a result of No-Expectation produces a value of NO.

There are six attributes in the Expectation area:

$A_{1}$ : Did the decision-maker break a promise or undertaking?

$A_{2}$ : Did the decision-maker go against an established course of practice?

$A_{3}$ : Did the decision involve a refusal to renew an existing interest?

$A_{4}:$ Did the decision-maker or a statutory provision suggest that an initial interest would be granted?

$A_{5}$ : Did the decision affect an established liberty or interest?

$A_{6}$ : Was there a standard administrative procedure which the decisionmaker did not follow?

There is a subtle distinction between $A_{1}, A_{2}$ and $A_{6}$ which is best illustrated by two examples.

In Haoucher $v$. Minister of State for Immigration and Ethnic Affairs, ${ }^{33}$ the Minister had told Parliament that recommendations of the Administrative Appeals Tribunal, on deportation matters, would be overturned only in "exceptional circumstances" and when justified by "strong evidence." The AAT recommended that the Minister's order to deport Haoucher be revoked, but the Minister rejected the recommendation, without giving Haoucher details of any "exceptional circumstances" or "strong evidence."

The value of $A_{1}$ is No: there may indeed have been "exceptional circumstances" - the Minister did not promise to give details of those circumstances. $A_{2}$ is YES: the Minister had an established course of practice of following AAT recommendations. However, $A_{6}$ is No: the Minister went against practice, not procedure.

In Attorney-General of Hong Kong v. $\mathrm{Ng}$ Yuen Shiu, ${ }^{34}$ an immigration official had announced that certain illegal immigrants would be interviewed, and each case "treated on its merits." Shiu came forward the next day and was questioned, but given no opportunity to put the merits of his case.

Although the decision-maker broke a promise $\left(A_{1}=\right.$ YES $)$, there had not been time to establish a course of practice $\left(A_{2}=\mathrm{NO}\right)$, and there was no standard procedure to be followed $\left(A_{6}=\mathrm{NO}\right)$. 


\section{Leading cases}

There are fifteen leading cases in the Natural area. Seven of them were decided by the High Court: FAI Insurances Ltd v. Winneke, ${ }^{35}$ Haoucher v. Minister of State for Immigration and Ethnic Affairs, ${ }^{36}$ Annetts v. McCann, ${ }^{37}$ Kioa v. West, ${ }^{38}$ Commissioner of Police v. Tanos, ${ }^{39}$ South Australia v. O'Shea ${ }^{40}$ and Bread Manufacturers of New South Wales v. Evans. ${ }^{41}$ Two were decided by the Federal Court of Australia: Marine Hull $\&$ Liability Insurance Co. Ltd v. Hurford ${ }^{42}$ and Minister for Arts Heritage and Environment v. Peko-Wallsend Ltd. ${ }^{43}$ Two were decided by the Supreme Court of New South Wales: Macrae v. Attorney-General for New South Wales ${ }^{44}$ and Nashua Australia Pty Ltd v. Channon. ${ }^{45}$ Two are decisions of the Judicial Committee of the Privy Council: Attorney-General of Hong Kong v. $\mathrm{Ng}$ Yuen Shiu ${ }^{46}$ and Durayappah v. Fernando. ${ }^{47}$ One was decided by the House of Lords: Council of Civil Service Unions v. Minister for the Civil Service. ${ }^{48}$ And one was a decision of the Chancery Division of the English Court of Appeal: McInnes v. Onslow Fane. ${ }^{49}$

Nine of these are also leading cases in the Affected area. And six of them, plus two further cases - Heatley v. Tasmanian Racing and Gaming Commission ${ }^{50}$ and Cole v. Cunningham ${ }^{51}$ - are used in the Expectation area. For reasons discussed in $§ 5.5 .3$ below, the prominent High Court cases of Twist v. Council of Municipality of Randwick ${ }^{52}$ and Salemi v. MacKellar ${ }^{53}$ were not used in the NATuraL specification.

A peculiarity of the NATURAL specification is that, as SHYSTER warns, there are three pairs of cases in the Natural area which have identical facts and different results. ${ }^{54}$ The existence of two or more leading cases with identical facts but different results indicates either that one of the cases was wrongly decided, or that there is a need for more attributes in the area (to distinguish between the cases). All of the leading cases in the Natural area were correctly decided, and the attributes in that area are based on the factors identified as being important by McMillan. Hence, no change is made to the specification. If two or more of these cases with identical facts but different results are the nearest cases, SHYSTER warns the user and chooses the most important of them as the case upon which to base its conclusion.

\section{Attribute dependence}

The probabilities matrices for the three areas in the NATURAL specification, as extracted from the probabilities file, are given in figure 5.9. SHYSTER detects no functional dependence, and no evidence of stochastic dependence, between any of the attributes in any of the three areas. 


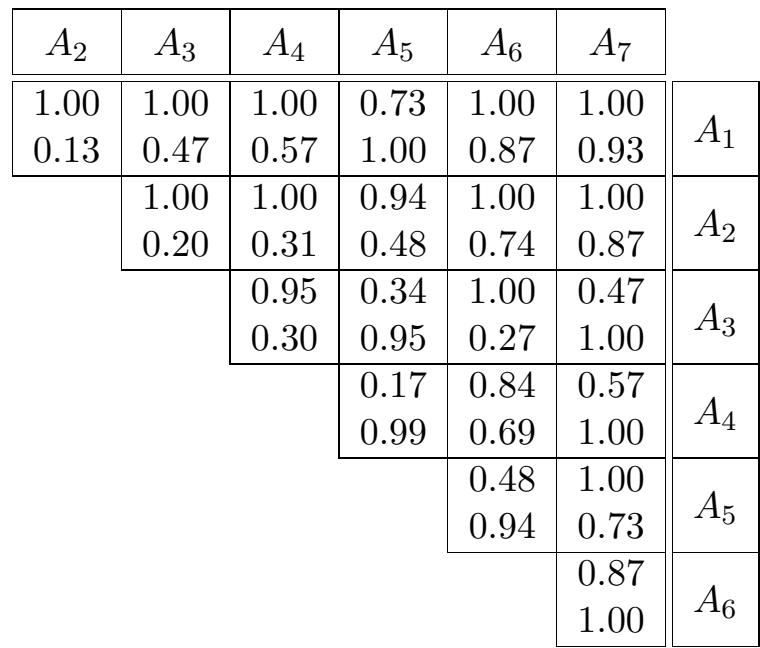

\begin{tabular}{|c|c|c|}
\hline$A_{2}$ & $A_{3}$ & $A_{4}$ \\
\hline 0.12 & 0.95 & 0.83 \\
\hline \multirow[t]{4}{*}{1.00} & 0.40 & 0.64 \\
\hline & 0.24 & 0.95 \\
\hline & 1.00 & 0.40 \\
\hline & & $\begin{array}{l}0.60 \\
088\end{array}$ \\
\hline
\end{tabular}

\begin{tabular}{|c|c|c|c|c|c|}
\hline$A_{2}$ & $A_{3}$ & $A_{4}$ & $A_{5}$ & $A_{6}$ & \\
\hline 0.36 & 0.75 & 1.00 & 0.46 & 0.75 & \multirow{2}{*}{$A_{1}$} \\
\hline \multirow[t]{9}{*}{1.00} & 1.00 & 1.00 & 0.96 & 1.00 & \\
\hline & 1.00 & 1.00 & 1.00 & 0.63 & \multirow[b]{2}{*}{$A$} \\
\hline & 0.38 & 1.00 & 0.36 & 1.00 & \\
\hline & & 1.00 & 1.00 & 0.88 & \multirow{2}{*}{$A_{3}$} \\
\hline & & 1.00 & 0.75 & 1.00 & \\
\hline & & & 1.00 & 1.00 & \multirow{2}{*}{$A_{4}$} \\
\hline & & & 1.00 & 1.00 & \\
\hline & & & & 1.00 & \\
\hline & & & & 0.75 & 15 \\
\hline
\end{tabular}

Figure 5.9: The probabilities matrices for the three areas in the NATURAL specification: the Natural area, the Affected area and the Expectation area (SHYSTER output). 


\begin{tabular}{|c|c|c|c|c|c|c|c|c|c|}
\hline \multirow{2}{*}{ Attr. } & \multicolumn{3}{|c|}{ Implied } & \multicolumn{3}{|c|}{ Not-Implied } & \multirow[b]{2}{*}{$\mu$} & \multirow{2}{*}{$\sigma^{2}$} & \multirow[b]{2}{*}{$w$} \\
\hline & $\mu$ & $\sigma^{2}$ & $w$ & $\mu$ & $\sigma^{2}$ & $w$ & & & \\
\hline$A_{1}$ & 1.00 & 0.00 & $\infty$ & 0.83 & 0.14 & 7.20 & 0.93 & 0.06 & 16.07 \\
\hline$A_{2}$ & 1.00 & 0.00 & $\infty$ & 0.67 & 0.22 & 4.50 & 0.87 & 0.12 & 8.65 \\
\hline$A_{3}$ & 0.67 & 0.22 & 4.50 & 0.33 & 0.22 & 4.50 & 0.53 & 0.25 & 4.02 \\
\hline$A_{4}$ & 0.63 & 0.23 & 4.27 & 0.17 & 0.14 & 7.20 & 0.43 & 0.24 & 4.08 \\
\hline$A_{5}$ & 0.78 & 0.17 & 5.79 & 0.67 & 0.22 & 4.50 & 0.73 & 0.20 & 5.11 \\
\hline$A_{6}$ & 0.22 & 0.17 & 5.79 & 0.00 & 0.00 & $\infty$ & 0.13 & 0.12 & 8.65 \\
\hline$A_{7}$ & 0.00 & 0.00 & $\infty$ & 0.17 & 0.14 & 7.20 & 0.07 & 0.06 & 16.07 \\
\hline
\end{tabular}

\begin{tabular}{|c|ccc|ccc|ccc|}
\hline \multirow{2}{*}{ Attr. } & \multicolumn{3}{|c|}{ Affected } & \multicolumn{3}{|c|}{ Unaffected } & \multicolumn{2}{c|}{$\sigma^{2}$} & $w$ \\
& $\mu$ & $\sigma^{2}$ & $w$ & $\mu$ & $\sigma^{2}$ & $w$ & & & \\
\hline \hline$A_{1}$ & 0.50 & 0.25 & 4.00 & 0.00 & 0.00 & $\infty$ & 0.44 & 0.25 & 4.05 \\
$A_{2}$ & 0.38 & 0.23 & 4.27 & 0.00 & 0.00 & $\infty$ & 0.33 & 0.22 & 4.50 \\
$A_{3}$ & 0.38 & 0.23 & 4.27 & 0.00 & 0.00 & $\infty$ & 0.33 & 0.22 & 4.50 \\
$A_{4}$ & 0.50 & 0.25 & 4.00 & 0.00 & 0.00 & $\infty$ & 0.44 & 0.25 & 4.05 \\
\hline
\end{tabular}

\begin{tabular}{|c|c|c|c|c|c|c|c|c|c|}
\hline \multirow{2}{*}{ Attr. } & \multicolumn{3}{|c|}{ Expectation } & \multicolumn{3}{|c|}{ No-Expectation } & \multirow[b]{2}{*}{$\mu$} & \multirow{2}{*}{$\sigma^{2}$} & \multirow[b]{2}{*}{$w$} \\
\hline & $\mu$ & $\sigma^{2}$ & $w$ & $\mu$ & $\sigma^{2}$ & $w$ & & & \\
\hline$A_{1}$ & 0.33 & $\overline{0.22}$ & 4.50 & 0.00 & 0.00 & $\infty$ & 0.25 & 0.19 & 5.33 \\
\hline$A_{2}$ & 0.50 & 0.25 & 4.00 & 0.00 & 0.00 & $\infty$ & 0.38 & 0.23 & 4.27 \\
\hline$A_{3}$ & 0.17 & 0.14 & 7.20 & 0.00 & 0.00 & $\infty$ & 0.13 & 0.11 & 9.14 \\
\hline$A_{4}$ & 0.00 & 0.00 & $\infty$ & 0.00 & 0.00 & $\infty$ & 0.00 & 0.00 & $\infty$ \\
\hline$A_{5}$ & 0.83 & 0.14 & 7.20 & 0.50 & 0.25 & 4.00 & 0.75 & 0.19 & 5.33 \\
\hline$A_{6}$ & 0.17 & 0.14 & 7.20 & 0.00 & 0.00 & $\infty$ & 0.13 & 0.11 & 9.14 \\
\hline
\end{tabular}

Figure 5.10: The tables of weights for the three areas in the NATURAL specification: the Natural area, the Affected area and the Expectation area (SHYSTER output).

\section{Weights}

The tables of weights for the three areas in the NATURAL specification, as extracted from the weights file, are given in figure 5.10.

In the Natural area, the rightmost column indicates that two attributes are of equal greatest importance: ${ }^{55}$

$A_{1}$ : Did the decision affect the property, right, interest, status, or legitimate expectation of the applicant?

$A_{7}$ : Were there circumstances which make an obligation to observe natural justice inappropriate? 
SHYSTER's weighting of attributes in this area is fairly good. $A_{1}$ and $A_{7}$ are very important attributes. Indeed if the value of $A_{1}$ is NO, then natural justice should never be implied. ${ }^{56}$ However, the legal expert advises that $A_{2}$ should be weighted more heavily than it is.

All four attributes in the Affected area are weighted (almost) the same. ${ }^{57}$ This is appropriate - each of these attributes is equally indicative of the applicant having been affected.

One of the attributes in the Expectation area is infinitely weighted:

$A_{4}$ : Did the decision-maker or a statutory provision suggest that an initial interest would be granted?

This is an important attribute, but its infinite weighting probably weights it too highly in relation to the other attributes in this area. ${ }^{58}$

\subsubsection{Test cases}

Eight cases are used to test the NATURAL specification - seven of which were considered for the specification, but deemed not to be leading cases. The last case, Ainsworth v. Criminal Justice Commission, ${ }^{59}$ is a very recently reported decision of the High Court of Australia. The results of these tests are summarized in figure 5.12 .

Twist v. Council of Municipality of Randwick

In Twist v. Council of Municipality of Randwick, ${ }^{60}$ Twist owned land in Randwick. The Council resolved, under s. 317B(1) of the Local Government Act 1919 (NSW), to demolish a building on his land. Twist was given sixty days to demolish the building, and then an extension of another three months.

Section $317 \mathrm{~B}(5)$ gave Twist a right of appeal to the District Court against the demolition order, but the Court's rules required that such appeals be made within sixty days. Twist did not appeal in time, and was not allowed an extension of time.

When the Council informed Twist that it would demolish the building, he appealed to the New South Wales Supreme Court, and then to the High Court. Twist claimed that the Council was bound by the rules of natural justice to have given him an opportunity to be heard before the demolition order was made. He relied on Ridge v. Baldwin ${ }^{61}$ a decision of the House of Lords. The Council relied on the nineteenth century English case of Vestry of St James and St John, Clerkenwell v. Feary. ${ }^{62}$ (Neither decision is part of the Natural specification.) 
Three judges of the High Court unanimously found against Twist. Barwick CJ and Mason J held that the existence of a right of appeal showed that Twist had no right to be heard before the Council made its demolition order. ${ }^{63}$ Jacobs J disagreed, but held that the existence of a right of appeal did show that the Council's failure to allow a hearing did not render its decision void. ${ }^{64}$

SHYSTER's fact vectors are (NNNNYN), (YNNN) and (YYYYNYN) - for the Expectation, Affected and Natural areas, respectively. To quote its report files:

... the decision-maker did not break a promise or undertaking; the decisionmaker did not go against an established course of practice; the decision did not involve a refusal to renew an existing interest; neither the decisionmaker nor a statutory provision suggested that an initial interest would be granted; the decision affected an established liberty or interest; and there was no standard administrative procedure which the decision-maker should have followed.

... the decision affected a financial, property or occupational interest of the applicant; the decision did not affect the applicant's personal liberty; the decision did not affect the applicant's reputation; and the applicant did not have a legitimate expectation which was affected by the decision.

... the decision affected the property, right, interest, status, or legitimate expectation of the applicant; the decision is apt to have a discrete impact on the interests of the applicant; the power is of a nature that would suggest that procedural fairness would be applied; the statutory or factual criteria focused on matters which were discrete to the interests of the applicant; the decision-maker was not a high-level policy-maker; there is a statutory right to appeal against the decision; and there were no circumstances which would have made an obligation to observe natural justice inappropriate.

In the Expectation area, SHYSTER concludes (as did the High Court) that Twist did not have a legitimate expectation which was affected by the decision. The nearest neighbour is South Australia v. O'Shea; the nearest others are Haoucher $v$. Minister of State for Immigration and Ethnic Affairs and Heatley v. Tasmanian Racing and Gaming Commission. SHYSTER warns that the specified directions suggest Expectation.

In the Affected area, SHYSTER concludes that the decision affected Twist's property, right, interest, status, or legitimate expectation. The nearest neighbour is Bread Manufacturers of New South Wales v. Evans; the nearest other is Minister for Arts Heritage and Environment v. Peko-Wallsend Ltd.

However, SHYSTER's conclusion in the Natural area is that a duty to observe natural justice is implied. Its table of distances, as extracted from the appropriate distances file, is given in figure 5.11. The nearest neighbour is Commissioner of Police v. Tanos $\left(C_{5}\right)$, one of the cases to which Mason J referred in coming to his decision; the nearest other is McInnes $v$. Onslow Fane $\left(C_{15}\right)$. 


\begin{tabular}{|c|c|c|c|c|c|}
\hline 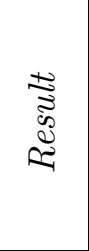 & & 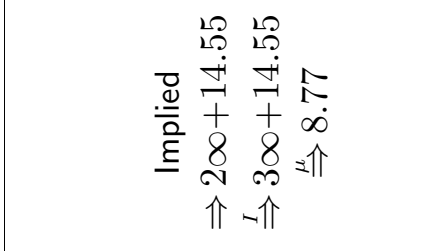 & & 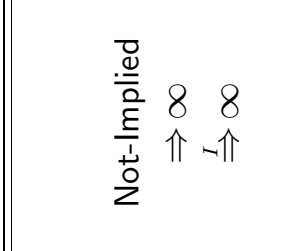 & \\
\hline$=$ & & 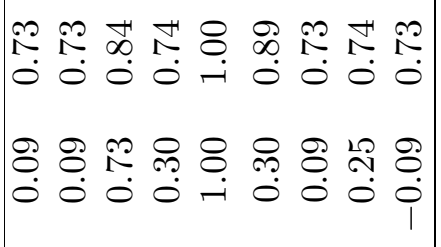 & $\left|\begin{array}{ll}\overrightarrow{1} & +1 \\
\infty & \infty \\
0 & 0 \\
0 & 0 \\
0 & 0 \\
0 & 0 \\
0 & 0\end{array}\right|$ & 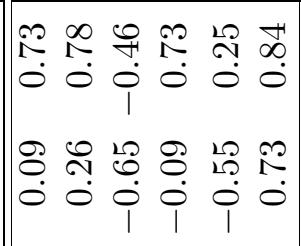 & 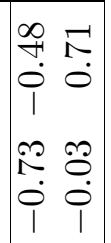 \\
\hline is & & 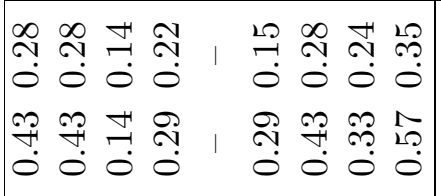 & 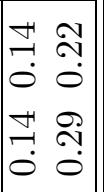 & 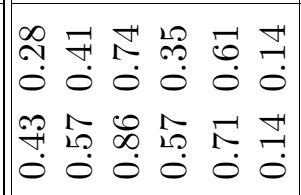 & $\begin{array}{ll}0 & 2 \\
\infty & 2 \\
0 & 0 \\
0 & 0 \\
0 & 1 \\
0 & 10 \\
0 & 0\end{array}$ \\
\hline$\triangleleft$ & & 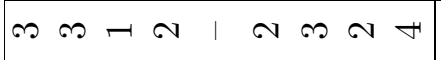 & $\neg \propto$ & 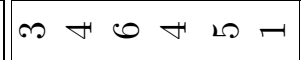 & $0 \%$ \\
\hline$\frac{1}{8}$ & & 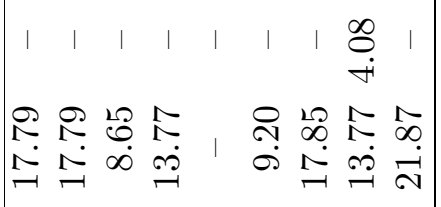 & 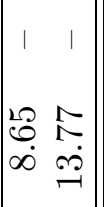 & 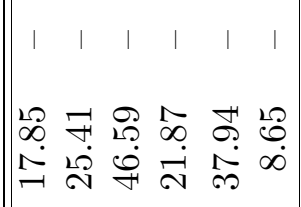 & 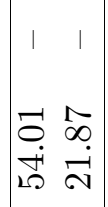 \\
\hline$u$ & & 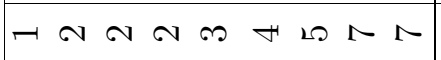 & & 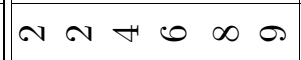 & \\
\hline 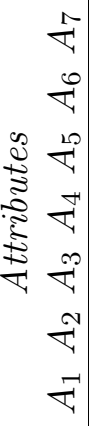 & $\begin{array}{l}x \\
\bullet\end{array}$ & $\begin{array}{lllllllll}\times & \times & \times & \times & \times & \times & \times & \times & \times \\
\times & \times & \times & \times & \bullet & \bullet & \times & \times & \times \\
\bullet & \bullet & \times & \bullet & \times & \bullet & \bullet & \bullet & \bullet \\
\bullet & \bullet & \bullet & \bullet & \bullet & \times & \times & & \times \\
\times & \times & \bullet & \bullet & \bullet & \bullet & \bullet & \bullet & \times \\
\bullet & \bullet & \bullet & \bullet & \bullet & \bullet & \bullet & \bullet & \bullet \\
\bullet & \bullet & \bullet & \bullet & \bullet & \bullet & \bullet & \bullet & \bullet\end{array}$ & \begin{tabular}{ll|}
$x$ & $x$ \\
$\times$ & $\times$ \\
$\times$ & $\bullet$ \\
$\bullet$ & $\bullet$ \\
$\bullet$ & $\bullet$ \\
$\bullet$ & $\bullet$ \\
$\bullet$ & $\bullet$
\end{tabular} & 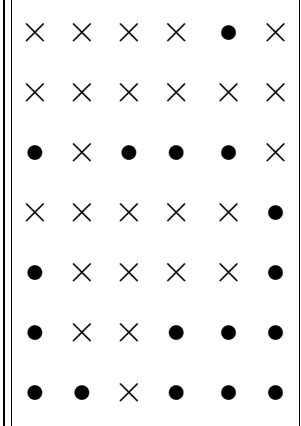 & $\begin{array}{ll}\bullet & \times \\
\bullet & x \\
\bullet & \bullet \\
x & \times \\
\times & \times \\
\times & \bullet \\
x & \bullet\end{array}$ \\
\hline$\underbrace{0}_{0}$ & $c^{2}$ & $U^{-1} \mho^{N} \mho^{\infty} U^{+1} U^{\infty} U^{\infty} U^{r} U^{\infty} U^{\infty}$ & 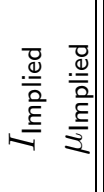 & 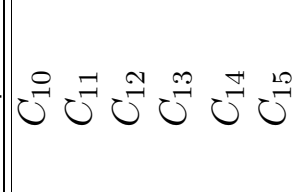 & 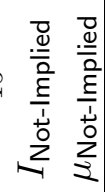 \\
\hline
\end{tabular}

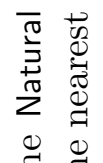

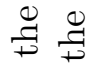

.$\exists \circ$

:

焉

क

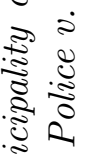

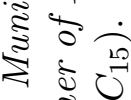

4 ป

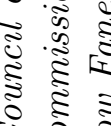

ठิ

इ. . క

.

న ธ․ำ

of

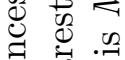

苟莺

웅 -

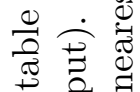
焉索

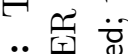

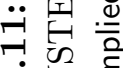
की 용 赵青 
This conclusion is at odds with the decision of the High Court. However, as discussed in $\S 5.5 .1$ above, the significance of the existence of a statutory right to appeal has changed over the years since Twist $v$. Randwick. The author respectfully submits (and the legal expert agrees) that Twist $v$. Randwick was wrongly decided, at least insofar as it concerns the significance of a statutory right to appeal. The case was not included in the NATURAL specification for this reason.

Apart from Commissioner of Police v. Tanos, all of SHYSTER's chosen cases were decided after Twist $v$. Randwick.

In Salemi v. MacKellar, ${ }^{65}$ Salemi was an Italian citizen who entered Australia under a temporary entry permit. He stayed in the country after the permit expired, and became a "prohibited immigrant" within the meaning of the Migration Act 1958 (Cth). The Minister of Immigration announced, in a press release, an amnesty under which prohibited immigrants who met certain conditions would be granted resident status if they came forward before a certain date. Salemi appeared to meet those conditions, but the Minister refused his application for the benefit of the amnesty and proposed to deport him under s. 18 of the Act.

Salemi cited Schmidt v. Secretary of State for Home Affairs ${ }^{66}$ and claimed that the news release had given him a "legitimate expectation" that he would be entitled to remain in Australia. Hence the Minister should have given him an opportunity to be heard before deciding to issue the deportation order.

A majority of the High Court held that Salemi had no "legitimate expectation," 67 and half of the Court held that the legislative scheme indicated that Parliament did not intend that people affected by deportation orders issued under s. 18 should have a right to be heard. ${ }^{68}$

SHYSTER's fact vectors are (YUNYYN), (UYNY) and (YYYYYNN).

In the Expectation area, SHYSTER concludes that the applicant did have a legitimate expectation which was affected by the decision. The nearest neighbour is Attorney-General of Hong Kong v. Ng Yuen Shiu; the nearest other is South Australia v. O'Shea. SHYSTER warns that the weighted correlation coefficients suggest that $S A v$. O'Shea should be nearest neighbour.

In the Affected area, SHYSTER concludes that the decision affected the property, right, interest, status, or legitimate expectation of the applicant. Although not discussed in the case (apart from the issue of legitimate expectation) this is clearly true. The nearest neighbours are Haoucher v. Minister of State for Immigration and Ethnic Affairs and Kioa v. West; the nearest other is Minister for Arts Heritage and Environment v. Peko-Wallsend Ltd. 
In the Natural area, SHYSTER disagrees with the High Court and concludes that a duty to observe natural justice is implied. The nearest neighbour is Kioa $v$. West; the nearest other is SA v. O'Shea. However, according to the legal expert, it is now generally believed that Salemi v. MacKellar was wrongly decided. Salemi did have a legitimate expectation, and natural justice should have been implied. The case was not included in the NATURAL specification for this reason.

All of SHYSTER's chosen cases were decided after Salemi v. MacKellar.

\section{Heatley v. Tasmanian Racing and Gaming Commission}

Heatley v. Tasmanian Racing and Gaming Commission ${ }^{69}$ is one of the leading cases in the Expectation area, and its facts are summarized in the dump file for the NATURAL specification in $\S$ A.5. It does not appear in either of the Affected or Natural areas, so it is used here to test those two areas.

SHYSTER's fact vectors are (UNYY) and (YYYUNNN).

In the Affected area, SHYSTER agrees with the High Court that the decision affected the property, right, interest, status, or legitimate expectation of the applicant. The nearest neighbour is FAI Insurances Ltd v. Winneke; the nearest other is Minister for Arts Heritage and Environment v. Peko-Wallsend Ltd.

In the Natural area, SHYSTER concludes, as did the High Court, that a duty to observe natural justice is implied. The nearest neighbour is Annetts v. McCann; the nearest other is McInnes $v$. Onslow Fane.

However, SHYSTER warns that these two cases are equidistant from Heatley $v$. TRC; it chooses Annetts v. McCann because it is a decision of five judges of the High Court whereas McInnes v. Onslow Fane is merely a decision of the Chancery Division of the English High Court. It also warns that both the weighted association and weighted correlation coefficients prefer McInnes v. Onslow Fane as nearest neighbour.

All of SHYSTER's chosen cases were decided after Heatley $v$. TRC, however the legal expert points out that the cases it has chosen in the Affected area both concerned licence interests; SHYSTER's choice would have been better if the chosen cases had been about a mere interest.

\section{Cole v. Cunningham}

Like Heatley v. Tasmanian Racing and Gaming Commission, Cole v. Cunning$\mathrm{ham}^{70}$ is one of the leading cases in the Expectation area, and its facts are summarized in the dump file for the NATURAL specification in $\S$ A.5. It does not appear in either of the Affected or Natural areas, so it is used here to test those two areas.

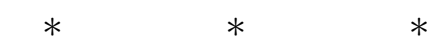


SHYSTER's fact vectors are (YNNY) and (YYNYNNN).

In the Affected area, SHYSTER concludes that the decision affected the property, right, interest, status, or legitimate expectation of the applicant - as did the Full Court of the Federal Court. The nearest neighbour is Council of Civil Service Unions v. Minister for the Civil Service; the nearest other is Minister for Environment v. Peko-Wallsend.

SHYSTER agrees with the Court that a duty to observe natural justice is implied. The nearest neighbour is Annetts v. McCann; the nearest other is McInnes v. Onslow Fane to which no reference was made.

As with Heatley $v$. TRC, SHYSTER warns that these two cases are equidistant from Cole v. Cunningham and chooses Annetts v. McCann over McInnes v. Onslow Fane because it was decided in a higher court. It also warns that both the weighted coefficients prefer McInnes v. Onslow Fane.

Apart from McInnes $v$. Onslow Fane, all of SHYSTER's chosen cases were decided after Cole v. Cunningham. The legal expert approves of SHYSTER's choice of nearest neighbour in the Affected area, but not its choice of nearest other: Minister for Environment v. Peko-Wallsend concerned a property interest, where Cole v. Cunningham concerned a promissory interest.

\section{Ackroyd v. Whitehouse (Director of National Parks $\&$ Wildlife Service)}

In Ackroyd v. Whitehouse (Director of National Parks 8 Wildlife Service), ${ }^{71}$ Ackroyd was a bird trapper. His licence was cancelled without notice, hearing or reasons, by the Director under s. 134(1) of the National Parks and Wildlife Act 1974 (Cth). Section 135 of the Act granted a person whose licence had been cancelled the right to appeal to the Minister. Ackroyd claimed that the Minister ought to have applied natural justice principles before making his decision. The Director cited Twist $v$. Council of Municipality of Randwick claiming that the right of appeal to the Minister meant that natural justice ought not to be implied. The New South Wales Court of Appeal disagreed, and found for Ackroyd.

Kirby P held that the fact that Ackroyd had had his licence renewed annually for several years "might reasonably give rise to a legitimate expectation" that the licence would continue in operation unless some misconduct was proved. ${ }^{72}$

SHYSTER's fact vectors are (NUYNYN), (YNNY) and (YYYYNYN).

In the Expectation area, SHYSTER agrees with Kirby $\mathrm{P}$ that Ackroyd had a legitimate expectation which was affected by the decision. The nearest neighbour is FAI Insurances Ltd v. Winneke; the nearest other is South Australia v. O'Shea, which was decided after Ackroyd v. Whitehouse. 
In the Affected area, SHYSTER concludes (as did the Court) that the decision affected the property, right, interest, status, or legitimate expectation of the applicant. The nearest neighbour is Council of Civil Service Unions v. Minister for the Civil Service; the nearest other is Minister for Arts Heritage and Environment $v$. Peko-Wallsend Ltd, which was decided afterwards.

Finally, in the Natural area, SHYSTER agrees with the Supreme Court that a duty to observe natural justice is implied. The nearest neighbour is Commissioner of Police v. Tanos; the nearest other is McInnes v. Onslow Fane.

Of those of SHYSTER's chosen cases which were decided before Ackroyd $v$. Whitehouse, only McInnes v. Onslow Fane was cited in argument-none was cited in the Court's judgment. However, the legal expert approves of SHYSTER's choice of cases in the Expectation area. SHYSTER issues no warnings.

\section{Hodgens v. Gunn}

In Hodgens v. Gunn, ${ }^{73}$ Hodgens had been convicted of ill-treating dogs. The Magistrates Court had made an order under s. 19(2) of the Animals Protection Act 1925 (Qld) permanently prohibiting him from having a dog. He continued to breed dogs, which were seized by the police. The Minister made an order under s. 11(4) of the Act forfeiting the dogs to the Crown.

Hodgens appealed to the Full Court of the Supreme Court of Queensland, claiming that the principles of natural justice required that he should have been given a hearing before the Minister made his order. The Court agreed: since the Minister's order involved the expropriation of property without compensation, and the Minister had absolute discretion in the exercise of this power, natural justice was implied. (However, Hodgens was denied the remedy he sought because he had waited six months before seeking a Court order.)

SHYSTER's fact vectors are (NNNNNN), (YNNN) and (YYNYYNY).

In the Expectation area, SHYSTER concludes that Hodgens did not have a legitimate expectation which was affected by the decision. This is appropriate; legitimate expectation was not an issue in Hodgens $v$. Gunn. The nearest neighbour is Minister for Arts Heritage and Environment v. Peko-Wallsend Ltd; the nearest other is Cole v. Cunningham.

In the Affected area, SHYSTER agrees with the Court that the decision affected Hodgens's property, right, interest, status, or legitimate expectation. The nearest neighbour is Bread Manufacturers of New South Wales v. Evans; the nearest other is Minister for Environment v. Peko-Wallsend.

However, in the Natural area SHYSTER concludes (unlike the Supreme Court) that a duty to observe natural justice is not implied. The nearest neighbour is Council of Civil Service Unions v. Minister for the Civil Service; the nearest others are FAI Insurances Ltd $v$. Winneke and Haoucher v. Minister of State for Immigration and Ethnic Affairs. 
Interestingly, all of SHYSTER's unweighted measures suggest that FAI $v$. Winneke and Haoucher $v$. Minister for Immigration should be the nearest neighbours. Furthermore the ideal points, centroids, and specified and ideal point directions all suggest that the result should be Implied, but only the specified directions give rise to a warning (see $\S 3.12 .4$ ).

Of SHYSTER's chosen cases, only FAI $v$. Winneke was judicially cited. ${ }^{74}$ The legal expert agrees with SHYSTER's choice of cases in the Affected area, and with its choice of nearest neighbour in the Expectation area. However, she disagrees with its choice of Cole v. Cunningham as nearest other in the Expectation area because that case did not concern property rights.

\section{Western Australia v. Bropho}

In Western Australia v. Bropho, ${ }^{75}$ land on the Swan River was converted into a Crown reserve and a proposal put forward to renovate an old brewery on the site. However, the Aboriginal Heritage Act 1972 (WA) required that a Committee be asked to recommend to the Minister as to whether there was any Aboriginal site on the land. The Committee invited public submissions, and recommended that the site was a significant Aboriginal site and that the development should not be allowed. Nevertheless, the Minister gave her consent to the development. Bropho was a custodian of the site, in accordance with the customs of his people. He claimed that the Minister was obliged to give him a hearing before making her decision.

The Full Court of the Supreme Court of Western Australia held that the fact that Bropho had not made a submission to the Committee, before it made its recommendation to the Minister, meant that he had no legitimate expectation of a hearing before the Minister. The Minister was obliged to follow natural justice principles in coming to her decision, but the opportunity to be heard before the Committee meant that this obligation had been met.

Malcolm CJ stated that Bropho had a "special interest in the subject matter of the action" and was "more particularly affected by the proposed development than ordinary members of the public". ${ }^{76}$ But Franklyn and Anderson JJ were not so sure. They said that Bropho may only have had a "spiritual concern rather than a special interest" in the matter. ${ }^{77}$

SHYSTER's fact vectors are (NNNNNN), (NNNN) and (NYYNYYN).

In the Expectation area, SHYSTER agrees with the Supreme Court that Bropho did not have a legitimate expectation which was affected by the decision. The nearest neighbour is Minister for Arts Heritage and Environment v. PekoWallsend Ltd; the nearest other is Cole v. Cunningham.

In the Affected area, SHYSTER concludes that the decision did not affect Bropho's property, right, interest, status, or legitimate expectation. The nearest neighbour is Minister for Environment v. Peko-Wallsend; the nearest other is 
Bread Manufacturers of New South Wales v. Evans. This conclusion is in line with the reservations expressed by Franklyn and Anderson JJ, although their honours appear to have continued on the assumption that Bropho did have an interest which was affected.

In the Natural area, SHYSTER concludes, as did the Court, that a duty to observe natural justice is implied. The nearest neighbour is Marine Hull \& Liability Insurance Co. Ltd v. Hurford; the nearest other is Minister for Environment $v$. Peko-Wallsend.

Of course, if a decision does not affect the interests of an applicant (as in this case) then natural justice should never be implied. This fact is captured, in a limited sense, by the specified direction towards Not-Implied of a value of NO for $A_{1}$ - and SHYSTER warns that the specified directions suggest Not-Implied in WA v. Bropho. ${ }^{78}$

None of SHYSTER's chosen cases was cited in the published judgment.

\section{Ainsworth v. Criminal Justice Commission}

In Ainsworth v. Criminal Justice Commission, ${ }^{79}$ the Commission's advice had been sought on the implementation of the introduction of poker machines into Queensland. Its report dealt with matters of general concern and with particular poker machine suppliers and manufacturers (including Ainsworth's companies). It was very critical of their conduct and recommended that "the Ainsworth group of companies not be permitted to participate in the gaming machine industry in Queensland."

The Commission had formed its opinion on the basis of reports of other bodies. Ainsworth had not been informed of the Commission's interest in his group of companies, and was not given any opportunity to make representations on the matter before the Commission made its report. Ainsworth claimed that the business reputation of the companies was an interest which was adversely affected by the Commission's report.

The Full Court of the High Court unanimously agreed. It followed Annetts v. McCann and held that the Commission ought to have applied natural justice principles. $^{80}$

The report files for Ainsworth $v$. CJC (one for each area) are given as examples in $§$ B.5. SHYSTER's fact vectors are (NNNNNN), (NNYN) and (YYYYNNN).

In the Expectation area, SHYSTER concludes that the applicant did not have a legitimate expectation which was affected by the decision. The nearest neighbour is Minister for Arts Heritage and Environment v. Peko-Wallsend Ltd; the nearest other is Cole v. Cunningham. This is appropriate; legitimate expectation was not an issue in Ainsworth v. CJC. 
In the Affected area, SHYSTER concludes, as did the High Court, that the decision affected the property, right, interest, status, or legitimate expectation of the applicant. The nearest neighbour is Annetts v. McCann: the same case as the High Court followed; the nearest other is Minister for Environment $v$. Peko-Wallsend.

In the Natural area, SHYSTER agrees with the Court that a duty to observe natural justice is implied. The nearest neighbour is also Annetts v. McCann; the nearest other is McInnes $v$. Onslow Fane.

However, SHYSTER warns that these two cases are equidistant from Ainsworth v. CJC; it chooses Annetts v. McCann because it is a decision of five judges of the High Court whereas McInnes v. Onslow Fane is merely a decision of the Chancery Division of the English High Court. It also warns that both the weighted association and weighted correlation coefficients prefer McInnes v. Onslow Fane as nearest neighbour.

\subsubsection{Generated tests}

The legal expert provided two examples for generating tests using the Natural area of the NATURAL specification: generated tests 7 and 8 . The results of these tests are summarized in figure 5.13.

\section{Generated test 7}

The legal expert advises that, if the decision did not affect the property, right, interest, status, or legitimate expectation of the applicant, then natural justice will not be implied. The fact vector is (NUUUUUU) ${ }^{81}$ SHYSTER generates 64 instantiations, half the search space, and in 56 of them the choice of result is good (i.e. Not-Implied).

In one of the 8 instantiations in which the choice of result is bad, SHYSTER warns that an ideal point suggesting the good result is at least as near to the instant case as is the nearest neighbour. In all 8 , the decision is apt to have a discrete impact on the interests of the applicant. This does not justify SHYSTER's choice of result in those instantiations.

\section{Generated test 8}

The legal expert also advises that, if the decision is not apt to have a discrete impact on the interests of the applicant, natural justice will not be implied. The required fact vector is (UNUUUUU). But this cannot be given to SHYSTER directly because the value of $A_{1}$ cannot be UNKNOwn: it is an external attribute defined by reference to the Affected area. So, this test is divided into two. In the first test ("8a" in figure 5.13), the value of $A_{1}$ is YEs; in the second ("8b") it is NO: i.e. the fact vectors are (YNUUUUU) and (NNUUUUU). ${ }^{82}$ Each of these two tests generates half of the required search space. 
In 55 of the total of 64 instantiations generated for test 8 , the choice of result is good (i.e. Not-Implied). In one of the 9 instantiations in which the choice of result is bad, SHYSTER issues an ideal point warning. In each of these 9 instantiations, the decision affected the property, right, interest, status, or legitimate expectation of the applicant. This does not justify SHYSTER's choice of result in those instantiations.

\subsubsection{Conclusion}

The tests described in $§ 5.5 .3$ indicate that, using the NATURAL specification, SHYSTER is exceptionally good at coming to the right conclusion $(95.46 \%$ good results), though not very good at choosing good cases to use in argument (40\%).

As shown in figure 5.13, SHYSTER's choice of result is good in $86.72 \%$ of the instantiations in both generated tests. If a bad choice of result is considered good where an ideal point warning is issued, SHYSTER's success rate rises only slightly to $89.06 \%$ and $85.94 \%$ for each test: $87.50 \%$ overall. (In none of the instantiations in these tests does SHYSTER issue a warning about a nearer ideal point when the choice of results is good; i.e. none of SHYSTER's ideal point warnings is bad.)

As discussed in $\S 5.5 .2$, three pairs of cases in the Natural area have identical facts yet different results. Because all of the leading cases in the Natural area were correctly decided, this inconsistency indicates a need for more attributes to distinguish between cases in the area. Extra attributes were not added, because the seven attributes were based on the seven factors identified by McMillan as being important. ${ }^{83}$

The Natural area is a good example of an area with attributes which require further definition by reference to other areas. The NATURAL specification represents - and represents effectively - an area of law that is quite fluid and expanding rapidly: as discussed in $\$ 5.5 .3$ above, both Twist v. Council of Municipality of Randwick and Salemi v. MacKellar would be decided differently today - seventeen years after they were heard by the High Court.

SHYSTER (when using the NATURAL specification) produces good advice in the test cases, and chooses good results in the vast majority of the generated tests. However, the reflexive tests performed in $\S$ D.5, and summarized in figure D.4, indicate that the NATURAL specification is not as capable of handling new fact situations as are the other three specifications.

\subsection{Conclusion}

The results of all the testing described in this chapter - test cases, generated tests and reflexive tests - are discussed below. General conclusions are drawn from these results in the next chapter. 


\subsubsection{Test cases}

The results of the testing using test cases are summarized in figure 5.12. Several special symbols are used in that figure. A tick is used to indicate a "good" result or choice of cases; a cross indicates a "bad" result or choice of cases. (These terms are defined in §3.13.2.) A dash indicates that a chosen case was not decided before the test case and that it is not possible to evaluate that choice. A blank space indicates that no symbol is applicable.

The "Warnings" column summarizes the safeguard warnings that were issued during each test. A warning that one of the weighted similarity measures suggested a different result is indicated by the appropriate symbol: $S^{\prime}$ for the weighted association coefficients; $r^{\prime}$ for the correlation coefficients. An $I$ or a $\mu$ indicates that an ideal point or a centroid suggesting a different result was at least as near to the instant case as was the nearest neighbour. ${ }^{84} \mathrm{~A} \Rightarrow$ indicates that the specified directions suggested a different result. An $\equiv$ indicates that the test produced equidistant results.

A $\forall$ in the warnings column indicates that one or more unknown values was instantiated. The fraction after each $\forall$ is the number of instantiations that produced a different result to that of the nearest neighbour, over the total number of instantiations; SHYSTER only issues a warning if an instantiation has a different result. At the right of the warnings column is a tick or a cross which indicates whether the warning (or absence thereof) was "good" or "bad" — as defined in $§ 3.13 .2$.

These symbols are also used in the figures in appendix D which summarize the reflexive testing described in that appendix.

As figure 5.12 illustrates, SHYSTER has proven itself exceptionally able to choose good results: a 95.24\% success rate (including ideal point tests). However, its ability to choose good cases with which to argue varies dramatically between the four different specifications.

For the FinDER specification, SHYSTER's choice of cases is excellent, but there is only one test case for that specification. For the Authorization specification, $75 \%$ of its chosen cases are good. However, only $41.67 \%$ and $40 \%$ of the cases chosen from the EMPlOyeE and NATURAL specifications were good.

One reason for this discrepancy may be the greater number of leading cases in the Employee and Natural specifications $\left(15 \mathrm{each}^{85}\right)$ than in the Finder and Authorization specifications ( 8 and 9 , respectively). Judges and lawyers will often refer only to a handful of cases; only rarely does a judge discuss exhaustively all of the important cases in a field. ${ }^{86}$ Given an area of law in which there were comparatively few leading cases, SHYSTER would be more likely to chose a good case (all other things being equal).

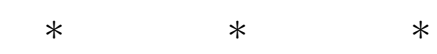




\begin{tabular}{|c|c|c|c|c|c|}
\hline Case & Result & $\begin{array}{l}\text { Nearest } \\
n^{\prime} \text { bours }\end{array}$ & $\begin{array}{c}\text { Nearest } \\
\text { others }\end{array}$ & \multicolumn{2}{|c|}{ Warnings } \\
\hline Parker v. British Airways & $\checkmark$ & $\checkmark$ & $\checkmark$ & & $\checkmark$ \\
\hline$C B S$ v. Ames & $\checkmark$ & $\checkmark$ & $\checkmark$ & $\Rightarrow$ & $x$ \\
\hline WEA v. Hanimex & $\checkmark$ & $\checkmark$ & $x$ & $\Rightarrow$ & $x$ \\
\hline$C B S$ v. Amstrad & $\checkmark$ & $\checkmark$ & $\checkmark$ & $\Rightarrow$ & $x$ \\
\hline APRA v. Jain & $\checkmark$ & $x$ & $x$ & & $\checkmark$ \\
\hline Hypothetical case 1 & $\checkmark$ & $\checkmark$ & $\checkmark$ & $\Rightarrow$ & $x$ \\
\hline Hypothetical case 2 & $\checkmark$ & $\checkmark$ & $\checkmark$ & $r^{\prime} \Rightarrow \equiv$ & $x$ \\
\hline$I_{\text {Auth }}$ & $\checkmark$ & & & $\Rightarrow$ & $x$ \\
\hline$I_{\text {Not-Auth }}$ & $\checkmark$ & & & & $\checkmark$ \\
\hline$I_{\text {Liable }}$ & $\checkmark$ & & & $r^{\prime} \forall \frac{0}{4}$ & $x$ \\
\hline Narich v. CPT & $\checkmark$ & $x$ & $x$ & $\Rightarrow$ & $x$ \\
\hline Stevens v. Brodribb & $\checkmark$ & $\checkmark$ & $x$ & $\forall \frac{0}{2}$ & $\checkmark$ \\
\hline Re Porter; Re TWU & $\checkmark$ & $x$ & $\checkmark$ & $\forall \frac{1}{4}$ & $\checkmark$ \\
\hline$B W I U v . O d c o$ & $\checkmark$ & $\checkmark$ & $\checkmark$ & $\forall \frac{0}{4}$ & $\checkmark$ \\
\hline Hypothetical case 3 & $\checkmark$ & $x$ & $x$ & $r^{\prime} \forall \frac{0}{2}$ & $x$ \\
\hline Hypothetical case 4 & $x$ & $x$ & $\checkmark$ & & $x$ \\
\hline$I_{\text {Employee }}$ & $\checkmark$ & & & $\forall \frac{0}{2}$ & $\checkmark$ \\
\hline$I_{\text {Contractor }}$ & $\checkmark$ & & & $r^{\prime} \forall \frac{0}{4}$ & $x$ \\
\hline Twist v. Randwick & $\checkmark \checkmark \checkmark$ & $--\checkmark$ & --- & $\Rightarrow$ & $x^{*}$ \\
\hline Salemi v. MacKellar & $\checkmark \checkmark \checkmark$ & --- & --- & $r^{\prime} \forall \frac{0}{2}$ & $x^{*}$ \\
\hline Heatley v. TRC & $\checkmark \checkmark$ & $\times-$ & $\times-$ & $S^{\prime} r^{\prime} \equiv \forall \frac{0}{2}$ & $x$ \\
\hline Cole v. Cunningham & $\checkmark \checkmark$ & $\checkmark-$ & $\times x$ & $S^{\prime} r^{\prime} \equiv$ & $x$ \\
\hline Ackroyd v. Whitehouse & $\checkmark \checkmark \checkmark$ & $\checkmark \times \times$ & $\checkmark-\checkmark$ & $\forall \frac{0}{2}$ & $\checkmark$ \\
\hline Hodgens v. Gunn & $\checkmark \checkmark \times$ & $\checkmark \times$ & $\checkmark \checkmark$ & $\Rightarrow$ & $\checkmark$ \\
\hline WA v. Bropho & $\checkmark \checkmark \checkmark$ & $\times \times \times$ & $\times \times \times$ & $\Rightarrow$ & $x$ \\
\hline Ainsworth v. CJC & $\checkmark \checkmark \checkmark$ & $\checkmark \checkmark$ & $\times \times$ & $S^{\prime} r^{\prime} \equiv$ & $x$ \\
\hline$I_{\text {Implied }}$ & $\checkmark$ & & & $S^{\prime} r^{\prime}$ & $x$ \\
\hline$I_{\text {Not-Implied }}$ & $\checkmark$ & & & & $\checkmark$ \\
\hline
\end{tabular}

Figure 5.12: A summary of the testing using test cases performed in this chapter. These tests are described in $\S 5.2 .3, \S 5.3 .3, \S 5.4 .3$ and $\S 5.5 .3$. The meaning of the symbols used here is explained in $\S 5.6 .1$.

Symbols for tests in the NATURAL specification refer to the Expectation, Affected and Natural areas, respectively. Safeguard warnings in the NATURAL specification are from the Natural area - except those marked with an asterisk, which are from the Expectation area. 
The appropriateness of SHYSTER's warnings also varies between specifications. In the single test case for the Finder specification, the (absence of a) warning is good. But warnings in the Authorization, Employee and Natural specifications are generally bad: only $22.22 \%, 50 \%$ and $30 \%$, respectively, good warnings.

For the Authorization specification, all but one of the seven bad warnings are due to specified directions. Across all four specifications, the rate of good warnings would rise from $35.71 \%$ to $60.71 \%$ if the specified direction warnings were ignored. Yet specified directions represent the expert's opinion as to which values for attributes suggest certain results; warnings based upon the specified directions should be sound.

It is possible that the preponderance of bad specified direction warnings in tests of the Authorization specification may be due to the fact that the Authorization area has more than two results. However, that is not the case in the other three specifications.

In each of the nine tests in which a warning was issued due to an extra similarity measure (i.e. $S^{\prime}$ or $r^{\prime}$ ) the warning was bad. Without those warnings due exclusively to either or both extra similarity measures, $53.57 \%$ of the warnings are good. That these warnings are so bad indicates that the choice of similarity measure made in $§ 3.9 .3$ was a good one: using known and unknown distance measures (variations on the weighted distance measure $d_{j k}^{\prime}$ ) SHYSTER produces better results than it would using either of the other two weighted measures.

In only one of the twenty-one tests did an instantiation of the instant case have a different result to that of the uninstantiated instant case: Re Porter; Re Transport Workers Union of Australia, ${ }^{87}$ a case used to test the EMPLOYEe specification in §5.4.3. As explained there, the legal expert agrees with SHYSTER that that instantiation would indeed lead to a different result.

\subsubsection{Generated tests}

The results of the generated testing are summarized in figure 5.13. The " $\checkmark$ Results" column gives the number of generated tests in which SHYSTER's choice of result is good, as a fraction of the total number of generated tests. That fraction is also expressed as a percentage. For tests in which SHYSTER's choice of result is bad (i.e. " $\times$ Results") the number of tests in which ideal point warnings were issued is given in the "Wrn" column. The "IP" column gives the number of tests in which the nearest ideal point was that of a different result, but in which no warning was issued because that ideal point was not nearer the instant case than was the nearest neighbour. Corresponding figures for those tests in which SHYSTER's choice of result is good are given in the next column. 


\begin{tabular}{|c|c|c|c|c|c|c|}
\hline No. & \multirow{2}{*}{$\frac{\checkmark \text { Results }}{14 / 16}$} & \multirow{2}{*}{$\begin{array}{c}\% \\
87.50\end{array}$} & $\begin{array}{l}\times \text { Results } \\
I P \text { Wrn }\end{array}$ & $\begin{array}{r}\checkmark \text { Results } \\
I P \text { Wrn }\end{array}$ & \multirow{2}{*}{$\begin{array}{c}\checkmark \text { Results } \\
\text { and warnings }\end{array}$} & \multirow{2}{*}{$\frac{\%}{87.50}$} \\
\hline 1 & & & 2 & 0 & & \\
\hline 2 & $12 / 16$ & 75.00 & 0 & 0 & $16 / 16$ & 100.00 \\
\hline \multirow[t]{2}{*}{3} & $51 / 64$ & 79.69 & $\begin{array}{ll}0 & 13\end{array}$ & 0 & $64 / 64$ & 100.00 \\
\hline & $77 / 96$ & 80.21 & $\begin{array}{ll}2 & 17 \\
\end{array}$ & 0 & $94 / 96$ & 97.92 \\
\hline 4 & $363 / 512$ & 70.90 & 4145 & 0 & $508 /$ & 99.22 \\
\hline 5 & $4198 / 6144$ & 68.33 & 861492 & 144111 & $4579 / 6144$ & 74.53 \\
\hline 6 & $875 / 1024$ & 85.45 & $37 \quad 112$ & $\begin{array}{ll}0 & 0\end{array}$ & $987 / 1024$ & 96.39 \\
\hline & $5436 / 7680$ & 70.78 & 902749 & 144111 & $6074 / 7680$ & 79.09 \\
\hline & $56 / 64$ & 87.50 & $\begin{array}{ll}1 & 1\end{array}$ & $\begin{array}{ll}10 & 0\end{array}$ & $57 / 64$ & 89.06 \\
\hline & $55 / 64$ & 85.94 & 1 & 16 & $55 / 64$ & 85.94 \\
\hline & $23 / 32$ & 71.87 & 1 & 12 & $23 / 32$ & 71.87 \\
\hline & $32 / 32$ & 100.00 & 0 & 4 & $32 / 32$ & 100.00 \\
\hline & $111 / 128$ & 86.72 & 2 & 26 & $112 / 128$ & 87.50 \\
\hline
\end{tabular}

Figure 5.13: A summary of the generated testing performed in this chapter. These tests are described in $\S 5.3 .4, \S 5.4 .4$ and $\S 5.5 .4$. This table is explained in §5.6.2. Note that results for generated tests of the EMPLOYEE specification take into account the removal of paradoxical cases from the search space (see $\S 5.4 .4)$.

In the " $\checkmark$ Results and warnings" column, a bad choice is considered good if an ideal point warning is issued. The number of good choices is also expressed as a fraction of the total number of generated tests, and as a percentage. Except in one generated test, SHYSTER never issues an ideal point warning when a good result has been chosen. The bad warnings issued in that test - generated test 5 are accounted for in this column because a good choice of result is considered bad if an ideal point warning is issued.

A total for each column is given after each group of tests. Ignoring warnings, SHYSTER has a success rate of $80.21 \%, 70.78 \%$ and $86.72 \%$, respectively, for each of the three specifications. If warnings are taken into account the success rate rises to $97.92 \%, 79.09 \%$ and $87.50 \%$.

\subsubsection{Reflexive tests}

The results of the reflexive tests are described in $\S \mathrm{D}$ and summarized in figures D.1, D.2, D.3 and D.4. As explained in $§ 3.13 .5$, reflexive tests do not test SHYSTER's approach to case law; they do, however, provide information about a specification. The reflexive tests indicate that the Finder, Authorization and EMPLOYEe specifications are capable of handling new fact situations, but that the Natural specification is less capable. 



\section{6}

\section{Conclusion}

Among the vital processes occurring at [the] intellectually intractable points of precedent growth are the modification, supplementation, or even abandonment of reigning legal precepts by reference to contemporary social ideas and ideals. Because the doctrine of precedent, as many lawyers and social scientists still misunderstand it, does not allow for the operation of these processes, their occurrence is an evercontinuing source of trauma.

Julius Stone (1964)

Man and Machine in the Search For Justice ${ }^{88}$

We do not use to judge of Cases by fractions.

John Finch (1637)

R v. Hampden ${ }^{89}$

Donne, I suppose, was such another Who found no substitute for sense,

To seize and clutch and penetrate;

Expert beyond experience...

T. S. Eliot (1920)

Whispers of Immortality ${ }^{90}$ 


\subsection{Introduction}

SHYSTER was developed to support the main argument of this thesis: that a useful, working legal expert system can be based upon a pragmatic approach to the law. In this chapter, SHYSTER and its approach to case law are evaluated ( $(6.2)$, and avenues of future research are identified ( $(6.3)$. The contribution made by this thesis to the field of legal expert systems development is discussed in $§ 6.4$.

\subsection{Evaluating SHYSTER}

This evaluation of SHYSTER is carried out under five headings: its usefulness, its generality, the quality of its advice, its limitations, and possible enhancements that could be made to it. Although there is some overlap between these five topics - and in the identification of areas of future research in $\S 6.3$ below - it is convenient to evaluate SHYSTER under these headings.

\subsubsection{A useful, working system}

Susskind claims that in 1986 there was "an embarrassing lack of demonstrable expert systems in law". 91 Although several systems have been developed since then, the embarrassment has continued. In 1990, Kowalski and Sergot announced that they had decided not to finish their system because their experience suggested that the final stage of the development process "can involve a considerable amount of work and extra programming effort." 92 That decision made feeble their claim that "there are no outstanding technical obstacles which need to be overcome", ${ }^{93}$ and left Kowalski and Sergot particularly vulnerable to Moles's suggestion that the real reason for the abandonment of the project was a realization of the inadequacy of their approach. ${ }^{94}$

The development of (at least) a working prototype of a system is an important part of any expert system project. In order to demonstrate the efficacy of a pragmatic approach to case law, SHYSTER's case-based system has been fully implemented, as explained in chapter 4. SHYSTER has been tested, as explained in chapter 5. That testing was not as comprehensive as is desirable but was as comprehensive as possible within the restrictions of this thesis project, and compares favourably with the testing of other comparable systems. ${ }^{95}$

Ashley, as quoted in $§ 2.5$, lists the following tasks as being general to all case-based reasoning:

... (1) ordering relevant cases and potentially relevant cases in terms of how analogous they are to the problem situation, (2) selecting the most analogous cases, (3) identifying configurations of counterexamples, (4) hy- 
pothetically modifying the problem situation to explore contingencies, and (5) comparing case-based analyses of different problem situations to explain differences. ${ }^{96}$

SHYSTER performs all five of these tasks, within the restrictions placed upon it by its simple knowledge representation.

SHYSTER's usefulness for the user - a lawyer - is due to the fact that it is based upon a pragmatic model of legal reasoning; it operates at the same pragmatic level of abstraction as do lawyers. SHYSTER's REPORTER module goes to some considerable trouble to ensure that its reports are in a form that might be produced by a lawyer. Details of SHYSTER's distance calculations are not part of its reports.

During the development and testing of SHYSTER, an unexpected aspect of the system's usefulness became apparent. Three of the four specifications described in chapter 5 were developed by the author in consultation with lawyers with expertise in the relevant fields. Two of those legal experts found that the process of specifying an area of law for SHYSTER, and examining and evaluating its reports, changed the way that they looked at that area. They felt that the process had contributed to their knowledge and understanding of the field. For example, the legal expert who was consulted about the EMPLOYEE specification found that the process showed that that area of law was more systematic than she had first thought.

Ashley says that a case-based legal expert system could be useful as part of a legal tutoring system. ${ }^{97}$ The experiences of the legal experts during the development of the specifications suggest that SHYSTER has the potential to assist users to gain similar insights.

\subsubsection{A general approach}

The general applicability of SHYSTER's approach to case law is an open question. SHYSTER has been designed so that it can provide advice in different areas of case law, specified by legal experts. However, as explained in $\S 3.3 .1$, the model of legal reasoning adopted for SHYSTER in $§ 3.3 .3$ reflects the way in which lawyers reason with statutes and cases in areas of private law. SHYSTER has not been designed to deal with areas of public law in which the doctrine of precedent is given less weight.

Nevertheless, the four specifications described and tested in chapter 5 suggest a wide application for SHYSTER's approach to case law. The specifications are quite different from each other: they are of differing sizes (in terms of the number of attributes and the number of cases), and they represent disparate areas of the law.

The Finder specification represents a completely case-based area of law. The AUthorizATION specification represents an area of case law which has developed 
from specific statutory provisions. The EMPLOYEE specification represents an area of case law which has developed alongside several different statutes. In the cases that make up the NATURAL specification, judges were overtly concerned with matters of policy. That specification is more like a public law area than any of the other three specifications. ${ }^{98}$

Mendelson applied dimensional analysis (as performed by HYPO) to the law governing government appeals in US criminal cases. ${ }^{99}$ His goal was to determine how "domain-sensitive" was HYPO's approach to case law. His dimensional analysis was unsuccessful, because, he concludes, the area of law "is unsettled, fact-sensitive and conflicting." ${ }^{1}$ As explained in $\S 5.5$, the NATURAL specification shares all three of these characteristics. This makes SHYSTER's success with that specification (although not as consistent as its success with other specifications) particularly interesting.

Although results vary between the four specifications (as discussed in $\S 5.6 .1$, and in $§ 6.2 .3$ below) they are generally good in all four specifications. This thesis does not demonstrate the generality of SHYSTER's approach to private case law, but its does suggest its broad applicability.

\subsubsection{Good advice}

As discussed in $§ 3.13 .2$, the quality of SHYSTER's advice is evaluated on the basis of its prediction as to the likely result, and its choice of cases for use in argument. Within the limitations imposed by its internal representation of case law, SHYSTER is capable of producing quite sophisticated reports - but these reports can only be as good as the cases it chooses. (What is meant by a "good" result or a "good" choice of cases is defined in $\S 3.13 .2$.)

As is shown in chapter 5, SHYSTER has proved remarkably good at choosing a result: in test cases and in generated tests. It is, however, quite inconsistent (across the four specifications) in the quality of its choice of cases.

It should be remembered, however, that the test cases used in chapter 5 are difficult tests. As explained in $§ 3.13 .3$, the same filters in the legal system which restrict the number of cases that are reported also ensure that those cases that are reported make difficult tests. ${ }^{2}$

Furthermore, it is a little harsh to rule SHYSTER's choice of a case "bad" on the basis that that case was not cited in judgment or argument by a judge or by counsel. Some judges will not look beyond the cases cited in argument by counsel. For all sorts of non-legal reasons (lack of time, incompetence, etc.) counsel may not cite all the good cases in argument. And, of course, opinion as to what is a good case to use in argument differs. However, harsh as it may be, some method of evaluating the quality of SHYSTER's choice of cases had to be adopted. ${ }^{3}$ 


\subsubsection{Limitations}

Although SHYSTER has proved quite successful, it has several limitations.

As well as being restricted (for the most part ${ }^{4}$ ) to areas of private law, there are some areas of private law to which SHYSTER is not suited. These restrictions upon domains - restrictions which apply to all legal expert systems - are examined in $\S 3.13 .1$.

"Reasonableness," for example, is a difficult concept. As discussed in $§ 5.3 .2$, it plays an important part in the Authorization specification, yet it is so nebulous a concept that it would probably be impossible to specify for SHYSTER. Furthermore, the meaning of "reasonableness" may well be very different within different areas of the law: even if it could be specified for one area, that specification might not be appropriate for another area within which the concept is also important.

SHYSTER's solution to this problem in the Authorization specification is to leave the user to answer the question of whether the accused took reasonable steps. $^{5}$ This is not a major limitation: it is quite possible that the user (a lawyer) knows the answer to that question, but not to the larger question of whether the accused authorized the infringement. In any event, the user has the option of answering UNKNOWN to difficult questions, forcing SHYSTER to examine instantiations.

As discussed in $§ 5.6 .1$, if many leading cases are specified then SHYSTER's ability to choose good cases is reduced. This problem can be avoided by choosing a different threshold within which SHYSTER will consider two cases to be equidistant. ${ }^{6}$ If such a change is made, SHYSTER will tend to choose more cases to use in argument. This increases the likelihood of SHYSTER choosing good cases, but also increases the number of bad cases chosen.

The fact that SHYSTER will construct arguments for all possible results means that sometimes it will produce extraneous arguments. For example, as explained in $\S 5.3 .3$, given an authorization case, SHYSTER will argue about the Liable result even if the question of direct or vicarious liability does not arise. In an area where there are several results, this problem would be exacerbated. One solution to this problem would be to restrict SHYSTER to arguing only about the nearest $n$ results (where $n$ is 2 or more): i.e. the result of the nearest neighbour, and the results of the $n-1$ nearest others. This solution reduces the likelihood of SHYSTER building an irrelevant argument, but increases the likelihood of it missing a relevant one. 


\subsubsection{Possible enhancements}

The model of legal reasoning adopted in $\S 3.3 .3$ allows for open-textured concepts to be defined by reference to case law. However, there may be circumstances in which a case-based open-textured concept is defined by reference to statute law.

SHYSTER's model of legal reasoning does not allow for such circumstances, though it could be augmented to allow resolution of open texture using legislation. Implementing such an augmented model should not pose any major difficulties. SHYSTER's case law specification language would have to be extended to allow external attributes to be linked to rules in the rule base in the same fashion that external attributes are linked to areas in the case base at present.

SHYSTER allows the specification of no more than one ideal point for each result in each area. It is possible that there might be more than one best combination of attribute values for a given result. There is no reason why SHYSTER could not be modified to allow multiple ideal points for the same result.

As shown in $\S 5.4 .5$, SHYSTER can produce clumsily worded sentences. The quality of SHYSTER's reports could be improved by changing the way in which it uses attribute strings. The number of attribute strings that are written could be restricted on the basis of their importance (defined by their weights), but sometimes the user may be interested in a less important attribute. Attribute strings could be combined in more sophisticated ways: rather than writing " $x$ is not true; and $y$ is not true,"SHYSTER could write "neither $x$ nor $y$ is true." This would require changes to SHYSTER's case law specification language, as well as to the REPORTER module.

\subsection{Future research}

The most obvious avenue of future research using SHYSTER is the development of a rule-based system, and the linking together of that rule-based system with the existing case-based system to form a hybrid system. As argued in chapter 2, a hybrid approach is desirable where the system seeks to represent statute law and case law. SHYSTER has been designed to facilitate such an articulation. The mechanism by which this could be achieved is explained in $\S 4.3$.

There are also several areas of further research which could be carried out using the existing SHYSTER system. New specifications could be written, and tested in the same manner as are the four specifications described in chapter 5 . As well as writing different specifications, different legal experts could be asked to 
specify the same area of law. It would be interesting to examine the differences (if any) between the specifications and the ways in which those specifications make SHYSTER behave.

Research could also be done into the process of updating specifications to take account of changes in the law. Most changes could be captured by the addition of new cases to, and the removal of out-of-date cases from, the specification-both operations are easily performed using SHYSTER's case law specification language. More significant changes in the law might require the addition of new attributes, or the complete rewriting of a specification.

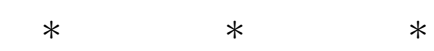

SHYSTER's method of assigning weights to attributes deserves further examination. As explained in $\S 3.7$, SHYSTER adopts FINDER's method of attribute weighting. This method contributes significantly to SHYSTER's distance calculations, and so has an important effect on SHYSTER's opinions. As has been shown, SHYSTER's opinions are generally good which suggests that its method of attribute weighting is also a good one. Yet, as demonstrated in chapter 5, SHYSTER's weighting of attributes is not always appropriate. ${ }^{7}$

Some experimentation by the author indicates that weighting attributes according to the inverse of the variance of each attribute across the leading cases (as SHYSTER does) produces better results than weighting according to the variance (the standard approach adopted in statistical classification problems ${ }^{8}$ ) or weighting each attribute equally.

Further testing could be performed. SHYSTER's ADJUSTER provides an ideal tool for experimenting with the effect of different weighting schemes.

The choice of SHYSTER's similarity measure, made in $\S 3.9 .3$, is vindicated by the number of bad warnings generated using the other similarity measures (see §5.6.1). Nevertheless, further research could also focus on the refinement of SHYSTER's similarity measure: its known and unknown distance. SHYSTER makes only limited use of the hierarchy of courts that can be part of any specification. ${ }^{9}$ It would be possible to take account of the rank of a leading case when quantifying the distance between it and the instant case. Another possibility would be to determine the court in which the instant case is likely to be heard, and to apply rigorously the doctrine of precedent as it relates to a court being bound by decisions of courts higher in its hierarchy. ${ }^{10}$

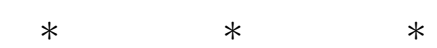

As demonstrated in several of the examples in chapter 5, there are occasions when specifying an area of law seems to call for a more disjunctive structure than SHYSTER permits: i.e. occasionally there is a need for a conditional attribute. 
For example, $A_{5}$ in the Authorization area of the Authorization specification concerns whether reasonable steps were taken to avoid an infringement of copyright. If the accused does take reasonable steps to avoid an infringement then she/he will not be said to have authorized the infringement, ${ }^{11}$ although she/he may still be directly or vicariously liable. Other examples can be found in the generated tests discussed in chapter 5: each of these relies on some attribute value or combination of attribute values implying a given result or results.

The idea of conditional attributes can be captured, in a limited way, by attribute direction. ${ }^{12}$ But the idea of allowing a given result to be conditional upon certain attribute values, in a strict sense, could be investigated. This would represent a major change to SHYSTER's operation, and raises questions of attribute dependence.

\subsection{The contribution of this thesis}

This thesis has argued that a legal expert system need not be based upon a complex or deep model of legal reasoning in order to be successful. It has recommended a pragmatic approach to legal expert system design.

SHYSTER is a working example - just one possible example - of such an expert system. Despite its simple knowledge representation structure, it has shown itself capable of producing good advice. And its simple structure has facilitated the specification of several different areas of law.

This thesis expands upon the work of previous researchers in this field. It has adopted the approach taken by the developers of FINDER, and expanded it so that it incorporates some of the capabilities of more sophisticated systems (e.g. HYPO) without greatly increasing the complexity of the underlying model.

SHYSTER incorporates a simple model of the way in which lawyers argue with cases. However, no attempt has been made to model the way in which lawyers decide which cases to use in those arguments. It is not claimed that lawyers choose the cases that they use in argument by reference to distance calculations in $n$-dimensional space. SHYSTER's is not a deep model of legal reasoning. As Ashley points out:

Even an only partially successful computational theory of jurisprudence may still prove useful. For example LEXIS and WESTLAW [legal retrieval systems ${ }^{13}$ ] represent a theory about legal reasoning: that relevance can be assessed by the appearance of keywords in past cases. No one would accept that theory as adequate, and yet it yields a practical tool. ${ }^{14}$

SHYSTER, too, is a practical tool. It demonstrates that a pragmatic approach to legal expert system development can be a successful one. 


\section{Appendices}





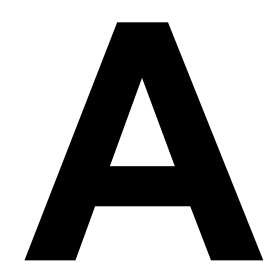

\section{Case law specifications}

The Law is the true embodiment

Of everything that's excellent.

It has no kind of fault or flaw,

And I, my Lords, embody the Law.

William Gilbert (1882)

Iolanthe; or The Peer and the Peri ${ }^{15}$

It is one of the maxims of the civil law, that definitions are hazardous. 


\section{A.1 Introduction}

The complete dump files for the four specifications used to test SHYSTER in chapter 5 are given in this appendix.

The Finder specification (described in $\S 5.2 .2$ ) is in $\S$ A.2. The Authorization specification ( $\S 5.3 .2)$ is in $\S$ A.3. The EMPLOYEE specification (§5.4.2) is in $\S$ A.4. (The complete specification file for the EMPLOYEe specification, written in SHYSTER's case law specification language and input by SHYSTER, is in $\S$ C.3.) The NATURAL specification ( $\S 5.5 .2)$ is in $\S$ A.5: external attributes in the Natural area are defined by reference to the Affected and Expectation areas.

Each dump file is shown exactly as output by $\mathrm{LAT}_{\mathrm{E}} \mathrm{X}$, except that the attribute matrix in the EMPLOYEE specification has been reduced in size so as to fit within the margins of this thesis.

\section{A.2 The Finder specification}

\section{Hierarchy}

\begin{tabular}{|c|l|}
\hline$c$ & \multicolumn{1}{|c|}{ Court } \\
\hline \hline 1 & the Chancery Division of the English High Court \\
& the King's Bench Division of the English High Court \\
& the Queen's Bench Division of the English High Court \\
\hline
\end{tabular}

\section{Finder area}

\begin{tabular}{|c|cccccccccc|c|c|}
\hline \multirow{2}{*}{ Case } & \multicolumn{1}{|c|}{ Attributes } & & \multirow{1}{|c|}{ Result } \\
& $A_{1}$ & $A_{2}$ & $A_{3}$ & $A_{4}$ & $A_{5}$ & $A_{6}$ & $A_{7}$ & $A_{8}$ & $A_{9}$ & $A_{10}$ & $c$ & R \\
\hline \hline$C_{1}$ & $\times$ & $\times$ & $\bullet$ & $\times$ & $\times$ & $\times$ & $\times$ & $\bullet$ & $\bullet$ & $\times$ & 1 & \\
$C_{2}$ & $\times$ & $\times$ & $\bullet$ & $\times$ & $\bullet$ & $\times$ & $\times$ & $\times$ & $\bullet$ & $\times$ & 1 & Win \\
$C_{3}$ & $\times$ & $\times$ & $\times$ & $\times$ & $\bullet$ & $\times$ & $\times$ & $\times$ & $\times$ & $\bullet$ & 1 & \\
\hline \hline$C_{4}$ & $\bullet$ & $\times$ & $\times$ & $\bullet$ & $\times$ & $\times$ & $\times$ & $\bullet$ & $\bullet$ & $\bullet$ & 1 & \\
$C_{5}$ & $\times$ & $\bullet$ & $\times$ & $\times$ & $\times$ & $\times$ & $\bullet$ & $\bullet$ & $\bullet$ & $\times$ & 1 & \\
$C_{6}$ & $\bullet$ & $\bullet$ & $\bullet$ & $\times$ & $\bullet$ & $\bullet$ & $\times$ & $\bullet$ & $\bullet$ & $\times$ & 1 & Lose \\
$C_{7}$ & $\times$ & $\bullet$ & $\bullet$ & $\times$ & $\times$ & $\times$ & $\bullet$ & $\bullet$ & $\bullet$ & $\times$ & 1 & \\
$C_{8}$ & $\bullet$ & $\bullet$ & $\bullet$ & $\times$ & $\times$ & $\bullet$ & $\times$ & $\bullet$ & $\bullet$ & $\times$ & 1 & \\
\hline
\end{tabular}

\section{Results}

Win: the finder wins.

Lose: the finder loses. 


\section{Attributes}

$A_{1}$ : Was the finder the occupier of the premises where the chattel was found?

YES: the finder was the occupier of the premises where the chattel was found.

NO: the finder was not the occupier of the premises where the chattel was found.

UNKNOWN: it is not known whether the finder was the occupier of the premises where the chattel was found.

$A_{2}$ : Was the chattel attached to the land or premises where it was found?

YES: the chattel was attached.

NO: the chattel was not attached.

UNKNOWN: it is not known whether the chattel was attached.

$A_{3}$ : Was the other claimant (the non-finder) the owner of the premises where the chattel was found?

YES: the other claimant was the owner of the premises where the chattel was found.

NO: the other claimant was not the owner of the premises where the chattel was found.

UNKNOWN: it is not known whether the other claimant was the owner of the premises where the chattel was found.

$A_{4}$ : Was the other claimant the true owner of the chattel or did she/he claim through the rights of the true owner?

YES: the other claimant was the true owner of the chattel or was claiming through the rights of the true owner.

NO: the other claimant was not the true owner of the chattel and was not claiming through the rights of the true owner.

UNKNOWN: it is not known whether the other claimant was the true owner of the chattel or was claiming through the rights of the true owner.

$A_{5}$ : Did the finder hand over the chattel to the other claimant after the finding?

YES: the finder handed over the chattel to the other claimant after the finding.

NO: the finder did not hand over the chattel to the other claimant after the finding.

UNKNOWN: it is not known whether the finder handed over the chattel to the other claimant after the finding. 
$A_{6}$ : Did one of the parties rely on the terms of an agreement made with the other which purported to give her/him the right to the chattel?

YES: one of the parties relied on the terms of an agreement made with the other which purported to give her/him the right to the chattel.

NO: neither party relied on the terms of an agreement regarding the right to the chattel.

UNKNOWN: it is not known whether one of the parties relied on the terms of an agreement regarding the right to the chattel.

$A_{7}$ : Was the finder a servant of the other claimant?

YES: the finder was a servant of the other claimant.

NO: the finder was not a servant of the other claimant.

UNKNOWN: it is not known whether the finder was a servant of the other claimant.

$A_{8}$ : Was the chattel hidden or in a position so as to be difficult to find?

YES: the chattel was hidden or was in a position so as to be difficult to find.

NO: the chattel was not hidden and was not in a position so as to be difficult to find.

UNKNOWN: it is not known whether the chattel was hidden or was in a position so as to be difficult to find.

$A_{9}$ : Was an attempt made to find the true owner of the chattel or, alternatively, was the chattel clearly abandoned?

YES: an attempt was made to find the true owner of the chattel or, alternatively, the chattel was clearly abandoned.

NO: no attempt was made to find the true owner of the chattel and the chattel was not clearly abandoned.

UNKNOWN: it is not known whether an attempt was made to find the true owner of the chattel or whether the chattel was clearly abandoned.

$A_{10}$ : Did either of the parties know of the existence of the chattel prior to the finding?

YES: one of the parties knew of the existence of the chattel prior to the finding.

NO: neither party knew of the existence of the chattel prior to the finding.

UNKNOWN: it is not known whether either of the parties knew of the existence of the chattel prior to the finding. 


\section{Cases in which the finder wins}

$C_{1}$ : Hannah v. Peel [1945] 1 KB 509

$A_{1}$ : the finder was not the occupier of the premises where the chattel was found.

$A_{2}$ : the chattel was not attached.

$A_{3}$ : the other claimant was the owner of the premises where the chattel was found.

$A_{4}$ : the other claimant was not the true owner of the chattel and was not claiming through the rights of the true owner.

$A_{5}$ : the finder did not hand over the chattel to the other claimant after the finding.

$A_{6}$ : neither party relied on the terms of an agreement regarding the right to the chattel.

$A_{7}$ : the finder was not a servant of the other claimant.

$A_{8}$ : the chattel was hidden or was in a position so as to be difficult to find.

$A_{9}$ : an attempt was made to find the true owner of the chattel or, alternatively, the chattel was clearly abandoned.

$A_{10}$ : neither party knew of the existence of the chattel prior to the finding.

In Hannah v. Peel, ${ }^{1}$ a 1945 decision of the King's Bench Division of the English High Court, a brooch was found by the plaintiff who was a lance-corporal stationed in a house owned by the defendant. The house had been requisitioned by the army during the war and had never been occupied by the defendant.

The plaintiff was adjusting the black-out curtains when he touched something on the top of the window-frame. He thought the object to be a piece of dirt or plaster and he dropped it on the outside window ledge. On the following morning, he saw that it was a brooch and, on the advice of his commanding officer, turned it over to the police for the purpose of finding the owner. In the following year, the police returned the brooch to the defendant who sold it to a jeweller. The plaintiff at all times maintained his rights to the brooch against all persons other than the true owner.

The Court found for the plaintiff on the basis of Bridges v. Hawkesworth ${ }^{2}$ after a thorough review of the authorities. The Court further noted that the defendant was never in possession of the premises, that the brooch was never his, and that he had no knowledge of it until it was brought to his notice by the finder.

\footnotetext{
1 [1945] 1 KB 509.

${ }^{2}$ (1851) 21 LJQB 75.
} 
$C_{2}$ : Bridges v. Hawkesworth (1851) 21 LJQB 75

$A_{1}$ : the finder was not the occupier of the premises where the chattel was found.

$A_{2}$ : the chattel was not attached.

$A_{3}$ : the other claimant was the owner of the premises where the chattel was found.

$A_{4}$ : the other claimant was not the true owner of the chattel and was not claiming through the rights of the true owner.

$A_{5}$ : the finder handed over the chattel to the other claimant after the finding.

$A_{6}$ : neither party relied on the terms of an agreement regarding the right to the chattel.

$A_{7}$ : the finder was not a servant of the other claimant.

$A_{8}$ : the chattel was not hidden and was not in a position so as to be difficult to find.

$A_{9}$ : an attempt was made to find the true owner of the chattel or, alternatively, the chattel was clearly abandoned.

$A_{10}$ : neither party knew of the existence of the chattel prior to the finding.

In Bridges v. Hawkesworth ${ }^{3}$ an 1851 decision of the Queen's Bench Division of the English High Court, the plaintiff found a bundle of banknotes on the floor of the public area of a shop. He handed the notes to the shopkeeper in order that the true owner of the notes might be found. Although the owner was never found, the shopkeeper refused to return the notes to the finder. The Court found for the finder, holding that there is a "general right of [a] finder to any article which has been lost as against all the world except the true owner". ${ }^{4}$ It was further noted that the notes had never been in the custody of the shopkeeper nor within the protection of his house as might be the case had they intentionally been deposited there.

$C_{3}$ : Armory v. Delamirie (1722) 1 Str 505

$A_{1}$ : the finder was not the occupier of the premises where the chattel was found.

$A_{2}$ : the chattel was not attached.

$A_{3}$ : the other claimant was not the owner of the premises where the chattel was found.

$A_{4}$ : the other claimant was not the true owner of the chattel and was not claiming through the rights of the true owner.

$A_{5}$ : the finder handed over the chattel to the other claimant after the finding.

$A_{6}$ : neither party relied on the terms of an agreement regarding the right to the chattel.

$A_{7}$ : the finder was not a servant of the other claimant.

3 (1851) 21 LJQB 75.

4 ibid. at 77 , per Patteson J. 
$A_{8}$ : the chattel was not hidden and was not in a position so as to be difficult to find.

$A_{9}$ : no attempt was made to find the true owner of the chattel and the chattel was not clearly abandoned.

$A_{10}$ : one of the parties knew of the existence of the chattel prior to the finding.

In Armory v. Delamirie, ${ }^{5}$ a 1722 decision of the King's Bench Division of the English High Court, a chimney sweep found a jewel. Although the report does not expressly say so, most commentators assume that the jewel was found in a chimney in the course of the sweep's occupation. The jewel was handed to a goldsmith for appraisal who, under the pretence of weighing it, extracted the stone from its setting and offered the sweep three halfpence for it. The sweep refused the offer, but the goldsmith refused to return the stone. The goldsmith was held liable in trover and ordered to return the stone to the sweep or, failing that, to pay him a sum equivalent to what a stone of that size "of the finest water" would be worth.

\section{Cases in which the finder loses}

$C_{4}$ : Moffatt v. Kazana [1969] 2 QB 152

$A_{1}$ : the finder was the occupier of the premises where the chattel was found.

$A_{2}$ : the chattel was not attached.

$A_{3}$ : the other claimant was not the owner of the premises where the chattel was found.

$A_{4}$ : the other claimant was the true owner of the chattel or was claiming through the rights of the true owner.

$A_{5}$ : the finder did not hand over the chattel to the other claimant after the finding.

$A_{6}$ : neither party relied on the terms of an agreement regarding the right to the chattel.

$A_{7}$ : the finder was not a servant of the other claimant.

$A_{8}$ : the chattel was hidden or was in a position so as to be difficult to find.

$A_{9}$ : an attempt was made to find the true owner of the chattel or, alternatively, the chattel was clearly abandoned.

$A_{10}$ : one of the parties knew of the existence of the chattel prior to the finding.

In Moffatt v. Kazana, ${ }^{6}$ a 1967 decision of the Queen's Bench Division of the English High Court, the occupant of a house found a biscuit tin which contained a large number of banknotes. The tin was discovered during the course of some work in the kitchen of the house. To do that work it was necessary to dislodge some bricks from a point at which the main kitchen chimney joined a false flue. The tin fell out when the bricks were dislodged.

$5(1722) 1$ Str 505.

${ }^{6}[1969] 2$ QB 152. 
It emerged from the evidence that the defendant occupant had purchased the house from the previous plaintiff owner who had secreted the tin of notes during the time before the current owner had occupied the house. In selling the bungalow, the seller had wholly forgotten the existence of the tin and the buyer was, of course, unaware of its existence.

The Court held that the conveyance of the house and land did not suffice to convey the chattels in the house, a consequence of s. 62 of the governing Law of Property Act 1965 (UK). Consequently, the plaintiff remained the true owner of the tin of notes and was entitled to prevail.

Although the decision depends upon the provisions of a specific statute, it is clear that the true owners of chattels may displace the prima facie title of the owner of the land and the possessory title of any finder.

$C_{5}$ : City of London Corporation v. Appleyard (1) [1963] 1 WLR 982 ("London v. Appleyard (1)")

$A_{1}$ : the finder was not the occupier of the premises where the chattel was found.

$A_{2}$ : the chattel was attached.

$A_{3}$ : the other claimant was not the owner of the premises where the chattel was found.

$A_{4}$ : the other claimant was not the true owner of the chattel and was not claiming through the rights of the true owner.

$A_{5}$ : the finder did not hand over the chattel to the other claimant after the finding.

$A_{6}$ : neither party relied on the terms of an agreement regarding the right to the chattel.

$A_{7}$ : the finder was a servant of the other claimant.

$A_{8}$ : the chattel was hidden or was in a position so as to be difficult to find.

$A_{9}$ : an attempt was made to find the true owner of the chattel or, alternatively, the chattel was clearly abandoned.

$A_{10}$ : neither party knew of the existence of the chattel prior to the finding.

In City of London Corporation v. Appleyard (1), ${ }^{7}$ a 1963 decision of the Queen's Bench Division of the English High Court, workmen employed by Wates Ltd were engaged in cutting a key-way into a cellar wall for the purposes of securing a foundation when they found an old wall-safe built into a recess of the old wall. Inside was a wooden box which contained a large number of Bank of England notes. The notes were handed over to the City of London police who sought interpleader proceedings to determine who was entitled to the possession of the notes. 
Wates Ltd was an independent contractor engaged by Yorkwin Investments Ltd for a construction project. Yorkwin was lessee in possession of the property which was owned in fee simple by the City of London.

The Court followed the decision in South Staffordshire Water Co. v. Sharman ${ }^{8}$ in holding that the occupier is, in the absence of a better title elsewhere, entitled to the possession of objects which are attached to or under the land. Consequently, since the notes were in a wooden box within a safe built into the wall of the old building, the safe formed part of the demised premises. Yorkwin, being in lawful possession of the premises, were in de facto possession of the safe, even though ignorant of its existence.

Although Yorkwin was entitled to possession as against the finders, they in turn were displaced by the City of London which relied successfully on a term in the lease which granted them the right to certain objects found on the premises.

$C_{6}$ : City of London Corporation v. Appleyard (2) [1963] 1 WLR 982 ("London v. Appleyard (2)")

$A_{1}$ : the finder was the occupier of the premises where the chattel was found.

$A_{2}$ : the chattel was attached.

$A_{3}$ : the other claimant was the owner of the premises where the chattel was found.

$A_{4}$ : the other claimant was not the true owner of the chattel and was not claiming through the rights of the true owner.

$A_{5}$ : the finder handed over the chattel to the other claimant after the finding.

$A_{6}$ : one of the parties relied on the terms of an agreement made with the other which purported to give her/him the right to the chattel.

$A_{7}$ : the finder was not a servant of the other claimant.

$A_{8}$ : the chattel was hidden or was in a position so as to be difficult to find.

$A_{9}$ : an attempt was made to find the true owner of the chattel or, alternatively, the chattel was clearly abandoned.

$A_{10}$ : neither party knew of the existence of the chattel prior to the finding.

In City of London Corporation v. Appleyard (2), ${ }^{9}$ a 1963 decision of the Queen's Bench Division of the English High Court, workmen employed by Wates Ltd were engaged in cutting a key-way into a cellar wall for the purposes of securing a foundation when they found an old wall-safe built into a recess of the old wall. Inside was a wooden box which contained a large number of Bank of England notes. The notes were handed over to the City of London police who sought interpleader proceedings to determine who was entitled to the possession of the notes. 
Wates Ltd was an independent contractor engaged by Yorkwin Investments Ltd for a construction project. Yorkwin was lessee in possession of the property which was owned in fee simple by the City of London. The Court found that the safe formed part of the demised premises and that, consequently, Yorkwin was entitled to the notes as against the workmen.

The lease contained a clause which purported to grant the rights to "every relic or article of antiquity rarity or value" to the City of London. The sole issue was to determine if the notes fell into that description. The Court could find no reason for limiting the generality of the words and so found for the City of London.

$C_{7}$ : South Staffordshire Water Co. v. Sharman [1896] 2 QB 44 ("South Staffordshire v. Sharman")

$A_{1}$ : the finder was not the occupier of the premises where the chattel was found.

$A_{2}$ : the chattel was attached.

$A_{3}$ : the other claimant was the owner of the premises where the chattel was found.

$A_{4}$ : the other claimant was not the true owner of the chattel and was not claiming through the rights of the true owner.

$A_{5}$ : the finder did not hand over the chattel to the other claimant after the finding.

$A_{6}$ : neither party relied on the terms of an agreement regarding the right to the chattel.

$A_{7}$ : the finder was a servant of the other claimant.

$A_{8}$ : the chattel was hidden or was in a position so as to be difficult to find.

$A_{9}$ : an attempt was made to find the true owner of the chattel or, alternatively, the chattel was clearly abandoned.

$A_{10}$ : neither party knew of the existence of the chattel prior to the finding.

In South Staffordshire Water Co. v. Sharman, ${ }^{10}$ an 1896 decision of the Queen's Bench Division of the English High Court, the defendant was a workman employed by the plaintiff to clean out a pool located on land owned by the plaintiff. During the operation the defendant found two gold rings embedded in the mud at the bottom of the pool. Although the plaintiff demanded the rings, the defendant refused to give them up. He placed them in the hands of police authorities who unsuccessfully endeavoured to find the owners of the rings. The police returned the rings to the defendant who was then sued in detinue for the recovery of the rings.

It was proved at the trial that there was no special contract between the parties which called upon the defendant to give up any articles which might be found.

Although the county court held in favour of the defendant on the basis of Bridges v. Hawkesworth, ${ }^{11}$ the appeal found for the plaintiff on the basis that

${ }^{10}[1896] 2$ QB 44.

${ }^{11}(1851) 21$ LJQB 75. 
they had, as owners of the land and pool, the right to exercise control over the same. Bridges $v$. Hawkesworth was distinguished on the grounds that the notes in that case were in a public part of the shop and the shopkeeper did not in any sense control them.

The Court stated a general principle: where a person has possession of a house or land with a manifest intention to exercise control over it and the things which may be upon or in it, then there is a presumption that things found there are in the possession of the owner.

$C_{8}$ : Elwes v. Brigg Gas Co. (1886) $33 \mathrm{ChD} 562$ ("Elwes v. Brigg Gas")

$A_{1}$ : the finder was the occupier of the premises where the chattel was found.

$A_{2}$ : the chattel was attached.

$A_{3}$ : the other claimant was the owner of the premises where the chattel was found.

$A_{4}$ : the other claimant was not the true owner of the chattel and was not claiming through the rights of the true owner.

$A_{5}$ : the finder did not hand over the chattel to the other claimant after the finding.

$A_{6}$ : one of the parties relied on the terms of an agreement made with the other which purported to give her/him the right to the chattel.

$A_{7}$ : the finder was not a servant of the other claimant.

$A_{8}$ : the chattel was hidden or was in a position so as to be difficult to find.

$A_{9}$ : an attempt was made to find the true owner of the chattel or, alternatively, the chattel was clearly abandoned.

$A_{10}$ : neither party knew of the existence of the chattel prior to the finding.

In Elwes v. Brigg Gas Co. ${ }^{12}$ an 1886 decision of the Chancery Division of the English High Court, a lessee of land found, during the course of some excavations, an ancient boat buried in the soil. The Court held that the boat belonged to the owner of the land at the time when the lease was granted since: (a) if the boat was regarded as a part of the soil or as a mineral in the soil, it was clearly a part of the land to which the plaintiff was entitled; alternatively, (b) if the boat was considered as a chattel, then the plaintiff was in lawful possession of everything that lay beneath the surface. Since a trespasser could not have taken possession of the boat, it followed that only the original owner could have a better title, but obviously such rights could no longer be established.

It followed from the above that the defendant's claim must rest on the terms of the lease. Although the lease contemplated the excavations which were done, it was silent as to what was to be done with the soil excavated. It was impossible to imply a term into the contract which would give the defendant lessee the rights to the boat.

${ }^{12}(1886) 33 \mathrm{ChD} 562$. 


\section{A.3 The Authorization specification}

\section{Hierarchy}

\begin{tabular}{|c|l|}
\hline$c$ & \multicolumn{1}{|c|}{ Court } \\
\hline \hline 1 & three judges of the High Court of Australia \\
2 & the Full Court of the Supreme Court of New South Wales \\
3 & the Supreme Court of New South Wales \\
& the Supreme Court of Victoria \\
4 & the Judicial Committee of the Privy Council \\
5 & the English Court of Appeal \\
6 & the Chancery Division of the English High Court \\
\hline
\end{tabular}

\section{Authorization area}

\begin{tabular}{|c|c|c|c|c|c|c|c|c|c|}
\hline Case & \multicolumn{7}{|c|}{ Attributes } & $c$ & Result \\
\hline$C_{1}$ & $x$ & $x$ & $\bullet$ & $\bullet$ & $x$ & - & $x$ & 1 & \multirow{6}{*}{ Auth } \\
\hline$C_{2}$ & & & $x$ & $\bullet$ & $x$ & $\bullet$ & $\bullet$ & 2 & \\
\hline$C_{3}$ & $\times$ & $\times$ & $\bullet$ & $\bullet$ & $x$ & $\bullet$ & $\bullet$ & 3 & \\
\hline$C_{4}$ & $x$ & $\bullet$ & $x$ & • & $x$ & • & $\bullet$ & 4 & \\
\hline \multirow{2}{*}{$\begin{array}{c}C_{5} \\
I_{\text {Auth }}\end{array}$} & $x$ & $\times$ & $\bullet$ & $x$ & $x$ & $\bullet$ & $\bullet$ & 5 & \\
\hline & $x$ & $\bullet$ & $\bullet$ & $\bullet$ & $x$ & $\bullet$ & $\bullet$ & & \\
\hline$C_{6}$ & $x$ & $\times$ & $x$ & $x$ & $x$ & $\bullet$ & $\times$ & 3 & \multirow{4}{*}{ Not-Auth } \\
\hline$C_{7}$ & $\times$ & $\bullet$ & $x$ & $\bullet$ & $x$ & $x$ & $\times$ & 5 & \\
\hline \multirow{2}{*}{$\begin{array}{c}C_{8} \\
I_{\text {Not-Auth }}\end{array}$} & $x$ & $x$ & $\bullet$ & $x$ & $x$ & $\bullet$ & $x$ & 6 & \\
\hline & $x$ & $x$ & $x$ & $x$ & $\bullet$ & $x$ & $x$ & & \\
\hline \multirow{2}{*}{$\begin{array}{c}C_{9} \\
I_{\text {Liable }}\end{array}$} & $\bullet$ & $x$ & $x$ & $\bullet$ & $x$ & $\bullet$ & $\bullet$ & 3 & \multirow[t]{2}{*}{ Liable } \\
\hline & $\bullet$ & $x$ & & $\bullet$ & $x$ & $\bullet$ & & & \\
\hline
\end{tabular}

\section{Opening}

The notion of authorization extends beyond the authority given to an agent. The word "authorize" should be "understood in its ordinary dictionary sense of "sanction, approve, and countenance." "1

"[A] person who has under his control the means by which an infringement of copyright may be committed ... and who makes it available to other persons, knowing, or having reason to suspect, that it is likely to be used for the purpose of committing an infringement, and omitting to take reasonable steps to limit its use to legitimate purposes, would authorize any infringement that resulted from its use." ${ }^{2}$

\footnotetext{
${ }^{1}$ Falcon v. Famous Players Film Co. [1926] 2 KB 474 at 491, per Bankes LJ.

${ }^{2}$ University of New South Wales v. Moorhouse (1975) 133 CLR 1 at 13, per Gibbs J.
} 


\section{Results}

Auth: the accused authorized the infringement.

Not-Auth: the accused did not authorize the infringement.

Liable: the accused is liable (directly or vicariously) for the infringement.

\section{Attributes}

$A_{1}$ : Was the infringer an employee of the accused?

YES: the infringer was an employee of the accused.

$$
\Rightarrow \text { Liable }
$$

NO: the infringer was not an employee of the accused.

$$
\Rightarrow \text { Auth } \vee \text { Not-Auth }
$$

UNKNOWN: it is not known whether the infringer was an employee of the accused.

If the accused had control over the infringer's manner of doing her/his work then the infringer was an employee of the accused. If the infringer undertook to do something for the accused and had discretion as to the manner in which it was to be done then the infringer was an independent contractor to the accused, not an employee.

$A_{2}$ : Was the infringer an independent contractor to the accused?

YES: the infringer was an independent contractor to the accused.

$$
\Rightarrow \text { Auth }
$$

NO: the infringer was not an independent contractor to the accused.

$$
\Rightarrow \text { Not-Auth } \vee \text { Liable }
$$

UNKNOWN: it is not known whether the infringer was an independent contractor to the accused.

If the infringer undertook to do something for the accused and had discretion as to the manner in which it was to be done then the infringer was an independent contractor to the accused. If the accused had control over the infringer's manner of doing her/his work then the infringer was an employee of the accused, not an independent contractor.

$A_{3}$ : Did the accused sell or hire the infringer the means of infringing?

YES: the accused sold or hired the infringer the means of infringing.

$\Rightarrow$ Auth

NO: the accused did not sell or hire the infringer the means of infringing.

UNKNOWN: it is not known whether the accused sold or hired the infringer the means of infringing. 
$A_{4}$ : Did the accused have the power to prevent the infringement?

YES: the accused had the power to prevent the infringement.

$\Rightarrow$ Auth $\vee$ Liable

NO: the accused did not have the power to prevent the infringement.

$\Rightarrow$ Not-Auth

UNKNOWN: it is not known whether the accused had the power to prevent the infringement.

If the accused had some control over the infringer, or the means by which the infringement was committed, then the accused had the power to prevent the infringement.

$A_{5}$ : Did the accused take reasonable steps to avoid the infringement?

YES: the accused took reasonable steps to avoid the infringement.

$\Rightarrow$ Not-Auth

NO: the accused did not take reasonable steps to avoid the infringement.

$\Rightarrow$ Auth $\vee$ Liable

UNKNOWN: it is not known whether the accused took reasonable steps to avoid the infringement.

Whether particular steps were reasonable depends on the facts of the case.

$A_{6}$ : Did the accused know, or have reason to anticipate or suspect, that the infringing act was to be, or was likely to be, done?

YES: the accused knew, or had reason to anticipate or suspect, that the infringing act was to be, or was likely to be, done.

$\Rightarrow$ Auth $\vee$ Liable

NO: the accused did not know, and had no reason to anticipate or suspect, that the infringing act was to be, or was likely to be, done.

$\Rightarrow$ Not-Auth

UNKNOWN: it is not known whether the accused knew, or had reason to anticipate or suspect, that the infringing act was to be, or was likely to be, done.

Authorization requires a mental element. However, it is not necessary for the accused to have known that a particular infringing act was to occur; merely that, from her or his awareness of the circumstances, she or he recognized that it was likely that such an act might occur.

$A_{7}$ : Was the specific infringement causally related to an incitement to infringe on the part of the accused?

YES: the specific infringement was causally related to an incitement to infringe on the part of the accused.

$\Rightarrow$ Auth $\vee$ Liable 
NO: the specific infringement was not causally related to an incitement to infringe on the part of the accused.

$\Rightarrow$ Not-Auth

UNKNOWN: it is not known whether the specific infringement was causally related to an incitement to infringe on the part of the accused.

General exhortations to infringe will not amount to authorization unless specific acts of infringement can be established. There must be some relationship creating a link or connection, however tenuous, between the authorizer and the infringer.

\section{Cases in which the accused authorized the infringement}

$C_{1}$ : University of New South Wales v. Moorhouse (1975) 133 CLR 1 ("UNSW v. Moorhouse")

$A_{1}$ : the infringer was not an employee of the accused.

$A_{2}$ : the infringer was not an independent contractor to the accused.

$A_{3}$ : the accused sold or hired the infringer the means of infringing.

$A_{4}$ : the accused had the power to prevent the infringement.

$A_{5}$ : the accused did not take reasonable steps to avoid the infringement.

$A_{6}$ : the accused knew, or had reason to anticipate or suspect, that the infringing act was to be, or was likely to be, done.

$A_{7}$ : the specific infringement was not causally related to an incitement to infringe on the part of the accused.

In University of New South Wales v. Moorhouse, ${ }^{3}$ a 1975 decision of three judges of the High Court of Australia, a graduate of the University used a photocopy machine in the University library to make two copies of a story from a library copy of a book of short stories.

McTiernan ACJ, Gibbs and Jacobs JJ held that the University had authorized the infringement within the meaning of s. 36(1) of the Copyright Act 1968 (Cth); it had the power to prevent infringements, but had not taken reasonable steps to prevent them. ${ }^{4}$ Gibbs J's statement about what constitutes authorization of an infringement is quoted above.

$C_{2}$ : Australasian Performing Right Association Ltd v. Canterbury-Bankstown League Club Ltd [1964-65] NSWR 138 ("APRA v. Canterbury-Bankstown")

$A_{1}$ : it is not known whether the infringer was an employee of the accused.

$A_{2}$ : it is not known whether the infringer was an independent contractor to the accused.

$A_{3}$ : the accused did not sell or hire the infringer the means of infringing.

$A_{4}$ : the accused had the power to prevent the infringement.

${ }^{3}(1975) 133$ CLR 1.

${ }^{4}$ The addition, in 1980, of s. 39A to the Copyright Act ameliorated the effect of UNSW $v$. Moorhouse as far as photocopying in libraries is concerned. 
$A_{5}$ : the accused did not take reasonable steps to avoid the infringement.

$A_{6}$ : the accused knew, or had reason to anticipate or suspect, that the infringing act was to be, or was likely to be, done.

$A_{7}$ : the specific infringement was causally related to an incitement to infringe on the part of the accused.

In Australasian Performing Right Association Ltd v. Canterbury-Bankstown League Club Ltd, ${ }^{5}$ a 1964 decision of the Full Court of the Supreme Court of New South Wales, the club engaged a dance band to play music for dances that it held at its premises. The choice of the music to be played was left to the band leader.

Herron CJ, Ferguson and Asprey JJ held that whether the bandleader was an employee or an independent contractor was immaterial. "He was authorized to play and was allowed a discretion to select whatever music he liked. He was thus given a general authority to play whatever music he liked irrespective of copyright." ${ }^{6}$ So (if he was an employee) the club was vicariously liable for-or (if he was an independent contractor) the club was liable for the authorization of - the bandleader's breach of the Copyright Act 1912 (Cth).

$C_{3}$ : Winstone v. Wurlitzer Automatic Phonograph Co. of Australia Pty Ltd [1946] VLR 338 ("Winstone v. Wurlitzer")

$A_{1}$ : the infringer was not an employee of the accused.

$A_{2}$ : the infringer was not an independent contractor to the accused.

$A_{3}$ : the accused sold or hired the infringer the means of infringing.

$A_{4}$ : the accused had the power to prevent the infringement.

$A_{5}$ : the accused did not take reasonable steps to avoid the infringement.

$A_{6}$ : the accused knew, or had reason to anticipate or suspect, that the infringing act was to be, or was likely to be, done.

$A_{7}$ : the specific infringement was causally related to an incitement to infringe on the part of the accused.

In Winstone v. Wurlitzer Automatic Phonograph Co. of Australia Pty Ltd, ${ }^{7}$ a 1946 decision of the Supreme Court of Victoria, Wurlitzer installed a juke-box in a shop and had an agreement with the shop's proprietor by which Wurlitzer maintained and repaired the machine, and supplied it with records which Wurlitzer selected. The juke-box played a musical work, the copyright in which was owned by Winstone.

Herring CJ held that the proprietor of the shop had publicly performed the musical work and-because of nature of the agreement between Wurlitzer and the shop's proprietor, and because Wurlitzer selected the records-Wurlitzer had

\footnotetext{
5 [1964-65] NSWR 138.

6 ibid. at 140, per Ferguson J, with whom Herron CJ agreed.

${ }^{7}[1946]$ VLR 338.
} 
authorized that infringing performance within the meaning of s. 1(2) of the Copyright Act 1911 (UK) which was in force in Australia by virtue of the Copyright Act 1912 (Cth).

$C_{4}$ : Mellor v. Australian Broadcasting Commission [1940] AC 491 ("Mellor v. ABC")

$A_{1}$ : the infringer was not an employee of the accused.

$A_{2}$ : the infringer was an independent contractor to the accused.

$A_{3}$ : the accused did not sell or hire the infringer the means of infringing.

$A_{4}$ : the accused had the power to prevent the infringement.

$A_{5}$ : the accused did not take reasonable steps to avoid the infringement.

$A_{6}$ : the accused knew, or had reason to anticipate or suspect, that the infringing act was to be, or was likely to be, done.

$A_{7}$ : the specific infringement was causally related to an incitement to infringe on the part of the accused.

In Mellor v. Australian Broadcasting Commission, ${ }^{8}$ a 1940 decision of the Judicial Committee of the Privy Council, Mellor and others held the sole right to perform in public in Australia some musical works arranged for performance by brass and military bands. They published and distributed advertising pamphlets which included a statement that all of their sheet music was "Free for Public Performance' anywhere ... We have paid for the performing rights of every piece we issue." 9 The ABC engaged bands to play some of this music, and broadcast the bands' performances on radio.

The Privy Council held that the ABC had authorized the bands to perform the musical works within the meaning of s. 1(2) of the Copyright Act 1911 (UK) which was in force in Australia by virtue of the Copyright Act 1912 (Cth). However, the $\mathrm{ABC}$ had not infringed the plaintiffs' sole right to authorize public performance because the statements made in the pamphlets amounted to consent.

$C_{5}$ : Falcon v. Famous Players Film Co. [1926] 2 KB 474 ("Falcon v. Famous Players")

$A_{1}$ : the infringer was not an employee of the accused.

$A_{2}$ : the infringer was not an independent contractor to the accused.

$A_{3}$ : the accused sold or hired the infringer the means of infringing.

$A_{4}$ : the accused did not have the power to prevent the infringement.

$A_{5}$ : the accused did not take reasonable steps to avoid the infringement.

$A_{6}$ : the accused knew, or had reason to anticipate or suspect, that the infringing act was to be, or was likely to be, done.

$A_{7}$ : the specific infringement was causally related to an incitement to infringe on the part of the accused.

\footnotetext{
${ }^{8}[1940]$ AC 491.

${ }^{9}$ ibid. at $498-9$.
} 
In Falcon v. Famous Players Film Co. ${ }^{10}$ a 1926 decision of the English Court of Appeal, the author of a play assigned to Falcon the sole right to perform the play in the United Kingdom and, twenty-one years later, sold to Famous Players the film rights to the play throughout the world. Famous Players made a film of the play in America, imported it to England, and purported to let the right to exhibit it to the proprietor of a cinema. Falcon brought an action to restrain Famous Players from infringing his performing right. Famous Players denied that Falcon had such an exclusive right and claimed that, even if he had, they had not infringed it.

The Court of Appeal held that Falcon did have an exclusive right to perform the play in the UK, and that Famous Players had infringed it.

Scrutton LJ referred to the hiring agreement that impliedly stipulated that the cinema proprietor should exhibit. "They have imposed an obligation upon him that he shall perform, and in my view persons who do that perform themselves." 11 Hence Scrutton LJ found no need to consider whether Famous Players had authorized the cinema proprietor to infringe.

Bankes and Atkin LJJ held that Famous Players had authorized the infringement within the meaning of s. 1(2) of the Copyright Act 1911 (UK). Bankes LJ's view (quoted above) that the word "authorize" should be understood in its ordinary dictionary sense of "sanction, approve, and countenance" has been adopted by most subsequent courts in the UK and in Australia.

$I_{\text {Auth }}$ (the ideal case in which the accused authorized the infringement):

$A_{1}$ : the infringer was not an employee of the accused.

$A_{2}$ : the infringer was an independent contractor to the accused.

$A_{3}$ : the accused sold or hired the infringer the means of infringing.

$A_{4}$ : the accused had the power to prevent the infringement.

$A_{5}$ : the accused did not take reasonable steps to avoid the infringement.

$A_{6}$ : the accused knew, or had reason to anticipate or suspect, that the infringing act was to be, or was likely to be, done.

$A_{7}$ : the specific infringement was causally related to an incitement to infringe on the part of the accused.

\section{Cases in which the accused did not authorize the infringement}

$C_{6}$ : RCA Corporation v. John Fairfax and Sons Ltd [1981] 1 NSWLR 251 ("RCA v. Fairfax")

$A_{1}$ : the infringer was not an employee of the accused.

$A_{2}$ : the infringer was not an independent contractor to the accused.

$A_{3}$ : the accused did not sell or hire the infringer the means of infringing.

${ }^{10}[1926] 2 \mathrm{~KB} 474$.

${ }^{11}$ ibid. at 495 . 
$A_{4}$ : the accused did not have the power to prevent the infringement.

$A_{5}$ : the accused did not take reasonable steps to avoid the infringement.

$A_{6}$ : the accused knew, or had reason to anticipate or suspect, that the infringing act was to be, or was likely to be, done.

$A_{7}$ : the specific infringement was not causally related to an incitement to infringe on the part of the accused.

In RCA Corporation v. John Fairfax and Sons Ltd, ${ }^{12}$ a 1981 decision of the Supreme Court of New South Wales, the Fairfax newspaper the Sun-Herald carried an article which pointed out that, using cassette tapes and good quality taping equipment, the same album can be taped by many people. It also discussed how the advent of FM radio had made it easy for people to tape new album and single releases without buying the discs: "Why spend nearly $\$ 10$ on the new David Bowie album when you can tape it from 2JJJ?" 13

Kearney J held that "authorization involves some element of causation - and hence the necessity for some relationship creating a link or connection however tenuous between the authorizer and the infringer." 14 There was no such link, so Fairfax had not authorized any infringement within the meaning of s. 13(2) of the Copyright Act 1968 (Cth).

$C_{7}$ : Performing Right Society Ltd $v$. Ciryl Theatrical Syndicate Ltd [1924] 1 KB 1 ("PRS v. Ciryl")

$A_{1}$ : the infringer was not an employee of the accused.

$A_{2}$ : the infringer was an independent contractor to the accused.

$A_{3}$ : the accused did not sell or hire the infringer the means of infringing.

$A_{4}$ : the accused had the power to prevent the infringement.

$A_{5}$ : the accused did not take reasonable steps to avoid the infringement.

$A_{6}$ : the accused did not know, and had no reason to anticipate or suspect, that the infringing act was to be, or was likely to be, done.

$A_{7}$ : the specific infringement was not causally related to an incitement to infringe on the part of the accused.

In Performing Right Society Ltd v. Ciryl Theatrical Syndicate Ltd, ${ }^{15}$ a 1923 decision of the English Court of Appeal, the syndicate was the lessee of a theatre. The managing-director of the syndicate produced a play at that theatre, and engaged a band to perform at the theatre under the direction of a bandmaster. In the absence of the managing-director, and without his knowledge, the band performed works the copyright in which was owned by the Performing Right Society.

12 [1981] 1 NSWLR 251.

13ibid. at 252 .

${ }^{14}$ ibid. at 259 .

${ }^{15}[1924] 1$ KB 1. 
Bankes, Scrutton and Atkin LJJ held that the managing-director had not authorized the infringing performances, within the meaning of s. 1(2) of the Copyright Act 1911 (UK), because the infringement occurred without his knowledge and he had no reason to anticipate or suspect that the band was likely to give performances which would breach copyright.

$C_{8}$ : A\&̈M Records Inc. v. Audio Magnetics Inc. (UK) Ltd [1979] FSR 1 ("A\&̈Mv. Audio Magnetics")

$A_{1}$ : the infringer was not an employee of the accused.

$A_{2}$ : the infringer was not an independent contractor to the accused.

$A_{3}$ : the accused sold or hired the infringer the means of infringing.

$A_{4}$ : the accused did not have the power to prevent the infringement.

$A_{5}$ : the accused did not take reasonable steps to avoid the infringement.

$A_{6}$ : the accused knew, or had reason to anticipate or suspect, that the infringing act was to be, or was likely to be, done.

$A_{7}$ : the specific infringement was not causally related to an incitement to infringe on the part of the accused.

In A\&M Records Inc. v. Audio Magnetics Inc. (UK) $L t d,{ }^{16}$ a 1978 decision of the Chancery Division of the English High Court, A\&M Records and twenty-three others alleged that Audio Magnetics was inciting the public to infringe their copyright in sound recording by advertising blank cassette tapes.

Foster J held that there was no "particular specific authorisation"; 17 there was not sufficient causal relationship between the alleged authorization and the actual breach. "It was not sufficient to allege authorisation at large. Authorisation meant sanctioning, express approval or countenancing of an actual breach of copyright by some act directly related to that breach." ${ }^{18}$

$I_{\text {Not-Auth }}$ (the ideal case in which the accused did not authorize the infringement):

$A_{1}$ : the infringer was not an employee of the accused.

$A_{2}$ : the infringer was not an independent contractor to the accused.

$A_{3}$ : the accused did not sell or hire the infringer the means of infringing.

$A_{4}$ : the accused did not have the power to prevent the infringement.

$A_{5}$ : the accused took reasonable steps to avoid the infringement.

$A_{6}$ : the accused did not know, and had no reason to anticipate or suspect, that the infringing act was to be, or was likely to be, done.

$A_{7}$ : the specific infringement was not causally related to an incitement to infringe on the part of the accused.

\footnotetext{
${ }^{16}$ [1979] FSR 1.

17 ibid. at 10 .

18 ibid. at 2 .
} 


\section{Cases in which the accused is liable (directly or vicariously) for the infringement}

$C_{9}$ : Australasian Performing Right Association Ltd v. Miles [1962] NSWR 405 ("APRA v. Miles")

$A_{1}$ : the infringer was an employee of the accused.

$A_{2}$ : the infringer was not an independent contractor to the accused.

$A_{3}$ : the accused did not sell or hire the infringer the means of infringing.

$A_{4}$ : the accused had the power to prevent the infringement.

$A_{5}$ : the accused did not take reasonable steps to avoid the infringement.

$A_{6}$ : the accused knew, or had reason to anticipate or suspect, that the infringing act was to be, or was likely to be, done.

$A_{7}$ : the specific infringement was causally related to an incitement to infringe on the part of the accused.

In Australasian Performing Right Association Ltd v. Miles, ${ }^{19}$ a 1961 decision of the Supreme Court of New South Wales, the Dee Why RSL Club engaged a band to play at a dance held at the club. During the dance the band played I've Got a Lovely Bunch of Coconuts, the copyright in which was owned by the Australasian Performing Right Association.

Jacobs $\mathrm{J}$ held that the members of the band were servants of the club, because "the club through its officers was exercising a control over the work performed in such a way as to show that there was an authority to command the orchestra in its performance." ${ }^{20}$ So the members of the club, through the band, performed the musical work and infringed the copyright under s. 2(1) of the Copyright Act 1911 (UK) which was in force in Australia by virtue of the Copyright Act 1912 (Cth).

$I_{\text {Liable }}$ (the ideal case in which the accused is liable (directly or vicariously) for the infringement):

$A_{1}$ : the infringer was an employee of the accused.

$A_{2}$ : the infringer was not an independent contractor to the accused.

$A_{3}$ : it is not known whether the accused sold or hired the infringer the means of infringing.

$A_{4}$ : the accused had the power to prevent the infringement.

$A_{5}$ : the accused did not take reasonable steps to avoid the infringement.

$A_{6}$ : the accused knew, or had reason to anticipate or suspect, that the infringing act was to be, or was likely to be, done.

$A_{7}$ : it is not known whether the specific infringement was causally related to an incitement to infringe on the part of the accused.

${ }^{19}$ [1962] NSWR 405.

${ }^{20}$ ibid. at 407. 


\section{A.4 The EMPloYeE specification}

\section{Hierarchy}

\begin{tabular}{|c|l|}
\hline$c$ & \multicolumn{1}{|c|}{ Court } \\
\hline \hline 1 & five judges of the High Court of Australia \\
2 & four judges of the High Court of Australia \\
3 & three judges of the High Court of Australia \\
4 & a single judge of the High Court of Australia \\
5 & three judges of the Federal Court of Australia \\
6 & the Judicial Committee of the Privy Council \\
7 & the English Court of Appeal \\
8 & the King's Bench Division of the English High Court \\
& the Queen's Bench Division of the English High Court \\
\hline
\end{tabular}

\section{Employee area}

\begin{tabular}{|c|c|c|c|c|c|c|c|c|c|c|c|c|c|c|c|c|c|c|c|c|c|c|c|}
\hline \multirow{2}{*}{ Case } & \multicolumn{21}{|c|}{ Attributes } & \multirow{2}{*}{$c$} & \multirow{2}{*}{ Result } \\
\hline & $A_{1}$ & $A_{2}$ & $A_{3}$ & $A_{4}$ & $A$ & 5 & $A_{6}$ & $A$ & 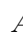 & & $A_{9}$ & $A_{1 \mathrm{C}}$ & & 11 & $A_{12}$ & $A_{13}$ & $A_{14}$ & $A_{15}$ & $A_{16}$ & $A_{17}$ & $A_{18}$ & & \\
\hline \multirow{8}{*}{$\begin{array}{c}C_{1} \\
C_{2} \\
C_{3} \\
C_{4} \\
C_{5} \\
C_{6} \\
C_{7} \\
I_{\text {Employee }} \\
\end{array}$} & $\times$ & $\bullet$ & $\bullet$ & $x$ & $\bullet$ & & & $\bullet$ & & x & $x$ & $x$ & & $\bullet$ & $\bullet$ & $\times$ & $\times$ & $\bullet$ & $\times$ & $x$ & $\times$ & 1 & \multirow{8}{*}{ Employee } \\
\hline & $\bullet$ & $\bullet$ & $\bullet$ & $x$ & $\bullet$ & & $\times$ & $\times$ & & K & $\bullet$ & $x$ & & $x$ & $\times$ & $\times$ & $\times$ & $\times$ & $\times$ & $\times$ & $\times$ & 2 & \\
\hline & $\bullet$ & $\times$ & & $x$ & $\bullet$ & & • & $\bullet$ & & & $x$ & $x$ & & $\bullet$ & $x$ & $\bullet$ & $\times$ & $\times$ & $\times$ & $x$ & $\times$ & 4 & \\
\hline & $\bullet$ & $\times$ & $\bullet$ & $\bullet$ & $x$ & & $\bullet$ & $\bullet$ & & x & & $x$ & & $x$ & $\times$ & $\times$ & $\times$ & $\times$ & $\times$ & $\times$ & $\times$ & 5 & \\
\hline & $\bullet$ & $\times$ & $\bullet$ & $x$ & $\bullet$ & & $\times$ & $\bullet$ & & r & $x$ & $x$ & & $\bullet$ & $\bullet$ & $\times$ & $\times$ & $\times$ & $\times$ & $\times$ & $\bullet$ & 7 & \\
\hline & $\times$ & $\bullet$ & $\bullet$ & $x$ & $\bullet$ & & $\times$ & & & x & $x$ & $x$ & & $\bullet$ & & & & & & & $\times$ & 7 & \\
\hline & $\bullet$ & $\bullet$ & $\bullet$ & $\bullet$ & $x$ & & $\bullet$ & $\bullet$ & & x & $x$ & $\bullet$ & & $\bullet$ & $\bullet$ & $\times$ & $\bullet$ & $\times$ & $\times$ & $x$ & $\times$ & 8 & \\
\hline & $\bullet$ & $\times$ & $\bullet$ & $\times$ & $\bullet$ & & $\bullet$ & $\bullet$ & & K & $x$ & $\bullet$ & & $\bullet$ & $\bullet$ & $x$ & $\bullet$ & $\bullet$ & $\bullet$ & \multicolumn{2}{|l|}{$\bullet$} & & \\
\hline$C_{8}$ & $x$ & $\bullet$ & $\times$ & $\bullet$ & $x$ & & $\times$ & $x$ & & x & $\bullet$ & $x$ & & $x$ & $x$ & $\times$ & $\times$ & $\times$ & $\times$ & $\times$ & $\times$ & 3 & \multirow{8}{*}{ Contractor } \\
\hline$C_{9}$ & $x$ & $\bullet$ & $x$ & $\bullet$ & $\times$ & & $\times$ & $\bullet$ & & b & $\bullet$ & $\times$ & & $x$ & $\times$ & $\times$ & $\times$ & $\times$ & $\times$ & $\bullet$ & $\times$ & 3 & \\
\hline$C_{10}$ & $x$ & $\bullet$ & $\bullet$ & $\bullet$ & $x$ & & $\times$ & $\times$ & & x & $\bullet$ & $x$ & & $x$ & $\times$ & $x$ & $\times$ & $\bullet$ & $\times$ & $x$ & $\times$ & 5 & \\
\hline$C_{11}$ & $\times$ & $\bullet$ & $\bullet$ & $\bullet$ & $x$ & & $\times$ & $\times$ & & b & $\bullet$ & $\bullet$ & & $x$ & $\times$ & $x$ & $\times$ & $\times$ & $\times$ & $x$ & $\bullet$ & 6 & \\
\hline$C_{12}$ & $\bullet$ & $\bullet$ & $\bullet$ & $\times$ & $\bullet$ & & 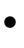 & $\times$ & & b & $\bullet$ & $x$ & & $\bullet$ & $\bullet$ & $\times$ & $\times$ & $\times$ & $\bullet$ & $\times$ & $\bullet$ & 7 & \\
\hline$C_{13}$ & $x$ & $\bullet$ & $x$ & $\times$ & $\bullet$ & & $\times$ & & & x & $x$ & $x$ & & $\bullet$ & & & & & & $\bullet$ & $x$ & 7 & \\
\hline \multirow{2}{*}{$\begin{array}{c}C_{14} \\
I_{\text {Contractor }}\end{array}$} & $\times$ & $\bullet$ & $\bullet$ & $\bullet$ & x & & $\times$ & $\times$ & & x & $\bullet$ & $\bullet$ & & $x$ & $\times$ & $x$ & $\times$ & $\times$ & $x$ & $x$ & $\bullet$ & 8 & \\
\hline & $\times$ & $\bullet$ & $\times$ & $\bullet$ & $x$ & & & $\times$ & & b & $\bullet$ & $\times$ & & $x$ & $\times$ & $\bullet$ & $\times$ & $\times$ & $\times$ & & $\bullet$ & & \\
\hline
\end{tabular}

\section{Opening}

The law distinguishes between a contract of service (between employer and employee) and a contract for services (between principal and independent contractor). This distinction affects the terms that will be implied in the absence of an express agreement, the liability of the employer to third parties, the applicability of industrial awards, the applicability of statutes which may affect workers' compensation, occupational health and safety, long-service leave, fringe benefits tax, etc.

The terms "employer" and "worker" are used here to mean "employer" and "employee" (in the case of a contract of service) or "principal" and "independent contractor" (in the case of a contract for services). 


\section{Results}

Employee: the worker is an employee.

Contractor: the worker is an independent contractor.

\section{Attributes}

$A_{1}$ : Did the employer direct not only what work was to be done, but also the manner in which it was to be done?

YES: the employer directed the manner in which the work was to be done.

$$
\Rightarrow \text { Employee }
$$

NO: the employer did not direct the manner in which the work was to be done.

$\Rightarrow$ Contractor

UNKNOWN: it is not known whether the employer directed the manner in which the work was to be done.

If the employer had a right of control over how the worker did the work then the employer had the power to direct not only what work was to be done, but also the manner in which it was to be done.

$A_{2}$ : Was the worker allowed to use her/his own discretion in doing an aspect of the work that was not specified beforehand?

YES: the worker was allowed to use her/his own discretion in doing an aspect of the work that was not specified beforehand.

$$
\Rightarrow \text { Contractor }
$$

NO: the worker was not allowed to use her/his own discretion in doing an aspect of the work that was not specified beforehand.

$\Rightarrow$ Employee

UNKNOWN: it is not known whether the worker was allowed to use her/his own discretion in doing an aspect of the work that was not specified beforehand.

$A_{3}$ : Was the worker an integral part of the employer's business?

YES: the worker was an integral part of the employer's business.

$\Rightarrow$ Employee

NO: the worker was not an integral part of the employer's business, but was accessory to it.

$$
\Rightarrow \text { Contractor }
$$

UNKNOWN: it is not known whether the worker was an integral part of the employer's business or was merely accessory to it.

If the worker was "part and parcel" of the employer's business then she/he was an integral part of the business, not merely accessory to it. 
$A_{4}$ : Did the worker own the tools or provide the transport with which she/he performed the work?

YES: the worker owned the tools or provided the transport with which she/he performed the work.

$\Rightarrow$ Contractor

NO: the worker neither owned the tools nor provided the transport with which she/he performed the work.

$\Rightarrow$ Employee

UNKNOWN: it is not known whether the worker owned the tools or provided the transport with which she/he performed the work.

$A_{5}$ : Would the employer make a profit/loss if the work performed by the worker cost less/more than expected?

YES: the employer would make a profit/loss if the work performed by the worker cost less/more than expected.

NO: the employer would not make a profit/loss if the work performed by the worker cost less/more than expected.

UNKNOWN: it is not known whether the employer would make a profit/loss if the work performed by the worker cost less/more than expected.

$A_{6}$ : Was the work performed on the employer's premises?

YES: the work was performed on the employer's premises.

NO: the work was not performed on the employer's premises.

$\Rightarrow$ Contractor

UNKNOWN: it is not known whether the work was performed on the employer's premises.

$A_{7}$ : Did the employer supervise or inspect the work?

YES: the employer supervised or inspected the work.

$\Rightarrow$ Employee

NO: the employer neither supervised nor inspected the work.

$\Rightarrow$ Contractor

UNKNOWN: it is not known whether the employer supervised or inspected the work.

$A_{8}$ : Was the worker in business on her/his own account?

YES: the worker was in business on her/his own account.

$\Rightarrow$ Contractor

NO: the worker was not in business on her/his own account.

$\Rightarrow$ Employee

UNKNOWN: it is not known whether the worker was in business on her/his own account. 
$A_{9}$ : Was the worker allowed to employ others to assist with her/his work?

YES: the worker was allowed to employ others to assist with her/his work.

$\Rightarrow$ Contractor

NO: the worker was not allowed to employ others to assist with her/his work.

$\Rightarrow$ Employee

UNKNOWN: it is not known whether the worker was allowed to employ others to assist with her/his work.

$A_{10}$ : Was the worker obliged to work only for the employer?

YES: the worker was obliged to work only for the employer.

$\Rightarrow$ Employee

NO: the worker was not obliged to work only for the employer.

$\Rightarrow$ Contractor

UNKNOWN: it is not known whether the worker was obliged to work only for the employer.

$A_{11}$ : Was the worker required to work at specified times?

YES: the worker was required to work at specified times.

$$
\Rightarrow \text { Employee }
$$

NO: the worker was not required to work at specified times.

$\Rightarrow$ Contractor

UNKNOWN: it is not known whether the worker was required to work at specified times.

$A_{12}$ : Did the employer pay the worker by time?

YES: the employer paid the worker by time.

$\Rightarrow$ Employee

NO: the employer did not pay the worker by time.

$\Rightarrow$ Contractor

UNKNOWN: it is not known whether the employer paid the worker by time.

The employer could pay the worker by time (e.g. by the hour, or by the week) or by results.

$A_{13}$ : Was the money that the employer paid to the worker stated to be a "fee"?

YES: the money that the employer paid to the worker was stated to be a "fee".

$\Rightarrow$ Contractor

NO: the money that the employer paid to the worker was not stated to be a "fee".

$\Rightarrow$ Employee

UNKNOWN: it is not known whether the money that the employer paid to the worker was stated to be a "fee". 
$A_{14}$ : Was the money that the employer paid to the worker stated to be "wages" or "salary"?

YES: the money that the employer paid to the worker was stated to be "wages" or "salary".

$\Rightarrow$ Employee

NO: the money that the employer paid to the worker was not stated to be "wages" or "salary".

$\Rightarrow$ Contractor

UNKNOWN: it is not known whether the money that the employer paid to the worker was stated to be "wages" or "salary".

$A_{15}$ : Did the employer deduct PAYE tax instalments from the worker's pay?

YES: the employer deducted PAYE tax instalments from the worker's pay. $\Rightarrow$ Employee

NO: the employer did not deduct PAYE tax instalments from the worker's pay.

$\Rightarrow$ Contractor

UNKNOWN: it is not known whether the employer deducted PAYE tax instalments from the worker's pay.

$A_{16}$ : Did the employer pay the worker sick pay or holiday pay?

YES: the employer paid the worker sick pay or holiday pay.

$\Rightarrow$ Employee

NO: the employer paid the worker neither sick pay nor holiday pay.

$\Rightarrow$ Contractor

UNKNOWN: it is not known whether the employer paid the worker sick pay or holiday pay.

$A_{17}$ : Did the employer and the worker express an intention that the relationship would be one of employer and employee?

YES: the employer and the worker expressed an intention that the relationship would be one of employer and employee.

$\Rightarrow$ Employee

NO: the employer and the worker did not express any intention that the relationship would be one of employer and employee.

UNKNOWN: it is not known whether the employer and the worker expressed an intention that the relationship would be one of employer and employee.

For example, if the employer and the worker characterized their agreement as being a "contract of service," that would be an expression of an intention that the relationship would be one of employer and employee. 
$A_{18}$ : Did the employer and the worker express an intention that the relationship would be one of principal and independent contractor?

YES: the employer and the worker expressed an intention that the relationship would be one of principal and independent contractor.

$\Rightarrow$ Contractor

NO: the employer and the worker did not express any intention that the relationship would be one of principal and independent contractor.

UNKNOWN: it is not known whether the employer and the worker expressed an intention that the relationship would be one of principal and independent contractor.

For example, if the employer and the worker characterized their agreement as being a "contract for services," that would be an expression of an intention that the relationship would be one of principal and independent contractor.

\section{Cases in which the worker is an employee}

\section{$C_{1}$ : Zuijs v. Wirth Brothers Pty Ltd (1955) 93 CLR 561 ("Zuijs v. Wirth Brothers")}

$A_{1}$ : the employer did not direct the manner in which the work was to be done.

$A_{2}$ : the worker was allowed to use her/his own discretion in doing an aspect of the work that was not specified beforehand.

$A_{3}$ : the worker was an integral part of the employer's business.

$A_{4}$ : the worker neither owned the tools nor provided the transport with which she/he performed the work.

$A_{5}$ : the employer would make a profit/loss if the work performed by the worker cost less/more than expected.

$A_{6}$ : the work was performed on the employer's premises.

$A_{7}$ : the employer supervised or inspected the work.

$A_{8}$ : the worker was not in business on her/his own account.

$A_{9}$ : the worker was not allowed to employ others to assist with her/his work.

$A_{10}$ : the worker was not obliged to work only for the employer.

$A_{11}$ : the worker was required to work at specified times.

$A_{12}$ : the employer paid the worker by time.

$A_{13}$ : the money that the employer paid to the worker was not stated to be a "fee".

$A_{14}$ : the money that the employer paid to the worker was not stated to be "wages" or "salary".

$A_{15}$ : the employer deducted PAYE tax instalments from the worker's pay.

$A_{16}$ : the employer paid the worker neither sick pay nor holiday pay.

$A_{17}$ : the employer and the worker did not express any intention that the relationship would be one of employer and employee.

$A_{18}$ : the employer and the worker did not express any intention that the relationship would be one of principal and independent contractor. 
In Zuijs v. Wirth Brothers Pty Ltd, ${ }^{1}$ a 1955 decision of five judges of the High Court of Australia, Zuijs was an acrobat who fell during a trapeze act at one of Wirth Brothers' circuses. He sought compensation under the Worker's Compensation Act 1926 (NSW), claiming to be an employee of Wirth Brothers. Wirth Brothers claimed that, because of the high degree of skill and personal judgment that he had to exercise in his work, Zuijs was an independent contractor and therefore not entitled to compensation.

The High Court unanimously agreed with Zuijs. "Even if [Wirth Brothers] could not interfere in the actual technique of the acrobats and in the character of the act, no reason appears why the appellant should not be subject to his directions in all other respects ... There are countless examples of highly specialized functions in modern life that must as a matter of practical necessity and sometimes even as a matter of law be performed on the responsibility of persons who possess particular knowledge and skill and who are accordingly qualified. But those engaged to perform the functions may nevertheless work under a contract of service." $^{2}$

$C_{2}$ : Cam and Sons Pty Ltd v. Sargent (1940) 14 ALJ 162 ("Cam v. Sargent")

$A_{1}$ : the employer directed the manner in which the work was to be done.

$A_{2}$ : the worker was allowed to use her/his own discretion in doing an aspect of the work that was not specified beforehand.

$A_{3}$ : the worker was an integral part of the employer's business.

$A_{4}$ : the worker neither owned the tools nor provided the transport with which she/he performed the work.

$A_{5}$ : the employer would make a profit/loss if the work performed by the worker cost less/more than expected.

$A_{6}$ : the work was not performed on the employer's premises.

$A_{7}$ : the employer neither supervised nor inspected the work.

$A_{8}$ : the worker was not in business on her/his own account.

$A_{9}$ : the worker was allowed to employ others to assist with her/his work.

$A_{10}$ : the worker was not obliged to work only for the employer.

$A_{11}$ : the worker was not required to work at specified times.

$A_{12}$ : the employer did not pay the worker by time.

$A_{13}$ : the money that the employer paid to the worker was not stated to be a "fee".

$A_{14}$ : the money that the employer paid to the worker was not stated to be "wages" or "salary".

\footnotetext{
${ }^{1}(1955) 93$ CLR 561.

2ibid. at 571-2, per Dixon CJ, Williams, Webb and Taylor JJ.
} 
$A_{15}$ : the employer did not deduct PAYE tax instalments from the worker's pay.

$A_{16}$ : the employer paid the worker neither sick pay nor holiday pay.

$A_{17}$ : the employer and the worker did not express any intention that the relationship would be one of employer and employee.

$A_{18}$ : the employer and the worker did not express any intention that the relationship would be one of principal and independent contractor.

In Cam and Sons Pty Ltd v. Sargent, ${ }^{3}$ a 1940 decision of four judges of the High Court of Australia, Sargent was the master of a ship. He entered into an agreement with Cam and Sons that claimed that the ship was hired by Cam and Sons to Sargent and his fellow contractors (called "the partnership"). However, it was doubtful whether that agreement actually deprived Cam and Sons of any control over the ship. The partnership was to use the ship only to carry coal from Swansea to Sydney. Cam and Sons were sole agents of the partnership for securing cargoes for the ship, and for collecting money due to the partnership. The partnership paid nothing for the "hire" of the ship, but received a specified sum for each return trip of a certain tonnage plus (in certain circumstances) $5 \%$ of the earnings, the balance of which was retained by Cam and Sons. Cam and Sons had to approve people employed by the partnership.

Sargent claimed that he (and others in the partnership) were employed by Cam and Sons, and therefore came within the terms of an industrial award. Cam and Sons claimed that members of the partnership were independent contractors.

The High Court unanimously agreed with Sargent. Rich J came to the conclusion that the agreement was an attempt to evade the terms of the industrial award. ${ }^{4}$

$C_{3}$ : Federal Commissioner of Taxation v. J. Walter Thompson (Australia) Pty Ltd (1944) 69 CLR 227 ("FCT v. Thompson")

$A_{1}$ : the employer directed the manner in which the work was to be done.

$A_{2}$ : the worker was not allowed to use her/his own discretion in doing an aspect of the work that was not specified beforehand.

$A_{3}$ : the worker was an integral part of the employer's business.

$A_{4}$ : the worker neither owned the tools nor provided the transport with which she/he performed the work.

$A_{5}$ : the employer would make a profit/loss if the work performed by the worker cost less/more than expected.

$A_{6}$ : the work was performed on the employer's premises.

$A_{7}$ : the employer supervised or inspected the work.

$A_{8}$ : it is not known whether the worker was in business on her/his own account.

3 (1940) 14 ALJ 162.

4 ibid. at 163 . 
$A_{9}$ : the worker was not allowed to employ others to assist with her/his work.

$A_{10}$ : the worker was not obliged to work only for the employer.

$A_{11}$ : the worker was required to work at specified times.

$A_{12}$ : the employer did not pay the worker by time.

$A_{13}$ : the money that the employer paid to the worker was stated to be a "fee".

$A_{14}$ : the money that the employer paid to the worker was not stated to be "wages" or "salary".

$A_{15}$ : the employer did not deduct PAYE tax instalments from the worker's pay.

$A_{16}$ : the employer paid the worker neither sick pay nor holiday pay.

$A_{17}$ : the employer and the worker did not express any intention that the relationship would be one of employer and employee.

$A_{18}$ : the employer and the worker did not express any intention that the relationship would be one of principal and independent contractor.

In Federal Commissioner of Taxation v. J. Walter Thompson (Australia) Pty $L t d,{ }^{5}$ a 1944 decision of a single judge of the High Court of Australia, the FCT claimed that payments made to radio artists by Thompson were "wages" within the meaning of the Pay-roll Tax Assessment Act 1941 (Cth) and therefore taxable. The artists were selected by a producer and paid to appear in radio plays. They were paid a "fee" for each performance, but were paid nothing for attending (compulsory) rehearsals. Thompson claimed that the artists were presumed to know their work and "to render services in the same manner as a professional man, such as a surgeon or an architect, not being subject ... to detailed control as to the manner in which those services are to be performed." ${ }^{6}$ Hence, Thompson claimed, they were independent contractors.

Latham CJ held that the radio actors were employed "to co-operate with others in a team under the control of the producer to bring about a result, the details of which must in great measure be determined by the producer." 7 Hence the artists were employed by Thompson; the fee they were paid was subject to payroll tax.

$C_{4}$ : Australian Timber Workers Union v. Monaro Sawmills Pty Ltd (1980) 29 ALR 322 ("ATWU v. Monaro Sawmills")

$A_{1}$ : the employer directed the manner in which the work was to be done.

$A_{2}$ : the worker was not allowed to use her/his own discretion in doing an aspect of the work that was not specified beforehand.

$A_{3}$ : the worker was an integral part of the employer's business.

$A_{4}$ : the worker owned the tools or provided the transport with which she/he performed the work.

\footnotetext{
5(1944) 69 CLR 227.

6 ibid. at 231 .

7 ibid. at 232 .
} 
$A_{5}$ : the employer would not make a profit/loss if the work performed by the worker cost less/more than expected.

$A_{6}$ : the work was performed on the employer's premises.

$A_{7}$ : the employer supervised or inspected the work.

$A_{8}$ : the worker was not in business on her/his own account.

$A_{9}$ : it is not known whether the worker was allowed to employ others to assist with her/his work.

$A_{10}$ : the worker was not obliged to work only for the employer.

$A_{11}$ : the worker was not required to work at specified times.

$A_{12}$ : the employer did not pay the worker by time.

$A_{13}$ : the money that the employer paid to the worker was not stated to be a "fee".

$A_{14}$ : the money that the employer paid to the worker was not stated to be "wages" or "salary".

$A_{15}$ : the employer did not deduct PAYE tax instalments from the worker's pay.

$A_{16}$ : the employer paid the worker neither sick pay nor holiday pay.

$A_{17}$ : the employer and the worker did not express any intention that the relationship would be one of employer and employee.

$A_{18}$ : the employer and the worker did not express any intention that the relationship would be one of principal and independent contractor.

In Australian Timber Workers Union v. Monaro Sawmills Pty Ltd, ${ }^{8}$ a 1980 decision of three judges of the Federal Court of Australia, Wales was a tree feller who cut timber exclusively for Monaro Sawmills. He performed his work in an area allotted to him by Monaro Sawmills. He, and other fellers, were paid by the amount of millable wood they cut. Wales provided his own tools and transport, but was (with the other fellers) covered by Monaro Sawmill's workers' compensation policy.

The union sought an order that a penalty be imposed on Monaro Sawmills for breaching the Timber Industries Consolidated Award 1974 by failing to pay Wales money in lieu of annual leave. Monaro Sawmills claimed that Wales was an independent contractor, and so was not subject to the award.

Sweeney and Evatt JJ examined the circumstances of Wales's employment and held that those circumstances clearly pointed to the existence of a relationship of employer and employee. They could not see "any sense in which it could be said that Wales was conducting some sort of business of his own." 9

8 (1980) 29 ALR 322.

9ibid. at 329 . 
$C_{5}$ : Ferguson v. John Dawson 83 Partners (Contractors) Ltd [1976] 1 WLR 1213 ("Ferguson v. Dawson")

$A_{1}$ : the employer directed the manner in which the work was to be done.

$A_{2}$ : the worker was not allowed to use her/his own discretion in doing an aspect of the work that was not specified beforehand.

$A_{3}$ : the worker was an integral part of the employer's business.

$A_{4}$ : the worker neither owned the tools nor provided the transport with which she/he performed the work.

$A_{5}$ : the employer would make a profit/loss if the work performed by the worker cost less/more than expected.

$A_{6}$ : the work was not performed on the employer's premises.

$A_{7}$ : the employer supervised or inspected the work.

$A_{8}$ : the worker was not in business on her/his own account.

$A_{9}$ : the worker was not allowed to employ others to assist with her/his work.

$A_{10}$ : the worker was not obliged to work only for the employer.

$A_{11}$ : the worker was required to work at specified times.

$A_{12}$ : the employer paid the worker by time.

$A_{13}$ : the money that the employer paid to the worker was not stated to be a "fee".

$A_{14}$ : the money that the employer paid to the worker was not stated to be "wages" or "salary".

$A_{15}$ : the employer did not deduct PAYE tax instalments from the worker's pay.

$A_{16}$ : the employer paid the worker neither sick pay nor holiday pay.

$A_{17}$ : the employer and the worker did not express any intention that the relationship would be one of employer and employee.

$A_{18}$ : the employer and the worker expressed an intention that the relationship would be one of principal and independent contractor.

In Ferguson v. John Dawson $\&$ Partners (Contractors) Ltd, ${ }^{10}$ a 1976 decision of the English Court of Appeal, Ferguson fell off a roof while removing some scaffolding boards. He claimed damages against John Dawson (the building contractors) for breach of statutory duty relying on the Construction (Working Places) Regulations 1966 (UK). This duty would only be owed if Ferguson was an employee of John Dawson.

Megaw and Browne LJJ held that, despite the fact that both parties labelled Ferguson a "self-employed labour only subcontractor" ${ }^{11}$ the reality of the relationship between them was that of employer/employee.

${ }^{10}[1976] 1$ WLR 1213.

${ }^{11}$ ibid. at 1219, per Megaw LJ; at 1225, per Lawton LJ; at 1228, per Browne LJ. 
$C_{6}$ : Stevenson Jordon and Harrison Ltd v. Macdonald and Evans (2) [1952] 1 TLR 101 ("Stevenson v. Macdonald (2)")

$A_{1}$ : the employer did not direct the manner in which the work was to be done.

$A_{2}$ : the worker was allowed to use her/his own discretion in doing an aspect of the work that was not specified beforehand.

$A_{3}$ : the worker was an integral part of the employer's business.

$A_{4}$ : the worker neither owned the tools nor provided the transport with which she/he performed the work.

$A_{5}$ : the employer would make a profit/loss if the work performed by the worker cost less/more than expected.

$A_{6}$ : the work was not performed on the employer's premises.

$A_{7}$ : it is not known whether the employer supervised or inspected the work.

$A_{8}$ : the worker was not in business on her/his own account.

$A_{9}$ : the worker was not allowed to employ others to assist with her/his work.

$A_{10}$ : the worker was not obliged to work only for the employer.

$A_{11}$ : the worker was required to work at specified times.

$A_{12}$ : it is not known whether the employer paid the worker by time.

$A_{13}$ : it is not known whether the money that the employer paid to the worker was stated to be a "fee".

$A_{14}$ : it is not known whether the money that the employer paid to the worker was stated to be "wages" or "salary".

$A_{15}$ : it is not known whether the employer deducted PAYE tax instalments from the worker's pay.

$A_{16}$ : it is not known whether the employer paid the worker sick pay or holiday pay.

$A_{17}$ : it is not known whether the employer and the worker expressed an intention that the relationship would be one of employer and employee.

$A_{18}$ : the employer and the worker did not express any intention that the relationship would be one of principal and independent contractor.

In Stevenson Jordon and Harrison Ltd v. Macdonald and Evans (2), ${ }^{12}$ a 1952 decision of the English Court of Appeal, Evans-Hemming was an accountant who had been employed (first as a servant, then as an executive officer) by Macdonald and Evans. Shortly after he left them, he wrote a textbook on business management and submitted the manuscript to Stevenson Jordon and Harrison (a firm of publishers). He died before the book was published. Macdonald and Evans claimed that the book was written while Evans-Hemming was their employee, and so they owned the copyright in the work under s. 5(1)(b) of the Copyright Act 1911 (UK). 
The book was divided into five sections. The second section was written in its final form while Evans-Hemming was employed by Macdonald and Evans. The Court of Appeal held that he wrote the second section as an employee, and hence the copyright in the second section was in Macdonald and Evans.

$C_{7}$ : Performing Right Society Ltd v. Mitchell \&3 Booker (Palais de Danse) Ltd [1924] 1 KB 762 ("PRS v. Palais de Danse")

$A_{1}$ : the employer directed the manner in which the work was to be done.

$A_{2}$ : the worker was allowed to use her/his own discretion in doing an aspect of the work that was not specified beforehand.

$A_{3}$ : the worker was an integral part of the employer's business.

$A_{4}$ : the worker owned the tools or provided the transport with which she/he performed the work.

$A_{5}$ : the employer would not make a profit/loss if the work performed by the worker cost less/more than expected.

$A_{6}$ : the work was performed on the employer's premises.

$A_{7}$ : the employer supervised or inspected the work.

$A_{8}$ : the worker was not in business on her/his own account.

$A_{9}$ : the worker was not allowed to employ others to assist with her/his work.

$A_{10}$ : the worker was obliged to work only for the employer.

$A_{11}$ : the worker was required to work at specified times.

$A_{12}$ : the employer paid the worker by time.

$A_{13}$ : the money that the employer paid to the worker was not stated to be a "fee".

$A_{14}$ : the money that the employer paid to the worker was stated to be "wages" or "salary".

$A_{15}$ : the employer did not deduct PAYE tax instalments from the worker's pay.

$A_{16}$ : the employer paid the worker neither sick pay nor holiday pay.

$A_{17}$ : the employer and the worker did not express any intention that the relationship would be one of employer and employee.

$A_{18}$ : the employer and the worker did not express any intention that the relationship would be one of principal and independent contractor.

In Performing Right Society Ltd v. Mitchell $\&$ Booker (Palais de Danse) Ltd, ${ }^{13}$ a 1924 decision of the King's Bench Division of the English High Court, the defendants were the occupiers of a dance hall. They engaged a band to provide music in the hall. The agreement provided that the band should not infringe 
copyright, and that the band would be liable for damages and costs caused by any such infringement. There was also a notice displayed in the hall stating that "[o]nly such music as may be played without fee or licence is allowed to be played in this Hall." 14

The band performed several pieces of music, the copyright in which was held by the Performing Right Society, without its permission. The defendants did not know, and had no reasonable grounds for suspecting, that the infringement was to take place.

The PRS abandoned its earlier claim that the defendants had "permitted" the infringement under s. 2(3) of the Copyright Act 1911 (UK). However, it claimed that the band were the defendants' employees, and so the defendants were vicariously liable for the infringement.

McCardie J examined the agreement and found that it gave "to the defendants the right of continuous, dominant, and detailed control on every point, including the nature of the music to be played". ${ }^{15}$ Hence the band members were employees of the defendant company, which was liable for the infringement.

$I_{\text {Employee }}$ (the ideal case in which the worker is an employee):

$A_{1}$ : the employer directed the manner in which the work was to be done.

$A_{2}$ : the worker was not allowed to use her/his own discretion in doing an aspect of the work that was not specified beforehand.

$A_{3}$ : the worker was an integral part of the employer's business.

$A_{4}$ : the worker neither owned the tools nor provided the transport with which she/he performed the work.

$A_{5}$ : the employer would make a profit/loss if the work performed by the worker cost less/more than expected.

$A_{6}$ : the work was performed on the employer's premises.

$A_{7}$ : the employer supervised or inspected the work.

$A_{8}$ : the worker was not in business on her/his own account.

$A_{9}$ : the worker was not allowed to employ others to assist with her/his work.

$A_{10}$ : the worker was obliged to work only for the employer.

$A_{11}$ : the worker was required to work at specified times.

$A_{12}$ : the employer paid the worker by time.

$A_{13}$ : the money that the employer paid to the worker was not stated to be a "fee".

$A_{14}$ : the money that the employer paid to the worker was stated to be "wages" or "salary".

$A_{15}$ : the employer deducted PAYE tax instalments from the worker's pay.

$A_{16}$ : the employer paid the worker sick pay or holiday pay.

\footnotetext{
14 ibid. at 764 .

15 ibid. at 771 .
} 
$A_{17}$ : the employer and the worker expressed an intention that the relationship would be one of employer and employee.

$A_{18}$ : it is not known whether the employer and the worker expressed an intention that the relationship would be one of principal and independent contractor.

\section{Cases in which the worker is an independent contractor}

\section{$C_{8}$ : Humberstone v. Northern Timber Mills (1949) 79 CLR 389 ("Humberstone v. NTM")}

$A_{1}$ : the employer did not direct the manner in which the work was to be done.

$A_{2}$ : the worker was allowed to use her/his own discretion in doing an aspect of the work that was not specified beforehand.

$A_{3}$ : the worker was not an integral part of the employer's business, but was accessory to it.

$A_{4}$ : the worker owned the tools or provided the transport with which she/he performed the work.

$A_{5}$ : the employer would not make a profit/loss if the work performed by the worker cost less/more than expected.

$A_{6}$ : the work was not performed on the employer's premises.

$A_{7}$ : the employer neither supervised nor inspected the work.

$A_{8}$ : the worker was not in business on her/his own account.

$A_{9}$ : the worker was allowed to employ others to assist with her/his work.

$A_{10}$ : the worker was not obliged to work only for the employer.

$A_{11}$ : the worker was not required to work at specified times.

$A_{12}$ : the employer did not pay the worker by time.

$A_{13}$ : the money that the employer paid to the worker was not stated to be a "fee".

$A_{14}$ : the money that the employer paid to the worker was not stated to be "wages" or "salary".

$A_{15}$ : the employer did not deduct PAYE tax instalments from the worker's pay.

$A_{16}$ : the employer paid the worker neither sick pay nor holiday pay.

$A_{17}$ : the employer and the worker did not express any intention that the relationship would be one of employer and employee.

$A_{18}$ : the employer and the worker did not express any intention that the relationship would be one of principal and independent contractor.

In Humberstone $v$. Northern Timber Mills ${ }^{16}$ a 1949 decision of three judges of the High Court of Australia, Humberstone carried goods for NTM. He had originally held himself out as a carrier, prepared to carry for anyone, but for over twenty years he had carried goods solely for NTM (although he would, infrequently, 
carry back-loads for NTM's customers). Humberstone owned the truck, and paid for petrol and repairs. He was paid weekly on a weight-mileage basis. He was a licenced carrier, and had his name printed on the side of his truck with the description "carrier."

On the way back from a job, he had a puncture. He went home to change the wheel, but exerted himself so strenuously in trying to remove the tyre from the wheel that he became ill and later lapsed into a coma, from which he did not recover. Section 3 of the Worker's Compensation Act 1928 (Vic) had been amended about a year before Humberstone's death so as to include independent contractors in its definition of a "worker" covered by the Act. However, the High Court held that the amendment applied only to contracts entered into after it came into operation. Further, the Court decided that Humberstone was not an employee of NTM. Hence he was not a "worker" under the Act and his widow was not entitled to compensation under the Act.

$C_{9}$ : Queensland Stations Pty Ltd v. Federal Commissioner of Taxation (1945) 70 CLR 539 ("Queensland Stations v. FCT")

$A_{1}$ : the employer did not direct the manner in which the work was to be done.

$A_{2}$ : the worker was allowed to use her/his own discretion in doing an aspect of the work that was not specified beforehand.

$A_{3}$ : the worker was not an integral part of the employer's business, but was accessory to it.

$A_{4}$ : the worker owned the tools or provided the transport with which she/he performed the work.

$A_{5}$ : the employer would not make a profit/loss if the work performed by the worker cost less/more than expected.

$A_{6}$ : the work was not performed on the employer's premises.

$A_{7}$ : the employer supervised or inspected the work.

$A_{8}$ : the worker was in business on her/his own account.

$A_{9}$ : the worker was allowed to employ others to assist with her/his work.

$A_{10}$ : the worker was not obliged to work only for the employer.

$A_{11}$ : the worker was not required to work at specified times.

$A_{12}$ : the employer did not pay the worker by time.

$A_{13}$ : the money that the employer paid to the worker was not stated to be a "fee".

$A_{14}$ : the money that the employer paid to the worker was not stated to be "wages" or "salary".

$A_{15}$ : the employer did not deduct PAYE tax instalments from the worker's pay.

$A_{16}$ : the employer paid the worker neither sick pay nor holiday pay.

$A_{17}$ : the employer and the worker expressed an intention that the relationship would be one of employer and employee.

$A_{18}$ : the employer and the worker did not express any intention that the relationship would be one of principal and independent contractor. 
In Queensland Stations Pty Ltd v. Federal Commissioner of Taxation, ${ }^{17}$ a 1945 decision of three judges of the High Court of Australia, agreements were entered into between Queensland Stations and some drovers. The agreements stated that the drovers would "serve" Queensland Stations and take charge of a specified number of cattle, and deliver them to a specified place. The drovers were paid a specified rate per head of cattle successfully delivered. Each drover was responsible for hiring help, and paying for feed for the cattle. The drovers were to "obey and carry out all lawful instructions and to use the whole of [their] time, energy and ability in the careful droving of the stock." 18 The FCT claimed that payments made to drovers were "wages" within the meaning of the Pay-roll Tax Assessment Act 1941 (Cth), and that Queensland Stations was liable to payroll tax.

The High Court held that the drovers were independent contractors, so the payments were not "wages". Rich J pointed out that drovers were traditionally free from the control of owners of cattle. "The obligation imposed on the drover to obey and carry out all lawful instructions is not a reservation of detailed control and possession having regard to the terms of the agreement as a whole." 19

$C_{10}$ : Price v. Grant Industries Pty Ltd (1978) 21 ALR 388 ("Price v. Grant Industries")

$A_{1}$ : the employer did not direct the manner in which the work was to be done.

$A_{2}$ : the worker was allowed to use her/his own discretion in doing an aspect of the work that was not specified beforehand.

$A_{3}$ : the worker was an integral part of the employer's business.

$A_{4}$ : the worker owned the tools or provided the transport with which she/he performed the work.

$A_{5}$ : the employer would not make a profit/loss if the work performed by the worker cost less/more than expected.

$A_{6}$ : the work was not performed on the employer's premises.

$A_{7}$ : the employer neither supervised nor inspected the work.

$A_{8}$ : the worker was not in business on her/his own account.

$A_{9}$ : the worker was allowed to employ others to assist with her/his work.

$A_{10}$ : the worker was not obliged to work only for the employer.

$A_{11}$ : the worker was not required to work at specified times.

$A_{12}$ : the employer did not pay the worker by time.

$A_{13}$ : the money that the employer paid to the worker was not stated to be a "fee".

$A_{14}$ : the money that the employer paid to the worker was not stated to be "wages" or "salary".

${ }^{17}$ (1945) 70 CLR 539.

18 ibid. at 540

${ }^{19}$ ibid. at 549 . 
$A_{15}$ : the employer deducted PAYE tax instalments from the worker's pay.

$A_{16}$ : the employer paid the worker neither sick pay nor holiday pay.

$A_{17}$ : the employer and the worker did not express any intention that the relationship would be one of employer and employee.

$A_{18}$ : the employer and the worker did not express any intention that the relationship would be one of principal and independent contractor.

In Price v. Grant Industries Pty Ltd, ${ }^{20}$ a 1978 decision of three judges of the Federal Court of Australia, Grant Industries manufactured and sold wardrobes, which Price (and others) delivered and installed. Price and each of the other "contractors" (as Grant Industries called them) had to provide and maintain a suitable truck to deliver the wardrobes, and provide the tools required to install them. Price sought an order that a penalty be imposed on Grant Industries for breaching the Furnishing Trades (Consolidated) Award 1975 by not paying Price the appropriate rate of wages, and not giving him annual leave. The award only applied to "employees" of specified employers.

The Federal Court examined the facts, and the provisions of the agreement, and held that Price was an independent contractor and, therefore, not subject to the award.

$C_{11}$ : Australian Mutual Provident Society v. Chaplin (1978) 18 ALR 385 ("AMP v. Chaplin")

$A_{1}$ : the employer did not direct the manner in which the work was to be done.

$A_{2}$ : the worker was allowed to use her/his own discretion in doing an aspect of the work that was not specified beforehand.

$A_{3}$ : the worker was an integral part of the employer's business.

$A_{4}$ : the worker owned the tools or provided the transport with which she/he performed the work.

$A_{5}$ : the employer would not make a profit/loss if the work performed by the worker cost less/more than expected.

$A_{6}$ : the work was not performed on the employer's premises.

$A_{7}$ : the employer neither supervised nor inspected the work.

$A_{8}$ : the worker was in business on her/his own account.

$A_{9}$ : the worker was allowed to employ others to assist with her/his work.

$A_{10}$ : the worker was obliged to work only for the employer.

$A_{11}$ : the worker was not required to work at specified times.

$A_{12}$ : the employer did not pay the worker by time.

$A_{13}$ : the money that the employer paid to the worker was not stated to be a "fee".

${ }^{20}(1978) 21$ ALR 388. 
$A_{14}$ : the money that the employer paid to the worker was not stated to be "wages" or "salary".

$A_{15}$ : the employer did not deduct PAYE tax instalments from the worker's pay.

$A_{16}$ : the employer paid the worker neither sick pay nor holiday pay.

$A_{17}$ : the employer and the worker did not express any intention that the relationship would be one of employer and employee.

$A_{18}$ : the employer and the worker expressed an intention that the relationship would be one of principal and independent contractor.

In Australian Mutual Provident Society v. Chaplin, ${ }^{21}$ a 1978 decision of the Judicial Committee of the Privy Council, Chaplin was a representative of AMP. A clause of the agreement between them stated that the relationship was one of "principal and agent" and not one of "master and servant". Chaplin claimed that he was employed under a contract of service, and therefore a "worker" under the Long Service Leave Act, 1967 (SA) and entitled to certain benefits.

The Privy Council found that there was no reason to think that the clause was not a genuine statement of the parties' intentions. Examining the agreement, their lordships concluded that it provided for a contract of agency. The fact that Chaplin was given the power of unlimited delegation of the whole performance of his work was "almost conclusive against the contract being a contract of service." 22

$C_{12}$ : Massey v. Crown Life Insurance Co. [1978] ICR 590 ("Massey v. Crown Life")

$A_{1}$ : the employer directed the manner in which the work was to be done.

$A_{2}$ : the worker was allowed to use her/his own discretion in doing an aspect of the work that was not specified beforehand.

$A_{3}$ : the worker was an integral part of the employer's business.

$A_{4}$ : the worker neither owned the tools nor provided the transport with which she/he performed the work.

$A_{5}$ : the employer would make a profit/loss if the work performed by the worker cost less/more than expected.

$A_{6}$ : the work was performed on the employer's premises.

$A_{7}$ : the employer neither supervised nor inspected the work.

$A_{8}$ : the worker was in business on her/his own account.

$A_{9}$ : the worker was allowed to employ others to assist with her/his work.

$A_{10}$ : the worker was not obliged to work only for the employer.

$A_{11}$ : the worker was required to work at specified times.

${ }^{21}(1978) 18$ ALR 385.

22 ibid. at 391 . 
$A_{12}$ : the employer paid the worker by time.

$A_{13}$ : the money that the employer paid to the worker was not stated to be a "fee".

$A_{14}$ : the money that the employer paid to the worker was not stated to be "wages" or "salary".

$A_{15}$ : the employer did not deduct PAYE tax instalments from the worker's pay.

$A_{16}$ : the employer paid the worker sick pay or holiday pay.

$A_{17}$ : the employer and the worker did not express any intention that the relationship would be one of employer and employee.

$A_{18}$ : the employer and the worker expressed an intention that the relationship would be one of principal and independent contractor.

In Massey v. Crown Life Insurance Co. ${ }^{23}$ a 1978 decision of the English Court of Appeal, Massey was the manager of a branch of Crown Life. He had been an employee for two years, then he and Crown Life entered into a new agreement whereby Massey continued to perform the same duties as before, but was selfemployed. This arrangement had tax advantages for Massey. After a further two years, Crown Life terminated the agreement and Massey sought compensation for unfair dismissal under the Trade Union and Labour Relations Act 1974 (UK). Compensation was only payable if Massey was employed under a contract of service.

Lord Denning MR stated that "if the true relationship of the parties is that of master and servant under a contract of service, the parties cannot alter the truth of that relationship by putting a different label upon it." ${ }^{24}$ However, he (and the rest of the Court of Appeal) held that the agreement was genuinely intended to establish Massey as being self-employed: an independent contractor.

$C_{13}$ : Stevenson Jordon and Harrison Ltd v. Macdonald and Evans (1) [1952] 1 TLR 101 ("Stevenson v. Macdonald (1)")

$A_{1}$ : the employer did not direct the manner in which the work was to be done.

$A_{2}$ : the worker was allowed to use her/his own discretion in doing an aspect of the work that was not specified beforehand.

$A_{3}$ : the worker was not an integral part of the employer's business, but was accessory to it.

$A_{4}$ : the worker neither owned the tools nor provided the transport with which she/he performed the work.

$A_{5}$ : the employer would make a profit/loss if the work performed by the worker cost less/more than expected.

$A_{6}$ : the work was not performed on the employer's premises.

${ }^{23}$ [1978] ICR 590.

24 ibid. at 594 . 
$A_{7}$ : it is not known whether the employer supervised or inspected the work.

$A_{8}$ : the worker was not in business on her/his own account.

$A_{9}$ : the worker was not allowed to employ others to assist with her/his work.

$A_{10}$ : the worker was not obliged to work only for the employer.

$A_{11}$ : the worker was required to work at specified times.

$A_{12}$ : it is not known whether the employer paid the worker by time.

$A_{13}$ : it is not known whether the money that the employer paid to the worker was stated to be a "fee".

$A_{14}$ : it is not known whether the money that the employer paid to the worker was stated to be "wages" or "salary".

$A_{15}$ : it is not known whether the employer deducted PAYE tax instalments from the worker's pay.

$A_{16}$ : it is not known whether the employer paid the worker sick pay or holiday pay.

$A_{17}$ : the employer and the worker expressed an intention that the relationship would be one of employer and employee.

$A_{18}$ : the employer and the worker did not express any intention that the relationship would be one of principal and independent contractor.

In Stevenson Jordon and Harrison Ltd v. Macdonald and Evans (1), ${ }^{25}$ a 1952 decision of the English Court of Appeal, Evans-Hemming was an accountant who had been employed (first as a servant, then as an executive officer) by Macdonald and Evans. Shortly after he left them, he wrote a textbook on business management and submitted the manuscript to Stevenson Jordon and Harrison (a firm of publishers). He died before the book was published. Macdonald and Evans claimed that the book was written while Evans-Hemming was their employee, and so they owned the copyright in the work under s. 5(1)(b) of the Copyright Act 1911 (UK).

The book was divided into five sections. The first section consisted of the text of three public lectures that Evans-Hemming had given while employed by Macdonald and Evans. The Court of Appeal held that he had given these lectures as an independent contractor. As Denning LJ said, "under a contract of service, a man is employed as part of the business, and his work is done as an integral part of the business; whereas, under a contract for services, his work, although done for the business, is not integrated into it but is only accessory to it ... The lectures were, in a sense, part of the services rendered by Mr Evans-Hemming for the benefit of the company. But they were in no sense part of his service. It follows that the copyright in the lectures was in Mr Evans-Hemming." 26

25 [1952] 1 TLR 101.

${ }^{26}$ ibid. at 111 
$C_{14}$ : Ready Mixed Concrete (South East) Ltd v. Minister of Pensions and National Insurance [1968] 2 QB 497 ("Ready Mixed v. Minister")

$A_{1}$ : the employer did not direct the manner in which the work was to be done.

$A_{2}$ : the worker was allowed to use her/his own discretion in doing an aspect of the work that was not specified beforehand.

$A_{3}$ : the worker was an integral part of the employer's business.

$A_{4}$ : the worker owned the tools or provided the transport with which she/he performed the work.

$A_{5}$ : the employer would not make a profit/loss if the work performed by the worker cost less/more than expected.

$A_{6}$ : the work was not performed on the employer's premises.

$A_{7}$ : the employer neither supervised nor inspected the work.

$A_{8}$ : the worker was not in business on her/his own account.

$A_{9}$ : the worker was allowed to employ others to assist with her/his work.

$A_{10}$ : the worker was obliged to work only for the employer.

$A_{11}$ : the worker was not required to work at specified times.

$A_{12}$ : the employer did not pay the worker by time.

$A_{13}$ : the money that the employer paid to the worker was not stated to be a "fee".

$A_{14}$ : the money that the employer paid to the worker was not stated to be "wages" or "salary".

$A_{15}$ : the employer did not deduct PAYE tax instalments from the worker's pay.

$A_{16}$ : the employer paid the worker neither sick pay nor holiday pay.

$A_{17}$ : the employer and the worker did not express any intention that the relationship would be one of employer and employee.

$A_{18}$ : the employer and the worker expressed an intention that the relationship would be one of principal and independent contractor.

In Ready Mixed Concrete (South East) Ltd v. Minister of Pensions and National Insurance, ${ }^{27}$ a 1967 decision of the Queen's Bench Division of the English High Court, Latimer worked for Ready Mixed as an "owner-driver." He was paid at mileage rates, and was obliged to buy the truck through a financial organization associated with Ready Mixed. The truck was painted in the company's colours, and he had to wear a Ready Mixed uniform. Latimer was obliged to meet the 
costs of maintenance, repair and insurance of the truck (and the attached mixing unit, which belonged to Ready Mixed). The Minister determined that Latimer was employed under a contract of service and therefore an "employed person" under s. 1(2) of the National Insurance Act 1965 (UK), making Ready Mixed liable to make weekly contributions.

MacKenna J examined the contract and held that the rights it conferred, and the duties it imposed, between Latimer and Ready Mixed were not such as to make it a contract of service.

$I_{\text {Contractor }}$ (the ideal case in which the worker is an independent contractor):

$A_{1}$ : the employer did not direct the manner in which the work was to be done.

$A_{2}$ : the worker was allowed to use her/his own discretion in doing an aspect of the work that was not specified beforehand.

$A_{3}$ : the worker was not an integral part of the employer's business, but was accessory to it.

$A_{4}$ : the worker owned the tools or provided the transport with which she/he performed the work.

$A_{5}$ : the employer would not make a profit/loss if the work performed by the worker cost less/more than expected.

$A_{6}$ : it is not known whether the work was performed on the employer's premises.

$A_{7}$ : the employer neither supervised nor inspected the work.

$A_{8}$ : the worker was in business on her/his own account.

$A_{9}$ : the worker was allowed to employ others to assist with her/his work.

$A_{10}$ : the worker was not obliged to work only for the employer.

$A_{11}$ : the worker was not required to work at specified times.

$A_{12}$ : the employer did not pay the worker by time.

$A_{13}$ : the money that the employer paid to the worker was stated to be a "fee".

$A_{14}$ : the money that the employer paid to the worker was not stated to be "wages" or "salary".

$A_{15}$ : the employer did not deduct PAYE tax instalments from the worker's pay.

$A_{16}$ : the employer paid the worker neither sick pay nor holiday pay.

$A_{17}$ : it is not known whether the employer and the worker expressed an intention that the relationship would be one of employer and employee.

$A_{18}$ : the employer and the worker expressed an intention that the relationship would be one of principal and independent contractor. 


\section{A.5 The Natural specification}

\section{Hierarchy}

\begin{tabular}{|c|l|}
\hline$c$ & \multicolumn{1}{|c|}{ Court } \\
\hline \hline 1 & seven judges of the High Court of Australia \\
2 & five judges of the High Court of Australia \\
3 & three judges of the High Court of Australia \\
4 & the Full Court of the Federal Court of Australia \\
5 & the New South Wales Court of Appeal \\
6 & the Supreme Court of New South Wales \\
7 & the Judicial Committee of the Privy Council \\
8 & the House of Lords \\
9 & the Chancery Division of the English High Court \\
\hline
\end{tabular}

\section{Natural area}

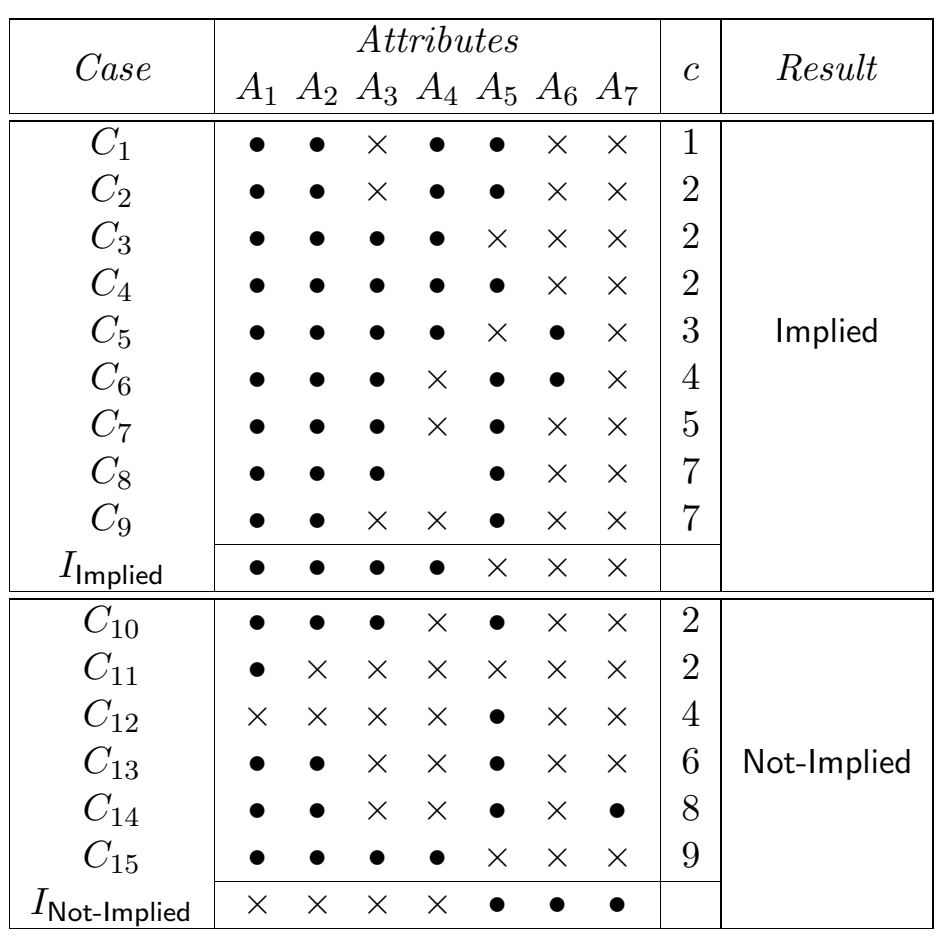

\section{Opening}

In recent years courts have tended to imply a duty to observe the principles of natural justice. It has been said that "[t]he law has now developed to a point where it may be accepted that there is a common law duty to act fairly, in the sense of according procedural fairness, in the making of administrative decisions which affect rights, interests 
and legitimate expectations, subject only to the clear manifestation of a contrary statutory intention." ${ }^{1}$ However, there are some circumstances in which a duty to observe natural justice will not be implied: "the law has not yet reached the stage of applying the obligation of natural justice to every decision which disadvantages individuals." 2

\section{Results}

Implied: a duty to observe natural justice is implied.

Not-Implied: a duty to observe natural justice is not implied.

\section{Attributes}

$A_{1}: \Leftrightarrow$ Affected area

YES: the decision affected the property, right, interest, status, or legitimate expectation of the applicant.

$\Leftarrow$ Affected

$\Rightarrow$ Implied

NO: the decision did not affect the property, right, interest, status, or legitimate expectation of the applicant.

$\Leftarrow$ Unaffected

$\Rightarrow$ Not-Implied

UNKNOWN: it is not known whether the decision affected the property, right, interest, status, or legitimate expectation of the applicant.

$A_{2}$ : Is the decision apt to have a discrete impact on the interests of the applicant?

YES: the decision is apt to have a discrete impact on the interests of the applicant.

$\Rightarrow$ Implied

NO: the decision is not apt to have a discrete impact on the interests of the applicant.

$\Rightarrow$ Not-Implied

UNKNOWN: it is not known whether the decision is apt to have a discrete impact on the interests of the applicant.

If the decision affects the applicant differently to the way in which it affects others then the decision has a discrete impact on the interest of the applicant. Note that the applicant must suffer the detriment as a direct and immediate effect of the decision, not as a contingent result.

${ }^{1}$ Kioa v. West (1985) 159 CLR 550 at 584, per Mason J.

${ }^{2}$ Minister for Arts Heritage and Environment v. Peko-Wallsend Ltd (1987) 75 ALR 218 at 251, per Wilcox J. 
$A_{3}$ : Is the power of a nature that would suggest that procedural fairness would be applied?

YES: the power is of a nature that would suggest that procedural fairness would be applied.

$\Rightarrow$ Implied

NO: the power is of a nature that would suggest that procedural fairness would not be applied.

$\Rightarrow$ Not-Implied

UNKNOWN: it is not known whether the power is of a nature that would suggest that procedural fairness would be applied.

Some prerogative powers by their nature suggest that procedural fairness would not apply in their exercise (e.g. international relations, security, defence, emergency). The exercise of a high-level policy-making power, a broad unfettered discretion, or (in some circumstances) a power which is recommendatory only, suggests that procedural fairness would not apply. However, the exercise of an administrative power or discretion that has been curtailed by statute suggests that procedural fairness would apply.

$A_{4}$ : Did the statutory or factual criteria focus on matters which were discrete to the interests of the applicant?

YES: the statutory or factual criteria focused on matters which were discrete to the interests of the applicant.

$\Rightarrow$ Implied

NO: the statutory or factual criteria focused on matters of policy or public interest.

$\Rightarrow$ Not-Implied

UNKNOWN: it is not known whether the statutory or factual criteria focused on matters which were discrete to the interests of the applicant, or on matters of policy or public interest.

The decisional criteria are of two kinds: the spectrum of considerations to which the decision maker was authorized to have regard (the statutory criteria), and the specific considerations to which regard was had in fact (the factual criteria). Either set of criteria can focus on matters which are discrete to the interests of the applicant, or on matters of policy or public interest.

$A_{5}$ : Was the decision-maker a high-level policy-maker?

YES: the decision-maker was a high-level policy-maker.

$\Rightarrow$ Not-Implied

NO: the decision-maker was not a high-level policy-maker.

$\Rightarrow$ Implied

UNKNOWN: it is not known whether the decision-maker was a high-level policymaker.

Ministers, members of Cabinet, Governors and the Governor-General are highlevel policy-makers. 
$A_{6}$ : Is there a statutory right to appeal against the decision?

YES: there is a statutory right to appeal against the decision. $\Rightarrow$ Not-Implied

NO: there is no statutory right to appeal against the decision. $\Rightarrow$ Implied

UNKNOWN: it is not known whether there is a statutory right to appeal against the decision.

$A_{7}$ : Were there circumstances which make an obligation to observe natural justice inappropriate?

YES: there were circumstances which made an obligation to observe natural justice inappropriate.

$\Rightarrow$ Not-Implied

NO: there were no circumstances which would have made an obligation to observe natural justice inappropriate.

UNKNOWN: it is not known whether there were circumstances which made an obligation to observe natural justice inappropriate.

For example, a prompt or urgent decision may have been necessary, or they may have been national security considerations.

\section{Cases in which a duty to observe natural justice is implied}

$C_{1}$ : FAI Insurances Ltd v. Winneke (1982) 151 CLR 342 ("FAI v. Winneke")

$A_{1}$ : the decision affected the property, right, interest, status, or legitimate expectation of the applicant.

$A_{2}$ : the decision is apt to have a discrete impact on the interests of the applicant.

$A_{3}$ : the power is of a nature that would suggest that procedural fairness would not be applied.

$A_{4}$ : the statutory or factual criteria focused on matters which were discrete to the interests of the applicant.

$A_{5}$ : the decision-maker was a high-level policy-maker.

$A_{6}$ : there is no statutory right to appeal against the decision.

$A_{7}$ : there were no circumstances which would have made an obligation to observe natural justice inappropriate.

In FAI Insurances Ltd v. Winneke, ${ }^{3}$ a 1982 decision of seven judges of the High Court of Australia, Winneke was the Governor of Victoria. The Workers Compensation Act 1958 (Vic) made accident insurance compulsory for all employers, and required them to obtain that insurance from the Insurance Commissioner or from an insurer approved by the Governor in Council. Regulations made under the Act allowed the Governor in Council to grant an approval for a period 
not exceeding twelve months, and (on application) to renew an approval for any period not exceeding twelve months "if the Governor in Council thinks fit." FAI had had its approval to provide worker's compensation renewed every year for twenty years, until 1981.

Gibbs CJ, Stephen, Mason, Aickin, Wilson and Brennan JJ (Murphy J dissenting) held that in deciding whether to renew an approval previously given, the Governor in Council is subject to the requirements of natural justice. "In these circumstances, a company which becomes an approved insurer has a legitimate expectation that its approval will be renewed unless some good reason exists for refusing to renew it. It would not be fair to deprive a company of the ability to carry on its business without revealing the reason for doing so". ${ }^{4}$

$C_{2}$ : Haoucher v. Minister of State for Immigration and Ethnic Affairs (1990) 169 CLR 648 ("Haoucher v. Minister for Immigration")

$A_{1}$ : the decision affected the property, right, interest, status, or legitimate expectation of the applicant.

$A_{2}$ : the decision is apt to have a discrete impact on the interests of the applicant.

$A_{3}$ : the power is of a nature that would suggest that procedural fairness would not be applied.

$A_{4}$ : the statutory or factual criteria focused on matters which were discrete to the interests of the applicant.

$A_{5}$ : the decision-maker was a high-level policy-maker.

$A_{6}$ : there is no statutory right to appeal against the decision.

$A_{7}$ : there were no circumstances which would have made an obligation to observe natural justice inappropriate.

In Haoucher v. Minister of State for Immigration and Ethnic Affairs, ${ }^{5}$ a 1990 decision of five judges of the High Court of Australia, Haoucher had been convicted of an offence for which he had been sentenced to imprisonment for a period not less than one year. Hence - as Haoucher was not an Australian citizen - the Minister had the power to order his deportation under s. 12 of the Migration Act 1958 (Cth), and did so. The Minister had previously told Parliament that the Government's policy was that a deportee had the right to appeal to the Administrative Appeals Tribunal, and that "recommendations of the Administrative Appeals Tribunal should be overturned by the Minister only in exceptional circumstances and only when strong evidence can be produced to justify his decision."

Haoucher appealed to the AAT which recommended that the deportation order be revoked. The Minister decided not to accept that recommendation. Deane, Toohey and McHugh (Dawson and Gaudron dissenting) held that Haoucher was entitled to know the matters which constituted "exceptional circumstances" and

4 ibid. at 348, per Gibbs CJ.

${ }^{5}$ (1990) 169 CLR 648. 
"strong evidence" sufficient for the Minister to depart from the general policy of following the AAT's recommendations. The fact that the Minister had not given Haoucher such details, and had given him no opportunity to make representations, was a denial of natural justice.

$C_{3}$ : Annetts v. McCann (1990) 170 CLR 596

$A_{1}$ : the decision affected the property, right, interest, status, or legitimate expectation of the applicant.

$A_{2}$ : the decision is apt to have a discrete impact on the interests of the applicant.

$A_{3}$ : the power is of a nature that would suggest that procedural fairness would be applied.

$A_{4}$ : the statutory or factual criteria focused on matters which were discrete to the interests of the applicant.

$A_{5}$ : the decision-maker was not a high-level policy-maker.

$A_{6}$ : there is no statutory right to appeal against the decision.

$A_{7}$ : there were no circumstances which would have made an obligation to observe natural justice inappropriate.

In Annetts v. McCann, ${ }^{6}$ a 1990 decision of five judges of the High Court of Australia, a coroner had been conducting an inquest into the death of a 16-year old boy. The boy's parents (Mr and Mrs Annetts) sought to make a submission before the coroner made a finding. The coroner decided that the Coroner's Act 1920 (WA) gave him the discretion (which he chose to exercise) to disallow their submission. The Annettses appealed.

The High Court (Mason CJ, Brennan, Deane, Toohey and McHugh JJ) held that their son's reputation gave the Annettses an interest in the Coroner's inquiry. "A finding in an inquest into a death is naturally likely to deal with the conduct of the deceased leading to death. An unfavourable reflection on the deceased is usually a matter of concern to her or his parents, spouse or children and, if they choose to appear at the inquest in order to safeguard the reputation of the deceased, the familial relationship suffices, in my view, to establish the deceased's reputation as a relevant interest which should not be adversely affected without according natural justice to those who are seeking to safeguard that reputation."7

The Court held that the fact that the coroner's decision was merely recommendatory (whether or not to prosecute) was not sufficient to avoid the implication of natural justice; the coroner was bound to hear the Annettses before making any finding adverse to them or their son. ${ }^{8}$

${ }^{6}(1990) 170$ CLR 596.

7 ibid. at 612 , per Brennan J.

8ibid. at 603, per Mason CJ, Deane and McHugh JJ; at 612 per Brennan J; at 621 per Toohey J. Note, however, that Brennan and Toohey JJ dismissed the appeal because they believed that the decision of the Full Court of the Supreme Court of Western Australia (from which the Annettses appealed) was right on the material before it. 
$C_{4}$ : Kioa v. West (1985) 159 CLR 550

$A_{1}$ : the decision affected the property, right, interest, status, or legitimate expectation of the applicant.

$A_{2}$ : the decision is apt to have a discrete impact on the interests of the applicant.

$A_{3}$ : the power is of a nature that would suggest that procedural fairness would be applied.

$A_{4}$ : the statutory or factual criteria focused on matters which were discrete to the interests of the applicant.

$A_{5}$ : the decision-maker was a high-level policy-maker.

$A_{6}$ : there is no statutory right to appeal against the decision.

$A_{7}$ : there were no circumstances which would have made an obligation to observe natural justice inappropriate.

In Kioa v. West, ${ }^{9}$ a 1985 decision of five judges of the High Court of Australia, Kioa and his wife were Tongan citizens. They were each granted a temporary entry permit. When their entry permits expired, they stayed in Australia and their daughter was born (becoming an Australian citizen). The Minister's delegate made an order for their deportation under the Migration Act 1958 (Cth). They were given no opportunity to answer prejudicial statements against them made to the Minister's delegate.

Mason, Wilson, Brennan and Deane JJ (Gibbs CJ dissenting) held that, in the absence of statutory provisions to the contrary, the requirements of natural justice should have been observed in relation to the making of the deportation order. The deportation order was set aside.

$C_{5}$ : Commissioner of Police v. Tanos (1958) 98 CLR 383

$A_{1}$ : the decision affected the property, right, interest, status, or legitimate expectation of the applicant.

$A_{2}$ : the decision is apt to have a discrete impact on the interests of the applicant.

$A_{3}$ : the power is of a nature that would suggest that procedural fairness would be applied.

$A_{4}$ : the statutory or factual criteria focused on matters which were discrete to the interests of the applicant.

$A_{5}$ : the decision-maker was not a high-level policy-maker.

$A_{6}$ : there is a statutory right to appeal against the decision.

$A_{7}$ : there were no circumstances which would have made an obligation to observe natural justice inappropriate.

In Commissioner of Police v. Tanos, ${ }^{10}$ a 1958 decision of three judges of the High Court of Australia, a judge had made a declaration that a restaurant run by Tanos was a "disorderly house" pursuant to s. 3(1)(b) of the Disorderly Houses

${ }^{9}$ (1985) 159 CLR 550.

10 (1958) 98 CLR 383. 
Act 1943 (NSW). A police inspector had sworn an affidavit as to his suspicion and belief that liquor had been unlawfully sold or supplied on the premises and was likely to unlawfully sold or supplied on the premises again. The judge made the declaration ex parte.

The stated grounds for the police inspector's suspicion concerned things that Tanos's husband had done when he had been running the restaurant. Tanos claimed that things had changed since she had taken over: "the patronage of a much more desirable class of customer was obtained, a class which would not demand wine with their food."11 Tanos appealed successfully to the Supreme Court.

Dixon CJ, Webb and Taylor JJ held that Tanos's appeal should have been dismissed by the Supreme Court; once the declaration was made, the Act placed the burden on Tanos to prove that liquor had never been sold or supplied on the premises, and she had not proved that. However, the High Court also held that, except in exceptional and special circumstances, an owner/occupier should have an opportunity to be heard before her/his premises are declared "disorderly". Tanos had been denied that opportunity, so the declaration was set aside.

$C_{6}$ : Marine Hull \& Liability Insurance Co. Ltd v. Hurford (1986) 67 ALR 77 ("Marine Hull v. Hurford")

$A_{1}$ : the decision affected the property, right, interest, status, or legitimate expectation of the applicant.

$A_{2}$ : the decision is apt to have a discrete impact on the interests of the applicant.

$A_{3}$ : the power is of a nature that would suggest that procedural fairness would be applied.

$A_{4}$ : the statutory or factual criteria focused on matters of policy or public interest.

$A_{5}$ : the decision-maker was a high-level policy-maker.

$A_{6}$ : there is a statutory right to appeal against the decision.

$A_{7}$ : there were no circumstances which would have made an obligation to observe natural justice inappropriate.

In Marine Hull \& Liability Insurance Co. Ltd v. Hurford, ${ }^{12}$ a 1986 decision of the Full Court of the Federal Court of Australia, Hurford was Acting Treasurer. He forbade Marine Hull from issuing or renewing insurance policies, using his powers under s. 62 of the Insurance Act 1973 (Cth). Marine Hull complained that they had not been granted a hearing before the Minister made his direction.

In the first case, Wilcox J held that the Treasurer should have applied the principles of natural justice before making his direction under s. 62 . However, because s. 63 of the Act provided for a review of any such directions by the Administrative Appeals Tribunal, "the legislature must be taken to have evinced an intention

${ }^{11}$ ibid. at 389, per Dixon CJ and Webb J.

12 (1986) 67 ALR 77. 
that, in the event of the Treasurer failing to so act, the directions are not to be regarded as being invalid in law. They are merely susceptible of challenge before the Tribunal." 13

Marine Hull appealed to the Full Court, which agreed with Wilcox J that natural justice had not been denied in this case; the Acting Treasurer's direction was not invalidated by his failing to allow Marine Hull a prior hearing. However, the Full Court also held that the "[t]he existence of a right to have a matter reconsidered ... may well affect the nature of the procedures which ought to be adopted in complying with the rules of natural justice but, ordinarily, it does not exclude them." 14

$C_{7}$ : Macrae v. Attorney-General for New South Wales (1987) 9 NSWLR 268 ("Macrae v. $A G$ for $N S W^{\prime \prime)}$

$A_{1}$ : the decision affected the property, right, interest, status, or legitimate expectation of the applicant.

$A_{2}$ : the decision is apt to have a discrete impact on the interests of the applicant.

$A_{3}$ : the power is of a nature that would suggest that procedural fairness would be applied.

$A_{4}$ : the statutory or factual criteria focused on matters of policy or public interest.

$A_{5}$ : the decision-maker was a high-level policy-maker.

$A_{6}$ : there is no statutory right to appeal against the decision.

$A_{7}$ : there were no circumstances which would have made an obligation to observe natural justice inappropriate.

In Macrae v. Attorney-General for New South Wales, ${ }^{15}$ a 1987 decision of the New South Wales Court of Appeal, five magistrates who had been appointed under the Justices Act 1902 (NSW) were not appointed under the Local Courts Act 1982 (NSW). The new Act had reorganized the magistracy in NSW, and magistrates appointed under the old Act were entitled to apply for appointment as magistrates under the new Act. The five had applied and were interviewed. Allegations were made privately to the Attorney-General claiming that they were unfit to be appointed, but these allegations were not brought to their notice at the time of the interviews.

The Court of Appeal held that the Attorney-General's decision not to recommend the appointment of the magistrates was void because they were denied their legitimate expectation of procedural fairness. "They have not been treated fairly." 16

${ }^{13}(1985) 62$ ALR 253 at 266.

${ }^{14}(1986) 67$ ALR 77 at 81, per Davies J.

${ }^{15}(1987) 9$ NSWLR 268.

${ }^{16}$ ibid. at 283, per Kirby P. 
$C_{8}$ : Attorney-General of Hong Kong v. Ng Yuen Shiu [1983] 2 AC 629 ("AG of Hong Kong v. Shiu")

$A_{1}$ : the decision affected the property, right, interest, status, or legitimate expectation of the applicant.

$A_{2}$ : the decision is apt to have a discrete impact on the interests of the applicant.

$A_{3}$ : the power is of a nature that would suggest that procedural fairness would be applied.

$A_{4}$ : it is not known whether the statutory or factual criteria focused on matters which were discrete to the interests of the applicant, or on matters of policy or public interest.

$A_{5}$ : the decision-maker was a high-level policy-maker.

$A_{6}$ : there is no statutory right to appeal against the decision.

$A_{7}$ : there were no circumstances which would have made an obligation to observe natural justice inappropriate.

In Attorney-General of Hong Kong v. Ng Yuen Shiu, ${ }^{17}$ a 1983 decision of the Judicial Committee of the Privy Council, Shiu was an illegal immigrant in Hong Kong. The Hong Kong Government's immigrant policy allowed illegal entrants to stay if they had reached the urban areas without being arrested. This policy was changed, and it was announced that illegal immigrants would be repatriated. Many illegal immigrants, who (like Shiu) had come from China via Macau, were fearful of being repatriated to China. A senior immigration official announced that each illegal immigrant from Macau would be interviewed, and each case "treated on its merits". Shiu was questioned by an immigration officer, but was given no opportunity to make representations, and the Director of Immigration ordered his removal.

The Privy Council held that, because the government had promised to follow a certain procedure before reaching its decision, it should honour that promise. The removal order was set aside because Shiu had not been asked whether he wished to make representations as to why he should not be removed.

C9: Durayappah v. Fernando [1967] 2 AC 337

$A_{1}$ : the decision affected the property, right, interest, status, or legitimate expectation of the applicant.

$A_{2}$ : the decision is apt to have a discrete impact on the interests of the applicant.

$A_{3}$ : the power is of a nature that would suggest that procedural fairness would not be applied.

$A_{4}$ : the statutory or factual criteria focused on matters of policy or public interest.

$A_{5}$ : the decision-maker was a high-level policy-maker. 
$A_{6}$ : there is no statutory right to appeal against the decision.

$A_{7}$ : there were no circumstances which would have made an obligation to observe natural justice inappropriate.

In Durayappah v. Fernando, ${ }^{18}$ a 1967 decision of the Judicial Committee of the Privy Council, following complaints as to the conduct of the Jaffna Municipal Council, the Ceylonese Minister of Local Government sent a Commissioner to Jaffna to inquire into the matter. The Commissioner examined the Council's records, but did not ask any questions of members of the Council, or give them any opportunity to put their views to him. The Commissioner reported to the Minister who, pursuant to s. 277(1) of the Municipal Councils Ordinance 1947 (Ceylon), made an order stating that the Council was not competent to perform its duties and dissolved it.

The Mayor of Jaffna (Durayappah) sought writs to quash the Minister's order and to annul the appointments of the special commissioners who had taken over the running of Jaffna, and a declaration that he was the duly elected mayor.

The Privy Council held that the Minister had no right to dissolve the Council without allowing it the right to be heard. However, the Minister's order was voidable only after a complaint by the Council. Durayappah (as Mayor) had no right to complain independently of the Council: he could only complain if he was representing the Council - and he was not.

$I_{\text {Implied }}$ (the ideal case in which a duty to observe natural justice is implied):

$A_{1}$ : the decision affected the property, right, interest, status, or legitimate expectation of the applicant.

$A_{2}$ : the decision is apt to have a discrete impact on the interests of the applicant.

$A_{3}$ : the power is of a nature that would suggest that procedural fairness would be applied.

$A_{4}$ : the statutory or factual criteria focused on matters which were discrete to the interests of the applicant.

$A_{5}$ : the decision-maker was not a high-level policy-maker.

$A_{6}$ : there is no statutory right to appeal against the decision.

$A_{7}$ : there were no circumstances which would have made an obligation to observe natural justice inappropriate.

\section{Cases in which a duty to observe natural justice is not implied}

$C_{10}$ : South Australia v. O'Shea (1987) 163 CLR 378 ("SA v. O'Shea")

$A_{1}$ : the decision affected the property, right, interest, status, or legitimate expectation of the applicant.

$A_{2}$ : the decision is apt to have a discrete impact on the interests of the applicant.

${ }^{18}[1967] 2$ AC 337. 
$A_{3}$ : the power is of a nature that would suggest that procedural fairness would be applied.

$A_{4}$ : the statutory or factual criteria focused on matters of policy or public interest.

$A_{5}$ : the decision-maker was a high-level policy-maker.

$A_{6}$ : there is no statutory right to appeal against the decision.

$A_{7}$ : there were no circumstances which would have made an obligation to observe natural justice inappropriate.

In South Australia v. O'Shea, ${ }^{19}$ a 1987 decision of five judges of the High Court of Australia, O'Shea had been convicted of two offences of indecent assault of young children. He was released on licence and remained at liberty after the licence expired. Over a year later, after allegations had been made against him, O'Shea was apprehended and detained. The parole board recommended his release on licence on various conditions, but the Governor in Council resolved to take no action. O'Shea had been given a hearing by the Parole Board, but he claimed he was entitled to a further hearing before the Governor in Council could exercise his discretionary powers under s. 77a(7a) of the Criminal Law Consolidation Act, 1935 (SA).

Mason CJ, Wilson, Brennan and Toohey JJ (Deane J dissenting) held that O'Shea was not entitled to a further hearing. "Given the nature of this decision, it cannot be said that Mr O'Shea could have more than a hope that the Governor would be prepared to act on the recommendation of the Board. Hope, of itself, is not sufficient to ground an expectation that will attract legal consequences. So far as the concept of legitimate expectation is concerned, Mr O'Shea must be taken to know that the Act committed to the Governor, with the advice and consent of the Executive Council, the responsibility for determining where the public interest lay ... The nature of the decision that they were required to make was such that participation by Mr O'Shea was inappropriate." ${ }^{20}$

$C_{11}$ : Bread Manufacturers of New South Wales v. Evans (1981) 38 ALR 93 ("Bread Manufacturers v. Evans")

$A_{1}$ : the decision affected the property, right, interest, status, or legitimate expectation of the applicant.

$A_{2}$ : the decision is not apt to have a discrete impact on the interests of the applicant.

$A_{3}$ : the power is of a nature that would suggest that procedural fairness would not be applied.

$A_{4}$ : the statutory or factual criteria focused on matters of policy or public interest.

$A_{5}$ : the decision-maker was not a high-level policy-maker.

19 (1987) 163 CLR 378.

${ }^{20}$ ibid. at 402, per Wilson and Toohey JJ. 
$A_{6}$ : there is no statutory right to appeal against the decision.

$A_{7}$ : there were no circumstances which would have made an obligation to observe natural justice inappropriate.

In Bread Manufacturers of New South Wales v. Evans, ${ }^{21}$ a 1981 decision of five judges of the High Court of Australia, the Bread Manufacturers claimed that an order made by the Prices Commission was void. The order affected the classification of bread products and had an incidental effect on the price of hamburger buns. The Bread Manufacturers complained that they should have been given the right to put their case to the Commission.

The Prices Regulation Act 1948 (NSW) provided that a public inquiry had to be held before an order could be made setting prices, except where the Minister consented to dispensing with the inquiry. The Minister had dispensed with an inquiry before this order was made. Hence, "[t]he argument that the Commission was bound to disclose to the Association the fact that it proposed to make an order which would have the incidental effect of reducing the price of hamburger buns can only succeed if the Commission, although not bound to hold an inquiry, was bound to observe the rules of natural justice". ${ }^{22}$

The High Court held that there was no denial of natural justice in relation to the order, because "the reduction of the maximum price in respect of one item was simply a minor incident in a major revision of the price framework covering the whole range of bread products. The effect of that major revision was generally to increase prices. There was, in our opinion, no obligation on the Commission to give advance notice of this development or of the possibility of its occurrence." 23

$C_{12}$ : Minister for Arts Heritage and Environment v. Peko-Wallsend Ltd (1987) 75 ALR 218 ("Minister for Environment v. Peko-Wallsend")

$A_{1}$ : the decision did not affect the property, right, interest, status, or legitimate expectation of the applicant.

$A_{2}$ : the decision is not apt to have a discrete impact on the interests of the applicant.

$A_{3}$ : the power is of a nature that would suggest that procedural fairness would not be applied.

$A_{4}$ : the statutory or factual criteria focused on matters of policy or public interest.

$A_{5}$ : the decision-maker was a high-level policy-maker.

$A_{6}$ : there is no statutory right to appeal against the decision.

$A_{7}$ : there were no circumstances which would have made an obligation to observe natural justice inappropriate.

${ }^{21}(1981) 38$ ALR 93.

${ }^{22}$ ibid. at 101, per Gibbs CJ.

${ }^{23}$ ibid. at 119, per Mason and Wilson JJ, with whom Murphy and Aickin JJ agreed on this point. 
In Minister for Arts Heritage and Environment v. Peko-Wallsend Ltd, ${ }^{24}$ a 1987 decision of the Full Court of the Federal Court of Australia, Peko-Wallsend held various mining interests in Stage 2 of Kakadu National Park. Federal Cabinet decided to nominate Stage 2 for inclusion in the World Heritage List, so it became "identified property" within the meaning of s. 3(2) of the World Heritage Properties Conservation Act 1983 (Cth). This meant that the Governor-General could, by proclamation, make mining operations unlawful in the area. The decision did not affect Peko-Wallsend's mining rights which were preserved under s. 8B of the National Parks and Wildlife Conservation Act 1975 (Cth).

Before Cabinet's decision, Peko-Wallsend had lobbied Ministers and other officials extensively, seeking to preserve their mining interests. After the decision they commenced proceedings to prevent the Government from taking any further steps to have Stage 2 nominated on the World Heritage List, claiming that Cabinet was bound by the rules of natural justice and had failed to give PekoWallsend an opportunity to be heard. Beaumont J (a Federal Court judge) agreed, and held the Cabinet decision void. ${ }^{25}$

The Full Court of the Federal Court disagreed. Bowen CJ decided that "it would ... be inappropriate for this court to interfere to set aside a Cabinet decision involving such complex policy considerations". ${ }^{26}$ Both Sheppard and Wilcox JJ held that Peko-Wallsend had had adequate opportunity to put their case to relevant Ministers and officials before the Cabinet decision, and were not denied natural justice. ${ }^{27}$ However, Wilcox $\mathrm{J}$ (with whose reasons the other two judges generally agreed) held that the Cabinet's decision in this case did not attract the obligations of natural justice. ${ }^{28}$

$C_{13}$ : Nashua Australia Pty Ltd v. Channon (1981) 36 ALR 215 ("Nashua v. Channon")

$A_{1}$ : the decision affected the property, right, interest, status, or legitimate expectation of the applicant.

$A_{2}$ : the decision is apt to have a discrete impact on the interests of the applicant.

$A_{3}$ : the power is of a nature that would suggest that procedural fairness would not be applied.

$A_{4}$ : the statutory or factual criteria focused on matters of policy or public interest.

$A_{5}$ : the decision-maker was a high-level policy-maker.

$A_{6}$ : there is no statutory right to appeal against the decision.

$A_{7}$ : there were no circumstances which would have made an obligation to observe natural justice inappropriate.

\footnotetext{
${ }^{24}(1987) 75$ ALR 218.

${ }^{25}$ Peko-Wallsend Ltd v. Minister for Arts Heritage and Environment (1986) 70 ALR 523.

${ }^{26}(1987) 75$ ALR 218 at 225.

27 ibid. at 228 per Sheppard J; at 253 per Wilcox J.

28 ibid. at 253 .
} 
In Nashua Australia Pty Ltd v. Channon, ${ }^{29}$ a 1981 decision of the Supreme Court of New South Wales, Nashua applied for a by-law to be made in respect of specially coated paper that they wanted to import from America. They claimed that suitable paper was not available in Australia. Channon, as delegate of the Minister, made a determination under s. 273 of the Customs Act 1966 (Cth) that enabled the paper to be imported at a duty of $2 \%$ (instead of $25 \%$ ) for a period of over two years. Less than four months into that period, the determination was revoked because (according to Channon) suitably equivalent paper was available from an Australian manufacturer. Nashua claimed that they had been denied natural justice as they had been given no notice of the intended revocation and no opportunity to make representations that the revocation should not be made.

Lee $\mathrm{J}$ held that, given the purposes and operation of the Act, the rules of natural justice did not apply to the revocation of a determination under s. 273. However, he held the revocation invalid because Channon himself had not exercised the discretion entrusted to him.

$C_{14}$ : Council of Civil Service Unions v. Minister for the Civil Service [1985] AC 374 ("CCSU v. Minister for the Civil Service")

$A_{1}$ : the decision affected the property, right, interest, status, or legitimate expectation of the applicant.

$A_{2}$ : the decision is apt to have a discrete impact on the interests of the applicant.

$A_{3}$ : the power is of a nature that would suggest that procedural fairness would not be applied.

$A_{4}$ : the statutory or factual criteria focused on matters of policy or public interest.

$A_{5}$ : the decision-maker was a high-level policy-maker.

$A_{6}$ : there is no statutory right to appeal against the decision.

$A_{7}$ : there were circumstances which made an obligation to observe natural justice inappropriate.

In Council of Civil Service Unions v. Minister for the Civil Service, ${ }^{30}$ a 1984 decision of the House of Lords, the Minister gave an instruction varying the terms and conditions for staff working at the Government Communications Headquarters. The effect of the variation was to prohibit staff from belonging to national trade unions. Staff had been allowed to belong to these unions for almost forty years. There was a well-established practice of consultation between the government and the trade unions about important alterations to the staff's terms and conditions, but there was no consultation with staff or the unions before the Minister issued her instruction.

The unions claimed that the Minister had been under a duty to act fairly by consulting those concerned before issuing the instruction. The Minister produced an affidavit from the Secretary to the Cabinet stating that recent industrial

29 (1981) 36 ALR 215.

30 [1985] AC 374 . 
action had led the Cabinet to believe that prior consultation about the Minister's instruction could have precipitated further disruption and would have indicated vulnerable areas of the Government Communications Headquarters' operations. The House of Lords held that the unions had a legitimate expectation that the Minister would consult with them on this matter. However, their Lordships held that the requirements of national security outweighed those of fairness: the Minister "had shown that her decision was one which not only could reasonably have been based, but was in fact based, on considerations of national security, which outweighed what would otherwise have been the reasonable expectation on the part of the [unions] for prior consultation." ${ }^{31}$ The Minister's instruction was valid.

\section{$C_{15}$ : McInnes v. Onslow Fane [1978] 3 All ER 211}

$A_{1}$ : the decision affected the property, right, interest, status, or legitimate expectation of the applicant.

$A_{2}$ : the decision is apt to have a discrete impact on the interests of the applicant.

$A_{3}$ : the power is of a nature that would suggest that procedural fairness would be applied.

$A_{4}$ : the statutory or factual criteria focused on matters which were discrete to the interests of the applicant.

$A_{5}$ : the decision-maker was not a high-level policy-maker.

$A_{6}$ : there is no statutory right to appeal against the decision.

$A_{7}$ : there were no circumstances which would have made an obligation to observe natural justice inappropriate.

In McInnes v. Onslow Fane, ${ }^{32}$ a 1978 decision of the Chancery Division of the English High Court, McInnes had held, at various times, licences to promote, train and act as master of ceremonies in professional boxing. All his licences were revoked by the British Boxing Board of Control. He made five unsuccessful applications for a manager's licence. With his sixth application he requested an oral hearing and prior notification of anything that might prevent the area council (to which he applied) making a favourable recommendation to the board. The board refused his applications without giving him an oral hearing or informing him of the case against him.

Megarry V-C held that the board was under no duty to provide reasons to McInnes or to allow him a hearing: "This is not a case in which there has been any suggestion of the board considering any alleged dishonesty or morally culpable conduct of the plaintiff. A man free from any moral blemish may nevertheless be wholly unsuitable for a particular type of work ... In such circumstances, in the absence of anything to suggest that the board have been affected by dishonesty or bias or caprice, or that there is any other impropriety, I think that the board are fully entitled to give no reasons for their decision, and to decide the application

\footnotetext{
31 ibid. at 403, per Lord Fraser of Tullybelton.

32 [1978] 3 All ER 211.
} 
without any preliminary indication to the plaintiff of those reasons. The board are the best judges of the desirability of granting a licence, and in the absence of any impropriety the court ought not to interfere." 33

$I_{\text {Not-Implied }}$ (the ideal case in which a duty to observe natural justice is not implied):

$A_{1}$ : the decision did not affect the property, right, interest, status, or legitimate expectation of the applicant.

$A_{2}$ : the decision is not apt to have a discrete impact on the interests of the applicant.

$A_{3}$ : the power is of a nature that would suggest that procedural fairness would not be applied.

$A_{4}$ : the statutory or factual criteria focused on matters of policy or public interest.

$A_{5}$ : the decision-maker was a high-level policy-maker.

$A_{6}$ : there is a statutory right to appeal against the decision.

$A_{7}$ : there were circumstances which made an obligation to observe natural justice inappropriate.

\section{Affected area}

\begin{tabular}{|c|cccc|c|c|}
\hline \multirow{2}{*}{ Case } & \multicolumn{3}{|c|}{ Attributes } & \multirow{2}{*}{ Result } \\
& $A_{1}$ & $A_{2}$ & $A_{3}$ & $A_{4}$ & & \\
\hline \hline$C_{1}$ & $\bullet$ & $\times$ & $\bullet$ & $\bullet$ & 1 & \\
$C_{2}$ & $\times$ & $\bullet$ & $\times$ & $\bullet$ & 2 & \\
$C_{3}$ & $\times$ & $\times$ & $\bullet$ & $\times$ & 2 & \\
$C_{4}$ & $\times$ & $\bullet$ & $\times$ & $\times$ & 2 & \\
$C_{5}$ & $\times$ & $\bullet$ & $\times$ & $\bullet$ & 2 & \multirow{2}{*}{ Affected } \\
$C_{6}$ & $\bullet$ & $\times$ & $\times$ & $\times$ & 2 & \\
$C_{7}$ & $\bullet$ & $\times$ & $\bullet$ & $\times$ & 3 & \\
$C_{8}$ & $\bullet$ & $\times$ & $\times$ & $\bullet$ & 8 & \\
$I_{\text {Affected }}$ & $\bullet$ & $\bullet$ & $\bullet$ & $\bullet$ & & \\
\hline \hline$C_{9}$ & $\times$ & $\times$ & $\times$ & $\times$ & 4 & Unaffected \\
\cline { 2 - 5 }$I_{\text {Unaffected }}$ & $\times$ & $\times$ & $\times$ & $\times$ & & \\
\hline
\end{tabular}

\section{Results}

Affected: the decision affected the property, right, interest, status, or legitimate expectation of the applicant.

Unaffected: the decision did not affect the property, right, interest, status, or legitimate expectation of the applicant.

33 ibid. at 223 . 


\section{Attributes}

$A_{1}$ : Did the decision affect a financial, property or occupational interest of the applicant?

YES: the decision affected a financial, property or occupational interest of the applicant.

$\Rightarrow$ Affected

NO: the decision did not affect a financial, property or occupational interest of the applicant.

UNKNOWN: it is not known whether or not the decision affected a financial, property or occupational interest of the applicant.

The interest must be an existing interest: one which existed when the decision was made.

$A_{2}$ : Did the decision affect the applicant's personal liberty?

YES: the decision affected the applicant's personal liberty.

$$
\Rightarrow \text { Affected }
$$

NO: the decision did not affect the applicant's personal liberty.

UNKNOWN: it is not known whether the decision affected the applicant's personal liberty.

$A_{3}$ : Did the decision affect the applicant's reputation?

YES: the decision affected the applicant's reputation.

$$
\Rightarrow \text { Affected }
$$

NO: the decision did not affect the applicant's reputation.

UNKNOWN: it is not known whether the decision affected the applicant's reputation.

$A_{4}: \Leftrightarrow$ Expectation area

YES: the applicant had a legitimate expectation which was affected by the decision.

$\Leftarrow$ Expectation

$\Rightarrow$ Affected

NO: the applicant did not have a legitimate expectation which was affected by the decision.

$\Leftarrow$ No-Expectation

UNKNOWN: it is not known whether the applicant had a legitimate expectation which was affected by the decision. 


\section{Cases in which the decision affected the property, right, interest, status, or legitimate expectation of the applicant}

$C_{1}$ : FAI Insurances Ltd v. Winneke (1982) 151 CLR 342 ("FAI v. Winneke")

$A_{1}$ : the decision affected a financial, property or occupational interest of the applicant.

$A_{2}$ : the decision did not affect the applicant's personal liberty.

$A_{3}$ : the decision affected the applicant's reputation.

$A_{4}$ : the applicant had a legitimate expectation which was affected by the decision.

In FAI Insurances Ltd $v$. Winneke, ${ }^{34}$ a 1982 decision of seven judges of the High Court of Australia, Winneke was the Governor of Victoria. The Workers Compensation Act 1958 (Vic) made accident insurance compulsory for all employers, and required them to obtain that insurance from the Insurance Commissioner or from an insurer approved by the Governor in Council. Regulations made under the Act allowed the Governor in Council to grant an approval for a period not exceeding twelve months, and (on application) to renew an approval for any period not exceeding twelve months "if the Governor in Council thinks fit." FAI had had its approval to provide worker's compensation renewed every year for twenty years, until 1981.

Gibbs CJ, Stephen, Mason, Aickin, Wilson and Brennan JJ (Murphy J dissenting) held that in deciding whether to renew an approval previously given, the Governor in Council is subject to the requirements of natural justice. "In these circumstances, a company which becomes an approved insurer has a legitimate expectation that its approval will be renewed unless some good reason exists for refusing to renew it. It would not be fair to deprive a company of the ability to carry on its business without revealing the reason for doing so". ${ }^{35}$

$C_{2}$ : Haoucher v. Minister of State for Immigration and Ethnic Affairs (1990) 169 CLR 648 ("Haoucher v. Minister for Immigration")

$A_{1}$ : the decision did not affect a financial, property or occupational interest of the applicant.

$A_{2}$ : the decision affected the applicant's personal liberty.

$A_{3}$ : the decision did not affect the applicant's reputation.

$A_{4}$ : the applicant had a legitimate expectation which was affected by the decision.

34 (1982) 151 CLR 342.

35 ibid. at 348 , per Gibbs CJ. 
In Haoucher $v$. Minister of State for Immigration and Ethnic Affairs, ${ }^{36}$ a 1990 decision of five judges of the High Court of Australia, Haoucher had been convicted of an offence for which he had been sentenced to imprisonment for a period not less than one year. Hence - as Haoucher was not an Australian citizen - the Minister had the power to order his deportation under s. 12 of the Migration Act 1958 (Cth), and did so. The Minister had previously told Parliament that the Government's policy was that a deportee had the right to appeal to the Administrative Appeals Tribunal, and that "recommendations of the Administrative Appeals Tribunal should be overturned by the Minister only in exceptional circumstances and only when strong evidence can be produced to justify his decision."

Haoucher appealed to the AAT which recommended that the deportation order be revoked. The Minister decided not to accept that recommendation. Deane, Toohey and McHugh (Dawson and Gaudron dissenting) held that Haoucher was entitled to know the matters which constituted "exceptional circumstances" and "strong evidence" sufficient for the Minister to depart from the general policy of following the AAT's recommendations. The fact that the Minister had not given Haoucher such details, and had given him no opportunity to make representations, was a denial of natural justice.

$C_{3}$ : Annetts v. McCann (1990) 170 CLR 596

$A_{1}$ : the decision did not affect a financial, property or occupational interest of the applicant.

$A_{2}$ : the decision did not affect the applicant's personal liberty.

$A_{3}$ : the decision affected the applicant's reputation.

$A_{4}$ : the applicant did not have a legitimate expectation which was affected by the decision.

In Annetts v. McCann, ${ }^{37}$ a 1990 decision of five judges of the High Court of Australia, a coroner had been conducting an inquest into the death of a 16-year old boy. The boy's parents (Mr and Mrs Annetts) sought to make a submission before the coroner made a finding. The coroner decided that the Coroner's Act 1920 (WA) gave him the discretion (which he chose to exercise) to disallow their submission. The Annettses appealed.

The High Court (Mason CJ, Brennan, Deane, Toohey and McHugh JJ) held that their son's reputation gave the Annettses an interest in the Coroner's inquiry. "A finding in an inquest into a death is naturally likely to deal with the conduct of the deceased leading to death. An unfavourable reflection on the deceased is usually a matter of concern to her or his parents, spouse or children and, if they choose to appear at the inquest in order to safeguard the reputation of the deceased, the 
familial relationship suffices, in my view, to establish the deceased's reputation as a relevant interest which should not be adversely affected without according natural justice to those who are seeking to safeguard that reputation." 38

The Court held that the fact that the coroner's decision was merely recommendatory (whether or not to prosecute) was not sufficient to avoid the implication of natural justice; the coroner was bound to hear the Annettses before making any finding adverse to them or their son. ${ }^{39}$

$C_{4}$ : South Australia v. O'Shea (1987) 163 CLR 378 ("SA v. O'Shea")

$A_{1}$ : the decision did not affect a financial, property or occupational interest of the applicant.

$A_{2}$ : the decision affected the applicant's personal liberty.

$A_{3}$ : the decision did not affect the applicant's reputation.

$A_{4}$ : the applicant did not have a legitimate expectation which was affected by the decision.

In South Australia v. O'Shea, ${ }^{40}$ a 1987 decision of five judges of the High Court of Australia, O'Shea had been convicted of two offences of indecent assault of young children. He was released on licence and remained at liberty after the licence expired. Over a year later, after allegations had been made against him, O'Shea was apprehended and detained. The parole board recommended his release on licence on various conditions, but the Governor in Council resolved to take no action. O'Shea had been given a hearing by the Parole Board, but he claimed he was entitled to a further hearing before the Governor in Council could exercise his discretionary powers under s. 77a(7a) of the Criminal Law Consolidation Act, 1935 (SA).

Mason CJ, Wilson, Brennan and Toohey JJ (Deane J dissenting) held that O'Shea was not entitled to a further hearing. "Given the nature of this decision, it cannot be said that Mr O'Shea could have more than a hope that the Governor would be prepared to act on the recommendation of the Board. Hope, of itself, is not sufficient to ground an expectation that will attract legal consequences. So far as the concept of legitimate expectation is concerned, Mr O'Shea must be taken to know that the Act committed to the Governor, with the advice and consent of the Executive Council, the responsibility for determining where the public interest lay ... The nature of the decision that they were required to make was such that participation by Mr O'Shea was inappropriate." ${ }^{41}$

\footnotetext{
${ }^{38}$ ibid. at 612 , per Brennan J.

39 ibid. at 603, per Mason CJ, Deane and McHugh JJ; at 612 per Brennan J; at 621 per Toohey J. Note, however, that Brennan and Toohey JJ dismissed the appeal because they believed that the decision of the Full Court of the Supreme Court of Western Australia (from which the Annettses appealed) was right on the material before it.

40 (1987) 163 CLR 378.

${ }^{41}$ ibid. at 402, per Wilson and Toohey JJ.
} 
$C_{5}$ : Kioa v. West (1985) 159 CLR 550

$A_{1}$ : the decision did not affect a financial, property or occupational interest of the applicant.

$A_{2}$ : the decision affected the applicant's personal liberty.

$A_{3}$ : the decision did not affect the applicant's reputation.

$A_{4}$ : the applicant had a legitimate expectation which was affected by the decision.

In Kioa v. West, ${ }^{42}$ a 1985 decision of five judges of the High Court of Australia, Kioa and his wife were Tongan citizens. They were each granted a temporary entry permit. When their entry permits expired, they stayed in Australia and their daughter was born (becoming an Australian citizen). The Minister's delegate made an order for their deportation under the Migration Act 1958 (Cth). They were given no opportunity to answer prejudicial statements against them made to the Minister's delegate.

Mason, Wilson, Brennan and Deane JJ (Gibbs CJ dissenting) held that, in the absence of statutory provisions to the contrary, the requirements of natural justice should have been observed in relation to the making of the deportation order. The deportation order was set aside.

$C_{6}$ : Bread Manufacturers of New South Wales v. Evans (1981) 38 ALR 93 ("Bread Manufacturers v. Evans")

$A_{1}$ : the decision affected a financial, property or occupational interest of the applicant.

$A_{2}$ : the decision did not affect the applicant's personal liberty.

$A_{3}$ : the decision did not affect the applicant's reputation.

$A_{4}$ : the applicant did not have a legitimate expectation which was affected by the decision.

In Bread Manufacturers of New South Wales v. Evans, ${ }^{43}$ a 1981 decision of five judges of the High Court of Australia, the Bread Manufacturers claimed that an order made by the Prices Commission was void. The order affected the classification of bread products and had an incidental effect on the price of hamburger buns. The Bread Manufacturers complained that they should have been given the right to put their case to the Commission.

The Prices Regulation Act 1948 (NSW) provided that a public inquiry had to be held before an order could be made setting prices, except where the Minister consented to dispensing with the inquiry. The Minister had dispensed with an inquiry before this order was made. Hence, "[t]he argument that the Commission was bound to disclose to the Association the fact that it proposed to make an 
order which would have the incidental effect of reducing the price of hamburger buns can only succeed if the Commission, although not bound to hold an inquiry, was bound to observe the rules of natural justice". 44

The High Court held that there was no denial of natural justice in relation to the order, because "the reduction of the maximum price in respect of one item was simply a minor incident in a major revision of the price framework covering the whole range of bread products. The effect of that major revision was generally to increase prices. There was, in our opinion, no obligation on the Commission to give advance notice of this development or of the possibility of its occurrence." ${ }^{45}$

$C_{7}$ : Commissioner of Police v. Tanos (1958) 98 CLR 383

$A_{1}$ : the decision affected a financial, property or occupational interest of the applicant.

$A_{2}$ : the decision did not affect the applicant's personal liberty.

$A_{3}$ : the decision affected the applicant's reputation.

$A_{4}$ : the applicant did not have a legitimate expectation which was affected by the decision.

In Commissioner of Police v. Tanos, ${ }^{46}$ a 1958 decision of three judges of the High Court of Australia, a judge had made a declaration that a restaurant run by Tanos was a "disorderly house" pursuant to s. 3(1)(b) of the Disorderly Houses Act 1943 (NSW). A police inspector had sworn an affidavit as to his suspicion and belief that liquor had been unlawfully sold or supplied on the premises and was likely to unlawfully sold or supplied on the premises again. The judge made the declaration ex parte.

The stated grounds for the police inspector's suspicion concerned things that Tanos's husband had done when he had been running the restaurant. Tanos claimed that things had changed since she had taken over: "the patronage of a much more desirable class of customer was obtained, a class which would not demand wine with their food." 47 Tanos appealed successfully to the Supreme Court.

Dixon CJ, Webb and Taylor JJ held that Tanos's appeal should have been dismissed by the Supreme Court; once the declaration was made, the Act placed the burden on Tanos to prove that liquor had never been sold or supplied on the premises, and she had not proved that. However, the High Court also held that, except in exceptional and special circumstances, an owner/occupier should have an opportunity to be heard before her/his premises are declared "disorderly". Tanos had been denied that opportunity, so the declaration was set aside.

\footnotetext{
${ }^{44}$ ibid. at 101, per Gibbs CJ.

45 ibid. at 119, per Mason and Wilson JJ, with whom Murphy and Aickin JJ agreed on this point.

46 (1958) 98 CLR 383.

47ibid. at 389, per Dixon C.J and Webb J.
} 
$C_{8}$ : Council of Civil Service Unions v. Minister for the Civil Service [1985] AC 374 ("CCSU v. Minister for the Civil Service")

$A_{1}$ : the decision affected a financial, property or occupational interest of the applicant.

$A_{2}$ : the decision did not affect the applicant's personal liberty.

$A_{3}$ : the decision did not affect the applicant's reputation.

$A_{4}$ : the applicant had a legitimate expectation which was affected by the decision.

In Council of Civil Service Unions v. Minister for the Civil Service, ${ }^{48}$ a 1984 decision of the House of Lords, the Minister gave an instruction varying the terms and conditions for staff working at the Government Communications Headquarters. The effect of the variation was to prohibit staff from belonging to national trade unions. Staff had been allowed to belong to these unions for almost forty years. There was a well-established practice of consultation between the government and the trade unions about important alterations to the staff's terms and conditions, but there was no consultation with staff or the unions before the Minister issued her instruction.

The unions claimed that the Minister had been under a duty to act fairly by consulting those concerned before issuing the instruction. The Minister produced an affidavit from the Secretary to the Cabinet stating that recent industrial action had led the Cabinet to believe that prior consultation about the Minister's instruction could have precipitated further disruption and would have indicated vulnerable areas of the Government Communications Headquarters' operations.

The House of Lords held that the unions had a legitimate expectation that the Minister would consult with them on this matter. However, their Lordships held that the requirements of national security outweighed those of fairness: the Minister "had shown that her decision was one which not only could reasonably have been based, but was in fact based, on considerations of national security, which outweighed what would otherwise have been the reasonable expectation on the part of the [unions] for prior consultation." 49 The Minister's instruction was valid.

$I_{\text {Affected }}$ (the ideal case in which the decision affected the property, right, interest, status, or legitimate expectation of the applicant):

$A_{1}$ : the decision affected a financial, property or occupational interest of the applicant.

$A_{2}$ : the decision affected the applicant's personal liberty.

$A_{3}$ : the decision affected the applicant's reputation.

$A_{4}$ : the applicant had a legitimate expectation which was affected by the decision.

${ }^{48}[1985]$ AC 374.

${ }^{49}$ ibid. at 403, per Lord Fraser of Tullybelton. 


\section{Cases in which the decision did not affect the property, right, interest, status, or legitimate expectation of the applicant}

$C_{9}$ : Minister for Arts Heritage and Environment v. Peko-Wallsend Ltd (1987) 75 ALR 218 ("Minister for Environment v. Peko-Wallsend")

$A_{1}$ : the decision did not affect a financial, property or occupational interest of the applicant.

$A_{2}$ : the decision did not affect the applicant's personal liberty.

$A_{3}$ : the decision did not affect the applicant's reputation.

$A_{4}$ : the applicant did not have a legitimate expectation which was affected by the decision.

In Minister for Arts Heritage and Environment v. Peko-Wallsend Ltd, ${ }^{50}$ a 1987 decision of the Full Court of the Federal Court of Australia, Peko-Wallsend held various mining interests in Stage 2 of Kakadu National Park. Federal Cabinet decided to nominate Stage 2 for inclusion in the World Heritage List, so it became "identified property" within the meaning of s. 3(2) of the World Heritage Properties Conservation Act 1983 (Cth). This meant that the Governor-General could, by proclamation, make mining operations unlawful in the area. The decision did not affect Peko-Wallsend's mining rights which were preserved under s. 8B of the National Parks and Wildlife Conservation Act 1975 (Cth).

Before Cabinet's decision, Peko-Wallsend had lobbied Ministers and other officials extensively, seeking to preserve their mining interests. After the decision they commenced proceedings to prevent the Government from taking any further steps to have Stage 2 nominated on the World Heritage List, claiming that Cabinet was bound by the rules of natural justice and had failed to give PekoWallsend an opportunity to be heard. Beaumont J (a Federal Court judge) agreed, and held the Cabinet decision void. ${ }^{51}$

The Full Court of the Federal Court disagreed. Bowen CJ decided that "it would ... be inappropriate for this court to interfere to set aside a Cabinet decision involving such complex policy considerations". ${ }^{52}$ Both Sheppard and Wilcox JJ held that Peko-Wallsend had had adequate opportunity to put their case to relevant Ministers and officials before the Cabinet decision, and were not denied natural justice. ${ }^{53}$ However, Wilcox $\mathrm{J}$ (with whose reasons the other two judges generally agreed) held that the Cabinet's decision in this case did not attract the obligations of natural justice. ${ }^{54}$

\footnotetext{
${ }^{50}(1987) 75$ ALR 218.

${ }^{51}$ Peko-Wallsend Ltd v. Minister for Arts Heritage and Environment (1986) 70 ALR 523.

52 (1987) 75 ALR 218 at 225.

53 ibid. at 228 per Sheppard J; at 253 per Wilcox J.

54 ibid. at 253 .
} 
$I_{\text {Unaffected }}$ (the ideal case in which the decision did not affect the property, right, interest, status, or legitimate expectation of the applicant):

$A_{1}$ : the decision did not affect a financial, property or occupational interest of the applicant.

$A_{2}$ : the decision did not affect the applicant's personal liberty.

$A_{3}$ : the decision did not affect the applicant's reputation.

$A_{4}$ : the applicant did not have a legitimate expectation which was affected by the decision.

\section{Expectation area}

\begin{tabular}{|c|ccccccc|c|c|}
\hline \multirow{2}{*}{ Case } & \multicolumn{7}{|c|}{ Attributes } & \multirow{2}{*}{ Result } \\
\hline \hline$C_{1}$ & $A_{1}$ & $A_{2}$ & $A_{3}$ & $A_{4}$ & $A_{5}$ & $A_{6}$ & & \\
$C_{2}$ & $\times$ & $\bullet$ & $\bullet$ & $\times$ & $\bullet$ & $\times$ & 1 & \\
$C_{3}$ & $\times$ & $\times$ & $\times$ & $\times$ & $\bullet$ & $\bullet$ & 2 & \multirow{2}{*}{ Expectation } \\
$C_{4}$ & $\times$ & $\bullet$ & $\times$ & $\times$ & $\bullet$ & $\times$ & 2 & \\
$C_{5}$ & $\bullet$ & $\times$ & $\times$ & $\times$ & $\times$ & $\times$ & 4 & \\
$C_{6}$ & $\bullet$ & $\times$ & $\times$ & $\times$ & $\bullet$ & $\times$ & 7 & \\
$I_{\text {Expectation }}$ & $\bullet$ & $\bullet$ & $\bullet$ & $\bullet$ & $\bullet$ & $\bullet$ & & \\
\hline \hline$C_{7}$ & $\times$ & $\times$ & $\times$ & $\times$ & $\bullet$ & $\times$ & 2 & \multirow{2}{*}{ No-Expectation } \\
$C_{8}$ & $\times$ & $\times$ & $\times$ & $\times$ & $\times$ & $\times$ & 4 & \\
\cline { 2 - 6 }$I_{\text {No-Expectation }}$ & $\times$ & $\times$ & $\times$ & $\times$ & $\times$ & $\times$ & & \\
\hline
\end{tabular}

\section{Opening}

If the applicant had a legitimate expectation - or a reasonable expectation - which was affected by the decision, natural justice may be implied. "‘L]egitimate expectations' ... are capable of including expectations which go beyond enforceable legal rights, provided they have some reasonable basis". ${ }^{55}$

\section{Results}

Expectation: the applicant had a legitimate expectation which was affected by the decision.

No-Expectation: the applicant did not have a legitimate expectation which was affected by the decision.

${ }^{55}$ Cole v. Cunningham (1983) 49 ALR 123 at 131, per Bowen CJ, Sheppard and Morling JJ. 


\section{Attributes}

$A_{1}$ : Did the decision-maker break a promise or undertaking?

YES: the decision-maker broke a promise or undertaking.

$$
\Rightarrow \text { Expectation }
$$

NO: the decision-maker did not break a promise or undertaking.

UNKNOWN: it is not known whether the decision-maker broke a promise or undertaking.

For example, if the decision went against a published policy then it was in breach of a promise or undertaking.

$A_{2}$ : Did the decision-maker go against an established course of practice?

YES: the decision-maker went against an established course of practice.

$$
\Rightarrow \text { Expectation }
$$

NO: the decision-maker did not go against an established course of practice.

UNKNOWN: it is not known whether the decision-maker went against an established course of practice.

There may have been an expectation that an established course of practice would be adopted, or that notice (and an opportunity to comment) would be given before the practice was abandoned.

$A_{3}$ : Did the decision involve a refusal to renew an existing interest?

YES: the decision involved the refusal to renew an existing interest.

$$
\Rightarrow \text { Expectation }
$$

NO: the decision did not involve a refusal to renew an existing interest.

UNKNOWN: it is not known whether the decision involved a refusal to renew an existing interest.

For example, if the decision was to refuse to renew a licence, then it was a refusal to renew an existing interest.

$A_{4}$ : Did the decision-maker or a statutory provision suggest that an initial interest would be granted?

YES: the decision-maker or a statutory provision suggested that an initial interest would be granted.

$\Rightarrow$ Expectation

NO: neither the decision-maker nor a statutory provision suggested that an initial interest would be granted.

UNKNOWN: it is not known whether the decision-maker or a statutory provision suggested that an initial interest would be granted. 
$A_{5}:$ Did the decision affect an established liberty or interest?

YES: the decision affected an established liberty or interest.

$\Rightarrow$ Expectation

NO: the decision did not affect an established liberty or interest.

UNKNOWN: it is not known whether the decision affected an established liberty or interest.

For example, a decision to warn the applicant off a racetrack would affect the applicant's established liberty to go to the races. And a deportation order would affect an applicant's established liberty to remain in the country.

$A_{6}$ : Was there a standard administrative procedure which the decision-maker did not follow?

YES: there was a standard administrative procedure which the decision-maker did not follow.

$\Rightarrow$ Expectation

NO: there was no standard administrative procedure which the decision-maker should have followed.

UNKNOWN: it is not known whether there was a standard administrative procedure which the decision-maker should have followed.

\section{Cases in which the applicant had a legitimate expectation which was affected by the decision}

$C_{1}$ : FAI Insurances Ltd v. Winneke (1982) 151 CLR 342 ("FAI v. Winneke")

$A_{1}$ : the decision-maker did not break a promise or undertaking.

$A_{2}$ : the decision-maker went against an established course of practice.

$A_{3}$ : the decision involved the refusal to renew an existing interest.

$A_{4}$ : neither the decision-maker nor a statutory provision suggested that an initial interest would be granted.

$A_{5}$ : the decision affected an established liberty or interest.

$A_{6}$ : there was no standard administrative procedure which the decision-maker should have followed.

In FAI Insurances Ltd $v$. Winneke ${ }^{56}$ a 1982 decision of seven judges of the High Court of Australia, Winneke was the Governor of Victoria. The Workers Compensation Act 1958 (Vic) made accident insurance compulsory for all employers, and required them to obtain that insurance from the Insurance Commissioner or from an insurer approved by the Governor in Council. Regulations made under the Act allowed the Governor in Council to grant an approval for a period

${ }^{56}$ (1982) 151 CLR 342. 
not exceeding twelve months, and (on application) to renew an approval for any period not exceeding twelve months "if the Governor in Council thinks fit." FAI had had its approval to provide worker's compensation renewed every year for twenty years, until 1981.

Gibbs CJ, Stephen, Mason, Aickin, Wilson and Brennan JJ (Murphy J dissenting) held that in deciding whether to renew an approval previously given, the Governor in Council is subject to the requirements of natural justice. "In these circumstances, a company which becomes an approved insurer has a legitimate expectation that its approval will be renewed unless some good reason exists for refusing to renew it. It would not be fair to deprive a company of the ability to carry on its business without revealing the reason for doing so". ${ }^{57}$

$C_{2}$ : Haoucher v. Minister of State for Immigration and Ethnic Affairs (1990) 169 CLR 648 ("Haoucher v. Minister for Immigration")

$A_{1}$ : the decision-maker did not break a promise or undertaking.

$A_{2}$ : the decision-maker went against an established course of practice.

$A_{3}$ : the decision did not involve a refusal to renew an existing interest.

$A_{4}$ : neither the decision-maker nor a statutory provision suggested that an initial interest would be granted.

$A_{5}$ : the decision affected an established liberty or interest.

$A_{6}$ : there was no standard administrative procedure which the decision-maker should have followed.

In Haoucher $v$. Minister of State for Immigration and Ethnic Affairs, ${ }^{58}$ a 1990 decision of five judges of the High Court of Australia, Haoucher had been convicted of an offence for which he had been sentenced to imprisonment for a period not less than one year. Hence - as Haoucher was not an Australian citizen - the Minister had the power to order his deportation under s. 12 of the Migration Act 1958 (Cth), and did so. The Minister had previously told Parliament that the Government's policy was that a deportee had the right to appeal to the Administrative Appeals Tribunal, and that "recommendations of the Administrative Appeals Tribunal should be overturned by the Minister only in exceptional circumstances and only when strong evidence can be produced to justify his decision."

Haoucher appealed to the AAT which recommended that the deportation order be revoked. The Minister decided not to accept that recommendation. Deane, Toohey and McHugh (Dawson and Gaudron dissenting) held that Haoucher was entitled to know the matters which constituted "exceptional circumstances" and "strong evidence" sufficient for the Minister to depart from the general policy of following the AAT's recommendations. The fact that the Minister had not given Haoucher such details, and had given him no opportunity to make representations, was a denial of natural justice.

57ibid. at 348, per Gibbs CJ.

58 (1990) 169 CLR 648. 
$C_{3}$ : Kioa v. West (1985) 159 CLR 550

$A_{1}$ : the decision-maker did not break a promise or undertaking.

$A_{2}$ : the decision-maker did not go against an established course of practice.

$A_{3}$ : the decision did not involve a refusal to renew an existing interest.

$A_{4}$ : neither the decision-maker nor a statutory provision suggested that an initial interest would be granted.

$A_{5}$ : the decision affected an established liberty or interest.

$A_{6}$ : there was a standard administrative procedure which the decision-maker did not follow.

In Kioa v. West, ${ }^{59}$ a 1985 decision of five judges of the High Court of Australia, Kioa and his wife were Tongan citizens. They were each granted a temporary entry permit. When their entry permits expired, they stayed in Australia and their daughter was born (becoming an Australian citizen). The Minister's delegate made an order for their deportation under the Migration Act 1958 (Cth). They were given no opportunity to answer prejudicial statements against them made to the Minister's delegate.

Mason, Wilson, Brennan and Deane JJ (Gibbs CJ dissenting) held that, in the absence of statutory provisions to the contrary, the requirements of natural justice should have been observed in relation to the making of the deportation order. The deportation order was set aside.

$C_{4}$ : Heatley v. Tasmanian Racing and Gaming Commission (1977) 137 CLR 487 ("Heatley v. TRC")

$A_{1}$ : the decision-maker did not break a promise or undertaking.

$A_{2}$ : the decision-maker went against an established course of practice.

$A_{3}$ : the decision did not involve a refusal to renew an existing interest.

$A_{4}$ : neither the decision-maker nor a statutory provision suggested that an initial interest would be granted.

$A_{5}$ : the decision affected an established liberty or interest.

$A_{6}$ : there was no standard administrative procedure which the decision-maker should have followed.

In Heatley v. Tasmanian Racing and Gaming Commission, ${ }^{60}$ a 1977 decision of five judges of the High Court of Australia, Heatley was warned off racecourses in Tasmania by the Commission, using its powers under s. 39(3) of the Racing and Gaming Act 1952 (Tas). Heatley had been given no notice that the Commission intended warning him off (and hence was given no opportunity to make representations to the Commission) and was given no reasons.

Stephen, Mason, Murphy and Aickin JJ (Barwick CJ dissenting) held that the Commission was bound by the rules of natural justice. In the absence of an emergency, the Commission should have given Heatley notice of its intention to warn 
him off, and the grounds for taking such action. Further, Heatley should have been given an opportunity to make representations to the Commission before it made its decision.

Aickin J (with whom Stephen and Mason JJ agreed) held that all members of the public - including Heatley - have a legitimate expectation that they will be allowed onto racecourses. ${ }^{61}$

$C_{5}$ : Cole v. Cunningham (1983) 49 ALR 123

$A_{1}$ : the decision-maker broke a promise or undertaking.

$A_{2}$ : the decision-maker did not go against an established course of practice.

$A_{3}$ : the decision did not involve a refusal to renew an existing interest.

$A_{4}$ : neither the decision-maker nor a statutory provision suggested that an initial interest would be granted.

$A_{5}$ : the decision did not affect an established liberty or interest.

$A_{6}$ : there was no standard administrative procedure which the decision-maker should have followed.

In Cole v. Cunningham, ${ }^{62}$ a 1983 decision of the Full Court of the Federal Court of Australia, Cunningham had been encouraged to resign from the Public Service because his superiors believed he had been guilty of misconduct in the performance of his duties. He had formed an attachment and begun to live with a Fijian women whose permit extension application he had processed. He was threatened with criminal prosecution and told that "[i]f you resign now it will be a normal resignation and you'll leave with a clean record." 63

About eighteen months later, Cunningham sought reappointment to the Public Service and was told that he would be offered a position subject to police and ASIO clearances. The next day he was told that he have been given an unsatisfactory report based on the earlier events.

Bowen CJ, Sheppard and Morling JJ held that, in general, applicants for appointment or reappointment to the public service are not entitled to natural justice because they have no legitimate expectation which can be affected by a refusal to appoint. However, Cunningham did have a legitimate expectation that any decision to reappoint him would not be made on the basis of his past record.

$C_{6}$ : Attorney-General of Hong Kong v. Ng Yuen Shiu [1983] 2 AC 629 ("AG of Hong Kong v. Shiu")

$A_{1}$ : the decision-maker broke a promise or undertaking.

$A_{2}$ : the decision-maker did not go against an established course of practice.

$A_{3}$ : the decision did not involve a refusal to renew an existing interest.

$A_{4}$ : neither the decision-maker nor a statutory provision suggested that an initial interest would be granted.

\footnotetext{
61 ibid. at 509 .

${ }^{62}$ (1983) 49 ALR 123.

${ }^{63}$ ibid. at 125 .
} 
$A_{5}$ : the decision affected an established liberty or interest.

$A_{6}$ : there was no standard administrative procedure which the decision-maker should have followed.

In Attorney-General of Hong Kong v. Ng Yuen Shiu, ${ }^{64}$ a 1983 decision of the Judicial Committee of the Privy Council, Shiu was an illegal immigrant in Hong Kong. The Hong Kong Government's immigrant policy allowed illegal entrants to stay if they had reached the urban areas without being arrested. This policy was changed, and it was announced that illegal immigrants would be repatriated. Many illegal immigrants, who (like Shiu) had come from China via Macau, were fearful of being repatriated to China. A senior immigration official announced that each illegal immigrant from Macau would be interviewed, and each case "treated on its merits". Shiu was questioned by an immigration officer, but was given no opportunity to make representations, and the Director of Immigration ordered his removal.

The Privy Council held that, because the government had promised to follow a certain procedure before reaching its decision, it should honour that promise. The removal order was set aside because Shiu had not been asked whether he wished to make representations as to why he should not be removed.

$I_{\text {Expectation }}$ (the ideal case in which the applicant had a legitimate expectation which was affected by the decision):

$A_{1}$ : the decision-maker broke a promise or undertaking.

$A_{2}$ : the decision-maker went against an established course of practice.

$A_{3}$ : the decision involved the refusal to renew an existing interest.

$A_{4}$ : the decision-maker or a statutory provision suggested that an initial interest would be granted.

$A_{5}$ : the decision affected an established liberty or interest.

$A_{6}$ : there was a standard administrative procedure which the decision-maker did not follow.

\section{Cases in which the applicant did not have a legitimate expectation which was affected by the decision}

$C_{7}$ : South Australia v. O'Shea (1987) 163 CLR 378 ("SA v. O'Shea")

$A_{1}$ : the decision-maker did not break a promise or undertaking.

$A_{2}$ : the decision-maker did not go against an established course of practice.

$A_{3}$ : the decision did not involve a refusal to renew an existing interest.

$A_{4}$ : neither the decision-maker nor a statutory provision suggested that an initial interest would be granted.

${ }^{64}[1983] 2$ AC 629. 
$A_{5}$ : the decision affected an established liberty or interest.

$A_{6}$ : there was no standard administrative procedure which the decision-maker should have followed.

In South Australia v. O'Shea, ${ }^{65}$ a 1987 decision of five judges of the High Court of Australia, O'Shea had been convicted of two offences of indecent assault of young children. He was released on licence and remained at liberty after the licence expired. Over a year later, after allegations had been made against him, O'Shea was apprehended and detained. The parole board recommended his release on licence on various conditions, but the Governor in Council resolved to take no action. O'Shea had been given a hearing by the Parole Board, but he claimed he was entitled to a further hearing before the Governor in Council could exercise his discretionary powers under s. 77a(7a) of the Criminal Law Consolidation Act, 1935 (SA).

Mason CJ, Wilson, Brennan and Toohey JJ (Deane J dissenting) held that O'Shea was not entitled to a further hearing. "Given the nature of this decision, it cannot be said that Mr O'Shea could have more than a hope that the Governor would be prepared to act on the recommendation of the Board. Hope, of itself, is not sufficient to ground an expectation that will attract legal consequences. So far as the concept of legitimate expectation is concerned, Mr O'Shea must be taken to know that the Act committed to the Governor, with the advice and consent of the Executive Council, the responsibility for determining where the public interest lay ... The nature of the decision that they were required to make was such that participation by Mr O'Shea was inappropriate." 66

$C_{8}$ : Minister for Arts Heritage and Environment v. Peko-Wallsend Ltd (1987) 75 ALR 218 ("Minister for Environment v. Peko-Wallsend")

$A_{1}$ : the decision-maker did not break a promise or undertaking.

$A_{2}$ : the decision-maker did not go against an established course of practice.

$A_{3}$ : the decision did not involve a refusal to renew an existing interest.

$A_{4}$ : neither the decision-maker nor a statutory provision suggested that an initial interest would be granted.

$A_{5}$ : the decision did not affect an established liberty or interest.

$A_{6}$ : there was no standard administrative procedure which the decision-maker should have followed.

In Minister for Arts Heritage and Environment v. Peko-Wallsend Ltd, ${ }^{67}$ a 1987 decision of the Full Court of the Federal Court of Australia, Peko-Wallsend held various mining interests in Stage 2 of Kakadu National Park. Federal Cabinet decided to nominate Stage 2 for inclusion in the World Heritage List, so it became "identified property" within the meaning of s. 3(2) of the World Heritage Properties Conservation Act 1983 (Cth). This meant that the Governor-General

65 (1987) 163 CLR 378.

66 ibid. at 402, per Wilson and Toohey JJ.

${ }^{67}(1987) 75$ ALR 218. 
could, by proclamation, make mining operations unlawful in the area. The decision did not affect Peko-Wallsend's mining rights which were preserved under s. 8B of the National Parks and Wildlife Conservation Act 1975 (Cth).

Before Cabinet's decision, Peko-Wallsend had lobbied Ministers and other officials extensively, seeking to preserve their mining interests. After the decision they commenced proceedings to prevent the Government from taking any further steps to have Stage 2 nominated on the World Heritage List, claiming that Cabinet was bound by the rules of natural justice and had failed to give PekoWallsend an opportunity to be heard. Beaumont J (a Federal Court judge) agreed, and held the Cabinet decision void. ${ }^{68}$

The Full Court of the Federal Court disagreed. Bowen CJ decided that "it would ... be inappropriate for this court to interfere to set aside a Cabinet decision involving such complex policy considerations". 69 Both Sheppard and Wilcox JJ held that Peko-Wallsend had had adequate opportunity to put their case to relevant Ministers and officials before the Cabinet decision, and were not denied natural justice. ${ }^{70}$ However, Wilcox $\mathrm{J}$ (with whose reasons the other two judges generally agreed) held that the Cabinet's decision in this case did not attract the obligations of natural justice. ${ }^{71}$

$I_{\text {No-Expectation }}$ (the ideal case in which the applicant did not have a legitimate expectation which was affected by the decision):

$A_{1}$ : the decision-maker did not break a promise or undertaking.

$A_{2}$ : the decision-maker did not go against an established course of practice.

$A_{3}$ : the decision did not involve a refusal to renew an existing interest.

$A_{4}$ : neither the decision-maker nor a statutory provision suggested that an initial interest would be granted.

$A_{5}$ : the decision did not affect an established liberty or interest.

$A_{6}$ : there was no standard administrative procedure which the decision-maker should have followed.

\footnotetext{
${ }^{68}$ Peko-Wallsend Ltd v. Minister for Arts Heritage and Environment (1986) 70 ALR 523.

${ }^{69}$ (1987) 75 ALR 218 at 225.

70 ibid. at 228 per Sheppard J; at 253 per Wilcox J.

${ }^{71}$ ibid. at 253 .
} 


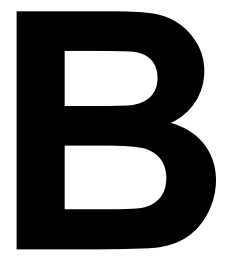

\section{Example reports}

The law is not a series of calculating machines where definitions and answers come tumbling out when the right levers are pushed.

William O. Douglas (1948) ${ }^{17}$

An argument which does not convince yourself, may convince the Judge to whom you urge it; and if it does convince him, why, then, Sir, you are wrong, and he is right.

Samuel Johnson $(1768)^{18}$ 


\section{B.1 Introduction}

This appendix presents six example reports from four test cases, demonstrating the use of each of the four specifications described in chapter 5 and given in appendix A. For each example, SHYSTER was requested to hypothesize with a limit of two changed attribute values, and to report on up to one hypothetical per result.

Parker v. British Airways ${ }^{19}$ is used as a test case for the Finder specification. SHYSTER's opinion on that case is evaluated in $\S 5.2 .3$, and its report file is in $\S$ B.2.

Australasian Performing Right Association Ltd v. Jain ${ }^{20}$ is used in $\S 5.3 .3$ as a test case for the AUthorization specification, and SHYSTER's advice is evaluated there. SHYSTER's report file for APRA v. Jain is in $\S \mathrm{B} .3$.

Re Porter; Re Transport Workers Union of Australia ${ }^{21}$ is used as a test case for the EmPloyeE specification. SHYSTER's opinion on Re Porter; Re TWU is evaluated in $\S 5.4 .3$ and its report file is in $\S$ B.4.

Ainsworth v. Criminal Justice Commission ${ }^{22}$ is used in $\S 5.5 .3$ as a test case for the NATURAL specification, and SHYSTER's advice is evaluated there. SHYSTER prepares a report file for each of the three areas in that specification: the Expectation, Affected and Natural areas. All three reports, combined, are in $\S$ B.5. ${ }^{23}$

(The report file for Building Workers' Industrial Union of Australia v. Odco Pty $L t d^{24}$ is given in $\S \mathrm{C} .13$ as part of the complete example, using the EMPLOYEe specification, in appendix C.)

\section{B.2 Report file for Parker v. British Airways}

\section{Finder area}

\section{Instant case}

In the instant case, the finder was not the occupier of the premises where the chattel was found; the chattel was not attached; the other claimant was not the owner of the premises where the chattel was found; the other claimant was not the true owner of the chattel and was not claiming through the rights of the true owner; the finder handed over the chattel to the other claimant after the finding; neither party relied on the terms of an agreement regarding the right to the chattel; the finder was not a servant of the other claimant; the chattel was not hidden and was not in a position so as to be difficult to find; an attempt was made to find the true owner of the chattel or, alternatively, the chattel was clearly abandoned; and neither party knew of the existence of the chattel prior to the finding.

In my opinion-following Bridges v. Hawkesworth - the finder wins.

In Bridges v. Hawkesworth, ${ }^{1}$ an 1851 decision of the Queen's Bench Division of the English High Court, the plaintiff found a bundle of banknotes on the floor of the public

${ }^{1}(1851) 21$ LJQB 75. 
area of a shop. He handed the notes to the shopkeeper in order that the true owner of the notes might be found. Although the owner was never found, the shopkeeper refused to return the notes to the finder. The Court found for the finder, holding that there is a "general right of [a] finder to any article which has been lost as against all the world except the true owner". ${ }^{2}$ It was further noted that the notes had never been in the custody of the shopkeeper nor within the protection of his house as might be the case had they intentionally been deposited there.

There are several significant similarities between the instant case and Bridges $v$. Hawkesworth: the finder was not the occupier of the premises where the chattel was found; the chattel was not attached; the other claimant was not the true owner of the chattel and was not claiming through the rights of the true owner; the finder handed over the chattel to the other claimant after the finding; neither party relied on the terms of an agreement regarding the right to the chattel; the finder was not a servant of the other claimant; the chattel was not hidden and was not in a position so as to be difficult to find; an attempt was made to find the true owner of the chattel or, alternatively, the chattel was clearly abandoned; and neither party knew of the existence of the chattel prior to the finding.

However, the instant case is not on all fours with Bridges v. Hawkesworth. In that case the other claimant was the owner of the premises where the chattel was found.

Nevertheless, I believe that Bridges v. Hawkesworth should be followed.

If City of London Corporation v. Appleyard (1) is followed then the finder loses.

In City of London Corporation v. Appleyard (1), ${ }^{3}$ a 1963 decision of the Queen's Bench Division of the English High Court, workmen employed by Wates Ltd were engaged in cutting a key-way into a cellar wall for the purposes of securing a foundation when they found an old wall-safe built into a recess of the old wall. Inside was a wooden box which contained a large number of Bank of England notes. The notes were handed over to the City of London police who sought interpleader proceedings to determine who was entitled to the possession of the notes.

Wates Ltd was an independent contractor engaged by Yorkwin Investments Ltd for a construction project. Yorkwin was lessee in possession of the property which was owned in fee simple by the City of London.

The Court followed the decision in South Staffordshire Water Co. v. Sharman ${ }^{4}$ in holding that the occupier is, in the absence of a better title elsewhere, entitled to the possession of objects which are attached to or under the land. Consequently, since the notes were in a wooden box within a safe built into the wall of the old building, the safe formed part of the demised premises. Yorkwin, being in lawful possession of the premises, were in de facto possession of the safe, even though ignorant of its existence.

Although Yorkwin was entitled to possession as against the finders, they in turn were displaced by the City of London which relied successfully on a term in the lease which granted them the right to certain objects found on the premises.

\footnotetext{
${ }^{2}$ ibid. at 77 , per Patteson J.

${ }^{3}$ [1963] 1 WLR 982.

4 [1896] 2 QB 44.
} 
There are several similarities between the instant case and London v. Appleyard (1): the finder was not the occupier of the premises where the chattel was found; the other claimant was not the owner of the premises where the chattel was found; the other claimant was not the true owner of the chattel and was not claiming through the rights of the true owner; neither party relied on the terms of an agreement regarding the right to the chattel; an attempt was made to find the true owner of the chattel or, alternatively, the chattel was clearly abandoned; and neither party knew of the existence of the chattel prior to the finding.

However, there are several significant differences between the instant case and London v. Appleyard (1). In that case the chattel was attached; the finder did not hand over the chattel to the other claimant after the finding; the finder was a servant of the other claimant; and the chattel was hidden or was in a position so as to be difficult to find.

Despite the fact that London v. Appleyard (1) and Bridges v. Hawkesworth are both decisions of the Queen's Bench Division of the English High Court, there is nothing in London v. Appleyard (1) to warrant any change in my conclusion.

\section{Hypothetical 1}

Consider the instant case changed so that the following is true: the other claimant was the owner of the premises where the chattel was found.

If that were so then I would be more strongly of the opinion that-following Bridges $v$. Hawkesworth - the finder wins.

Details of Bridges v. Hawkesworth are summarized above. The hypothetical case is on all fours with Bridges v. Hawkesworth.

If City of London Corporation v. Appleyard (2) or South Staffordshire Water Co. v. Sharman are followed then the finder loses.

In City of London Corporation v. Appleyard (2), ${ }^{5}$ a 1963 decision of the Queen's Bench Division of the English High Court, workmen employed by Wates Ltd were engaged in cutting a key-way into a cellar wall for the purposes of securing a foundation when they found an old wall-safe built into a recess of the old wall. Inside was a wooden box which contained a large number of Bank of England notes. The notes were handed over to the City of London police who sought interpleader proceedings to determine who was entitled to the possession of the notes.

Wates Ltd was an independent contractor engaged by Yorkwin Investments Ltd for a construction project. Yorkwin was lessee in possession of the property which was owned in fee simple by the City of London. The Court found that the safe formed part of the demised premises and that, consequently, Yorkwin was entitled to the notes as against the workmen.

${ }^{5}[1963] 1$ WLR 982. 
The lease contained a clause which purported to grant the rights to "every relic or article of antiquity rarity or value" to the City of London. The sole issue was to determine if the notes fell into that description. The Court could find no reason for limiting the generality of the words and so found for the City of London.

There are several similarities between the hypothetical case and London v. Appleyard (2): the other claimant was the owner of the premises where the chattel was found; the other claimant was not the true owner of the chattel and was not claiming through the rights of the true owner; the finder handed over the chattel to the other claimant after the finding; the finder was not a servant of the other claimant; an attempt was made to find the true owner of the chattel or, alternatively, the chattel was clearly abandoned; and neither party knew of the existence of the chattel prior to the finding.

However, there are several significant differences between the hypothetical case and London v. Appleyard (2). In that case the finder was the occupier of the premises where the chattel was found; the chattel was attached; one of the parties relied on the terms of an agreement made with the other which purported to give her/him the right to the chattel; and the chattel was hidden or was in a position so as to be difficult to find.

Despite the fact that London v. Appleyard (2) and Bridges v. Hawkesworth are both decisions of the Queen's Bench Division of the English High Court, there is nothing in London v. Appleyard (2) to warrant any change in my conclusion.

In 1896, the Queen's Bench Division of the English High Court also decided South Staffordshire Water Co. v. Sharman. ${ }^{6}$ (Note, however, that London v. Appleyard (2) is 67 years more recent than South Staffordshire v. Sharman.)

In South Staffordshire v. Sharman, the defendant was a workman employed by the plaintiff to clean out a pool located on land owned by the plaintiff. During the operation the defendant found two gold rings embedded in the mud at the bottom of the pool. Although the plaintiff demanded the rings, the defendant refused to give them up. He placed them in the hands of police authorities who unsuccessfully endeavoured to find the owners of the rings. The police returned the rings to the defendant who was then sued in detinue for the recovery of the rings.

It was proved at the trial that there was no special contract between the parties which called upon the defendant to give up any articles which might be found.

Although the county court held in favour of the defendant on the basis of Bridges $v$. Hawkesworth, ${ }^{7}$ the appeal found for the plaintiff on the basis that they had, as owners of the land and pool, the right to exercise control over the same. Bridges v. Hawkesworth was distinguished on the grounds that the notes in that case were in a public part of the shop and the shopkeeper did not in any sense control them.

The Court stated a general principle: where a person has possession of a house or land with a manifest intention to exercise control over it and the things which may be upon or in it, then there is a presumption that things found there are in the possession of the owner.

${ }^{6}[1896] 2$ QB 44.

${ }^{7}(1851) 21$ LJQB 75. 
There are several similarities between the hypothetical case and South Staffordshire v. Sharman: the finder was not the occupier of the premises where the chattel was found; the other claimant was the owner of the premises where the chattel was found; the other claimant was not the true owner of the chattel and was not claiming through the rights of the true owner; neither party relied on the terms of an agreement regarding the right to the chattel; an attempt was made to find the true owner of the chattel or, alternatively, the chattel was clearly abandoned; and neither party knew of the existence of the chattel prior to the finding.

However, there are several significant differences between the hypothetical case and South Staffordshire $v$. Sharman. In that case the chattel was attached; the finder did not hand over the chattel to the other claimant after the finding; the finder was a servant of the other claimant; and the chattel was hidden or was in a position so as to be difficult to find.

Despite the fact that South Staffordshire v. Sharman and Bridges v. Hawkesworth are both decisions of the Queen's Bench Division of the English High Court, there is nothing in South Staffordshire v. Sharman to warrant any change in my conclusion.

\section{Hypothetical 2}

Consider the instant case changed so that the following is true: the finder was a servant of the other claimant; and the chattel was hidden or was in a position so as to be difficult to find.

If that were so then my opinion would be that-following City of London Corporation v. Appleyard (1) - the finder loses.

Details of London v. Appleyard (1) are summarized above. There are several significant similarities between the hypothetical case and London v. Appleyard (1): the finder was not the occupier of the premises where the chattel was found; the other claimant was not the owner of the premises where the chattel was found; the other claimant was not the true owner of the chattel and was not claiming through the rights of the true owner; neither party relied on the terms of an agreement regarding the right to the chattel; the finder was a servant of the other claimant; the chattel was hidden or was in a position so as to be difficult to find; an attempt was made to find the true owner of the chattel or, alternatively, the chattel was clearly abandoned; and neither party knew of the existence of the chattel prior to the finding.

However, the hypothetical case is not on all fours with London v. Appleyard (1). In that case the chattel was attached; and the finder did not hand over the chattel to the other claimant after the finding.

Nevertheless, I believe that London v. Appleyard (1) should be followed.

If Hannah $v$. Peel is followed then the finder wins.

In Hannah v. Peel, ${ }^{8}$ a 1945 decision of the King's Bench Division of the English High Court, a brooch was found by the plaintiff who was a lance-corporal stationed in a

${ }^{8}$ [1945] 1 KB 509. 
house owned by the defendant. The house had been requisitioned by the army during the war and had never been occupied by the defendant.

The plaintiff was adjusting the black-out curtains when he touched something on the top of the window-frame. He thought the object to be a piece of dirt or plaster and he dropped it on the outside window ledge. On the following morning, he saw that it was a brooch and, on the advice of his commanding officer, turned it over to the police for the purpose of finding the owner. In the following year, the police returned the brooch to the defendant who sold it to a jeweller. The plaintiff at all times maintained his rights to the brooch against all persons other than the true owner.

The Court found for the plaintiff on the basis of Bridges v. Hawkesworth ${ }^{9}$ after a thorough review of the authorities. The Court further noted that the defendant was never in possession of the premises, that the brooch was never his, and that he had no knowledge of it until it was brought to his notice by the finder.

There are several similarities between the hypothetical case and Hannah v. Peel: the finder was not the occupier of the premises where the chattel was found; the chattel was not attached; the other claimant was not the true owner of the chattel and was not claiming through the rights of the true owner; neither party relied on the terms of an agreement regarding the right to the chattel; the chattel was hidden or was in a position so as to be difficult to find; an attempt was made to find the true owner of the chattel or, alternatively, the chattel was clearly abandoned; and neither party knew of the existence of the chattel prior to the finding.

However, there are several significant differences between the hypothetical case and Hannah v. Peel. In that case the other claimant was the owner of the premises where the chattel was found; the finder did not hand over the chattel to the other claimant after the finding; and the finder was not a servant of the other claimant.

Despite the fact that Hannah v. Peel is a decision of the King's Bench Division of the English High Court (and as good authority as a case decided by the Queen's Bench Division of the English High Court-like London v. Appleyard (1)), there is nothing in Hannah v. Peel to warrant any change in my conclusion.

\section{B.3 Report file for Australasian Performing Right Association Ltd v. Jain}

\section{Authorization area}

\section{Instant case}

The notion of authorization extends beyond the authority given to an agent. The word "authorize" should be "understood in its ordinary dictionary sense of "sanction, approve, and countenance." "1

${ }^{9}(1851) 21$ LJQB 75.

${ }^{1}$ Falcon v. Famous Players Film Co. [1926] 2 KB 474 at 491, per Bankes LJ. 
"[A] person who has under his control the means by which an infringement of copyright may be committed ... and who makes it available to other persons, knowing, or having reason to suspect, that it is likely to be used for the purpose of committing an infringement, and omitting to take reasonable steps to limit its use to legitimate purposes, would authorize any infringement that resulted from its use." ${ }^{2}$

In the instant case, the infringer was not an employee of the accused; the infringer was an independent contractor to the accused; the accused did not sell or hire the infringer the means of infringing; the accused had the power to prevent the infringement; the accused did not take reasonable steps to avoid the infringement; the accused knew, or had reason to anticipate or suspect, that the infringing act was to be, or was likely to be, done; and the specific infringement was not causally related to an incitement to infringe on the part of the accused.

In my opinion-following Mellor v. Australian Broadcasting Commission-the accused authorized the infringement.

In Mellor v. Australian Broadcasting Commission, ${ }^{3}$ a 1940 decision of the Judicial Committee of the Privy Council, Mellor and others held the sole right to perform in public in Australia some musical works arranged for performance by brass and military bands. They published and distributed advertising pamphlets which included a statement that all of their sheet music was " 'Free for Public Performance' anywhere ... We have paid for the performing rights of every piece we issue." 4 The ABC engaged bands to play some of this music, and broadcast the bands' performances on radio.

The Privy Council held that the ABC had authorized the bands to perform the musical works within the meaning of s. 1(2) of the Copyright Act 1911 (UK) which was in force in Australia by virtue of the Copyright Act 1912 (Cth). However, the ABC had not infringed the plaintiffs' sole right to authorize public performance because the statements made in the pamphlets amounted to consent.

There are several significant similarities between the instant case and Mellor $v$. $A B C$ : the infringer was not an employee of the accused; the infringer was an independent contractor to the accused; the accused did not sell or hire the infringer the means of infringing; the accused had the power to prevent the infringement; the accused did not take reasonable steps to avoid the infringement; and the accused knew, or had reason to anticipate or suspect, that the infringing act was to be, or was likely to be, done.

However, the instant case is not on all fours with Mellor v. ABC. In that case the specific infringement was causally related to an incitement to infringe on the part of the accused.

Nevertheless, I believe that Mellor v. $A B C$ should be followed.

If $R C A$ Corporation v. John Fairfax and Sons Ltd is followed then the accused did not authorize the infringement.

\footnotetext{
${ }^{2}$ University of New South Wales v. Moorhouse (1975) 133 CLR 1 at 13, per Gibbs J.

${ }^{3}[1940]$ AC 491.

4 ibid. at $498-9$.
} 
In RCA Corporation v. John Fairfax and Sons Ltd, ${ }^{5}$ a 1981 decision of the Supreme Court of New South Wales, the Fairfax newspaper the Sun-Herald carried an article which pointed out that, using cassette tapes and good quality taping equipment, the same album can be taped by many people. It also discussed how the advent of FM radio had made it easy for people to tape new album and single releases without buying the discs: "Why spend nearly $\$ 10$ on the new David Bowie album when you can tape it from 2JJJ?" 6

Kearney J held that "authorization involves some element of causation - and hence the necessity for some relationship creating a link or connection however tenuous between the authorizer and the infringer." 7 There was no such link, so Fairfax had not authorized any infringement within the meaning of s. 13(2) of the Copyright Act 1968 (Cth).

There are several similarities between the instant case and $R C A v$. Fairfax: the infringer was not an employee of the accused; the accused did not sell or hire the infringer the means of infringing; the accused did not take reasonable steps to avoid the infringement; the accused knew, or had reason to anticipate or suspect, that the infringing act was to be, or was likely to be, done; and the specific infringement was not causally related to an incitement to infringe on the part of the accused.

However, there are two very significant differences between the instant case and $R C A v$. Fairfax. In that case the infringer was not an independent contractor to the accused; and the accused did not have the power to prevent the infringement.

Despite the fact that RCA v. Fairfax is a decision of the Supreme Court of New South Wales (and better authority than a case decided by the Judicial Committee of the Privy Council-like Mellor v. $A B C$ ), there is nothing in RCA v. Fairfax to warrant any change in my conclusion.

If Australasian Performing Right Association Ltd v. Miles is followed then the accused is liable (directly or vicariously) for the infringement.

In Australasian Performing Right Association Ltd v. Miles, ${ }^{8}$ a 1961 decision of the Supreme Court of New South Wales, the Dee Why RSL Club engaged a band to play at a dance held at the club. During the dance the band played I've Got a Lovely Bunch of Coconuts, the copyright in which was owned by the Australasian Performing Right Association.

Jacobs $\mathrm{J}$ held that the members of the band were servants of the club, because "the club through its officers was exercising a control over the work performed in such a way as to show that there was an authority to command the orchestra in its performance." 9 So the members of the club, through the band, performed the musical work and infringed the copyright under s. 2(1) of the Copyright Act 1911 (UK) which was in force in Australia by virtue of the Copyright Act 1912 (Cth).

\footnotetext{
${ }^{5}$ [1981] 1 NSWLR 251.

6 ibid. at 252 .

7 ibid. at 259 .

8 [1962] NSWR 405.

9 ibid. at 407 .
} 
There are several similarities between the instant case and APRA v. Miles: the accused did not sell or hire the infringer the means of infringing; the accused had the power to prevent the infringement; the accused did not take reasonable steps to avoid the infringement; and the accused knew, or had reason to anticipate or suspect, that the infringing act was to be, or was likely to be, done.

However, there are several significant differences between the instant case and APRA $v$. Miles. In that case the infringer was an employee of the accused; the infringer was not an independent contractor to the accused; and the specific infringement was causally related to an incitement to infringe on the part of the accused.

Despite the fact that APRA v. Miles is a decision of the Supreme Court of New South Wales (and better authority than a case decided by the Judicial Committee of the Privy Council-like Mellor v. ABC), there is nothing in APRA v. Miles to warrant any change in my conclusion.

\section{Hypothetical 1}

Consider the instant case changed so that the following is true: the specific infringement was causally related to an incitement to infringe on the part of the accused.

If that were so then I would be more strongly of the opinion that-following Mellor $v$. Australian Broadcasting Commission - the accused authorized the infringement.

Details of Mellor $v . A B C$ are summarized above. The hypothetical case is on all fours with Mellor v. $A B C$.

If RCA Corporation v. John Fairfax and Sons Ltd is followed then the accused did not authorize the infringement.

Details of $R C A$ v. Fairfax are summarized above. There are several similarities between the hypothetical case and $R C A v$. Fairfax: the infringer was not an employee of the accused; the accused did not sell or hire the infringer the means of infringing; the accused did not take reasonable steps to avoid the infringement; and the accused knew, or had reason to anticipate or suspect, that the infringing act was to be, or was likely to be, done.

However, there are several significant differences between the hypothetical case and $R C A v$. Fairfax. In that case the infringer was not an independent contractor to the accused; the accused did not have the power to prevent the infringement; and the specific infringement was not causally related to an incitement to infringe on the part of the accused.

Despite the fact that RCA v. Fairfax is a decision of the Supreme Court of New South Wales (and better authority than a case decided by the Judicial Committee of the Privy Council-like Mellor v. ABC), there is nothing in RCA v. Fairfax to warrant any change in my conclusion.

If Australasian Performing Right Association Ltd v. Miles is followed then the accused is liable (directly or vicariously) for the infringement. 
Details of APRA v. Miles are summarized above. There are several similarities between the hypothetical case and APRA $v$. Miles: the accused did not sell or hire the infringer the means of infringing; the accused had the power to prevent the infringement; the accused did not take reasonable steps to avoid the infringement; the accused knew, or had reason to anticipate or suspect, that the infringing act was to be, or was likely to be, done; and the specific infringement was causally related to an incitement to infringe on the part of the accused.

However, there are two very significant differences between the hypothetical case and APRA v. Miles. In that case the infringer was an employee of the accused; and the infringer was not an independent contractor to the accused.

Despite the fact that APRA v. Miles is a decision of the Supreme Court of New South Wales (and better authority than a case decided by the Judicial Committee of the Privy Council-like Mellor v. ABC), there is nothing in APRA v. Miles to warrant any change in my conclusion.

\section{Hypothetical 2}

Consider the instant case changed so that the following is true: the accused did not know, and had no reason to anticipate or suspect, that the infringing act was to be, or was likely to be, done.

If that were so then my opinion would be that-following Performing Right Society Ltd v. Ciryl Theatrical Syndicate Ltd - the accused did not authorize the infringement.

In Performing Right Society Ltd v. Ciryl Theatrical Syndicate $L t d,{ }^{10}$ a 1923 decision of the English Court of Appeal, the syndicate was the lessee of a theatre. The managingdirector of the syndicate produced a play at that theatre, and engaged a band to perform at the theatre under the direction of a bandmaster. In the absence of the managing-director, and without his knowledge, the band performed works the copyright in which was owned by the Performing Right Society.

Bankes, Scrutton and Atkin LJJ held that the managing-director had not authorized the infringing performances, within the meaning of s. 1(2) of the Copyright Act 1911 (UK), because the infringement occurred without his knowledge and he had no reason to anticipate or suspect that the band was likely to give performances which would breach copyright.

The hypothetical case is on all fours with PRS v. Ciryl.

If Mellor v. Australian Broadcasting Commission is followed then the accused authorized the infringement.

Details of Mellor v. $A B C$ are summarized above. There are several similarities between the hypothetical case and Mellor v. $A B C$ : the infringer was not an employee of the accused; the infringer was an independent contractor to the accused; the accused did not sell or hire the infringer the means of infringing; the accused had the power to prevent the infringement; and the accused did not take reasonable steps to avoid the infringement.

${ }^{10}[1924] 1$ KB 1. 
However, there are two very significant differences between the hypothetical case and Mellor v. $A B C$. In that case the accused knew, or had reason to anticipate or suspect, that the infringing act was to be, or was likely to be, done; and the specific infringement was causally related to an incitement to infringe on the part of the accused.

Despite the fact that Mellor $v . A B C$ is a decision of the Judicial Committee of the Privy Council (and better authority than a case decided by the English Court of Appeal-like PRS v. Ciryl), there is nothing in Mellor $v$. ABC to warrant any change in my conclusion.

If Australasian Performing Right Association Ltd v. Miles is followed then the accused is liable (directly or vicariously) for the infringement.

Details of APRA v. Miles are summarized above. There are several similarities between the hypothetical case and APRA $v$. Miles: the accused did not sell or hire the infringer the means of infringing; the accused had the power to prevent the infringement; and the accused did not take reasonable steps to avoid the infringement.

However, there are several significant differences between the hypothetical case and APRA $v$. Miles. In that case the infringer was an employee of the accused; the infringer was not an independent contractor to the accused; the accused knew, or had reason to anticipate or suspect, that the infringing act was to be, or was likely to be, done; and the specific infringement was causally related to an incitement to infringe on the part of the accused.

Despite the fact that APRA v. Miles is a decision of the Supreme Court of New South Wales (and better authority than a case decided by the English Court of Appeallike PRS v. Ciryl), there is nothing in APRA v. Miles to warrant any change in my conclusion.

\section{Hypothetical 3}

Consider the instant case changed so that the following is true: the infringer was an employee of the accused; and the infringer was not an independent contractor to the accused.

If that were so then my opinion would be that-following Australasian Performing Right Association Ltd v. Miles - the accused is liable (directly or vicariously) for the infringement.

Details of APRA v. Miles are summarized above. There are several significant similarities between the hypothetical case and APRA $v$. Miles: the infringer was an employee of the accused; the infringer was not an independent contractor to the accused; the accused did not sell or hire the infringer the means of infringing; the accused had the power to prevent the infringement; the accused did not take reasonable steps to avoid the infringement; and the accused knew, or had reason to anticipate or suspect, that the infringing act was to be, or was likely to be, done.

However, the hypothetical case is not on all fours with APRA $v$. Miles. In that case the specific infringement was causally related to an incitement to infringe on the part of the accused.

Nevertheless, I believe that APRA v. Miles should be followed. 
If University of New South Wales v. Moorhouse is followed then the accused authorized the infringement.

In University of New South Wales v. Moorhouse, ${ }^{11}$ a 1975 decision of three judges of the High Court of Australia, a graduate of the University used a photocopy machine in the University library to make two copies of a story from a library copy of a book of short stories.

McTiernan ACJ, Gibbs and Jacobs JJ held that the University had authorized the infringement within the meaning of s. 36(1) of the Copyright Act 1968 (Cth); it had the power to prevent infringements, but had not taken reasonable steps to prevent them. ${ }^{12}$ Gibbs J's statement about what constitutes authorization of an infringement is quoted above.

There are several similarities between the hypothetical case and UNSW v. Moorhouse: the infringer was not an independent contractor to the accused; the accused had the power to prevent the infringement; the accused did not take reasonable steps to avoid the infringement; the accused knew, or had reason to anticipate or suspect, that the infringing act was to be, or was likely to be, done; and the specific infringement was not causally related to an incitement to infringe on the part of the accused.

However, there are two very significant differences between the hypothetical case and $U N S W$ v. Moorhouse. In that case the infringer was not an employee of the accused; and the accused sold or hired the infringer the means of infringing.

Despite the fact that $U N S W v$. Moorhouse is a decision of three judges of the High Court of Australia (and better authority than a case decided by the Supreme Court of New South Wales-like APRA v. Miles), there is nothing in UNSW v. Moorhouse to warrant any change in my conclusion.

If RCA Corporation v. John Fairfax and Sons Ltd is followed then the accused did not authorize the infringement.

Details of RCA $v$. Fairfax are summarized above. There are several similarities between the hypothetical case and RCA $v$. Fairfax: the infringer was not an independent contractor to the accused; the accused did not sell or hire the infringer the means of infringing; the accused did not take reasonable steps to avoid the infringement; the accused knew, or had reason to anticipate or suspect, that the infringing act was to be, or was likely to be, done; and the specific infringement was not causally related to an incitement to infringe on the part of the accused.

However, there are two very significant differences between the hypothetical case and $R C A v$. Fairfax. In that case the infringer was not an employee of the accused; and the accused did not have the power to prevent the infringement.

Despite the fact that RCA v. Fairfax and APRA v. Miles are both decisions of the Supreme Court of New South Wales, there is nothing in $R C A v$. Fairfax to warrant any change in my conclusion.

${ }^{11}(1975) 133$ CLR 1.

${ }^{12}$ The addition, in 1980, of s. 39A to the Copyright Act ameliorated the effect of UNSW $v$. Moorhouse as far as photocopying in libraries is concerned. 


\section{B.4 Report file for Re Porter; Re Transport Workers Union of Australia}

\section{Employee area}

\section{Instant case}

The law distinguishes between a contract of service (between employer and employee) and a contract for services (between principal and independent contractor). This distinction affects the terms that will be implied in the absence of an express agreement, the liability of the employer to third parties, the applicability of industrial awards, the applicability of statutes which may affect workers' compensation, occupational health and safety, long-service leave, fringe benefits tax, etc.

The terms "employer" and "worker" are used here to mean "employer" and "employee" (in the case of a contract of service) or "principal" and "independent contractor" (in the case of a contract for services).

In the instant case, the employer directed the manner in which the work was to be done; the worker was not allowed to use her/his own discretion in doing an aspect of the work that was not specified beforehand; the worker was an integral part of the employer's business; the worker owned the tools or provided the transport with which she/he performed the work; it is not known whether the employer would make a profit/loss if the work performed by the worker cost less/more than expected; the work was not performed on the employer's premises; it is not known whether the employer supervised or inspected the work; the worker was not in business on her/his own account; the worker was not allowed to employ others to assist with her/his work; the worker was obliged to work only for the employer; the worker was not required to work at specified times; the employer paid the worker by time; the money that the employer paid to the worker was not stated to be a "fee"; the money that the employer paid to the worker was not stated to be "wages" or "salary"; the employer deducted PAYE tax instalments from the worker's pay; the employer paid the worker neither sick pay nor holiday pay; the employer and the worker did not express any intention that the relationship would be one of employer and employee; and the employer and the worker expressed an intention that the relationship would be one of principal and independent contractor.

In my opinion-following Ferguson v. John Dawson $\mathcal{E}$ Partners (Contractors) Ltd-the worker is an employee.

In Ferguson v. John Dawson $\&$ Partners (Contractors) Ltd, ${ }^{1}$ a 1976 decision of the English Court of Appeal, Ferguson fell off a roof while removing some scaffolding boards. He claimed damages against John Dawson (the building contractors) for breach of statutory duty relying on the Construction (Working Places) Regulations 1966 (UK). This duty would only be owed if Ferguson was an employee of John Dawson.

\footnotetext{
${ }^{1}[1976] 1$ WLR 1213.
} 
Megaw and Browne LJJ held that, despite the fact that both parties labelled Ferguson a "self-employed labour only subcontractor", ${ }^{2}$ the reality of the relationship between them was that of employer/employee.

There are several significant similarities between the instant case and Ferguson $v$. Dawson: the employer directed the manner in which the work was to be done; the worker was not allowed to use her/his own discretion in doing an aspect of the work that was not specified beforehand; the worker was an integral part of the employer's business; the work was not performed on the employer's premises; the worker was not in business on her/his own account; the worker was not allowed to employ others to assist with her/his work; the employer paid the worker by time; the money that the employer paid to the worker was not stated to be a "fee"; the money that the employer paid to the worker was not stated to be "wages" or "salary"; the employer paid the worker neither sick pay nor holiday pay; the employer and the worker did not express any intention that the relationship would be one of employer and employee; and the employer and the worker expressed an intention that the relationship would be one of principal and independent contractor.

However, the instant case is not on all fours with Ferguson v. Dawson. In that case the worker neither owned the tools nor provided the transport with which she/he performed the work; the employer would make a profit/loss if the work performed by the worker cost less/more than expected; the employer supervised or inspected the work; the worker was not obliged to work only for the employer; the worker was required to work at specified times; and the employer did not deduct PAYE tax instalments from the worker's pay.

Nevertheless, I believe that Ferguson v. Dawson should be followed.

If Ready Mixed Concrete (South East) Ltd v. Minister of Pensions and National Insurance is followed then the worker is an independent contractor.

In Ready Mixed Concrete (South East) Ltd v. Minister of Pensions and National Insurance, ${ }^{3}$ a 1967 decision of the Queen's Bench Division of the English High Court, Latimer worked for Ready Mixed as an "owner-driver." He was paid at mileage rates, and was obliged to buy the truck through a financial organization associated with Ready Mixed. The truck was painted in the company's colours, and he had to wear a Ready Mixed uniform. Latimer was obliged to meet the costs of maintenance, repair and insurance of the truck (and the attached mixing unit, which belonged to Ready Mixed). The Minister determined that Latimer was employed under a contract of service and therefore an "employed person" under s. 1(2) of the National Insurance Act 1965 (UK), making Ready Mixed liable to make weekly contributions.

MacKenna J examined the contract and held that the rights it conferred, and the duties it imposed, between Latimer and Ready Mixed were not such as to make it a contract of service.

There are several similarities between the instant case and Ready Mixed v. Minister: the worker was an integral part of the employer's business; the worker owned the tools or provided the transport with which she/he performed the work; the work was not

${ }^{2}$ ibid. at 1219, per Megaw LJ; at 1225, per Lawton LJ; at 1228, per Browne LJ.

${ }^{3}$ [1968] 2 QB 497. 
performed on the employer's premises; the worker was not in business on her/his own account; the worker was obliged to work only for the employer; the worker was not required to work at specified times; the money that the employer paid to the worker was not stated to be a "fee"; the money that the employer paid to the worker was not stated to be "wages" or "salary"; the employer paid the worker neither sick pay nor holiday pay; the employer and the worker did not express any intention that the relationship would be one of employer and employee; and the employer and the worker expressed an intention that the relationship would be one of principal and independent contractor.

However, there are several significant differences between the instant case and Ready Mixed $v$. Minister. In that case the employer did not direct the manner in which the work was to be done; the worker was allowed to use her/his own discretion in doing an aspect of the work that was not specified beforehand; the employer would not make a profit/loss if the work performed by the worker cost less/more than expected; the employer neither supervised nor inspected the work; the worker was allowed to employ others to assist with her/his work; the employer did not pay the worker by time; and the employer did not deduct PAYE tax instalments from the worker's pay. Note also that Ready Mixed v. Minister is only a decision of the Queen's Bench Division of the English High Court and not as good authority as a case decided by the English Court of Appeal-like Ferguson v. Dawson.

Consequently, there is nothing in Ready Mixed $v$. Minister to warrant any change in my conclusion.

\section{Instantiation 4}

It may be that the following is true of the instant case: the employer would not make a profit/loss if the work performed by the worker cost less/more than expected; and the employer neither supervised nor inspected the work.

If that is so then in my opinion-following Ready Mixed Concrete (South East) Ltd v. Minister of Pensions and National Insurance - the worker is an independent contractor.

Details of Ready Mixed $v$. Minister are summarized above. There are several significant similarities between the instantiated case and Ready Mixed $v$. Minister: the worker was an integral part of the employer's business; the worker owned the tools or provided the transport with which she/he performed the work; the employer would not make a profit/loss if the work performed by the worker cost less/more than expected; the work was not performed on the employer's premises; the employer neither supervised nor inspected the work; the worker was not in business on her/his own account; the worker was obliged to work only for the employer; the worker was not required to work at specified times; the money that the employer paid to the worker was not stated to be a "fee"; the money that the employer paid to the worker was not stated to be "wages" or "salary"; the employer paid the worker neither sick pay nor holiday pay; the employer and the worker did not express any intention that the relationship would be one of employer and employee; and the employer and the worker expressed an intention that the relationship would be one of principal and independent contractor. 
However, the instantiated case is not on all fours with Ready Mixed v. Minister. In that case the employer did not direct the manner in which the work was to be done; the worker was allowed to use her/his own discretion in doing an aspect of the work that was not specified beforehand; the worker was allowed to employ others to assist with her/his work; the employer did not pay the worker by time; and the employer did not deduct PAYE tax instalments from the worker's pay.

Nevertheless, I believe that Ready Mixed v. Minister should be followed.

If Ferguson v. John Dawson $\mathscr{G}$ Partners (Contractors) Ltd is followed then the worker is an employee.

Details of Ferguson v. Dawson are summarized above. There are several similarities between the instantiated case and Ferguson v. Dawson: the employer directed the manner in which the work was to be done; the worker was not allowed to use her/his own discretion in doing an aspect of the work that was not specified beforehand; the worker was an integral part of the employer's business; the work was not performed on the employer's premises; the worker was not in business on her/his own account; the worker was not allowed to employ others to assist with her/his work; the employer paid the worker by time; the money that the employer paid to the worker was not stated to be a "fee"; the money that the employer paid to the worker was not stated to be "wages" or "salary"; the employer paid the worker neither sick pay nor holiday pay; the employer and the worker did not express any intention that the relationship would be one of employer and employee; and the employer and the worker expressed an intention that the relationship would be one of principal and independent contractor.

However, there are several significant differences between the instantiated case and Ferguson v. Dawson. In that case the worker neither owned the tools nor provided the transport with which she/he performed the work; the employer would make a profit/loss if the work performed by the worker cost less/more than expected; the employer supervised or inspected the work; the worker was not obliged to work only for the employer; the worker was required to work at specified times; and the employer did not deduct PAYE tax instalments from the worker's pay.

Despite the fact that Ferguson v. Dawson is a decision of the English Court of Appeal (and better authority than a case decided by the Queen's Bench Division of the English High Court-like Ready Mixed v. Minister), there is nothing in Ferguson v. Dawson to warrant any change in my conclusion.

\section{Hypothetical 1}

Consider the instant case changed so that the following is true: the worker was not obliged to work only for the employer; and the employer did not deduct PAYE tax instalments from the worker's pay.

If that were so then I would be more strongly of the opinion that-following Ferguson $v$. John Dawson 8 Partners (Contractors) Ltd - the worker is an employee.

Details of Ferguson v. Dawson are summarized above. There are several significant similarities between the hypothetical case and Ferguson v. Dawson: the employer directed the manner in which the work was to be done; the worker was not allowed to use her/his own discretion in doing an aspect of the work that was not specified beforehand; 
the worker was an integral part of the employer's business; the work was not performed on the employer's premises; the worker was not in business on her/his own account; the worker was not allowed to employ others to assist with her/his work; the worker was not obliged to work only for the employer; the employer paid the worker by time; the money that the employer paid to the worker was not stated to be a "fee"; the money that the employer paid to the worker was not stated to be "wages" or "salary"; the employer did not deduct PAYE tax instalments from the worker's pay; the employer paid the worker neither sick pay nor holiday pay; the employer and the worker did not express any intention that the relationship would be one of employer and employee; and the employer and the worker expressed an intention that the relationship would be one of principal and independent contractor.

However, the hypothetical case is not on all fours with Ferguson v. Dawson. In that case the worker neither owned the tools nor provided the transport with which she/he performed the work; the employer would make a profit/loss if the work performed by the worker cost less/more than expected; the employer supervised or inspected the work; and the worker was required to work at specified times.

Nevertheless, I believe that Ferguson v. Dawson should be followed.

If Ready Mixed Concrete (South East) Ltd v. Minister of Pensions and National Insurance is followed then the worker is an independent contractor.

Details of Ready Mixed $v$. Minister are summarized above. There are several similarities between the hypothetical case and Ready Mixed $v$. Minister: the worker was an integral part of the employer's business; the worker owned the tools or provided the transport with which she/he performed the work; the work was not performed on the employer's premises; the worker was not in business on her/his own account; the worker was not required to work at specified times; the money that the employer paid to the worker was not stated to be a "fee"; the money that the employer paid to the worker was not stated to be "wages" or "salary"; the employer did not deduct PAYE tax instalments from the worker's pay; the employer paid the worker neither sick pay nor holiday pay; the employer and the worker did not express any intention that the relationship would be one of employer and employee; and the employer and the worker expressed an intention that the relationship would be one of principal and independent contractor.

However, there are several significant differences between the hypothetical case and Ready Mixed $v$. Minister. In that case the employer did not direct the manner in which the work was to be done; the worker was allowed to use her/his own discretion in doing an aspect of the work that was not specified beforehand; the employer would not make a profit/loss if the work performed by the worker cost less/more than expected; the employer neither supervised nor inspected the work; the worker was allowed to employ others to assist with her/his work; the worker was obliged to work only for the employer; and the employer did not pay the worker by time. Note also that Ready Mixed v. Minister is only a decision of the Queen's Bench Division of the English High Court and not as good authority as a case decided by the English Court of Appeal-like Ferguson v. Dawson.

Consequently, there is nothing in Ready Mixed $v$. Minister to warrant any change in my conclusion. 


\section{Hypothetical 2}

Consider the instant case changed so that the following is true: the worker was allowed to use her/his own discretion in doing an aspect of the work that was not specified beforehand; and the employer did not deduct PAYE tax instalments from the worker's pay.

If that were so then my opinion would be that-following Ready Mixed Concrete (South East) Ltd $v$. Minister of Pensions and National Insurance - the worker is an independent contractor.

Details of Ready Mixed $v$. Minister are summarized above. There are several significant similarities between the hypothetical case and Ready Mixed v. Minister: the worker was allowed to use her/his own discretion in doing an aspect of the work that was not specified beforehand; the worker was an integral part of the employer's business; the worker owned the tools or provided the transport with which she/he performed the work; the work was not performed on the employer's premises; the worker was not in business on her/his own account; the worker was obliged to work only for the employer; the worker was not required to work at specified times; the money that the employer paid to the worker was not stated to be a "fee"; the money that the employer paid to the worker was not stated to be "wages" or "salary"; the employer did not deduct PAYE tax instalments from the worker's pay; the employer paid the worker neither sick pay nor holiday pay; the employer and the worker did not express any intention that the relationship would be one of employer and employee; and the employer and the worker expressed an intention that the relationship would be one of principal and independent contractor.

However, the hypothetical case is not on all fours with Ready Mixed v. Minister. In that case the employer did not direct the manner in which the work was to be done; the employer would not make a profit/loss if the work performed by the worker cost less/more than expected; the employer neither supervised nor inspected the work; the worker was allowed to employ others to assist with her/his work; and the employer did not pay the worker by time.

Nevertheless, I believe that Ready Mixed $v$. Minister should be followed.

If Ferguson v. John Dawson 83 Partners (Contractors) Ltd is followed then the worker is an employee.

Details of Ferguson v. Dawson are summarized above. There are several similarities between the hypothetical case and Ferguson v. Dawson: the employer directed the manner in which the work was to be done; the worker was an integral part of the employer's business; the work was not performed on the employer's premises; the worker was not in business on her/his own account; the worker was not allowed to employ others to assist with her/his work; the employer paid the worker by time; the money that the employer paid to the worker was not stated to be a "fee"; the money that the employer paid to the worker was not stated to be "wages" or "salary"; the employer did not deduct PAYE tax instalments from the worker's pay; the employer paid the worker neither sick pay nor holiday pay; the employer and the worker did not express 
any intention that the relationship would be one of employer and employee; and the employer and the worker expressed an intention that the relationship would be one of principal and independent contractor.

However, there are several significant differences between the hypothetical case and Ferguson v. Dawson. In that case the worker was not allowed to use her/his own discretion in doing an aspect of the work that was not specified beforehand; the worker neither owned the tools nor provided the transport with which she/he performed the work; the employer would make a profit/loss if the work performed by the worker cost less/more than expected; the employer supervised or inspected the work; the worker was not obliged to work only for the employer; and the worker was required to work at specified times.

Despite the fact that Ferguson v. Dawson is a decision of the English Court of Appeal (and better authority than a case decided by the Queen's Bench Division of the English High Court-like Ready Mixed v. Minister), there is nothing in Ferguson v. Dawson to warrant any change in my conclusion.

\section{B.5 Report files for Ainsworth v. Criminal Justice Commission}

\section{Expectation area}

\section{Instant case}

If the applicant had a legitimate expectation — or a reasonable expectation - which was affected by the decision, natural justice may be implied. "‘[L]egitimate expectations' ... are capable of including expectations which go beyond enforceable legal rights, provided they have some reasonable basis". ${ }^{1}$

In the instant case, the decision-maker did not break a promise or undertaking; the decision-maker did not go against an established course of practice; the decision did not involve a refusal to renew an existing interest; neither the decision-maker nor a statutory provision suggested that an initial interest would be granted; the decision did not affect an established liberty or interest; and there was no standard administrative procedure which the decision-maker should have followed.

In my opinion-following Minister for Arts Heritage and Environment v. Peko-Wallsend $L t d$ - the applicant did not have a legitimate expectation which was affected by the decision.

In Minister for Arts Heritage and Environment v. Peko-Wallsend Ltd, ${ }^{2}$ a 1987 decision of the Full Court of the Federal Court of Australia, Peko-Wallsend held various mining interests in Stage 2 of Kakadu National Park. Federal Cabinet decided to nominate Stage 2 for inclusion in the World Heritage List, so it became "identified property"

\footnotetext{
${ }^{1}$ Cole v. Cunningham (1983) 49 ALR 123 at 131, per Bowen CJ, Sheppard and Morling JJ.

${ }^{2}(1987) 75$ ALR 218.
} 
within the meaning of s. 3(2) of the World Heritage Properties Conservation Act 1983 (Cth). This meant that the Governor-General could, by proclamation, make mining operations unlawful in the area. The decision did not affect Peko-Wallsend's mining rights which were preserved under s. 8B of the National Parks and Wildlife Conservation Act 1975 (Cth).

Before Cabinet's decision, Peko-Wallsend had lobbied Ministers and other officials extensively, seeking to preserve their mining interests. After the decision they commenced proceedings to prevent the Government from taking any further steps to have Stage 2 nominated on the World Heritage List, claiming that Cabinet was bound by the rules of natural justice and had failed to give Peko-Wallsend an opportunity to be heard. Beaumont J (a Federal Court judge) agreed, and held the Cabinet decision void. ${ }^{3}$

The Full Court of the Federal Court disagreed. Bowen CJ decided that "it would ... be inappropriate for this court to interfere to set aside a Cabinet decision involving such complex policy considerations". ${ }^{4}$ Both Sheppard and Wilcox JJ held that PekoWallsend had had adequate opportunity to put their case to relevant Ministers and officials before the Cabinet decision, and were not denied natural justice. ${ }^{5}$ However, Wilcox J (with whose reasons the other two judges generally agreed) held that the Cabinet's decision in this case did not attract the obligations of natural justice. ${ }^{6}$

The instant case is on all fours with Minister for Environment v. Peko-Wallsend.

If Cole v. Cunningham is followed then the applicant had a legitimate expectation which was affected by the decision.

In Cole v. Cunningham, ${ }^{7}$ a 1983 decision of the Full Court of the Federal Court of Australia, Cunningham had been encouraged to resign from the Public Service because his superiors believed he had been guilty of misconduct in the performance of his duties. He had formed an attachment and begun to live with a Fijian women whose permit extension application he had processed. He was threatened with criminal prosecution and told that "[i]f you resign now it will be a normal resignation and you'll leave with a clean record." 8

About eighteen months later, Cunningham sought reappointment to the Public Service and was told that he would be offered a position subject to police and ASIO clearances. The next day he was told that he have been given an unsatisfactory report based on the earlier events.

Bowen CJ, Sheppard and Morling JJ held that, in general, applicants for appointment or reappointment to the public service are not entitled to natural justice because they have no legitimate expectation which can be affected by a refusal to appoint. However, Cunningham did have a legitimate expectation that any decision to reappoint him would not be made on the basis of his past record.

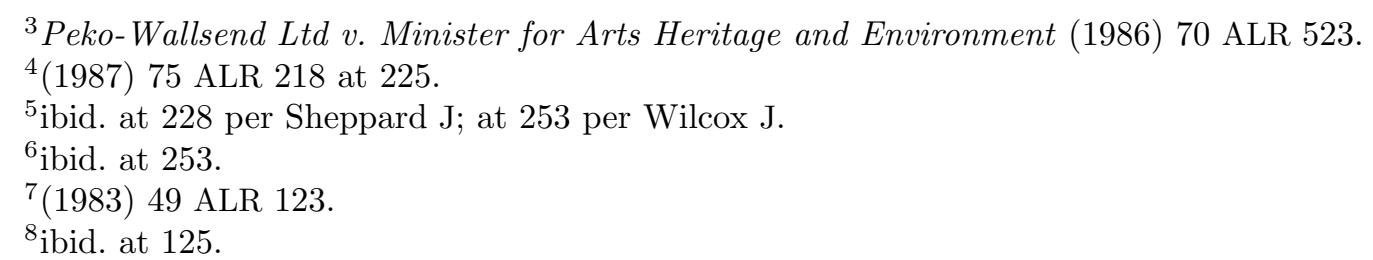


There are several similarities between the instant case and Cole v. Cunningham: the decision-maker did not go against an established course of practice; the decision did not involve a refusal to renew an existing interest; neither the decision-maker nor a statutory provision suggested that an initial interest would be granted; the decision did not affect an established liberty or interest; and there was no standard administrative procedure which the decision-maker should have followed.

However, there is one extremely significant difference between the instant case and Cole v. Cunningham. In that case the decision-maker broke a promise or undertaking.

Despite the fact that Cole v. Cunningham and Minister for Environment v. PekoWallsend are both decisions of the Full Court of the Federal Court of Australia, there is nothing in Cole v. Cunningham to warrant any change in my conclusion.

\section{Hypothetical 1}

Consider the instant case changed so that the following is true: the decision-maker broke a promise or undertaking.

If that were so then my opinion would be that-following Cole v. Cunningham-the applicant had a legitimate expectation which was affected by the decision.

Details of Cole v. Cunningham are summarized above. The hypothetical case is on all fours with Cole v. Cunningham.

If Minister for Arts Heritage and Environment v. Peko-Wallsend Ltd is followed then the applicant did not have a legitimate expectation which was affected by the decision.

Details of Minister for Environment v. Peko-Wallsend are summarized above. There are several similarities between the hypothetical case and Minister for Environment $v$. Peko-Wallsend: the decision-maker did not go against an established course of practice; the decision did not involve a refusal to renew an existing interest; neither the decisionmaker nor a statutory provision suggested that an initial interest would be granted; the decision did not affect an established liberty or interest; and there was no standard administrative procedure which the decision-maker should have followed.

However, there is one extremely significant difference between the hypothetical case and Minister for Environment v. Peko-Wallsend. In that case the decision-maker did not break a promise or undertaking.

Despite the fact that Minister for Environment v. Peko-Wallsend and Cole v. Cunningham are both decisions of the Full Court of the Federal Court of Australia, there is nothing in Minister for Environment v. Peko-Wallsend to warrant any change in my conclusion.

\section{Affected area}

\section{Instant case}

In the instant case, the decision did not affect a financial, property or occupational interest of the applicant; the decision did not affect the applicant's personal liberty; the decision affected the applicant's reputation; and the applicant did not have a legitimate expectation which was affected by the decision. 
In my opinion - following Annetts v. McCann - the decision affected the property, right, interest, status, or legitimate expectation of the applicant.

In Annetts v. McCann,${ }^{9}$ a 1990 decision of five judges of the High Court of Australia, a coroner had been conducting an inquest into the death of a 16-year old boy. The boy's parents ( $\mathrm{Mr}$ and Mrs Annetts) sought to make a submission before the coroner made a finding. The coroner decided that the Coroner's Act 1920 (WA) gave him the discretion (which he chose to exercise) to disallow their submission. The Annettses appealed.

The High Court (Mason CJ, Brennan, Deane, Toohey and McHugh JJ) held that their son's reputation gave the Annettses an interest in the Coroner's inquiry. "A finding in an inquest into a death is naturally likely to deal with the conduct of the deceased leading to death. An unfavourable reflection on the deceased is usually a matter of concern to her or his parents, spouse or children and, if they choose to appear at the inquest in order to safeguard the reputation of the deceased, the familial relationship suffices, in my view, to establish the deceased's reputation as a relevant interest which should not be adversely affected without according natural justice to those who are seeking to safeguard that reputation." 10

The Court held that the fact that the coroner's decision was merely recommendatory (whether or not to prosecute) was not sufficient to avoid the implication of natural justice; the coroner was bound to hear the Annettses before making any finding adverse to them or their son. ${ }^{11}$

The instant case is on all fours with Annetts v. McCann.

If Minister for Arts Heritage and Environment v. Peko-Wallsend Ltd is followed then the decision did not affect the property, right, interest, status, or legitimate expectation of the applicant.

In Minister for Arts Heritage and Environment v. Peko-Wallsend Ltd, ${ }^{12}$ a 1987 decision of the Full Court of the Federal Court of Australia, Peko-Wallsend held various mining interests in Stage 2 of Kakadu National Park. Federal Cabinet decided to nominate Stage 2 for inclusion in the World Heritage List, so it became "identified property" within the meaning of s. 3(2) of the World Heritage Properties Conservation Act 1983 (Cth). This meant that the Governor-General could, by proclamation, make mining operations unlawful in the area. The decision did not affect Peko-Wallsend's mining rights which were preserved under s. 8B of the National Parks and Wildlife Conservation Act 1975 (Cth).

\footnotetext{
${ }^{9}(1990) 170$ CLR 596.

10 ibid. at 612 , per Brennan J.

${ }^{11}$ ibid. at 603, per Mason CJ, Deane and McHugh JJ; at 612 per Brennan J; at 621 per Toohey J. Note, however, that Brennan and Toohey JJ dismissed the appeal because they believed that the decision of the Full Court of the Supreme Court of Western Australia (from which the Annettses appealed) was right on the material before it.

12 (1987) 75 ALR 218.
} 
Before Cabinet's decision, Peko-Wallsend had lobbied Ministers and other officials extensively, seeking to preserve their mining interests. After the decision they commenced proceedings to prevent the Government from taking any further steps to have Stage 2 nominated on the World Heritage List, claiming that Cabinet was bound by the rules of natural justice and had failed to give Peko-Wallsend an opportunity to be heard. Beaumont J (a Federal Court judge) agreed, and held the Cabinet decision void. ${ }^{13}$

The Full Court of the Federal Court disagreed. Bowen CJ decided that "it would ... be inappropriate for this court to interfere to set aside a Cabinet decision involving such complex policy considerations". ${ }^{14}$ Both Sheppard and Wilcox JJ held that PekoWallsend had had adequate opportunity to put their case to relevant Ministers and officials before the Cabinet decision, and were not denied natural justice. ${ }^{15}$ However, Wilcox $J$ (with whose reasons the other two judges generally agreed) held that the Cabinet's decision in this case did not attract the obligations of natural justice. ${ }^{16}$

There are several similarities between the instant case and Minister for Environment v. Peko-Wallsend: the decision did not affect a financial, property or occupational interest of the applicant; the decision did not affect the applicant's personal liberty; and the applicant did not have a legitimate expectation which was affected by the decision.

However, there is one extremely significant difference between the instant case and Minister for Environment v. Peko-Wallsend. In that case the decision did not affect the applicant's reputation. Note also that Minister for Environment v. Peko-Wallsend is only a decision of the Full Court of the Federal Court of Australia and not as good authority as a case decided by five judges of the High Court of Australia-like Annetts $v$. McCann.

Consequently, there is nothing in Minister for Environment v. Peko-Wallsend to warrant any change in my conclusion.

\section{Hypothetical 1}

Consider the instant case changed so that the following is true: the decision did not affect the applicant's reputation.

If that were so then my opinion would be that-following Minister for Arts Heritage and Environment v. Peko-Wallsend Ltd - the decision did not affect the property, right, interest, status, or legitimate expectation of the applicant.

Details of Minister for Environment v. Peko-Wallsend are summarized above. The hypothetical case is on all fours with Minister for Environment v. Peko-Wallsend.

If Bread Manufacturers of New South Wales v. Evans is followed then the decision affected the property, right, interest, status, or legitimate expectation of the applicant.

\footnotetext{
${ }^{13}$ Peko-Wallsend Ltd v. Minister for Arts Heritage and Environment (1986) 70 ALR 523.

14 (1987) 75 ALR 218 at 225.

15 ibid. at 228 per Sheppard J; at 253 per Wilcox J.

16 ibid. at 253 .
} 
In Bread Manufacturers of New South Wales v. Evans, ${ }^{17}$ a 1981 decision of five judges of the High Court of Australia, the Bread Manufacturers claimed that an order made by the Prices Commission was void. The order affected the classification of bread products and had an incidental effect on the price of hamburger buns. The Bread Manufacturers complained that they should have been given the right to put their case to the Commission.

The Prices Regulation Act 1948 (NSW) provided that a public inquiry had to be held before an order could be made setting prices, except where the Minister consented to dispensing with the inquiry. The Minister had dispensed with an inquiry before this order was made. Hence, "[t]he argument that the Commission was bound to disclose to the Association the fact that it proposed to make an order which would have the incidental effect of reducing the price of hamburger buns can only succeed if the Commission, although not bound to hold an inquiry, was bound to observe the rules of natural justice". ${ }^{18}$

The High Court held that there was no denial of natural justice in relation to the order, because "the reduction of the maximum price in respect of one item was simply a minor incident in a major revision of the price framework covering the whole range of bread products. The effect of that major revision was generally to increase prices. There was, in our opinion, no obligation on the Commission to give advance notice of this development or of the possibility of its occurrence." 19

There are several similarities between the hypothetical case and Bread Manufacturers v. Evans: the decision did not affect the applicant's personal liberty; the decision did not affect the applicant's reputation; and the applicant did not have a legitimate expectation which was affected by the decision.

However, there is one extremely significant difference between the hypothetical case and Bread Manufacturers v. Evans. In that case the decision affected a financial, property or occupational interest of the applicant.

Despite the fact that Bread Manufacturers v. Evans is a decision of five judges of the High Court of Australia (and better authority than a case decided by the Full Court of the Federal Court of Australia-like Minister for Environment v. Peko-Wallsend), there is nothing in Bread Manufacturers v. Evans to warrant any change in my conclusion.

\section{Natural area}

\section{Instant case}

In recent years courts have tended to imply a duty to observe the principles of natural justice. It has been said that "[t]he law has now developed to a point where it may be accepted that there is a common law duty to act fairly, in the sense of according procedural fairness, in the making of administrative decisions which affect rights, interests

\footnotetext{
17 (1981) 38 ALR 93.

18 ibid. at 101, per Gibbs CJ.

19 ibid. at 119, per Mason and Wilson JJ, with whom Murphy and Aickin JJ agreed on this point.
} 
and legitimate expectations, subject only to the clear manifestation of a contrary statutory intention." 20 However, there are some circumstances in which a duty to observe natural justice will not be implied: "the law has not yet reached the stage of applying the obligation of natural justice to every decision which disadvantages individuals." ${ }^{21}$

In the instant case, the decision affected the property, right, interest, status, or legitimate expectation of the applicant; the decision is apt to have a discrete impact on the interests of the applicant; the power is of a nature that would suggest that procedural fairness would be applied; the statutory or factual criteria focused on matters which were discrete to the interests of the applicant; the decision-maker was not a high-level policy-maker; there is no statutory right to appeal against the decision; and there were no circumstances which would have made an obligation to observe natural justice inappropriate.

In my opinion-following Annetts v. McCann-a duty to observe natural justice is implied.

In Annetts v. McCann ${ }^{22}$ a 1990 decision of five judges of the High Court of Australia, a coroner had been conducting an inquest into the death of a 16-year old boy. The boy's parents ( $\mathrm{Mr}$ and Mrs Annetts) sought to make a submission before the coroner made a finding. The coroner decided that the Coroner's Act 1920 (WA) gave him the discretion (which he chose to exercise) to disallow their submission. The Annettses appealed.

The High Court (Mason CJ, Brennan, Deane, Toohey and McHugh JJ) held that their son's reputation gave the Annettses an interest in the Coroner's inquiry. "A finding in an inquest into a death is naturally likely to deal with the conduct of the deceased leading to death. An unfavourable reflection on the deceased is usually a matter of concern to her or his parents, spouse or children and, if they choose to appear at the inquest in order to safeguard the reputation of the deceased, the familial relationship suffices, in my view, to establish the deceased's reputation as a relevant interest which should not be adversely affected without according natural justice to those who are seeking to safeguard that reputation." 23

The Court held that the fact that the coroner's decision was merely recommendatory (whether or not to prosecute) was not sufficient to avoid the implication of natural justice; the coroner was bound to hear the Annettses before making any finding adverse to them or their son. ${ }^{24}$

The instant case is on all fours with Annetts v. McCann.

${ }^{20}$ Kioa v. West (1985) 159 CLR 550 at 584, per Mason J.

${ }^{21}$ Minister for Arts Heritage and Environment v. Peko-Wallsend Ltd (1987) 75 ALR 218 at 251, per Wilcox J.

22 (1990) 170 CLR 596.

${ }^{23}$ ibid. at 612 , per Brennan J.

24 ibid. at 603, per Mason CJ, Deane and McHugh JJ; at 612 per Brennan J; at 621 per Toohey J. Note, however, that Brennan and Toohey JJ dismissed the appeal because they believed that the decision of the Full Court of the Supreme Court of Western Australia (from which the Annettses appealed) was right on the material before it. 
If McInnes v. Onslow Fane is followed then a duty to observe natural justice is not implied.

In McInnes v. Onslow Fane, ${ }^{25}$ a 1978 decision of the Chancery Division of the English High Court, McInnes had held, at various times, licences to promote, train and act as master of ceremonies in professional boxing. All his licences were revoked by the British Boxing Board of Control. He made five unsuccessful applications for a manager's licence. With his sixth application he requested an oral hearing and prior notification of anything that might prevent the area council (to which he applied) making a favourable recommendation to the board. The board refused his applications without giving him an oral hearing or informing him of the case against him.

Megarry V-C held that the board was under no duty to provide reasons to McInnes or to allow him a hearing: "This is not a case in which there has been any suggestion of the board considering any alleged dishonesty or morally culpable conduct of the plaintiff. A man free from any moral blemish may nevertheless be wholly unsuitable for a particular type of work ... In such circumstances, in the absence of anything to suggest that the board have been affected by dishonesty or bias or caprice, or that there is any other impropriety, I think that the board are fully entitled to give no reasons for their decision, and to decide the application without any preliminary indication to the plaintiff of those reasons. The board are the best judges of the desirability of granting a licence, and in the absence of any impropriety the court ought not to interfere." 26

The instant case is on all fours with McInnes $v$. Onslow Fane. Note, however, that McInnes v. Onslow Fane is only a decision of the Chancery Division of the English High Court and not as good authority as a case decided by five judges of the High Court of Australia-like Annetts v. McCann.

Consequently, there is nothing in McInnes $v$. Onslow Fane to warrant any change in my conclusion.

\section{Hypothetical 1}

Consider the instant case changed so that the following is true: the statutory or factual criteria focused on matters of policy or public interest; and the decision-maker was a high-level policy-maker.

If that were so then my opinion would be that-following South Australia v. O'Shea-a duty to observe natural justice is not implied.

In South Australia v. O'Shea, ${ }^{27}$ a 1987 decision of five judges of the High Court of Australia, O'Shea had been convicted of two offences of indecent assault of young children. He was released on licence and remained at liberty after the licence expired. Over a year later, after allegations had been made against him, O'Shea was apprehended and detained. The parole board recommended his release on licence on various conditions,

25 [1978] 3 All ER 211.

26 ibid. at 223 .

27 (1987) 163 CLR 378. 
but the Governor in Council resolved to take no action. O'Shea had been given a hearing by the Parole Board, but he claimed he was entitled to a further hearing before the Governor in Council could exercise his discretionary powers under s. 77a(7a) of the Criminal Law Consolidation Act, 1935 (SA).

Mason CJ, Wilson, Brennan and Toohey JJ (Deane J dissenting) held that O'Shea was not entitled to a further hearing. "Given the nature of this decision, it cannot be said that Mr O'Shea could have more than a hope that the Governor would be prepared to act on the recommendation of the Board. Hope, of itself, is not sufficient to ground an expectation that will attract legal consequences. So far as the concept of legitimate expectation is concerned, Mr O'Shea must be taken to know that the Act committed to the Governor, with the advice and consent of the Executive Council, the responsibility for determining where the public interest lay ... The nature of the decision that they were required to make was such that participation by Mr O'Shea was inappropriate." 28

The hypothetical case is on all fours with $S A v$. O'Shea.

If Macrae v. Attorney-General for New South Wales is followed then a duty to observe natural justice is implied.

In Macrae v. Attorney-General for New South Wales ${ }^{29}$ a 1987 decision of the New South Wales Court of Appeal, five magistrates who had been appointed under the Justices Act 1902 (NSW) were not appointed under the Local Courts Act 1982 (NSW). The new Act had reorganized the magistracy in NSW, and magistrates appointed under the old Act were entitled to apply for appointment as magistrates under the new Act. The five had applied and were interviewed. Allegations were made privately to the Attorney-General claiming that they were unfit to be appointed, but these allegations were not brought to their notice at the time of the interviews.

The Court of Appeal held that the Attorney-General's decision not to recommend the appointment of the magistrates was void because they were denied their legitimate expectation of procedural fairness. "They have not been treated fairly." 30

The hypothetical case is on all fours with Macrae v. AG for NSW. Note, however, that Macrae v. AG for NSW is only a decision of the New South Wales Court of Appeal and not as good authority as a case decided by five judges of the High Court of Australia-like $S A$ v. O'Shea.

Consequently, there is nothing in Macrae v. AG for $N S W$ to warrant any change in my conclusion.

\footnotetext{
${ }^{28}$ ibid. at 402, per Wilson and Toohey JJ.

29 (1987) 9 NSWLR 268.

30 ibid. at 283, per Kirby P.
} 


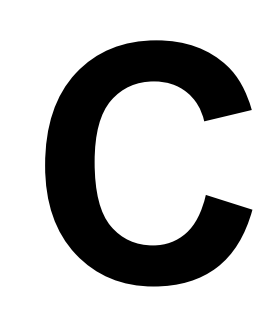

\section{A complete example}

... in order to enter the magic circle of employees, the worker has to solve not only the riddle of "contract" but also that of "employment." And no sooner has the hapless worker tried to answer the riddles, but the judicial wizards have changed the question.

Bob Hepple (1986)

Restructuring Employment Rights ${ }^{25}$

Fury said to a mouse, That he met in the house,

"Let us both go to law: I will prosecute you.

Come, I'll take no denial: We must have the trial;

For really this morning I've nothing to do."

Said the mouse to the cur, "Such a trial, dear sir,

With no jury or judge, would be wasting our breath."

"I'll be judge, I'll be jury," said cunning old Fury:

"I'll try the whole cause, and condemn you to death."

Lewis Carroll (1865)

Alice's Adventures in Wonderland ${ }^{26}$ 


\section{C.1 Introduction}

This appendix gives a complete example of SHYSTER's input and output files for one of the cases used to test the EMPLOYEe specification in $\S 5.4 .3$ : Building Workers' Industrial Union of Australia v. Odco Pty Ltd. ${ }^{27}$

The log file ( $\S$ C.2) summarizes SHYSTER's operation for this test case. The complete case law specification file for the EMPLOYEE specification (Employee.cls) is given in $\S$ C.3. The only other input to SHYSTER for this example is the test case's fact vector as entered by the user, one attribute value at a time, in response to the attribute questions asked by SHYSTER. The fact vector for BWIU v. Odco is given in the log file. SHYSTER was requested to hypothesize with a limit of two changed attribute values, and to report on up to one hypothetical per result.

The dump file Dump.tex is shown in raw form as output by SHYSTER (i.e. ready to be input by $\mathrm{LAT}_{\mathrm{E}} \mathrm{X}$ ) in $\S \mathrm{C} .4$; the dump file as output by $\mathrm{LAT}_{\mathrm{E}} \mathrm{X}$ appears with those of the other specifications in appendix A (§A.4). The remaining files are shown here as both $\mathrm{LAT}_{\mathrm{E}} \mathrm{X}$ input and $\mathrm{LAT}_{\mathrm{E}} \mathrm{X}$ output: viz. the probabilities file Probabilities.tex ( $\S$ C.6 and $\S$ C.7), the weights file Weights.tex ( C.8 and $\S$ C.9), the distances file Distances-Employee.tex $(\S \mathrm{C} .10$ and $\S \mathrm{C} .11)$ and the report file Report-Employee.tex ( $\S \mathrm{C} .12$ and $\S$ C.13).

Most of the $\mathrm{LAT}_{\mathrm{E}} \mathrm{X}$ input shown here is in expurgated form $;{ }^{28}$ vertical ellipses are used to indicate elision. $\mathrm{A} \hookrightarrow$ symbol indicates that the line above is too wide to fit on the page, and continues after the symbol. The level of indentation of a long line is maintained by indenting the continuation appropriately.

LAT $_{\mathrm{E}} \mathrm{X}$ output is complete, and unaltered - except that two formatting changes were made so that all of the output would fit within the margins of this thesis: the probabilities matrix in $\S \mathrm{C} .7$ has been reduced in size, and all seven distance tables in $\S$ C.11 have been rotated anti-clockwise by 90 degrees.

\section{C.2 Log file for Building Workers' Industrial Union of Australia v. Odco Pty Ltd}

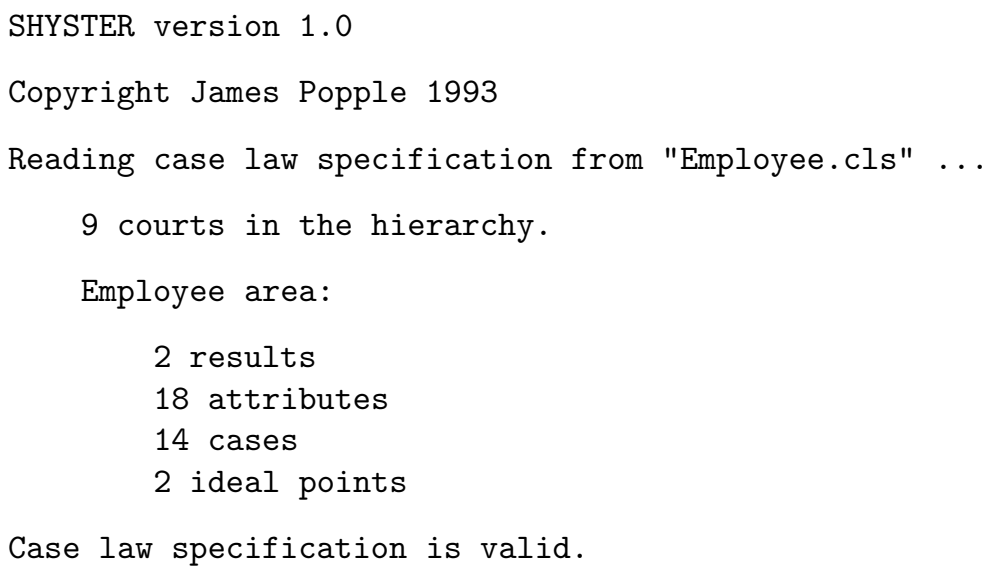




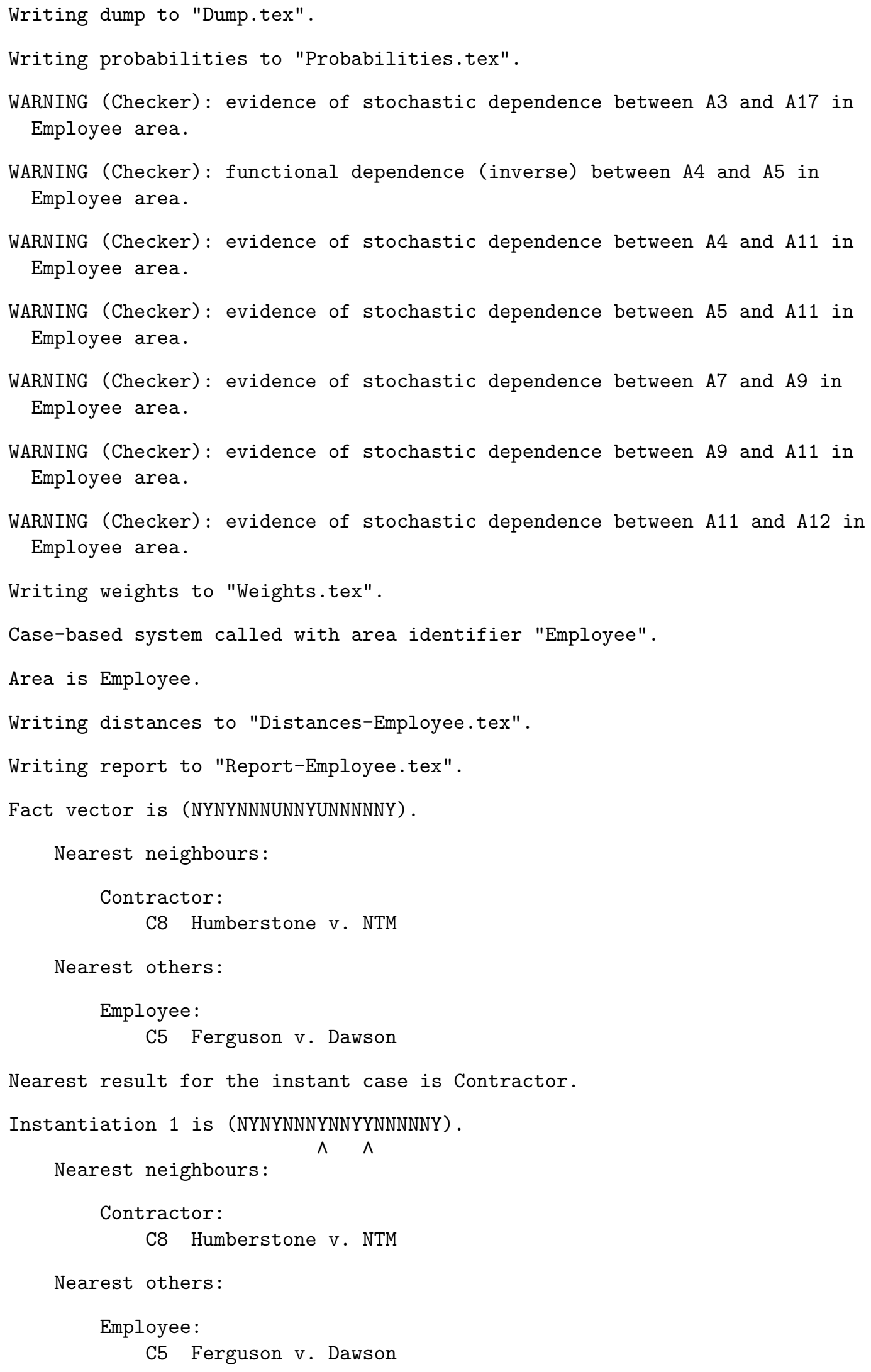


Safeguards :

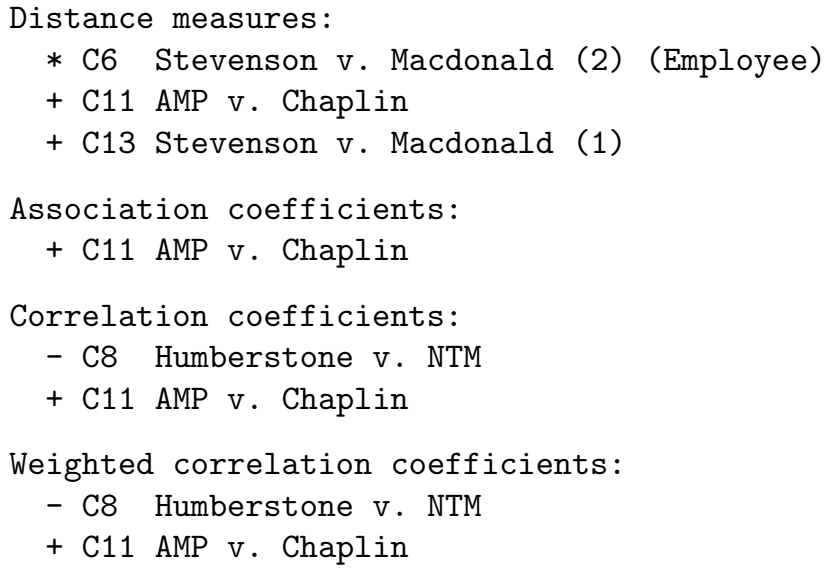


Safeguards :

Distance measures:

* C6 Stevenson V. Macdonald (2) (Employee)

+ C13 Stevenson V. Macdonald (1)

Nearest result for instantiation 3 is Contractor.

Instantiation 4 is (NYNYNNNNNNYNNNNNNY).

Nearest neighbours:

Contractor:

C8 Humberstone v. NTM

Nearest others:

Employee :

C2 Cam v. Sargent

Nearest result for instantiation 4 is Contractor.

Hypothetical 1 is (NYNYNNNUYNYUNNNNNN).

Nearest neighbours:

Contractor:

C8 Humberstone v. NTM

Nearest others:

Employee:

C2 Cam v. Sargent

Nearest result for hypothetical 1 is Contractor.

Hypothetical 2 is (NNYYNNNUNNYUNNNNNY).

Nearest neighbours:

Employee :

C5 Ferguson v. Dawson

Nearest others:

Contractor:

C11 AMP v. Chaplin

C14 Ready Mixed v. Minister

Safeguards :

Distance measures:

+ C6 Stevenson V. Macdonald (2)

* C11 AMP v. Chaplin (Contractor)

* C14 Ready Mixed v. Minister (Contractor)

Association coefficients:

* C11 AMP v. Chaplin (Contractor)

* C14 Ready Mixed V. Minister (Contractor)

Correlation coefficients:

* C11 AMP v. Chaplin (Contractor)

* C14 Ready Mixed v. Minister (Contractor) 


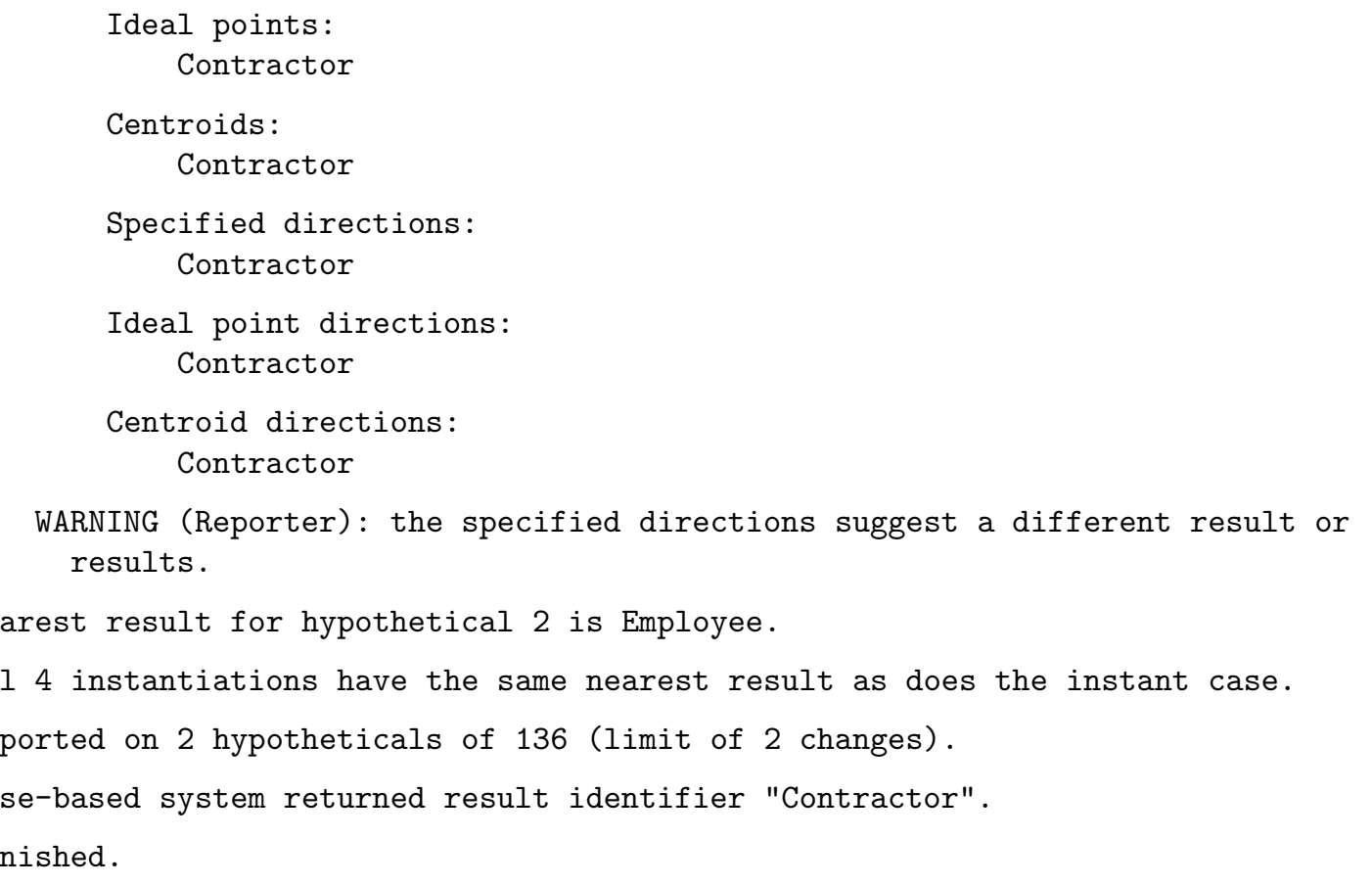

\section{C.3 EMPlOYeE specification file}

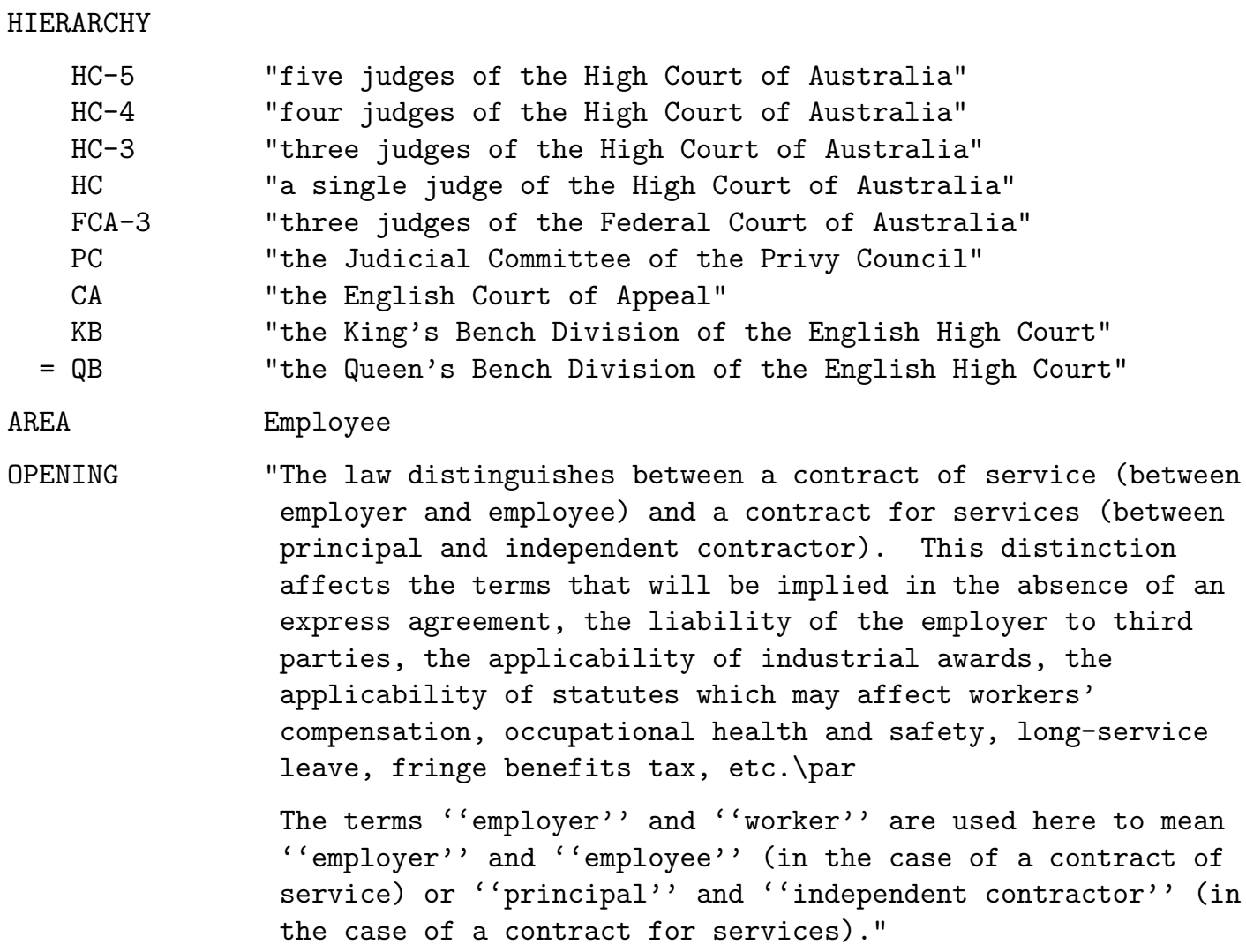


RESULTS

Employee "the worker is an employee"

Contractor "the worker is an independent contractor"

ATTRIBUTE \% Employer had control over manner in which work was done

QUESTION "Did the employer direct not only what work was to be done, but also the manner in which it was to be done"

YES "the employer directed the manner in which the work was to be done" Employee

NO

"the employer did not direct the manner in which the work was to be done" Contractor

UNKNOWN "it is not known whether the employer directed the manner in which the work was to be done"

HELP "If the employer had a right of control over how the worker did the work then the employer had the power to direct not only what work was to be done, but also the manner in which it was to be done."

ATTRIBUTE $\quad \%$ Worker had discretion as to how to do work

QUESTION "Was the worker allowed to use her/his own discretion in doing an aspect of the work that was not specified beforehand"

YES

"the worker was allowed to use her/his own discretion in doing an aspect of the work that was not specified beforehand" Contractor

NO

"the worker was not allowed to use her/his own discretion in doing an aspect of the work that was not specified beforehand" Employee

UNKNOWN "it is not known whether the worker was allowed to use her/his own discretion in doing an aspect of the work that was not specified beforehand"

ATTRIBUTE

$\%$ Worker was an integral part of employer's business

QUESTION

"Was the worker an integral part of the employer's business"

YES

"the worker was an integral part of the employer's business" Employee

NO

"the worker was not an integral part of the employer's business, but was accessory to it" Contractor

UNKNOWN "it is not known whether the worker was an integral part of the employer's business or was merely accessory to it"

HELP "If the worker was 'part and parcel', of the employer's business then she/he was an integral part of the business, not merely accessory to it."

ATTRIBUTE

$\%$ Worker used own tools or provided transport

QUESTION "Did the worker own the tools or provide the transport with which she/he performed the work"

YES

"the worker owned the tools or provided the transport with which she/he performed the work" Contractor

NO

"the worker neither owned the tools nor provided the transport with which she/he performed the work" Employee

UNKNOWN "it is not known whether the worker owned the tools or provided the transport with which she/he performed the work" 


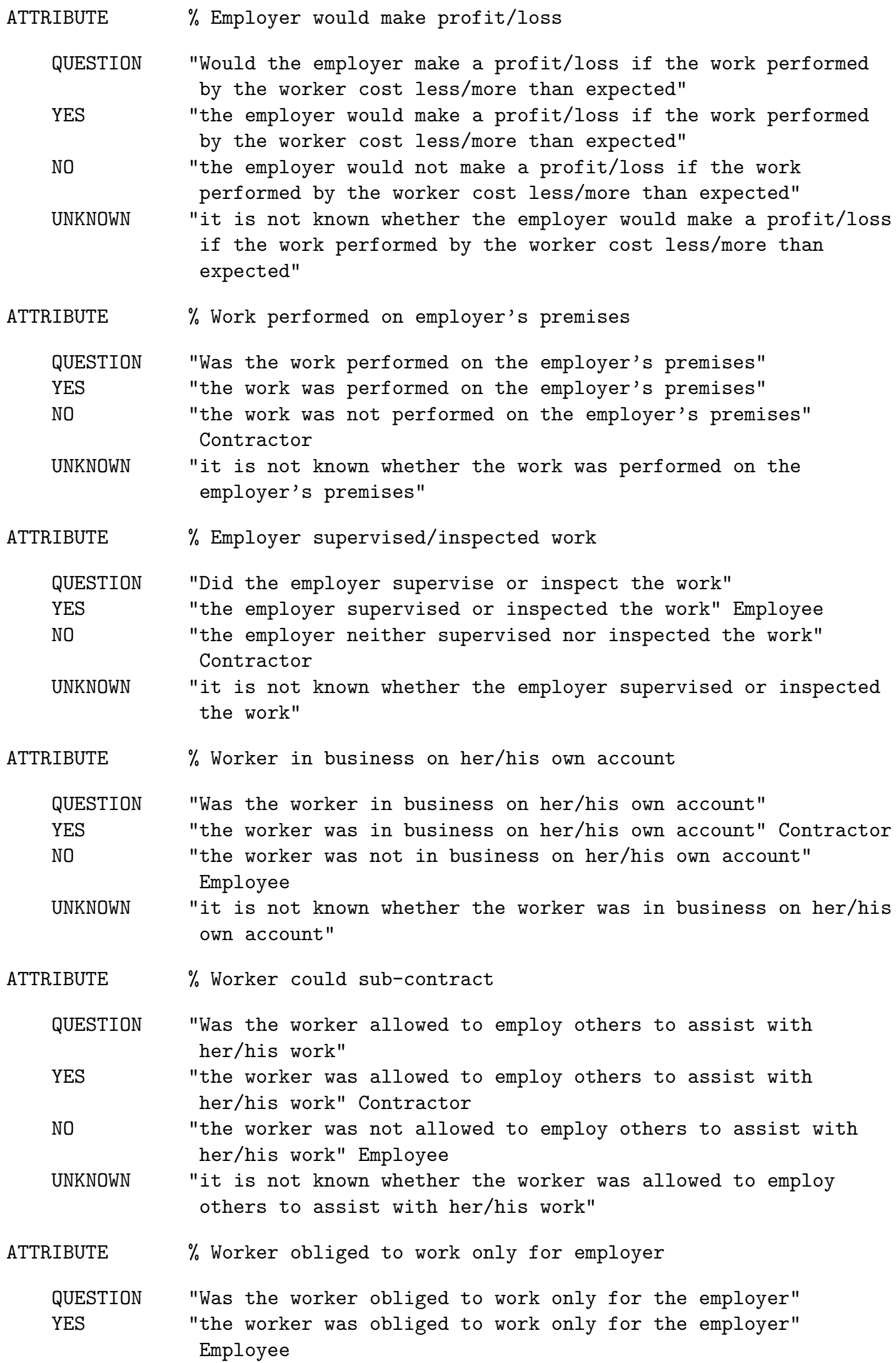




\begin{tabular}{|c|c|}
\hline NO & $\begin{array}{l}\text { "the worker was not obliged to work only for the employer" } \\
\text { Contractor }\end{array}$ \\
\hline UNKNOWN & $\begin{array}{l}\text { "it is not known whether the worker was obliged to work only } \\
\text { for the employer" }\end{array}$ \\
\hline ATTRIBUTE & $\%$ Worker required to work at specified times \\
\hline QUESTION & "Was the worker required to work at specified times" \\
\hline YES & "the worker was required to work at specified times" Employee \\
\hline NO & $\begin{array}{l}\text { "the worker was not required to work at specified times" } \\
\text { Contractor }\end{array}$ \\
\hline UNKNOWN & $\begin{array}{l}\text { "it is not known whether the worker was required to work at } \\
\text { specified times" }\end{array}$ \\
\hline ATTRIBUTE & $\%$ Worker paid by time \\
\hline QUESTION & "Did the employer pay the worker by time" \\
\hline YES & "the employer paid the worker by time" Employee \\
\hline NO & "the employer did not pay the worker by time" Contractor \\
\hline UNKNOWN & "it is not known whether the employer paid the worker by time" \\
\hline HELP & $\begin{array}{l}\text { "The employer could pay the worker by time (e.g. by the hour, } \\
\text { or by the week) or by results." }\end{array}$ \\
\hline ATTRIBUTE & $\%$ Payment was called a "fee" \\
\hline QUESTION & $\begin{array}{l}\text { "Was the money that the employer paid to the worker stated to } \\
\text { be a "fee")" }\end{array}$ \\
\hline YES & $\begin{array}{l}\text { "the money that the employer paid to the worker was stated to } \\
\text { be a "fee"," Contractor }\end{array}$ \\
\hline NO & $\begin{array}{l}\text { "the money that the employer paid to the worker was not stated } \\
\text { to be a "fee"," Employee }\end{array}$ \\
\hline UNKNOWN & $\begin{array}{l}\text { "it is not known whether the money that the employer paid to } \\
\text { the worker was stated to be a "fee"," }\end{array}$ \\
\hline ATTRIBUTE & \% Payment was called "wages" or "salary" \\
\hline QUESTION & $\begin{array}{l}\text { "Was the money that the employer paid to the worker stated to } \\
\text { be "'wages", or "'salary"," }\end{array}$ \\
\hline YES & $\begin{array}{l}\text { "the money that the employer paid to the worker was stated to } \\
\text { be "'wages', or "'salary'," Employee }\end{array}$ \\
\hline NO & $\begin{array}{l}\text { "the money that the employer paid to the worker was not stated } \\
\text { to be "wages', or "'salary'," Contractor }\end{array}$ \\
\hline UNKNOWN & $\begin{array}{l}\text { "it is not known whether the money that the employer paid to } \\
\text { the worker was stated to be "'wages'" or "salary"," }\end{array}$ \\
\hline ATTRIBUTE & \% Employer deducted PAYE tax instalments from worker's pay \\
\hline QUESTION & $\begin{array}{l}\text { "Did the employer deduct PAYE tax instalments from the } \\
\text { worker's pay" }\end{array}$ \\
\hline YES & $\begin{array}{l}\text { "the employer deducted PAYE tax instalments from the worker's } \\
\text { pay" Employee }\end{array}$ \\
\hline NO & $\begin{array}{l}\text { "the employer did not deduct PAYE tax instalments from the } \\
\text { worker's pay" Contractor }\end{array}$ \\
\hline UNKNOWN & $\begin{array}{l}\text { "it is not known whether the employer deducted PAYE tax } \\
\text { instalments from the worker's pay" }\end{array}$ \\
\hline
\end{tabular}




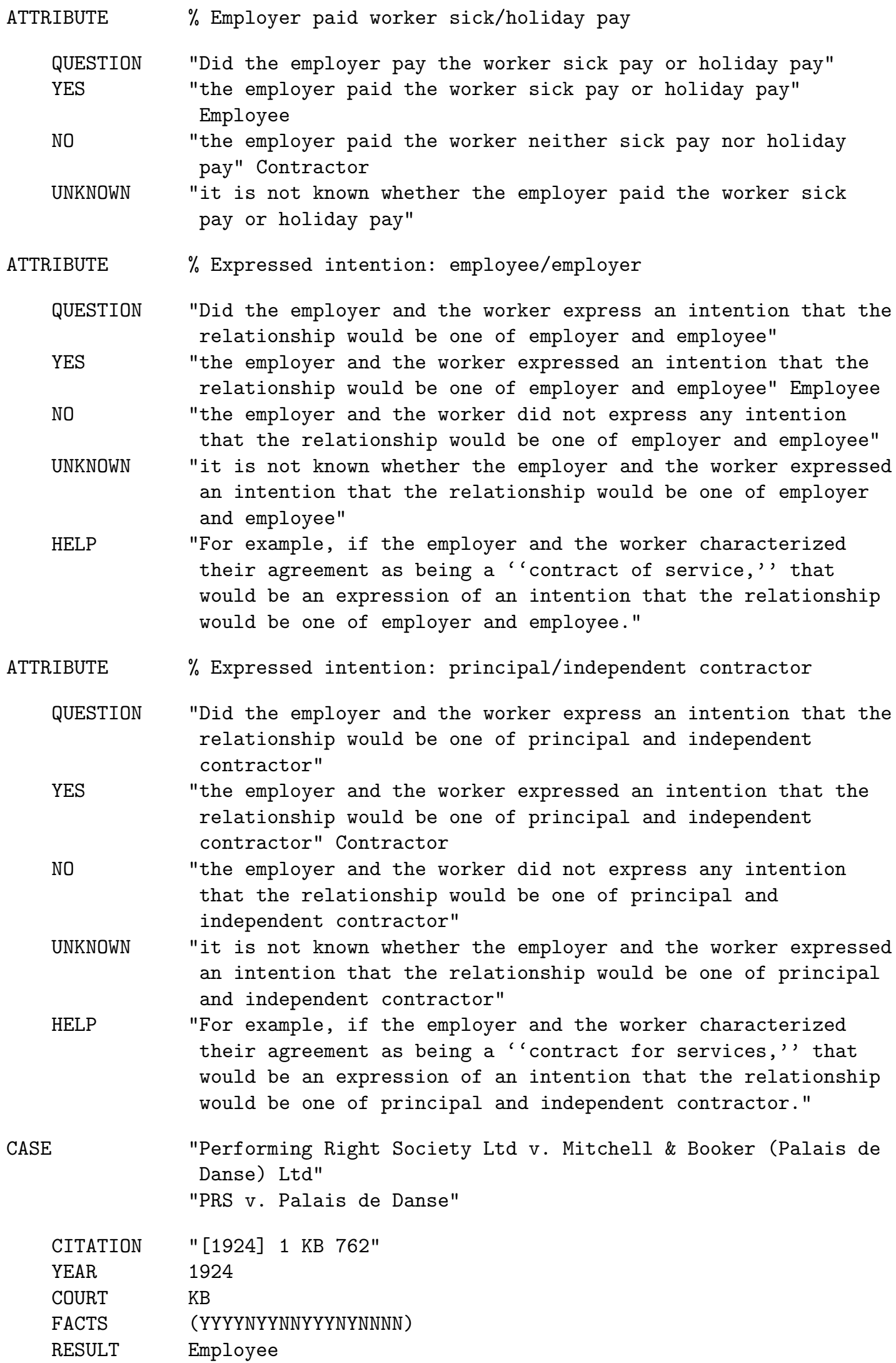


SUMMARY "the defendants were the occupiers of a dance hall. They engaged a band to provide music in the hall. The agreement provided that the band should not infringe copyright, and that the band would be liable for damages and costs caused by any such infringement. There was also a notice displayed in the hall stating that " [0]nly such music as may be played without fee or licence is allowed to be played in this Hall. ' \footnote\{ibid. at 764.\}\par

The band performed several pieces of music, the copyright in which was held by the Performing Right Society, without its permission. The defendants did not know, and had no reasonable grounds for suspecting, that the infringement was to take place. \par

The PRS abandoned its earlier claim that the defendants had ' 'permitted', the infringement under s. 2(3) of the Copyright Act 1911 (UK). However, it claimed that the band were the defendants' employees, and so the defendants were vicariously liable for the infringement. \par

McCardie $J$ examined the agreement and found that it gave " to the defendants the right of continuous, dominant, and detailed control on every point, including the nature of the music to be played', \footnote\{ibid. at 771.$\}$ Hence the band members were employees of the defendant company, which was liable for the infringement."

"Cam and Sons Pty Ltd v. Sargent"

"Cam v. Sargent"

$\begin{array}{ll}\text { CITATION } & "(1940) \text { 14 ALJ 162" } \\ \text { YEAR } & 1940 \\ \text { COURT } & \text { HC-4 } \\ \text { FACTS } & \text { (YYYNYNNNYNNNNNNNNN) } \\ \text { RESULT } & \text { Employee }\end{array}$

SUMMARY "Sargent was the master of a ship. He entered into an agreement with Cam and Sons that claimed that the ship was hired by Cam and Sons to Sargent and his fellow contractors (called "the partnership"'). However, it was doubtful whether that agreement actually deprived Cam and Sons of any control over the ship. The partnership was to use the ship only to carry coal from Swansea to Sydney. Cam and Sons were sole agents of the partnership for securing cargoes for the ship, and for collecting money due to the partnership. The partnership paid nothing for the ' hire') of the ship, but received a specified sum for each return trip of a certain tonnage plus (in certain circumstances) $5 \%$ of the earnings, the balance of which was retained by Cam and Sons. Cam and Sons had to approve people employed by the partnership. Ipar

Sargent claimed that he (and others in the partnership) were employed by Cam and Sons, and therefore came within the terms of an industrial award. Cam and Sons claimed that members of the partnership were independent contractors.।par 
The High Court unanimously agreed with Sargent. Rich J came to the conclusion that the agreement was an attempt to evade the terms of the industrial award. \footnote\{ibid. at 163.\}"

"Federal Commissioner of Taxation v. J. Walter Thompson (Australia) Pty Ltd"

"FCT v. Thompson"

$\begin{array}{ll}\text { CITATION } & "(1944) 69 \text { CLR 227" } \\ \text { YEAR } & 1944 \\ \text { COURT } & \text { HC } \\ \text { FACTS } & \text { (YNYNYYYUNNYNYNNNNN) } \\ \text { RESULT } & \text { Employee }\end{array}$

SUMMARY "the FCT claimed that payments made to radio artists by Thompson were '"wages') within the meaning of the $\{\backslash$ it Pay-roll Tax Assessment Act 1941\/\} (Cth) and therefore taxable. The artists were selected by a producer and paid to appear in radio plays. They were paid a 'fee', for each performance, but were paid nothing for attending (compulsory) rehearsals. Thompson claimed that the artists were presumed to know their work and "to render services in the same manner as a professional man, such as a surgeon or an architect, not being subject \dots $\backslash$ to detailed control as to the manner in which those services are to be performed.' '\footnote\{ibid. at 231.\} Hence, Thompson claimed, they were independent contractors. \par

Latham CJ held that the radio actors were employed "to co-operate with others in a team under the control of the producer to bring about a result, the details of which must in great measure be determined by the producer.' '\footnote\{ibid. at 232.\} Hence the artists were employed by Thompson; the fee they were paid was subject to payroll tax."

"Queensland Stations Pty Ltd v. Federal Commissioner of Taxation" "Queensland Stations v. FCT"

CITATION

"(1945) 70 CLR 539"

YEAR 1945

COURT

$\mathrm{HC}-3$

FACTS

RESULT

(NYNYNNYYYNNNNNNNYN)

SUMMARY "agreements were entered into between Queensland Stations and some drovers. The agreements stated that the drovers would ' 'serve"' Queensland Stations and take charge of a specified number of cattle, and deliver them to a specified place. The drovers were paid a specified rate per head of cattle successfully delivered. Each drover was responsible for hiring help, and paying for feed for the cattle. The drovers were to "obey and carry out all lawful instructions and to use the whole of [their] time, energy and ability in the 
careful droving of the stock.' '\footnote\{ibid. at 540\} The FCT claimed that payments made to drovers were "wages', within the meaning of the \{\it Pay-roll Tax Assessment Act $1941 \backslash /\}$ (Cth), and that Queensland Stations was liable to payroll tax. \par

The High Court held that the drovers were independent contractors, so the payments were not ' wages''. Rich J pointed out that drovers were traditionally free from the control of owners of cattle. "The obligation imposed on the drover to obey and carry out all lawful instructions is not a reservation of detailed control and possession having regard to the terms of the agreement as a whole.' '\footnote\{ibid. at 549.$\} "$

"Humberstone v. Northern Timber Mills"

"Humberstone v. NTM"

$\begin{array}{ll}\text { CITATION } & \text { "(1949) 79 CLR 389" } \\ \text { YEAR } & 1949 \\ \text { COURT } & \text { HC-3 } \\ \text { FACTS } & \text { (NYNYNNNNYNNNNNNNNN) } \\ \text { RESULT } & \text { Contractor }\end{array}$

SUMMARY "Humberstone carried goods for NTM. He had originally held himself out as a carrier, prepared to carry for anyone, but for over twenty years he had carried goods solely for NTM (although he would, infrequently, carry back-loads for NTM's customers). Humberstone owned the truck, and paid for petrol and repairs. He was paid weekly on a weight-mileage basis. He was a licenced carrier, and had his name printed on the side of his truck with the description 'carrier.' $\backslash$ par

On the way back from a job, he had a puncture. He went home to change the wheel, but exerted himself so strenuously in trying to remove the tyre from the wheel that he became ill and later lapsed into a coma, from which he did not recover. Section 3 of the \{\it Worker's Compensation Act $\backslash /\} 1928$ (Vic) had been amended about a year before Humberstone's death so as to include independent contractors in its definition of a "'worker') covered by the Act. However, the High Court held that the amendment applied only to contracts entered into after it came into operation. Further, the Court decided that Humberstone was not an employee of NTM. Hence he was not a "'worker') under the Act and his widow was not entitled to compensation under the Act."

CASE

"Stevenson Jordon and Harrison Ltd v. Macdonald and Evans (1)" "Stevenson v. Macdonald (1)"

$\begin{array}{ll}\text { CITATION } & \text { "[1952] 1 TLR 101" } \\ \text { YEAR } & 1952 \\ \text { COURT } & \text { CA } \\ \text { FACTS } & \text { (NYNNYNUNNNYUUUUUYN) } \\ \text { RESULT } & \text { Contractor }\end{array}$


SUMMARY "Evans-Hemming was an accountant who had been employed (first as a servant, then as an executive officer) by Macdonald and Evans. Shortly after he left them, he wrote a textbook on business management and submitted the manuscript to Stevenson Jordon and Harrison (a firm of publishers). He died before the book was published. Macdonald and Evans claimed that the book was written while Evans-Hemming was their employee, and so they owned the copyright in the work under s. 5(1)(b) of the Copyright Act 1911 (UK). \par

The book was divided into five sections. The first section consisted of the text of three public lectures that Evans-Hemming had given while employed by Macdonald and Evans. The Court of Appeal held that he had given these lectures as an independent contractor. As Denning LJ said, " "under a contract of service, a man is employed as part of the business, and his work is done as an integral part of the business; whereas, under a contract for services, his work, although done for the business, is not integrated into it but is only accessory to it \dots\ The lectures were, in a sense, part of the services rendered by Mr Evans-Hemming for the benefit of the company. But they were in no sense part of his service. It follows that the copyright in the lectures was in Mr Evans-Hemming. "\footnote\{ibid. at 111\}"

"Stevenson Jordon and Harrison Ltd V. Macdonald and Evans (2)" "Stevenson V. Macdonald (2)"

$\begin{array}{ll}\text { CITATION } & \text { "[1952] 1 TLR 101" } \\ \text { YEAR } & 1952 \\ \text { COURT } & \text { CA } \\ \text { FACTS } & \text { (NYYNYNUNNNYUUUUUUN) } \\ \text { RESULT } & \text { Employee }\end{array}$

SUMMARY "Evans-Hemming was an accountant who had been employed (first as a servant, then as an executive officer) by Macdonald and Evans. Shortly after he left them, he wrote a textbook on business management and submitted the manuscript to Stevenson Jordon and Harrison (a firm of publishers). He died before the book was published. Macdonald and Evans claimed that the book was written while Evans-Hemming was their employee, and so they owned the copyright in the work under s. 5(1)(b) of the Copyright Act 1911 (UK). \par

The book was divided into five sections. The second section was written in its final form while Evans-Hemming was employed by Macdonald and Evans. The Court of Appeal held that he wrote the second section as an employee, and hence the copyright in the second section was in Macdonald and Evans." 
"Zuijs v. Wirth Brothers"

$\begin{array}{ll}\text { CITATION } & \text { "(1955) 93 CLR 561" } \\ \text { YEAR } & 1955 \\ \text { COURT } & \text { HC-5 } \\ \text { FACTS } & \text { (NYYNYYYNNNYYNNYNNN) } \\ \text { RESULT } & \text { Employee }\end{array}$

SUMMARY "Zuijs was an acrobat who fell during a trapeze act at one of Wirth Brothers' circuses. He sought compensation under the Worker's Compensation Act 1926 (NSW), claiming to be an employee of Wirth Brothers. Wirth Brothers claimed that, because of the high degree of skill and personal judgment that he had to exercise in his work, Zuijs was an independent contractor and therefore not entitled to compensation. Ipar

The High Court unanimously agreed with Zuijs. "Even if [Wirth Brothers] could not interfere in the actual technique of the acrobats and in the character of the act, no reason appears why the appellant should not be subject to his directions in all other respects \dots\ There are countless examples of highly specialized functions in modern life that must as a matter of practical necessity and sometimes even as a matter of law be performed on the responsibility of persons who possess particular knowledge and skill and who are accordingly qualified. But those engaged to perform the functions may nevertheless work under a contract of service.' '\footnote\{ibid. at 571--2, per Dixon CJ, Williams, Webb and Taylor JJ.\}"

"Ready Mixed Concrete (South East) Ltd v. Minister of Pensions and National Insurance"

"Ready Mixed v. Minister"

CITATION

YEAR

COURT

FACTS

RESULT

SUMMARY
"[1968] 2 QB 497"

1967

QB

(NYYYNNNNYYNNNNNNNY)

Contractor

"Latimer worked for Ready Mixed as an 'owner-driver', He was paid at mileage rates, and was obliged to buy the truck through a financial organization associated with Ready Mixed. The truck was painted in the company's colours, and he had to wear a Ready Mixed uniform. Latimer was obliged to meet the costs of maintenance, repair and insurance of the truck (and the attached mixing unit, which belonged to Ready Mixed). The Minister determined that Latimer was employed under a contract of service and therefore an "employed person"' under s. 1(2) of the National Insurance Act 1965 (UK), making Ready Mixed liable to make weekly contributions. Ipar 
MacKenna $J$ examined the contract and held that the rights it conferred, and the duties it imposed, between Latimer and Ready Mixed were not such as to make it a contract of service."

"Ferguson v. John Dawson \& Partners (Contractors) Ltd"

"Ferguson v. Dawson"

$\begin{array}{ll}\text { CITATION } & "[1976] \text { 1 WLR 1213" } \\ \text { YEAR } & 1976 \\ \text { COURT } & \text { CA } \\ \text { FACTS } & \text { (YNYNYNYNNNYYNNNNNY) } \\ \text { RESULT } & \text { Employee }\end{array}$

SUMMARY "Ferguson fell off a roof while removing some scaffolding boards. He claimed damages against John Dawson (the building contractors) for breach of statutory duty relying on the $\{\backslash$ it Construction (Working Places) Regulations 1966\/\} (UK). This duty would only be owed if Ferguson was an employee of John Dawson. \par

Megaw and Browne LJJ held that, despite the fact that both parties labelled Ferguson a 'self-employed labour only subcontractor' ', \footnote\{ibid. at 1219, per Megaw LJ; at 1225, per Lawton LJ; at 1228, per Browne LJ.\} the reality of the relationship between them was that of employer/employee."

"Massey v. Crown Life Insurance Co."

"Massey v. Crown Life"

$\begin{array}{ll}\text { CITATION } & \text { "[1978] ICR 590" } \\ \text { YEAR } & 1978 \\ \text { COURT } & \text { CA } \\ \text { FACTS } & \text { (YYYNYYNYYNYYNNNYNY) } \\ \text { RESULT } & \text { Contractor }\end{array}$

SUMMARY "Massey was the manager of a branch of Crown Life. He had been an employee for two years, then he and Crown Life entered into a new agreement whereby Massey continued to perform the same duties as before, but was self-employed. This arrangement had tax advantages for Massey. After a further two years, Crown Life terminated the agreement and Massey sought compensation for unfair dismissal under the Trade Union and Labour Relations Act 1974 (UK). Compensation was only payable if Massey was employed under a contract of service.\par

Lord Denning MR stated that " if the true relationship of the parties is that of master and servant under a contract of service, the parties cannot alter the truth of that relationship by putting a different label upon 
CASE

CITATION

YEAR

COURT

FACTS

RESULT

SUMMARY

$\begin{array}{ll}\text { CITATION } & "(1978) \quad 21 \text { ALR 388" } \\ \text { YEAR } & 1978 \\ \text { COURT } & \text { FCA-3 } \\ \text { FACTS } & \text { (NYYYNNNNYNNNNNNNN) } \\ \text { RESULT } & \text { Contractor }\end{array}$

it.' '\footnote\{ibid. at 594.\} However, he (and the rest of the Court of Appeal) held that the agreement was genuinely intended to establish Massey as being self-employed: an independent contractor."

"Australian Mutual Provident Society v. Chaplin"

"AMP v. Chaplin"

"(1978) 18 ALR 385"

1978

$\mathrm{PC}$

(NYYYNNNYYYNNNNNNNY)

Contractor

"Chaplin was a representative of AMP. A clause of the agreement between them stated that the relationship was one of '"principal and agent', and not one of 'master and servant' '. Chaplin claimed that he was employed under a contract of service, and therefore a ' worker', under the \{\it Long Service Leave Act, 1967\/\} (SA) and entitled to certain benefits. \par

The Privy Council found that there was no reason to think that the clause was not a genuine statement of the parties' intentions. Examining the agreement, their lordships concluded that it provided for a contract of agency. The fact that Chaplin was given the power of unlimited delegation of the whole performance of his work was "almost conclusive against the contract being a contract of service." \footnote\{ibid. at 391.\}"

"Price v. Grant Industries Pty Ltd"

"Price v. Grant Industries"

SUMMARY "Grant Industries manufactured and sold wardrobes, which Price (and others) delivered and installed. Price and each of the other ' "contractors') (as Grant Industries called them) had to provide and maintain a suitable truck to deliver the wardrobes, and provide the tools required to install them. Price sought an order that a penalty be imposed on Grant Industries for breaching the Furnishing Trades (Consolidated) Award 1975 by not paying Price the appropriate rate of wages, and not giving him annual leave. The award only applied to ' 'employees', of specified employers. \par

The Federal Court examined the facts, and the provisions of the agreement, and held that Price was an independent contractor and, therefore, not subject to the award." 
CITATION "(1980) 29 ALR 322"

YEAR $\quad 1980$

COURT FCA-3

FACTS (YNYYNYYNUNNNNNNNNN)

RESULT Employee

SUMMARY "Wales was a tree feller who cut timber exclusively for Monaro Sawmills. He performed his work in an area allotted to him by Monaro Sawmills. He, and other fellers, were paid by the amount of millable wood they cut. Wales provided his own tools and transport, but was (with the other fellers) covered by Monaro Sawmill's workers' compensation policy. \par

The union sought an order that a penalty be imposed on Monaro Sawmills for breaching the Timber Industries Consolidated Award 1974 by failing to pay Wales money in lieu of annual leave. Monaro Sawmills claimed that Wales was an independent contractor, and so was not subject to the award. \par

Sweeney and Evatt JJ examined the circumstances of Wales's employment and held that those circumstances clearly pointed to the existence of a relationship of employer and employee. They could not see " any sense in which it could be said that Wales was conducting some sort of business of his own. "\footnote\{ibid. at 329.\}"

IDEAL

FACTS (YNYNYYYNNYYYNYYYYU)

RESULT Employee

IDEAL

FACTS (NYNYNUNYYNNNYNNNUY)

RESULT Contractor

\section{C.4 Dump file for EMPloyee specification ( $\mathrm{AT}_{\mathrm{E}} \mathrm{X}$ input)}

$\%$ Dump file

$\%$ Produced by SHYSTER version 1.0

\% Copyright James Popple 1993

$\%$ This is not a stand-alone LaTeX file.

$\%$ Include it in a LaTeX document using the \input command.

\% Use LaTeX version $2.09<25$ March 1992> and TeX version 3.141. 


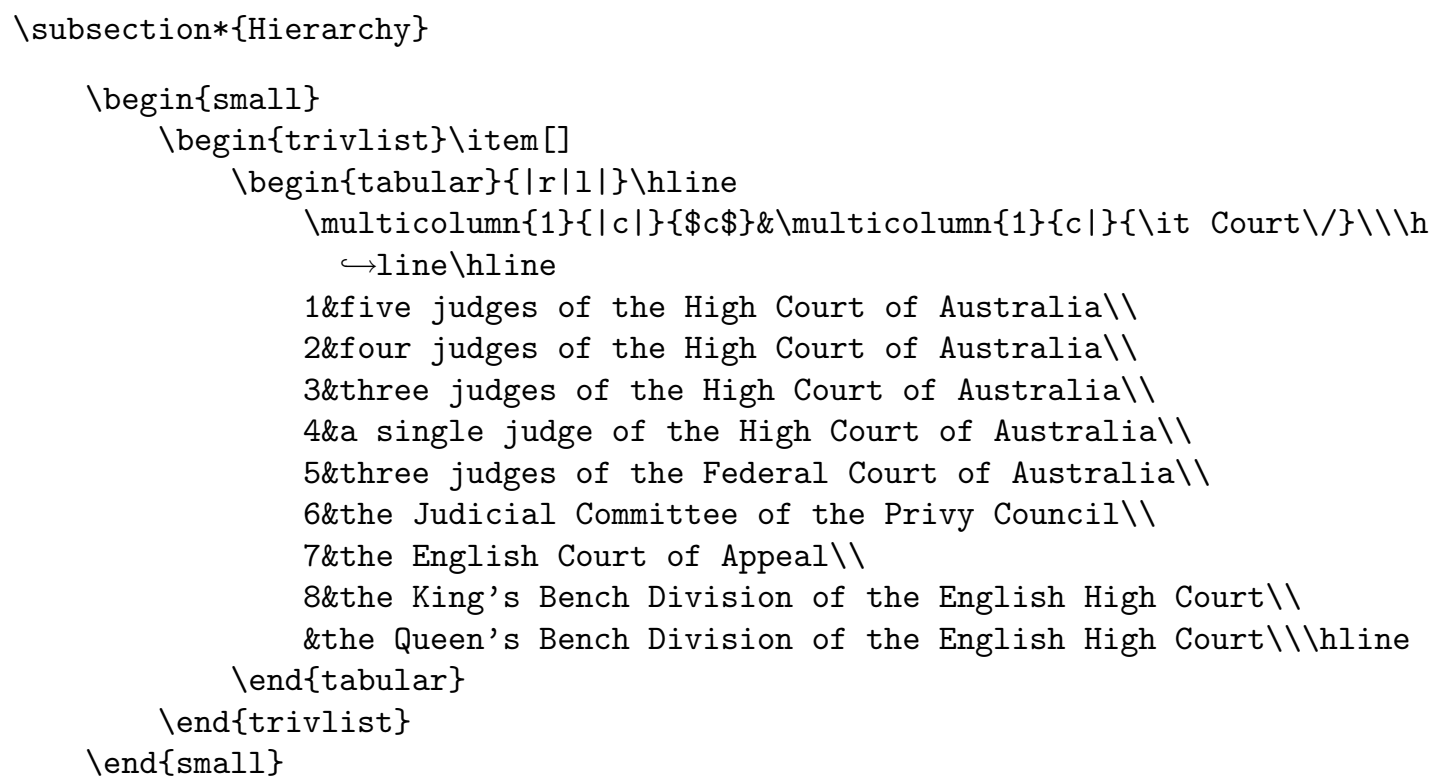

$\backslash$ subsection*\{Employee area\}

\begin } \{ \text { small\} }

$\backslash$ begin $\{$ tabular $\}\{*\{2\}\{\mid c\} *\{17\}\{@\{\backslash$ hspace $\{0.4 \mathrm{em}\}\} \mathrm{c}\}|\mathrm{r}| \mathrm{c} \mid\} \backslash \mathrm{hl}$ ine $\& \backslash \operatorname{multicolumn}\{18\}\{|c|\}\{\backslash$ it Attributes $\backslash /\} \& \& \backslash \backslash$

$\backslash$ smash $\{\backslash$ raisebox $\{0.6 \backslash$ ht $\backslash$ strutbox $\}\{\backslash$ it Case $\backslash /\}\} \& \$ A_{-}\{1\} \$ \& \$ A_{-}\{2\} \$ \& \$ A_{-}$ $\hookrightarrow\{3\} \$ \& \$ A_{-}\{4\} \$ \& \$ A_{-}\{5\} \$ \& \$ A_{-}\{6\} \$ \& \$ A_{-}\{7\} \$ \& \$ A_{-}\{8\} \$ \& \$ A_{-}\{9\} \$ \& \$ A_{-}\{10\} \$ \&$ $\hookrightarrow \$ A_{-}\{11\} \$ \& \$ A_{-}\{12\} \$ \& \$ A_{-}\{13\} \$ \& \$ A_{-}\{14\} \$ \& \$ A_{-}\{15\} \$ \& \$ A_{-}\{16\} \$ \& \$ A_{-}\{17\} \$$ $\hookrightarrow \& \$ A_{-}\{18\} \$ \& \backslash \operatorname{multicolumn}\{1\}\{c \mid\}\{\backslash \operatorname{smash}\{\backslash$ raisebox $\{0.6 \backslash$ ht $\backslash$ strutbox $\hookrightarrow\}\{\$ c \$\}\}\} \& \backslash \operatorname{smash}\{\backslash$ raisebox $\{0.6 \backslash$ ht $\backslash$ strutbox $\}\{\backslash$ it Result $\backslash /\}\} \backslash \backslash \backslash h l$ $\hookrightarrow$ ine $\backslash$ hline

$\$ C_{-}\{1\} \$ \& \$ \backslash$ times $\$ \& \$ \backslash$ bullet $\$ \& \$ \backslash$ bullet $\$ \& \$ \backslash$ times $\$ \& \$ \backslash$ bullet $\$ \& \$ \backslash$ bullet $\$ \&$

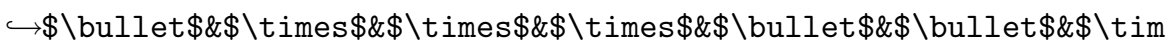
$\hookrightarrow$ es $\$ \& \$ \backslash$ times $\$ \& \$ \backslash$ bullet $\$ \& \$ \backslash$ times $\$ \& \$ \backslash$ times $\$ \& \$ \backslash$ times $\$ \& 1 \& \backslash \backslash$

$\$ C_{-}\{14\} \$ \& \$ \backslash$ times $\$ \& \$ \backslash$ bullet $\$ \& \$ \backslash$ bullet $\$ \& \$ \backslash$ bullet $\$ \& \$ \backslash$ times $\$ \& \$ \backslash$ times $\$ \&$ $\hookrightarrow$ $\$$ times $\$ \& \$ \backslash$ times $\$ \& \$ \backslash$ bullet $\$ \& \$ \backslash$ bullet $\$ \& \$ \backslash$ times $\$ \& \$ \backslash$ times $\$ \& \$ \backslash$ time

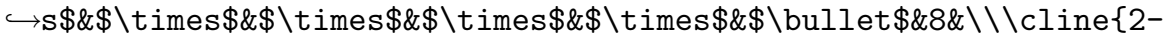
$\hookrightarrow 20\}$

$\$ I_{-}\{\backslash \operatorname{mbox}\{\backslash$ scriptsize $\backslash$ sf Contractor $\}\} \$ \& \$$ times $\$ \& \$ \backslash$ bullet $\$ \& \$ \backslash$ times $\$$ $\hookrightarrow \& \$ \backslash$ bullet $\$ \& \$ \backslash$ times $\$ \& \& \$ \backslash$ times $\$ \& \$ \backslash$ bullet $\$ \& \$ \backslash$ bullet $\$ \& \$ \backslash$ times $\$ \& \$ \backslash t$ $\hookrightarrow$ imes $\$ \& \$ \backslash$ times $\$ \& \$ \backslash$ bullet $\$ \& \$ \backslash$ times $\$ \& \$ \backslash$ times $\$ \& \$ \backslash$ times $\$ \& \& \backslash$ bullet $\$$ $\hookrightarrow \& \& \backslash \backslash \backslash$ hline

\end } \{ \text { tabular } \}

lend $\{$ small $\}$

$\backslash$ subsubsection*\{0pening\}

$\backslash$ begin $\{$ list $\}\{\}\{\backslash$ leftmargin=0mm $\} \backslash$ item []

The law distinguishes between a contract of service (between employer and employee) and a contract for services (between principal and independent contractor). This distinction affects the terms that 
will be implied in the absence of an express agreement, the liability of the employer to third parties, the applicability of industrial awards, the applicability of statutes which may affect workers' compensation, occupational health and safety, long-service leave, fringe benefits tax, etc.lpar The terms ' employer', and

' 'worker', are used here to mean 'employer', and 'employee', (in the case of a contract of service) or '

" 'independent contractor', (in the case of a contract for services). lend\{list\}

$\backslash$ subsubsection $*\{$ Results $\}$

\begin\{description\} }

\item $[\backslash \mathrm{rm}\{\backslash$ sf Employee\}:] the worker is an employee.

\item $[\backslash \mathrm{rm}\{\backslash \mathrm{sf}$ Contractor\}:] the worker is an independent contractor.

\end } \{ \text { description } \}

\subsubsection*\{Attributes\}

\begin\{description\} }

$\backslash$ item $\left[\backslash r m \$ A_{-}\{1\} \$:\right]$

Did the employer direct not only what work was to be done, but also the manner in which it was to be done?

\begin\{description\} }

\item [\sc yes:] the employer directed the manner in which the work was to be done.

$\$ \backslash$ Rightarrow $\$$ \{ $\backslash$ sf Employee $\}$

\item [\sc no:]

\end\{description\} }

If the employer had a right of control over how the worker did the work then the employer had the power to direct not only what work was to be done, but also the manner in which it was to be done.

$\backslash$ item $\left[\backslash \mathrm{rm} \$ \mathrm{~A}_{-}\{2\} \$:\right]$

Was the worker allowed to use her/his own discretion in doing an aspect of the work that was not specified beforehand? 


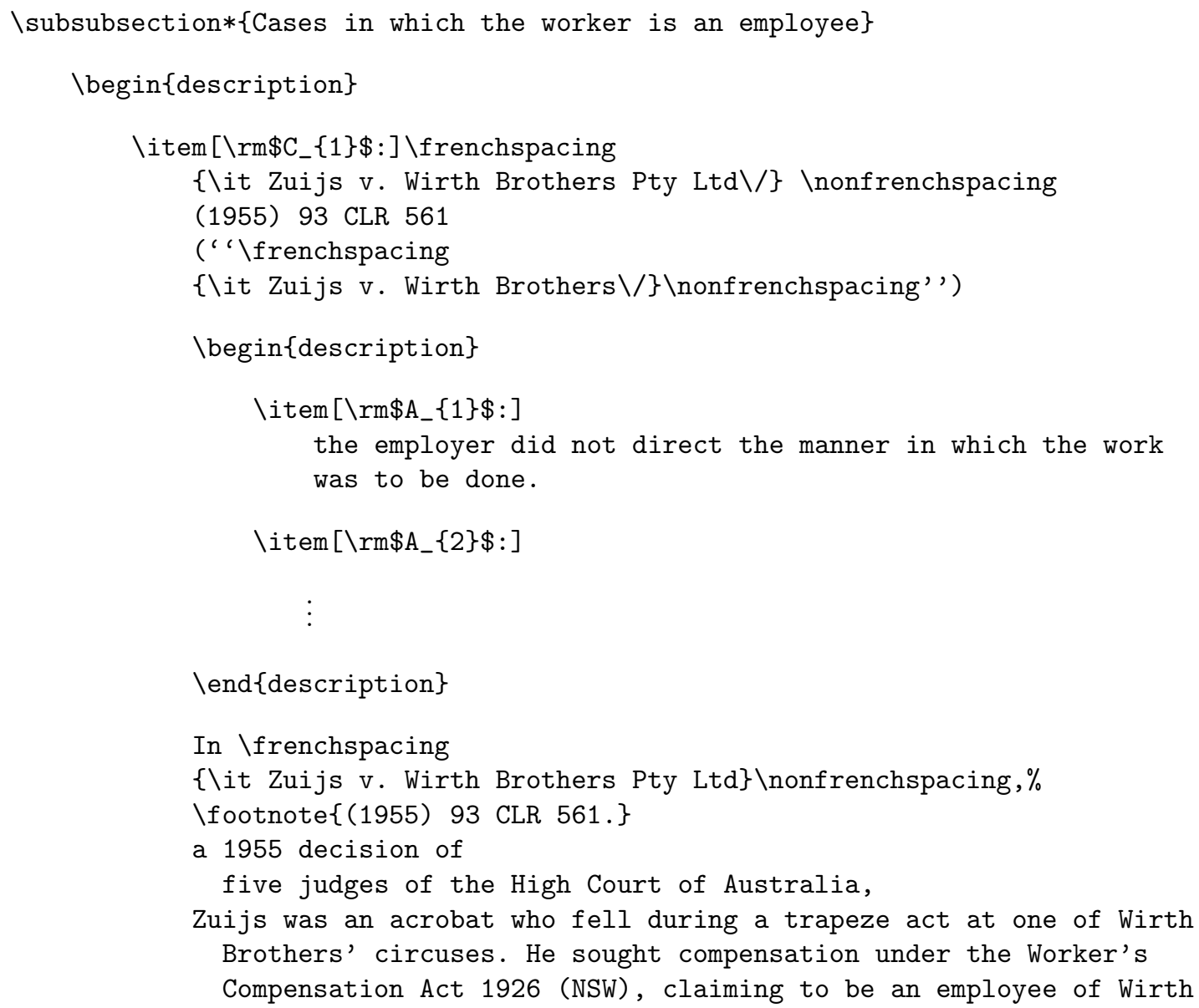

\section{C.5 Dump file for EMPlOYEe specification ( $\mathrm{L}^{\mathrm{T}} \mathrm{T}_{\mathrm{E}} \mathrm{X}$ output)}

The dump file for the EMPLOYEE specification, as output by $\mathrm{IAT}_{\mathrm{E}} \mathrm{X}$, appears with those of the other specifications in appendix A (§A.4). 


\section{C.6 Probabilities file for EMPLOYEe specification ( $\mathrm{IAT}_{\mathrm{E}} \mathrm{X}$ input)}

$\%$ Probabilities file

$\%$ Produced by SHYSTER version 1.0

\% Copyright James Popple 1993

$\%$ This is not a stand-alone LaTeX file.

$\%$ Include it in a LaTeX document using the \input command.

\% Use LaTeX version $2.09<25$ March 1992> and TeX version 3.141.

\subsection*\{Employee area\}

$\backslash$ begin $\{$ small\}

$\backslash$ def \arraystretch $\{0\}$

$\backslash$ begin $\{$ tabular $\}\{*\{17\}\{\mid c\} \mid @\{\} p\{\backslash$ doublerulesep $\} @\{\}|c|\} \backslash c l i n e\{1-17\}$

$\backslash \operatorname{smash}\left\{\backslash\right.$ raisebox $\left\{0.6 \backslash\right.$ ht $\backslash$ strutbox $\left.\left\{\$ A_{-}\{2\} \$\right\}\right\} \& \backslash \operatorname{smash}\{\backslash$ raisebox $\{0.6 \backslash h$ $\hookrightarrow$ t\strutbox $\left.\left\{\$ A_{-}\{3\} \$\right\}\right\} \& \backslash$ smash $\left\{\backslash\right.$ raisebox $\{0.6 \backslash$ ht $\backslash$ strutbox $\}\left\{\$ A_{-}\{4\}\right.$ $\hookrightarrow \$\}\} \& \backslash \operatorname{smash}\left\{\backslash\right.$ raisebox $\{0.6 \backslash$ ht $\backslash$ strutbox $\left.\}\left\{\$ A_{-}\{5\} \$\right\}\right\} \& \backslash$ smash $\{\backslash$ raiseb $\hookrightarrow$ ox $\{0.6 \backslash h t \backslash$ strutbox $\left.\}\left\{\$ A_{-}\{6\} \$\right\}\right\} \& \backslash \operatorname{smash}\{\backslash$ raisebox $\{0.6 \backslash h t \backslash$ strutbox $\left.\hookrightarrow\}\left\{\$ A_{-}\{7\} \$\right\}\right\} \& \backslash \operatorname{smash}\left\{\backslash\right.$ raisebox $\{0.6 \backslash$ ht $\backslash$ strutbox $\left.\}\left\{\$ A_{-}\{8\} \$\right\}\right\} \& \backslash$ smash $\hookrightarrow\left\{\backslash\right.$ raisebox $\{0.6 \backslash$ ht $\backslash$ strutbox $\left.\}\left\{\$ A_{-}\{9\} \$\right\}\right\} \& \backslash \operatorname{smash}\{\backslash$ raisebox $\{0.6 \backslash h t \backslash$ $\hookrightarrow$ strutbox $\left\{\left\{\$ A_{-}\{10\} \$\right\}\right\} \& \backslash \operatorname{smash}\left\{\backslash\right.$ raisebox $\{0.6 \backslash$ ht $\backslash$ strutbox $\}\left\{\$ A_{-}\{11\}\right.$ $\hookrightarrow \$\}\} \& \backslash \operatorname{smash}\left\{\backslash\right.$ raisebox $\{0.6 \backslash h t \backslash$ strutbox $\left.\}\left\{\$ A_{-}\{12\} \$\right\}\right\} \& \backslash \operatorname{smash}\{\backslash$ raise $\hookrightarrow$ box $\left\{0.6 \backslash\right.$ ht $\backslash$ strutbox $\left\{\left\{\$ A_{-}\{13\} \$\right\}\right\} \& \backslash \operatorname{smash}\{\backslash$ raisebox $\{0.6 \backslash$ ht $\backslash$ strutb $\hookrightarrow$ ox $\left.\}\left\{\$ A_{-}\{14\} \$\right\}\right\} \& \backslash \operatorname{smash}\left\{\backslash\right.$ raisebox $\{0.6 \backslash h t \backslash$ strutbox $\left.\}\left\{\$ A_{-}\{15\} \$\right\}\right\} \& \backslash \mathrm{s}$ $\hookrightarrow \operatorname{mash}\left\{\backslash\right.$ raisebox $\left\{0.6 \backslash h t \backslash\right.$ strutbox $\left\{\left\{\$ A_{-}\{16\} \$\right\}\right\} \& \backslash \operatorname{smash}\{\backslash$ raisebox $\{0$. $\hookrightarrow 6 \backslash$ ht $\backslash$ strutbox $\left.\}\left\{\$ A_{-}\{17\} \$\right\}\right\} \& \backslash \operatorname{smash}\{\backslash$ raisebox $\{0.6 \backslash$ ht $\backslash$ strutbox $\}\{\$ A$ $\hookrightarrow\{\{18\} \$\}\} \& \backslash \operatorname{multicolumn}\{2\}\{c\}\{\backslash$ raisebox $\{\backslash$ ht $\backslash$ strutbox $\}\{\backslash$ strut $\}\} \backslash \backslash$ $\hookrightarrow \backslash c$ line $\{1-17\}$

$\backslash \operatorname{multicolumn}\{19\}\{c\}\{\backslash$ rule $\{0 \mathrm{~mm}\}\{\backslash$ doublerulesep $\}\} \backslash \backslash \backslash \mathrm{cline}\{1-17\} \backslash \mathrm{clin}$ $\hookrightarrow \mathrm{e}\{19-19\}$

$0.05 \& 1.00 \& 0.30 \& 0.95 \& 1.00 \& 0.96 \& 0.69 \& 0.41 \& 0.62 \& 0.95 \& 0.97 \& 1.00 \& 1.00 \& 0$ $\hookrightarrow .23 \& 1.00 \& 0.27 \& 0.83 \& \& \backslash \backslash$

$1.00 \& 0.15 \& 0.95 \& 0.30 \& 0.06 \& 0.28 \& 0.80 \& 0.91 \& 0.85 \& 0.30 \& 0.27 \& 0.50 \& 0.50 \& 1$ $\hookrightarrow .00 \& 0.50 \& 1.00 \& 0.59 \& \& \backslash \operatorname{smash}\left\{\backslash\right.$ raisebox $\{0.6 \backslash$ ht $\backslash$ strutbox $\}\left\{\$ A_{-}\{1\} \$\right\}$ $\hookrightarrow\} \backslash \backslash \backslash c l i n e\{1-17\} \backslash c l i n e\{19-19\}$

$\backslash$ multicolumn $\{1\}\{c \mid\}\{\} \& 0.45 \& 0.90 \& 0.50 \& 0.27 \& 0.09 \& 1.00 \& 1.00 \& 1.00 \& 0.50$ $\hookrightarrow \& 0.75 \& 0.25 \& 1.00 \& 1.00 \& 1.00 \& 1.00 \& 0.67 \& \& \backslash \backslash$

$\backslash$ multicolumn $\{1\}\{c \mid\}\{\} \& 1.00 \& 0.50 \& 0.90 \& 0.97 \& 1.00 \& 0.58 \& 0.19 \& 0.45 \& 0.90$ $\hookrightarrow \& 0.76 \& 1.00 \& 0.75 \& 0.55 \& 0.75 \& 0.58 \& 0.82 \& \& \backslash \operatorname{smash}\{\backslash$ raisebox $\{0.6 \backslash h t \backslash \mathrm{s}$ $\hookrightarrow$ trutbox $\left\{\left\{\$ A_{-}\{2\} \$\right\}\right\} \backslash \backslash \backslash \mathrm{cline}\{2-17\} \backslash \mathrm{cline}\{19-19\}$

$\backslash \operatorname{multicolumn}\{2\}\{c \mid\}\{\} \& 0.50 \& 0.90 \& 1.00 \& 0.77 \& 0.58 \& 0.56 \& 1.00 \& 0.90 \& 1.00$ $\hookrightarrow \& 1.00 \& 1.00 \& 1.00 \& 1.00 \& 0.04 \backslash \mathrm{rlap}\{\backslash$ makebox $[\backslash$ tabcolsep $]\{\$ \backslash$ bullet $\$\}$ $\hookrightarrow\} \& 1.00 \& \& \backslash \backslash$

$\backslash$ multicolumn $\{2\}\{c \mid\}\{\} \& 0.90 \& 0.50 \& 0.23 \& 0.77 \& 0.89 \& 0.88 \& 0.45 \& 0.50 \& 0.42$ $\hookrightarrow \& 0.83 \& 0.83 \& 0.68 \& 0.83 \& 1.00 \& 0.33 \& \& \backslash \operatorname{smash}\{\backslash$ raisebox $\{0.6 \backslash$ ht $\backslash$ strutb $\hookrightarrow$ ox $\left.\}\left\{\$ A_{-}\{3\} \$\right\}\right\} \backslash \backslash \backslash \mathrm{cline}\{3-17\} \backslash \mathrm{cline}\{19-19\}$

$\backslash \operatorname{multicolumn}\{3\}\{\mathrm{c} \mid\}\{\} \& 0.00 \backslash \mathrm{rlap}\{\backslash \operatorname{makebox}[\backslash$ tabcolsep] $\{\backslash \mathrm{rule}[0.25 \mathrm{ex}]$ $\hookrightarrow\{0.35 \mathrm{em}\}\{0.35 \mathrm{em}\}\}\} \& 0.50 \& 0.50 \& 0.88 \& 1.00 \& 1.00 \& 0.01 \backslash \mathrm{rlap}\{\backslash$ makebox $\hookrightarrow[\backslash$ tabcolsep $]\{\$ \backslash$ bullet $\$\} \& 0.15 \& 0.42 \& 1.00 \& 0.68 \& 0.42 \& 0.73 \& 0.72 \& \& \backslash$ $\hookrightarrow \backslash$ 
$\backslash$ multicolumn $\{3\}\{c \mid\}\{\} \& 1.00 \& 0.87 \& 0.88 \& 0.56 \& 0.08 \& 0.10 \& 1.00 \& 0.99 \& 1.00$ $\hookrightarrow \& 0.58 \& 0.85 \& 1.00 \& 0.81 \& 0.72 \& \& \backslash \operatorname{smash}\{\backslash$ raisebox $\{0.6 \backslash$ ht $\backslash$ strutbox $\}\{\$$ $\left.\left.\hookrightarrow A_{-}\{4\} \$\right\}\right\} \backslash \backslash \backslash$ cline $\{4-17\} \backslash c l i n e\{19-19\}$

$\backslash \operatorname{multicolumn}\{15\}\{c \mid\}\{\} \& 0.92 \& 1.00 \& \& \backslash \backslash$

$\backslash \operatorname{multicolumn}\{15\}\{c \mid\}\{\} \& 1.00 \& 0.33 \& \& \backslash \operatorname{smash}\{\backslash$ raisebox $\{0.6 \backslash$ ht $\backslash$ strutbox $\left.\hookrightarrow\}\left\{\$ A_{-}\{16\} \$\right\}\right\} \backslash \backslash \backslash c l i n e\{16-17\} \backslash c l i n e\{19-19\}$

$\backslash$ multicolumn $\{16\}\{c \mid\}\{\} \& 0.46 \& \& \backslash \backslash$

$\backslash \operatorname{multicolumn}\{16\}\{c \mid\}\{\} \& 1.00 \& \& \backslash \operatorname{smash}\left\{\backslash\right.$ raisebox $\{0.6 \backslash$ ht $\backslash$ strutbox $\}\left\{\$ A_{-}\right.$ \end } \{ \text { tabular\} }

lend $\{$ small\}

\section{C.7 Probabilities file for EMPLOYEe specification ( $\mathrm{LAT}_{\mathrm{E}} \mathrm{X}$ output)}

\section{Employee area}

\begin{tabular}{|c|c|c|c|c|c|c|c|c|c|c|c|c|c|c|c|c|c|}
\hline$A_{2}$ & $A_{3}$ & $A_{4}$ & $A_{5}$ & $A_{6}$ & $A_{7}$ & $A_{8}$ & $A_{9}$ & $A_{10}$ & $A_{11}$ & $A_{12}$ & $A_{13}$ & $A_{14}$ & $A_{15}$ & $A_{16}$ & $A_{17}$ & $A_{18}$ & \\
\hline 0.05 & 1.00 & 0.30 & 0.95 & 1.00 & $\overline{0.96}$ & 0.69 & 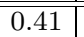 & $\overline{c 0.62}$ & 0.95 & $\overline{0.97}$ & 1.00 & 1.00 & $\overline{c 0.23}$ & 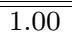 & 0.27 & $\overline{c 0.83}$ & \multirow[b]{2}{*}{$A_{1}$} \\
\hline 1.00 & 0.15 & 0.95 & 0.30 & 0.06 & 0.28 & 0.80 & 0.91 & 0.85 & 0.30 & 0.27 & 0.50 & 0.50 & 1.00 & 0.50 & 1.00 & 0.59 & \\
\hline & 0.45 & 0.90 & 0.50 & 0.27 & 0.09 & 1.00 & 1.00 & 1.00 & 0.50 & 0.75 & 0.25 & 1.00 & 1.00 & 1.00 & 1.00 & 0.67 & \multirow{2}{*}{$A_{2}$} \\
\hline & 1.00 & 0.50 & 0.90 & 0.97 & 1.00 & 0.58 & 0.19 & 0.45 & 0.90 & 0.76 & 1.00 & 0.75 & 0.55 & 0.75 & 0.58 & 0.82 & \\
\hline & & 0.50 & 0.90 & 1.00 & 0.77 & 0.58 & 0.56 & 1.00 & 0.90 & 1.00 & 1.00 & 1.00 & 1.00 & 1.00 & $0.04 \bullet$ & 1.00 & \multirow[b]{2}{*}{$A_{3}$} \\
\hline & & 0.90 & 0.50 & 0.23 & 0.77 & 0.89 & 0.88 & 0.45 & 0.50 & 0.42 & 0.83 & 0.83 & 0.68 & 0.83 & 1.00 & 0.33 & \\
\hline & & & $0.00 \llbracket$ & 0.50 & 0.50 & 0.88 & 1.00 & 1.00 & $0.01 \bullet$ & 0.15 & 0.42 & 1.00 & 0.68 & 0.42 & 0.73 & 0.72 & \multirow{2}{*}{$A_{4}$} \\
\hline & & & 1.00 & 0.87 & 0.88 & 0.56 & 0.08 & 0.10 & 1.00 & 0.99 & 1.00 & 0.58 & 0.85 & 1.00 & 0.81 & 0.72 & \\
\hline & & & & 0.87 & 0.88 & 0.56 & 0.08 & 0.10 & 1.00 & 0.99 & 1.00 & 0.58 & 0.85 & 1.00 & 0.81 & 0.72 & \multirow[b]{2}{*}{$A_{5}$} \\
\hline & & & & 0.50 & 0.50 & 0.88 & 1.00 & 1.00 & $0.01 \bullet$ & 0.15 & 0.42 & 1.00 & 0.68 & 0.42 & 0.73 & 0.72 & \\
\hline & & & & & 0.99 & 0.80 & 0.22 & 0.73 & 0.99 & 0.99 & 1.00 & 1.00 & 0.85 & 1.00 & 0.36 & 0.55 & \multirow[b]{2}{*}{$A_{6}$} \\
\hline & & & & & 0.12 & 0.71 & 0.98 & 0.77 & 0.13 & 0.15 & 0.42 & 0.42 & 0.68 & 0.42 & 1.00 & 0.87 & \\
\hline & & & & & & 0.58 & $0.02 \bullet$ & 0.50 & 0.99 & 0.97 & 1.00 & 1.00 & 0.77 & 0.50 & 1.00 & 0.27 & \multirow{2}{*}{$A_{7}$} \\
\hline & & & & & & 0.88 & 1.00 & 0.91 & 0.12 & 0.27 & 0.50 & 0.50 & 0.77 & 1.00 & 0.50 & 0.97 & \\
\hline & & & & & & & 1.00 & 0.89 & 0.56 & 0.72 & 1.00 & 0.73 & 0.51 & 1.00 & 0.95 & 0.99 & \multirow[b]{2}{*}{$A_{8}$} \\
\hline & & & & & & & 0.16 & 0.58 & 0.88 & 0.79 & 1.00 & 1.00 & 1.00 & 0.27 & 0.45 & 0.20 & \\
\hline & & & & & & & & 0.88 & $0.00 \bullet$ & 0.09 & 0.36 & 0.36 & 0.62 & 1.00 & 0.68 & 0.95 & \multirow{2}{*}{$A_{9}$} \\
\hline & & & & & & & & 0.56 & 1.00 & 1.00 & 1.00 & 1.00 & 0.89 & 0.64 & 0.85 & 0.34 & \\
\hline & & & & & & & & & 0.50 & 0.76 & 0.75 & 1.00 & 0.55 & 0.75 & 0.58 & 0.99 & \multirow[b]{2}{*}{$A_{10}$} \\
\hline & & & & & & & & & 0.90 & 0.75 & 1.00 & 0.25 & 1.00 & 1.00 & 1.00 & 0.18 & \\
\hline & & & & & & & & & & 1.00 & 1.00 & 1.00 & 0.85 & 1.00 & 0.81 & 0.72 & \multirow{2}{*}{$A_{11}$} \\
\hline & & & & & & & & & & $0.01 \bullet$ & 0.42 & 0.42 & 0.68 & 0.42 & 0.73 & 0.72 & \\
\hline & & & & & & & & & & & 0.67 & 1.00 & 0.91 & 1.00 & 0.67 & 0.93 & \multirow[b]{2}{*}{$A_{12}$} \\
\hline & & & & & & & & & & & 1.00 & 0.33 & 0.58 & 0.33 & 1.00 & 0.41 & \\
\hline & & & & & & & & & & & & 0.92 & 0.83 & 0.92 & 0.92 & 0.67 & \multirow[b]{2}{*}{$A_{13}$} \\
\hline & & & & & & & & & & & & 1.00 & 1.00 & 1.00 & 1.00 & 1.00 & \\
\hline & & & & & & & & & & & & & 0.83 & 0.92 & 0.92 & 0.67 & \multirow{2}{*}{$A_{14}$} \\
\hline & & & & & & & & & & & & & 1.00 & 1.00 & 1.00 & 1.00 & \\
\hline & & & & & & & & & & & & & & 0.83 & 0.83 & 0.42 & \multirow{2}{*}{$A_{15}$} \\
\hline & & & & & & & & & & & & & & 1.00 & 1.00 & 1.00 & \\
\hline & & & & & & & & & & & & & & & 0.92 & 1.00 & \multirow{2}{*}{$A_{16}$} \\
\hline & & & & & & & & & & & & & & & 1.00 & 0.33 & \\
\hline & & & & & & & & & & & & & & & & $\begin{array}{l}0.46 \\
1.00\end{array}$ & $A_{17}$ \\
\hline
\end{tabular}




\section{C.8 Weights file for EMPLOYEE specification ( $\mathrm{IAT}_{\mathrm{E}} \mathrm{X}$ input)}

$\%$ Weights file

$\%$ Produced by SHYSTER version 1.0

\% Copyright James Popple 1993

$\%$ This is not a stand-alone LaTeX file.

$\%$ Include it in a LaTeX document using the \input command.

\% Use LaTeX version $2.09<25$ March 1992> and TeX version 3.141.

$\backslash$ subsection*\{Employee area\}

$\backslash$ begin $\{$ small\}

$\backslash$ begin $\{$ tabular $\}\{|c| *\{3\}\{c @\{\backslash$ hspace $\{\backslash$ tabcolsep $\}$ c@ $\{\backslash$ hspace $\{\backslash$ tabcolsep $\}\}$

$\hookrightarrow \mathrm{r} \mid\}\} \backslash$ hline

$\& \backslash \operatorname{multicolumn}\{3\}\{c \mid\}\{\backslash$ sf Employee $\& \backslash \operatorname{multicolumn}\{3\}\{c \mid\}\{\backslash$ sf Contrac $\hookrightarrow$ tor $\} \& \& \& \backslash \backslash$

$\backslash \operatorname{smash}\{\backslash$ raisebox $\{0.6 \backslash$ ht $\backslash$ strutbox $\}\{\backslash$ it Attr. $\}\} \& \$ \backslash \operatorname{mu} \$ \& \$ \backslash$ sigma^ $2 \$ \& \backslash m u$ $\hookrightarrow$ lticolumn $\{1\}\{c \mid\}\{\$ \mathrm{w} \$\} \& \$ \backslash \operatorname{mu} \$ \& \$ \backslash \operatorname{sigma} \wedge 2 \$ \& \backslash \operatorname{multicolumn}\{1\}\{\mathrm{c} \mid\}\{\$ \mathrm{w} \$$

$\hookrightarrow\} \& \backslash \operatorname{smash}\{\backslash$ raisebox $\{0.6 \backslash$ ht $\backslash$ strutbox $\}\{\$ \backslash \operatorname{mu} \$\}\} \& \backslash \operatorname{smash}\{\backslash$ raisebox $\{0$

$\hookrightarrow .6 \backslash$ ht $\backslash$ strutbox $\}\{\$ \backslash$ sigma $\wedge 2 \$\}\} \& \backslash \operatorname{multicolumn}\{1\}\{c \mid\}\{\backslash \operatorname{smash}\{\backslash$ raise $\hookrightarrow$ box $\{0.6 \backslash$ ht $\backslash$ strutbox $\}\{\$ \mathrm{w} \$\}\}\} \backslash \backslash \backslash$ hline $\backslash$ hline

$\$ A_{-}\{1\} \$ \& 0.71 \& 0.20 \& 4.90 \& 0.14 \& 0.12 \& 8.17 \& 0.43 \& 0.24 \& 4.08 \backslash \backslash$

$\$ A_{-}\{2\} \$ \& 0.57 \& 0.24 \& 4.08 \& 1.00 \& 0.00 \& \backslash \operatorname{multicolumn}\{1\}\{c \mid\}\{\$ \backslash$ infty $\$\} \& 0.7$ $\hookrightarrow 9 \& 0.17 \& 5.94 \backslash \backslash$

$\$ A_{-}\{3\} \$ \& 1.00 \& 0.00 \& \backslash \operatorname{multicolumn}\{1\}\{c \mid\}\{\$ \backslash$ infty $\$\} \& 0.57 \& 0.24 \& 4.08 \& 0.7$ $\hookrightarrow 9 \& 0.17 \& 5.94 \backslash \backslash$

$\$ A_{-}\{4\} \$ \& 0.29 \& 0.20 \& 4.90 \& 0.71 \& 0.20 \& 4.90 \& 0.50 \& 0.25 \& 4.00 \backslash \backslash$

$\$ A_{-}\{5\} \$ \& 0.71 \& 0.20 \& 4.90 \& 0.29 \& 0.20 \& 4.90 \& 0.50 \& 0.25 \& 4.00 \backslash \backslash$

$\$ A_{-}\{6\} \$ \& 0.57 \& 0.24 \& 4.08 \& 0.14 \& 0.12 \& 8.17 \& 0.36 \& 0.23 \& 4.36 \backslash \backslash$

$\$ A_{-}\{7\} \$ \& 0.83 \& 0.14 \& 7.20 \& 0.17 \& 0.14 \& 7.20 \& 0.50 \& 0.25 \& 4.00 \backslash \backslash$

$\$ A_{-}\{8\} \$ \& 0.00 \& 0.00 \& \backslash \operatorname{multicolumn}\{1\}\{c \mid\}\{\$ \backslash$ infty $\$\} \& 0.43 \& 0.24 \& 4.08 \& 0.2$ $\hookrightarrow 3 \& 0.18 \& 5.63 \backslash \backslash$

$\$ A_{-}\{9\} \$ \& 0.17 \& 0.14 \& 7.20 \& 0.86 \& 0.12 \& 8.17 \& 0.54 \& 0.25 \& 4.02 \backslash \backslash$

$\$ A_{-}\{10\} \$ \& 0.14 \& 0.12 \& 8.17 \& 0.29 \& 0.20 \& 4.90 \& 0.21 \& 0.17 \& 5.94 \backslash \backslash$

$\$ A_{-}\{11\} \$ \& 0.71 \& 0.20 \& 4.90 \& 0.29 \& 0.20 \& 4.90 \& 0.50 \& 0.25 \& 4.00 \backslash \backslash$

$\$ A_{-}\{12\} \$ \& 0.50 \& 0.25 \& 4.00 \& 0.17 \& 0.14 \& 7.20 \& 0.33 \& 0.22 \& 4.50 \backslash \backslash$

$\$ A_{-}\{13\} \$ \& 0.17 \& 0.14 \& 7.20 \& 0.00 \& 0.00 \& \backslash \operatorname{multicolumn}\{1\}\{c \mid\}\{\$ \backslash$ infty $\$\} \& 0$. $\hookrightarrow 08 \& 0.08 \& 13.09 \backslash \backslash$

$\$ A_{-}\{14\} \$ \& 0.17 \& 0.14 \& 7.20 \& 0.00 \& 0.00 \& \backslash \operatorname{multicolumn}\{1\}\{c \mid\}\{\$ \backslash$ infty $\$\} \& 0$. $\hookrightarrow 08 \& 0.08 \& 13.09 \backslash \backslash$

$\$ A_{-}\{15\} \$ \& 0.17 \& 0.14 \& 7.20 \& 0.17 \& 0.14 \& 7.20 \& 0.17 \& 0.14 \& 7.20 \backslash \backslash$

$\$ A_{-}\{16\} \$ \& 0.00 \& 0.00 \& \backslash m u l t i c o l u m n\{1\}\{c \mid\}\{\$ \backslash$ infty $\$\} \& 0.17 \& 0.14 \& 7.20 \& 0$. $\hookrightarrow 08 \& 0.08 \& 13.09 \backslash \backslash$

$\$ A_{-}\{17\} \$ \& 0.00 \& 0.00 \& \backslash \operatorname{multicolumn}\{1\}\{c \mid\}\{\$ \backslash \operatorname{infty} \$\} \& 0.29 \& 0.20 \& 4.90 \& 0$. $\hookrightarrow 15 \& 0.13 \& 7.68 \backslash \backslash$

$\$ A_{-}\{18\} \$ \& 0.14 \& 0.12 \& 8.17 \& 0.43 \& 0.24 \& 4.08 \& 0.29 \& 0.20 \& 4.90 \backslash \backslash \backslash \mathrm{hl}$ ine $\backslash$ end $\{$ tabular $\}$

\end } \{ \text { small } \} 


\section{C.9 Weights file for EMPLOYEE specification ( ${ }^{A} \mathrm{~T}_{\mathrm{E}} \mathrm{X}$ output)}

\section{Employee area}

\begin{tabular}{|c|ccc|ccc|ccc|}
\hline \multirow{2}{*}{ Attr. } & \multicolumn{4}{|c|}{ Employee } & \multicolumn{3}{c|}{ Contractor } & \multicolumn{3}{c|}{$\sigma^{2}$} & \multirow{2}{*}{$w$} \\
& $\mu$ & $\sigma^{2}$ & $w$ & $\mu$ & $\sigma^{2}$ & $w$ & & & \\
\hline \hline$A_{1}$ & 0.71 & 0.20 & 4.90 & 0.14 & 0.12 & 8.17 & 0.43 & 0.24 & 4.08 \\
$A_{2}$ & 0.57 & 0.24 & 4.08 & 1.00 & 0.00 & $\infty$ & 0.79 & 0.17 & 5.94 \\
$A_{3}$ & 1.00 & 0.00 & $\infty$ & 0.57 & 0.24 & 4.08 & 0.79 & 0.17 & 5.94 \\
$A_{4}$ & 0.29 & 0.20 & 4.90 & 0.71 & 0.20 & 4.90 & 0.50 & 0.25 & 4.00 \\
$A_{5}$ & 0.71 & 0.20 & 4.90 & 0.29 & 0.20 & 4.90 & 0.50 & 0.25 & 4.00 \\
$A_{6}$ & 0.57 & 0.24 & 4.08 & 0.14 & 0.12 & 8.17 & 0.36 & 0.23 & 4.36 \\
$A_{7}$ & 0.83 & 0.14 & 7.20 & 0.17 & 0.14 & 7.20 & 0.50 & 0.25 & 4.00 \\
$A_{8}$ & 0.00 & 0.00 & $\infty$ & 0.43 & 0.24 & 4.08 & 0.23 & 0.18 & 5.63 \\
$A_{9}$ & 0.17 & 0.14 & 7.20 & 0.86 & 0.12 & 8.17 & 0.54 & 0.25 & 4.02 \\
$A_{10}$ & 0.14 & 0.12 & 8.17 & 0.29 & 0.20 & 4.90 & 0.21 & 0.17 & 5.94 \\
$A_{11}$ & 0.71 & 0.20 & 4.90 & 0.29 & 0.20 & 4.90 & 0.50 & 0.25 & 4.00 \\
$A_{12}$ & 0.50 & 0.25 & 4.00 & 0.17 & 0.14 & 7.20 & 0.33 & 0.22 & 4.50 \\
$A_{13}$ & 0.17 & 0.14 & 7.20 & 0.00 & 0.00 & $\infty$ & 0.08 & 0.08 & 13.09 \\
$A_{14}$ & 0.17 & 0.14 & 7.20 & 0.00 & 0.00 & $\infty$ & 0.08 & 0.08 & 13.09 \\
$A_{15}$ & 0.17 & 0.14 & 7.20 & 0.17 & 0.14 & 7.20 & 0.17 & 0.14 & 7.20 \\
$A_{16}$ & 0.00 & 0.00 & $\infty$ & 0.17 & 0.14 & 7.20 & 0.08 & 0.08 & 13.09 \\
$A_{17}$ & 0.00 & 0.00 & $\infty$ & 0.29 & 0.20 & 4.90 & 0.15 & 0.13 & 7.68 \\
$A_{18}$ & 0.14 & 0.12 & 8.17 & 0.43 & 0.24 & 4.08 & 0.29 & 0.20 & 4.90 \\
\hline
\end{tabular}

\section{C.10 Distances file for Building Workers' Industrial Union of Australia v. Odco Pty Ltd ( $\mathrm{I}^{A} \mathrm{~T}_{\mathrm{E}} \mathrm{X}$ input)}

$\%$ Distances file

$\%$ Produced by SHYSTER version 1.0

\% Copyright James Popple 1993

$\%$ This is not a stand-alone LaTeX file.

$\%$ Include it in a LaTeX document using the \input command.

\% Use LaTeX version 2.09 <25 March 1992> and TeX version 3.141.

\subsection*\{Employee area\}

$\backslash$ subsubsection*\{Instant case\}

$\backslash$ begin\{small\}

$\backslash$ begin $\{$ tabular $\}\{*\{2\}\{\mid c\} *\{17\}\{@\{\backslash$ hspace $\{0.4 \mathrm{em}\}\} \mathrm{c}\}|\mathrm{r}| \mathrm{r} @\{\backslash$ hspace $\{\backslash$ tabcol

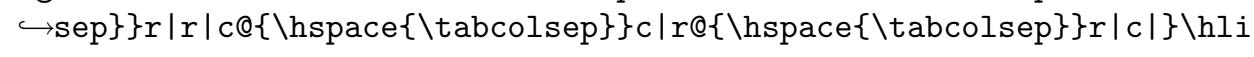
$\hookrightarrow$ ne 
$\& \backslash \operatorname{multicolumn}\{18\}\{|c|\}\{\backslash$ it Attributes $\backslash /\} \& \& \& \& \& \& \& \& \backslash \backslash$

$\backslash \operatorname{smash}\{\backslash$ raisebox $\{0.6 \backslash$ ht $\backslash$ strutbox $\}\{\backslash$ it Case $\backslash /\}\} \& \$ A_{-}\{1\} \$ \& \$ A_{-}\{2\} \$ \& \$ A_{-}$ $\hookrightarrow\{3\} \$ \& \$ A_{-}\{4\} \$ \& \$ A_{-}\{5\} \$ \& \$ A_{-}\{6\} \$ \& \$ A_{-}\{7\} \$ \& \$ A_{-}\{8\} \$ \& \$ A_{-}\{9\} \$ \& \$ A_{-}\{10\} \$ \&$ $\hookrightarrow \$ A_{-}\{11\} \$ \& \$ A_{-}\{12\} \$ \& \$ A_{-}\{13\} \$ \& \$ A_{-}\{14\} \$ \& \$ A_{-}\{15\} \$ \& \$ A_{-}\{16\} \$ \& \$ A_{-}\{17\} \$$ $\hookrightarrow \& \$ A_{-}\{18\} \$ \& \backslash \operatorname{multicolumn}\{1\}\{c \mid\}\{\backslash \operatorname{smash}\{\backslash$ raisebox $\{0.6 \backslash$ ht $\backslash$ strutbox $\hookrightarrow\}\{\$ c \$\}\}\} \& \backslash \operatorname{multicolumn}\{1\}\{c\}\{\backslash \operatorname{smash}\{\backslash$ raisebox $\{0.6 \backslash$ ht $\backslash$ strutbox $\}\{$ $\left.\left.\left.\hookrightarrow \$ \mathrm{~d}_{-}\{\backslash \mathrm{rm} \mathrm{K}\} \$\right\}\right\}\right\} \& \backslash \operatorname{multicolumn}\{1\}\{\mathrm{c} \mid\}\{\backslash \operatorname{smash}\{\backslash$ raisebox $\{0.6 \backslash$ ht $\backslash$ str $\hookrightarrow$ utbox $\left.\left.\left\{\$ \mathrm{~d}_{-}\{\backslash \mathrm{rm} \mathrm{U}\} \$\right\}\right\}\right\} \& \backslash \operatorname{multicolumn}\{1\}\{\mathrm{c} \mid\}\{\backslash \operatorname{smash}\{\backslash$ raisebox $\{0.6$ $\hookrightarrow \backslash$ ht $\backslash$ strutbox $\}\{\$ \backslash$ Delta $\$\}\}\} \& \backslash$ smash $\{\backslash$ raisebox $\{0.6 \backslash$ ht $\backslash$ strutbox $\}\{\$ S$ $\hookrightarrow \$\}\} \& \backslash \operatorname{smash}\left\{\backslash\right.$ raisebox $\left\{0.6 \backslash\right.$ ht $\backslash$ strutbox $\left.\left\{\$ S^{\prime} \$\right\}\right\} \& \backslash \operatorname{multicolumn}\{1\}\{\mathrm{c}$ $\hookrightarrow\}\{\backslash \operatorname{smash}\{\backslash$ raisebox $\{0.6 \backslash$ ht $\backslash$ strutbox $\}\{\$ r \$\}\}\} \& \backslash \operatorname{multicolumn}\{1\}\{c \mid\}$ $\hookrightarrow\{\backslash \operatorname{smash}\{\backslash$ raisebox $\{0.6 \backslash$ ht $\backslash$ strutbox $\}\{\$ r, \$\}\}\} \& \backslash \operatorname{smash}\{\backslash$ raisebox $\{0$. $\hookrightarrow 6 \backslash$ ht $\backslash$ strutbox $\}\{\backslash$ it Result $\backslash /\}\} \backslash \backslash \backslash h l i n e \backslash h l i n e$

\$C_ $\{\backslash$ rm Instant $\} \& \& \$ \backslash$ times $\$ \& \$ \backslash$ bullet $\$ \& \$ \backslash$ times $\$ \& \$ \backslash$ bullet $\$ \& \$ \backslash$ times $\$ \& \$$

$\hookrightarrow \backslash$ times $\$$ \& $\$$ times $\$ \& \& \backslash$ times $\$ \& \$$ times $\$ \& \$$ bullet $\$ \& \& \backslash$ times $\$ \& \$ \backslash$ time $\hookrightarrow$ s\$\&\$times $\$ \& \$ \backslash$ times $\$ \& \$ \backslash$ times $\$ \& \$ \backslash$ bullet $\$ \& \backslash$ multicolumn $\{9\}\{c \mid\}\{\} \backslash$ $\hookrightarrow \backslash \backslash \mathrm{hline} \backslash \mathrm{hline}$

\$C_ $\{1\} \$ \& \$ \backslash$ times $\$ \& \$$ bullet $\$ \& \$ \backslash$ bullet $\$ \& \$ \backslash$ times $\$ \& \$ \backslash$ bullet $\$ \& \backslash$ bullet $\$ \&$

$\hookrightarrow$ $\backslash$ bullet $\$ \& \$ \backslash$ times $\$ \& \$ \backslash$ times $\$ \& \$ \backslash$ times $\$ \& \$ \backslash$ bullet $\$ \& \$ \backslash$ bullet $\$ \& \$ \backslash$ tim $\hookrightarrow$ es $\$ \& \$$ times $\$ \& \$ \backslash$ bullet $\$ \& \$ \backslash$ times $\$ \& \$$ times $\$ \& \$ \backslash$ times $\$ 1 \& 34$. 39\&10.1 $\hookrightarrow 3 \& 7 \& 0.44 \& 0.33 \& 0.07 \& 0.11 \& \backslash \backslash$

$\$ C_{-}\{12\} \$ \& \$ \backslash$ bullet $\$ \& \$ \backslash$ bullet $\$ \& \$ \backslash$ bullet $\$ \& \$ \backslash$ times $\$ \& \$ \backslash$ bullet $\$ \& \$ \backslash$ bullet $\hookrightarrow \$ \& \$ \backslash$ times $\$ \& \$ \backslash$ bullet $\$ \& \$ \backslash$ bullet $\$ \& \$ \backslash$ times $\$ \& \$ \backslash$ bullet $\$ \& \$ \backslash$ bullet $\$ \& \$ \backslash$ $\hookrightarrow$ times $\$ \& \$ \backslash$ times $\$ \& \$ \backslash$ times $\$ \& \$ \backslash$ bullet $\$ \& \$$ times $\$ \& \$ \backslash$ bullet $\$$ \& 7 39. 49\& $\hookrightarrow 10.13 \& 7 \& 0.44 \& 0.37 \& 0.22 \& 0.14 \& \$ \backslash$ stackrel $\left\{\backslash\right.$ scriptscriptstyle $\left.I^{\sim}\right\}\{$

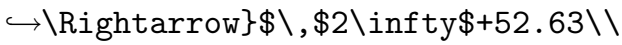

\$I_ $\{\backslash \operatorname{mbox}\{\backslash$ scriptsize \sf Contractor $\}$ \}\& $\$ \backslash$ times $\$ \& \$ \backslash$ bullet $\$ \& \$ \backslash$ times $\$$ $\hookrightarrow \& \$ \backslash$ bullet $\$ \& \backslash$ times $\$ \& \& \backslash$ times $\$ \& \$ \backslash$ bullet $\$ \& \$ \backslash$ bullet $\$$ \& $\$$ times $\$$ \& $\$ \backslash t$ $\hookrightarrow$ imes $\$ \& \$ \backslash$ times $\$ \& \$ \backslash$ bullet $\$ \& \$ \backslash$ times $\$ \& \$ \backslash$ times $\$ \& \$ \backslash$ times $\$ \& \& \$ \backslash$ bullet $\$$ $\hookrightarrow \& \& 21.11 \& 22.17 \& 3 \& 0.21 \& 0.23 \& 0.52 \& 0.29 \& \backslash \backslash$

$\$ \backslash m u_{-}\{\backslash \operatorname{mbox}\{\backslash$ scriptsize $\backslash$ sf Contractor $\}$ \} $\$$ \& $\$$ times $\$ \& \$ \backslash$ bullet $\$ \& \$ \backslash$ bull $\hookrightarrow$ et $\$ \& \$ \backslash$ bullet $\$ \& \$ \backslash$ times $\$ \& \$ \backslash$ times $\$ \& \$ \backslash$ times $\$ \& \$ \backslash$ times $\$ \& \$ \backslash$ bullet $\$ \& \$ \backslash$ $\hookrightarrow$ times $\$ \& \$ \backslash$ times $\$ \& \$ \backslash$ times $\$ \& \$ \backslash$ times $\$ \& \$ \backslash$ times $\$ \& \$ \backslash$ times $\$ \& \$$ times $\$ \& \$$

$\hookrightarrow \backslash$ times $\$ \& \backslash$ times $\$ \& \& 18.86 \& 10.13 \& 4 \& 0.25 \& 0.18 \& 0.53 \& 0.55 \& \backslash \backslash \backslash$ hline $\backslash$ end $\{$ tabular\} \end } \{ \text { small } \}

\subsubsection $*$ Instantiation 1$\}$

\section{C.11 Distances file for Building Workers' Industrial Union of Australia v. Odco Pty Ltd ( $\mathrm{IT}_{\mathrm{E}} \mathrm{X}$ output)}




\section{Employee area}

\section{Instant case}

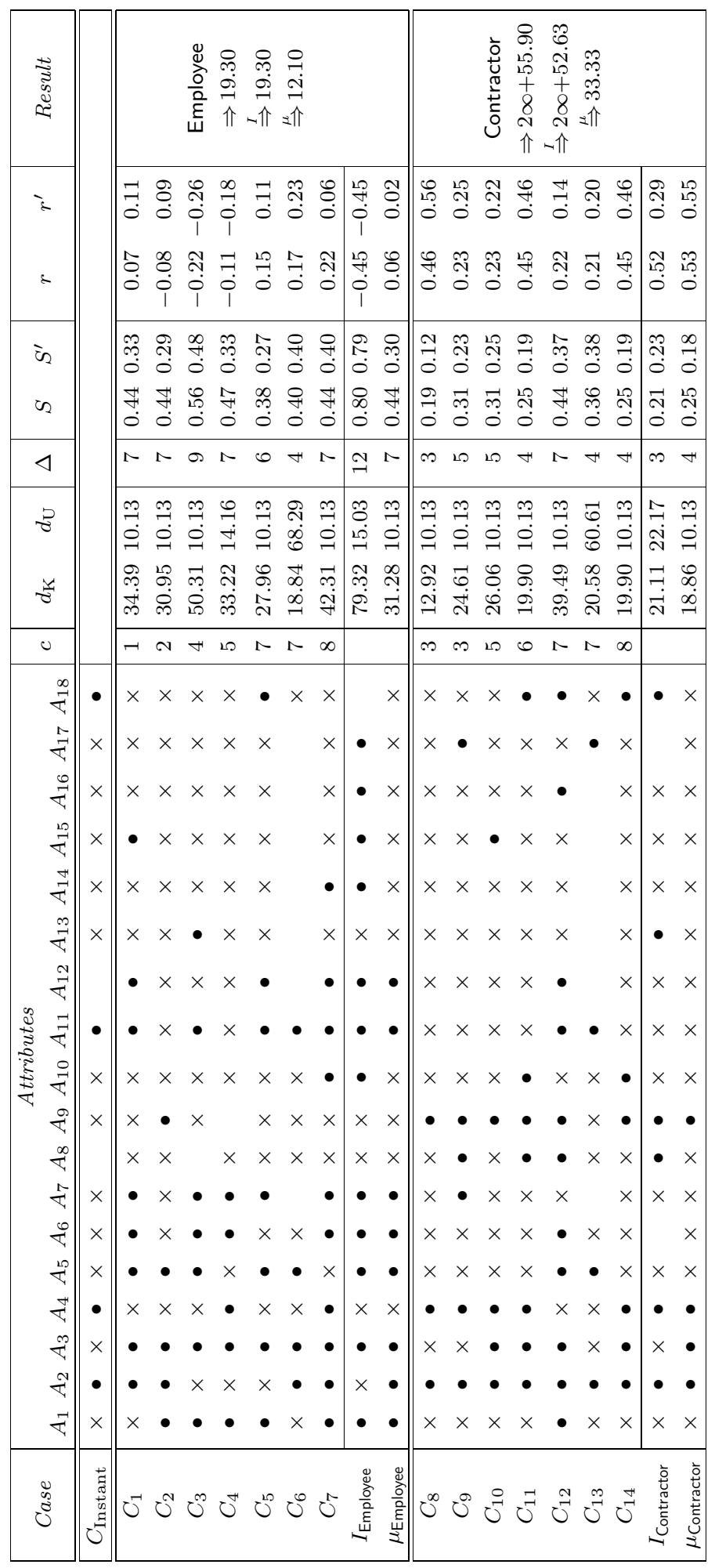




\section{Instantiation 1}

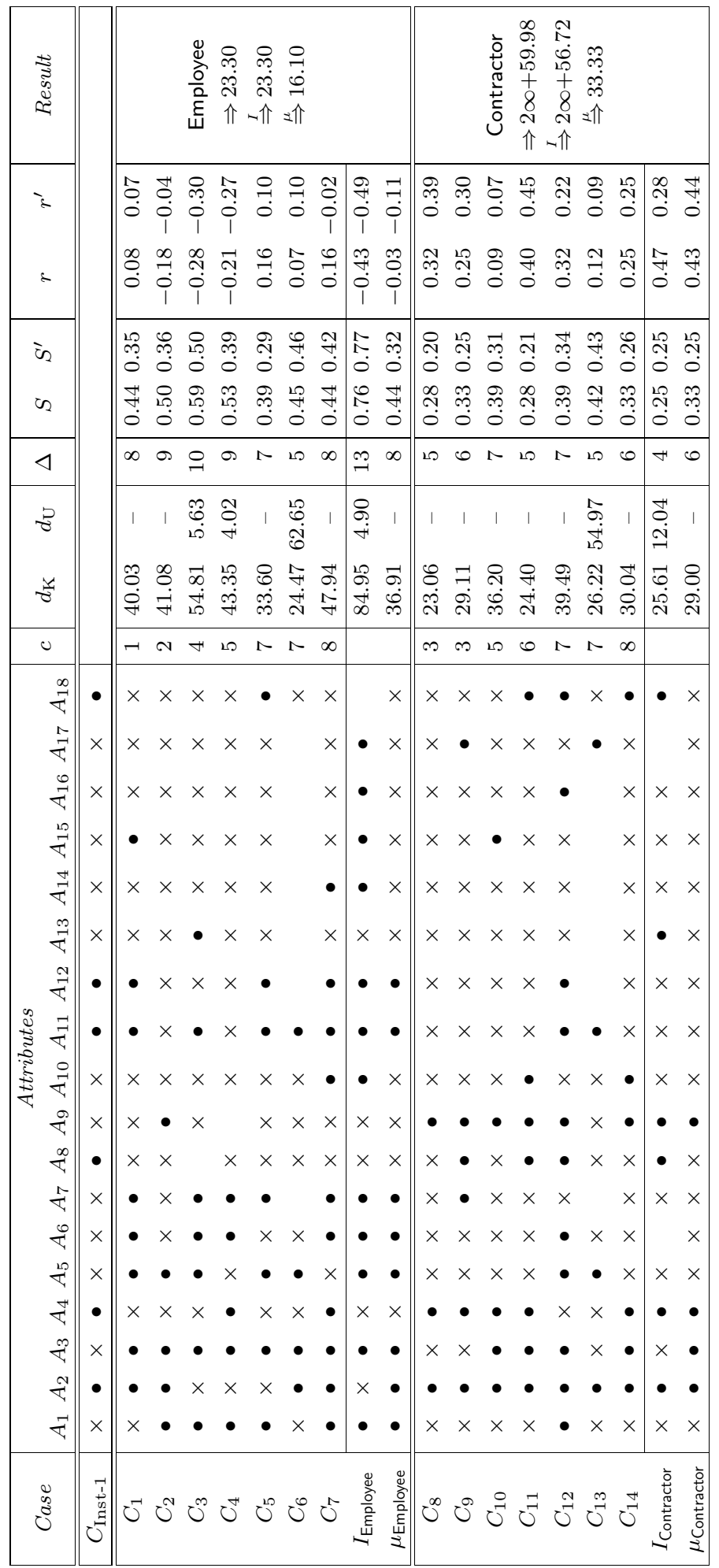




\section{Instantiation 2}

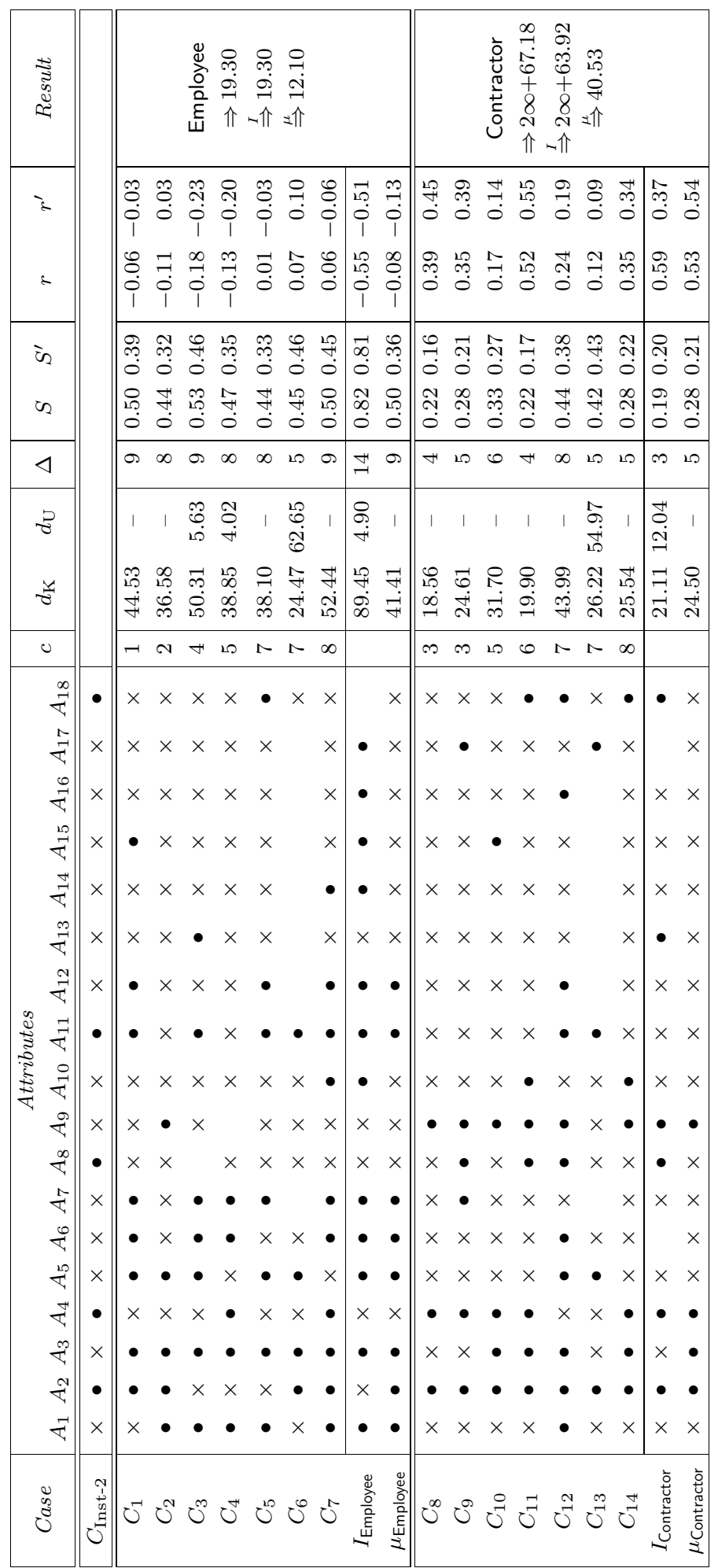




\section{Instantiation 3}

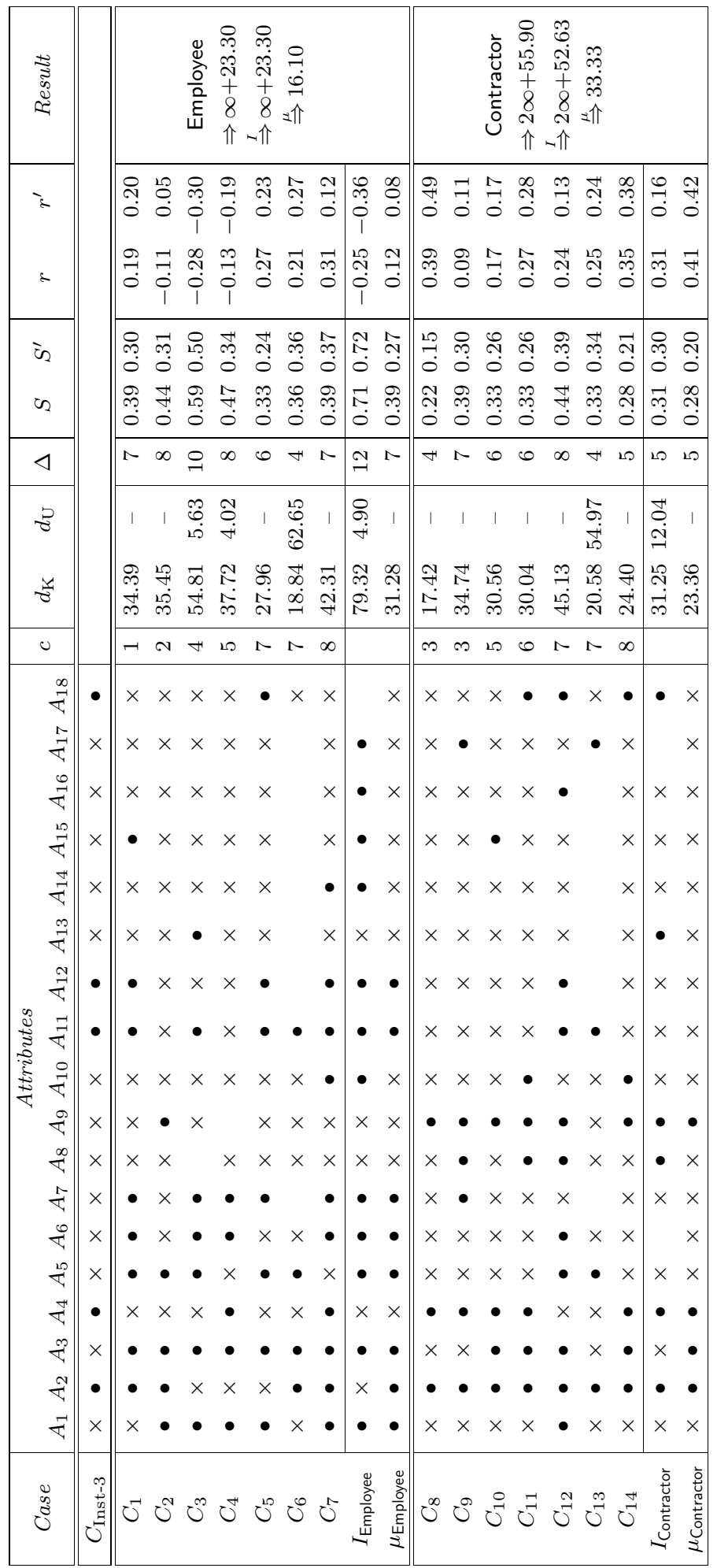




\section{Instantiation 4}

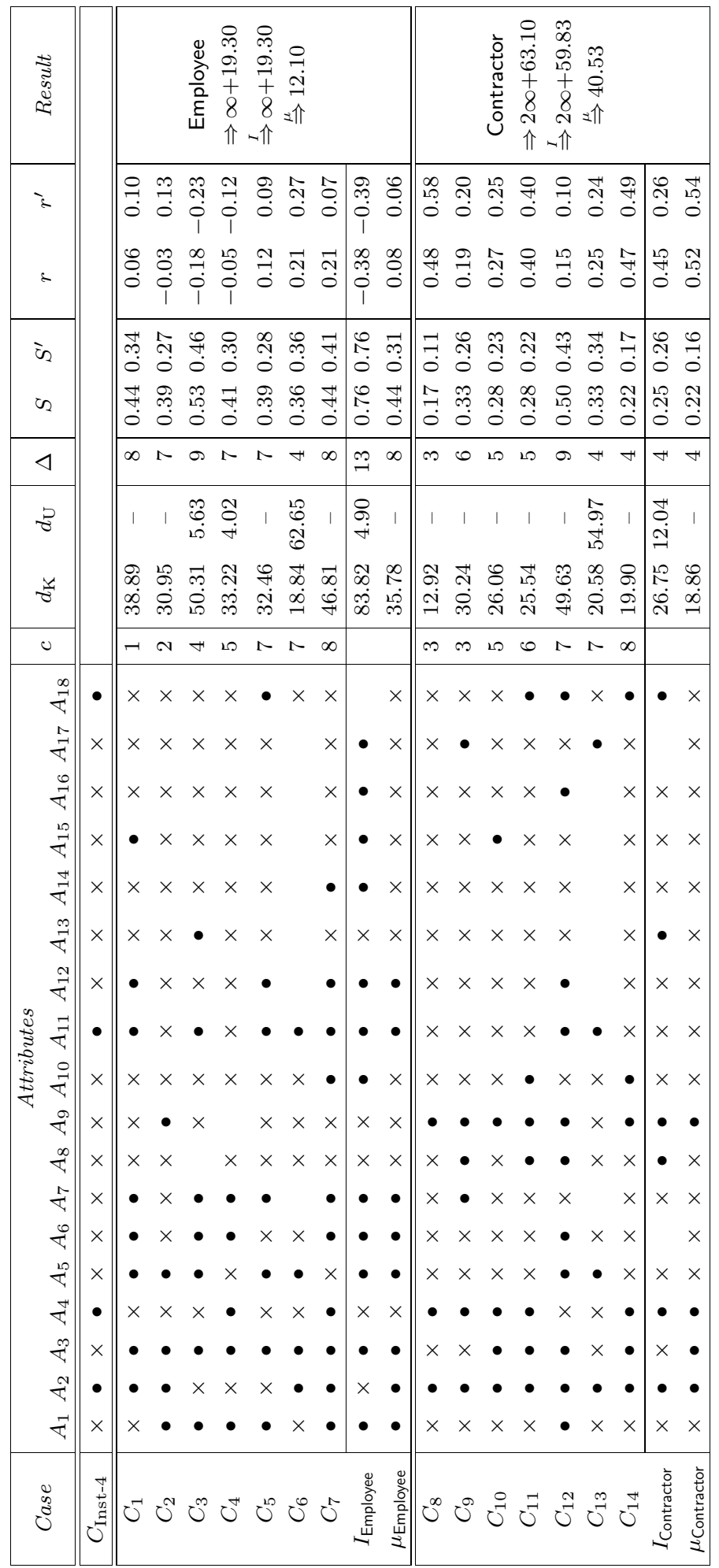




\section{Hypothetical 1}

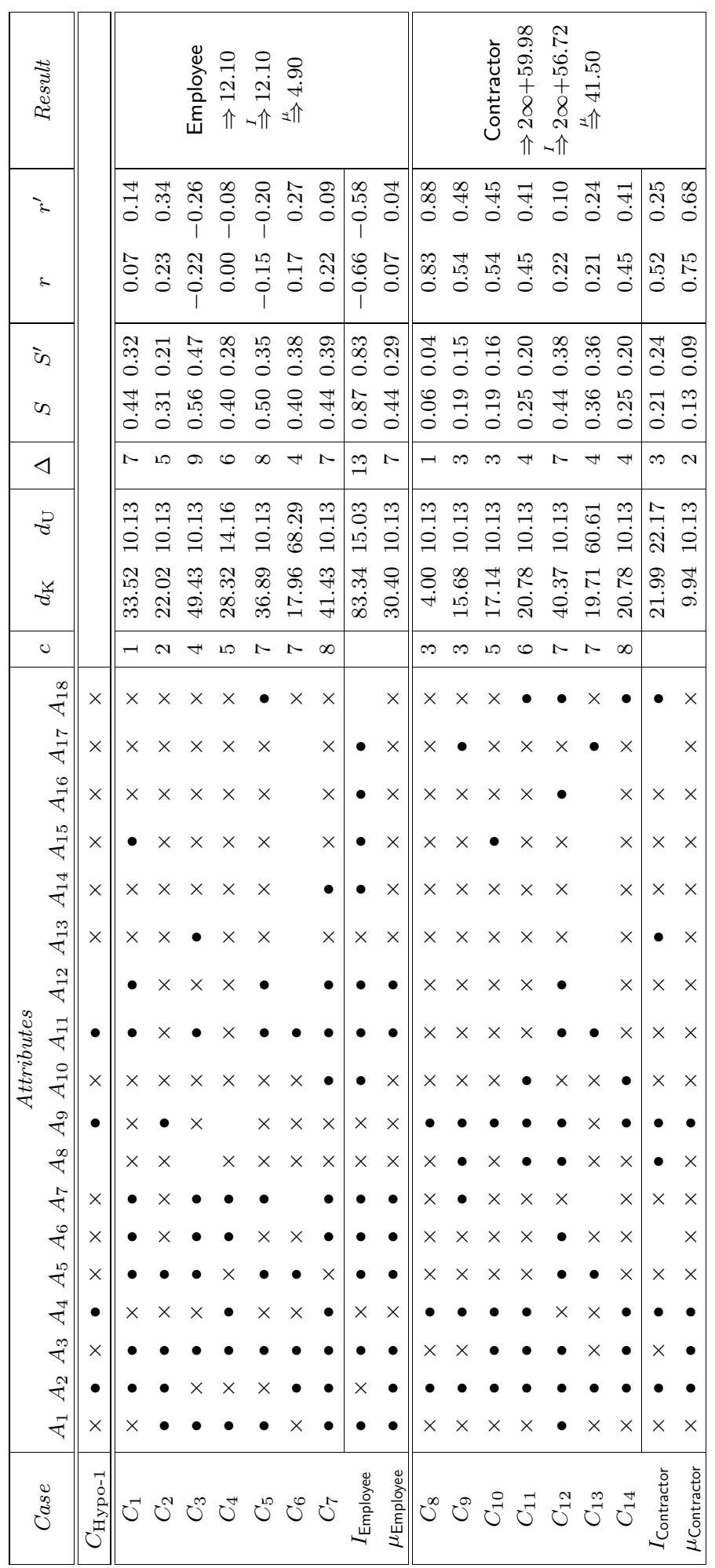


Hypothetical 2

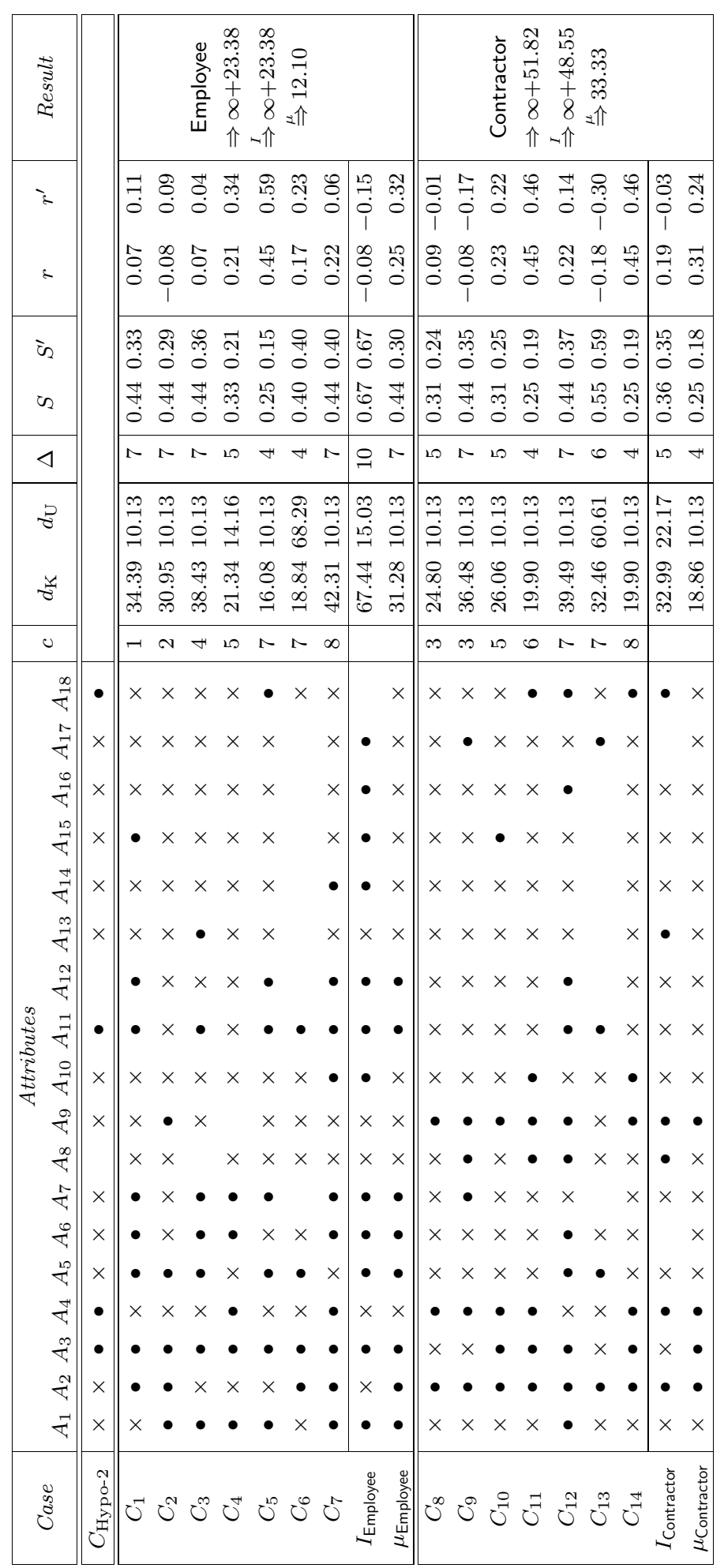




\section{C.12 Report file for Building Workers' Industrial Union of Australia v. Odco Pty Ltd ( $\mathrm{I}_{\mathrm{T}} \mathrm{T}_{\mathrm{E}} \mathrm{X}$ input)}

$\%$ Report file

$\%$ Produced by SHYSTER version 1.0

\% Copyright James Popple 1993

$\%$ This is not a stand-alone LaTeX file.

$\%$ Include it in a LaTeX document using the \input command.

\% Use LaTeX version 2.09 <25 March 1992> and TeX version 3.141.

$\backslash$ subsection*\{Employee area\}

$\backslash$ subsubsection*\{Instant case $\}$

The law distinguishes between a contract of service (between employer and employee) and a contract for services (between principal and independent

$\backslash$ medskip \noindent In the instant case,

the employer did not direct the manner in which the work was to be done;

the worker was allowed to use her/his own discretion in doing an aspect of the work that was not specified beforehand;

$\backslash$ medskip \noindent In my opinion---following \frenchspacing

$\{\backslash$ it Humberstone v. Northern Timber Mills $\backslash /\} \backslash$ nonfrenchspacing---\%

the worker is an independent contractor.

$\backslash$ medskip \noindent In \frenchspacing

$\{\backslash$ it Humberstone v. Northern Timber Mills\}\nonfrenchspacing, $\%$

$\backslash$ footnote\{(1949) 79 CLR 389.\}

a 1949 decision of three judges of the High Court of Australia,

Humberstone carried goods for NTM. He had originally held himself out as a carrier, prepared to carry for anyone, but for over twenty years he had

There are several significant similarities

between the instant case and \frenchspacing

$\{\backslash$ it Humberstone v. NTM $\backslash /\} \backslash$ null \nonfrenchspacing:

the employer did not direct the manner in which the work was to be done;

the worker was allowed to use her/his own discretion in doing an aspect of the work that was not specified beforehand;

However, the instant case is not on all fours with \frenchspacing $\{\backslash$ it Humberstone v. NTM $\backslash \backslash$ null \nonfrenchspacing. 
In that case

the worker was not in business on her/his own account;

the worker was allowed to employ others to assist with her/his work;

Nevertheless, I believe that \frenchspacing

$\{\backslash$ it Humberstone V. NTM \/\} \nonfrenchspacing

should be followed.

$\backslash$ medskip \noindent If \frenchspacing

$\{\backslash$ it Ferguson v. John Dawson \\& Partners (Contractors) Ltd $\backslash /\} \backslash$ nonfrenchspacing is followed then the worker is an employee.

$\backslash$ medskip \noindent In \frenchspacing

$\{\backslash$ it Ferguson v. John Dawson \\& Partners (Contractors) Ltd\} \nonfrenchspacing, \% $\backslash$ footnote $\{[1976] 1$ WLR 1213.\}

a 1976 decision of the English Court of Appeal,

Ferguson fell off a roof while removing some scaffolding boards. He claimed damages against John Dawson (the building contractors) for

There are several similarities

between the instant case and Ifrenchspacing

$\{\backslash$ it Ferguson v. Dawson $\backslash /\} \backslash$ null \nonfrenchspacing:

the work was not performed on the employer's premises;

the worker was not allowed to employ others to assist with her/his work;

However, there are several significant differences

between the instant case and \frenchspacing

$\{\backslash$ it Ferguson v. Dawson $\} \backslash$ null \nonfrenchspacing.

In that case

the employer directed the manner in which the work was to be done;

the worker was not allowed to use her/his own discretion in doing an aspect of the work that was not specified beforehand;

Note also that $\backslash$ frenchspacing

$\{\backslash$ it Ferguson v. Dawson $\backslash /\}$ \nonfrenchspacing

is only a decision of

the English Court of Appeal

and not as good authority as a case decided by

three judges of the High Court of Australia\%

---like \frenchspacing

$\{\backslash$ it Humberstone v. NTM $\} \backslash$ null $\backslash$ nonfrenchspacing.

Consequently, there is nothing in \frenchspacing

$\{\backslash$ it Ferguson v. Dawson $\backslash /\}$ \nonfrenchspacing

to warrant any change in my conclusion.

\subsubsection*\{Hypothetical 1\} 


\section{C.13 Report file for Building Workers' Industrial Union of Australia v. Odco Pty Ltd ( $\mathrm{LAT}_{\mathrm{E}} \mathrm{X}$ output)}

\section{Employee area}

\section{Instant case}

The law distinguishes between a contract of service (between employer and employee) and a contract for services (between principal and independent contractor). This distinction affects the terms that will be implied in the absence of an express agreement, the liability of the employer to third parties, the applicability of industrial awards, the applicability of statutes which may affect workers' compensation, occupational health and safety, long-service leave, fringe benefits tax, etc.

The terms "employer" and "worker" are used here to mean "employer" and "employee" (in the case of a contract of service) or "principal" and "independent contractor" (in the case of a contract for services).

In the instant case, the employer did not direct the manner in which the work was to be done; the worker was allowed to use her/his own discretion in doing an aspect of the work that was not specified beforehand; the worker was not an integral part of the employer's business, but was accessory to it; the worker owned the tools or provided the transport with which she/he performed the work; the employer would not make a profit/loss if the work performed by the worker cost less/more than expected; the work was not performed on the employer's premises; the employer neither supervised nor inspected the work; it is not known whether the worker was in business on her/his own account; the worker was not allowed to employ others to assist with her/his work; the worker was not obliged to work only for the employer; the worker was required to work at specified times; it is not known whether the employer paid the worker by time; the money that the employer paid to the worker was not stated to be a "fee"; the money that the employer paid to the worker was not stated to be "wages" or "salary"; the employer did not deduct PAYE tax instalments from the worker's pay; the employer paid the worker neither sick pay nor holiday pay; the employer and the worker did not express any intention that the relationship would be one of employer and employee; and the employer and the worker expressed an intention that the relationship would be one of principal and independent contractor.

In my opinion-following Humberstone v. Northern Timber Mills - the worker is an independent contractor.

In Humberstone v. Northern Timber Mills, ${ }^{1}$ a 1949 decision of three judges of the High Court of Australia, Humberstone carried goods for NTM. He had originally held himself out as a carrier, prepared to carry for anyone, but for over twenty years he had carried 
goods solely for NTM (although he would, infrequently, carry back-loads for NTM's customers). Humberstone owned the truck, and paid for petrol and repairs. He was paid weekly on a weight-mileage basis. He was a licenced carrier, and had his name printed on the side of his truck with the description "carrier."

On the way back from a job, he had a puncture. He went home to change the wheel, but exerted himself so strenuously in trying to remove the tyre from the wheel that he became ill and later lapsed into a coma, from which he did not recover. Section 3 of the Worker's Compensation Act 1928 (Vic) had been amended about a year before Humberstone's death so as to include independent contractors in its definition of a "worker" covered by the Act. However, the High Court held that the amendment applied only to contracts entered into after it came into operation. Further, the Court decided that Humberstone was not an employee of NTM. Hence he was not a "worker" under the Act and his widow was not entitled to compensation under the Act.

There are several significant similarities between the instant case and Humberstone v. NTM: the employer did not direct the manner in which the work was to be done; the worker was allowed to use her/his own discretion in doing an aspect of the work that was not specified beforehand; the worker was not an integral part of the employer's business, but was accessory to it; the worker owned the tools or provided the transport with which she/he performed the work; the employer would not make a profit/loss if the work performed by the worker cost less/more than expected; the work was not performed on the employer's premises; the employer neither supervised nor inspected the work; the worker was not obliged to work only for the employer; the money that the employer paid to the worker was not stated to be a "fee"; the money that the employer paid to the worker was not stated to be "wages" or "salary"; the employer did not deduct PAYE tax instalments from the worker's pay; the employer paid the worker neither sick pay nor holiday pay; and the employer and the worker did not express any intention that the relationship would be one of employer and employee.

However, the instant case is not on all fours with Humberstone v. NTM. In that case the worker was not in business on her/his own account; the worker was allowed to employ others to assist with her/his work; the worker was not required to work at specified times; the employer did not pay the worker by time; and the employer and the worker did not express any intention that the relationship would be one of principal and independent contractor.

Nevertheless, I believe that Humberstone v. NTM should be followed.

If Ferguson v. John Dawson \& Partners (Contractors) Ltd is followed then the worker is an employee.

In Ferguson v. John Dawson $\mathcal{G}$ Partners (Contractors) Ltd, ${ }^{2}$ a 1976 decision of the English Court of Appeal, Ferguson fell off a roof while removing some scaffolding boards. He claimed damages against John Dawson (the building contractors) for breach of statutory duty relying on the Construction (Working Places) Regulations 1966 (UK). This duty would only be owed if Ferguson was an employee of John Dawson.

${ }^{2}[1976] 1$ WLR 1213. 
Megaw and Browne LJJ held that, despite the fact that both parties labelled Ferguson a "self-employed labour only subcontractor", 3 the reality of the relationship between them was that of employer/employee.

There are several similarities between the instant case and Ferguson v. Dawson: the work was not performed on the employer's premises; the worker was not allowed to employ others to assist with her/his work; the worker was not obliged to work only for the employer; the worker was required to work at specified times; the money that the employer paid to the worker was not stated to be a "fee"; the money that the employer paid to the worker was not stated to be "wages" or "salary"; the employer did not deduct PAYE tax instalments from the worker's pay; the employer paid the worker neither sick pay nor holiday pay; the employer and the worker did not express any intention that the relationship would be one of employer and employee; and the employer and the worker expressed an intention that the relationship would be one of principal and independent contractor.

However, there are several significant differences between the instant case and Ferguson v. Dawson. In that case the employer directed the manner in which the work was to be done; the worker was not allowed to use her/his own discretion in doing an aspect of the work that was not specified beforehand; the worker was an integral part of the employer's business; the worker neither owned the tools nor provided the transport with which she/he performed the work; the employer would make a profit/loss if the work performed by the worker cost less/more than expected; the employer supervised or inspected the work; the worker was not in business on her/his own account; and the employer paid the worker by time. Note also that Ferguson v. Dawson is only a decision of the English Court of Appeal and not as good authority as a case decided by three judges of the High Court of Australia-like Humberstone v. NTM.

Consequently, there is nothing in Ferguson v. Dawson to warrant any change in my conclusion.

\section{Hypothetical 1}

Consider the instant case changed so that the following is true: the worker was allowed to employ others to assist with her/his work; and the employer and the worker did not express any intention that the relationship would be one of principal and independent contractor.

If that were so then I would be more strongly of the opinion that-following Humberstone v. Northern Timber Mills - the worker is an independent contractor.

Details of Humberstone v. NTM are summarized above. There are several significant similarities between the hypothetical case and Humberstone v. NTM: the employer did not direct the manner in which the work was to be done; the worker was allowed to use her/his own discretion in doing an aspect of the work that was not specified beforehand; the worker was not an integral part of the employer's business, but was accessory to it; the worker owned the tools or provided the transport with which she/he performed the work; the employer would not make a profit/loss if the work performed by the worker

\footnotetext{
${ }^{3}$ ibid. at 1219, per Megaw LJ; at 1225, per Lawton LJ; at 1228, per Browne LJ.
} 
cost less/more than expected; the work was not performed on the employer's premises; the employer neither supervised nor inspected the work; the worker was allowed to employ others to assist with her/his work; the worker was not obliged to work only for the employer; the money that the employer paid to the worker was not stated to be a "fee"; the money that the employer paid to the worker was not stated to be "wages" or "salary"; the employer did not deduct PAYE tax instalments from the worker's pay; the employer paid the worker neither sick pay nor holiday pay; the employer and the worker did not express any intention that the relationship would be one of employer and employee; and the employer and the worker did not express any intention that the relationship would be one of principal and independent contractor.

However, the hypothetical case is not on all fours with Humberstone v. NTM. In that case the worker was not in business on her/his own account; the worker was not required to work at specified times; and the employer did not pay the worker by time.

Nevertheless, I believe that Humberstone v. NTM should be followed.

If Cam and Sons Pty Ltd v. Sargent is followed then the worker is an employee.

In Cam and Sons Pty Ltd v. Sargent, ${ }^{4}$ a 1940 decision of four judges of the High Court of Australia, Sargent was the master of a ship. He entered into an agreement with Cam and Sons that claimed that the ship was hired by Cam and Sons to Sargent and his fellow contractors (called "the partnership"). However, it was doubtful whether that agreement actually deprived Cam and Sons of any control over the ship. The partnership was to use the ship only to carry coal from Swansea to Sydney. Cam and Sons were sole agents of the partnership for securing cargoes for the ship, and for collecting money due to the partnership. The partnership paid nothing for the "hire" of the ship, but received a specified sum for each return trip of a certain tonnage plus (in certain circumstances) $5 \%$ of the earnings, the balance of which was retained by Cam and Sons. Cam and Sons had to approve people employed by the partnership.

Sargent claimed that he (and others in the partnership) were employed by Cam and Sons, and therefore came within the terms of an industrial award. Cam and Sons claimed that members of the partnership were independent contractors.

The High Court unanimously agreed with Sargent. Rich J came to the conclusion that the agreement was an attempt to evade the terms of the industrial award. ${ }^{5}$

There are several similarities between the hypothetical case and Cam v. Sargent: the worker was allowed to use her/his own discretion in doing an aspect of the work that was not specified beforehand; the work was not performed on the employer's premises; the employer neither supervised nor inspected the work; the worker was allowed to employ others to assist with her/his work; the worker was not obliged to work only for the employer; the money that the employer paid to the worker was not stated to be a "fee"; the money that the employer paid to the worker was not stated to be "wages" or "salary"; the employer did not deduct PAYE tax instalments from the worker's pay; the employer paid the worker neither sick pay nor holiday pay; the employer and the

\footnotetext{
${ }^{4}$ (1940) 14 ALJ 162.

5ibid. at 163 .
} 
worker did not express any intention that the relationship would be one of employer and employee; and the employer and the worker did not express any intention that the relationship would be one of principal and independent contractor.

However, there are several significant differences between the hypothetical case and Cam v. Sargent. In that case the employer directed the manner in which the work was to be done; the worker was an integral part of the employer's business; the worker neither owned the tools nor provided the transport with which she/he performed the work; the employer would make a profit/loss if the work performed by the worker cost less/more than expected; the worker was not in business on her/his own account; the worker was not required to work at specified times; and the employer did not pay the worker by time.

Despite the fact that Cam v. Sargent is a decision of four judges of the High Court of Australia (and better authority than a case decided by three judges of the High Court of Australia-like Humberstone v. NTM), there is nothing in Cam v. Sargent to warrant any change in my conclusion.

\section{Hypothetical 2}

Consider the instant case changed so that the following is true: the worker was not allowed to use her/his own discretion in doing an aspect of the work that was not specified beforehand; and the worker was an integral part of the employer's business.

If that were so then my opinion would be that-following Ferguson v. John Dawson 8 Partners (Contractors) $L t d$ - the worker is an employee.

Details of Ferguson v. Dawson are summarized above. There are several significant similarities between the hypothetical case and Ferguson v. Dawson: the worker was not allowed to use her/his own discretion in doing an aspect of the work that was not specified beforehand; the worker was an integral part of the employer's business; the work was not performed on the employer's premises; the worker was not allowed to employ others to assist with her/his work; the worker was not obliged to work only for the employer; the worker was required to work at specified times; the money that the employer paid to the worker was not stated to be a "fee"; the money that the employer paid to the worker was not stated to be "wages" or "salary"; the employer did not deduct PAYE tax instalments from the worker's pay; the employer paid the worker neither sick pay nor holiday pay; the employer and the worker did not express any intention that the relationship would be one of employer and employee; and the employer and the worker expressed an intention that the relationship would be one of principal and independent contractor.

However, the hypothetical case is not on all fours with Ferguson $v$. Dawson. In that case the employer directed the manner in which the work was to be done; the worker neither owned the tools nor provided the transport with which she/he performed the work; the employer would make a profit/loss if the work performed by the worker cost less/more than expected; the employer supervised or inspected the work; the worker was not in business on her/his own account; and the employer paid the worker by time.

Nevertheless, I believe that Ferguson v. Dawson should be followed. 
If Australian Mutual Provident Society v. Chaplin or Ready Mixed Concrete (South East) Ltd v. Minister of Pensions and National Insurance are followed then the worker is an independent contractor.

In Australian Mutual Provident Society v. Chaplin, ${ }^{6}$ a 1978 decision of the Judicial Committee of the Privy Council, Chaplin was a representative of AMP. A clause of the agreement between them stated that the relationship was one of "principal and agent" and not one of "master and servant". Chaplin claimed that he was employed under a contract of service, and therefore a "worker" under the Long Service Leave Act, 1967 (SA) and entitled to certain benefits.

The Privy Council found that there was no reason to think that the clause was not a genuine statement of the parties' intentions. Examining the agreement, their lordships concluded that it provided for a contract of agency. The fact that Chaplin was given the power of unlimited delegation of the whole performance of his work was "almost conclusive against the contract being a contract of service." ${ }^{7}$

There are several similarities between the hypothetical case and AMP v. Chaplin: the employer did not direct the manner in which the work was to be done; the worker was an integral part of the employer's business; the worker owned the tools or provided the transport with which she/he performed the work; the employer would not make a profit/loss if the work performed by the worker cost less/more than expected; the work was not performed on the employer's premises; the employer neither supervised nor inspected the work; the money that the employer paid to the worker was not stated to be a "fee"; the money that the employer paid to the worker was not stated to be "wages" or "salary"; the employer did not deduct PAYE tax instalments from the worker's pay; the employer paid the worker neither sick pay nor holiday pay; the employer and the worker did not express any intention that the relationship would be one of employer and employee; and the employer and the worker expressed an intention that the relationship would be one of principal and independent contractor.

However, there are several significant differences between the hypothetical case and $A M P$ v. Chaplin. In that case the worker was allowed to use her/his own discretion in doing an aspect of the work that was not specified beforehand; the worker was in business on her/his own account; the worker was allowed to employ others to assist with her/his work; the worker was obliged to work only for the employer; the worker was not required to work at specified times; and the employer did not pay the worker by time.

Despite the fact that AMP $v$. Chaplin is a decision of the Judicial Committee of the Privy Council (and better authority than a case decided by the English Court of Appeal-like Ferguson v. Dawson), there is nothing in AMP v. Chaplin to warrant any change in my conclusion.

In 1967, Ready Mixed Concrete (South East) Ltd v. Minister of Pensions and National Insurance $^{8}$ was decided by the Queen's Bench Division of the English High Court. (A case decided by the Queen's Bench Division of the English High Court is not as

\footnotetext{
${ }^{6}$ (1978) 18 ALR 385.

7 ibid. at 391 .

${ }^{8}$ [1968] 2 QB 497.
} 
good authority as a case decided by the Judicial Committee of the Privy Council—like $A M P$ v. Chaplin; furthermore Ready Mixed v. Minister is 11 years older than AMP $v$. Chaplin.)

In Ready Mixed v. Minister, Latimer worked for Ready Mixed as an "owner-driver." He was paid at mileage rates, and was obliged to buy the truck through a financial organization associated with Ready Mixed. The truck was painted in the company's colours, and he had to wear a Ready Mixed uniform. Latimer was obliged to meet the costs of maintenance, repair and insurance of the truck (and the attached mixing unit, which belonged to Ready Mixed). The Minister determined that Latimer was employed under a contract of service and therefore an "employed person" under s. 1(2) of the National Insurance Act 1965 (UK), making Ready Mixed liable to make weekly contributions.

MacKenna J examined the contract and held that the rights it conferred, and the duties it imposed, between Latimer and Ready Mixed were not such as to make it a contract of service.

There are several similarities between the hypothetical case and Ready Mixed $v$. Minister: the employer did not direct the manner in which the work was to be done; the worker was an integral part of the employer's business; the worker owned the tools or provided the transport with which she/he performed the work; the employer would not make a profit/loss if the work performed by the worker cost less/more than expected; the work was not performed on the employer's premises; the employer neither supervised nor inspected the work; the money that the employer paid to the worker was not stated to be a "fee"; the money that the employer paid to the worker was not stated to be "wages" or "salary"; the employer did not deduct PAYE tax instalments from the worker's pay; the employer paid the worker neither sick pay nor holiday pay; the employer and the worker did not express any intention that the relationship would be one of employer and employee; and the employer and the worker expressed an intention that the relationship would be one of principal and independent contractor.

However, there are several significant differences between the hypothetical case and Ready Mixed v. Minister. In that case the worker was allowed to use her/his own discretion in doing an aspect of the work that was not specified beforehand; the worker was not in business on her/his own account; the worker was allowed to employ others to assist with her/his work; the worker was obliged to work only for the employer; the worker was not required to work at specified times; and the employer did not pay the worker by time. Note also that Ready Mixed $v$. Minister is only a decision of the Queen's Bench Division of the English High Court and not as good authority as a case decided by the English Court of Appeal-like Ferguson v. Dawson.

Consequently, there is nothing in Ready Mixed $v$. Minister to warrant any change in my conclusion. 


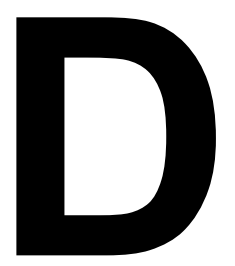

\section{Reflexive tests}

Great cases, like hard cases, make bad law.

Oliver Wendell Holmes (1904)

Northern Securities Co. v. United States ${ }^{29}$

This is the tale of the 1401 ,

The law clerk that was nobody's son.

It spends its days in a furious hunt

For authorities, dictum, and argumunt.

But after it found them, it burned with shame;

The Supreme Court reversed it just the same.

T. H. Lassagne $(1963)^{30}$ 


\section{D.1 Introduction}

A reflexive test is performed by removing one of the leading cases from a specification, and using it as a test case with the diminished specification. Reflexive tests, and their limitations, are discussed in $\S 3.13 .5$.

A reflexive test was performed for every leading case in each of the four specifications described in chapter 5. Details of each of the cases are summarized in the dump files of the specifications given in appendix A.

The results of these reflexive tests are described below ( $\S \mathrm{D} .2-\S \mathrm{D} .5)$, and summarized in four figures in this appendix. The symbols used in these figures are the same as those used in figure 5.12 and are explained in $§ 5.6$.

Conclusions are drawn from the results of this reflexive testing in $§ 5.6 .3$.

\section{D.2 The FINDER specification}

The results of the reflexive testing of all cases in the Finder specification are described here, and summarized in figure D.1.

Hannah v. Peel

In Hannah v. Peel ${ }^{31}\left(C_{1}\right)$, Birkett J of the King's Bench Division of the English High Court followed Bridges v. Hawkesworth, and distinguished South Staffordshire Water Co. v. Sharman and Elwes v. Brigg Gas Co. ${ }^{32}$ Hannah, the finder, won.

SHYSTER concludes that Hannah should have lost. The nearest neighbour is South Staffordshire v. Sharman; the nearest other is Bridges v. Hawkesworth. Its choice of cases is good, although SHYSTER chooses to follow the wrong one.

However, SHYSTER warns that these two cases are equidistant from Hannah $v$. Peel; it only chooses South Staffordshire $v$. Sharman because it is the more recent of the two-both were decided by the Queen's Bench Division. It also warns that the weighted association coefficients suggest that Bridges $v$. Hawkesworth should be the nearest neighbour.

Bridges v. Hawkesworth

1851

In Bridges v. Hawkesworth ${ }^{33}\left(C_{2}\right)$, Patteson J of the Queen's Bench Division could find no authority "directly in point" and found no reason not to apply the general rule, as stated in Armory $v$. Delamirie, that the finder is entitled to the found chattel as against all parties except the real owner. ${ }^{34}$

SHYSTER agrees that Bridges should have won. The nearest neighbour is Hannah v. Peel; the nearest other is City of London Corporation v. Appleyard (2). Both of these cases were decided after Bridges $v$. Hawkesworth. The results of this test are consistent with the results of a similar reflexive test performed by Tyree, Greenleaf and Mowbray using FINDER. ${ }^{35}$ 
(With Bridges v. Hawkesworth removed from the specification, there is an equivalent functional dependence between $A_{8}$ and $A_{9}$.)

Armory v. Delamirie

Armory v. Delamirie ${ }^{36}\left(C_{3}\right)$ is the oldest of all the finder cases. Pratt CJ of the King's Bench Division had no previous authorities upon which to base his decision, which was in favour of the finder, Armory.

SHYSTER agrees that the finder should have won. The nearest neighbour is Bridges $v$. Hawkesworth; the nearest other is Moffatt v. Kazana. Of course, both of these cases were decided after Armory $v$. Delamirie.

However, SHYSTER warns that the weighted correlation coefficients suggest that six other cases should be the nearest neighbour - in five of which, the finder lost.

(Without Armory v. Delamirie, there is an equivalent functional dependence between $A_{4}$ and $A_{10}$. Further, $A_{9}$ has infinite weight.)

In Moffatt v. Kazana ${ }^{37}\left(C_{4}\right)$, Wrangham J of the Queen's Bench Division followed City of London Corporation v. Appleyard and found against Kazana (the finder). ${ }^{38}$

SHYSTER concludes, unlike the Court, that Kazana should have won. The nearest neighbour is Hannah v. Peel; the nearest others are City of London Corporation v. Appleyard (1) and Elwes v. Brigg Gas Co.

(With Moffatt v. Kazana removed from the specification, there are two functional dependencies: equivalence between $A_{1}$ and $A_{6}$, and inverse between $A_{9}$ and $A_{10}$. Further, $A_{4}$ has infinite weight.)

City of London Corporation v. Appleyard (1)

As discussed in $§ 5.2 .2$, City of London Corporation v. Appleyard appears in the specification twice.

In London v. Appleyard $(1)^{39}\left(C_{5}\right)$, McNair J resolved the conflict between Appleyard (as finder) and Yorkwin by finding against Appleyard. In coming to this decision, he followed South Staffordshire Water Co. v. Sharman and Hannah v. Peel. $^{40}$

SHYSTER agrees. The nearest neighbour is South Staffordshire v. Sharman; the nearest other is Hannah v. Peel.

(With London v. Appleyard (1) removed from the specification, there is an inverse functional dependence between $A_{3}$ and $A_{10}$.) 


\begin{tabular}{|c|c|c|c|c|c|}
\hline Case & Result & $\begin{array}{l}\text { Nearest } \\
n^{\prime} \text { bours }\end{array}$ & $\begin{array}{l}\text { Nearest } \\
\text { others }\end{array}$ & \multicolumn{2}{|c|}{ Warnings } \\
\hline Hannah v. Peel & $\times$ & $\checkmark$ & $\checkmark$ & $S^{\prime} \equiv$ & $\checkmark$ \\
\hline Bridges v. Hawkesworth & $\checkmark$ & - & - & & $\checkmark$ \\
\hline Armory v. Delamirie & $\checkmark$ & - & - & $r^{\prime}$ & $x$ \\
\hline Moffatt v. Kazana & $x$ & $x$ & $\checkmark$ & & $x$ \\
\hline London v. Appleyard (1) & $\checkmark$ & $\checkmark$ & $\checkmark$ & & $\checkmark$ \\
\hline London v. Appleyard (2) & $\checkmark$ & $x$ & $\times$ & & $\checkmark$ \\
\hline South Staffordshire v. Sharman & $\checkmark$ & - & - & & $\checkmark$ \\
\hline Elwes v. Brigg Gas & $\checkmark$ & - & - & & $\checkmark$ \\
\hline
\end{tabular}

Figure D.1: A summary of the reflexive testing of the Finder specification, described in $\S$ D.2. The meaning of the symbols used here is explained in $\S 5.6 .1$.

City of London Corporation v. Appleyard (2)

In City of London Corporation v. Appleyard (2) ${ }^{41}\left(C_{6}\right)$, McNair J resolved the conflict between Yorkwin (as finder) and the City of London by finding against Yorkwin. His decision was based on the construction of a clause of the lease agreement between the two parties.

SHYSTER agrees that the finder should lose. The nearest neighbour is Elwes $v$. Brigg Gas Co.; the nearest others are Hannah v. Peel and Bridges v. Hawkesworth.

(With London v. Appleyard (2) removed from the specification, there is an inverse functional dependence between $A_{5}$ and $A_{8}$.)

South Staffordshire Water Co. v. Sharman

1896

In South Staffordshire Water Co. v. Sharman ${ }^{42}\left(C_{7}\right)$, Lord Russell of Killowen CJ and Wills J distinguished Bridges v. Hawkesworth and found against Sharman (the finder). ${ }^{43}$

SHYSTER agrees that Sharman should have lost. The nearest neighbour is City of London Corporation v. Appleyard (1); the nearest other is Hannah v. Peel. Both of these cases were decided after South Staffordshire v. Sharman.

Elwes v. Brigg Gas Co.

In Elwes v. Brigg Gas Co. $^{44}\left(C_{8}\right)$, Chitty J found against the finder.

SHYSTER agrees that Brigg Gas should have lost. The nearest neighbour is City of London Corporation v. Appleyard (2); the nearest other is Hannah $v$. Peel. Both of these cases were decided after Elwes v. Brigg Gas Co. 


\section{D.3 The Authorization specification}

The results of the reflexive testing of all cases in the Authorization specification are described here, and summarized in figure D.2.

\section{University of New South Wales v. Moorhouse}

In University of New South Wales v. Moorhouse ${ }^{45}\left(C_{1}\right)$, the High Court applied Falcon v. Famous Players Film Co. and held that the infringement was authorized. ${ }^{46}$

SHYSTER agrees that the University authorized the infringement. The nearest neighbour is Winstone $v$. Wurlitzer: a case of which Gibbs J approved; ${ }^{47}$ the nearest others are A\&MM Records Inc. v. Audio Magnetics Inc. (UK) Ltd (NotAuth), which was decided after UNSW v. Moorhouse, and Australasian Performing Right Association Ltd v. Miles (Liable).

However, SHYSTER warns that two of these cases are equidistant from UNSW $v$. Moorhouse; it chooses Winstone v. Wurlitzer because it is a decision of the Supreme Court of Victoria whereas A\&M v. Audio Magnetics is merely a decision of the Chancery Division of the English High Court. It also warns that the weighted correlation coefficients suggest that A\&BM v. Audio Magnetics should be the nearest neighbour, and that the specified directions suggest Liable.

\section{Australasian Performing Right Association Ltd $v$. Canterbury-Bankstown League Club Ltd}

Australasian Performing Right Association Ltd v. Canterbury-Bankstown League $C l u b \operatorname{Ltd}^{48}\left(C_{2}\right)$, appears in the specification as a case in which the accused authorized an infringement. However, in that case Asprey J cited Australasian Performing Right Association Ltd v. Miles and held that the club had performed the work, through the orchestra, and was directly liable for the infringement or, if that were not so, that the club had authorized the infringement. ${ }^{49}$ Ferguson J (with whom Herron CJ agreed) came to a similar conclusion. ${ }^{50}$

SHYSTER concludes that the Club is liable (directly or vicariously) for the infringement. The nearest neighbour is APRA $v$. Miles; the nearest others are $\mathrm{Mel}$ lor v. Australian Broadcasting Commission (Auth) and RCA Corporation v. John Fairfax and Sons Ltd (Not-Auth), which was decided after APRA v. CanterburyBankstown.

However, SHYSTER warns that two of these cases are equidistant from APRA $v$. Canterbury-Bankstown; it chooses APRA v. Miles because it is a decision of the Supreme Court of New South Wales whereas Mellor v. ABC is merely a decision of the Judicial Committee of the Privy Council. It also warns that the weighted correlation coefficients suggest that Mellor $v$. $A B C$ should be the nearest neighbour. Furthermore, two of the four instantiations have a nearest result of Auth. 


\section{Winstone v. Wurlitzer Automatic Phonograph Co. of Australia Pty Ltd}

In Winstone v. Wurlitzer Automatic Phonograph Co. of Australia Pty Ltd ${ }^{51}\left(C_{3}\right)$, Herring CJ applied Bankes LJ's test, from Falcon v. Famous Players Film Co., and decided that Wurlitzer had authorized the infringement. ${ }^{52}$

SHYSTER agrees, but all of its chosen cases were decided after Winstone $v$. Wurlitzer. The nearest neighbour is University of New South Wales v. Moorhouse; the nearest others are A\&MM Records Inc. v. Audio Magnetics Inc. (UK) Ltd (Not-Auth) and Australasian Performing Right Association Ltd v. Miles (Liable). SHYSTER warns that the specified directions suggest Liable.

\section{Mellor v. Australian Broadcasting Commission}

In Mellor v. Australian Broadcasting Commission ${ }^{53}\left(C_{4}\right)$, the Judicial Committee of the Privy Council made reference to no authorization cases in deciding that the $\mathrm{ABC}$ had authorized the infringement (although Mellor was held to have consented to that infringement).

SHYSTER also concludes that the $\mathrm{ABC}$ authorized the infringement. The nearest neighbours are Winstone v. Wurlitzer Automatic Phonograph Co. of Australia Pty Ltd and Australasian Performing Right Association Ltd v. CanterburyBankstown League Club Ltd; the nearest others are Performing Right Society Ltd v. Ciryl Theatrical Syndicate Ltd (Not-Auth) and Australasian Performing Right Association Ltd v. Miles (Liable). With the exception of PRS v. Ciryl, all of these cases were decided after Mellor $v$. ABC.

(With Mellor v. $A B C$ removed from the specification, there is an inverse functional dependence between $A_{2}$ and $A_{6}$.)

Falcon v. Famous Players Film Co.

In Falcon v. Famous Players Film $C_{0}{ }^{54}\left(C_{5}\right)$, a majority of the English Court of Appeal held that Famous Players had authorized the infringement; Bankes LJ made his oft-quoted statement that "authorize" should be "understood in its ordinary dictionary sense of 'sanction, approve, and countenance.'" 55

SHYSTER disagrees with the Court of Appeal and concludes that Famous Players did not authorize the infringement. It warns that the specified directions suggest Liable. The nearest neighbour is A $\mathscr{E} M$ Records Inc. v. Audio Magnetics Inc. (UK) Ltd; the nearest others are Winstone v. Wurlitzer Automatic Phonograph Co. of Australia Pty Ltd (Auth) and Australasian Performing Right Association Ltd $v$. Miles (Liable). All of these cases were decided after Falcon $v$. Famous Players. 
In $R C A$ Corporation v. John Fairfax and Sons $\operatorname{Ltd}^{56}\left(C_{6}\right)$, Kearney J referred to several cases, especially University of New South Wales v. Moorhouse and A\&M Records Inc. v. Audio Magnetics Inc. (UK) Ltd, before deciding that Fairfax had not authorized any infringement.

SHYSTER also concludes that Fairfax did not authorize the infringement, although it warns that the specified directions suggest Auth or Liable. The nearest neighbour is $A \& M v$. Audio Magnetics: one of the cases to which his honour referred. The nearest others are Falcon v. Famous Players Film Co. (Auth) and Australasian Performing Right Association Ltd v. Miles (Liable), neither of which was mentioned in the judgment.

\section{Performing Right Society Ltd $v$.} Ciryl Theatrical Syndicate Ltd

Performing Right Society Ltd v. Ciryl Theatrical Syndicate $\operatorname{Ltd}^{57}\left(C_{7}\right)$ is the oldest of the leading cases. The only authorization case cited in argument was Performing Right Society Ltd v. Bradford Corporation. As discussed in §5.3.2, PRS v. Bradford had an identical fact vector to Mellor v. Australian Broadcasting Commission, and was omitted from the Authorization specification.

SHYSTER comes to a different conclusion to that of the English Court of Appeal; it concludes that Ciryl authorized the infringement. The nearest neighbour is Mellor $v$. ABC which (being identical to the omitted PRS v. Bradford) is a good choice. The nearest others are RCA Corporation v. John Fairfax and Sons Ltd (Not-Auth) and Australasian Performing Right Association Ltd v. Miles (Liable). Of course, all of these cases were decided after PRS v. Ciryl.

However, SHYSTER warns that the weighted correlation coefficients suggest that six other cases should be the nearest neighbour-including RCA v. Fairfax and another case in which the accused did not authorize the infringement, and APRA $v$. Miles. It also warns that the specified directions suggest Not-Auth.

(With PRS $v$. Ciryl removed from the specification, there is an inverse functional dependence between $A_{5}$ and $A_{6}$. Further, both of those attributes have infinite weight-instead of just $A_{5}$, as before.)

A $G M$ Records Inc. v. Audio Magnetics Inc. (UK) Ltd

In A\&M Records Inc. v. Audio Magnetics Inc. (UK) $L t d^{58}\left(C_{8}\right)$, Foster J applied Bankes LJ's definition of "authorization" from Falcon v. Famous Players Film Co. and held that Audio Magnetics had not authorized any specific infringement. ${ }^{59}$ 


\begin{tabular}{|l|c|c|c|cc|}
\hline \multicolumn{1}{|c|}{ Case } & Result & $\begin{array}{c}\text { Nearest } \\
n^{\prime} \text { 'bours }\end{array}$ & $\begin{array}{c}\text { Nearest } \\
\text { others }\end{array}$ & Warnings \\
\hline \hline UNSW v. Moorhouse & $\checkmark$ & $\checkmark$ & - & $r^{\prime} \Rightarrow \equiv$ & $\times$ \\
APRA v. Canterbury-Bankstown & $\checkmark$ & $\checkmark$ & - & $r^{\prime} \equiv \forall \frac{2}{4}$ & $\times$ \\
Winstone v. Wurlitzer & $\checkmark$ & - & - & $\Rightarrow$ & $\times$ \\
Mellor v. ABC & $\checkmark$ & - & - & & $\checkmark$ \\
Falcon v. Famous Players & $\times$ & - & - & & $\times$ \\
RCA v. Fairfax & $\checkmark$ & $\checkmark$ & $\times$ & $\Rightarrow$ & $\times$ \\
PRS v. Ciryl & $\times$ & $\checkmark$ & - & $r^{\prime} \Rightarrow$ & $\checkmark$ \\
A\&M v. Audio Magnetics & $\checkmark$ & - & $\times$ & $r^{\prime} \Rightarrow \equiv$ & $\times$ \\
APRA v. Miles & & $\checkmark$ & - & & \\
\hline
\end{tabular}

Figure D.2: A summary of the reflexive testing of the Authorization specification, described in $\S$ D.3. The meaning of the symbols used here is explained in $\S 5.6 .1$.

SHYSTER agrees with Foster J that Audio Magnetics did not authorize the infringement. The nearest neighbour is RCA Corporation v. John Fairfax and Sons Ltd, which was decided after A\&M $v$. Audio Magnetics; the nearest others are Falcon v. Famous Players (Auth) and Australasian Performing Right Association Ltd v. Miles (Liable).

However, SHYSTER warns that two of these cases are equidistant from $A \& B M v$. Audio Magnetics; it chooses RCA v. Fairfax because it is a decision of the Supreme Court of New South Wales whereas Falcon v. Famous Players is merely a decision of the English Court of Appeal. It also warns that the weighted correlation coefficients suggest that Falcon v. Famous Players should be the nearest neighbour, and that the specified directions suggest Auth.

In Australasian Performing Right Association Ltd $v$. Miles ${ }^{60}\left(C_{9}\right)$, Jacobs J followed Performing Right Society Ltd v. Mitchell $\&$ Booker (Palais de Danse) Ltdnot a leading case in this specification, though it does appear in the EMPLOYEe specification - and held the members of the Dee Why RSL Club directly liable for the infringing acts of the orchestra.

SHYSTER concludes that Miles authorized the infringement. This is not surprising. With APRA v. Miles removed from the specification, there is no case in which the result was Liable-and SHYSTER warns that this is so. Further, SHYSTER warns that the ideal point for Liable is at least as near to the instant case as is the nearest neighbour. 
The nearest neighbours are Winstone v. Wurlitzer Automatic Phonograph Co. of Australia Pty Ltd and Australasian Performing Right Association Ltd $v$. Canterbury-Bankstown League Club Ltd; the nearest other is RCA Corporation v. John Fairfax and Sons Ltd (Not-Auth). Apart from Winstone v. Wurlitzer, these were decided after APRA $v$. Miles.

The choice of APRA $v$. Canterbury-Bankstown is a good one, despite the fact that its result is Auth. As discussed above, the judges in that case held that the club was either directly liable for, or had authorized, the infringement.

(Without APRA v. Miles there is an equivalent functional dependence between $A_{1}$ and $A_{5}$, and each of those attributes - not just $A_{5}$ - has infinite weight.)

\section{D.4 The EMPlOYEe specification}

The results of the reflexive testing of all cases in the EMPLOYEE specification are described here, and summarized in figure D.3.

\section{Zuijs v. Wirth Brothers Pty Ltd}

In Zuijs v. Wirth Brothers Pty $\operatorname{Ltd}^{61}\left(C_{1}\right)$, Zuijs cited several cases, notably Performing Right Society Ltd v. Mitchell 85 Booker (Palais de Danse) Ltd and Stevenson Jordon and Harrison Ltd v. Macdonald and Evans. In response, Wirth Brothers relied on Humberstone v. Northern Timber Mills. The Full Court of the High Court found for Zuijs: he was held to be an employee of Wirth Brothers.

SHYSTER disagrees. The nearest neighbours are Price v. Grant Industries Pty Ltd and Stevenson Jordon and Harrison Ltd v. Macdonald and Evans (1); the nearest other is Ferguson v. John Dawson 85 Partners (Contractors) Ltd. Apart from Stevenson v. Macdonald, these cases were decided after Zuijs v. Wirth Brothers.

However, SHYSTER issues four safeguard warnings. It warns that the weighted association coefficients and the weighted correlation coefficients suggest that Stevenson v. Macdonald (2) - in which the result was Employee - should be the nearest neighbour. It also warns that the ideal point and the centroid for Employee are both at least as near to the instant case as is the nearest neighbour, and that the specified directions suggest Employee.

\section{Cam and Sons Pty Ltd v. Sargent}

In Cam and Sons Pty Ltd v. Sargent ${ }^{62}\left(C_{2}\right)$, four judges of the High Court agreed with Sargent who claimed that he was an employee of Cam.

SHYSTER concludes, instead, that Cam was an independent contractor. The nearest neighbour is Humberstone $v$. Northern Timber Mills; the nearest others are Australian Timber Workers Union v. Monaro Sawmills Pty Ltd and Ferguson v. John Dawson $\&$ Partners (Contractors) Ltd. All three cases were decided after Cam v. Sargent. 
SHYSTER warns that the weighted correlation coefficients suggest that Stevenson Jordon and Harrison Ltd v. Macdonald and Evans (2) - in which the result was Employee - should be the nearest neighbour.

\section{Federal Commissioner of Taxation $v$.}

\section{J. Walter Thompson (Australia) Pty Ltd}

In Federal Commissioner of Taxation v. J. Walter Thompson (Australia) Pty $L t d^{63}\left(C_{3}\right)$, Latham CJ of the High Court held that the radio artists were employees of J. Walter Thompson, and hence their fees were subject to payroll tax.

SHYSTER agrees. The nearest neighbour is Ferguson v. John Dawson $\&$ Partners (Contractors) Ltd; the nearest other is Massey v. Crown Life Insurance Co., both of which were decided after FCT $v$. Thompson. In both instantiations, the result is the same as in the instant case.

SHYSTER warns that the ideal point for Contractor is at least as near to the instant case as is the nearest neighbour, and that the specified directions suggest Contractor.

(With FCT $v$. Thompson removed from the specification, there is an equivalent functional dependence between $A_{11}$ and $A_{12}$. Further, $A_{13}$ has infinite weight.)

\section{Australian Timber Workers Union v. Monaro Sawmills Pty Ltd}

In Australian Timber Workers Union v. Monaro Sawmills Pty Ltd ${ }^{64}\left(C_{4}\right)$, Sweeney and Evatt JJ of the Federal Court applied the "organization test" as proposed by Denning LJ in Stevenson Jordon and Harrison Ltd v. Macdonald and Evans and Bank Voor Handel en Scheepvaart NV v. Slatford. ${ }^{65}$

SHYSTER agrees with their honours and concludes that Wales (the worker) was an employee. However its chosen cases were not cited in the judgment: the nearest neighbour is Cam and Sons Pty Ltd $v$. Sargent; the nearest other is Humberstone $v$. Northern Timber Mills. SHYSTER reaches the same conclusion in both instantiations.

SHYSTER warns that the centroid for Contractor is at least as near to the instant case as is the nearest neighbour.

Ferguson v. John Dawson E Partners (Contractors) Ltd

Megaw and Brown JJ in Ferguson v. John Dawson \& Partners (Contractors) $L_{t d}{ }^{66}\left(C_{5}\right)$ held (Lawton LJ dissenting) that despite the label that the parties put on their relationship, it was in fact one of employer and employee. They based their decision on Ready Mixed Concrete (South East) Ltd v. Minister of Pensions and National Insurance and Market Investigations Ltd $v$. Minister of Social Security ${ }^{67}$ 
SHYSTER agrees that Ferguson was an employee. The nearest neighbours are Zuijs v. Wirth Brothers Pty Ltd and Australian Timber Workers Union v. Monaro Sawmills Pty Ltd; the nearest other is Massey v. Crown Life Insurance Co. Apart from Zuijs v. Wirth Brothers, these cases were decided after Ferguson v. Dawson.

\section{Stevenson Jordon and Harrison Ltd $v$. Macdonald and Evans (2)}

As discussed in §5.4.2, Stevenson Jordon and Harrison Ltd v. Macdonald and Evans appears in the specification twice.

In Stevenson v. Macdonald (2) ${ }^{68}\left(C_{6}\right)$, the Court of Appeal held that EvansHemming was an employee of Macdonald and Evans when he wrote the second section of his book. Scant reference was made to previously decided cases.

SHYSTER agrees with the Court that Evans-Hemming was an employee. The nearest neighbour is Zuijs v. Wirth Brothers Pty Ltd, which was decided after Stevenson v. Macdonald. The nearest other is Stevenson v. Macdonald (1). In 120 of the 128 instantiations, SHYSTER reaches the same conclusion.

\section{Performing Right Society Ltd $v$. Mitchell \& Booker (Palais de Danse) Ltd}

Performing Right Society Ltd v. Mitchell \& Booker (Palais de Danse) Ltd ${ }^{69}\left(C_{7}\right)$ is the oldest of the cases in the Employee specification. McCardie J applied the control test and held that the band members were employees of Palais de Danse.

SHYSTER agrees. The nearest neighbours are Australian Timber Workers Union v. Monaro Sawmills Pty Ltd are Zuijs v. Wirth Brothers Pty Ltd; the nearest others are Stevenson Jordon and Harrison Ltd v. Macdonald and Evans (1) and Ready Mixed Concrete (South East) Ltd $v$. Minister of Pensions and National Insurance. All, of course, were decided after PRS v. Palais de Danse.

(Without PRS v. Palais de Danse, $A_{14}$ has infinite weight.)

Humberstone v. Northern Timber Mills

In Humberstone $v$. Northern Timber Mills ${ }^{70}\left(C_{8}\right)$, three High Court judges followed Queensland Stations Pty Ltd v. Federal Commissioner of Taxation and applied the control test. ${ }^{71}$ They concluded that Humberstone was not an employee of NTM.

SHYSTER agrees that Humberstone was an independent contractor. The nearest neighbour is Price v. Grant Industries Pty Ltd, which was decided after Humberstone v. NTM; the nearest other is Cam and Sons Pty Ltd v. Sargent.

(With Humberstone $v$. NTM removed from the specification, there is an inverse functional dependence between $A_{3}$ and $A_{17}$.) 


\section{Queensland Stations Pty Ltd v. Federal Commissioner of Taxation}

In Queensland Stations Pty Ltd v. Federal Commissioner of Taxation ${ }^{72}\left(C_{9}\right)$, three judges of the High Court based their arguments on Logan v. Gilchrist, Watt and Cunningham, ${ }^{73}$ another droving case. Latham CJ and Dixon J followed that case, and held that the drovers were independent contractors.

SHYSTER agrees with their honours' decision. The nearest neighbours are Humberstone v. Northern Timber Mills and Stevenson Jordon and Harrison Ltd v. Macdonald and Evans (1), both of which were decided after Queensland Stations v. FCT; the nearest other is Cam and Sons Pty Ltd v. Sargent.

(With Queensland Stations v. FCT removed from the specification, there is an inverse functional dependence between $A_{7}$ and $A_{9}$.)

Price v. Grant Industries Pty Ltd

In Price v. Grant Industries Pty $L_{t d}^{74}\left(C_{10}\right)$, Smithers, Evatt and Keely JJ of the Federal Court followed Humberstone v. Northern Timber Mills and held that Price was an independent contractor.

SHYSTER agrees. The nearest neighbour is Humberstone $v$. NTM; the nearest other is Cam and Sons Pty Ltd v. Sargent. However, SHYSTER warns that the weighted correlation coefficients suggest that Zuijs v. Wirth Brothers Pty Ltd-in which the result was Employee - should be the nearest neighbour.

\section{Australian Mutual Provident Society v. Chaplin}

In Australian Mutual Provident Society v. Chaplin ${ }^{75}\left(C_{11}\right)$, the Judicial Committee of the Privy Council held that Chaplin's power of unlimited delegation was almost conclusive against Chaplin being an employee. ${ }^{76}$

SHYSTER agrees that Chaplin was an independent contractor. The nearest neighbour is Ready Mixed Concrete (South East) Ltd v. Minister of Pensions and National Insurance; the nearest other is Cam and Sons Pty Ltd v. Sargent. The Privy Council mentioned neither of these cases.

Massey v. Crown Life Insurance Co.

In Massey v. Crown Life Insurance Co. ${ }^{77}\left(C_{12}\right)$, the English Court of Appeal unanimously distinguished Ferguson v. John Dawson \& Partners (Contractors) Ltd, ${ }^{78}$ and Lord Denning MR referred to Stevenson Jordon and Harrison Ltd $v$. Macdonald and Evans, before holding that Massey was an independent contractor.

SHYSTER disagrees, however its choice of cases is good. It chooses both Ferguson v. Dawson and Stevenson v. Macdonald (1): the former is the nearest neighbour, which the latter should be. Australian Mutual Provident Society $v$. 


\begin{tabular}{|l|c|c|c|cc|}
\hline \multicolumn{1}{|c|}{ Case } & Result & $\begin{array}{c}\text { Nearest } \\
n^{\prime} \text { bours }\end{array}$ & $\begin{array}{c}\text { Nearest } \\
\text { others }\end{array}$ & Warnings \\
\hline \hline Zuijs v. Wirth Brothers & $\times$ & - & - & $S^{\prime} r^{\prime} I \mu$ & $\checkmark$ \\
Cam v. Sargent & $\times$ & - & - & $r^{\prime}$ & $\checkmark$ \\
FCT v. Thompson & $\checkmark$ & - & - & $I \Rightarrow \forall \frac{0}{2}$ & $\times$ \\
ATWU v. Monaro Sawmills & $\checkmark$ & $\times$ & $\times$ & $\mu \forall \frac{0}{2}$ & $\times$ \\
Ferguson v. Dawson & $\checkmark$ & - & - & & $\checkmark$ \\
Stevenson v. Macdonald (2) & $\checkmark$ & - & & $\forall \frac{8}{128}$ & $\times$ \\
PRS v. Palais de Danse & $\checkmark$ & - & - & & $\checkmark$ \\
Humberstone v. NTM & $\checkmark$ & - & $\times$ & & $\checkmark$ \\
Queensland Stations v. FCT & $\checkmark$ & - & $\times$ & & $\checkmark$ \\
Price v. Grant Industries & $\checkmark$ & $\checkmark$ & $\times$ & $r^{\prime}$ & $\times$ \\
AMP v. Chaplin & $\checkmark$ & $\times$ & $\times$ & & $\checkmark$ \\
Massey v. Crown Life & $\times$ & $\checkmark$ & $\checkmark$ & $\Rightarrow$ & $\checkmark$ \\
Stevenson v. Macdonald (1) & $\times$ & & $\times$ & $r^{\prime} \forall \frac{48}{64}$ & $\checkmark$ \\
Ready Mixed v. Minister & $\checkmark$ & - & $\times$ & & $\checkmark$ \\
\hline
\end{tabular}

Figure D.3: A summary of the reflexive testing of the EMPLOYEe specification, described in $\S$ D.4. The meaning of the symbols used here is explained in $\S 5.6 .1$.

Chaplin, which was decided in the same year as was Massey $v$. Crown Life, is also chosen as a nearest other. SHYSTER warns that the specified directions suggest Contractor.

(With Massey v. Crown Life removed from the specification, there is an inverse functional dependence between $A_{9}$ and $A_{11}$, and $A_{16}$ has infinite weight.)

\section{Stevenson Jordon and Harrison Ltd $v$. Macdonald and Evans (1)}

As discussed in $§ 5.4 .2$, Stevenson Jordon and Harrison Ltd v. Macdonald and Evans appears in the specification twice.

In Stevenson v. Macdonald $(1)^{79}\left(C_{13}\right)$, the Court of Appeal held that EvansHemming was not an employee of Macdonald and Evans when he wrote the first section of his book. The court made very few references to previously decided cases in making its decision.

SHYSTER disagrees with the Court. The nearest neighbour is Stevenson Jordon and Harrison Ltd v. Macdonald and Evans (2) - an unsurprising choice. The nearest other is Queensland Stations Pty Ltd v. Federal Commissioner of Taxation. 
However, SHYSTER warns that the weighted correlation coefficients suggest that Queensland Stations v. FCT should be the nearest neighbour. And in 48 of the 64 instantiations, the nearest result is Contractor.

\section{Ready Mixed Concrete (South East) Ltd v. Minister of Pensions and National Insurance}

In Ready Mixed Concrete (South East) Ltd v. Minister of Pensions and National Insurance ${ }^{80}\left(C_{14}\right)$, held that the contract between Latimer and Ready Mixed was a contract of carriage and not a contract of service. ${ }^{81}$

SHYSTER agrees that Latimer was an independent contractor. The nearest neighbour is Australian Mutual Provident Society v. Chaplin, which was decided after Ready Mixed v. Minister; the nearest other is Cam and Sons Pty Ltd $v$. Sargent, which was not referred to in argument or in MacKenna J's judgment.

\section{D.5 The Natural specification}

Reflexive testing of the NATURAL specification was limited to those cases in the Natural area - the principal area in that specification. The results of those reflexive tests are described here, and summarized in figure D.4.

FAI Insurances Ltd v. Winneke

In FAI Insurances Ltd v. Winneke ${ }^{82}\left(C_{1}\right)$, Gibbs CJ, Stephen, Mason, Aickin, Wilson and Brennan JJ (Murphy J dissenting) held that FAI had a legitimate expectation that its approval would be renewed. Hence the Governor in Council was required to apply principles of natural justice before deciding not to renew that approval.

SHYSTER agrees that a duty to observe natural justice is implied. The nearest neighbour is Haoucher v. Minister of State for Immigration and Ethnic Affairs, which was decided after FAI v. Winneke; the nearest other is Nashua Australia Pty Ltd v. Channon, which was not referred to by any of the judges.

\section{Haoucher $v$. Minister of State for} Immigration and Ethnic Affairs

In Haoucher $v$. Minister of State for Immigration and Ethnic Affairs ${ }^{83}\left(C_{2}\right)$, the majority of the High Court ${ }^{84}$ applied several cases, including FAI Insurances Ltd v. Winneke, ${ }^{85}$ in concluding that Haoucher had been entitled to make representations to the Minister before the Minister ordered his deportation.

SHYSTER agrees that a duty to observe natural justice is implied. The nearest neighbour is FAI $v$. Winneke; the nearest other is Nashua Australia Pty Ltd $v$. Channon, to which no reference was made. 
In Annetts v. McCann ${ }^{86}\left(C_{3}\right)$, five judges of the High Court unanimously held that the coroner ought to have accorded natural justice to the parents of the boy who was the subject of the inquest. ${ }^{87}$

SHYSTER disagrees, and concludes that a duty to observe natural justice is not implied. The nearest neighbour is McInnes $v$. Onslow Fane to which no reference was made; the nearest other is Kioa $v$. West, to which all but one of their honours referred in their judgments. ${ }^{88}$

SHYSTER warns that the ideal point for Implied is at least as near to the instant case as is the nearest neighbour, and that the specified directions suggest Implied.

In Kioa v. West ${ }^{89}\left(C_{4}\right)$, Mason, Wilson, Brennan and Deane JJ (Gibbs CJ dissenting) held that natural justice ought to have been observed in the making of the deportation order.

SHYSTER agrees that a duty to observe natural justice is implied. The nearest neighbours are FAI Insurances Ltd $v$. Winneke, Haoucher $v$. Minister of State for Immigration and Ethnic Affairs and Attorney-General of Hong Kong v. Ng Yuen Shiu; the nearest other is South Australia v. O'Shea. Gibbs CJ, Mason and Brennan JJ made reference to FAI v. Winneke and AG of Hong Kong v. Shiu. ${ }^{90}$ The other two of SHYSTER's chosen cases were decided after Kioa v. West.

Commissioner of Police v. Tanos

Commissioner of Police v. Tanos ${ }^{91}\left(C_{5}\right)$ is the oldest of all the natural justice cases. Dixon CJ, Webb and Taylor JJ held that Tanos ought to have been accorded natural justice before her restaurant was declared a "disorderly house."

SHYSTER agrees that a duty to observe natural justice is implied. The nearest neighbour is Marine Hull $\&$ Liability Insurance Co. Ltd v. Hurford; the nearest other is McInnes $v$. Onslow Fane. Of course, both of these cases were decided after Commissioner of Police v. Tanos.

Marine Hull \& Liability Insurance Co. Ltd v. Hurford

In Marine Hull \& Liability Insurance Co. Ltd v. Hurford ${ }^{92}\left(C_{6}\right)$, Fox, Davies and Morling JJ held that natural justice was implied, but that Marine Hull had not been denied it in this case.

SHYSTER also concludes that a duty to observe natural justice is implied. The nearest neighbour is Commissioner of Police v. Tanos, to which Morling J referred $;^{93}$ the nearest other is South Australia $v$. O'Shea, which was decided after Marine Hull v. Hurford. 
In Macrae v. Attorney-General for New South Wales $^{94}\left(C_{7}\right)$, Kirby P, Mahoney and Priestly JJA held that the magistrates were denied their legitimate expectation of procedural fairness. The legal expert advises that this was a somewhat surprising outcome.

SHYSTER disagrees with their honours. It concludes that a duty to observe natural justice is not implied. The nearest neighbour is South Australia v. O'Shea which was decided in the same year as was Macrae $v$. AG for NSW; the nearest other is Durayappah v. Fernando to which no reference was made.

SHYSTER warns that both the weighted coefficients prefer Attorney-General of Hong Kong v. $\mathrm{Ng}$ Yuen Shiu (in which natural justice was implied) as the nearest neighbour. It also warns that the specified directions suggest Implied.

\section{Attorney-General of Hong Kong v. Ng Yuen Shiu}

1983

In Attorney-General of Hong Kong v. $N g$ Yuen Shiu ${ }^{95}\left(C_{8}\right)$, the Judicial Committee of the Privy Council held that Shiu ought to have been given an opportunity to make a representation to the Minister.

SHYSTER disagrees. The nearest neighbour is South Australia v. O'Shea; the nearest others are Kioa v. West and Macrae v. Attorney-General for New South Wales.

However, SHYSTER warns that all three chosen cases are equidistant from $A G$ of Hong Kong v. Shiu; SA v. O'Shea and Kioa v. West are both decisions of five judges of the High Court, but SHYSTER prefers the former because it is two years more recent. (Macrae v. AG for NSW is merely a decision of the New South Wales Court of Appeal.)

SHYSTER also warns that both the weighted coefficients prefer SA $v$. O'Shea and Kioa v. West as the nearest neighbours, that the centroid for Implied is at least as near to the instant case as is the nearest neighbour, and that the specified directions suggest Implied. In one of the two instantiations, the result was Implied.

Kioa $v$. West was not referred to in any of the judgments, or in argument, and the other two chosen cases were decided after AG of Hong Kong v. Shiu.

Durayappah v. Fernando

In Durayappah v. Fernando ${ }^{96}\left(C_{9}\right)$, the Judicial Committee of the Privy Council held that the Minister ought to have applied natural justice principles before dissolving the Jaffna Municipal Council.

Unlike the Privy Council, SHYSTER concludes that a duty to observe natural justice is not implied. The nearest neighbour is Nashua Australia Pty Ltd $v$. Channon; the nearest others are FAI Insurances Ltd v. Winneke and Haoucher $v$. Minister of State for Immigration and Ethnic Affairs. All of these cases were decided after Durayappah v. Fernando.

SHYSTER warns that the specified directions suggest Implied. 
In South Australia v. O'Shea ${ }^{97}\left(C_{10}\right)$, Mason CJ, Wilson, Brennan and Toohey JJ (Deane J dissenting) held that O'Shea was not entitled to a further hearing.

SHYSTER disagrees: it concludes that a duty to observe natural justice is implied. The nearest neighbour is Macrae v. Attorney-General for New South Wales, which was decided in the same year as was $S A v$. O'Shea; the nearest other is Nashua Australia Pty Ltd $v$. Channon, to which no reference was made.

Bread Manufacturers of New South Wales v. Evans

In Bread Manufacturers of New South Wales v. Evans ${ }^{98}\left(C_{11}\right)$, Gibbs CJ, Mason, Murphy, Aickin and Wilson JJ held that the Prices Commission was not obliged to apply natural justice principles.

SHYSTER agrees. The nearest neighbours are Minister for Arts Heritage and Environment v. Peko-Wallsend Ltd and Nashua Australia Pty Ltd v. Channon, which were decided after Bread Manufacturers $v$. Evans; the nearest other is Durayappah $v$. Fernando to which none of their honours referred.

However, SHYSTER warns that all three chosen cases are equidistant from Bread Manufacturers v. Evans; SHYSTER prefers both Minister for Environment v. Peko-Wallsend and Nashua v. Channon (decisions of the Full Court of the Federal Court and the New South Wales Supreme Court, respectively) to Durayappah v. Fernando (a Privy Council decision).

SHYSTER warns that the weighted association coefficients prefer Durayappah v. Fernando as the nearest neighbour, and that the weighted correlation coefficients prefer Annetts v. McCann (in which natural justice was implied). It also warns that the specified directions suggest Implied.

\section{Minister for Arts Heritage and Environment $v$.} Peko-Wallsend Ltd

In Minister for Arts Heritage and Environment v. Peko-Wallsend Ltd ${ }^{99}\left(C_{12}\right)$, Wilcox J (with whom Bowen CJ and Sheppard J generally agreed) held that Federal Cabinet was not obliged to apply natural justice principles.

SHYSTER agrees that a duty to observe natural justice is not implied. The nearest neighbour is Bread Manufacturers of New South Wales v. Evans; the nearest other is Durayappah v. Fernando. Neither case was mentioned in the Federal Court's judgment.

However, SHYSTER warns that the weighted correlation coefficients suggest that twelve other cases should be the nearest neighbour - in eight of which, natural justice was implied. 
In Nashua Australia Pty Ltd v. Channon ${ }^{1}\left(C_{13}\right)$, Lee J held that the Minister was not required to afford Nashua natural justice before making his determination.

SHYSTER disagrees. The nearest neighbour is Durayappah v. Fernando, which Lee J applied; ${ }^{2}$ the nearest other is South Australia $v$. O'Shea, which was decided after Nashua v. Channon.

However, it warns that the centroid for Not-Implied is at least as near to the instant case as is the nearest neighbour.

\section{Council of Civil Service Unions $v$. Minister for the Civil Service}

In Council of Civil Service Unions v. Minister for the Civil Service ${ }^{3}\left(C_{14}\right)$, the House of Lords held that the Minister was not obliged to accord the unions natural justice.

SHYSTER agrees. The nearest neighbour is Nashua Australia Pty Ltd v. Channon; the nearest other is Durayappah v. Fernando. Neither case was mentioned in the law lords' judgment.

However, SHYSTER warns that these two cases are equidistant from CCSU v. Minister for the Civil Service; it chooses Nashua v. Channon because it is a decision of the Supreme Court of New South Wales, while Durayappah v. Fernando is a Privy Council decision. It also warns that the specified directions suggest Implied.

In McInnes v. Onslow Fane ${ }^{4}\left(C_{15}\right)$, Megarry V-C held that the British Boxing Board of Control had no duty to afford McInnes natural justice.

SHYSTER comes to the opposite conclusion. The nearest neighbour is $A n$ netts v. McCann; the nearest other is South Australia v. O'Shea. Both cases were decided after McInnes v. Onslow Fane. 
$\S D .5$ The NATURAL specification

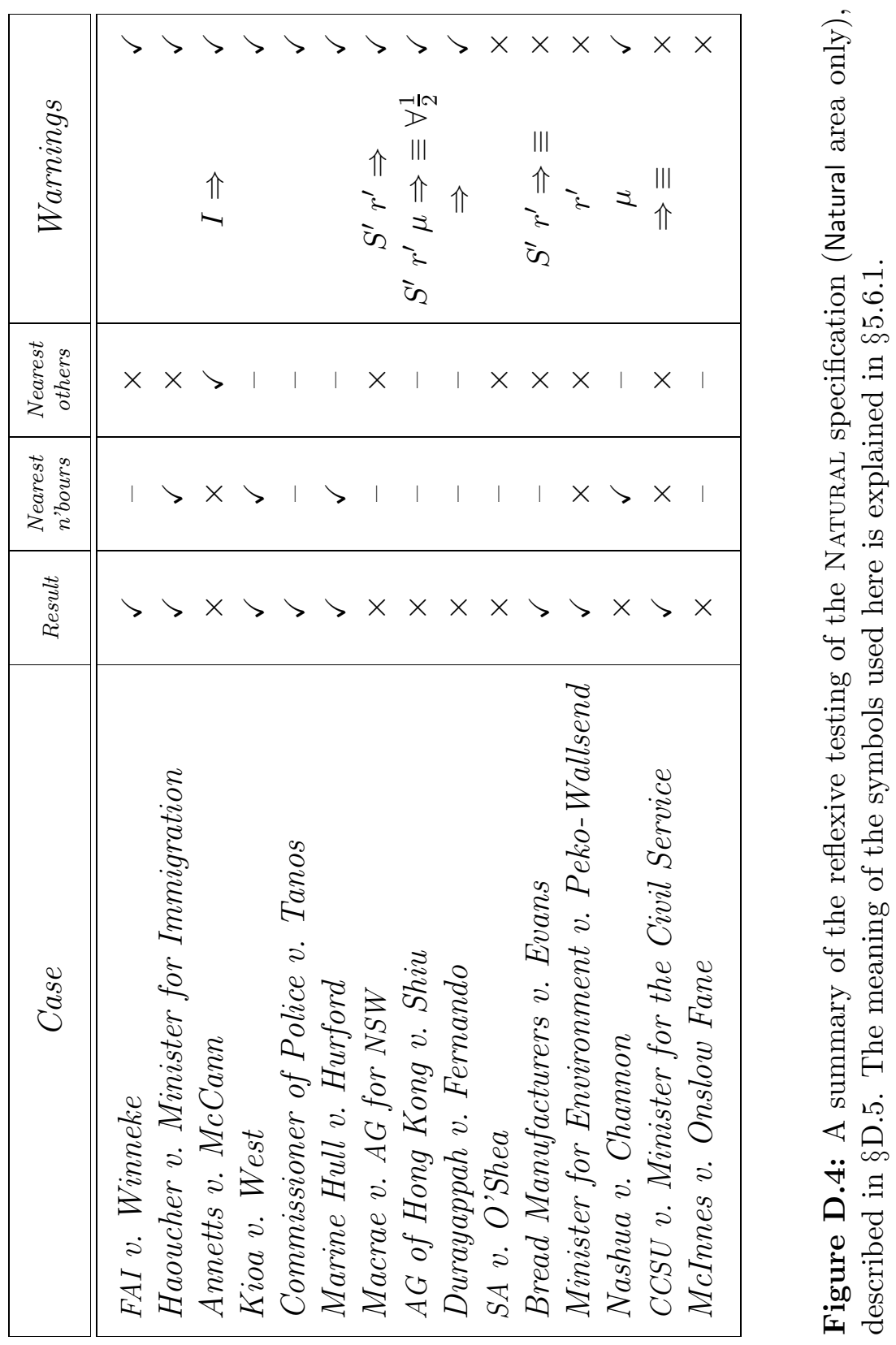





\section{Notes \\ Cases Statutes Bibliography}





\section{Notes}

1. at 2110 .

2. Second edition, vol. XV at 403.

3. Rawson 1991 at $355-6$.

\section{Chapter 1: Introduction}

4. Wells and Taylor 1986, 4.2.78/2238. Original title: The First Part of the Contention of the Two Famous Houses of Yorke and Lancaster. Dated by Wells and Taylor 1987 at 111.

5. Quoted by Muir 1976 at 81 . Muir does not date this quotation but, according to Twiss 1844 at 16, Lord Eldon finished his anecdote book in 1827.

6. Plater 1985 at 57.

\section{Chapter 2: Legal analysis systems}

7. Leith 1986 b at 97 .

8. Moles 1987 at 271.

9. Montesquieu 1973, liv. VI, ch. II at 84, footnote omitted. Translation by Peter Brown, Lecturer, Department of Modern European Languages, Faculty of Arts, The Australian National University.

10. McCarty 1980a identifies a third category of legal AI systems: "integrated legal systems." He cites, as an example, computerized title registration systems which make decisions about people's rights and obligations (at 2). It is hard to see why such a system would not be better classified as a legal analysis system, albeit with some of the features of a legal retrieval system.

11. On legal retrieval systems see Tapper 1963, 1984; Bing and Selmer 1980, chs 8-15; Bing 1984a, 1984b, 1987, 1989; Croydon 1980; Dick 1991. For an example of early work in the field see Allen, Brooks and James 1962. Sprowl 1979 describes a doc- 
ument preparation system (see also Cook, Hafner, McCarty, Meldman, Peterson, Sprowl, Sridharan and Waterman 1981). For a detailed summary of Australian legal retrieval systems (CLIRS, SCALE, etc.) see Greenleaf, Mowbray and Lewis 1988.

12. Mehl 1959 at 759 .

13. Shannon and Golshani 1988 at 306.

14. Susskind 1987 a at 3.

15. This is, of course, a variation on the famous test for machine intelligence proposed by Turing 1950 .

16. For example, HYPO (§2.5.3) and the Latent Damage System (see §2.4.4). Apropos SHYSTER and legal expertise, see $\S 3.4$.

17. The doctrine of parliamentary supremacy over the courts developed in England in the seventeenth century: see Morris, Cook, Creyke and Geddes 1992 at 8.

18. Morris et al. 1992 at 31.

19. ibid. at 40 .

20. Cross 1961 at 4 .

21. See Stone 1964a, 1985; Harris 1980; Krygier 1986. See also the discussion in $\S 2.2 .5$.

22. Stone 1964 a at 325 .

23. In Queensland v. Commonwealth of Australia (1977) 139 CLR 585, Gibbs J (at 601) and Stephen J (at 604) of the High Court of Australia felt bound by the principle of stare decisis to follow Western Australia v. Commonwealth of Australia (1975) 134 CLR 201 - a decision in which they had been in the minority - despite still believing that that case had been wrongly decided.

24. Bain's JUDGE system, although strictly speaking a rule-based system, adopts an approach which is similar to that of a case-based system. It analyzes the similarities and differences between cases to give advice about sentencing for certain crimes including murder, assault and manslaughter. JUDGE starts with a rule about a crime (provided by the user) and an appropriate sentence taken from a case. When a new case is added, JUDGE finds similar crimes and generates a rule about the new case on the basis of the similarities and differences between the new case and those similar crimes.

25. Thomas 1979; Campbell 1984; Boden 1985; Willick 1985; Clarke 1988; Capper and Susskind 1988 at $101-38$.

26. Susskind 1987a at 19.

27. Niblett 1981 at 3.

28. Susskind 1987 a at 20 , emphasis in the original.

29. In 1987, only four of the twelve law schools in Australia had compulsory courses in jurisprudence: Pearce, Campbell and Harding 1987 at 106-7.

30. Susskind 1987 a at 26.

31. Harris 1980 at 2.

32. Niblett 1988 at 33.

33. Susskind 1987a at 20, as quoted above.

34. The Oxford English Dictionary 1989, second edition, vol. VIII at 321.

35. Pound 1908 at 605.

36. Loevinger 1949 at 472, citing Frank 1930.

37. Pound 1908 at 607. 
38. ibid. at 605 .

39. Loevinger 1949 at 483, footnote omitted. See also Loevinger 1963.

40. Loevinger 1963 at 35 .

41. Lasswell 1955 at 398. As Gardner 1987 comments, "[w] hy one robot was not enough was not discussed; the idea may have been that hard cases would be decided by a vote of the random number generators the robots were to incorporate" (at 81-2).

42. Lasswell 1955 at 398.

43. Frank 1949 at 207.

44. Mehl 1959 at 758.

45. D'Amato 1977 at 1280.

46. ibid. at $1290-1$.

47. Weizenbaum 1976 at 226-7.

48. D'Amato 1977 at 1281.

49. Stone $1964 \mathrm{~b}$ at 555 .

50. This increased level of certainty would be a result of freely available (automated) judicial advisory opinions.

51. D'Amato 1977 at 1278.

52. ibid. at 1279 .

53. ibid. at 1290 .

54. Kayton 1964 at 302.

55. Schubert 1968 at 63, emphasis omitted.

56. Tyree 1980.

57. Pound 1908 at 606.

58. Stone 1964b at 554. See also Spengler 1963: an earlier cautionary article about judgment machines.

59. Stone 1964 b at 558, emphasis omitted.

60 . ibid. at 559 .

61. ibid. at 560 .

62. Hart 1983 at 106 .

63. Hart 1961 at 132 . As discussed in $§ 2.2 .6$, a true rule sceptic would agree with Hart that the rules embodied in cases are "as determinate as any statutory rule" - that is, not at all.

64. Susskind 1986 at $189-90$.

65. Hart 1983 at 106.

66. Section 13(2) of the Domestic Violence and Matrimonial Proceedings Act 1976 (UK). See Moles 1987 at $142-57$.

67. [1978] 1 All ER 821.

68. Cantliff v. Jenkins [1978] 1 All ER 836.

69. Davis v. Johnson [1978] 1 All ER 841.

70. Moles 1987 at 155, footnotes added.

Compare Moles's analysis with that of MacCormick 1978. In order to demonstrate the possibility of purely deductive legal justification he provides a detailed analysis of the judgment of Lewis $\mathrm{J}$ in Daniels and Daniels v. R. White and Sons and Tarbard [1938] 4 All ER 258. He argues that that judgment is an example of deductive reasoning - a series of applications of modus ponens: $p \supset q ; p ; \therefore q$.

71. Moles 1987 at 172. 
72. id.

73. Leith's system was called ELI (see also $§ 3.13 .1$ ).

74. Leith 1985 b at 9 . See also Leith 1985a, 1986a.

75. See Llewellyn 1931, 1951, 1962. See also Holmes 1897.

76. Drahos and Parker 1992 at 110, referring to Llewellyn 1931 at 1237.

77. Kripke 1982 bases his argument on Wittgenstein's Philosophical Investigations (1974), but Drahos and Parker 1992 cite devotees of Wittgenstein who deny that he was the source of Kripke's argument or conclusion (at 111).

78. Kripke 1982 at $8-9$.

79. ibid. at 13,97 .

80. Drahos and Parker 1992 at 112.

81. For example Yablon 1987 who agrees with Kripke, and Bjarup 1988 who does not.

82. Drahos and Parker 1992 at 113. It also puts paid to Susskind's claim of a consensus in legal theory (see §2.2.7): Hart's and Kripke's viewpoints are totally irreconcilable.

83. Drahos and Parker 1992 at 112.

84. ibid. at 114 .

85. id.

86. ibid. at 115 .

87. ibid. at 114 .

88. Susskind $1987 \mathrm{a}$.

89. ibid. at 27-8. See also Susskind $1987 \mathrm{~b}$ at 2.

90. Susskind 1987 a at vii.

91. ibid. at 27.

92. Susskind's development work, as opposed to his jurisprudential work, is discussed in $\S 2.4 .4$.

93. Moles 1991 at $153-4$.

94. Susskind $1987 \mathrm{~b}$ at 2 .

95. ibid. at 1 .

96. ibid. at 2. Unaccountably, Moles 1991 does not make this point in his criticism of Susskind.

97. Clark 1988 at 429

98. Kelsen 1945, 1967; Hart 1961, 1983; Dworkin 1977, 1978.

99. Leith 1987 at $131-2$, emphases omitted, footnote added.

1. Loevinger 1961 at 259 .

2. ibid. at 266, 269-70, 274 .

3. Loevinger 1949 at 493 .

4. Gardner 1987 at 69 . Note, however, that the Jurimetrics Journal (ISSN 0022-6793 and ISSN 0897-1277) is published quarterly, and has been published since 1966.

5. As well as the references made in this section, see Tanenhaus, Schick, Muraskin and Rosen 1963.

6. Lawlor 1963 at 341, footnote omitted.

7. Kort 1963a, 1963b.

8. Kort $1963 \mathrm{~b}$ at 145 .

9. Lawlor 1963, 1968, 1981.

10. (1942) 316 US 455. 
11. (1963) 372 US 335.

12. Wiener 1962 at 1023 .

13. ibid. at 1024 .

14. Kayton 1964 at 312, some emphasis omitted, some emphasis added.

15. Nagel 1960, 1963, 1986, 1989; Schubert 1963a, 1963b. See also Spaeth 1963 and Aubert 1963.

16. Nagel 1963 at 40, emphases and footnotes omitted.

17. Haar, Sawyer and Cummings 1977.

18. ibid. at 758 .

19. id.

20. See Gardner's comments quoted in $\S 3.14$. Note, however, that Gardner is less critical of Haar et al. because "[b]y reflecting on their statistical results in the light of a traditional legal analysis of the same cases, the authors are able to provide recommendations for attorneys and litigants in zoning cases and to raise questions about zoning law for the attention of both court and legislature" (Gardner 1987 at 76, referring especially to Haar et al. 1977 at 742-50).

21. Loevinger 1949 at 461 citing Holmes 1897.

22. Loevinger 1961 at 269.

23. Lawlor 1963 at 339.

24. Susskind 1987 a at $95-6$.

25. Tapper 1973 at 249 , footnote omitted.

26. ibid. at 250 .

27. Gardner 1987 at 196 (a note to p. 75). Gardner cites as examples Schubert 1975 and Goldman and Sarat 1978, though her criticism clearly applies to Nagel's work too.

28. Stone $1964 \mathrm{~b}$ at 518 . See also Stone 1966 at 688 . Stone refers to Schubert 1963b and Ulmer 1963 (see also Ulmer 1964).

29. Stone $1964 \mathrm{~b}$ at 550 , emphases in the original.

30. ibid. at 553, emphasis in the original.

31. Buchanan and Headrick 1970 at 40.

32. ibid. at 41 .

33. ibid. at 45 .

34. id.

35. Maggs and deBessonet 1972.

36. Popp and Schlink 1975.

37. Buchanan and Shortliffe 1984.

38. Popp and Schlink 1975 at 309.

39. Michaelsen and Michie 1983.

40. More precisely, EMYCIN: MYCIN with its domain knowledge removed.

41. The domain of taxation law had been widely used in rule-based expert systems research. Apart from McCarty's TAXMAN project (§2.4.1), see Torsun 1987 and Sherman 1987, 1989. See also the hybrid CABARET system $(\S 2.6)$.

42. Meldman 1977. See also Cook et al. 1981.

43. Cook et al. 1981 at 692 .

44. Waterman and Peterson 1980. See also Cook et al. 1981 and Waterman, Paul and Peterson 1987. 
45. Waterman et al. 1987 at 26.

46. ibid. at 27-8. These "informal rules" are similar to the "conventions" proposed by Drahos and Parker 1992, as discussed in $\S 2.2 .6$.

47. Waterman et al. 1987 at 28.

48. Bing 1980; Borchgrevink and Hansen 1980.

49. Bing 1980 at 132 .

50. Stamper 1980, 1982. See also Cook et al. 1981. Stamper later decided that LEGOL was inadequate, and developed NORMA (1986).

51. Pattison and Ciesielski 1990. See also Ciesielski 1990.

52. Johnson and Mead 1991.

53. Susskind $1989 \mathrm{a}$ at 29.

54. McCarty 1977, 1980a, 1980b, 1980c.

55. McCarty 1980a at 3 .

56. id.

57. id.

58. ibid. at 4 .

59. id.

60 . id.

61. Moles 1987 at 270.

62. McCarty 1980a at 4-5.

63 . ibid. at 5 .

64. McCarty 1977 at 839.

65. ibid. at 839-40.

66. Austin 1885.

67. Moles 1987 at 270-1, footnote added.

68. ibid. at 269 .

69. McCarty 1980 c at 29-30.

70. McCarty and Sridharan 1981.

71. Eisner v. Macomber (1920) 252 US 189 (see McCarty 1982).

72. Ashley 1990 at $224-5$, footnote added, extraneous punctuation removed. Ashley's (i.e. HYPO's) mechanism for determining how "on point" are two cases is discussed in $§ 3.9 .3$.

73. Moles 1987 at 270 , referring to the structure of TAXMAN I.

74. Ashley 1990 at 226-7. Ashley criticizes Goldman, Dyer and Flowers 1987 for a similar use of primitives in their STARE system.

75. Kowalski and Sergot 1985, 1990; Sergot, Cory, Hammond, Kowalski, Kriwaczek and Sadri 1986a, 1986b; Bench-Capon, Robinson, Routen and Sergot 1987; BenchCapon and Sergot 1988; Kowalski 1989; Bench-Capon 1991.

76. Sergot et al. 1986a at 49. Note that this statement was qualified in another paper by the same authors published in the same year: "The knowledge elicitation problem is almost entirely absent in the formalization of legislation" (Sergot et al. 1986b at 383 , emphasis added).

77. Moles 1991 at 144.

78. Sergot et al. 1986a, 1986b; Kowalski and Sergot 1985; Bench-Capon and Sergot 1988; Kowalski 1989.

79. Sergot et al. 1986a at 41. 
80. Bench-Capon et al. 1987 at 192.

81. Moles 1991 at 159. He also refers to Bench-Capon 1991 (a collection of articles by Bench-Capon, Sergot, and others) and notes that, "[a]lthough the book is called Knowledge-Based Systems and Legal Applications, it would appear from [the biographical notes] that there was very little emphasis on involving people with legal expertise" (at 146). Moles's article sparked a debate: see Tyree 1992 and Moles and Dayal 1992.

82. Kowalski and Sergot 1990 at 207.

83. Hayes-Roth, Waterman and Lenat 1983 at 165, quoted by Susskind 1986 at 176 .

84. Sergot et al. 1986a at 46 .

85. Leith 1986b at 97, some extraneous punctuation removed. Leith's remarks are particularly interesting given Susskind's criticism of Leith for having built a legal expert system without legal expertise (see §3.13.1).

86. Gardner 1983, 1984, 1985, 1987.

87. Gardner 1987 at 4.

88. ibid. at 6 .

89. Susskind 1990 at 222 .

90. Gardner 1987 at 120.

91. ibid. at 119 .

92. Susskind 1990 at 225. Grunbaum 1988 is similarly critical of Gardner's "courageous" attempt (at 639). Jones 1988 also doubts "[t]he utility of the system outside this domain" (at 32).

93. Ashley 1990 at 226.

94. See Levi 1949.

95. Gardner 1987 at 22, footnote added.

96. id.

97. id.

98. ibid. at 33 .

99. Susskind 1989a at 30 .

1. Gold and Susskind 1986; Susskind 1986, 1987a, 1987b.

2. Capper and Susskind 1988; Susskind 1989b.

3. Capper and Susskind 1988 at vii. See also Susskind 1989b at 24.

4. Susskind 1987a at 255 .

5. ibid. at 87, citing Simpson 1986.

6. Susskind 1987 a at 256 .

7. ibid. at 87 .

8. Feigenbaum 1981 at 226 . See also Feigenbaum and McCorduck 1983 at 75.

9. Sergot et al. 1986a at 49.

10. Allen 1963, 1965, 1968, 1981, 1982; Allen and Caldwell 1963; Allen and Saxon 1985, 1988. See also Bench-Capon 1987.

11. Shannon and Golshani 1988 at 308. They also warn that ad hoc rule formulation means that subsequent designers/users cannot trace the evolution of a set of rules from the words of the statute. However, the author suggests that this could be obviated by the sensible use of comments.

12. ibid. at $308-9$. 
13. However, Dworkin 1978, for example, contends that legal problems do have a correct answer.

14. deBessonet and Cross 1985, 1986, 1988.

15. Shannon and Golshani 1988 at 310, footnotes omitted, one footnote added. An extraneous right parenthesis has been removed from the ANF representation.

16. This criticism of Shannon and Golshani's modified example does not apply to the original example given by deBessonet and Cross 1985. They refer, in much less ambiguous terms, to the lessee's belief about damage caused by her/himself (at 208).

17. Tyree, Greenleaf and Mowbray 1988 at 232.

18. id.

19. Stone 1985 at 56 . See also Tyree 1989 at 136.

20. Tyree et al. 1988 at 232 .

21. Kowalski 1991 at 30.

22. Tyree et al. 1988 at 232 .

Quinlan, Compton, Horn and Lazarus 1987 give two examples of the use of an inductive technique. The first involves 2647 cases, from which a training set of 2000 and a test set of 647 are extracted (at 165-6). The second involves 3066 cases: a training set of 2300 and a test set of 766 (at 168-70). These examples use an inductive inference tool called C4: a descendent of ID3. See also Quinlan 1986, 1988.

23. Susskind 1986 at 190.

24. Sergot et al. $1986 \mathrm{~b}$ at 370 .

25. Pearce and Geddes 1988 at 56-62.

26. Bankowski and MacCormick 1991 at 377.

27. Pearce and Geddes 1988 at 1.

28. Ashley 1990 at 127.

29. Lambert and Grunewald 1991 at 194.

30. Ashley 1985 at 116. See Ashley and Aleven 1991 for a description of their research into developing an intelligent tutoring system for teaching law students to argue with cases.

31. Mackaay and Robillard 1974. They did not actually develop an expert system, but their work is relevant to the development of legal expert systems and, hence, is discussed here.

32. The first formulation of the nearest neighbour rule appears to have been made by Fix and Hodges in 1951.

33. Cover and Hart 1967 at 21.

34. Lawlor reported his results in a paper presented to the 1971 Annual Meeting of the American Political Science Association. Excerpts from that paper, published in 1972, omit details of his work with these cases.

Lawlor's approach was to find a weight for each of the attributes such that the sum of those weights, where the attribute is present, is less than 1 if the case was decided against a given party, and greater than 1 otherwise.

35. Mackaay and Robillard 1974 at 308.

36. See Schmidt 1971 and Claudy 1972. 
37. Mackaay and Robillard 1974 at 322 . They also examine the visual representation of cases, scaling the cases onto one- and two-dimensional maps using the same similarity metric as they use for their nearest neighbour analysis. These maps allow easy visual identification of the borderline cases.

38. Tyree 1985, 1986, 1989 and Tyree, Greenleaf and Mowbray 1988, 1989. FINDER's approach to case law is based upon a mathematical concept of the similarity of cases described by Tyree 1977 .

39. Treating each YEs as a 1 , and each NO as a 0.

40. Tyree 1989 at $12-13$.

41. The complete Finder specification is given in $\S$ A.2.

42. Ashley 1985, 1986, 1989a, 1989b, 1990; Rissland 1983, 1985, 1990; Rissland, Valcarce and Ashley 1984; Ashley and Rissland 1987a, 1987b, 1988; Rissland and Ashley 1987, 1989.

43. Ashley 1990 at $2-3$.

44. ibid. at 37 .

45. id.

46. For example, a finding that there has been trade secrets misappropriation.

47. Ashley 1990 at $112-3$.

48. ibid. at 279. "On pointness" in HYPO is discussed in $\S 3.9 .3$.

49. ibid. at 20 .

50. ibid. at 142 .

51. id.

52. See Rissland 1989 for a discussion of argument with hypotheticals.

53. Hafner 1981. See also Cook et al. 1981. Negotiable instruments are cheques and promissory notes.

54. GREBE is described in $\S 2.6$.

55. Branting 1989 at 109.

56. id.

57. Rissland and Skalak 1989a at 48.

58. Rissland and Skalak 1989a, 1989b; Skalak 1989; Skalak and Rissland 1991, 1992. See also Rissland 1990.

59. Rissland and Skalak $1989 \mathrm{~b}$ at 527.

60. Branting 1989, 1991.

61. See $\S 2.5 .4$ for a discussion of the inadequacy of semantic networks for representing case law.

62. Branting 1991 at 150 .

63. Oskamp, Walker, Schrickx and van den Berg 1989; Walker, Zeinstra and van den Berg 1989.

64. van Opdorp, Walker, Schrickx, Groendijk and van den Berg 1991 at 280.

65. Oskamp 1989; Oskamp et al. 1989; Walker et al. 1989; Wolstenholme 1989; van Opdorp et al. 1991.

66. van Opdorp et al. 1991 at 280.

67. ibid. at 285 .

68. Berman and Hafner 1991; Vossos, Zeleznikow, Dillon and Vossos 1991. See also Sanders 1991 (a hybrid planner).

69. Susskind 1987a at 151. 
70. ibid. at 153 .

71. Hafner 1981, 1987; deBessonet and Cross 1985, 1986, 1988.

72. McCarty 1984, 1986, 1989, 1990, 1991.

73. McCarty 1984 at 126 .

74. Greenleaf, Mowbray and Tyree 1987 at 11.

75. McCarty 1983 at 267-8. See also Bench-Capon 1989.

76. Shannon and Golshani 1988 at 311. They propose a "happy medium" between shallow and deep conceptual models.

77. Greenleaf et al. 1987 at 11.

78. Susskind 1987 a at 153 .

79. Meta-rules are similar to the "conventions" proposed by Drahos and Parker 1992, as discussed in $\S 2.2 .6$.

80. Greenleaf et al. 1987 at 11.

81. Stone 1985 at 46 , footnotes omitted, emphases in the original.

82. McCarty 1989 at 180.

83. Moles 1991 at 163.

84. Susskind $1987 \mathrm{a}$ at 20.

85. Stone $1964 \mathrm{~b}$ at 560 , as quoted in $\S 2.2 .3$.

86. Susskind 1987 a at 20.

87. Moles 1987 at 7.

88. See $\S 2.4 .2$.

\section{Chapter 3: A pragmatic approach to case law}

89. Galsworthy 1941 , act II at 36 .

90. Murphy 1980 at 5. Opening address delivered at the First National Conference of Labor Lawyers, Adelaide, 29 June 1979.

91. Susskind 1987a at 12 .

92. The difference between private and public law is explained in $§ 3.3 .1$.

93. For example, Susskind 1987a at 255.

94. See Stone 1964a at 120; Kelman 1987; Bottomley, Gunningham and Parker 1991.

95. For example, Kelsen 1945, 1967, and the authors referred to in the previous note.

96. See $\S 2.1 .2$.

97. Susskind 1987a at 41.

98. id. Susskind also claims that law reformers use legal reasoning to try to persuade the legislature to change the law. This is debatable. In a sense, law reformers operate outside the realm of legal reasoning; their concern is that the application of legal reasoning to the existing law produces undesirable results.

99. id.

1. ibid. at 42 .

2. See $\S 2.2 .7$.

3. See Morris, Cook, Creyke and Geddes 1992, especially chs 3-4, 8 .

4. Hart 1961 at 131.

5. Ashley 1990 at 229, citing Burton 1985 at 40. 
6. Ashley 1990 at 230, capitalization added.

7. See $\S 2.4 .2$, and the beginning of $\S 2.5$.

8. BA (Hons); LLB; Barrister of the High Court of Australia and of the Supreme Court of New South Wales; Barrister and Solicitor of the Supreme Court of the Australian Capital Territory.

9. See chapter 5 .

10. Shannon and Golshani 1988 at 315.

11. See the discussion of $A_{5}$ in the Authorization area of the Authorization specification $(\S 5.3 .2)$.

12. The word "hypothetical" is used here in its general sense; ideal points are different to the hypotheticals which SHYSTER uses in argument (see §3.11.3).

13. For example, the Employee area of the EmployeE specification (§5.4.2) represents the open-textured concept of a worker's employment status. There are two results: the worker is an employee or the worker is an independent contractor.

14. The Natural specification ( $\$ 5.5 .2)$ contains three areas. The root node (whether a duty to observe natural justice is implied in a particular decision-making process) is represented by the Natural area. One of its seven children is an internal node (whether the decision affected an interest of the applicant) represented by the Affected area. One of the four children of that node is also internal (whether the applicant had a legitimate expectation which was affected by the decision) and is represented by the Expectation area, which has six children - all leaf nodes. The relationship between the attributes in these areas is indicated in figure 5.8.

15. Mehl 1959 at 768.

16. id.

17. Susskind 1987a at 54, emphasis omitted. He continues: "[b]ut that is not a job for the near future."

18. Berman and Hafner 1991 discuss a formal model for distinguishing between questions of fact, questions of law, and mixed questions.

19. See, for example, the development of the Natural specification $(\S 5.5 .2)$ from the work of McMillan 1991 (discussed in §5.5.1).

20. Mackaay and Robillard 1974 at 308. See $\S 2.5 .1$.

21. As discussed in $\S 3.5 .2$.

22. An example of this can be found in the EMPLOYEE specification described in §5.4.2. The legal expert identified the question of whether the worker's employer deducted "pay as you earn" (PAYE) tax instalments from the worker's pay as being an attribute in the Employee area $\left(A_{15}\right)$. In Zuijs v. Wirth Brothers Pty Ltd (1955) 93 CLR 561, Wirth Brothers deducted PAYE tax instalments from Zuijs's pay. Dixon CJ, Williams, Webb and Taylor JJ of the High Court mention this fact (at 567), but do not use it in coming to their decision.

23. Blackstone 1773 (especially vol. 1 at 69-70); Krygier 1986.

24. For example, Stone 1964a, 1985.

25. The Natural area of the Natural specification (§5.5.2) affords an example of this. $A_{3}$ concerns the question whether the administrative power in question is of a nature that would suggest that procedural fairness would be applied in its exercise. When 
Bread Manufacturers of New South Wales v. Evans (1981) 38 ALR 93 was decided, the answer in that case would have been YEs. The law has developed to the extent that, if that case were to be heard now, the answer would be No.

26. Ideal points are so-named because, as explained in $§ 3.9 .1$, SHYSTER treats cases as points in space, the dimensionality of which is the number of attributes.

27. Apropos specifying more than one ideal point, see $\S 6.2 .5$.

28. Lambert and Grunewald 1989. LESTER deals with unjust discharge from employment under collective bargaining agreements.

29. ibid. at 91 .

30. Ashley and Rissland 1988 at 239.

31. Williams 1971.

32. Sneath and Sokal 1973.

33. Aldenderfer and Blashfield 1984 at 21, emphases added, footnotes added.

34. Everitt 1974 at 50. Note that Haar, Sawyer and Cummings 1977 use statistical methods to determine which are the important attributes, and ignore the unimportant ones.

35. Tyree 1989 at 149, n. 16 .

36. ibid. at 141, emphases added. Tyree refers to separating the cases into two classes because FINDER allows only two results for each leading case. SHYSTER allows an arbitrary number of results in each specified area of law.

37. Tyree, Greenleaf and Mowbray 1988 at 245, n. 8.

38. Neter, Wasserman and Whitmore 1982 at 185. This formula defines the variance of a finite number of values - a finite population - and is different from the formula that defines the variance of a sample of values.

39. FINDER does not deal with infinite weight as none of its attributes has zero variance.

40. Real examples are $A_{5}$ in the Authorization area of the Authorization specification (§5.3.2), and $A_{4}$ in the Expectation area of the NATURAL specification (§5.5.2).

41. Ashley 1990 at 175 .

42. Bing 1992 at 104.

43. id. Bing's reference is to FINDER's method, but his comment applies to SHYSTER's method too.

44. Ashley 1990 at $175-6$.

45. Ashley and Rissland 1988.

46. Ashley 1990 at $176-8$, capitalization added.

47. Lambert and Grunewald 1991 at 194.

48. There is only a stochastic dependence in this example if there is some variation of values within the attributes. In the extreme circumstance where all of the attribute values are the same, the two attributes are (strictly) stochastically independent.

49. An example of this can be found in the discussion of attribute dependence in the EMPLOYEE specification in $\S 5.4 .2$.

50. If an attribute's known values are all YESs, or all NOs, the legal expert is warned that the attribute has been given infinite weight (as explained in §3.7).

51. These attributes are $A_{4}$ and $A_{9}$, respectively, from the Employee area of the EMPLOYEE specification explained in $§ 5.4 .2$ (see also the attribute value matrix in figure 4.4). 
52. Conover 1971 at $154-8$.

53. Fisher 1970.

54. Aldenderfer and Blashfield 1984 at 18, emphasis omitted.

55. id. Everitt 1974 at 56 lists all but the third criterion.

56. Aldenderfer and Blashfield 1984 at 18-19.

57. ibid. at 19.

58. Sneath and Sokal 1973 at 140-5; Aldenderfer and Blashfield 1984 at 33.

59. Attribute values are often standardized before Euclidean distance is calculated. Standardization is not necessary in SHYSTER as all of the attribute values are of the same type.

60. In SHYSTER, $n$ is the number of known pairs of attribute values. Attribute pairs with one or two UNKNowns are ignored for these purposes; they are taken into account in the calculation of unknown distance.

61. Aldenderfer and Blashfield 1984 at 28.

62. Everitt 1974 at 51.

63. Gower's coefficient, another measure of similarity, is identical to Jaccard's coefficient in the binary case: Aldenderfer and Blashfield 1984 at 31.

64. Aldenderfer and Blashfield 1984 at 22.

65. See ibid. at 23-4, and Everitt 1974 at 53-4.

66. Identical cases do not have a coefficient of zero, and the coefficient often fails to satisfy the triangle inequality.

67. Aldenderfer and Blashfield 1984 at 23.

68. A correlation coefficient of -1 indicates a negative linear association; a value of 1 indicates a positive linear association. A value of zero indicates no linear association.

69. Ashley 1990 at 127.

70. ibid. at 128 .

71. As explained in $\S 3.7$, HYPO adopts a "symbolic least commitment approach" to attribute weighting.

72. Sneath and Sokal 1973 at 146.

73. These distances are for Narich Pty Ltd v. Commissioner of Pay-roll Tax: one of the cases used to test the EmployeE specification described in §5.4.2. Narich v. $C P T$ is described in $\S 5.4 .3$. Its complete table of distances, as extracted from SHYSTER's distances file, is given in figure 5.7.

74. The Employee and Contractor results, respectively (see $§ 5.4 .2$ ).

75. Mackaay and Robillard 1974 at 309-10. They term this the problem of "tied distances amongst neighbours."

76. Tyree, Greenleaf and Mowbray 1989 at 47.

77. Ashley 1990 at 39.

78. Insofar as a program can have an opinion.

79. Ashley 1990 at $230-1$, capitalization added.

80. ibid. at 232 .

81. Note that the use of unknown distance as a measurement of error (discussed in §3.9) does not account for the possibility of an instantiation producing a different result. An UNKNOWn attribute value in the instant case has the effect of adding the weight 
of the attribute to the unknown distances of all the leading cases: i.e. the leading cases are all pushed away from the instant case by the same (unknown) distance.

82. Used in this fashion, instantiation is similar to SHYSTER's use of hypotheticals (§3.11.3), only with a higher degree of user control.

83. Ashley 1990 at 85.

84. ibid. at 233. See also Rissland 1989.

85. Ashley 1990 at 232.

86. UNKNOWN values are dealt with by instantiation: see $\S 3.11 .2$.

87. The number of hypotheticals with exactly $k$ differences from the instant case, given $n$ attributes, is $\left(\begin{array}{l}n \\ k\end{array}\right)=\frac{n(n-1) \cdots(n-k+1)}{k !}$. So, the number of hypotheticals $h$ with no more than $k$ differences is $\left(\begin{array}{l}n \\ 1\end{array}\right)+\left(\begin{array}{l}n \\ 2\end{array}\right)+\cdots+\left(\begin{array}{l}n \\ k\end{array}\right)$.

The Employee area of the EMPLOYEE specification ( $(5.4 .2)$, for example, has 18 attributes. Allowing no more than 2 differences $(k=2)$ restricts SHYSTER to 171 hypotheticals. If $k=3, h=987$; and if $k=4, h=4047$ - still only a small fraction of the maximum: $2^{18}=262144$ hypotheticals.

88. Each YES is assigned a value of 1 , and each NO is assigned a value of 0 . For the purposes of constructing the centroid, each mean (a number $n$ in the range 0 to 1$)$ is rounded to the nearest attribute value $(0 \leq n<0.5$ : NO; $0.5 \leq n \leq 1$ : YES). However, the unrounded means are used when calculating the correlation coefficients $r$ and $r^{\prime}$ for those centroids.

89. Tyree et al. 1988 at 241 .

90. SHYSTER uses the attribute's result weight (explained in $§ 4.9 .1$ ) when calculating the strength of directions.

91. Susskind 1987 a at 55 .

92. ibid. at $53-5$.

93. Leith 1985a, 1986a.

94. Susskind 1986 at $175-6$. It is probably unnecessary to point out that Susskind wrote this article for a legal audience.

He continues: "This is not to belittle Leith's achievements, for he was clearly working with limited resources" (at 176). Leith bitterly disputes Susskind's comments, including his definition of what constitutes a legal expert system. "If there is no agreement [over suitable terminology in the field], why should computer scientists accept the definition proposed by a lawyer? Will Susskind accept the definition of 'divorce' proposed by a computer scientist?" (1987 at 130). Susskind's early experimental work was with the Scottish law of divorce.

95. Clark 1988 at 428.

96. Gardner 1987 at 33. Gardner's chosen domain was one aspect of the law of contract (see $\S 2.4 .3$ ).

97. There are two exceptions to this rule - two tests where SHYSTER's choice of nearest result is deemed "good" despite disagreeing with the actual cases: Twist $v$. Council of Municipality of Randwick (1976) 136 CLR 106 and Salemi v. MacKellar (1977) 137 CLR 396. These exceptions are explained and justified in $\S 5.5 .3$.

98. The case upon which SHYSTER bases its argument is the nearest neighbour; the case upon which the counterargument is based is the nearest other (see §3.10.1).

99. (1977) 137 CLR 396. 
1. As discussed in $\S 2.4 .7$, the number of decided cases in any given area of law is usually so small that inductive inference algorithms cannot be used.

2. And bad legal advice may filter out a hopeful case.

3. Gardner 1987 at 6 .

4. Tyree 1986, 1989 and Tyree et al. 1988, 1989. Tyree et al. 1989 also describe (at 49-50) a reflexive test using Bridges v. Hawkesworth (1851) 21 LJQB 75.

5. Ashley 1990 at 183-93: Crown Industries, Inc. v. Kawneer Co. (1971) 335 F Supp 749; Structural Dynamics Research Corporation v. Engineering Mechanics Research Corporation (1975) 401 F Supp 1102; USM Corporation v. Marson Fastener Corporation (1979) 379 Mass 90; Amoco Production Co. v. Lindley (1980) 609 P 2d 733.

6. For example, the Employee area of the EMPLOYEe specification ( $\$ 5.4 .2)$ has 18 attributes and, hence, a search space of $2^{18}=262144$ different cases.

7. Some of the cases generated for the EMPLOYEe specification represent paradoxes. These impossible cases are discussed in $\S 5.4 .4$.

8. SHYSTER issues a warning if the instant case is nearer to the ideal point for another result than it is to that of the nearest result (see $\S 3.12 .2$ ).

9. Mackaay and Robillard 1974 at 312. Lawlor 1968 terms this reflexive approach "cyclical sampling" (at 110-11).

10. Compare this with the approach taken by Mackaay and Robillard 1974. They say that cases which are "incorrectly" predicted in a reflexive test are "suspect" because "[s]uch cases are difficult to explain on the basis of the remaining ones" (at 312). Mackaay and Robillard were dealing with a large set of cases, not a smaller set of expertly chosen cases as in SHYSTER.

11. The Finder specification ( $\$ 5.2 .2)$ uses only English cases.

12. Greenleaf, Mowbray and Tyree 1987 at 11.

13. Gardner 1987 at 22.

14. See $\S 2.4 .3$.

15. Gardner 1987 at $74-5$. Gardner refers to several of the researchers discussed in chapter 2, including Kort 1963a, Lawlor 1963, 1972, Mackaay and Robillard 1974, Haar et al. 1977, Borchgrevink and Hansen 1980, and Tyree 1981.

16. Greenleaf, Mowbray and Tyree 1991 propose a similar mechanism for their DATALEX project (at 223), though the extent to which it has been implemented is not clear. See also Tyree et al. 1989 at 49.

17. Ashley 1990 at 254.

18. Gardner 1987 at 75. Questions of fact and questions of law are discussed in §3.5.2.

19. HYPO's knowledge representation is explained in $\S 2.5 .3$.

20. Tyree 1977 at 414, n. 40. There are two unknown values coded as No in FINDER (see $\S 5.2 .2$ ).

21. Ashley 1990 at 128.

22. For example, the Authorization area of the Authorization specification (§5.3.2) has three results.

23. See $§ 5.2 .2$.

24. Because FINDER does not allow unknown values, it has no unknown distance. None of its attributes is infinitely weighted. 
25. Tyree et al. 1988 at 241 .

26. Ashley 1990 at 249.

27. An example is the Authorization area of the Authorization specification (§5.3.2). An answer of yes to $A_{3}$ ("Did the accused sell or hire the infringer the means of infringing?") can be directed towards the result Auth, but an answer of NO or UNKNOWN for that attribute cannot be directed towards either of the other results: Not-Auth or Liable. The fact that the accused did not sell or hire the infringer the means of infringing $\left(A_{3}=\right.$ NO) suggests neither that the accused did not authorize the infringement (Not-Auth) nor that the accused is directly or vicariously liable for the infringement (Liable); similarly for $A_{3}=$ UnKNown.

Another example is $A_{6}$ in the Employee area of the EMPLOYEe specification described in $\S 5.4 .2$.

28. Berman 1991 at 308 .

29. See $\S 2.7$ and $\S 2.8$.

30. Berman 1991.

31. Including Gardner 1987, Goldman, Dyer and Flowers 1987, Hafner 1987, Rissland and Ashley 1987, Branting 1989, and Ashley and Aleven 1991.

32. For example, several attributes in areas in the NATURAL specification (§5.5.2) embody political considerations.

33. Berman 1991 at $307-8$.

34. ibid. at 308 .

\section{Chapter 4: Implementing SHYSTER}

35. Gay 1967, vol. II at 1-2 (fable I).

36. Lucas 1935 at 394. Letter to Samuel Rogers, probably 21 December 1833.

37. Paramount. Screenplay by Arthur Sheekman.

38. International standard ISO/IEC 9899: 1990; Australian standard AS 3955-1991. Kernighan and Ritchie 1988 describe ANSI C which is the same as ISO C.

39. Popple 1993.

40. 256 characters and 16 characters, respectively.

41. UNIX's stderr stream.

42. Lamport 1986 describes $\mathrm{LAT}_{\mathrm{E}} \mathrm{X}$ which is a set of macros for Knuth's $\mathrm{T}_{\mathrm{E}} \mathrm{X}$ system (1984). SHYSTER's $\mathrm{LAT}_{\mathrm{E}} \mathrm{X}$ output is suitable for processing by $\mathrm{LAT}_{\mathrm{E}} \mathrm{X}$ version 2.09 $\left\langle 25\right.$ March 1992〉 and $\mathrm{TEX}_{\mathrm{X}}$ version 3.141; it should also be suitable for processing by later versions.

43. Figures extracted from SHYSTER output are marked with a star in the list of figures that immediately precedes chapter 1 .

44. The SHYSTER output that appears in appendices A, B and C was produced using the $-i$ switch which causes SHYSTER to write $\mathrm{IAT}_{\mathrm{E}} \mathrm{X}$ code suitable for inclusion in another $\mathrm{LAT}_{\mathrm{E}} \mathrm{X}$ document.

45. UNIX's stdout stream.

46. A string, as defined in figure 4.3, includes the enclosing quotation marks. The ToKenizer discards these quotation marks when the specification is read. Hence, the word string is used in two different ways in this description of SHYSTER: before 
being tokenized, a string includes the enclosing quotation marks; once internally represented by SHYSTER, a string is only those characters which appeared between the enclosing quotation marks in the specification file.

47. A string may contain commands which will be processed by $\mathrm{LAT}_{\mathrm{E}} \mathrm{X}$ if the string is included in one of SHYSTER's files. $\mathrm{LAT}_{\mathrm{E}} \mathrm{X}$ commands are prefixed with a $\backslash$ character. The $\$$, \& and \% characters have special meanings in $\mathrm{L}_{\mathrm{E}} \mathrm{T} \mathrm{X}$; if one of these characters appears in a string, SHYSTER converts it into the command that produces the appropriate character: $\backslash \$$, $\backslash \&$ or $\backslash \%$.

48. As with strings (see the note before last), attribute vectors are defined in figure 4.3 to include the enclosing parentheses, but the Tokenizer discards these parentheses when the specification is read.

49. Whitespace is any sequence of spaces, carriage returns, tabs, vertical tabs, or form feeds.

50. SHYSTER's is an LL(1) parser.

51. EBNF is described by Wirth 1977.

52. Note that a result identifier from another (explicitly named) area can be referred to within the specification of an external attribute, as explained in $\S 4.6 .3$.

53. This is $A_{12}$ from the Employee area of the EmployeE specification (see $\S \mathrm{A} .4$ and $\S$ C.3).

54. This is $A_{4}$ from the Affected area of the Natural specification (see $\S$ A.5).

55. The external area does not need to have been specified before it is used in an external attribute. Forward references are allowed, and are not checked until invocation. Recursive references (circular definitions) are not allowed but SHYSTER does not detect them, unless the reference is to the same area in which the attribute is specified.

56. The specification of an UNKNOWN string is not strictly necessary; no external result identifier can produce a value of UNKNOWN in this attribute. However, if it is not specified and one of the leading cases or ideal points has a value of UNKNOwN for this attribute, the Dumper module will issue a warning.

57. Result identifiers are not checked for validity: i.e. the legal expert should ensure that these identifiers match result identifiers in the external area. If the same result identifier is specified for two or all of the possible attribute values it is linked to the first specified (the order being YES, NO then UNKNOWN).

58. The order in which cases are specified is unimportant. SHYSTER groups cases by their result, and orders the cases in each group according to the importance of the court in which each case was decided. The cases in the EMPLOYEe specification appear in the specification file (§C.3) in the order in which they were decided.

59. See, for example, Moffatt $v$. Kazana [1969] 2 QB 152 in the Finder area of the Finder specification ( $\$ 5.2 .2)$, and Ready Mixed Concrete (South East) Ltd v. Minister of Pensions and National Insurance [1968] 2 QB 497 in the Employee area of the EMPLOYEe specification (§5.4.2). Both cases were decided in 1967.

60. This warning is actually issued by the CASES module, not the PARSER, but it is convenient to mention it here.

61. This happens in the Natural specification ( $\S 5.5 .2)$, but note the discussion there as to why that specification was not changed. 
62. Some experimentation by the author has shown that this sort of graphical representation conveys its information more clearly than does one using Ys, Ns and Us, or ones and zeros.

63. As with the example specification of a local attribute in $\S 4.6 .3$, this is $A_{12}$ from the Employee area of the EMPLOYEE specification (see $\S$ A.4).

64. If an attribute value is directed towards more than one result, result identifiers are displayed separated by a $\vee$ symbol.

65. As with the example specification of an external attribute in $\S 4.6 .3$, this is $A_{4}$ from the Affected area of the NATURAL specification (see $\S$ A.5).

66. (1945) 70 CLR 539.

67. This cell contains the probabilities for the attribute values in figure 3.2 , used as an example in $\S 3.8 .2$. These example attributes are $A_{4}$ and $A_{9}$ from the Employee area of the EmployeE specification (§5.4.2). Figure 4.5 is an extract from the probabilities matrix for that area.

68. Because all of SHYSTER's known attribute values are assigned numerical values of 0 or 1 , the variance $\sigma_{i}{ }^{2}$ (defined in $\S 3.7$ ) simplifies to $\bar{A}_{i}-\bar{A}_{i}{ }^{2}$. SHYSTER uses this simplified formula to calculate the variance.

69. If an attribute has no known attribute values, i.e. all its values are UnKNown, it is given no weight and a warning message is issued. A further warning is issued (by the Odometer) if the instant case or an ideal point has a known attribute value for the weightless attribute.

70. Distance comparisons, like other comparisons, are precise to two decimal places. They need not be; by changing one constant, SHYSTER can be rebuilt with a different distance comparison threshold.

71. The Odometer is invoked twice for some hypotheticals. The first time it performs its calculations without writing anything to the distances file. Only some hypotheticals are chosen to be reported upon; for these hypotheticals the OdOMETER is called a second time to perform the calculations again and to write details to the distances file. Only a fraction of the hypotheticals are chosen; performing the calculations twice for the chosen hypotheticals avoids having to store the results of calculations from previous hypotheticals.

72. How hypotheticals are chosen is explained in $\S 3.11 .3$.

73. If one of the attributes is infinitely weighted, the values obtained for $S^{\prime}$ become meaningless because both the numerator and the denominator are infinite. In this event, values for $S^{\prime}$ are not written to the dump file, and $S^{\prime}$ is not used as a safeguard.

Calculation of the weighted correlation coefficient $r^{\prime}$ is complicated when one of the attributes is infinitely weighted. If this happens, a very large weight is used in place of an infinite one.

If either of two cases has all attribute values the same, the correlation coefficients $r$ and $r^{\prime}$ are meaningless and are ignored for safeguard purposes.

74. How hypotheticals are chosen to be reported on is explained in $\S 3.11 .3$.

75. Cases are not summarized more than once in the same report - and not summarized at all if the user has enabled quiet mode (using the - q switch on the command line). In quiet mode, opening and closing strings are not written either.

76. (1949) 79 CLR 389. 
77. The rather quaint expression "on all fours" is used by FINDER (see, for example, Tyree 1989 at 160). Although this expression may seem twee, it is preferable to "the same," "identical," etc., because two cases are never completely identical. Its meaning is clear from the context, and its oddness draws the reader's attention to the fact that something less than sameness is meant.

78. [1968] 2 QB 497.

79. [1976] 1 WLR 1213.

80. If there is more than one nearest neighbour, the comparison is made with the most important nearest neighbour.

81. This quotation is taken from the report file for Narich Pty Ltd v. Commissioner of Pay-roll Tax (1983) 50 ALR 417 which is used as a test case in $\S 5.4 .3$.

82. The opening and closing strings are not written again when arguing with instantiations.

83. This quotation is taken from the report on the fourth instantiation in the report file for Re Porter; Re Transport Workers Union of Australia (1989) 34 IR 179 which is given in full in $\S$ B.4. Re Porter; Re $T W U$ is used as a test case in $\S 5.4 .3$.

84. As with instantiations, the opening and closing strings are not written again when arguing with hypotheticals.

85. "Weighted association coefficients" does not appear as a subheading in this example because those coefficients agree with the choice of nearest neighbour.

\section{Chapter 5: Case studies}

86. Wiener 1962 at $1023-4$.

87. Doonesbury copyright G. B. Trudeau. Reprinted with permission of Universal Press Syndicate. All rights reserved.

88. (1722) 1 Str 505.

89. Bridges v. Hawkesworth (1851) 21 LJQB 75 at 77, per Patteson J.

90. (1851) 21 LJQB 75.

91. (1886) $33 \mathrm{ChD} 562$.

92. [1896] 2 QB 44.

93. [1945] 1 KB 509.

94. Hibbert v. McKiernan [1948] 2 KB 142 at 149 (footnotes omitted). Lord Goddard CJ, Humphreys and Pritchard JJ decided that the law of trover did not apply in that case.

95. Tyree 1989 at 119.

96. [1982] 1 All ER 834.

97. ibid. at 843 .

98. id. Tyree 1989 says that Donaldson LJ's statement of the principles to be applied is "a good candidate for a direct translation into a rule-based system" (at 119). He develops some rules to reflect this statement and concludes that "building even small rule-based systems is not a task which may be completed easily" (at 126); the rule base is considerably more complicated than both FINDER and SHYSTER's specification of FINDER.

99. [1948] 2 KB 142 at 149.

1. Tyree 1989 at 131, n. 12 . 
2. In City of London Corporation v. Appleyard (1), "the finders ... were not the servants of Yorkwin but the servants of Wates Ltd, who were engaged by Yorkwin as independent contractors": [1963] 1 WLR 982 at 988, per McNair J.

In South Staffordshire Water Co. v. Sharman, the finder was "employed" by the company. It is not clear whether the finder was an employee or an independent contractor, although some of what Lord Russell of Killowen CJ says in the case suggests the latter: [1896] 2 QB 44 at 46.

3. (1722) 1 Str 505.

4. (1851) 21 LJQB 75.

5. (1886) $33 \mathrm{ChD} 562$.

6. [1896] 2 QB 44.

7. [1945] 1 KB 509.

8. [1963] 1 WLR 982.

9. [1969] 2 QB 152.

10. In fact, the premises were leased by Venture Property and Development Co. Ltd which held the premises on trust and to the order of Yorkwin. Venture and Yorkwin were "associated companies." This added complication is not relevant for FINDER's (or SHYSTER's) purposes, and is ignored.

11. In FINDER these "two cases" are referred to as Yorkwin v. Appleyard and Corporation of London v. Yorkwin, respectively.

12. See $\S 3.14 .1$.

13. $A_{2}$ and $A_{8}$ in Armory v. Delamirie.

14. $A_{10}$ in Armory v. Delamirie, and $A_{5}$ in City of London Corporation v. Appleyard (2); both are Yess and both should be Nos.

15. In the Finder area, $w_{4}=w_{9}>w_{6}=w_{7}=w_{8}=w_{10}>w_{1}=w_{3}=w_{5}>w_{2}$ (where $w_{i}$ is the weight of $A_{i}$ ).

16. [1982] 1 All ER 834.

17. Donaldson LJ put it somewhat melodramatically: "On 15 November $1978 \mathrm{Mr}$ Alan George Parker had a date with fate, and perhaps with legal immortality" (ibid. at 835$)$.

18. [1982] 1 All ER 834 at 838, per Donaldson LJ.

19. ibid. at 838-40. His lordship was surprised to find the law so complex. "It is astonishing that there should be any doubt as to who is right. But there is. Indeed, it seems that academics have been debating this problem for years" (at 836).

20. ibid. at 845 .

21. ibid. at 843, per Donaldson LJ (with whom Eveleigh LJ and Sir David Cairns agreed).

22. [1963] 1 WLR 982 at 986, per McNair J.

23. Tyree 1986 at 18; 1989 at 160; Tyree, Greenleaf and Mowbray 1988 at 246-7; 1989 at $50-1$.

24. e.g. University of New South Wales v. Moorhouse (1975) 133 CLR 1; RCA Corporation v. John Fairfax and Sons Ltd [1981] 1 NSWLR 251; WEA International Inc. v. Hanimex Corporation Ltd (1987) AIPC \90-428; Australasian Performing Right Association Ltd v. Jain (1990) AIPC $990-718$.

25. [1924] $1 \mathrm{~KB} 1$ at 12 .

26. [1926] $2 \mathrm{~KB} 474$ at 491. 
27. Adelaide Corporation v. Australasian Performing Right Association Ltd (1928) 40 CLR 489.

28. Ricketson 1984 at 229 .

29. (1975) 133 CLR 1 at 12-13.

30. ibid. at 12, citing Adelaide Corporation v. Australasian Performing Right Association Ltd (1928) 40 CLR 481 at 497-8, 503. Although Adelaide v. APRA is cited as authority for several propositions in this area, it is not one of the leading cases in the Authorization specification because it was not actually a case about authorization. The question in that case was whether the City of Adelaide had "permitted" a song to be sung contrary to s. 2(3) of the Copyright Act 1911 (UK), in force in Australia by virtue of the Copyright Act 1912 (Cth).

31. (1975) 133 CLR 1 at 12-13.

32. ibid. at 13 .

33. ibid. at 12 .

34. Ricketson 1984 at $229-34$.

35. Peter Drahos, Lecturer, Faculty of Law, The Australian National University.

36. Ricketson 1984 states the law as it was on 1 May 1983.

37. The Employee specification would have to be modified before it could be linked to the Authorization specification in this fashion. At present, the Employee area assumes that there is a worker who is either an employee or an independent contractor (see $\S 5.4 .2$ ). In the Authorization area, the infringer may not work for the accused at all.

38. (1975) 133 CLR 1 at 13, per Gibbs J.

39. See $\S 6.3$ for a discussion of conditional attributes.

40. See $\S 6.2 .4$.

41. [1924] $1 \mathrm{~KB} 1$.

42. [1926] $2 \mathrm{~KB}$ and 474.

43. [1979] FSR 1.

44. [1940] AC 491.

45. [1946] VLR 338.

46. [1962] NSWR 405.

47. [1964-65] NSWR 138.

48. (1975) 133 CLR 1.

49. [1981] 1 NSWLR 251.

50. [1917-23] MacGillivray's Copyright Cases 309.

51. In the Authorization area, $w_{5}>w_{6}>w_{1}>w_{2}>w_{4}>w_{3}=w_{7}$ (where $w_{i}$ is the weight of $A_{i}$ ).

52. [1982] Ch 91.

53. id.

54. [1945] AC 108, a decision of the Judicial Committee of the Privy Council on appeal from the Supreme Court of Canada.

55. [1982] Ch 91 at 95.

56. ibid. at $95-6$.

57. (1987) AIPC $990-428$.

58. ibid. at $32764-5$. 
59. ibid. at 37764 .

60. ibid. at 37766 .

61. [1981] 1 NSWLR 251 at 257, per Kearney J.

62. [1988] AC 1013.

63. (1990) AIPC $990-718$.

64. ibid. at 36648 .

65. id.

66. In the Authorization specification, a value of yes for $A_{7}$ in the Authorization area is directed towards Auth or Liable.

67. Creighton, Ford and Mitchell 1983 at 25.

68. Brooks 1990 at 1.

69. id.

70. Creighton et al. 1983 at 25 .

71. This simplified statement of the law is incorporated in the help strings for $A_{1}$ and $A_{2}$ in the Authorization specification (see §5.3.2).

72. (1978) 21 ALR 388 at 399-402, per Smithers, Evatt and Keely JJ.

73. Creighton et al. 1983 at $25-44$.

74. Brooks 1990; Smith 1992.

75. Phillipa Weeks, Senior Lecturer, Faculty of Law, The Australian National University.

76. Creighton et al. 1983 state the law as it was on 31 March 1982.

77. "Pay as you earn."

78. (1940) 14 ALJ 162.

79. (1980) 29 ALR 322.

80. (1978) 21 ALR 388.

81. (1944) 69 CLR 227.

82. (1945) 70 CLR 539.

83. [1968] 2 QB 497.

84. (1955) 93 CLR 561.

85. (1949) 79 CLR 389.

86. [1952] 1 TLR 101.

87. [1924] 1 KB 762.

88. [1976] 1 WLR 1213.

89. (1978) 18 ALR 385.

90. [1978] ICR 590.

91. In the Employee area, $w_{13}=w_{14}=w_{16}>w_{17}>w_{15}>w_{2}=w_{3}=w_{10}>w_{8}>$ $w_{18}>w_{12}>w_{6}>w_{1}>w_{9}>w_{4}>w_{5}>w_{7}>w_{11}$ (where $w_{i}$ is the weight of $A_{i}$ ).

92. (1983) 50 ALR 417.

93. (1986) 160 CLR 16.

94. ibid. at 24, 26, per Mason J (with whom Brennan and Deane JJ generally agreed); at 38 , per Wilson and Dawson JJ.

95. (1989) 34 IR 179.

96. (1954) 94 CLR 409.

97. [1985] VR 577.

98. (1989) 34 IR 179 at 181. 
99. There were five different owner-drivers in Re Porter; Re TWU, and the circumstances for all five were very similar. These attribute values are for the first driver mentioned in the judgment-Newman: (1989) 34 IR 179 at 188-92.

The facts of Re Porter; Re TWU are summarized in the third paragraph of SHYSTER's report file in $\S$ B.4: "In the instant case ..."

1. (1991) 99 ALR 735.

2. In this matter, Odco was trading as "Trouble Shooters Available."

3. (1991) 99 ALR 735 at 736 .

4. ibid. at $752-6$.

5. ibid. at 756 .

6. The facts of Building Workers' Industrial Union of Australia v. Odco Pty Ltd are summarized in the third paragraph of SHYSTER's report file in $\S$ C.13: "In the instant case ..."

7. Individual Employment Law, 1991, Faculty of Law, The Australian National University, question 4 .

8. [1976] 1 WLR 1213 at $1217-19$.

9. These paradoxes could not be removed from the search space by giving $A_{13}$ and $A_{14}$ known values in the initial fact vector. It is only a YES/YEs pair which is impossible; the other three combinations are possible.

10. Generated test 5 contributes $80 \%$ of the instantiations in these three tests.

11. Clark and Wedderburn 1983 at 153.

12. McMillan 1991 at 63.

13. (1985) 159 CLR 550 at 584.

14. McMillan 1991 at 68.

15. Either term can be used: e.g. in Haoucher v. Minister of State for Immigration and Ethnic Affairs (1990) 169 CLR 648, McHugh J used "legitimate" and Deane J used "reasonable."

16. Cole v. Cunningham (1983) 49 ALR 123 at 131, per Bowen CJ, Sheppard and Morling JJ.

17. McMillan 1991 at 70, citing (1990) 169 CLR 648 at 653.

18. (1987) 75 ALR 218 at 251.

19. [1967] 2 AC 337.

20. (1977) 137 CLR 396.

21. (1982) 151 CLR 342.

22. McMillan 1991 at 71.

23. ibid. at $71-4$.

24. Twist v. Council of Municipality of Randwick (1976) 136 CLR 106.

25. ibid. at 117. The section references are to the Local Government Act 1919 (NSW).

26. (1985) 62 ALR 253 at 266.

27. (1986) 67 ALR 77 at 81.

28. (1990) 21 ALD 730 at 735 .

29. (1991) 24 ALD 768.

30. Robin Creyke, Senior Lecturer, Faculty of Law, The Australian National University.

31. McMillan 1991.

32. The significance of this attribute is discussed in $\S 5.5 .1$. For reasons discussed there, the specified directions direct a value of YEs towards Not-Implied, and a value of NO 
towards Implied. Note that there are only two leading cases in the Natural area with a value of yes for $A_{6}$-Commissioner of Police $v$. Tanos and Marine Hull 8 Liability Insurance Co. Ltd v. Hurford - and natural justice was implied in both, but for other reasons.

33. (1990) 169 CLR 648.

34. [1983] 2 AC 629.

35. (1982) 151 CLR 342.

36. (1990) 169 CLR 648.

37. (1990) 170 CLR 596.

38. (1985) 159 CLR 550.

39. (1958) 98 CLR 383.

40. (1987) 163 CLR 378.

41. (1981) 38 ALR 93.

42. (1986) 67 ALR 77.

43. (1987) 75 ALR 218.

44. (1987) 9 NSWLR 268.

45. (1981) 36 ALR 215.

46. [1983] 2 AC 629.

47. [1967] 2 AC 337.

48. [1985] AC 374.

49. [1978] 3 All ER 211.

50. (1990) 169 CLR 648.

51. (1983) 49 ALR 123.

52. (1976) 136 CLR 106.

53. (1977) 137 CLR 396.

54. Annetts v. McCann $\left(C_{3}\right)$ and McInnes v. Onslow Fane $\left(C_{15}\right)$, Macrae v. AttorneyGeneral for New South Wales $\left(C_{7}\right)$ and South Australia v. O'Shea $\left(C_{10}\right)$, and Durayappah v. Fernando $\left(C_{9}\right)$ and Nashua Australia Pty Ltd v. Channon $\left(C_{13}\right)$.

55. In the Natural area, $w_{1}=w_{7}>w_{2}=w_{6}>w_{5}>w_{4}>w_{3}$ (where $w_{i}$ is the weight of $A_{i}$ ).

56. This is the basis of generated test 7 (see $\S 5.5 .4$ ).

57. In the Affected area, $w_{2}=w_{3}>w_{1}=w_{4}$.

58. In the Expectation area, $w_{4}>w_{3}=w_{6}>w_{1}=w_{5}>w_{2}$.

59. (1992) 175 CLR 564.

60. (1976) 136 CLR 106.

61. [1964] AC 40.

62. (1890) 24 QBD 703.

63. (1976) 136 CLR 106 at 111-12, per Barwick CJ; at 117, per Mason J.

64. ibid. at $118-19$.

65. (1977) 137 CLR 396.

66. [1969] 2 Ch 149.

67. (1977) 137 CLR 396, per Barwick CJ, Gibbs, Stephen, Jacobs and Aickin JJ (Murphy J dissenting).

68. ibid. per Barwick CJ, Gibbs and Aickin JJ.

69. (1977) 137 CLR 487.

70. (1983) 49 ALR 123. 
71. [1985] 2 NSWLR 239.

72. ibid. at 246 .

73. (1989) 18 ALD 536.

74. ibid. at 537, per Thomas J (with whom Shepherdson and Williams JJ generally agreed).

75. (1991) 24 ALD 768.

76. ibid. at 770 .

77. ibid. at 768 .

78. See $\S 6.3$ for a discussion of conditional attributes.

79. (1992) 175 CLR 564.

80. ibid. at 578, per Mason CJ, Dawson, Toohey and Gaudron JJ; at 585, per Brennan J.

81. A value of No for $A_{1}$, an external attribute in the Natural area, is achieved as a result of a fact vector of (NNNNNN) in the Affected area. Similarly, a value of No for $A_{4}$, an external attribute in the Affected area, is achieved as a result of a fact vector of (NNNN) in the Expectation area.

82. The way in which $A_{1}$ in the Natural area is given a value of no is explained in the previous note. Similarly, fact vectors of (YYYYYY) and (YYYY) for the Affected and Expectation areas result in a value of yes for $A_{1}$ in the Natural area.

83. McMillan 1991 at 71-4. See $§ 5.5 .1$.

84. Neither $I$ nor $\mu$ appear in figure 5.12. They appear in the figures in appendix D which summarize the reflexive testing, so they are explained here with the other symbols.

85. The Natural specification has 15 cases in the Implied area, 9 in the Affected area and 8 in the Expectation area. There are 17 different cases in the specification.

86. For example, Donaldson LJ's discussion of finder cases in Parker v. British Airways (see $\S 5.2 .1$ and $\S 5.2 .3$ ), and Gummow J's discussion of authorization cases in WEA International Inc. v. Hanimex Corporation Ltd (see §5.3.3).

87. (1989) 34 IR 179.

\section{Chapter 6: Conclusion}

88. Stone $1964 \mathrm{~b}$ at 556 .

89. (1637) 3 How St Tr 825 at 969.

90. Eliot 1963 at 55 .

91. Susskind $1989 \mathrm{~b}$ at 24 .

92. Kowalski and Sergot 1990 at 208.

93. id.

94. Moles 1991 at $162-3$.

95. See $§ 3.13 .3$.

96. Ashley 1990 at 127.

97. Ashley 1985 at 116, as quoted in $\S 2.5$. See also Ashley and Aleven 1991.

98. The distinction between private and public law is discussed in $§ 3.3 .1$.

99. Mendelson 1989.

1. ibid. at 137 . 
2. The hypothetical test cases are also difficult, although the ideal points (which are also used as test cases) are not.

3. The harshness of this method is mitigated somewhat by taking the legal expert's opinion into account (see, for example, the discussions on Cole v. Cunningham and Ackroyd v. Whitehouse (Director of National Parks $\mathcal{G}$ Wildlife Service) in §5.5.3).

4. Note the comments on the NATURAL specification in $\S 6.2 .2$.

5. See the discussion on $A_{5}$ in the Authorization area of the Authorization specification $(\S 5.3 .2)$.

6. SHYSTER's use of precision thresholds is discussed in $§ 4.12 .1$.

7. See $\S 5.2 .2, \S 5.3 .2, \S 5.4 .2$ and $\S 5.5 .2$.

8. $\quad$ See $\S 3.7$.

9. The rank of courts is used as the first step in the resolution of equidistance (§3.10.3), and when referring to the relative importance of different leading cases (see $\S 4.13$ ).

10. See $\S 2.1 .2$.

11. (1975) 133 CLR 1 at 13, per Gibbs J. This is the basis of generated test 3 (see $§ 5.3 .4)$.

12. See, for example, the discussion on Western Australia v. Bropho in $§ 5.5 .3$.

13. LEXIS: Stanley 1980; WESTLAW: Herman 1980.

14. Ashley 1990 at 254, capitalization added.

\section{Appendix A: Case law specifications}

15. Act I; Gilbert 1977 at 212.

16. No. 125, 28 May 1751, emphasis omitted; Johnson 1823 at 344.

\section{Appendix B: Example reports}

17. Douglas 1948 at 105.

18. Quoted by Boswell 1960 at 342 .

19. [1982] 1 All ER 834.

20. (1990) AIPC $990-718$.

21. (1989) 34 IR 179.

22. (1992) 175 CLR 564.

23. The REPORTER module will not summarize the same case twice in the same report file. However, there is some repetition in $\S$ B.5, because there are three separate report files for Ainsworth v. CJC - one for each area. (Minister for Arts Heritage and Environment v. Peko-Wallsend Ltd (1987) 75 ALR 218 and Annetts v. McCann (1990) 170 CLR 596 are both summarized twice.)

24. (1991) 99 ALR 735.

\section{Appendix C: A complete example}

25. Hepple 1986 at 71.

26. Carroll 1982 at 19.

27. (1991) 99 ALR 735.

28. The complete $\mathrm{LAT}_{\mathrm{E}} \mathrm{X}$ input is shown for the weights file in $\S \mathrm{C} .8$. 


\section{Appendix D: Reflexive tests}

29. 193 US 197 at 400.

30. Quoted by Lawlor 1963 at 344 .

31. [1945] $1 \mathrm{~KB} 509$.

32 . ibid. at $520-1$.

33. (1851) 21 LJQB 75.

34. ibid. at $77-8$.

35. Tyree, Greenleaf and Mowbray 1989 at 49.

36. (1722) 1 Str 505.

37. [1969] 2 QB 152.

38. ibid. at 157 .

39. [1963] 1 WLR 982.

40. ibid. at $986-7$.

41. ibid.

42. [1896] 2 QB 44.

43. ibid. at $46,48$.

44. (1886) $33 \mathrm{ChD} 562$.

45. (1975) 133 CLR 1.

46. ibid. at 12, per Gibbs J; at 20-1, per Jacobs J (with whom McTiernan ACJ agreed).

47. ibid. at 13 .

48. [1964-65] NSWR 138.

49. ibid. at $149-50$.

50. ibid. at 140 .

51. [1946] VLR 338.

52. ibid. at 355 .

53. [1940] AC 491.

54. [1926] 2 KB 474.

55. ibid. at 491.

56. [1981] 1 NSWLR 251.

57. [1924] $1 \mathrm{~KB} 1$.

58. [1979] FSR 1.

59. ibid. at 9-10.

60. [1962] NSWR 405.

61. (1955) 93 CLR 561.

62. (1940) 14 ALJ 162.

63. (1944) 69 CLR 227.

64. (1980) 29 ALR 322.

65. [1952] 2 All ER 956.

66. [1976] 1 WLR 1213.

67. [1969] 2 QB 173.

68. [1952] 1 TLR 101.

69. [1924] $1 \mathrm{~KB} 762$.

70. (1949) 79 CLR 389. 
71. ibid. at 396-7, per Latham CJ; at 399, per Rich J; at 405, per Dixon J. Latham CJ also followed Performing Right Society Ltd v. Mitchell \& Booker (Palais de Danse) Ltd.

72. (1945) 70 CLR 539.

73. (1927) 33 Argus LR 321.

74. (1978) 21 ALR 388.

75. (1978) 18 ALR 385.

76. ibid. at 391.

77. [1978] ICR 590.

78. ibid. at 596, per Lord Denning MR; at 597, per Lawton LJ; Eveleigh LJ agreed with both.

79. [1952] 1 TLR 101.

80. [1968] 2 QB 497.

81. ibid. at 525 .

82. (1982) 151 CLR 342.

83. (1990) 169 CLR 648.

84. Deane, Toohey and McHugh JJ (Dawson and Gaudron JJ dissenting).

85. (1990) 169 CLR 648 at 651, per Deane J; at 679, per McHugh J.

86. (1990) 170 CLR 596.

87. Note, however, that Brennan and Toohey JJ dismissed the appeal on the basis that the decision of the Full Court of the Supreme Court of Western Australia (from which the Annettses had appealed) was right on the material before it.

88. (1990) 170 CLR 596 at 598, per Mason CJ, Deane and McHugh JJ; at 606, per Brennan J.

89. (1985) 159 CLR 550.

90. ibid. at 563, 567, per Gibbs CJ; at 582-3, 587 per Mason J; at 610, 616-7, per Brennan J.

91. (1958) 98 CLR 383.

92. (1986) 67 ALR 77.

93. ibid. at 88 .

94. (1987) 9 NSWLR 268.

95. [1983] 2 AC 629.

96. [1967] 2 AC 337.

97. (1987) 163 CLR 378.

98. (1981) 38 ALR 93.

99. (1987) 75 ALR 218.

1. (1981) 36 ALR 215.

2. ibid. at 229 .

3. [1985] AC 374.

4. [1978] 3 All ER 211. 


\section{Cases}

Page numbers set in boldface type refer to summaries of case details. Page numbers set in italics refer to SHYSTER output. " $400_{3}$ " refers to note 3 on page 400. " $150_{5.6}$ " refers to figure 5.6 on page 150 .

Leading cases in SHYSTER specifications are marked with a star. Test cases are marked with a diamond.

* A\&M Records Inc. v. Audio Magnetics Inc. (UK) Ltd [1979] FSR 1: 146, 148, 149, $150_{5.6}, 151,152,224,357-359,360,360_{\mathrm{D} .2}$

$\diamond$ Ackroyd v. Whitehouse (Director of National Parks \& Wildlife Service) [1985] 2

NSWLR 239: 184, 185, 1915.12, 400 3

Adelaide Corporation v. Australasian Performing Right Association Ltd (1928) 40

CLR 481: $395_{27}, 395_{30}$

AG of Hong Kong v. Shiu: see Attorney-General of Hong Kong v. Ng Yuen Shiu

$\diamond$ Ainsworth v. Criminal Justice Commission (1992) 175 CLR 564: 179, 187, 188,

$191_{5.12}, 284,302-310,400_{23}$

Amoco Production Co. v. Lindley (1980) 609 P 2d 733: 389

AMP v. Chaplin: see Australian Mutual Provident Society v. Chaplin

* Annetts v. McCann (1990) 170 CLR 596: 176, 183, 184, 187, 188, 254, 268, 305,

306, 308, 309, 367, 369, 370, 371 D. $, 398_{54}, 400_{23}$

APRA v. Canterbury-Bankstown: see Australasian Performing Right Association

Ltd v. Canterbury-Bankstown League Club Ltd

APRA v. Jain: see Australasian Performing Right Association Ltd v. Jain

APRA v. Miles: see Australasian Performing Right Association Ltd v. Miles

* Armory v. Delamirie (1722) 1 Str 505: 132, 133, 136, 210, 211, 354, 355, 356 D.1, $394_{13}, 394_{14}$

* Attorney-General of Hong Kong v. Ng Yuen Shiu [1983] 2 AC 629: 175, 176, 182, 258, 279, 280, 367, 368, 371 D.4 
404

$[\mathrm{ATW}-\mathrm{DAV}] \quad$ Cases

ATWU v. Monaro Sawmills: see Australian Timber Workers Union v. Monaro

Sawmills Pty Ltd

* Australasian Performing Right Association Ltd v. Canterbury-Bankstown League Club Ltd [1964-65] NSWR 138: 146, 219, 220, 357, 358, 360 $2.2,361$

$\diamond$ Australasian Performing Right Association Ltd v. Jain (1990) AIPC 990-718: 152, 1915.12, 284, 289-295, 394 24

* Australasian Performing Right Association Ltd v. Miles [1962] NSWR 405: 146, 149, 1505.6, 151-153, 225, 291, 292-295, 357-360, 360 D.2, 361

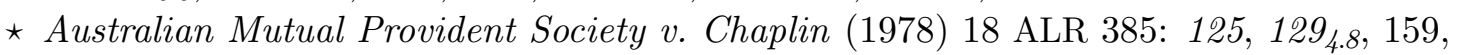
161, 163, 243, 244, 314, 315, 327, 351, 352, 364, 365 D.3, 366

* Australian Timber Workers Union v. Monaro Sawmills Pty Ltd (1980) 29 ALR 322: 159, 234, 235, 328, 361, 362, 363, 365 D.3

$B$ v. $B$ [1978] 1 All ER 821: 18

Bank Voor Handel en Scheepvaart NV v. Slatford [1952] 2 All ER 956: 362

Barro Group Pty Ltd v. Fraser [1985] VR 577: 163

Betts v. Brady (1942) 316 US 455: 24

* Bread Manufacturers of New South Wales v. Evans (1981) 38 ALR 93: 176, 180, 185, 187, 260, 261, 270, 306, 307, 369, 371 D.4, 386 25

* Bridges v. Hawkesworth (1851) 21 LJQB 75: 133, 136, 138-140, 1415.3, 209, 210, 214, 215, 284, 285-289, 354, 355, 356, 356 $\mathrm{D} .1,389_{4}, 393_{89}$

Bropho v. Western Australia (1990) 21 ALD 730: 172

$\diamond$ Building Workers' Industrial Union of Australia v. Odco Pty Ltd (1991) 99 ALR 735: $122,1234.7,124-126,128,129_{4.8}, 164,165,166,169,191_{5.12}, 284,312,312-316$, 335-352, $397_{6}$

BWIU v. Odco: see Building Workers' Industrial Union of Australia v. Odco Pty Ltd

* Cam and Sons Pty Ltd v. Sargent (1940) 14 ALJ 162: 126, 159, 163, 232, 233, 314, 315, 321, 349, 350, 361, 362-364, 365 $\mathrm{D} .3,366$

Cantliff v. Jenkins [1978] 1 All ER 836: 18, $377_{68}$

$\diamond$ CBS Inc. v. Ames Records and Tapes Ltd [1982] Ch 91: 147, 148, 149, 1505.6, 151, $191_{5.12}$

$\diamond$ CBS Songs Ltd v. Amstrad Consumer Electronics plc [1988] AC 1013: 151, $191_{5.12}$ CCSU v. Minister for the Civil Service: see Council of Civil Service Unions v.

Minister for the Civil Service

* City of London Corporation v. Appleyard [1963] 1 WLR 982: 136, 140, 1415.3, 212, 213, 285, 286, 287-289, 354, 355, 356, 356 D.1 $_{1} 394_{11}, 394_{14}, 394_{2}$

$\star \diamond$ Cole v. Cunningham (1983) 49 ALR 123: 176, 183-187, 1915.12, 27455, 279, 302 1 , 303, 304, 397 $16,400_{3}$

* Commissioner of Police v. Tanos (1958) 98 CLR 383: 176, 180, 1815.11, 182, 185, 255, 271, 367, 371 D., $398_{32}$

* Council of Civil Service Unions v. Minister for the Civil Service [1985] AC 374: 176, 184, 185, 263, 272, 370, 371 D.4

Crown Industries, Inc. v. Kawneer Co. (1971) 335 F Supp 749: 3895

Daniels and Daniels v. R. White and Sons and Tarbard [1938] 4 All ER 258: $377_{70}$ Davis v. Johnson [1978] 1 All ER 841: $377_{69}$ 
* Durayappah v. Fernando [1967] 2 AC 337: 171, 176, 258, 259, 368, 369, 370, 371 D.4, $398_{54}$

Eisner v. Macomber (1920) 252 US 189: $380_{71}, 422$

* Elwes v. Brigg Gas Co. (1886) 33 ChD 562: 133, 136, 215, 354-356, 356 D.1

* FAI Insurances Ltd v. Winneke (1982) 151 CLR 342: 171, 176, 183-186, 252, 267, 276, 366, 367, 368, 371 D.4

* Falcon v. Famous Players Film Co. [1926] 2 KB 474: 143, 146, 148, 149, 1505.6, 151-153, 2161, 221, 222, 2891, 357, 358, 359, 360, 360 D.2

FCT v. Thompson: see Federal Commissioner of Taxation v. J. Walter Thompson (Australia) Pty Ltd

* Federal Commissioner of Taxation v. J. Walter Thompson (Australia) Pty Ltd (1944) 69 CLR 227: 159, 233, 234, 322, 362, 365 $\mathrm{D} .3$

* Ferguson v. John Dawson ES Partners (Contractors) Ltd [1976] 1 WLR 1213: 126, 128, 1294.8, 159, 161, 162 $5.7,164-166,236,296,297-302,313-315,326,345$, 347, 348, 350-352, 361, 362, 363, 364, 365 D.3

Gideon v. Wainright (1963) 372 US 335: 24

$\star$ Hannah v. Peel [1945] 1 KB 509: 133, 136, 209, 288, 289, 354, 355, 356, 356 D.1 $_{\text {D }}$

* Haoucher v. Minister of State for Immigration and Ethnic Affairs (1990) 169 CLR 648: 171, 175, 176, 180, 182, 185, 186, 253, 267, 268, 277, 366, 367, 368, 371 D.4, $397_{15}$

$\star \diamond$ Heatley v. Tasmanian Racing and Gaming Commission (1977) 137 CLR 487: 176, 180, 183, 184, 191 $5.12,278$

Hibbert v. McKiernan [1948] 2 KB 142: 39394

$\diamond$ Hodgens v. Gunn (1989) 18 ALD 536: 185, $191_{5.12}$

* Humberstone v. Northern Timber Mills (1949) 79 CLR 389: 124, 124-126, 128, 1294.8, 159, 163, 164, 240, 313-315, 323, 344-346, 347-350, 361-363, 364, 365 D.3

* Kioa v. West (1985) 159 CLR 550: 170, 176, 182, 183, 2501, 255, 270, 278, 30820, $\mathbf{3 6 7}, 368,371_{\mathrm{D} .4}$

Logan v. Gilchrist, Watt and Cunningham (1927) 33 Argus LR 321: 364

London v. Appleyard: see City of London Corporation v. Appleyard

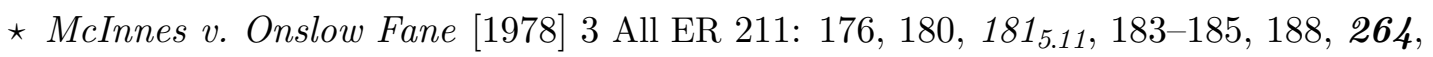
309, 367, 370, 371 D., $398_{54}$

* Macrae v. Attorney-General for New South Wales (1987) 9 NSWLR 268: 176, 257, 310, 368, 369, 371 D.4, 39854

Marine Hull \& Liability Insurance Co. Ltd v. Hurford (1985) 62 ALR 253: 172

* Marine Hull \&3 Liability Insurance Co. Ltd v. Hurford (1986) 67 ALR 77: 172, 176, 187, 256, 367, 371 D.4, $398_{32}$

Market Investigations Ltd v. Minister of Social Security [1969] 2 QB 173: 362

* Massey v. Crown Life Insurance Co. [1978] ICR 590: 127, 159, 161, 162 $5.7,165,166$, 244, 245, 326, 362, 363, 364, 365, 365 D.3

* Mellor v. Australian Broadcasting Commission [1940] AC 491: 146, 152, 221, 290, 291-294, 357, 358, 359, 360 D.2 
406

[MIN-SOU] Cases

* Minister for Arts Heritage and Environment v. Peko-Wallsend Ltd (1987) 75 ALR

218: 171, 176, 180, 182-188, 2502, 261, 262, 273, 281, 302, 303-305, 306, 307, $308_{21}, \mathbf{3 6 9}, 371_{\mathrm{D} .4}, 400_{23}$

* Moffatt v. Kazana [1969] 2 QB 152: 136, 137, 211, 355, 356 D.1 $_{1}, 391_{59}$

$\diamond$ Narich Pty Ltd v. Commissioner of Pay-roll Tax (1983) 50 ALR 417: 160, 162.7.7, $191_{5.12}, 387_{73}, 393_{81}$

* Nashua Australia Pty Ltd v. Channon (1981) 36 ALR 215: 176, 262, 263, 366, 368-370, 371 D.4, 398

Northern Securities Co. v. United States (1904) 193 US 197: 353

$\diamond$ Parker v. British Airways [1982] 1 All ER 834: 133, 138, 139, 140, 141 $1_{5.3}, 191_{5.12}$, 284, 284-289, 39986

Peko-Wallsend Ltd v. Minister for Arts Heritage and Environment (1986) 70 ALR 523: 262 $25,273_{51}, 282_{68}, 303_{3}, 306_{13}$

Performing Right Society Ltd v. Bradford Corporation [1917-23] MacGillivray's Copyright Cases 309: 146, 359

* Performing Right Society Ltd v. Ciryl Theatrical Syndicate Ltd [1924] 1 KB 1: 143, 146, 223, 293, 294, 358, 359, 360 D.2

* Performing Right Society Ltd v. Mitchell 83 Booker (Palais de Danse) Ltd [1924] 1

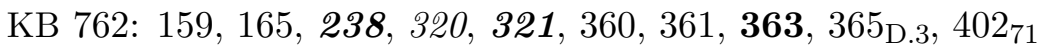

$\diamond$ Porter, Re; Re Transport Workers Union of Australia (1989) 34 IR 179: 163, 164, $191_{5.12}, 192,284$, 296-302, 393 $83,397_{99}$

* Price v. Grant Industries Pty Ltd (1978) 21 ALR 388: 157, 159, 242, 243, 327, 361, $363, \mathbf{3 6 4}, 365_{\mathrm{D} .3}$

PRS v. Bradford: see Performing Right Society Ltd v. Bradford Corporation

PRS v. Ciryl: see Performing Right Society Ltd v. Ciryl Theatrical Syndicate Ltd

PRS v. Palais de Danse: see Performing Right Society Ltd v. Mitchell $\&$ Booker (Palais de Danse) Ltd

Queensland v. Commonwealth of Australia (1977) 139 CLR 585: $376_{23}$

* Queensland Stations Pty Ltd v. Federal Commissioner of Taxation (1945) 70 CLR 539: 113, 117, 159, 241, 242, 322, 363, 364, 365, 365

$R v$. Hampden (1637) 3 How St $\operatorname{Tr} 825: 195$

$\star$ RCA Corporation v. John Fairfax and Sons Ltd [1981] 1 NSWLR 251: 146, 149, 151-153, 222, 223, 290, 291, 292, 295, 357, 359, 360, 360‥2, 361, 394 24

* Ready Mixed Concrete (South East) Ltd v. Minister of Pensions and National Insurance [1968] 2 QB 497: 125, 127, 159, 163, 164, 247, 297, 298-302, 315, 325, 351, 352, 362-364, 365 $\mathrm{D} .3, \mathbf{3 6 6}, 391_{59}$

Ridge v. Baldwin [1964] AC 40: 179

SA v. O'Shea: see South Australia v. O'Shea

$\diamond$ Salemi v. MacKellar (No. 2) (1977) 137 CLR 396: 92, 171, 176, 182, 183, 189, $191_{5.12}, 38897$

Schmidt v. Secretary of State for Home Affairs [1969] 2 Ch 149: 182

* South Australia v. O'Shea (1987) 163 CLR 378: 176, 180, 182-184, 259, 260, 269, 280, 281, 309, 310, 367-369, 370, 371 D.4, 39854 
* South Staffordshire Water Co. v. Sharman [1896] 2 QB 44: 133, 136, 138, 140, 213, 214, 285-287, 288, 354-356, 356 D.1 $_{1} 394_{2}$

$\diamond$ Stevens v. Brodribb Sawmilling Company Pty Ltd (1986) 160 CLR 16: 163, 164, 165, $169,191_{5.12}$

* Stevenson Jordon and Harrison Ltd v. Macdonald and Evans [1952] 1 TLR 101: 127, $1_{4.8}, 159,165,237,245,246,314,315,323,324,361-\mathbf{3 6 3}, 364, \mathbf{3 6 5}, 365_{\mathrm{D} .3}$

Structural Dynamics Research Corporation v. Engineering Mechanics Research Corporation (1975) 401 F Supp 1102: $389_{5}$

Timmons v. McCall (1939): 131

$\diamond$ Twist v. Council of Municipality of Randwick (1976) 136 CLR 106: 172, 176, 179, $181_{5.11}, 182,184,189,191_{5.12}, 388_{97}, 397_{24}$

* University of New South Wales v. Moorhouse (1975) 133 CLR 1: 143, 146, 148, 149, 152, 153, 216 2 219, 2194, 290 2 295, 29512, 357, 358, 359, 360 D.2, 394 24 UNSW v. Moorhouse: see University of New South Wales v. Moorhouse USM Corporation v. Marson Fastener Corporation (1979) 379 Mass 90: $389_{5}$

Vestry of St James and St John, Clerkenwell v. Feary (1890) 24 QBD 703: 179

Vigneux v. Canadian Performing Right Society Ltd [1945] AC 108: 148

WA v. Bropho: see Western Australia v. Bropho

$\diamond$ WEA International Inc. v. Hanimex Corporation Ltd (1987) AIPC 990-428: 149, 151, $191_{5.12}, 394_{24}, 399_{86}$

$\diamond$ Western Australia v. Bropho (1991) 24 ALD 768: 172, 186, 187, 1915.12, 400 12

Western Australia v. Commonwealth of Australia (1975) 134 CLR 201: $376_{23}$

* Winstone v. Wurlitzer Automatic Phonograph Co. of Australia Pty Ltd [1946] VLR 338: 146, 148, 220, 357, 358, 360 $\mathrm{D} .2,361$

Wright v. Attorney-General for Tasmania (1954) 94 CLR 409: 163

* Zuijs v. Wirth Brothers Pty Ltd (1955) 93 CLR 561: 159, 231, 232, 325, 331, 361, $363,364,365_{\text {D. } 3}, 385_{22}$ 



\section{Statutes}

Page numbers set in boldface type refer to statute extracts. Page numbers set in italics refer to SHYSTER output. "395 30 " refers to note 30 on page 395.

Aboriginal Heritage Act 1972 (WA): 186 Administrative Decisions (Judicial Review) Act 1977 (Cth): 423 Animals Protection Act 1925 (Qld)

s. 11(4): 185

s. 19(2): 185

British Nationality Act 1981 (UK): 32, $33,39,427$

Copyright Act 1911 (UK): 142, 146

s. 1(2): 142, 143, 221, 222, 224, 290, 293

s. 2(1): 225, 291

s. $2(3)$ : 239, $321,395_{30}$

s. 5(1)(b): 237, 246, 324

Copyright Act 1912 (Cth): 142, 146, 220, 221, 225, 290, 291, 395 30

Copyright Act 1956 (UK)

s. 1(2): 148

Copyright Act 1968 (Cth): 140, 142, 144, 146, 149, 155

s. 13(2): 140, 155, 223, 291

s. 36(1): 140, 142, 155, 219, 295

s. 39 A: 2194,29512

s. 101(1): 140, 142, 155
Coroner's Act 1920 (WA): 254, 268, 305, 308

Criminal Law Consolidation Act, 1935 (SA)

s. $77 \mathrm{a}(7 \mathrm{a}): 260,269,281,310$

Customs Act 1966 (Cth)

s. 273: 263

Disorderly Houses Act 1943 (NSW) s. 3(1)(b): 255, 271

Domestic Violence and Matrimonial Proceedings Act 1976 (UK) s. $13(2): 377_{66}$

Insurance Act 1973 (Cth)

s. $62: 256$

s. $63: 256$

Justices Act 1902 (NSW): 257, 310

Law of Property Act 1965 (UK) s. 62: 212

Local Courts Act 1982 (NSW): 257, 310

Local Government Act 1919 (NSW):

$397_{25}$

s. $317 \mathrm{~B}: 172,179$ 
Long Service Leave Act, 1967 (SA): 244 , 327, 351

Migration Act 1958 (Cth): 182, 255, 270, 278

s. 12: 253, 268, 277

s. 18: 182

Municipal Councils Ordinance 1947

(Ceylon)

s. $277(1): 259$

National Insurance Act 1965 (UK)

s. 1(2): 248, 297, 325, 352

National Parks and Wildlife Act 1974 (Cth)

s. $134(1): 184$

s. $135: 184$

National Parks and Wildlife

Conservation Act 1975 (Cth)

s. 8B: 262, 273, 282, 303, 305

Pay-roll Tax Act 1971 (NSW): 161

Pay-roll Tax Assessment Act 1941

(Cth): 234, 242, 322, 323
Prices Regulation Act 1948 (NSW): 261, 270, 307

Racing and Gaming Act 1952 (Tas) s. $39(3): 278$

Supplementary Benefits Act 1976 (UK): 32

Trade Practices Act 1974 (Cth) s. 45D: 164

Trade Union and Labour Relations Act 1974 (UK): 245, 326

Worker's Compensation Act 1926

(NSW): 232, 325, 331

Worker's Compensation Act 1928 (Vic) s. 3: 241, 323, 347

Worker's Compensation Act 1958 (Vic): 252, 267, 276

World Heritage Properties Conservation Act 1983 (Cth) s. 3(2): 262, 273, 281, 303, 305 


\section{Bibliography}

Aldenderfer, Mark S. and Blashfield, Roger K. 1984, Cluster Analysis, Sage University Paper Series on Quantitative Applications in the Social Sciences, no. 07-044, Sage, Beverly Hills. ISBN 0803923767.

Allen, Layman E. 1963, "Beyond document retrieval toward information retrieval", Minnesota Law Review, vol. 47, pp. 713-67. ISSN 0026-5535.

AlLen, Layman E. 1965, "Usefulness of modern logic to the readers and writers of legal documents", in Communication Sciences and Law: Reflections from the Jurimetrics Conference, eds Layman E. Allen and Mary E. Caldwell, Bobbs-Merrill, Indianapolis, ch. 13, pp. 83-112.

From the Conference on the Implications of Developments in the Communication Sciences for Legal Education in the Next Decade of the Jurimetrics Committee of the Association of American Law Schools, Yale Law School, New Haven, Connecticut, 5-7 September 1963.

AlLen, Layman E. 1968, "A language-normalization approach to information retrieval in law", Jurimetrics Journal, vol. 9, no. 1, September, pp. 41-56. ISSN 0022-6793.

Allen, Layman E. 1981, "Analysis of law by symbolic logic", in Computers and the Law: An Introductory Handbook (third edition), ed. Robert P. Bigelow, American Bar Association, pp. 84-90.

AlLen, Layman Edward 1982, "Towards a normalized language to clarify the structure of legal discourse", in Deontic Logic, Computational Linguistics and Legal Information Systems, ed. Antonio A. Martino, vol. II, North-Holland, Amsterdam, pp. 349-407. ISBN 044486415 6, 044486413 X (set).

From the International Conference on "Logic, Informatics, Law", Florence, Italy, April 1981.

Allen, Layman E., Brooks, Robin B.S. and James, Patricia A. 1962, Automatic Retrieval of Legal Literature: Why and How, Walter E. Meyer Research Institute of Law, New Haven, Connecticut. 
Allen, Layman E. and Caldwell, Mary Ellen 1963, "Modern logic and judicial decision making: A sketch of one view", Law and Contemporary Problems, vol. 28, no. 1, Winter, pp. 213-70. ISSN 0023-9186.

Republished in Baade 1963.

Allen, Layman E. and SAxon, Charles S. 1985, "Computer aided normalizing and unpacking: Some interesting machine-processable transformations of legal rules", in Computing Power and Legal Reasoning, ed. Charles Walter, West Publishing Company, St Paul, ch. 20, pp. 495-572. ISBN 0314955704.

Allen, Layman and SAXon, Charles 1988, "Exploring computer-aided generation of questions for normalizing legal rules", in Computer Power and Legal Language: The Use of Computational Linguistics, Artificial Intelligence, and Expert Systems in the Law, ed. Charles Walter, Quorum, New York, ch. 19, pp. 243-316. ISBN 0899303064 .

From the Second Annual Conference on Law and Technology, University of Houston, 24-28 June 1985.

Ashley, Kevin D. 1985, "Reasoning by analogy: A survey of selected AI research with implications for legal expert systems", in Computing Power and Legal Reasoning, ed. Charles Walter, West Publishing Company, St Paul, ch. 6, pp. 105-27. ISBN 0314955704.

Ashley, Kevin D. 1989a, "Toward a computational theory of arguing with precedents: Accomodating [sic] multiple interpretations of cases", in Proceedings of the Second International Conference on Artificial Intelligence and Law (ICAIL-89), University of British Columbia, Vancouver, 13-16 June, pp. 93-102. ISBN 0897913221.

Ashley, Kevin D. 1989b, "Defining salience in case-based arguments", in Proceedings of the Eleventh International Joint Conference on Artificial Intelligence (IJCAI-89), Detroit, Michigan, 20-25 August, vol. 1, pp. 537-42. ISBN 155860094 9. ISSN 1045-0823.

Ashley, Kevin D. 1990, Modeling Legal Argument: Reasoning with Cases and Hypotheticals, Artificial Intelligence and Legal Reasoning Series, MIT Press (Bradford), Cambridge, Massachusetts. ISBN 026201114 X.

Ashley, Kevin D. and Aleven, Vincent 1991, "Toward an intelligent tutoring system for teaching law students to argue with cases", in Proceedings of the Third International Conference on Artificial Intelligence and Law (ICAIL-91), St Catherine's College, Oxford, 25-28 June, pp. 42-52. ISBN 089791399 X.

Ashley, Kevin D. and Rissland, Edwina L. 1986, "Toward modelling legal argument", in Automated Analysis of Legal Texts: Logic, Informatics, Law, eds Antonio A. Martino and Fiorenza Socci Natali, Elsevier Science (North-Holland), Amsterdam, pp. 19-30. ISBN 0444701117.

From the Second International Conference on 'Logic, Informatics, Law', Florence, Italy, September 1985. 
Ashley, Kevin D. and Rissland, Edwina L. 1987a, "But, see, accord: Generating 'blue book' citations in HYPO", in Proceedings of the First International Conference on Artificial Intelligence and Law (ICAIL-87), Boston, Massachusetts, 27-29 May, pp. 67-74. ISBN 0897912306.

Ashley, Kevin D. and Rissland, Edwina L. 1987b, "Compare and contrast, a test of expertise", in Proceedings of the Sixth National Conference on Artificial Intelligence (AAAI-87), Seattle, Washington, 13-17 July, vol. 1, pp. 273-8. ISBN 0934613427.

Ashley, Kevin D. and Rissland, Edwina L. 1988, "Waiting on weighting: A symbolic least commitment approach", in Proceedings of the Seventh National Conference on Artificial Intelligence (AAAI-88), St Paul, Minnesota, 21-26 August, vol. 1, pp. 23944. ISBN 0929280008.

Aubert, Vilhelm 1963, "Conscientious objectors before Norwegian military courts", in Judicial Decision-Making, ed. Glendon Schubert, International Yearbook of Political Behavior Research, vol. 4, Free Press of Glencoe, New York, ch. vii, pp. 201-19.

Austin, John 1885, Lectures on Jurisprudence or The Philosophy of Positive Law (fifth edition), John Murray, London.

Two volumes, revised and edited by Robert Campbell.

BaAde, Hans W. (ed.) 1963, Jurimetrics, Basic Books, New York.

Originally published as Law and Contemporary Problems, vol. 28, no. 1, Winter 1963.

BAIN, William M. 1986, "A case-based reasoning system for subjective assessment", in Proceedings of the Fifth National Conference on Artificial Intelligence (AAAI-86), Philadelphia, Pennsylvania, 11-15 August, vol. 1, pp. 523-7. ISBN 0934613133.

BANKowski, Zenon and MaCCoRmick, D. Neil 1991, "Statutory interpretation in the United Kingdom", in Interpreting Statutes: A Comparative Study, eds D. Neil MacCormick and Robert S. Summers, Dartmouth Series in Applied Legal Philosophy, Dartmouth, Aldershot, ch. 10, pp. 359-406. ISBN 1855211831.

BENCH-CAPON, T. J. M. 1987, "Support for policy makers: Formulating legislation with the aid of logical models", in Proceedings of the First International Conference on Artificial Intelligence and Law (ICAIL-87), Boston, Massachusetts, 27-29 May, pp. 181-9. ISBN 0897912306.

Bench-Capon, T.J.M. 1989, "Deep models, normative reasoning and legal expert systems", in Proceedings of the Second International Conference on Artificial Intelligence and Law (ICAIL-89), University of British Columbia, Vancouver, 13-16 June, pp. 37-45. ISBN 0897913221.

Bench-Capon, T. J. M. (ed.) 1991, Knowledge-Based Systems and Legal Applications, APIC Series, no. 36, Academic Press, London. ISBN 012086441 X.

Bench-Capon, T. J. M., Robinson, G. O., Routen, T. W. and Sergot, M. J. 1987, "Logic programming for large scale applications in law: A formalisation of supplementary benefit legislation", in Proceedings of the First International Conference on Artificial Intelligence and Law (ICAIL-87), Boston, Massachusetts, 27-29 May, pp. 190-8. ISBN 0897912306. 
Bench-Capon, Trevor and Sergot, Marek 1988, "Toward a rule-based representation of open texture in law", in Computer Power and Legal Language: The Use of Computational Linguistics, Artificial Intelligence, and Expert Systems in the Law, ed. Charles Walter, Quorum, New York, ch. 6, pp. 39-60. ISBN 0899303064.

From the Second Annual Conference on Law and Technology, University of Houston, 24-28 June 1985.

Berman, Donald H. 1991, "Developer's choice in the legal domain: The Sisyphean journey with CBR or down hill with rules", in Proceedings of the Third International Conference on Artificial Intelligence and Law (ICAIL-91), St Catherine's College, Oxford, 25-28 June, pp. 307-9. ISBN 089791399 X.

A working paper for the Case-Rules Panel.

Berman, Donald H. and Hafner, Carole D. 1991, "Incorporating procedural context into a model of case-based legal reasoning", in Proceedings of the Third International Conference on Artificial Intelligence and Law (ICAIL-91), St Catherine's College, Oxford, 25-28 June, pp. 12-20. ISBN 089791399 X.

BING, Jon 1980, "Legal norms, discretionary rules and computer programs", in Computer Science and Law, ed. Bryan Niblett, Cambridge University Press, Cambridge, ch. 7, pp. 119-36. ISBN 0521234514.

Bing, Jon (ed.) 1984a, Handbook of Legal Information Retrieval, Elsevier Science (North-Holland), Amsterdam. ISBN 044487576 X.

BING, Jon 1984b, "Legal information services: Some trends and characteristics", in Data Processing and the Law, ed. Colin Campbell, Sweet and Maxwell, London, pp. 29-45. ISBN 0421320001.

BING, Jon 1987, "Designing text retrieval systems for "conceptual searching'", in Proceedings of the First International Conference on Artificial Intelligence and Law (ICAIL-87), Boston, Massachusetts, 27-29 May, pp. 43-51. ISBN 0897912306.

Bing, Jon 1989, "The law of the books and the law of the files: Possibilities and problems of legal information systems", in Advanced Topics of Law and Information Technology, ed. G.P. V. Vandenberghe, Computer/Law Series, no. 3, Kluwer Law and Taxation Publishers, Deventer, pp. 151-82. ISBN 9065443916.

BIng, Jon 1992, [Review of Ashley 1990], Artificial Intelligence and Law, vol. 1, no. 1, pp. 103-7. ISSN 0924-8463.

Bing, Jon and Selmer, Knut S. (eds) 1980, A Decade of Computers and Law, Universitetsforlaget, Oslo. ISBN 8200053768.

BJARUP, Jes 1988, "Kripke's case: Some remarks on rules, their interpretation and application", Rechtstheorie, vol. 19, pp. 39-49. ISSN 0034-1398.

Blackstone, William 1773, Commentaries on the Laws of England (fifth edition), Oxford (Clarendon).

Four volumes. 
Boden, Margaret A. 1985, "Panel: Artificial intelligence and legal responsibility", in Proceedings of the Ninth International Joint Conference on Artificial Intelligence (IJCAI-85), Los Angeles, California, 18-23 August, vol. 2, pp. 1267-8. ISBN 093461302 8. ISSN 1045-0823.

Borchgrevink, Mette and Hansen, Johannes 1980, "SARA: A system for the analysis of legal decisions", in A Decade of Computers and Law, eds Jon Bing and Knut S. Selmer, Universitetsforlaget, Oslo, ch. 18, pp. 342-75. ISBN 8200053768.

Boswell, James 1960?, The Life of Samuel Johnson, vol. I, Heron Books, London. First published in 1791.

Bottomley, Stephen, Gunningham, Neil and Parker, Stephen 1991, Law in Context, Federation Press, Leichhardt. ISBN 1862870616.

Branting, L. Karl 1989, "Representing and reusing explanations of legal precedents", in Proceedings of the Second International Conference on Artificial Intelligence and Law (ICAIL-89), University of British Columbia, Vancouver, 13-16 June, pp. 10310. ISBN 0897913221.

Branting, L. Karl 1991, "Reasoning with portions of precedents", in Proceedings of the Third International Conference on Artificial Intelligence and Law (ICAIL-91), St Catherine's College, Oxford, 25-28 June, pp. 145-54. ISBN 089791399 X.

Brooks, Adrian 1990, "Employment", in Australian Commentary on Halsbury's Laws of England (fourth edition), ed. Garfield Barwick, Butterworths, Sydney, ch. 56. ISBN 0409309303.

Buchanan, Bruce G. and Headrick, Thomas E. 1970, "Some speculation about artificial intelligence and legal reasoning", Stanford Law Review, vol. 23, no. 1, November, pp. 40-62. ISSN 0038-9765.

Buchanan, Bruce G. and Shortliffe, Edward H. (eds) 1984, Rule-Based Expert Systems: The MYCIN Experiments of the Stanford Heuristic Programming Project, Addison-Wesley Series in Artificial Intelligence, Addison-Wesley, Reading, Massachusetts. ISBN 0201101726.

Burton, Steven J. 1985, An Introduction to Law and Legal Reasoning, Little, Brown and Company, Boston. ISBN 0316117862.

CAmpbell, J.A. 1984, "The expert computer and professional negligence: who is liable?", in Artificial Intelligence: Human Effects, eds Masoud Yazdani and Ajit Narayanan, Ellis Horwood Series in Artificial Intelligence, Ellis Horwood, Chichester, pp. 37-51. ISBN 085312577 5, 0470200928.

Capper, Phillip and Susskind, Richard E. 1988, Latent Damage Law-The Expert System, Butterworths, London. ISBN 040602362 X.

Carroll, Lewis 1982, The Complete Illustrated Works of Lewis Carroll, Avenel Books, New York. ISBN 051738566 X.

Ciesielski, Victor B. 1990, Some Experiments in the Use of Expert Systems for Legislation and Regulations, Technical Report 21, Key Centre for Knowledge Based Systems, Departments of Computer Science, Royal Melbourne Institute of Technology and The University of Melbourne, 27 April. 
Clark, Andrew 1988, [Review of Susskind 1987a], Journal of Law and Society, vol. 15, no. 4, Winter, pp. 426-30. ISSN 0263-323X.

Clark, Jon and Wedderburn, Kenneth 1983, "Modern labour law: Problems, functions, and policies", in Labour Law and Industrial Relations: Building on KahnFreund, eds Kenneth Wedderburn, Roy Lewis and Jon Clark, Oxford University Press (Clarendon), Oxford, ch. 6, pp. 127-242. ISBN 0198253931.

Clarke, Roger 1988, "Legal aspects of knowledge-based technology", Journal of Information Technology, vol. 3, no. 1, March, pp. 9-16. ISSN 0268-3962.

Claudy, John G. 1972, "A comparison of five variable weighting procedures", Educational and Psychological Measurement, vol. 32, no. 2, Summer, pp. 311-22. ISSN 0013-1644.

Conover, W.J. 1971, Practical Nonparametric Statistics, John Wiley, New York. ISBN 0471168513.

Cook, Sandra, Hafner, Carole D., McCarty, L. Thorne, Meldman, Jeffrey A., Peterson, Mark, Sprowl, James A., Sridharan, N.S. and Waterman, D. A. 1981, "The applications of artificial intelligence to law: A survey of six current projects", AFIPS Conference Proceedings, vol. 50, pp. 689-96. ISSN 0449-1173.

From the National Computer Conference, Chicago, Illinois, 4-7 May 1981.

Cover, T.M. and HART, P.E. 1967, "Nearest neighbor pattern classification", IEEE Transactions on Information Theory, vol. IT-13, no. 1, January, pp. 21-7. ISSN 0018-9448.

Creighton, W.B., Ford, W.J. and Mitchell, R. J. 1983, Labour Law: Materials and Commentary, Law Book Company, Sydney. ISBN 0455204136.

Cross, Rupert 1961, Precedent in English Law, Clarendon Law Series, Oxford University Press (Clarendon), London.

Croydon, Stanley O., Jr 1980, "JURIS: A tool for legal research", in Legal and Legislative Information Processing, ed. Beth Krevitt Eres, Greenwood Press, Westport, Connecticut, ch. 11, pp. 163-72. ISBN 0313213437.

D'Amato, Anthony 1977, "Can/should computers replace judges?", Georgia Law Review, vol. 11, no. 5, September, pp. 1277-1301. ISSN 0016-8300.

DeBessonet, Cary G. and Cross, George R. 1985, "Representation of some aspects of legal causality", in Computing Power and Legal Reasoning, ed. Charles Walter, West Publishing Company, St Paul, ch. 11, pp. 205-14. ISBN 0314955704.

deBessonet, Cary G. and Cross, George R. 1986, "Conceptual retrieval and legal decision-making", in Automated Analysis of Legal Texts: Logic, Informatics, Law, eds Antonio A. Martino and Fiorenza Socci Natali, Elsevier Science (NorthHolland), Amsterdam, pp. 219-27. ISBN 0444701117.

From the Second International Conference on 'Logic, Informatics, Law', Florence, Italy, September 1985. 
DeBessonet, Cary and Cross, George 1988, "Distinguishing legal language-types for conceptual retrieval", in Computer Power and Legal Language: The Use of Computational Linguistics, Artificial Intelligence, and Expert Systems in the Law, ed. Charles Walter, Quorum, New York, ch. 14, pp. 155-66. ISBN 0899303064.

From the Second Annual Conference on Law and Technology, University of Houston, 24-28 June 1985.

Dick, Judith P. 1991, "Representation of legal text for conceptual retrieval", in Proceedings of the Third International Conference on Artificial Intelligence and Law (ICAIL-91), St Catherine's College, Oxford, 25-28 June, pp. 244-53. ISBN $089791399 \mathrm{X}$.

Douglas, William O. 1948, "The dissent: A safeguard of democracy", Journal of the American Judicature Society, vol. 32, December, pp. 104-7. ISSN 0022-5800.

Drahos, Peter and Parker, Stephen 1992, "Rule following, rule scepticism and indeterminacy in law: A conventional account", Ratio Juris, vol. 5, no. 1, March, pp. 109-19. ISSN 0952-1917.

Dworkin, Ronald 1977, Taking Rights Seriously, Duckworth, London. ISBN 0715607154.

Dworkin, Ronald 1978, "No right answer?", New York University Law Review, vol. 53, no. 1, April, pp. 1-32. ISSN 0028-7881.

Eliot, T.S. 1963, Collected Poems 1909-1962, Faber and Faber, London. ISBN 0571055494.

Everitt, Brian 1974, Cluster Analysis, Social Science Research Council Reviews of Current Research, no. 11, Heinemann Educational Books, London. ISBN 0435822977.

Feigenbaum, E. A. 1981, "Expert systems in the 1980s", in Machine Intelligence, ed. A. H. Bond, Infotech State of the Art Report Series 9, no. 3, Pergamon Infotech, Maidenhead, Berkshire, pp. 219-29. ISBN 008028556 2. ISSN 0734-8487.

Feigenbaum, Edward A. and McCorduck, Pamela 1983, The Fifth Generation: Artificial Intelligence and Japan's Computer Challenge to the World, Addison-Wesley, Reading, Massachusetts. ISBN 0201115190.

Fisher, Ronald A. 1970, Statistical Methods for Research Workers (fourteenth edition), Hafner, New York.

FIX, E. and Hodges, J. L., Jr 1951, Discriminatory Analysis, Non-parametric Discrimination, Project 21-49-004, Report 4, United States Air Force School of Aviation Medicine, Randolph Field, Texas, February.

Frank, Jerome 1930, Law and the Modern Mind, Brentano's, New York.

Frank, Jerome 1949, Courts on Trial: Myth and Reality in American Justice, Princeton University Press, Princeton, New Jersey.

Galsworthy, John 1941, Ten Famous Plays, Duckworth, London. 
Gardner, Anne von der Lieth 1983, "The design of a legal analysis program", in Proceedings of the National Conference on Artificial Intelligence (AAAI-83), Washington, DC, 22-26 August, pp. 114-18. ISBN 0865760659.

Gardner, Anne von der Lieth 1984, An Artificial Intelligence Approach to Legal Reasoning, PhD Thesis STAN-CS-85-1045, Department of Computer Science, Stanford University, Stanford, California, June.

Gardner, Anne v.d. L. 1985, "Overview of an artificial intelligence approach to legal reasoning", in Computing Power and Legal Reasoning, ed. Charles Walter, West Publishing Company, St Paul, ch. 13, pp. 247-74. ISBN 0314955704.

Gardner, Anne von der Lieth 1987, An Artificial Intelligence Approach to Legal Reasoning, Artificial Intelligence and Legal Reasoning Series, MIT Press (Bradford), Cambridge, Massachusetts. ISBN 0262071045.

GAY, John 1967, Fables, William Andrews Clark Memorial Library, University of California, Los Angeles.

Reprint of the 1727 (vol. I) and 1738 (vol. II) editions.

Gilbert, W. S. 1977, The Savoy Operas: Being the Complete Text of the Gilbert and Sullivan Operas as originally produced in the years 1875-1896, Macmillan, London. ISBN 0333069013.

Gold, David I. and Susskind, Richard E. 1986, "Expert systems in law: A jurisprudential and formal specification approach", in Automated Analysis of Legal Texts: Logic, Informatics, Law, eds Antonio A. Martino and Fiorenza Socci Natali, Elsevier Science (North-Holland), Amsterdam, pp. 625-42. ISBN 0444701117.

From the Second International Conference on 'Logic, Informatics, Law', Florence, Italy, September 1985.

Goldman, Seth R., Dyer, Michael G. and Flowers, Margot 1987, "Precedent-based legal reasoning and knowledge acquisition in contract law: A process model", in Proceedings of the First International Conference on Artificial Intelligence and Law (ICAIL-87), Boston, Massachusetts, 27-29 May, pp. 210-21. ISBN 0897912306.

Goldman, Sheldon and Sarat, Austin (eds) 1978, American Court Systems: Readings in Judicial Process and Behavior, W. H. Freeman, San Francisco. ISBN 0716700611.

Greenleaf, G. W., Mowbray, A. S. and Lewis, D. P. 1988, Australasian Computerised Legal Information Handbook, Butterworths, Sydney. ISBN 0409494453.

Greenleaf, Graham, Mowbray, Andrew and Tyree, Alan L. 1987, "Expert systems in law: The DataLex project", in Proceedings of the First International Conference on Artificial Intelligence and Law (ICAIL-87), Boston, Massachusetts, 27-29 May, pp. 9-17. ISBN 0897912306.

Greenleaf, Graham, Mowbray, Andrew and Tyree, Alan 1991, "The DataLex legal workstation - integrating tools for lawyers", in Proceedings of the Third International Conference on Artificial Intelligence and Law (ICAIL-91), St Catherine's College, Oxford, 25-28 June, pp. 215-24. ISBN 089791399 X. 
Grunbaum, W.F. 1988, [Review of Gardner 1987], Computing Reviews, vol. 29, no. 12, December, p. 639. ISSN 0010-4884.

Haar, Charles M., Sawyer, John P., Jr and Cummings, Stephen J. 1977, "Computer power and legal reasoning: A case study of judicial decision prediction in zoning amendment cases", American Bar Foundation Research Journal, no. 3, Summer, pp. 651-768. ISSN 0361-9486.

HAfner, Carole D. 1981, "Representation of knowledge in a legal information retrieval system", in Information Retrieval Research, eds R. N. Oddy, S. E. Robertson, C. J. van Rijsbergen and P. W. Williams, Butterworths, London, ch. 9, pp. 139-53. ISBN 0408107758.

From a symposium "Research and Development in Information Retrieval", St John's College, Cambridge, June 1980.

HAFner, Carole D. 1987, "Conceptual organization of case law knowledge bases", in Proceedings of the First International Conference on Artificial Intelligence and Law (ICAIL-87), Boston, Massachusetts, 27-29 May, pp. 35-42. ISBN 0897912306.

Harris, J. W. 1980, Legal Philosophies, Butterworths, London. ISBN 0406593612.

Hart, H. L. A. 1961, The Concept of Law, Clarendon Law Series, Oxford University Press (Clarendon), London.

Hart, H. L. A. 1983, Essays in Jurisprudence and Philosophy, Oxford University Press (Clarendon), London. ISBN 0198253877.

Hayes-Roth, Frederick, Waterman, Donald A. and Lenat, Douglas B. (eds) 1983, Building Expert Systems, Teknowledge Series in Knowledge Engineering, AddisonWesley, Reading, Massachusetts. ISBN 0201106868.

Hepple, Bob 1986, "Restructuring employment rights", The Industrial Law Journal, vol. 15, pp. 69-83. ISBN 0421373407.

HeRman, Theodor 1980, "WESTLAW: Computerized legal research program of West Publishing Company", in Legal and Legislative Information Processing, ed. Beth Krevitt Eres, Greenwood Press, Westport, Connecticut, ch. 10, pp. 157-61. ISBN 0313213437.

Holmes, O. W. 1897, "The path of the law", Harvard Law Review, vol. 10, no. 8, 25 March, pp. 457-78. ISSN 0017-811X.

Johnson, Peter and MeAD, David 1991, "Legislative knowledge base systems for public administration-Some practical issues", in Proceedings of the Third International Conference on Artificial Intelligence and Law (ICAIL-91), St Catherine's College, Oxford, 25-28 June, pp. 108-17. ISBN 089791399 X.

Johnson, Samuel 1823, The Works of Samuel Johnson, vol. III, Rivington et al., London.

Jones, R. 1988, [Review of Gardner 1987], Computers and Law, no. 56, June, p. 32. ISSN 0140-3249.

KaYton, Irving 1964, "Can jurimetrics be of value to jurisprudence?", The George Washington Law Review, vol. 33, no. 1, October, pp. 287-317. ISSN 0016-8076. 
Kelman, Mark 1987, A Guide to Critical Legal Studies, Harvard University Press, Cambridge, Massachusetts. ISBN 0674367553.

Kelsen, Hans 1945, General Theory of Law and State, 20th Century Legal Philosophy Series, vol. I, Russell and Russell, New York. ISBN 0846202158.

Kelsen, Hans 1967, Pure Theory of Law, University of California Press, Berkeley. Translation of Reine Rechtslehre (1960, second revised edition). Translated by Max Knight.

Kernighan, Brian W. and Ritchie, Dennis M. 1988, The C Programming Language (second edition), Prentice Hall, Englewood Cliffs, New Jersey. ISBN 0131103628.

Knuth, Donald E. 1984, The TEXbook, Addison-Wesley, Reading, Massachusetts. ISBN 0201134489.

KorT, Fred 1963a, "Content analysis of judicial opinions and rules of law", in Judicial Decision-Making, ed. Glendon Schubert, International Yearbook of Political Behavior Research, vol. 4, Free Press of Glencoe, New York, ch. vi, pp. 133-97.

Kort, Fred 1963b, "Simultaneous equations and boolean algebra in the analysis of judicial decisions", Law and Contemporary Problems, vol. 28, no. 1, Winter, pp. 14363. ISSN 0023-9186.

Republished in Baade 1963.

KowALSKI, Andrzej 1991, "Case-based reasoning and the deep structure approach to knowledge representation", in Proceedings of the Third International Conference on Artificial Intelligence and Law (ICAIL-91), St Catherine's College, Oxford, 25-28 June, pp. 21-30. ISBN 089791399 X.

KowALSKI, Robert 1989, "The treatment of negation in logic programs for representing legislation", in Proceedings of the Second International Conference on Artificial Intelligence and Law (ICAIL-89), University of British Columbia, Vancouver, 13-16 June, pp. 11-15. ISBN 0897913221.

Kowalski, Robert and Sergot, Marek 1985, "Computer representation of the law", in Proceedings of the Ninth International Joint Conference on Artificial Intelligence (IJCAI-85), Los Angeles, California, 18-23 August, vol. 2, pp. 1269-70. ISBN 093461302 8. ISSN 1045-0823.

Kowalski, Robert and Sergot, Marek 1990, "The use of logical models in legal problem solving", Ratio Juris, vol. 3, no. 2, July, pp. 201-18. ISSN 0952-1917.

KrIPKe, Saul A. 1982, Wittgenstein on Rules and Private Language: An Elementary Exposition, Basil Blackwell, Oxford. ISBN 0631130772.

Krygier, Martin 1986, "Julius Stone: Leeways of choice, legal tradition and the declaratory theory of law", University of New South Wales Law Journal, vol. 9, no. 2, pp. 26-38. ISSN 0313-0096.

Lambert, Kenneth A. and Grunewald, Mark H. 1989, "LESTER: Using paradigm cases in a quasi-precedential legal domain", in Proceedings of the Second International Conference on Artificial Intelligence and Law (ICAIL-89), University of British Columbia, Vancouver, 13-16 June, pp. 87-92. ISBN 0897913221. 
Lambert, Kenneth A. and Grunewald, Mark H. 1991, "Legal theory and case-based reasoners: The importance of context and the process of focusing", in Proceedings of the Third International Conference on Artificial Intelligence and Law (ICAIL-91), St Catherine's College, Oxford, 25-28 June, pp. 191-5. ISBN 089791399 X.

LAmport, Leslie 1986, LAT $_{E} X$ : A Document Preparation System, Addison-Wesley, Reading, Massachusetts. ISBN $020115790 \mathrm{X}$.

Lasswell, Harold D. 1955, "Current studies of the decision process: Automation versus creativity", The Western Political Quarterly, vol. 8, no. 3, September, pp. 381-99. ISSN 0043-4078.

LAWLOR, Reed C. 1963, "What computers can do: Analysis and prediction of judicial decisions", American Bar Association Journal, vol. 49, no. 4, April, pp. 337-44. ISSN 0002-7596.

Lawlor, Reed C. 1968, "Personal stare decisis", Southern California Law Review, vol. 41, pp. 73-118. ISSN 0038-3910.

LAWLOR, Reed C. 1972, "Fact content of cases and precedent-A modern theory of precedent", Jurimetrics Journal, vol. 12, no. 4, June, pp. 245-70. ISSN 0022-6793.

Excerpts from a paper presented at the September 1971 Annual Meeting of the American Political Science Association.

LAWLOR, Reed C. 1981, "Analysis and prediction of judicial decisions", in Computers and the Law: An Introductory Handbook (third edition), ed. Robert P. Bigelow, American Bar Association, pp. 81-4.

LeITH, Philip 1985a, "An IKBS implementation", Software-Practice and Experience, vol. 15, no. 1, January, pp. 65-86. ISSN 0038-0644.

LeITh, Philip 1985b, Law and Computer Program: The Limits to Logic, Paper, Department of Jurisprudence, Queen's University, Belfast, March.

LEITH, Philip 1986a, "Clear rules and legal expert systems", in Automated Analysis of Legal Texts: Logic, Informatics, Law, eds Antonio A. Martino and Fiorenza Socci Natali, Elsevier Science (North-Holland), Amsterdam, pp. 661-79.

ISBN 0444701117.

From the Second International Conference on 'Logic, Informatics, Law', Florence, Italy, September 1985.

Leith, Philip 1986b, [Review of Michie and Johnston 1984, and Yazdani and Narayanan 1984], Northern Ireland Legal Quarterly, vol. 37, no. 1, Spring, pp. 97-100. ISSN 0029-3105.

LeITH, Philip 1987, "The Emperor's new expert system", The Modern Law Review, vol. 50, no. 1, January, pp. 128-32. ISSN 0026-7961.

Levi, Edward H. 1949, An Introduction to Legal Reasoning, University of Chicago Press (Phoenix), Chicago.

Llewellyn, Karl N. 1931, "Some realism about realism-Responding to Dean Pound", Harvard Law Review, vol. 44, no. 8, June, pp. 1222-64. ISSN 0017-811X. 
Llewellyn, K. N. 1951, The Bramble Bush: On Our Law and its Study, Oceana Publications, New York.

Llewellyn, Karl N. 1962, Jurisprudence: Realism in Theory and Practice, University of Chicago Press, Chicago.

Loevinger, Lee 1949, "Jurimetrics: The next step forward", Minnesota Law Review, vol. 33, no. 5, April, pp. 455-93. ISSN 0026-5535.

Loevinger, Lee 1961, "Jurimetrics: Science and prediction in the field of law", Minnesota Law Review, vol. 46, no. 2, December, pp. 255-75. ISSN 0026-5535.

Loevinger, Lee 1963, "Jurimetrics: The methodology of legal inquiry", Law and Contemporary Problems, vol. 28, no. 1, Winter, pp. 5-35. ISSN 0023-9186.

Republished in Baade 1963.

Loevinger, Lee 1964, "Jurimetrics", in Judicial Behavior: A Reader in Theory and Research, ed. Glendon Schubert, Rand McNally Political Science Series, Rand McNally and Company, Chicago, ch. I.C.7, pp. 72-6.

Extracts from Loevinger 1949.

LuCAS, E. V. (ed.) 1935, The Letters of Charles Lamb to which are added those of his sister Mary Lamb, vol. III, Dent and Methuen, London.

MCCARTY, L. Thorne 1977, "Reflections on TAXMAN: An experiment in artificial intelligence and legal reasoning", Harvard Law Review, vol. 90, no. 5, March, pp. 83793. ISSN 0017-811X.

McCarty, L. Thorne 1980a, Some Requirements for a Computer-Based Legal Consultant, Technical Report LRP-TR-8, Laboratory for Computer Science Research, Rutgers University, New Brunswick, New Jersey, 1 July.

Published, with minor abbreviations, as McCarty 1980b.

MCCARTy, L. Thorne 1980b, "Some requirements for a computer-based legal consultant", in Proceedings of the First Annual National Conference on Artificial Intelligence (AAAI-80), Stanford University, 18-21 August, pp. 298-300.

MCCARTY, L. Thorne 1980c, "The TAXMAN project: Towards a cognitive theory of legal argument", in Computer Science and Law, ed. Bryan Niblett, Cambridge University Press, Cambridge, ch. 3, pp. 23-43. ISBN 0521234514.

MCCARTy, L. Thorne 1982, "A computational theory of Eisner v. Macomber", in Artificial Intelligence and Legal Information Systems, ed. Constantino Ciampi, vol. I, North-Holland, Amsterdam, pp. 329-55. ISBN 044486414 8, 044486413 X (set). From the International Conference on "Logic, Informatics, Law", Florence, Italy, April 1981.

MCCARTy, L. Thorne 1983, "Intelligent legal information systems: Problems and prospects", Rutgers Computer and Technology Law Journal, vol. 9, no. 2, pp. 265-94. ISSN 0735-8938.

Republished as McCarty 1984.

MCCARTy, L. Thorne 1984, "Intelligent legal information systems: Problems and prospects", in Data Processing and the Law, ed. Colin Campbell, Sweet and Maxwell, London, pp. 125-51. ISBN 0421320001. 
MCCARTy, L. Thorne 1986, "Permissions and obligations: An informal introduction", in Automated Analysis of Legal Texts: Logic, Informatics, Law, eds Antonio A. Martino and Fiorenza Socci Natali, Elsevier Science (North-Holland), Amsterdam, pp. 307-37. ISBN 0444701117.

From the Second International Conference on 'Logic, Informatics, Law', Florence, Italy, September 1985.

MCCARTy, L. Thorne 1989, "A language for legal discourse: I. Basic features", in Proceedings of the Second International Conference on Artificial Intelligence and Law (ICAIL-89), University of British Columbia, Vancouver, 13-16 June, pp. 1809. ISBN 0897913221.

McCARTy, L. Thorne 1990, "Artificial intelligence and law: How to get there from here", Ratio Juris, vol. 3, no. 2, July, pp. 189-200. ISSN 0952-1917.

MCCARTY, L. Thorne 1991, "On the role of prototypes in appellate legal argument (abstract)", in Proceedings of the Third International Conference on Artificial Intelligence and Law (ICAIL-91), St Catherine's College, Oxford, 25-28 June, pp. 18590. ISBN $089791399 \mathrm{X}$.

MCCarty, L. Thorne and SRidharan, N. S. 1981, "The representation of an evolving system of legal concepts: II. Prototypes and deformations", in Proceedings of the Seventh International Joint Conference on Artificial Intelligence (IJCAI-81), University of British Columbia, Vancouver, British Columbia, Canada, 24-28 August, vol. I, pp. 246-53. ISBN 086576059 4. ISSN 1045-0823.

MacCormick, Neil 1978, Legal Reasoning and Legal Theory, Clarendon Law Series, Oxford University Press (Clarendon), Oxford. ISBN 0198760809.

MackaAy, Ejan and Robillard, Pierre 1974, "Predicting judicial decisions: The nearest neighbour rule and visual representation of case patterns", Datenverarbeitung im Recht, vol. 3, no. 3/4, November, pp. 302-31. ISSN 0301-2980.

McMillan, John 1991, "Developments under the ADJR Act: The grounds of review", Federal Law Review, vol. 20, no. 1, pp. 50-82. ISSN 0067-205X.

Revised version of a paper presented at the Conference "Ten Years of the Federal Administrative Decisions (Judicial Review) Act", Canberra, September 1990.

Maggs, Peter B. and DeBessonet, Cary G. 1972, "Automated logical analysis of systems of legal rules", Jurimetrics Journal, vol. 12, no. 3, March, pp. 158-69. ISSN 0022-6793.

MeHL, Lucien 1959, "Automation in the legal world: From the machine processing of legal information to the "law machine", in Mechanisation of Thought Processes, vol. II, Her Majesty's Stationery Office, London, pp. 755-87.

From a symposium held at the National Physical Laboratory, 24-27 November 1958.

Meldman, Jeffrey A. 1977, "A structural model for computer-aided legal analysis", Rutgers Journal of Computers and the Law, vol. 6, no. 1, pp. 27-71. ISSN 0735-8938. 
Mendelson, Simon 1989, "An attempted dimensional analysis of the law governing government appeals in criminal cases", in Proceedings of the Second International Conference on Artificial Intelligence and Law (ICAIL-89), University of British Columbia, Vancouver, 13-16 June, pp. 128-37. ISBN 0897913221.

Michaelsen, Robert and Michie, Donald 1983, "Expert systems in business", Datamation, vol. 29, no. 11, November, pp. 240-6. ISSN 0011-6963.

Michie, Donald and Johnston, Rory 1984, The Creative Computer: Machine Intelligence and Human Knowledge, Penguin (Viking), Harmondsworth. ISBN 0670800600 .

Moles, Robert N. 1987, Definition and Rule in Legal Theory: A Reassessment of H. L. A. Hart and the Positivist Tradition, Basil Blackwell, Oxford. ISBN $063115342 \mathrm{X}$.

Moles, Robert N. 1991, "Logic programming-An assessment of its potential for artificial intelligence applications in law", Journal of Law and Information Science, vol. 2, no. 2, pp. 137-64. ISSN 0729-1485.

Moles, Robert N. and Dayal, Surendra 1992, "There is more to life than logic", Journal of Law and Information Science, vol. 3, no. 2, pp. 188-218. ISSN 0729-1485.

Montesquieu 1973, De l'Esprit des lois, vol. I, Garnier Frères, Paris. First published in 1748 .

Morris, Gwen, Cook, Catriona, Creyke, Robin and Geddes, Robert 1992, Laying Down the Law: The Foundations of Legal Reasoning, Research and Writing in Australia and New Zealand (third edition), Butterworths, Sydney. ISBN 0409305413.

Muir, Frank 1976, The Frank Muir Book: An Irreverent Companion to Social History, Heinemann, London. ISBN 0434481505.

Murphy, Lionel 1980, "The responsibility of judges", in Law, Politics and the Labor Movement, ed. Gareth Evans, Legal Service Bulletin, Law Faculty, Monash University, Clayton, ch. 1, pp. 2-10. ISBN 0959472703.

NAGEL, Stuart 1960, "Using simple calculations to predict judicial decisions", American Behavioral Scientist, vol. 4, no. 4, December, pp. 24-8. ISSN 0002-7642.

NAGEL, Stuart S. 1963, "Off-the-bench judicial attitudes", in Judicial Decision-Making, ed. Glendon Schubert, International Yearbook of Political Behavior Research, vol. 4, Free Press of Glencoe, New York, ch. ii, pp. 29-53.

NAGEL, Stuart S. 1986, "Microcomputers and judicial prediction", in Automated Analysis of Legal Texts: Logic, Informatics, Law, eds Antonio A. Martino and Fiorenza Socci Natali, Elsevier Science (North-Holland), Amsterdam, pp. 681-702. ISBN 0444701117.

From the Second International Conference on 'Logic, Informatics, Law', Florence, Italy, September 1985.

Nagel, Stuart S. 1989, Decision-Aiding Software and Legal Decision-Making: A Guide to Skills and Applications Throughout the Law, Quorum, New York. ISBN $089930382 \mathrm{X}$. 
Neter, John, Wasserman, William and Whitmore, G. A. 1982, Applied Statistics (second edition), Allyn and Bacon, Boston. ISBN 0205076130.

Niblett, Bryan 1981, "Expert systems for lawyers", Computers and Law, no. 29, August, pp. 2-4. ISSN 0140-3249.

Niblett, Bryan 1988, [Review of Susskind 1987a], Computers and Law, no. 56, June, pp. 32-3. ISSN 0140-3249.

OsKamp, A. 1989, "Knowledge representation and legal expert systems", in Advanced Topics of Law and Information Technology, ed. G. P. V. Vandenberghe, Computer/Law Series, no. 3, Kluwer Law and Taxation Publishers, Deventer, pp. 195211. ISBN 9065443916.

Oskamp, A., Walker, R.F., Schrickx, J.A. and van den Berg, P.H. 1989, "PROLEXS, divide and rule: a legal application", in Proceedings of the Second International Conference on Artificial Intelligence and Law (ICAIL-89), University of British Columbia, Vancouver, 13-16 June, pp. 54-62. ISBN 0897913221.

Pattison, Michael and Ciesielski, Victor B. 1990, Using Expert Systems and Conceptual Graphs to Review Legal Contracts, Technical Report 22, Key Centre for Knowledge Based Systems, Departments of Computer Science, Royal Melbourne Institute of Technology and The University of Melbourne, 10 May.

Pearce, Dennis, Campbell, Enid and Harding, Don 1987, Australian Law Schools: A Discipline Assessment for the Commonwealth Tertiary Education Commission, vol. 1, Australian Government Publishing Service, Canberra. ISBN 064406182 0, 0644061863 (set).

Pearce, D. C. and Geddes, R. S. 1988, Statutory Interpretation in Australia (third edition), Butterworths, Sydney. ISBN 0409495255.

Plater, Alan 1985, The Beiderbecke Affair, Methuen, London. ISBN 0413577503.

Popp, Walter G. and Schlink, Bernhard 1975, "JUDITH, a computer program to advise lawyers in reasoning a case", Jurimetrics Journal, vol. 15, no. 4, Summer, pp. 303-14. ISSN 0022-6793.

Popple, James 1990a, "Legal expert systems: The inadequacy of a rule-based approach", in Proceedings of the Thirteenth Australian Computer Science Conference (ACSC-13), Monash University, Melbourne, 7-9 February, pp. 303-13. ISSN 0157-3055.

Australian Computer Science Communications, vol. 12, no. 1.

Popple, James 1990b, "Legal expert systems: The inadequacy of a rule-based approach", in Advances in Computing and Information: Proceedings of the International Conference on Computing and Information (ICCI-90), eds Selim G. Akl, Frank Fiala and Waldemar W. Koczkodaj, Niagara Falls, Canada, 23-26 May, Canadian Scholars' Press, Toronto, pp. 348-51. ISBN 092162770 X.

Popple, James 1991, "Legal expert systems: The inadequacy of a rule-based approach", The Australian Computer Journal, vol. 23, no. 1, February, pp. 11-16. ISSN 0004-8917. 
Popple, James 1993, SHYSTER: The Program, Technical Report TR-CS-93-13, Department of Computer Science, Faculty of Engineering and Information Technology, The Australian National University, Canberra, December.

Pound, Roscoe 1908, "Mechanical jurisprudence", Columbia Law Review, vol. 8, no. 8, December, pp. 605-23. ISSN 0010-1958.

Quinlan, J.R. 1986, "Induction of decision trees", Machine Learning, vol. 1, no. 1, pp. 81-106. ISSN 0885-6125.

Quinlan, J. R. 1988, "Induction, knowledge and expert systems", in Artificial Intelligence Developments and Applications, eds John S. Gero and Robin Stanton, Elsevier Science (North-Holland), Amsterdam, ch. 17, pp. 253-71. ISBN 0444704655.

From the Australian Joint Artificial Intelligence Conference, Sydney, Australia, 2-4 November 1987.

Quinlan, J.R., Compton, P. J., Horn, K.A. and Lazarus, L. 1987, "Inductive knowledge acquisition: a case study", in Applications of Expert Systems, ed. J. Ross Quinlan, Addison-Wesley, Sydney, ch. 9, pp. 157-73. ISBN 0201174499.

Based on the proceedings of the Second Australian Conference on Applications of Expert Systems, May 1986.

Rawson, Hugh 1991, A Dictionary of Invective: A Treasury of Curses, Insults, PutDowns, and Other Formerly Unprintable Terms from Anglo-Saxon Times to the Present, Robert Hale, London. ISBN 0709043996.

Ricketson, Staniforth 1984, The Law of Intellectual Property, Law Book Company, Sydney. ISBN 0455205531.

Rissland, Edwina L. 1983, "Examples in legal reasoning: Legal hypotheticals", in Proceedings of the Eighth International Joint Conference on Artificial Intelligence (IJCAI-83), Karlsruhe, West Germany, 8-12 August, vol. 1, pp. 90-3. ISBN 086576064 0. ISSN 1045-0823.

Rissland, Edwina L. 1985, "Argument moves and hypotheticals", in Computing Power and Legal Reasoning, ed. Charles Walter, West Publishing Company, St Paul, ch. 7, pp. 129-43. ISBN 0314955704.

Rissland, Edwina L. 1989, "Dimension-based analysis of hypotheticals from Supreme Court oral argument", in Proceedings of the Second International Conference on Artificial Intelligence and Law (ICAIL-89), University of British Columbia, Vancouver, 13-16 June, pp. 111-20. ISBN 0897913221.

Rissland, Edwina L. 1990, "Artificial intelligence and law: Stepping stones to a model of legal reasoning", The Yale Law Journal, vol. 99, no. 8, June, pp. 195781. ISSN 0044-0094.

Rissland, Edwina L. and Ashley, Kevin D. 1987, "A case-based system for trade secrets law", in Proceedings of the First International Conference on Artificial Intelligence and Law (ICAIL-87), Boston, Massachusetts, 27-29 May, pp. 60-6. ISBN 0897912306. 
Rissland, Edwina L. and Ashley, Kevin D. 1989, "HYPO: A precedent-based legal reasoner", in Advanced Topics of Law and Information Technology, ed. G.P.V. Vandenberghe, Computer/Law Series, no. 3, Kluwer Law and Taxation Publishers, Deventer, pp. 213-34. ISBN 9065443916.

Rissland, Edwina L. and Skalak, David B. 1989a, "Interpreting statutory predicates", in Proceedings of the Second International Conference on Artificial Intelligence and Law (ICAIL-89), University of British Columbia, Vancouver, 13-16 June, pp. 46-53. ISBN 0897913221.

Rissland, Edwina L. and Skalak, David B. 1989b, "Combining case-based and rulebased reasoning: A heuristic approach", in Proceedings of the Eleventh International Joint Conference on Artificial Intelligence (IJCAI-89), Detroit, Michigan, 20-25 August, vol. 1, pp. 524-30. ISBN 155860094 9. ISSN 1045-0823.

Rissland, Edwina L., Valcarce, Eduardo M. and Ashley, Kevin D. 1984, "Explaining and arguing with examples", in Proceedings of the National Conference on Artificial Intelligence (AAAI-84), University of Texas at Austin, 6-10 August, pp. 288-94. ISBN 0865760802.

SANDERS, Kathryn E. 1991, "Representing and reasoning about open-textured predicates", in Proceedings of the Third International Conference on Artificial Intelligence and Law (ICAIL-91), St Catherine's College, Oxford, 25-28 June, pp. 137-44. ISBN $089791399 \mathrm{X}$.

SCHMIDT, Frank L. 1971, "The relative efficiency of regression and simple unit predictor weights in applied differential psychology", Educational and Psychological Measurement, vol. 31, no. 3, Autumn, pp. 699-714. ISSN 0013-1644.

SCHUBert, Glendon 1963a, "Civilian control and stare decisis in the Warren Court", in Judicial Decision-Making, ed. Glendon Schubert, International Yearbook of Political Behavior Research, vol. 4, Free Press of Glencoe, New York, ch. iii, pp. 55-77.

SchUBeRT, Glendon 1963b, "Judicial attitudes and voting behavior: The 1961 term of the United States Supreme Court", Law and Contemporary Problems, vol. 28, no. 1, Winter, pp. 100-42. ISSN 0023-9186.

Republished in Baade 1963.

SCHUBERT, Glendon 1968, "The importance of computer technology to political science research in judicial behavior", Jurimetrics Journal, vol. 8, no. 3, March, pp. 56-63. ISSN 0022-6793.

Schubert, Glendon 1975, Human Jurisprudence: Public Law as Political Science, University Press of Hawaii, Honolulu. ISBN 0824802942.

Sergot, Marek, Cory, Therese, Hammond, Peter, Kowalski, Robert, Kriwaczek, Frank and SADRI, Fariba 1986a, "Formalisation of the British Nationality Act", in Yearbook of Law Computers and Technology, ed. Christopher Arnold, vol. 2, Butterworths, London, pp. 40-52. ISBN 0406187010.

Sergot, M.J., Sadri, F., Kowalski, R.A., Kriwaczek, F., Hammond, P. and Cory, H. T. 1986b, "The British Nationality Act as a logic program", Communications of the ACM, vol. 29, no. 5, May, pp. 370-86. ISSN 0001-0782. 
Shannon, David T. and Golshani, Forouzan 1988, "On the automation of legal reasoning", Jurimetrics Journal, vol. 28, no. 3, Spring, pp. 305-15. ISSN 0897-1277.

Sherman, David M. 1987, "A PROLOG model of the Income Tax Act of Canada", in Proceedings of the First International Conference on Artificial Intelligence and Law (ICAIL-87), Boston, Massachusetts, 27-29 May, pp. 127-36. ISBN 0897912306.

Sherman, David M. 1989, "Expert systems and ICAI in tax law: Killing two birds with one AI stone", in Proceedings of the Second International Conference on Artificial Intelligence and Law (ICAIL-89), University of British Columbia, Vancouver, 13-16 June, pp. 74-80. ISBN 0897913221.

Simpson, Brian 1986, "The common law and legal theory", in Legal Theory and Common Law, ed. William Twining, Basil Blackwell, Oxford, ch. 2, pp. 8-25. ISBN 0631144773.

Skalak, David B. 1989, "Taking advantage of models for legal classification", in Proceedings of the Second International Conference on Artificial Intelligence and Law (ICAIL-89), University of British Columbia, Vancouver, 13-16 June, pp. 234-41. ISBN 0897913221.

Skalak, David B. and Rissland, Edwina L. 1991, "Argument moves in a rule-guided domain", in Proceedings of the Third International Conference on Artificial Intelligence and Law (ICAIL-91), St Catherine's College, Oxford, 25-28 June, pp. 1-11. ISBN $089791399 \mathrm{X}$.

Skalak, David B. and Rissland, Edwina L. 1992, "Arguments and cases: An inevitable intertwining", Artificial Intelligence and Law, vol. 1, no. 1, pp. 3-44. ISSN 0924-8463.

Sмith, Ian 1992, "Employment", in Halsbury's Laws of England (fourth edition reissue), ed. Lord Hailsham of St Marylebone, vol. 16, Butterworths, London. ISBN 040603465 5, 0406034001 (set).

Sneath, Peter H. A. and Sokal, Robert R. 1973, Numerical Taxonomy: The Principles and Practice of Numerical Classification, W. H. Freeman, San Francisco. ISBN 0716706970.

SpAeth, Harold J. 1963, "Warren Court attitudes toward business: The 'B' scale", in Judicial Decision-Making, ed. Glendon Schubert, International Yearbook of Political Behavior Research, vol. 4, Free Press of Glencoe, New York, ch. iv, pp. 79-108.

Spengler, Joseph J. 1963, "Machine-made justice: Some implications", Law and Contemporary Problems, vol. 28, no. 1, Winter, pp. 36-52. ISSN 0023-9186.

Republished in Baade 1963.

Sprowl, James A. 1979, "Automating the legal reasoning process: A computer that uses regulations and statutes to draft legal documents", American Bar Foundation Research Journal, no. 1, Winter, pp. 1-81. ISSN 0361-9486.

StAmper, Ronald 1980, "LEGOL: Modelling legal rules by computer", in Computer Science and Law, ed. Bryan Niblett, Cambridge University Press, Cambridge, ch. 4, pp. 45-71. ISBN 0521234514. 
StAmper, Ronald 1986, "A non-classical logic for based on the structures of behaviour [sic]", in Automated Analysis of Legal Texts: Logic, Informatics, Law, eds Antonio A. Martino and Fiorenza Socci Natali, Elsevier Science (North-Holland), Amsterdam, pp. 115-39. ISBN 0444701117.

From the Second International Conference on 'Logic, Informatics, Law', Florence, Italy, September 1985.

Stamper, Ronald, Tagg, Clare, Mason, Peter, Cook, Sandra and Marks, Jo 1982, "Developing the LEGOL semantic grammar", in Artificial Intelligence and Legal Information Systems, ed. Constantino Ciampi, vol. I, North-Holland, Amsterdam, pp. 357-79. ISBN 044486414 8, $044486413 \mathrm{X}$ (set).

From the International Conference on "Logic, Informatics, Law", Florence, Italy, April 1981.

StAnLEY, Karlyn D. 1980, "LEXIS: Legal research and litigation support", in Legal and Legislative Information Processing, ed. Beth Krevitt Eres, Greenwood Press, Westport, Connecticut, ch. 9, pp. 149-56. ISBN 0313213437.

Stone, Julius 1964a, Legal System and Lawyers' Reasonings, Maitland, Sydney.

Stone, Julius 1964b, "Man and machine in the search for justice", Stanford Law Review, vol. 16, May, pp. 515-60. ISSN 0038-9765.

Stone, Julius 1966, Social Dimensions of Law and Justice, Maitland, Sydney.

Stone, Julius 1985, Precedent and Law: Dynamics of Common Law Growth, Butterworths, Sydney. ISBN 040949304 X.

Susskind, Richard E. 1986, "Expert systems in law: A jurisprudential approach to artificial intelligence and legal reasoning", The Modern Law Review, vol. 49, no. 2, March, pp. 168-94. ISSN 0026-7961.

Susskind, Richard E. 1987a, Expert Systems in Law: A Jurisprudential Inquiry, Oxford University Press (Clarendon), Oxford. ISBN 0198255829.

Susskind, Richard E. 1987b, "Expert systems in law: Out of the research laboratory and into the marketplace", in Proceedings of the First International Conference on Artificial Intelligence and Law (ICAIL-87), Boston, Massachusetts, 27-29 May, pp. 1-8. ISBN 0897912306.

SussKIND, Richard 1989a, "Pragmatism and purism in artificial intelligence and legal reasoning", AI and Society, vol. 3, no. 1, January-March, pp. 28-38. ISSN 0951-5666.

Susskind, Richard E. 1989b, "The Latent Damage System: A jurisprudential analysis", in Proceedings of the Second International Conference on Artificial Intelligence and Law (ICAIL-89), University of British Columbia, Vancouver, 13-16 June, pp. 23-32. ISBN 0897913221.

Susskind, Richard E. 1990, [Review of Gardner 1987], in Yearbook of Law Computers and Technology, ed. Kenneth Russell, vol. 4, Butterworths, London, pp. 221-8. ISBN 0406187037.

Tanenhaus, Joseph, Schick, Marvin, Muraskin, Matthew and Rosen, Daniel 1963, "The Supreme Court's certiorari jurisdiction: Cue theory", in Judicial DecisionMaking, ed. Glendon Schubert, International Yearbook of Political Behavior Research, vol. 4, Free Press of Glencoe, New York, ch. v, pp. 111-32. 
TAPPer, Colin 1963, "Lawyers and machines", The Modern Law Review, vol. 26, no. 2, March, pp. 121-37. ISSN 0026-7961.

TAPPER, Colin 1973, Computers and the Law, Law in Context, Weidenfeld and Nicolson, London. ISBN 029776571 X.

TAPPeR, Colin 1984, "An experiment with citation vectors", in Data Processing and the Law, ed. Colin Campbell, Sweet and Maxwell, London, pp. 90-109. ISBN 0421320001.

Thomas, Bart T. 1979, "Unauthorized practice and computer aided legal analysis systems", Jurimetrics Journal, vol. 20, no. 1, Fall, pp. 41-51. ISSN 0897-1277.

Torsun, I.S. 1987, "PAYE: A tax expert system", in Research and Development in Expert Systems III, ed. M. A. Bramer, British Computer Society Workshop Series, Cambridge University Press, Cambridge, pp. 69-80. ISBN 052134145 X.

From Expert Systems '86: the Sixth Annual Technical Conference of the British Computer Society Specialist Group on Expert Systems, Brighton, 15-18 December 1986.

Trudeau, G. B. 1975, The Doonesbury Chronicles, Holt, Rinehart and Winston, New York. ISBN 0030152569.

Turing, A. M. 1950, "Computing machinery and intelligence", Mind, vol. 59, no. 236, October, pp. 433-60. ISSN 0026-4423.

Twiss, Horace 1844, The Public and Private Life of Lord Chancellor Eldon, with Selections from his Correspondence (second edition), vol. III, John Murray, London.

Tyree, Alan L. 1977, "The geometry of case law", Victoria University of Wellington Law Review, vol. 8, pp. 403-20. ISSN 0042-5117.

Tyree, Alan L. 1980, "Can a 'deterministic' computer judge overrule himself?", Rutgers Journal of Computers, Technology and the Law, vol. 7, no. 2, pp. 381-4. ISSN 0735-8938.

Tyree, Alan L. 1981, "Fact content analysis of case law: Methods and limitations", Jurimetrics Journal, vol. 22, no. 1, Fall, pp. 1-33. ISSN 0897-1277.

Tyree, A. 1985, "FINDER: An expert system", in Proceedings of the Fortieth Annual Conference of the Australasian Universities Law Schools Association, University of Adelaide, 26-29 August.

Tyree, Alan 1986, "Expert systems and the law: Will justice fall to bits?", Current Affairs Bulletin, vol. 62, no. 10, March, pp. 13-18. ISSN 0011-3182.

Tyree, Alan 1989, Expert Systems in Law, Prentice Hall, New York. ISBN 0132956500.

Tyree, Alan L. 1992, "The logic programming debate", Journal of Law and Information Science, vol. 3, no. 1, pp. 111-15. ISSN 0729-1485. 
Tyree, Alan L., Greenleaf, Graham and Mowbray, Andrew 1988, "Legal reasoning: the problem of precedent", in Artificial Intelligence Developments and Applications, eds John S. Gero and Robin Stanton, Elsevier Science (North-Holland), Amsterdam, ch. 16, pp. 231-47. ISBN 0444704655.

From the Australian Joint Artificial Intelligence Conference, Sydney, Australia, 2-4 November 1987.

Tyree, Alan L., Greenleaf, Graham and Mo[w]Bray, Andrew 1989, "Generating legal arguments", Knowledge-Based Systems, vol. 2, no. 1, March, pp. 46-51. ISSN 0950-7051.

Ulmer, S. Sidney 1963, "Quantitative analysis of judicial processes: Some practical and theoretical applications", Law and Contemporary Problems, vol. 28, no. 1, Winter, pp. 164-84. ISSN 0023-9186.

Republished in Baade 1963.

Ulmer, S. Sidney 1964, "Homeostasis in the Supreme Court", in Judicial Behavior: A Reader in Theory and Research, ed. Glendon Schubert, Rand McNally Political Science Series, Rand McNally and Company, Chicago, ch. II.B.6, pp. 162-80.

van Opdorp, G. J., Walker, R.F., Schrickx, J. A., Groendijk, C. and van den BERG, P. H. 1991, "Networks at work: A connectionist approach to non-deductive legal reasoning", in Proceedings of the Third International Conference on Artificial Intelligence and Law (ICAIL-91), St Catherine's College, Oxford, 25-28 June, pp. 278-87. ISBN $089791399 \mathrm{X}$.

Vossos, George, Zeleznikow, John, Dillon, Tharam and Vossos, Vivian 1991, "An example of integrating legal case based reasoning with object-oriented rulebased systems: IKBALS II", in Proceedings of the Third International Conference on Artificial Intelligence and Law (ICAIL-91), St Catherine's College, Oxford, 2528 June, pp. 31-41. ISBN 089791399 X.

Walker, R. F., Zeinstra, P. G. M. and van Den Berg, P. H. 1989, "A model to model knowledge about knowledge or implementing meta-knowledge in PROLEXS", in $A d$ vanced Topics of Law and Information Technology, ed. G. P. V. Vandenberghe, Computer/Law Series, no. 3, Kluwer Law and Taxation Publishers, Deventer, pp. 235-60. ISBN 9065443916.

Waterman, Donald A., Paul, Jody and Peterson, Mark 1987, "Expert systems for legal decision making", in Applications of Expert Systems, ed. J. Ross Quinlan, Addison-Wesley, Sydney, ch. 2, pp. 23-47. ISBN 0201174499.

Based on the proceedings of the Second Australian Conference on Applications of Expert Systems, May 1986.

Waterman, D. A. and Peterson, Mark 1980, "Rule-based models of legal expertise", in Proceedings of the First Annual National Conference on Artificial Intelligence (AAAI-80), Stanford University, 18-21 August, pp. 272-5.

Weizenbaum, Joseph 1976, Computer Power and Human Reason: From Judgment to Calculation, W.H. Freeman, San Francisco. ISBN 0716704641. 
Wells, Stanley and TAYlor, Gary (eds) 1986, William Shakespeare: The Complete Works (original-spelling edition), Oxford University Press (Clarendon), Oxford. ISBN 019812919 X.

Wells, Stanley and TaYlor, Gary 1987, William Shakespeare: A Textual Companion, Oxford University Press (Clarendon), Oxford. ISBN 0198129149.

WiEneR, Frederick Bernays 1962, "Decision prediction by computers: Nonsense cubed - and worse", American Bar Association Journal, vol. 48, no. 11, November, pp. 1023-8. ISSN 0002-7596.

Williams, W. T. 1971, "Principles of clustering", Annual Review of Ecology and Systematics, vol. 2, pp. 303-26. ISSN 0066-4162.

WILLICK, Marshal S. 1985, "Professional malpractice and the unauthorized practice of professions: Some legal and ethical aspects of the use of computers as decision-aids", in Computing Power and Legal Reasoning, ed. Charles Walter, West Publishing Company, St Paul, ch. 28, pp. 817-63. ISBN 0314955704.

WIRTH, Niklaus 1977, "What can we do about the unnecessary diversity of notation for syntactic definitions?", Communications of the ACM, vol. 20, no. 11, November, pp. 822-3. ISSN 0001-0782.

Wittgenstein, Ludwig 1974, Philosophical Investigations (third edition), Basil Blackwell, Oxford. ISBN 0631146709.

Translation of Philosophische Untersuchungen (1953). Translated by G. E. M. Anscombe.

Wolstenholme, David E. 1989, "Amalgamating regulation- and case-based advice systems through suggested answers", in Proceedings of the Second International Conference on Artificial Intelligence and Law (ICAIL-89), University of British Columbia, Vancouver, 13-16 June, pp. 63-7. ISBN 0897913221.

YABlon, Charles M. 1987, "Law and metaphysics", The Yale Law Journal, vol. 96, no. 3, January, pp. 613-36. ISSN 0044-0094.

Review of Kripke 1982.

YaZdani, Masoud and Narayanan, Ajit (eds) 1984, Artificial Intelligence: Human Effects, Ellis Horwood Series in Artificial Intelligence, Ellis Horwood, Chichester. ISBN 085312577 5, 0470200928. 